\title{
Linear Collider Physics
}

Resource Book

\section{for}

Snowmass 2001

American Linear Collider

Working Group 


\title{
BNL-52627, CLNS 01/1729, FERMILAB-Pub-01/058-E, LBNL-47813, SLAC-R-570, UCRL-ID-143810-DR \\ $\mathrm{UC}-414$
}

June 2001

\begin{abstract}
This document, and the material and data contained therein, was developed under sponsorship of the United States Government. Neither the United States nor the Department of Energy, nor the Leland Stanford Junior University, nor their employees, nor their respective contractors, subcontractors, or their employees, makes any warranty, express or implied, or assumes any liability of responsibility for accuracy, completeness or usefulness of any information, apparatus, product or process disclosed, or represents that its use will not infringe privately owned rights. Mention of any product, its manufacturer, or suppliers shall not, nor is intended to imply approval, disapproval, or fitness for any particular use. A royalty-free, nonexclusive right to use and disseminate same for any purpose whatsoever, is expressly reserved to the United States and the University.
\end{abstract}

Cover: Events of $e^{+} e^{-} \rightarrow Z^{0} h^{0}$, simulated with the Large linear collider detector described in Chapter 15. Front cover: $h^{0} \rightarrow \tau^{+} \tau^{-}, Z^{0} \rightarrow b \bar{b}$. Back cover: $h^{0} \rightarrow b \bar{b}$, $Z^{0} \rightarrow \mu^{+} \mu^{-}$.

Typset in $\mathrm{LT}_{\mathrm{E}} \mathrm{X}$ by S. Jensen.

Prepared for the Department of Energy under contract number DE-AC03-76SF00515 by Stanford Linear Accelerator Center, Stanford University, Stanford, California. Printed in the United State of America. Available from National Technical Information Services, US Department of Commerce, 5285 Port Royal Road, Springfield, Virginia 22161. 


\section{American Linear Collider Working Group}

T. Abe ${ }^{52}$, N. Arkani-Hamed ${ }^{29}$, D. Asner ${ }^{30}$, H. Baer ${ }^{22}$, J. Bagger ${ }^{26}$, C. Balazs ${ }^{23}$, C. Baltay ${ }^{59}$, T. Barker ${ }^{16}$, T. Barklow ${ }^{52}$, J. Barron ${ }^{16}$, U. Baur ${ }^{38}$, R. Beach ${ }^{30}$

R. Bellwied ${ }^{57}$, I. Bigi ${ }^{41}$, C. Blöchinger ${ }^{58}$, S. Boege ${ }^{47}$, T. Bolton ${ }^{27}$, G. Bower $^{52}$, J. Brau ${ }^{42}$, M. Breidenbach ${ }^{52}$, S. J. Brodsky ${ }^{52}$, D. Burke ${ }^{52}$, P. Burrows ${ }^{43}$, J. N. Butler ${ }^{21}$, D. Chakraborty ${ }^{40}$, H. C. Cheng ${ }^{14}$, M. Chertok ${ }^{6}$, S. Y. Choi ${ }^{15}$, D. Cinabro ${ }^{57}$, G. Corcella ${ }^{50}$, R. K. Cordero ${ }^{16}$, N. Danielson ${ }^{16}$, H. Davoudiasl ${ }^{52}$, S. Dawson ${ }^{4}$, A. Denner ${ }^{44}$, P. Derwent ${ }^{21}$, M. A. Diaz ${ }^{12}$, M. Dima ${ }^{16}$, S. Dittmaier ${ }^{18}$, M. Dixit ${ }^{11}$, L. Dixon ${ }^{52}$, B. Dobrescu ${ }^{59}$, M. A. Doncheski ${ }^{46}$, M. Duckwitz ${ }^{16}$, J. Dunn ${ }^{16}$, J. Early ${ }^{30}$, J. Erler ${ }^{45}$, J. L. Feng ${ }^{35}$, C. Ferretti ${ }^{37}$, H. E. Fisk ${ }^{21}$, H. Fraas ${ }^{58}$, A. Freitas ${ }^{18}$, R. Frey ${ }^{42}$, D. Gerdes ${ }^{37}$, L. Gibbons ${ }^{17}$, R. Godbole ${ }^{24}$, S. Godfrey $^{11}$, E. Goodman ${ }^{16}$, S. Gopalakrishna ${ }^{29}$, N. Graf ${ }^{52}$, P. D. Grannis ${ }^{39}$, J. Gronberg ${ }^{30}$, J. Gunion ${ }^{6}$, H. E. Haber ${ }^{9}$, T. Han ${ }^{55}$, R. Hawkings ${ }^{13}$, C. Hearty ${ }^{3}$, S. Heinemeyer ${ }^{4}$, S. S. Hertzbach ${ }^{34}$, C. Heusch ${ }^{9}$, J. Hewett ${ }^{52}$, K. Hikasa ${ }^{54}$, G. Hiller ${ }^{52}$, A. Hoang ${ }^{36}$, R. Hollebeek ${ }^{45}$, M. Iwasaki ${ }^{42}$, R. Jacobsen ${ }^{29}$, J. Jaros ${ }^{52}$, A. Juste ${ }^{21}$, J. Kadyk ${ }^{29}$, J. Kalinowski ${ }^{57}$, P. Kalyniak ${ }^{11}$, T. Kamon ${ }^{53}$, D. Karlen ${ }^{11}$, L. Keller ${ }^{52}$ D. Koltick ${ }^{48}$, G. Kribs ${ }^{55}$, A. Kronfeld ${ }^{21}$, A. Leike ${ }^{32}$, H. E. Logan ${ }^{21}$, J. Lykken ${ }^{21}$, C. Macesanu ${ }^{50}$,

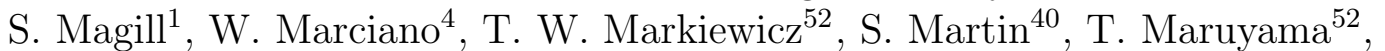

K. Matchev ${ }^{13}$, K. Moenig ${ }^{19}$, H. E. Montgomery ${ }^{21}$, G. Moortgat-Pick ${ }^{18}$, G. Moreau ${ }^{33}$, S. Mrenna ${ }^{6}$, B. Murakami ${ }^{6}$, H. Murayama ${ }^{29}$, U. Nauenberg ${ }^{16}$, H. Neal ${ }^{59}$, B. Newman ${ }^{16}$, M. Nojiri ${ }^{28}$, L. H. Orr ${ }^{50}$, F. Paige ${ }^{4}$, A. Para ${ }^{21}$, S. Pathak ${ }^{45}$, M. E. Peskin ${ }^{52}$, T. Plehn ${ }^{55}$, F. Porter ${ }^{10}$, C. Potter ${ }^{42}$, C. Prescott ${ }^{52}$, D. Rainwater ${ }^{21}$,

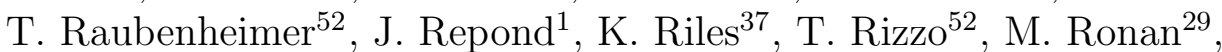
L. Rosenberg ${ }^{35}$, J. Rosner ${ }^{14}$, M. Roth ${ }^{31}$, P. Rowson ${ }^{52}$, B. Schumm ${ }^{9}$, L. Seppala ${ }^{30}$,

A. Seryi ${ }^{52}$, J. Siegrist ${ }^{29}$, N. Sinev ${ }^{42}$, K. Skulina ${ }^{30}$, K. L. Sterner ${ }^{45}$, I. Stewart ${ }^{8}$,

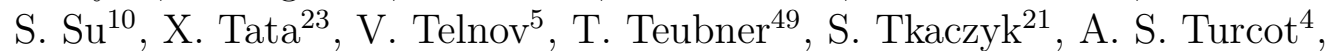
K. van Bibber $^{30}$, R. van Kooten ${ }^{25}$, R. Vega ${ }^{51}$, D. Wackeroth ${ }^{50}$, D. Wagner ${ }^{16}$, A. Waite ${ }^{52}$, W. Walkowiak ${ }^{9}$, G. Weiglein ${ }^{13}$, J. D. Wells ${ }^{6}$, W. Wester, III ${ }^{21}$, B. Williams ${ }^{16}$, G. Wilson ${ }^{13}$, R. Wilson ${ }^{2}$, D. Winn ${ }^{20}$, M. Woods ${ }^{52}$, J. Wudka ${ }^{7}$, O. Yakovlev ${ }^{37}$, H. Yamamoto ${ }^{23}$ H. J. Yang ${ }^{37}$ 
${ }^{1}$ Argonne National Laboratory, Argonne, IL 60439

${ }^{2}$ Universitat Autonoma de Barcelona, E-08193 Bellaterra,Spain

${ }^{3}$ University of British Columbia, Vancouver, BC V6T 1Z1, Canada

${ }^{4}$ Brookhaven National Laboratory, Upton, NY 11973

${ }^{5}$ Budker INP, RU-630090 Novosibirsk, Russia

${ }^{6}$ University of California, Davis, CA 95616

${ }^{7}$ University of California, Riverside, CA 92521

${ }^{8}$ University of California at San Diego, La Jolla, CA 92093

${ }^{9}$ University of California, Santa Cruz, CA 95064

${ }^{10}$ California Institute of Technology, Pasadena, CA 91125

${ }^{11}$ Carleton University, Ottawa, ON K1S 5B6, Canada

${ }^{12}$ Universidad Catolica de Chile, Chile

${ }^{13}$ CERN, CH-1211 Geneva 23, Switzerland

${ }^{14}$ University of Chicago, Chicago, IL 60637

${ }^{15}$ Chonbuk National University, Chonju 561-756, Korea

${ }^{16}$ University of Colorado, Boulder, CO 80309

${ }^{17}$ Cornell University, Ithaca, NY 14853

${ }^{18}$ DESY, D-22063 Hamburg, Germany

${ }^{19}$ DESY, D-15738 Zeuthen, Germany

${ }^{20}$ Fairfield University, Fairfield, CT 06430

${ }^{21}$ Fermi National Accelerator Laboratory, Batavia, IL 60510

${ }^{22}$ Florida State University, Tallahassee, FL 32306

${ }^{23}$ University of Hawaii, Honolulu, HI 96822

${ }^{24}$ Indian Institute of Science, Bangalore, 560 012, India

${ }^{25}$ Indiana University, Bloomington, IN 47405

${ }^{26}$ Johns Hopkins University, Baltimore, MD 21218

${ }^{27}$ Kansas State University, Manhattan, KS 66506

${ }^{28}$ Kyoto University, Kyoto 606, Japan

${ }^{29}$ Lawrence Berkeley National Laboratory, Berkeley, CA 94720

${ }^{30}$ Lawrence Livermore National Laboratory, Livermore, CA 94551

${ }^{31}$ Universität Leipzig, D-04109 Leipzig, Germany

32 Ludwigs-Maximilians-Universität, München, Germany

${ }^{32 a}$ Manchester University, Manchester M13 9PL, UK

${ }^{33}$ Centre de Physique Theorique, CNRS, F-13288 Marseille, France

${ }^{34}$ University of Massachusetts, Amherst, MA 01003

${ }^{35}$ Massachussetts Institute of Technology, Cambridge, MA 02139

${ }^{36}$ Max-Planck-Institut für Physik, München, Germany

${ }^{37}$ University of Michigan, Ann Arbor MI 48109

38 State University of New York, Buffalo, NY 14260

${ }^{39}$ State University of New York, Stony Brook, NY 11794

${ }^{40}$ Northern Illinois University, DeKalb, IL 60115 
${ }^{41}$ University of Notre Dame, Notre Dame, IN 46556

${ }^{42}$ University of Oregon, Eugene, OR 97403

${ }^{43}$ Oxford University, Oxford OX1 3RH, UK

${ }^{44}$ Paul Scherrer Institut, CH-5232 Villigen PSI, Switzerland

${ }^{45}$ University of Pennsylvania, Philadelphia, PA 19104

${ }^{46}$ Pennsylvania State University, Mont Alto, PA 17237

${ }^{47}$ Perkins-Elmer Bioscience, Foster City, CA 94404

${ }^{48}$ Purdue University, West Lafayette, IN 47907

${ }^{49}$ RWTH Aachen, D-52056 Aachen, Germany

${ }^{50}$ University of Rochester, Rochester, NY 14627

${ }^{51}$ Southern Methodist University, Dallas, TX 75275

${ }^{52}$ Stanford Linear Accelerator Center, Stanford, CA 94309

53 Texas A\&M University, College Station, TX 77843

${ }^{54}$ Tokoku University, Sendai 980, Japan

${ }^{55}$ University of Wisconsin, Madison, WI 53706

${ }^{57}$ Uniwersytet Warszawski, 00681 Warsaw, Poland

${ }^{57}$ Wayne State University, Detroit, MI 48202

58 Universität Würzburg, Würzburg 97074, Germany

${ }^{59}$ Yale University, New Haven, CT 06520

Work supported in part by the US Department of Energy under contracts DE-AC0276CH03000, DE-AC02-98CH10886, DE-AC03-76SF00098, DE-AC03-76SF00515, and W-7405-ENG-048, and by the National Science Foundation under contract PHY9809799. 


\section{Contents}

Introduction 1

\section{Linear Collider Physics Report}

"The Case for a $500 \mathrm{GeV} e^{+} e^{-}$Linear Collider" 7

1 Introduction . . . . . . . . . . . . . . . . 7

2 Lepton colliders and the long-term future of high energy physics . . . 10

2.1 A 20-year goal for high energy physics . . . . . . . . . . . . 10

2.2 A 20-year program for accelerators . . . . . . . . . . . . 12

3 Parameters of a $500 \mathrm{GeV}$ linear collider . . . . . . . . . . . . . 14

4 Why we expect new physics below $500 \mathrm{GeV}$. . . . . . . . . . 16

4.1 A fundamental versus composite Higgs boson . . . . . . . . . 17

4.2 A fundamental Higgs boson should be light . . . . . . . . . . . 20

4.3 The constraint on the Higgs mass from precision electroweak data .......................... 21

4.4 The lightest supersymmetry partners are likely to appear at 500 $\mathrm{GeV}$........................ 22

4.5 What if there is no fundamental Higgs boson? . . . . . . . . . 24

4.6 What if the LHC sees no new physics? . . . . . . . . . . 26

5 Physics at a $500 \mathrm{GeV}$ linear collider . . . . . . . . . . . . . 27

5.1 Study of the Higgs boson . . . . . . . . . . . . . . . . . 29

5.2 Studies of supersymmetry . . . . . . . . . . . . . . . . . 37

5.3 Studies of the top quark . . . . . . . . . . . . . . 47

5.4 Studies of $W$ boson couplings . . . . . . . . . . . . . . . . . 49

5.5 Studies of QCD . . . . . . . . . . . . . . . . . 51

5.6 Precision electroweak studies . . . . . . . . . . . . . 52

6 Further topics from the linear collider physics program . . . . . . . 53

6.1 Extended Higgs sector . . . . . . . . . . . . . . . 54

6.2 Supersymmetric particle studies . . . . . . . . . . . 56

6.3 New $Z^{\prime}$ bosons . . . . . . . . . . . . . . . . . . 58 
$6.4 \quad$ Large extra dimensions . . . . . . . . . . . . . . . . . . 59

\section{Sourcebook for Linear Collider Physics}

Higgs Bosons at the Linear Collider $\quad 73$

1 Introduction . . . . . . . . . . . . . . . . 73

2 Expectations for electroweak symmetry breaking . . . . . . . . . 75

3 The Standard Model Higgs boson — theory . . . . . . . . . . . . . 79

$3.1 \quad$ Standard Model Higgs boson decay modes . . . . . . . . . 79

3.2 Standard Model Higgs boson production at the LC . . . . . 80

4 SM Higgs searches before the linear collider . . . . . . . . . . 83

4.1 Direct search limits from LEP . . . . . . . . . . . . . . 83

4.2 Implications of precision electroweak measurements . . . . . . 83

4.3 Expectations for Tevatron searches . . . . . . . . . . . . 84

4.4 Expectations for LHC searches _. . . . . . . . . . 86

5 Higgs bosons in low-energy supersymmetry . . . . . . . . . . . 88

$5.1 \quad$ MSSM Higgs sector at tree-level . . . . . . . . . . . . . . . 89

5.2 The radiatively corrected MSSM Higgs sector . . . . . . . 90

5.3 MSSM Higgs boson decay modes . . . . . . . . . . . . . 95

5.4 MSSM Higgs boson production at the LC . . . . . . . . 96

6 MSSM Higgs boson searches before the LC . . . . . . . . . . . 97

6.1 Review of direct search limits . . . . . . . . . . . . . 97

6.2 MSSM Higgs searches at the Tevatron . . . . . . . . 98

6.3 MSSM Higgs searches at the LHC . . . . . . . . . . . . . . 99

7 Non-exotic extended Higgs sectors . . . . . . . . . . . . . . . . . . . 102

7.1 The decoupling limit . . . . . . . . . . . . . . 103

7.2 Constraints from precision electroweak data and LC implications103

7.3 Constraints on Higgs bosons with $V V$ coupling . . . . . . . 105

7.4 Detection of non-exotic extended Higgs sector scalars at the Tevatron and LHC . . . . . . . . . . . . . . 106

7.5 LC production mechanisms for non-exotic extended Higgs sector scalars . . . . . . . . . . . . . . . . . . 107

8 Measurements of Higgs boson properties at the LC . . . . . . . . . 109

$8.1 \quad$ Mass . . . . . . . . . . . . . . . . . . . . . . . 109

8.2 Coupling determinations - light Higgs bosons . . . . . . . . 112

8.3 Coupling determinations - intermediate mass Higgs bosons . . 117

8.4 Coupling determinations - heavy Higgs bosons . . . . . . . . . 119

8.5 Summary of couplings . . . . . . . . . . . . . 120

8.6 Total width . . . . . . . . . . . . . . . . . . . . . . . 121

8.7 Quantum numbers . . . . . . . . . . . . . . . . 121 
8.8 Precision studies of non-SM-like Higgs bosons . . . . . . . . . 124

9 The Giga-Z option-implications for the Higgs sector . . . . . . . . . 124

9.1 The MSSM context . . . . . . . . . . . . . . . 125

9.2 Non-exotic extended Higgs sector context . . . . . . . . . . . . 126

10 The $\gamma \gamma$ collider option . . . . . . . . . . . . . . . . . . . 127

11 Exotic Higgs sectors and other possibilities . . . . . . . . . . . . . 130

11.1 A triplet Higgs sector . . . . . . . . . . . . . . . . . . . 130

11.2 Pseudo Nambu Goldstone bosons . . . . . . . . . . . . . . . . 131

$\begin{array}{ll}\text { Supersymmetry Studies at the Linear Collider } & 141\end{array}$

1 Introduction . . . . . . . . . . . . . . . . . 141

2 The scale of supersymmetry . . . . . . . . . . . . . . . . . . . . . . . . . . . . . . . . 142

2.1 Naturalness . . . . . . . . . . . . . . . . . . . . . 142

2.2 Neutralino relic abundance . . . . . . . . . . . . . . . . . 145

2.3 Higgs mass and precision electroweak constraints . . . . . . . 146

2.4 Evidence for new physics . . . . . . . . . . . . . 146

3 Determination of masses and couplings . . . . . . . . . . . . . . . . . 148

3.1 Measurement of superpartner masses . . . . . . . . . . . . . . 148

3.2 Measurement of supersymmetry parameters . . . . . . . . . 153

4 Tests of supersymmetry . . . . . . . . . . . . . . . 155

4.1 Confirming supersymmetry . . . . . . . . . . . . . . 156

4.2 Super-oblique corrections . . . . . . . . . . . . . 156

4.3 Measurements at linear colliders . . . . . . . . . . . . . 157

5 Symmetry violating phenomena . . . . . . . . . . . . . . . 159

$5.1 \quad$ R-parity violation . . . . . . . . . . . . . . . . 159

5.2 Lepton flavor violation . . . . . . . . . . . . . . . . . . . . . . . . . . . . . . . . 162

$5.3 \quad \mathrm{CP}$ violation . . . . . . . . . . . . . . . . 163

$6 \quad$ Supersymmetry and $e^{-} e^{-}, e^{-} \gamma$, and $\gamma \gamma$ colliders . . . . . . . . . . . 166

6.1 Supersymmetry and $e^{-} e^{-}$colliders . . . . . . . . . . . . . 166

6.2 Supersymmetry and $e^{-} \gamma$ colliders . . . . . . . . . . . . . 168

6.3 Supersymmetry at $\gamma \gamma$ colliders . . . . . . . . . . . . . . . . . . 168

$7 \quad$ Comparison with LHC . . . . . . . . . . . . . . . . 169

New Physics at the TeV Scale and Beyond 185

1 Introduction . . . . . . . . . . . . . . . . . . 185

2 Gauge boson self-couplings . . . . . . . . . . . . . . . . 187

2.1 Triple gauge boson coupling overview . . . . . . . . . . . . . . 187

2.2 Triple gauge boson measurements . . . . . . . . . . . . . . . . 188

2.3 Electroweak radiative corrections to $e^{+} e^{-} \rightarrow 4$ fermions . . . . 191

2.4 Quartic gauge boson couplings . . . . . . . . . . . . 193

3 Strongly coupled theories . . . . . . . . . . . . . . . 195 
$3.1 \quad$ Strong $W W$ scattering and technicolor . . . . . . . . . . . 195

3.2 Composite Higgs models . . . . . . . . . . . . . . . . . . . 198

4 Contact interactions and compositeness . . . . . . . . . . . . . . 201

5 New particles in extended gauge sectors and GUTs . . . . . . . . . 202

$5.1 \quad$ Extended gauge sectors . . . . . . . . . . . . . . . . 202

5.2 Leptoquarks . . . . . . . . . . . . . . . . . . . . . . 208

5.3 Exotic fermions . . . . . . . . . . . . . . . . . . . . . 209

6 Extra dimensions . . . . . . . . . . . . . . . . . . . . . . 210

$6.1 \quad$ Large extra dimensions . . . . . . . . . . . . . . . . . . . 212

6.2 Localized gravity . . . . . . . . . . . . . . . . . . . 218

$6.3 \mathrm{TeV}$-scale extra dimensions . . . . . . . . . . . . . 222

7 Highly non-conventional theories and possible surprises . . . . . . . 225

7.1 String resonances . . . . . . . . . . . . . . 225

7.2 Non-commutative field theories . . . . . . . . . . 226

8 Determining the origin of new physics . . . . . . . . . . . . . 228

9 Conclusions . . . . . . . . . . . . . . . . . . . . . . 230

$\begin{array}{ll}\text { Top Quark Physics } & 239\end{array}$

1 Introduction . . . . . . . . . . . . . . . . . . . . . 239

2 Physics in the threshold region . . . . . . . . . . . . . . . . 239

$2.1 \quad$ Introduction . . . . . . . . . . . . . . . . . . . . . 239

2.2 QCD dynamics and cross section . . . . . . . . . . . 239

2.3 Top width . . . . . . . . . . . . . . . . . . . . . . 241

2.4 Top quark Yukawa coupling . . . . . . . . . . . . . . . . . . 241

2.5 Experimental issues . . . . . . . . . . . . . . . . . . . . 242

3 Physics above the top threshold . . . . . . . . . . . . . . . . . . 242

3.1 Determination of the top quark-Higgs Yukawa coupling . . . . 242

3.2 Top mass reconstruction . . . . . . . . . . . . . . . . . 245

3.3 Anomalous couplings . . . . . . . . . . . . . . . . . . 247

3.4 QCD and electroweak radiative corrections . . . . . . . 248

4 Conclusions . . . . . . . . . . . . . . . . . . . . . . . 251

QCD and Two-Photon Physics 255

1 Introduction . . . . . . . . . . . . . . . . 255

2 QCD from annihilation processes .............. 255

2.1 The precise determination of $\alpha_{s} \ldots \ldots \ldots \ldots \ldots$

$2.2 \quad Q^{2}$ evolution of $\alpha_{s} \ldots \ldots \ldots \ldots \ldots \ldots \ldots$

2.3 Top quark strong moments . . . . . . . . . . . . . . . . . . 259

3 Two-photon physics . . . . . . . . . . . . . . . . . 260

3.1 Experimental requirements . . . . . . . . . . . . . . . . 261

3.2 Bremsstrahlung photon beam . . . . . . . . . . . . . 261 
3.3 Photon structure . . . . . . . . . . . . . . . . . . 262

$3.4 \gamma \gamma$ scattering - total cross section . . . . . . . . . 264

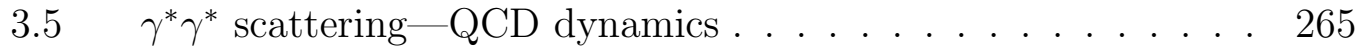

3.6 Summary of two-photon physics . . . . . . . . . . 268

4 Overall summary and conclusions . . . . . . . . . . . . . 268

Precision Studies at the $Z$ and the $W W$ Threshold 271

1 Electroweak observables on the $Z$ resonance . . . . . . . . . . 271

$1.1 \quad$ Machine issues . . . . . . . . . . . . . . . . . . 272

1.2 Electroweak observables . . . . . . . . . . . . . . . 273

$2 \quad m_{W}$ from $W W$ threshold running . . . . . . . . . . . . 277

$2.1 m_{W}$ from a polarized threshold scan . . . . . . . . . 278

2.2 Conclusion . . . . . . . . . . . . . . . . . . . . . 279

3 Electroweak tests of the Standard Model . . . . . . . . . . . . . . 279

3.1 Parameterizations of deviations from the Standard Model . . . 282

3.2 Tests of supersymmetry . . . . . . . . . . . . . . . . 284

4 Heavy flavor physics . . . . . . . . . . . . . . 286

$4.1 \quad$ Measurement prospects for $\mathcal{B}\left(B \rightarrow \pi^{0} \pi^{0}\right) \ldots \ldots \ldots 287$

$4.2 \quad B \rightarrow X_{q} \nu \bar{\nu} \ldots \ldots \ldots \ldots \ldots \ldots \ldots \ldots \ldots \ldots \ldots$

$4.3 \quad$ Semileptonic $B_{s}$ decays . . . . . . . . . . . . . 288

4.4 Weak decays of polarized beauty baryons . . . . . . . . . 289

5 Summary . . . . . . . . . . . . . . . . . . 291

\section{Pathways Beyond the Standard Model}

$\begin{array}{ll}\text { Pathways Beyond the Standard Model } & 299\end{array}$

1 Introduction . . . . . . . . . . . . . . . . . . . . . 299

2 Beyond the Standard Model . . . . . . . . . . . . . . . 300

3 Supersymmetry . . . . . . . . . . . . . . . . . . 301

4 New strong interactions at the $\mathrm{TeV}$ scale . . . . . . . . . . 305

$4.1 \quad$ Composite Higgs models . . . . . . . . . . . . . 306

4.2 Technicolor theories . . . . . . . . . . . . . . 307

5 Extra spatial dimensions . . . . . . . . . . . . . . . . . . 309

$5.1 \quad$ Flat extra dimensions, containing only gravity . . . . . . . 310

5.2 Warped extra dimensions, containing only gravity . . . . . 311

5.3 Flat extra dimensions, containing SM gauge fields . . . . . . 311

5.4 Flat extra dimensions, containing all SM particles . . . . . . 311

6 Surprises . . . . . . . . . . . . . . . . . . . . . . . . . 312 


\section{Experimental Program Issues}

Scenarios for Linear Collider Running $\quad 315$

1 Preliminaries . . . . . . . . . . . . . . . . . . . . 315

2 Illustrative scenarios . . . . . . . . . . . . . . . . . . . 317

2.1 A Higgs boson, but no other new physics, is seen at the LHC . 317

$2.2 \quad$ No Higgs boson or other new particles are seen at the LHC . . 318

2.3 Light Higgs and superpartners are seen at the LHC . . . . . 318

Interaction Regions $\quad 321$

1 Introduction . . . . . . . . . . . . . . . . . . . . 321

2 The two interaction region design at TESLA . . . . . . . . . . 322

3 The dual-energy interaction region design at the NLC . . . . . . . . 322

3.1 The low-energy interaction region at the NLC . . . . . . 326

3.2 The high-energy interaction region at the NLC . . . . . . . . . 329

3.3 Alternative interaction region scenarios . . . . . . . . . . . 330

3.4 Simultaneous operation . . . . . . . . . . . . . . . 332

Positron Polarization 333

$1 \quad$ Introduction . . . . . . . . . . . . . . . . . . . . . 333

2 The physics perspective . . . . . . . . . . . . . 334

2.1 The structure of electroweak interactions at high energies . . . 334

2.2 Standard Model-like Higgs boson . . . . . . . . . . . . . . 336

2.3 Supersymmetric particle production . . . . . . . . . . . 337

2.4 Some other new physics . . . . . . . . . . . . . . . . . . 339

2.5 Transverse polarization . . . . . . . . . . . . . . . . . . 339

3 Experimental issues . . . . . . . . . . . . . . . . . . . . 340

$3.1 \quad$ Polarimetry . . . . . . . . . . . . . . . . . . . . 340

$3.2 \quad$ Frequency of spin flips . . . . . . . . . . . . . . . . 341

3.3 Run time strategy for LL, LR, RL, RR . . . . . . . . . 341

4 Sources of polarized positrons . . . . . . . . . . . . . . . 341

4.1 Helical undulator . . . . . . . . . . . . . . . . . . . . . 342

4.2 Backscattered laser . . . . . . . . . . . . . . . . . . 343

5 Conclusions . . . . . . . . . . . . . . . . . . . . 343

$\begin{array}{ll}\text { Photon Collider } & 347\end{array}$

1 Introduction . . . . . . . . . . . . . . . . . 347

2 Physics Studies at a $\gamma \gamma$ Collider . . . . . . . . . . . . . . . 348

2.1 Production of Higgs bosons . . . . . . . . . . . . . 348

2.2 Supersymmetric particle production . . . . . . . . . . . 349

$2.3 \gamma \gamma \rightarrow W^{+} W^{-}$and $\gamma e \rightarrow W \nu \ldots \ldots \ldots \ldots \ldots$ 
$2.4 \quad \gamma \gamma \rightarrow t \bar{t} \ldots \ldots \ldots \ldots \ldots \ldots \ldots \ldots \ldots \ldots \ldots$

2.5 Other processes . . . . . . . . . . . . . . . . 352

3 Compton Backscattering for $\gamma \gamma$ Collisions . . . . . . . . . . . 352

3.1 Introduction . . . . . . . . . . . . . . . . . . . 352

3.2 Photon spectra . . . . . . . . . . . . . . . . . 352

3.3 Interaction region design and backgrounds . . . . . . . 357

$4 \quad$ IR optical system . . . . . . . . . . . . . . . . . . . . . . . 357

$4.1 \quad$ Optics design . . . . . . . . . . . . . . . . 357

4.2 Beam pipe modifications . . . . . . . . . . . . 358

$5 \quad$ Laser system . . . . . . . . . . . . . . . . . . . . . . . . . . . . 359

$5.1 \quad$ Requirements and overview . . . . . . . . . . . . 359

5.2 Laser system front end . . . . . . . . . . . . . . . . . . 359

5.3 Mercury amplifier . . . . . . . . . . . . . . . . . . 361

$5.4 \quad$ Multiplexer and beam transport . . . . . . . . . . . . 363

$5.5 \quad$ Compressor / stretcher . . . . . . . . . . . . . . 363

5.6 Laser facility, systems design and risk reduction . . . . . . . 364

$\begin{array}{ll}e^{-} e^{-} \text {Collisions } & 369\end{array}$

1 General characteristics of $e^{-} e^{-}$collisions . . . . . . . . . . 369

2 Physics at $e^{-} e^{-}$colliders . . . . . . . . . . . . . . 370

$2.1 \quad$ Møller scattering . . . . . . . . . . . . . . . . . 370

$2.2 \quad$ Higgs bosons . . . . . . . . . . . . . . . . . . . . . . 370

2.3 Supersymmetry . . . . . . . . . . . . . . . . . 371

2.4 Bileptons . . . . . . . . . . . . . . . . . . . . 371

$2.5 \quad$ Other physics . . . . . . . . . . . . . . . . . . 372

3 Accelerator and experimental issues . . . . . . . . . . . . 373

$3.1 \quad$ Machine $\operatorname{design} \ldots \ldots \ldots \ldots . \ldots \ldots$

3.2 Interaction region . . . . . . . . . . . . . . . . . 374

3.3 Detectors . . . . . . . . . . . . . . . . . 375

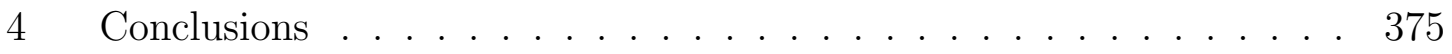

\section{Detectors for the Linear Collider}

$\begin{array}{ll}\text { Detectors for the Linear Collider } & 379\end{array}$

1 Introduction . . . . . . . . . . . . . . . . . . 379

2 Interaction region issues for the detector . . . . . . . . . . 379

2.1 Time structure. . . . . . . . . . . . . . . . . . . . . . 379

$2.2 \quad$ IR layout $\ldots \ldots \ldots \ldots$

$2.3 \quad$ Small spot size issues . . . . . . . . . . . . . . . . . . 382

2.4 The beam-beam interaction . . . . . . . . . . . . . 382

3 Subsystem considerations . . . . . . . . . . . . . . . . . . . 385 
$3.1 \quad$ Vertex detector . . . . . . . . . . . . . . . . 385

3.2 Tracking . . . . . . . . . . . . . . . . . . . . . 387

3.3 Calorimetry . . . . . . . . . . . . . . . . . . . . . . 388

3.4 Muon detection . . . . . . . . . . . . . . . . . . . . 392

3.5 Solenoid . . . . . . . . . . . . . . . . . . . . . . . . 394

$3.6 \quad$ Particle ID . . . . . . . . . . . . . . . . . . . . . 394

3.7 Electronics and data acquisition . . . . . . . . . . . . . . 395

4 Detectors . . . . . . . . . . . . . . . . . . . 397

$4.1 \quad$ L detector for the high-energy IR . . . . . . . . . . . 397

4.2 SD detector for the high energy IR . . . . . . . . . . . 404

4.3 P detector for the lower-energy IR . . . . . . . . . . . . . . . 409

4.4 Cost estimates . . . . . . . . . . . . . . . . . 412

\section{Questions for Further Study}

Suggested Study Questions on LC Physics and Experimentation 447

1 Physics issues . . . . . . . . . . . . . . . . . . 417

$1.1 \quad$ Higgs physics . . . . . . . . . . . . . . . . . . . . 417

$1.2 \quad$ Supersymmetry . . . . . . . . . . . . . . . . . . . . . . 418

1.3 New physics at the TeV scale . . . . . . . . . . . . . . 419

1.4 Top quark physics . . . . . . . . . . . . . . . . . . . . . 419

1.5 QCD and two-photon physics . . . . . . . . . 420

1.6 Precision electroweak measurements . . . . . . . . . . . . 420

2 Accelerator issues . . . . . . . . . . . . . . . . . . . . . 421

$2.1 \quad$ Running scenarios . . . . . . . . . . . . . . . . . . 421

2.2 Machine configuration . . . . . . . . . . . . . . 421

2.3 Positron polarization . . . . . . . . . . . . . . . . . . 422

2.4 Photon collider . . . . . . . . . . . . . . . . . . 422

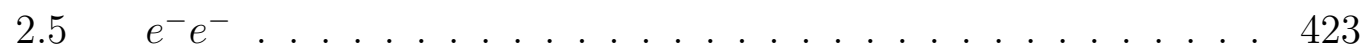

$2.6 \quad$ Fixed Target . . . . . . . . . . . . . . . . . . . . 423

3 Detector issues . . . . . . . . . . . . . . . . . . . . . . . . . 423

3.1 Detectors . . . . . . . . . . . . . . . . . . . . 423 



\section{Chapter 1 Introduction}

The American particle physics community can look forward to a well- conceived and vital program of experimentation for the next ten years, using both colliders and fixed target beams to study a wide variety of pressing questions. Beyond 2010, these programs will be reaching the end of their expected lives. The CERN LHC will provide an experimental program of the first importance. But beyond the LHC, the American community needs a coherent plan. The Snowmass 2001 Workshop and the deliberations of the HEPAP subpanel offer a rare opportunity to engage the full community in planning our future for the next decade or more.

A major accelerator project requires a decade from the beginning of an engineering design to the receipt of the first data. So it is now time to decide whether to begin a new accelerator project that will operate in the years soon after 2010. We believe that the world high-energy physics community needs such a project. With the great promise of discovery in physics at the next energy scale, and with the opportunity for the uncovering of profound insights, we cannot allow our field to contract to a single experimental program at a single laboratory in the world.

We believe that an $e^{+} e^{-}$linear collider is an excellent choice for the next major project in high-energy physics. Applying experimental techniques very different from those used at hadron colliders, an $e^{+} e^{-}$linear collider will allow us to build on the discoveries made at the Tevatron and the LHC, and to add a level of precision and clarity that will be necessary to understand the physics of the next energy scale. It is not necessary to anticipate specific results from the hadron collider programs to argue for constructing an $e^{+} e^{-}$linear collider; in any scenario that is now discussed, physics will benefit from the new information that $e^{+} e^{-}$experiments can provide.

This last point merits further emphasis. If a new accelerator could be designed and built in a few years, it would make sense to wait for the results of each accelerator before planning the next one. Thus, we would wait for the results from the Tevatron before planning the LHC experiments, and wait for the LHC before planning any later stage. In reality accelerators require a long time to construct, and they require such specialized resources and human talent that delay can cripple what would be promising opportunities. In any event, we believe that the case for the linear collider is so compelling and robust that we can justify this facility on the basis of our current knowledge, even before the Tevatron and LHC experiments are done.

The physics prospects for the linear collider have been studied intensively for more than a decade, and arguments for the importance of its experimental program have been developed from many different points of view. This book provides an introduction and a guide to this literature. We hope that it will allow physicists 
new to the consideration of linear collider physics to start from their own personal perspectives and develop their own assessments of the opportunities afforded by a linear collider.

The materials in this book are organized as follows. In Chapter 2, we reprint the 'Linear Collider Whitepaper', a document prepared last summer by the linear collider supporters for the Gilman writing group of HEPAP [1]. This document presents a distilled argument for the first phase of the linear collider at $500 \mathrm{GeV}$ in the center of mass. Though it describes a number of physics scenarios, it emphasizes a particular perspective on the physics to be expected at the next scale. Considerable space is given to the analysis of a light Higgs boson - as called for by the precision electroweak measurements - and to measurements of supersymmetry, motivated, for example, by the precisely known values of the Standard Model coupling constants. There is no question that, in these scenarios, the linear collider would provide a program of beautiful and illuminating experiments.

The 'Sourcebook for LC Physics', Chapters 3-8 gives a more complete overview of the physics measurements proposed for the linear collider program. In separate sections, we review the literature that describes the measurements that the linear collider will make available on the full variety of physics topics: Higgs, supersymmetry, other models of the electroweak symmetry breaking (including new $Z$ bosons, exotic particles, and extra dimensions), top quark physics, $\mathrm{QCD}$, and the new precision electroweak physics available at linear colliders. The chapter on Higgs physics includes a thorough review of the capabilities of a linear collider for the study of the Standard Model Higgs boson as a function of its mass.

Chapter 9 gives a survey of theoretical approaches to the next scale in physics and the implications of each for the linear collider physics case. This chapter attempts to cover the full range of possibilities for physics at the next energy scale. We hope that this review will be useful in putting each particular physics scenario into a larger perspective.

The discussion of experimental program issues in Chapters 10-14 presents a number of options for the linear collider experimental program, weighing their merits and requirements. We begin by presenting some typical scenarios for operation of the linear collider, with suggested choices for energy and luminosity to meet specific physics goals. We then discuss the baseline experimental facilities. Our baseline design is an accelerator of $500 \mathrm{GeV}$ center-of-mass energy, with polarized $e^{-}$beams, and with two interaction regions that share the luminosity. The design envisions a number of upgrade paths. These include low-energy precision measurements in one of the two regions and $e^{+} e^{-}$collisions at multi-TeV energies in the other. The logic of these plans is described in some detail. In the subsequent chapters, we discuss the possible options of positron polarization, operation of a $\gamma \gamma$ collider by laser backscattering from electron beams, and operation for $e^{-} e^{-}$collisions. In each case, we review the promise and the technological problems of the approach. 
Introduction

Chapter 15 discusses detectors for the linear collider experiments. We present and cost three detector models. We also discuss issues for the linear collider detector design. Though a generic LEP-style detector could carry out the basic measurements, the linear collider environment offers the opportunity for exceptional detection efficiencies and precision in the study of physics processes. We list a number of research problems whose solution would allow us to realize the full potential that high energy $e^{+} e^{-}$collisions offer.

The final chapter gives a list of suggested questions that could be taken up at Snowmass or in other studies. Many of these arise from the specific discussions of the earlier chapters. They range from questions of accelerator and detector optimizations to physics issues that require first study or more careful scrutiny.

We do not discuss linear collider accelerator designs in this book, but a number of useful reports on the various current proposals are available. TESLA, based on superconducting rf cavities, has been submitted to the German goverment as a formal TDR [2]. A detailed proposal for the warm cavity accelerator developed by the NLC and JLC groups was presented in the 1996 ZDR [3], and the current NLC baseline is described in a separate paper for the Snowmass 2001 workshop [4]. These two approaches have different emphases and differ in many details. However, both designs meet the requirements to achieve the physics goals that we discuss in this book.

We believe that it is urgent that the American high-energy physics community come to grips now with the issues related to the linear collider. There are several reasons for this. First, the proposals for a linear collider in Europe and in Asia are now becoming explicit. Inevitably, such proposals will raise the question of how the American community will participate. We are approaching the time when the nature of our involvement will be decided by default, not by our design. Second, the high energy frontier of accelerator-based research will pass to the LHC in only a few years. Since the health of any region's particle physics community depends on its central participation in a frontier facility, the US community needs to address how it will participate in the major facilities of the coming era. Third-and most importantly - the linear collider is very likely, in our opinion, to make major progress on the most pressing physics questions before us today. We can offer no guarantee of this, since it is the nature of our field that each new frontier accelerator steps into the unknown. But for all the ways that are foreseen to resolve the mystery of the origin of electroweak symmetry breaking, measurements at the linear collider would be of crucial importance. 
Chapter 1

\section{References}

[1] J. Bagger et al. [American Linear Collider Working Group], hep-ex/0007022.

[2] TESLA Technical Design Report, http://tesla.desy.de/new_pages/TDR_CD/ start.html.

[3] NLC Zeroth-Order Design Report, SLAC-R-474, http://www.slac.stanford. $\mathrm{edu} / \mathrm{accel} / \mathrm{nlc} / \mathrm{zdr} /$.

[4] US NLC Collaboration, 2001 Report on the Next Linear Collider, FERMILABConf-01/075-E, LBNL-47935, SLAC-R-571. 


\section{Linear Collider Physics Report}




\section{Chapter 2 "The Case for a $500 \mathrm{GeV} e^{+} e^{-}$Linear Collider"}

(Report to the HEPAP Writing Group, July 2000)

Authors: J. Bagger, C. Baltay, T. Barker, T. Barklow, U. Baur, T. Bolton, J. Brau, M. Breidenbach, D. Burke, P. Burrows, L. Dixon, H. E. Fisk, R. Frey,

D. Gerdes, N. Graf, P. D. Grannis, H. E. Haber, C. Hearty, S. Hertzbach, C. Heusch, J. Hewett, R. Hollebeek, R. Jacobsen, J. Jaros, T. Kamon, D. Karlen, D. Koltick, A. Kronfeld, W. Marciano, T. Markiewicz, H. Murayama, U. Nauenberg, L. Orr, F. Paige, A. Para, M. E. Peskin, F. Porter, K. Riles, M. Ronan, L. Rosenberg, B. Schumm, R. Stroynowski, S. Tkaczyk, A. S. Turcot, K. van Bibber, R. van Kooten, J. D. Wells, H. Yamamoto

Several proposals are being developed around the world for an $e^{+} e^{-}$linear collider with an initial center of mass energy of $500 \mathrm{GeV}$. In this paper, we will discuss why a project of this type deserves priority as the next major initiative in high energy physics.

\section{Introduction}

Those of us who have chosen to work in elementary particle physics have taken on the task of uncovering the laws of Nature at the smallest distance scales. The process is an excavation, and as such, the work proceeds through various stages. During the past ten years, experiments have clarified the basic structure of the strong, weak, and electromagnetic interactions through measurements of exquisite precision. Now the next stage is about to begin.

The structure of the electroweak interactions, confirmed in great detail by recent experiments, requires a new threshold in fundamental physics at distances or energies within a factor of ten beyond those we can currently probe. More detailed aspects of the data argue that this threshold is close at hand. In the next decade, we will carry out the first experiments that move beyond this threshold, perhaps at the Fermilab Tevatron, almost certainly at the CERN LHC.

Many measurements of this new physics will be made at these hadron colliders. In this document we will argue that electron-positron colliders also have an important role to play. Because the electron is an essentially structureless particle which interacts through the precisely calculable weak and electromagnetic interactions, an $e^{+} e^{-}$ 
collider can unambiguously determine the spins and quantum numbers of new particles. Cross section and branching ratio measurements are straightforward and can be readily compared to models for the underlying physics. Electron beam polarization allows experiments to distinguish electroweak quantum numbers and measure important mixing angles. During the next few years, hadron colliders will likely discover the agents of electroweak symmetry breaking. But electron-positron experiments will also be necessary to completely determine the properties of the new particles.

We believe that a number of new developments call for the start of construction of a high luminosity $500 \mathrm{GeV} e^{+} e^{-}$collider in this decade. First, precision measurements from experiments at CERN, Fermilab and SLAC suggest that important new physics is within range of this machine. Second, the necessary technologies have been developed to the point where it is feasible to construct the collider. Third, these technologies, and others still under development, should allow the collider to be upgraded to $\mathrm{TeV}$ and even multi-TeV energies. For all of these reasons, we believe that the time is right to design and construct a high luminosity $500 \mathrm{GeV} e^{+} e^{-}$linear collider.

In this paper, we formulate the physics case for this machine. The elements of the argument are:

1. New physics processes should appear at a $500 \mathrm{GeV}$ collider. In particular, precision data indicate that the Higgs boson should be accessible to this machine. If it is, the collider will definitively test whether the Higgs boson is responsible for generating the masses of the quarks, leptons, and gauge bosons of the Standard Model.

2. There are good reasons to believe that there is other new physics at the $\mathrm{TeV}$ scale. Across the range of models, $e^{+} e^{-}$collider experiments add crucial information to that available from hadron collider experiments. They will dramatically clarify our understanding of $\mathrm{TeV}$ scale physics.

3. A $500 \mathrm{GeV}$ collider is a critical first step toward a higher energy $e^{+} e^{-}$collider. We believe that such a machine is likely to be needed for the complete elucidation of the next set of physical laws.

This paper will proceed as follows: In Section 2, we will discuss the future of high energy physics from a long-term perspective. We will briefly review the recent developments that have clarified the structure of elementary particle interactions, the challenges posed by the next scale in physics, and the need for higher energy lepton and hadron colliders. In Section 3, we will briefly describe the current designs of $500 \mathrm{GeV} e^{+} e^{-}$colliders and the technologies that will enable them to be upgraded to higher energy. This discussion will define the basic accelerator specifications that we will explore in this study: center of mass energies up to $500 \mathrm{GeV}$, and luminosity samples of $200 \mathrm{fb}^{-1}$ to $600 \mathrm{fb}^{-1}$. In Section 4, we will give the arguments that 
new physics should appear at $500 \mathrm{GeV}$. In Section 5, we will describe some of the important measurements that could be made at a $500 \mathrm{GeV}$ collider, or with high luminosity measurements at the $Z$ pole or the $W W$ threshold. In Section 6, we will describe additional measurements for which the required energy is less certain but which, when they are kinematically accessible in $e^{+} e^{-}$collisions, will beautifully enhance the results of the LHC. Section 7 contains our conclusions.

There is an enormous literature on the physics capabilities of $e^{+} e^{-}$colliders at energies of $500 \mathrm{GeV}$ and above. Our goal in this document is to summarize and focus this information. Much more information about the capabilities of a high energy $e^{+} e^{-}$ linear collider can be found in [1-4] and references therein.

Before beginning our discussion, we would like to comment on three related issues. The first is the role of the LHC. The ATLAS and CMS experiments at the LHC are likely to be the most important high energy physics experiments of the decade, precisely because they will be the first experiments whose energy is clearly in the regime of new physics. The linear collider does not need to compete directly with the LHC in terms of energy; instead, its physics program should complement the LHC by adding important new information. It is just for this reason that we must look at the strengths and weaknesses of the LHC when we build the case for an $e^{+} e^{-}$linear collider.

The second concerns the competing linear collider technologies, the approach of NLC and JLC, with warm copper accelerating structures, and that of TESLA, with superconducting RF cavities. From the point of view of the physics, the similarities of these proposals are more important than their differences. Both schemes are capable of high luminosity $\left(2 \times 10^{34} \mathrm{~cm}^{-2} \mathrm{sec}^{-1}\right.$ for NLC/JLC, $3 \times 10^{34} \mathrm{~cm}^{-2} \mathrm{sec}^{-1}$ for TESLA) and lead to similar backgrounds from beamstrahlung, pair production, and other machine-related effects. The physics case we will develop applies to both schemes. A decision between them must eventually be made on the basis of cost, detailed technical advantages, and upgradability, but we will not argue for either particular approach in this report.

The third issue concerns the ultimate upgrade of the energy of the $e^{+} e^{-}$collider to multi- $\mathrm{TeV}$ center of mass energies. Recent $\mathrm{R} \& \mathrm{D}$ suggests that this may be achievable. It is likely that the needs of physics will eventually call for experiments at such high energies, and so the collider should be planned to support a program of successive energy upgrades. However, the first stage of any program toward multi-TeV $e^{+} e^{-}$ collisions will be a $500 \mathrm{GeV}$ linear collider. This first-stage machine now has a clear physics justification, and that will be the main focus of this report. 


\section{Lepton colliders and the long-term future of high energy physics}

The accelerators at CERN, Fermilab, DESY, and SLAC, which today provide the highest energy particle collisions, were originally envisioned and justified in an era when the fundamental structures of the strong and weak interactions were completely mysterious. These facilities provided much of the data that allowed these mysteries to be understood. Through successive upgrades and improvements, they also provided the data that allowed the resulting theories to be tested with precision. We have learned that with time, accelerators and individual experiments outstrip predictions of their physics reach. This history implies that we should think about future accelerators from a long-term perspective. We begin this report with that discussion. Where may we expect to be, 20 years from now, in our exploration of fundamental physics? How can we get there?

\subsection{A 20-year goal for high energy physics}

The beautiful experiments in particle physics over the past 20 years have brought us to the point where we are poised to discover the microphysical origin of mass. In the Standard Model, the electroweak interactions are built on the foundation of an $S U(2) \times U(1)$ gauge symmetry. All of the mass terms in the Standard Model necessarily violate this symmetry. Masses can only appear because some new fields cause this symmetry to be spontaneously broken.

The spontaneous symmetry breaking cannot be explained in terms of the known strong, weak, and electromagnetic interactions. In the 1980s, it was possible to believe that the $W$ and $Z$ bosons were composite particles [5-8]. In the 1990s, when electroweak radiative corrections were measured to be in agreement with the $S U(2) \times U(1)$ gauge theory [9], this possibility was swept away. At the same time, the fundamental couplings of the strong, weak, and electromagnetic interactions were precisely measured. At the weak interaction scale, these couplings are too small to create a new state of spontaneously broken symmetry. Thus, the breaking of the electroweak gauge symmetry must come from new fundamental interactions. To explain the magnitude of the $W$ and $Z$ masses, these interactions must operate at the $\mathrm{TeV}$ scale.

Over the next 20 years, a primary goal for high energy physics will be to discover these new fundamental interactions, to learn their qualitative character, and to describe them quantitatively by new physical laws. Today, although we can guess, we do not know what form these laws will take. It is logically possible that the electroweak symmetry is broken by a single Higgs boson. More likely, the agent of symmetry breaking will be accompanied by other new physics. A popular hypothesis is a supersymmetric generalization of the Standard Model. Other suggestions include models with new gauge interactions, leading to a strongly-coupled theory at $\mathrm{TeV}$ energies, and models with extra spatial dimensions and quantum gravity at the $\mathrm{TeV}$ scale. 
Aside from their own intrinsic importance, the study of these new interactions will play a crucial role in our understanding of the universe. For example, supersymmetry is a theory of space-time structure which requires modification of the theory of gravity. Other types of models, in particular those with large extra space dimensions, necessarily invoke new space-time physics at the TeV scale.

New physics is also needed to address one of the mysteries of cosmology. There is substantial evidence that a large fraction of the total energy density of the universe is composed of non-baryonic dark matter. Recent estimates require that dark matter should make up more than $80 \%$ of the total matter in the universe [10]. A new stable particle with a mass of about $100 \mathrm{GeV}$ and an annihilation cross section of electroweak size is an excellent candidate for this dark matter. Models of electroweak symmetry breaking typically contain a particle filling this description. During recent years, an enormous amount has been learned about the early universe, back to a time of about 1 second after the Big Bang, by the detailed comparison of primordial element abundances with a kinetic theory of nucleosynthesis based on measured nuclear physics cross sections [11]. In 20 years, we could have a precise knowledge of these new interactions that would allow a predictive kinetic theory of the dark matter. This would push our detailed knowledge of the early universe back to $10^{-12}$ seconds after the Big Bang.

High energy physics has many concerns aside from the nature of electroweak symmetry breaking. The origin of the quark and lepton flavors is mysterious; the pattern of masses and flavor mixings is not understood. The discovery that neutrinos have mass [12] has added a new dimension to this puzzle. In this decade, there will be a significant effort, with contributions from many laboratories, to measure the parameters of flavor mixing and $\mathrm{CP}$ violation. These questions are all intimately related to the puzzle of electroweak symmetry breaking.

There are two reasons for this. First, in the Standard Model all mass terms are forbidden by symmetry, and therefore all masses, mixings, and $\mathrm{CP}$ violating terms must involve the symmetry-breaking fields. For example, in a model in which this breaking is due to fundamental Higgs bosons, the quark and lepton masses, mixings, and $\mathrm{CP}$ violating angles originate in the fermion couplings to the Higgs fields. We will need to know what Higgs bosons exist, or what replaces them, in order to build a theory of flavor. Second, deviations from the conventional expectations for flavor physics are necessarily due to new particles from outside the Standard Model. If such deviations are to be visible in the study of CP violation, for example, the new particles must typically have masses of one to several hundred GeV. Given this mass scale, it is likely that those particles are associated with the physics of electroweak symmetry breaking.

Precision low energy experiments are designed to search for deviations from the Standard Model. Such deviations indicate the presence of new particles which must be found at high energies. Models of new physics do not always predict such deviations, 
and observed effects can be interpreted in multiple ways. So, there is no way to escape the need to search for new particles directly at high energy. In fact, we are already in a situation where our current knowledge requires that new physics be found at the next step in energy. The need for new accelerators can be seen from our study of the weak interactions, as a consequence of the laws that we have established experimentally in the past decade.

Thus, the elucidation of electroweak symmetry breaking should be the key central goal for particle physics research in the next 20 years.

\subsection{A 20-year program for accelerators}

As we have just seen, electroweak symmetry breaking requires new fundamental interactions; it is our task to find and understand them. In every example we know of a fundamental law of Nature (with the possible exception of Einstein's general relativity), the correct theoretical understanding arose only with the accumulation of a large stock of experimental data and the resolution of paradoxes within that data. New and varied experimental techniques were needed, both to accumulate the basic data, and to crucially check or refute intermediate hypotheses.

For the direct exploration of the $\mathrm{TeV}$ energy scale, only two types of collision processes are feasible - proton-proton and lepton-lepton reactions. Proton-proton collisions have the advantage of very high center of mass energies and high rates. However, this environment also has large backgrounds, mainly from Standard Model gluon-gluon collisions. Uncertainties from parton distributions and from perturbative calculations limit the accuracy possible in many precision measurements. Leptonlepton collisions have a complementary set of advantages and disadvantages. The cross sections are low, requiring high luminosity. However, new physics processes, if they occur, typically form a large fraction of the total cross section. Final states can be observed above well understood backgrounds, allowing unambiguous theoretical interpretation. Cross sections for signal and background processes can be computed to part-per-mil accuracy. Lepton-lepton collisions provide precise and modelindependent measurements which complement those from hadron machines.

It is well appreciated that, in developing our understanding of the strong and electroweak interactions, proton and electron colliders made distinct and complementary contributions. As representative examples, recall the discovery of nucleon and meson resonances, the $\Upsilon$, and the $Z^{0}$ and $W^{ \pm}$at proton facilities and the corresponding studies of deep inelastic scattering, the charmonium and bottomonium systems, the $Z^{0}$ resonance, and the $W^{+} W^{-}$threshold at electron machines. In a natural evolution, results from $e^{+} e^{-}$have pointed to new processes in $D$ and $B$ meson decays which have been probed further in high-rate hadron experiments. In the later sections of this report, we will discuss a number of specific models that illustrate the way this complementarity might play out at higher energies. 
This logic leads us to plan, over the next 20 years, to study the new interactions responsible for electroweak symmetry breaking in both proton-proton and leptonlepton collisions. From our experience with the strong and electroweak interactions, it is likely that these new interactions will not be thoroughly understood until we can look at them experimentally from energies above the relevant particle masses. In some supersymmetric models, it is possible to stand above the whole spectrum at a center of mass energy of $1 \mathrm{TeV}$. But quite possibly - and necessarily for models of strong-interaction electroweak symmetry breaking - this requires much higher energies, perhaps 5-10 $\mathrm{TeV}$ in parton-parton collisions.

This challenge was the motivation for building the SSC. With the anticipated start of the LHC experimental program in 2005, the proton-proton program will at last begin. The LHC, operating at $14 \mathrm{TeV}$ and a luminosity of $10^{34} \mathrm{~cm}^{-2} \mathrm{sec}^{-1}$, has parton collisions of sufficiently high energy that it is expected to produce some signature of the new physics that underlies electroweak symmetry breaking [13-15].

For electron-positron colliders, all schemes for achieving high energy collisions involve linear colliders. The technology of $e^{+} e^{-}$linear colliders is relatively new, but important expertise was gained through operation of the SLC [16], which operated at the $Z^{0}$ pole. The natural next step for this technology is a collider with $500 \mathrm{GeV}$ center of mass energy. A collider providing this energy, and delivering the required luminosity, above $10^{34} \mathrm{~cm}^{-2} \mathrm{sec}^{-1}$, would be a critical step on the path toward multi$\mathrm{TeV}$ energies and very high luminosities. At the same time, as we shall see, a 500 GeV collider has sufficient energy to make decisive contributions to the study of electroweak symmetry breaking.

The design of a $500 \mathrm{GeV}$ linear collider must not preclude extension to higher energies. Indeed, both the current warm and superconducting linear collider proposals explicitly include adiabatic extensions to somewhat higher energies. TESLA allows a stage of operation at $800 \mathrm{GeV}$. The NLC/JLC plan includes ready expansion to $1 \mathrm{TeV}$ and allows for an upgrade to $1.5 \mathrm{TeV}$. The pace of such an upgrade would depend on the physics found at the LHC, as well as on results from the first phase of $500 \mathrm{GeV}$ operation.

In the context of a 20-year plan, however, we must go even further, and contemplate partonic collision energies of 5-10 TeV. For hadron colliders, the VLHC program of R\&D now underway, or potential upgrades to the LHC, could provide this; however it seems premature to propose such a machine until the initial LHC results are available. A multi-TeV muon collider has received much recent attention, but there remain important $\mathrm{R} \& \mathrm{D}$ issues to be resolved before its feasibility can be determined. In the past few years, a promising route to multi-TeV collisions has emerged for $e^{+} e^{-}$ colliders. The possibility of a $5 \mathrm{TeV} e^{+} e^{-}$linear collider was studied at Snowmass '96 [17], where three outstanding problems were identified: the lack of a feasible RF power source for high frequency accelerating structures, the large length of the Final Focus sections, and the tight manufacturing and alignment tolerances for the accelerating 
structures. Since then, there has been considerable progress. A major rethinking of the two-beam (CLIC) acceleration scheme makes this concept, in which a low-energy, high-current beam is used to generate high-frequency RF, look promising as a power source for very high energy acceleration [18]. Indeed, such schemes now look feasible for lower RF frequencies (for example, at X band), and this could provide a natural evolution path to higher accelerating gradients [19]. New compact Final Focus layouts [20] have been recently incorporated into the NLC design.

The issue of manufacturing and alignment tolerances is central to the successful operation of any high-luminosity linear collider. This issue is presented in a more manageable form in the design of a $500 \mathrm{GeV}$ collider with either warm or superconducting RF. Moreover, the experience of building and running this machine will be an invaluable prerequisite to eventual $e^{+} e^{-}$experimentation at multi-TeV energies. In addition, any multi- $\mathrm{TeV} e^{+} e^{-}$linear collider will be placed in a long, straight tunnel exactly like the one on the site of a $500 \mathrm{GeV}$ machine and perhaps could reuse the damping rings and injector complex of the $500 \mathrm{GeV}$ stage. Thus, a $500 \mathrm{GeV}$ linear collider is the first stage of a 20-year exploration in $e^{+} e^{-}$physics.

\section{Parameters of a $500 \mathrm{GeV}$ linear collider}

The designs of linear colliders have evolved dramatically over the past five years, based in part on experience from the SLAC Linear Collider operating at $91 \mathrm{GeV}$, and in part on extensive collaborative R\&D efforts in Europe, Japan and the United States. At this writing, the machine parameters are still being evaluated; this section is intended to give the currently envisioned scope of the possible accelerator projects.

The TESLA collider, developed by a collaboration led by DESY, would employ superconducting RF accelerating cavities operating in L-band $(1.3 \mathrm{GHz})$. The JLC (KEK) and NLC (SLAC, LBNL, LLNL, FNAL) designs are based on warm accelerating structures operating in X-band $(11.4 \mathrm{GHz})$. Initial construction of each of these is expected for a $500 \mathrm{GeV}$ machine. A variety of important differences in the designs follow from the basic choice of accelerating frequency. (KEK is also considering a C-band variant operating at $5.7 \mathrm{GHz}$.)

The main parameters of TESLA and the X-band NLC/JLC are shown in Table 1. For all proposals, electron beam polarization of $80 \%$ is expected. Production of polarized positrons can be envisioned by creating polarized photons in sophisticated undulator magnets, or by backscattering polarized high-power lasers, but these possibilities require further development. In all proposals, the collider can also be operated for $e^{-} e^{-}$collisions with some loss in luminosity. By backscattering laser beams, it may be possible to create a high-luminosity gamma-gamma collider with a center of mass energy of about $80 \%$ of that for $e^{+} e^{-}$.

The U.S. design of the NLC underwent a DOE readiness review to initiate the 


\begin{tabular}{|c|c|c|}
\hline & TESLA & NLC/JLC \\
\hline$E_{\mathrm{CM}}(\mathrm{GeV})$ & 500 & 500 \\
\hline $\mathrm{RF}$ frequency $(\mathrm{GHz})$ & 1.3 & 11.4 \\
\hline Repetition rate $(\mathrm{Hz})$ & 5 & 120 \\
\hline Luminosity $\left(10^{34} \mathrm{~cm}^{-2} \mathrm{sec}^{-1}\right)$ & 3.4 & 2.2 \\
\hline Bunch separation (ns) & 337 & 1.4 \\
\hline Effective gradient $(\mathrm{MV} / \mathrm{m})$ & 22 & 50.2 \\
\hline Beamstrahlung (\%) & 3.3 & 4.6 \\
\hline Linac length $(\mathrm{km})$ & 31 & 10.8 \\
\hline
\end{tabular}

Table 2.1: Basic parameters of the high-luminosity TESLA and NLC/JLC accelerator designs.

Conceptual Design Report in May 1999. The Review Committee was positive in its assessment of the technical design. The cost was estimated at $\$ 7.9 \mathrm{~B}$. After subtraction of contingency, escalation, and detectors, these costs were distributed over the major subsystems as follows: injectors (19\%), main linacs (39\%), beam delivery (11\%), global costs (17\%), management/business (14\%). The DOE decided not to proceed with the official CD-1 milestone in view of this cost. Present work is focused on cost and possible scope reductions. In the past year, progress has been made in identifying areas of savings, including the use of permanent magnets for the beam lines, electronics distributed along the linacs, modifications to the injectors, and considerable reduction of the length of the Final Focus. Demonstrated improvements in the klystrons and modulators should give a reduction of RF power costs. Taken together, these developments are estimated to reduce the cost by $30 \%$. Scope reductions, including building the linacs initially for $500 \mathrm{GeV}$ operation, with subsequent civil construction for higher energy, could yield a further $10-15 \%$ reduction in the initial cost.

The luminosity expected for the NLC design depends critically on the precision with which one can build and align the disk-loaded accelerating structures of the main $\mathrm{X}$-band linac. Recent tests have demonstrated that structures can be produced with 2-3 times better accuracy than projected in the 1999 review, and that monitors built into these structures can measure their position with respect to the beam to within a few microns. Re-examination of the beam parameters in the light of these results has led to the realization that the luminosity of the collider can be expected to be 3-4 times higher than projected in 1999, although it is likely to require some period of running to carry out the needed beam-based alignment of the accelerator. It is reasonable to assume that the collider will begin operation at $5 \times 10^{33} \mathrm{~cm}^{-2} \mathrm{sec}^{-1}$ and that, over a period of time, it will reach the design luminosity of $2.2 \times 10^{34} \mathrm{~cm}^{-2} \mathrm{sec}^{-1}$ shown in Table 1 . This would yield $100 \mathrm{fb}^{-1}$ of accumulated data in the first year of 
operation and $200 \mathrm{fb}^{-1} / \mathrm{yr}$ in subsequent years.

Each of these proposals includes possible adiabatic upgrades in energy. The TESLA collider can be expanded to $800 \mathrm{GeV}$ through higher accelerating gradients. The NLC/JLC energy upgrade to $1 \mathrm{TeV}$ could be achieved through an increase in the linac lengths and the addition of more RF structures. Improvements in RF gradients or further increases in length could allow operation at $1.5 \mathrm{TeV}$. It is important for the long term evolution of the linear collider that the flexibility to implement these options be included in the initial machine design.

Work has been done at CERN (CLIC) to develop the RF power for acceleration to even higher energies. The idea is to generate wakefield power for the main linacs using a high current, low energy drive beam operating at low (L-band) frequencies. Recent work at SLAC has expanded this concept to incorporate a recycling drive beam train that is cheaper, more compact and efficient than the original CLIC concept. Accelerating gradients of about $100 \mathrm{MV} / \mathrm{m}$ are envisioned for this two beam design. The two beam linear collider offers an attractive possibility for later expansion of the linear collider to multi-TeV operation, and suggests the potential for an evolving accelerator facility that can follow the initial phase of physics results. Recent R\&D suggests that the use of the two beam drive technology is as well suited for linacs operating in the X-band as for the $30 \mathrm{GHz}$ structures originally envisioned by CLIC, although the limits to feasible gradients are not clear.

For the NLC design with permanent magnets in the beam lines, the energy for operation cannot be decreased below half its maximum. As discussed in the next sections, physics considerations may dictate that a wider range of energies is needed. In particular, a return to the $Z^{0}$ pole may be desirable to improve the precision of the electroweak measurements. Similarly, if the Higgs boson is in the low mass region favored by the Standard Model or supersymmetry, it may be advantageous to accumulate substantial integrated luminosity at the energy of the maximum Higgs cross section and, at the same time, explore the high energy region. Recently, consideration has been given to providing a second beam operating at lower energies. This beam would be extracted from the main accelerator and accelerated in unused time slices of the AC duty cycle. The extra power needed for this operation could be low because of the reduced energy of the beams. Low and high energy beams would be delivered to dedicated detectors installed at separate interaction points in the beam delivery region.

\section{Why we expect new physics below $500 \mathrm{GeV}$}

At Snowmass '96, it was argued that a $1.5 \mathrm{TeV} e^{+} e^{-}$collider is roughly equivalent to the LHC in its ability to detect the new physics related to electroweak symmetry breaking [15]. However, this point will certainly be moot by the time such a linear 
collider operates. The real question that we must address is different: In an era in which the LHC is already exploring the new interactions responsible for electroweak symmetry breaking, what critical information must $e^{+} e^{-}$experiments add, and at what $e^{+} e^{-}$center of mass energies should this information be sought?

Today, there is considerable evidence that an $e^{+} e^{-}$collider program should begin at a center of mass energy of $500 \mathrm{GeV}$. This evidence is indirect and will remain so until the new particles responsible for electroweak symmetry breaking are discovered. The case rests on the large body of precision data acquired over the past ten years. These data agree remarkably with the minimal Standard Model. When interpreted using this model, they require that the Higgs boson be light. The data also place strong constraints on possible new physics associated with electroweak symmetry breaking. These constraints define distinct pathways for new physics which will be tested at the next generation of colliders.

Following the guidance of the precision data, we will argue in this section that a $500 \mathrm{GeV}$ linear collider will be needed whatever the outcome of the LHC experiments might be. In Sections 4.1-4.3, we will outline why there should be a light Higgs boson with mass below about $200 \mathrm{GeV}$. In Section 4.4, we will argue that, if the new physics includes supersymmetry, the lightest superpartners should be found at a $500 \mathrm{GeV}$ collider. There are known ways to evade these arguments, but they too give rise to crucial tests in $e^{+} e^{-}$collisions at $500 \mathrm{GeV}$, as we will discuss in Section 4.5. Finally, in Section 4.6, we will address the question: what if the LHC sees no new physics?

\subsection{A fundamental versus composite Higgs boson}

Models of electroweak symmetry breaking divide into two groups at the first step. Is the symmetry breaking induced by a fundamental scalar field or by a composite object? Is electroweak symmetry breaking a weak-coupling phenomenon, or does it require new strong interactions? These basic questions have driven the study of electroweak symmetry breaking for 20 years [21,22]. Many people use analogies from QCD or superconductivity to argue against the plausibility of fundamental scalars, or use the perceived beauty of supersymmetry to motivate a fundamental scalar Higgs field. We believe that it is possible to make a preliminary judgment - in favor of a fundamental Higgs field - on the basis of the data. This will be important, because models in which the Higgs is fundamental favor a light Higgs boson, while other models favor a heavy Higgs resonance, or none at all.

The simplest model of electroweak symmetry breaking is the minimal version of the Standard Model, which introduces one elementary Higgs field and nothing else. This model is consistent with the present data, but it is totally inadequate as a physical theory. In this model, the mass parameter $m^{2}$ of the Higgs field is a free parameter which cannot be computed as a matter of principle, because it receives an infinite additive renormalization. Electroweak symmetry is broken or not according to 
whether this parameter, after renormalization, is positive or negative. If the infinite radiative corrections are made finite by a cutoff at some energy $M, m^{2}$ can be much less than $M^{2}$ only if the radiative corrections are finely tuned to cancel. If $M$ is taken to be the Planck scale, these corrections must cancel in the first 30 decimal places. Theorists often consider this to be a problem in its own right (the 'gauge hierarchy problem'). This problem is a symptom of the fact that the Standard Model is only a parametrization, and not an explanation, of electroweak symmetry breaking.

Theories of electroweak symmetry breaking can be constructed either with or without fundamental Higgs particles. The preference we have expressed for a fundamental Higgs particle is reflected in the history of the subject. Phenomenological models of supersymmetry introduced in the early 1980s [23-26] are as valid today as when they were first created. On the other hand, the predictions of the early dynamical models (as reviewed, for example, in [27]) have been found to be inconsistent with experiment, requiring major changes in model-building strategies.

To discuss this point, we must define what we mean by a 'fundamental scalar field'. A particle which looks fundamental and structureless on one length scale can be seen to be composite on a smaller length scale. In nuclear physics, and more generally in scattering processes with energies of a few hundred $\mathrm{MeV}$, the pion can be treated as a structureless particle. However, in hard QCD processes, the pion must be treated as a quark-antiquark bound state. At the other extreme, string theory predicts that even quarks and leptons have a finite size and an internal structure at the Planck scale. In almost any theory, a particle can at best be considered fundamental at some particular distance scale. The question here is whether the Higgs boson is elementary well above the scale of the new interactions responsible for electroweak symmetry breaking. In the following discussion, we use the term 'fundamental Higgs' for the case that there is a scalar Higgs field in the Lagrangian at an energy scale of $20 \mathrm{TeV}$.

The answer to this question has direct implications for the theory of the quark and lepton masses. These masses arise through $S U(2) \times U(1)$ symmetry breaking, from terms in the effective Lagrangian that couple left-handed to right-handed fermions. If there is a fundamental Higgs field, a typical term has the form

$$
\delta \mathcal{L}=\lambda_{f} \bar{f}_{L} \phi f_{R}+\text { h.c. },
$$

where $\phi$ is an $S U(2)$-doublet Higgs field and the coupling $\lambda_{f}$ is dimensionless. The fermion $f$ obtains mass when $\phi$ acquires a vacuum expectation value. To explain the size of the mass, a theory must contain new interactions that fix the value of $\lambda_{f}$. Because $\lambda_{f}$ is dimensionless, these interactions can occur, without prejudice, at any energy scale larger than $20 \mathrm{TeV}$. In typical models with a fundamental Higgs boson, these interactions occur at the scale of grand unification, or even above.

If there is no fundamental $S U(2)$-doublet scalar field, the interaction (2.1) does not exist. Instead, one must write a more complicated interaction that couples $\bar{f}_{L} f_{R}$ 
to other new fields. For example, in technicolor models, one writes

$$
\delta \mathcal{L}=\frac{g^{2}}{M_{E}^{2}} \bar{f}_{L} f_{R} \bar{Q}_{R} Q_{L}+\text { h.c. },
$$

where $Q$ is a new heavy fermion with strong interactions at the $\mathrm{TeV}$ scale. This is a dimension-6 operator, and therefore we have written a coefficient with the dimensions (mass $)^{-2}$. If the operator $\left(\bar{Q}_{R} Q_{L}\right)$ acquires a vacuum expectation value at the $\mathrm{TeV}$ scale and this operator is expected to generate a $1 \mathrm{GeV}$ fermion mass, $M_{E}$ must be roughly $30 \mathrm{TeV}$. The four-fermion operator (2.2) can be induced by the exchange of a heavy boson of mass $M_{E}$. However, whatever the mechanism that leads to this operator, the physical interactions responsible must operate at some energy scale not too far above $M_{E}$. This means that, unlike the previous case, the interactions that determine the quark and lepton masses and mixings must occur at energies not so far above those we now probe experimentally.

In fact, these interactions must occur at sufficiently low energies that they would be expected to contribute significantly to $\mu \rightarrow e \gamma$ and $K \rightarrow \mu e$, and to $K-\bar{K}, B-\bar{B}$, and $D-\bar{D}$ mixing. The fact that these processes are not observed is a severe problem for dynamical theories. A further problem arises from the large size of the top quark mass. To produce a mass as large as is observed, the mass scale $M_{E}$ for the top quark - and, by symmetry, for the $b_{L}$-must be close to $1 \mathrm{TeV}$. This new interaction would be expected to lead to enhanced flavor-changing neutral current amplitudes, and to few-percent corrections to the $Z b \bar{b}$ coupling [28].

These experimental observations have eliminated essentially all simple models of dynamical symmetry breaking. The only models that survive have complex new dynamics (e.g., [29-31]) or, below energies of several TeV, behave almost exactly like the Standard Model with a scalar Higgs field (e.g., [32]). Neither type of model resembles the attractive intuitive picture that first led people to explore electroweak symmetry breaking by new strong interactions.

Generalizations of the simplest Standard Model with additional fundamental scalar fields have also been proposed. But these have little motivation, and like the minimal Standard Model, the Higgs vacuum expectation value, and even the existence of electroweak symmetry breaking, cannot be predicted as a matter of principle.

The simplest models with a fundamental Higgs field in which electroweak symmetry breaking results from a calculation, rather than a parameter choice, are those with supersymmetry. Without debating the virtues or deficits of supersymmetric models, what is relevant here is that supersymmetric models have not been significantly constrained by the precise experimental measurements of the past 20 years. Supersymmetric particles give very small effects in electroweak precision measurements because the masses of the superparticles preserve $S U(2) \times U(1)$ gauge symmetry, and so do not require electroweak symmetry breaking. In models that decouple in this way, new particles with mass $M$ give corrections to the Standard Model predictions at the $Z^{0}$ 
which are of size

$$
\frac{\alpha}{\pi} \frac{m_{Z}^{2}}{M^{2}}
$$

As long as we stay below the energy at which the new particles actually appear, their influence is very small. Then, as we pass the threshold, new physics appears suddenly. Supersymmetry thus naturally suppresses deviations from the Standard Model - until we begin to produce the supersymmetric particles. Models with dynamical electroweak symmetry breaking almost always contain heavy matter states which have chiral couplings and thus do not decouple from electroweak symmetry breaking. In these models, one expects significant corrections to the Standard Model well below the energy scale of the new particles.

In addition to this decoupling, the early supersymmetry models made two important predictions. The first was that the top quark mass should be heavy. This tendency arises from the fact that, in supersymmetric models, electroweak symmetry breaking can be triggered by radiative corrections due to the top quark Yukawa coupling. The papers [23-26] all quoted lower bounds on the top quark mass, ranging from 50 to $65 \mathrm{GeV}$. (Later, corners of parameter space were found in which the top quark mass could be lower.) Supersymmetry readily accommodates a top quark mass as large as $175 \mathrm{GeV}$. The second prediction was that the value of $\sin ^{2} \theta_{w}$ should be close to 0.23 (as now observed), rather than the value 0.21 preferred in the early 1980's. This prediction arises from grand unification with the renormalization group equations of supersymmetry [33-35]. The precise determination of $\alpha_{s}$ and the electroweak couplings at the $Z^{0}$ has given even stronger support to the idea of supersymmetric grand unification, with the issue now at the level of detailed higher-order corrections [36].

Of course it is premature to make a final decision between the different models. For this, we must discover and study the Higgs boson, or whatever takes its place. But, in planning where we should look for these phenomena, we should take into account that models with fundamental Higgs bosons passed the first tests presented by the data, while the early dynamical models did not.

\subsection{A fundamental Higgs boson should be light}

In the previous section, we noted that in models with fundamental Higgs bosons, the Higgs is typically light. In this section, we will quantify that statement with upper bounds on the Higgs mass.

In the Standard Model, the mass of the Higgs boson is determined in terms of the Higgs field expectation value $v$ and the Higgs self-coupling $\lambda$ by the relation

$$
m_{h}=\sqrt{2 \lambda} v
$$

with $v=246 \mathrm{GeV}$ determined by the values of the $W$ and $Z$ masses. A bound on $\lambda$ 
thus implies a bound on $m_{h}$. For example, $\lambda<1$ implies $m_{h}<350 \mathrm{GeV}$. How large can $\lambda$ reasonably be?

Like $\alpha_{s}, \lambda$ is a running coupling constant, but in this case radiative corrections drive $\lambda$ to larger values at higher energies. Just as the running $\alpha_{s}$ diverges at $\Lambda_{\overline{M S}}$, signaling the onset of nonperturbative QCD effects, the running $\lambda$ diverges at a high energy scale $\Lambda_{h}$. Presumably, this must signal the breakdown of the fundamental Higgs picture. The relation between $\Lambda_{h}$ and the value of $\lambda$ at the weak interaction scale can be computed from the Standard Model [37]. It is conveniently written, using (2.4), as

$$
m_{h}=\frac{1000 \mathrm{GeV}}{\sqrt{\ln \left(\Lambda_{h} / v\right)}}
$$

The value of $m_{h}$ in (2.5) is the largest Higgs boson mass compatible with a Higgs field which is elementary at the scale $\Lambda_{h}$. For $\Lambda_{h}=20 \mathrm{TeV}, m_{h}<500 \mathrm{GeV}$.

A much stronger limit on $m_{h}$ is obtained if one takes seriously the experimental evidence for grand unification and assumes that the Higgs boson is a fundamental particle at the grand unification (GUT) scale. If we naively put $\Lambda_{h}>10^{16} \mathrm{GeV}$ into (2.5), we find $m_{h}<180 \mathrm{GeV}$. Successful grand unification requires supersymmetry and brings in ingredients that make the computation of $m_{h}$ more complex. But, detailed analysis of supersymmetric grand unified models has shown that the idea of an upper bound on $m_{h}$ remains valid. In 1992, two groups presented systematic scans of the parameter space of supersymmetric grand unified theories, demonstrating the bound $m_{h}<150 \mathrm{GeV}[38,39]$. Exceptions to this constraint were later found, but still all known models satisfy $m_{h}<205 \mathrm{GeV}$ [40].

The Minimal Supersymmetric Standard Model is a special case. In this model, the tree-level potential for the lightest Higgs boson is determined completely by supersymmetry. Radiative corrections to this potential are important. Nevertheless, it can be shown that $m_{h}<130 \mathrm{GeV}$ in this model [41]. Here the conclusion is independent of any assumptions about grand unification.

\subsection{The constraint on the Higgs mass from precision electroweak data}

The previous two sections did not make any reference to the determination of the Higgs boson mass from the precision electroweak data. Those data give a second, independent argument for a light Higgs boson. The Higgs field contributes to electroweak observables through loop corrections to the $W$ and $Z$ propagators. The effect is small, of order $\alpha \ln \left(m_{h} / m_{W}\right)$, but the accuracy of the measurements makes this effect visible. A fit of the current data to the Standard Model, using the measured value of the top quark mass, is consistent only if $\ln \left(m_{h} / m_{W}\right)$ is sufficiently small. The LEP Electroweak Working Group finds upper limits $m_{h}<188 \mathrm{GeV}$ at the $95 \%$ CL and $m_{h}<291 \mathrm{GeV}$ at the $99 \%$ CL [42]. Even using more conservative estimates of 
the theoretical errors [43], the limit on the Higgs boson mass is well within the range of a $500 \mathrm{GeV} e^{+} e^{-}$collider.

This Standard Model limit does not obviously apply to more general models of electroweak symmetry breaking. In what follows we will discuss its validity in various models. As previously, the result depends on whether or not the Higgs is fundamental.

We have noted in Section 4.1 that models with a fundamental Higgs boson typically satisfy decoupling. The practical effect of this is that, if new particles are sufficiently massive that they cannot be produced at LEP 2, their contributions to electroweak corrections are too small to affect the current global fits. In particular, fits to models of supersymmetry produce upper bounds on the Higgs mass similar to those from the Standard Model.

It is difficult to make a model with dynamical electroweak symmetry breaking that is consistent with precision electroweak measurements. The simplest technicolor models, for example, give several-percent corrections to electroweak observables [4446]; effects this large are completely excluded. Even models with one $S U(2)$ doublet of techni-fermions give corrections of a size roughly double that for a $1000 \mathrm{GeV}$ Higgs boson. With models of this type, it is typically necessary to invoke some mechanism that compensates the large corrections that appear in these models, and then to adjust the compensation so that the precision electroweak constraint is obeyed. In this process, the constraint on the Higgs boson mass can be relaxed.

A recent review [47] describes the three different compensation strategies that have been presented in the literature. One of these strategies leads to a lower value of the $W$ mass and a larger $Z$ width than predicted in the Standard Model. It can be distinguished by the improved precision electroweak measurements that we describe in Section 5.6. The other two strategies predict either new light particles with electroweak charge or other perturbations of Standard Model cross sections visible below $500 \mathrm{GeV}$. Thus, models based on new strong interactions can avoid having Higgs bosons below $500 \mathrm{GeV}$, but they predict phenomena observable at a $500 \mathrm{GeV}$ linear collider.

\subsection{The lightest supersymmetry partners are likely to appear at $500 \mathrm{GeV}$}

For supersymmetric models of electroweak symmetry breaking, the arguments of the previous two sections give us confidence that we will be able to produce the lightest Higgs boson. But we also need to study the supersymmetry partners of quarks, leptons, and gauge bosons. Thus, we must also explore how heavy these particles are likely to be.

Because supersymmetric generalizations of the Standard Model revert to the Standard Model when the superpartner masses are taken to be heavy, it is not possible to obtain upper limits on the masses of supersymmetric particles by precision measurements. One must take a different approach, related to the problems of the Standard Model discussed at the beginning of Section 4.1. As we noted there, it is a property of 
"The Case for a $500 \mathrm{GeV}$ Linear Collider"

\begin{tabular}{|l|c|c|c|c|}
\hline \hline & $\widetilde{\chi}_{1}^{+}$ & $\widetilde{g}$ & $\widetilde{e}_{R}$ & $\widetilde{u}, \tilde{d}$ \\
\hline \hline Barbieri-Giudice [48] & 110 & 350 & 250 & 420 \\
Ross-Roberts [49] & 110 & 560 & 200 & 520 \\
de Carlos-Casas [50] & 250 & 1100 & 450 & 900 \\
Anderson-Castano [51] & 270 & 750 & 400 & 900 \\
Chan-Chattopadhyay-Nath [52] & 250 & 930 & 550 & 900 \\
Giusti-Romanino-Strumia [53] & 500 & 1700 & 600 & 1700 \\
Feng-Matchev-Moroi [54] & $240 / 340$ & $860 / 1200$ & $1700 / 2200$ & $2000 / 2300$ \\
\hline \hline
\end{tabular}

Table 2.2: Upper limits on supersymmetry particle masses (in $\mathrm{GeV}$ ) from the fine-tuning criterion found by various groups. In the last line, we have chosen two different breakpoints in fine-tuning from the results given in the paper.

the Standard Model that radiative corrections from a high mass scale $M$ contribute additively to the Higgs mass and vacuum expectation value, affecting $m_{W}$ in the form

$$
m_{W}^{2}=\frac{g^{2} v^{2}}{4}+\frac{\alpha}{\pi} M^{2}+\cdots .
$$

It is possible to obtain a value of the $W$ mass much less than $M$ only if the various contributions cancel to high accuracy. For example, these terms must cancel to 3 decimal places for $M=20 \mathrm{TeV}$ or to 30 decimal places for $M=10^{18} \mathrm{GeV}$. Supersymmetry solves this problem by forbidding such additive corrections to $m_{W}^{2}$. But this restriction applies only if supersymmetry is unbroken. If the masses of the superpartners are much greater than $m_{W}$, the fine-tuning problem returns.

This theoretical motivation leads us to expect that supersymmetric particles are most natural if they are light, of order a few hundred GeV. One can try to quantify this argument by limiting the amount of accidental cancelation permitted in the calculation of $m_{W}$. By now, many authors have studied this cancelation in a variety of supersymmetric models. In Table 2, we show the upper limits on supersymmetry particle masses found by seven groups for the parameter space of minimal gravity-mediated supersymmetry models (mSUGRA). The detailed calculations leading to these limits are different and, in many cases, involve conflicting assumptions. These differences are reflected in the wide variation of the limits on first- and secondgeneration slepton and squark masses evident in the table.

Nevertheless, these analyses are in general agreement about the required scale of the gaugino masses and (except for [53]) expect chargino pair production to be kinematically accessible at or near $500 \mathrm{GeV}$. A simplified but quantitative argument for this bound can be made [54] by writing the expression for $m_{W}^{2}$ in terms of the underlying parameters of the model, and eliminating these in terms of physical particle 
masses. For the representative value $\tan \beta=10$, one finds

$$
m_{W}^{2}=-1.3 \mu^{2}+0.3 m^{2}(\widetilde{g})+\cdots,
$$

where the terms displayed involve the supersymmetric Higgs mass parameter and the gluino mass. The omitted terms involving scalar masses are more model-dependent. The gluino mass enters through its effect on the renormalization of the stop mass. For a gluino mass of $1 \mathrm{TeV}$, the requirement that the $W$ mass is no larger than 80 $\mathrm{GeV}$ requires a fine-tuning of 1 part in 50. A similar level of fine-tuning is needed if $\mu$ is greater than $500 \mathrm{GeV}$.

As we will discuss in Section 5.2, the masses of the two charginos are closely related to the wino mass parameter $m_{2}$ and the Higgs mass parameter $\mu$. In particular, the lighter chargino mass lies close to the smaller of these two values. The parameter $m_{2}$ is connected to the gluino mass in mSUGRA models by the grand unification relation

$$
m_{2} / m(\widetilde{g}) \approx \alpha_{w} / \alpha_{s} \approx 1 / 3.5 .
$$

This relation also holds in gauge-mediation, where, in addition, the masses of sleptons are predicted to be roughly the same size as the mass of the chargino. In other schemes of supersymmetry breaking, the chargino/gluino mass ratio can differ; for example, in anomaly-mediation, $m_{2} / m(\widetilde{g}) \approx 1 / 8$. In all of these models, the bound on $m(\widetilde{g})$ implies a strong bound on the lightest chargino mass. The fact that both $m_{2}$ and $\mu$ are bounded by the fine-tuning argument implies that there is also a bound on the mass of the heavier chargino. Indeed, one typically finds that the full set of chargino and neutralino states can be produced at an $800 \mathrm{GeV} e^{+} e^{-}$collider [54].

Although the fine-tuning limits are by no means rigorous, they indicate a preference for light supersymmetry partners. They encourage us to expect that we will be able to study the lighter chargino and neutralinos at the initial stage of the linear collider program, and all gauginos with a modest upgrade of the energy.

\subsection{What if there is no fundamental Higgs boson?}

Despite our arguments given in Section 4.1 for preferring a fundamental Higgs boson, electroweak symmetry breaking could result from a new strong interaction. Whereas for supersymmetry we have a well-defined minimal model, albeit one with many free parameters, here even the basic structure of the model is unknown and we will need more guidance from experiment. It is thus important to identify measurements that probe possible new strong interactions in a variety of ways.

In models with a composite Higgs boson, the Higgs mass can be large, $500 \mathrm{GeV}$ or higher. If the Higgs is very heavy, there is no distinct Higgs resonance. A heavy but narrow Higgs boson can be studied at the LHC in its $Z^{0} Z^{0}$ decay mode, and at a higher energy $e^{+} e^{-}$collider. A broad resonance or more general new strong 
interactions can be studied through $W W$ scattering at TeV energies. This study can also be done at the LHC and at a higher energy linear collider [15]. However, in this case, the experiments are expected to be very challenging. Certain classes of models which are preferred by the arguments of Section 4.1 (e.g., [32] ) predict that no effect will be seen in these reactions.

In view of this, it is essential to have another way to probe models with a composite Higgs boson. This can be done by studying the effects of the new physics on the Standard Model particles that couple most strongly to it - the $W, Z$, and top quark. Because the $Z$ couples to light fermions through a gauge current, effects of the new strong interactions are not expected to appear in $Z$ decays, except possibly in $Z \rightarrow b \bar{b}$. The first real opportunity to observe these effects will come in the study of the $W$, $Z$, and $t$ couplings. Effects of strong-interaction electroweak symmetry breaking can appreciably modify the Standard Model predictions for these couplings.

Without a specific model, it is difficult to predict how large these effects should be, but some estimates provide guidance. For example, triple gauge boson couplings can be related to parameters of the effective chiral Lagrangian describing the nonperturbative $S U(2) \times U(1)$ symmetry breaking. The parameter $\Delta \kappa_{\gamma}$ which contributes to the $W$ anomalous magnetic dipole moment, is given by [15]

$$
\Delta \kappa_{\gamma}=-2 \pi \alpha_{w}\left(L_{9 L}+L_{9 R}+L_{10}\right),
$$

where the $L_{i}$ are dimensionless parameters analogous to the Gasser-Leutwyler parameters of low energy QCD [55]. Naively putting in the QCD values, we find

$$
\Delta \kappa_{\gamma} \sim-3 \times 10^{-3} .
$$

A deviation of this size cannot be seen at LEP or the Tevatron. It is close to the expected error from the LHC. However, a $500 \mathrm{GeV} e^{+} e^{-}$collider can reach this sensitivity by the precision study of $e^{+} e^{-} \rightarrow W^{+} W^{-}$, as we will discuss in Section 5.5 .

For the top quark, somewhat larger effects are expected, specifically in the $Z \bar{t} t$ coupling. As we noted in Section 4.1, it is already a problem for these models that the decay width for $Z \rightarrow b \bar{b}$ agrees with the Standard Model. However, models can contain several competing effects which add destructively in the $Z \bar{b} b$ coupling but constructively in the $Z \bar{t} t$ coupling [56-58]. In that case, $5-10 \%$ corrections to the $Z \bar{t} t$ coupling would be expected. These would produce corrections to the cross section for $e^{+} e^{-} \rightarrow t \bar{t}$ which would be observed through the measurement of this cross section at a $500 \mathrm{GeV} e^{+} e^{-}$collider. We will discuss the program of precision measurements of anomalous top quark couplings in Section 5.3.

In the past few years, there has been a theoretical preference for supersymmetry and other weakly-coupled models of electroweak symmetry breaking. If supersymmetric particles are not discovered at the LHC, this situation will change dramatically. 
In that case, anomalous $W$ and $t$ coupling measurements at an $e^{+} e^{-}$collider will be among the most central issues in high-energy physics.

\subsection{What if the LHC sees no new physics?}

Though we expect that the LHC will reveal a rich spectrum of new particles, it is possible that the LHC will see no new phenomena. How could the LHC see no sign of the interactions responsible for electroweak symmetry breaking? The LHC should not fail to find supersymmetry if it exists. The LHC, at full luminosity, should be sensitive to resonances in $W W$ scattering beyond the limit set by $s$-channel unitarity. Thus, if the LHC fails to find signatures of electroweak symmetry breaking, it will not be because this collider does not have high enough energy. The scenarios in which the LHC fails - which, we emphasize, are very special scenarios occupying a tiny volume of typical parameter spaces - are those in which there is a light Higgs boson that does not have the decay modes important for detection at the LHC.

A Higgs boson with mass larger than about $150 \mathrm{GeV}$ has a large production cross section from $W W$ fusion and a substantial branching ratio to decay back to $W W$. Even if the $h W W$ coupling is diluted as described below, it is hard for us to imagine that this signature will not be seen at the LHC.

But for Higgs bosons with mass below $150 \mathrm{GeV}$, it is possible that there are new particles with masses tuned so that their loop contributions to the $h \gamma \gamma$ coupling cancel the Standard Model contribution. This can happen, for example, at specific points in the parameter space of the Minimal Supersymmetric Standard Model [59]. It is also possible that a substantial fraction of the Higgs decays are to invisible final states such as $\widetilde{\chi}_{1}^{0} \widetilde{\chi}_{1}^{0}$. Finally, if there are several neutral Higgs fields, each of which has a vacuum expectation value, the strength of the squared $h W W$ coupling for any individual field will be divided by the number of fields participating. Any of these three possibilities would compromise the ability of the LHC experiments to find and study the Higgs boson. The ability of an $e^{+} e^{-}$collider to see the Higgs boson does not depend on the Higgs decay pattern, but only on measurement of missing mass recoiling against a produced $Z^{0}$ boson. Thus, a $500 \mathrm{GeV} e^{+} e^{-}$collider would be the ideal instrument to study the Higgs boson under these special circumstances, as discussed in Section 5.1.

There is another way that the LHC could 'discover nothing' which we must confront. It could be that the Standard Model is correct up to a mass scale above $10^{16}$ $\mathrm{GeV}$, and that the only new physics below that scale is one standard Higgs boson. This conclusion would be extremely vexing, because it would imply that the reason for the spontaneous breaking of electroweak symmetry and the values of the quark and lepton masses could not be understood as a matter of principle. In that case, before giving up the quest for a fundamental theory, we should search in detail for non-standard properties of the observed Higgs boson. We will show in Section 5.1 that this study is ideally done at an $e^{+} e^{-}$linear collider. In this scenario, the mass 
of the Higgs boson must lie in a narrow window between 140 and $180 \mathrm{GeV}$, so an energy of $500 \mathrm{GeV}$ would be sufficient. The final confirmation of the Standard Model would be compelling only after the Higgs boson has passed all of the precision tests possible at an $e^{+} e^{-}$collider.

\section{$5 \quad$ Physics at a $500 \mathrm{GeV}$ linear collider}

We have argued in the previous section that there is a high probability that new physics associated with electroweak symmetry breaking will appear at a $500 \mathrm{GeV}$ $e^{+} e^{-}$collider. We have given two different arguments that the Higgs boson should appear in $e^{+} e^{-}$annihilation at this energy. For models with TeV-scale supersymmetry, it is likely that the lighter chargino and neutralino states can also be found. For models with strong-coupling electroweak symmetry breaking, important precision measurements on the $W, Z$, and top quark can be made at these energies. In this section, we will describe these experiments and estimate the accuracy they can achieve for the realistic luminosity samples set out in Section 3.

To introduce this discussion, we should recall the advantageous features of $e^{+} e^{-}$ collisions that have made them so useful in the past to provide a detailed understanding of the underlying physics. We will see that these features can also be used to great advantage in the experimental program for $500 \mathrm{GeV}$ :

- The cross sections for new Standard Model and exotic processes, and those of the dominant backgrounds, are all within about 2 orders of magnitude of one another (see Fig. 2.1). Thus, the desired signals have large production rates and favorable signal to background ratios. This situation contrasts with that at hadron colliders, where the interesting signals are typically very tiny fractions of the total cross section.

- Most of the interesting processes have simple two-body kinematics, from an initial state with well-defined quantum numbers.

- The cross sections for these processes are due to the electroweak interactions and can be predicted theoretically to part per mil accuracy.

- These processes also have known total energy and momentum at the level of the parton-parton interaction, with well understood and measurable smearing from initial-state radiation and beamstrahlung.

- The electron beam may be polarized, allowing selective suppression of backgrounds, separation of overlapping signals and measurement of parity-violating couplings. 


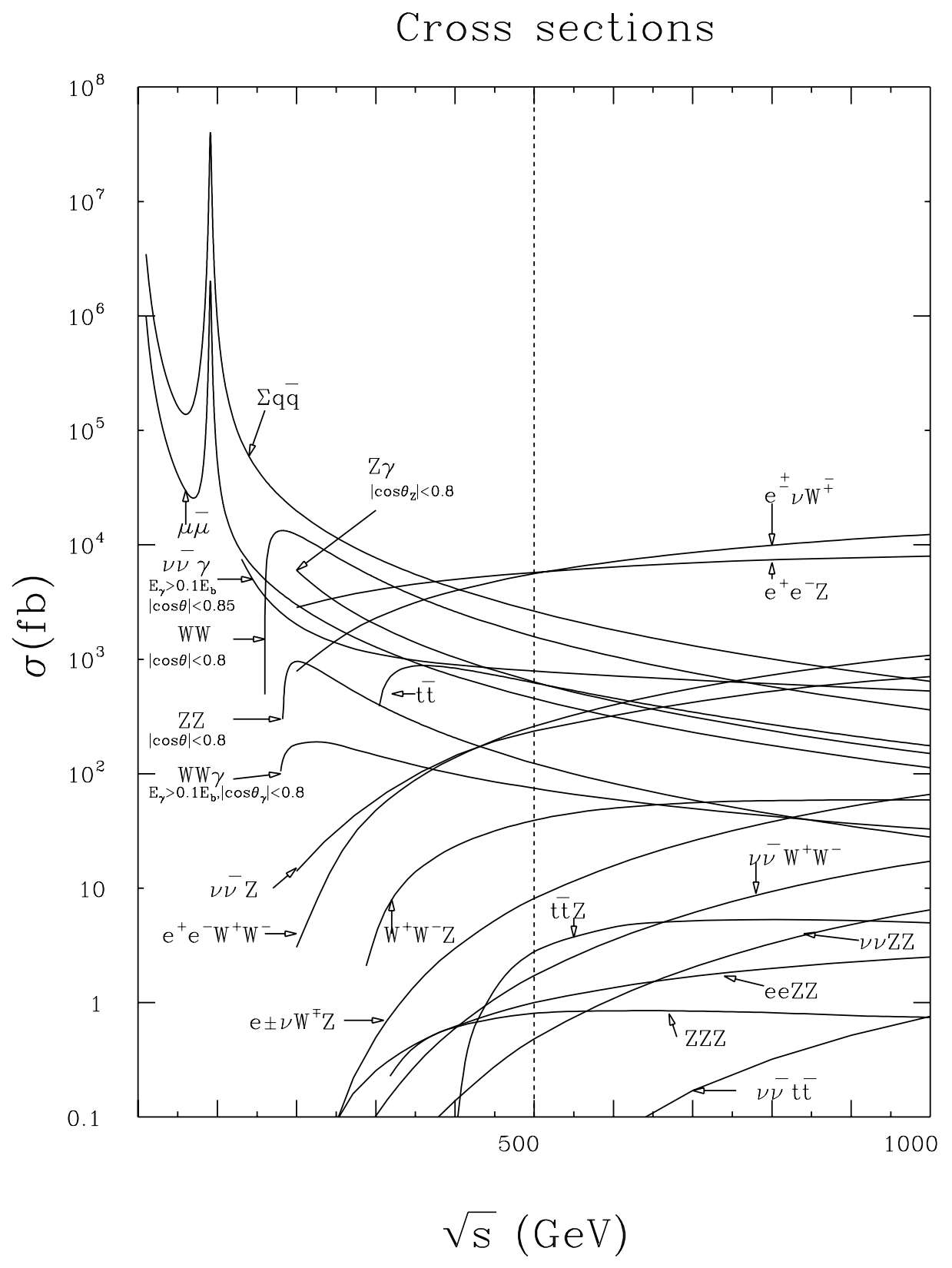

Figure 2.1: Cross sections for a variety of physics processes at an $e^{+} e^{-}$linear collider, from [60]. 
- The collider energy may be varied to optimize the study of particular reactions.

These features of $e^{+} e^{-}$collisions allow the study of heavy particles and their decays in many difficult circumstances, including detection of decays that are rare or have less distinct signatures, measurement of particle masses when some decays are invisible, measurement of spin, parity, $\mathrm{CP}$, and electroweak quantum numbers, measurement of widths and coupling constants, and measurement of mixing angles.

An extensive program studying physics at future high energy $e^{+} e^{-}$colliders has been carried out over the past few years as a collaborative effort of scientists in Europe, Asia, and America. In this section and the next, we will report on some highlights of that program. Much more detail on all of these studies can be found from the reviews $[1-4]$.

\subsection{Study of the Higgs boson}

The Higgs boson plays the central role in electroweak symmetry breaking and the generation of masses for quarks, leptons, and vector bosons. In the Standard Model, the Higgs boson is a simple scalar particle which couples to each fermion and boson species proportionately to its mass. Higher-order processes which couple the Higgs boson to $g g, \gamma \gamma$, and $\gamma Z^{0}$ add richness to its phenomenology. If the Standard Model is not correct, the surprises could come at many different points. Several scalar bosons could have large vacuum expectation values and thus could share responsibility for the $W$ and $Z$ masses. Different scalar bosons could be responsible for the up- and down-quark masses, or a different boson could produce the masses of third-generation fermions. These deviations from the standard picture might be large effects, or they might appear only in precision measurements.

One of the most remarkable features of the experimental environment of the linear collider is its ability to probe these issues directly. Each piece of information-from cross sections, angular distributions, and branching ratios - connects directly to a fundamental coupling of the Higgs particle. In this section, we will review how measurements at a linear collider can assemble a complete phenomenological profile of the Higgs boson.

It is almost certain that the Higgs boson will have been discovered before the linear collider begins operation. Results from LEP 2 presently imply that $m_{h} \geq 108$ $\mathrm{GeV}$ at the $95 \%$ confidence level [42]. It is expected that this limit will go up to about $115 \mathrm{GeV}$ as LEP 2 reaches its maximum energy. The Tevatron may be able to discover a Higgs boson up to about $180 \mathrm{GeV}$ [61]. This already covers most of the range of Higgs boson masses favored by the arguments of Section 4 .

The LHC studies have shown that a Higgs boson with the properties expected in the Standard Model can be discovered at that facility for any value of its mass. In addition, in models with an extended Higgs sector - for example, the Minimal

Supersymmetric Standard Model - the LHC should be able to find one and possibly 


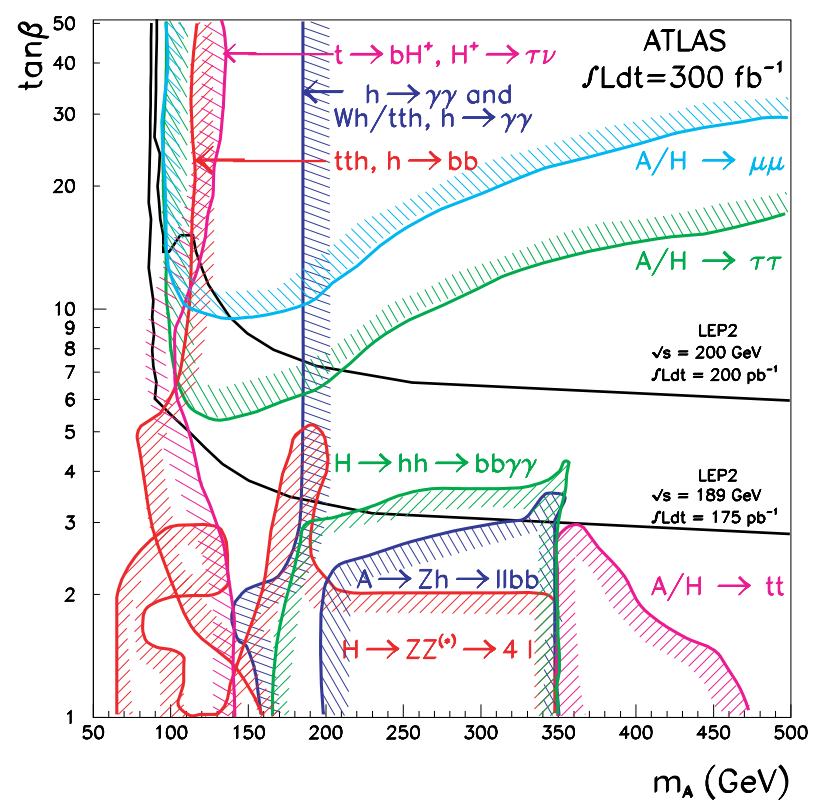

Figure 2.2: Capability of the ATLAS experiment to study the Higgs sector of the MSSM [62].

several of the Higgs particles. A recent summary of the LHC sensitivity to various MSSM Higgs processes is shown in Fig. 2.2. There are some regions of parameter space for which only one channel can be observed; in any case, it is typical that considerable luminosity is required for positive observation. In Section 4.6, we have noted some specific scenarios in which it is difficult to find the Higgs boson at the LHC. But, more generally, the LHC is limited in its ability to assemble a complete picture of the Higgs boson properties by the fact that Higgs boson production is such a tiny fraction of the LHC cross section that the Higgs particle must be reconstructed in order to study its production and decay.

\subsubsection{Discovery of the Higgs independent of its decay modes}

As a first step, we will argue that the Higgs boson can be found at a linear collider whatever its decay scheme might be. It is not necessary to reconstruct a Higgs boson to discover the particle or to measure its coupling to the $Z^{0}$. At low energies, the dominant Higgs production process in $e^{+} e^{-}$collisions is $e^{+} e^{-} \rightarrow Z^{0} h^{0}$, shown as the first diagram in Fig. 2.3. If the $Z^{0}$ is reconstructed from any one of its well-known decay modes, the Higgs is seen as a peak in the missing mass distribution recoiling against the $Z^{0}$. This detection is independent of the Higgs decay mode, visible or 


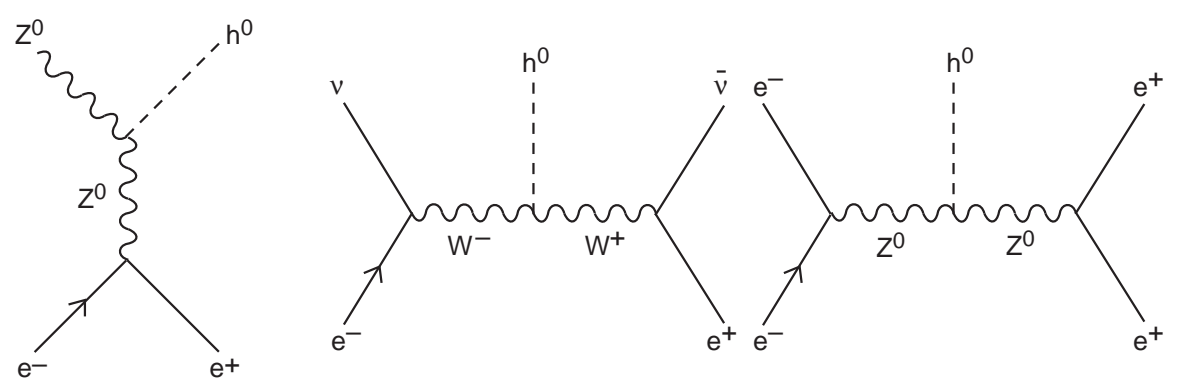

Figure 2.3: Processes for production of the Higgs boson at an $e^{+} e^{-}$linear collider.

invisible. Simulations show that this process is very clean, with minimal backgrounds. Figure 2.4 shows the expected signal of the Higgs boson using lepton, neutrino, and hadronic $Z$ decays for a $30 \mathrm{fb}^{-1}$ event sample [63].

The cross section for $Z^{0} h^{0}$ production depends on the magnitude of the $Z Z h$ coupling. Thus, the observation of the Higgs boson in this process measures the size of that coupling. If we replace the Higgs field $h^{0}$ by its vacuum expectation value, we see that this same coupling generates the mass of the $Z$ through the Higgs mechanism. Thus, determination of the absolute magnitude of the cross section for $e^{+} e^{-} \rightarrow Z^{0} h^{0}$ tests whether the observed $h^{0}$ generates the complete mass of the $Z^{0}$. Since Higgs measurements at the LHC require reconstruction of the Higgs boson, the LHC experiments can only measure ratios of couplings and cannot determine the $Z Z h$ coupling directly.

If there are several Higgs bosons contributing to the mass of the $Z^{0}$, the $e^{+} e^{-}$cross section for production of the lightest Higgs will be smaller, but heavier Higgs bosons must appear at higher values of the recoil mass. To discuss this quantitatively, let the coupling of the boson $h_{i}$ be $g_{Z Z i}$. (For simplicity, we assume that all of the $h_{i}$ are $S U(2)$ doublets; this assumption can be checked by searching for multiply-charged Higgs states.) Then the statement that the sum of the contributions from the vacuum expectation values of the $h_{i}$ generates the full mass of the $Z^{0}$ can be expressed as the sum rule [64]

$$
\sum_{i} g_{Z Z i}^{2}=4 m_{Z}^{4} / v^{2}
$$

where $v=246 \mathrm{GeV}$. With a $200 \mathrm{fb}^{-1}$ event sample at $500 \mathrm{GeV}$, Higgs particles $h_{i}$ can be discovered in recoil against the $Z^{0}$ down to a cross section of 0.2 of the Standard Model value for $m\left(h_{i}\right)=350 \mathrm{GeV}$, and below 0.01 of the Standard Model value for $m\left(h_{i}\right)=150 \mathrm{GeV}$ [3]. If all contributing Higgs bosons have masses below 150 $\mathrm{GeV}$, the sum rule can be checked in a $200 \mathrm{fb}^{-1}$ experiment to $5 \%$ accuracy, with dominantly statistical uncertainty. When we have saturated the sum rule (2.11), we will have discovered all of the Higgs states that contribute to the $Z^{0}$ mass. 

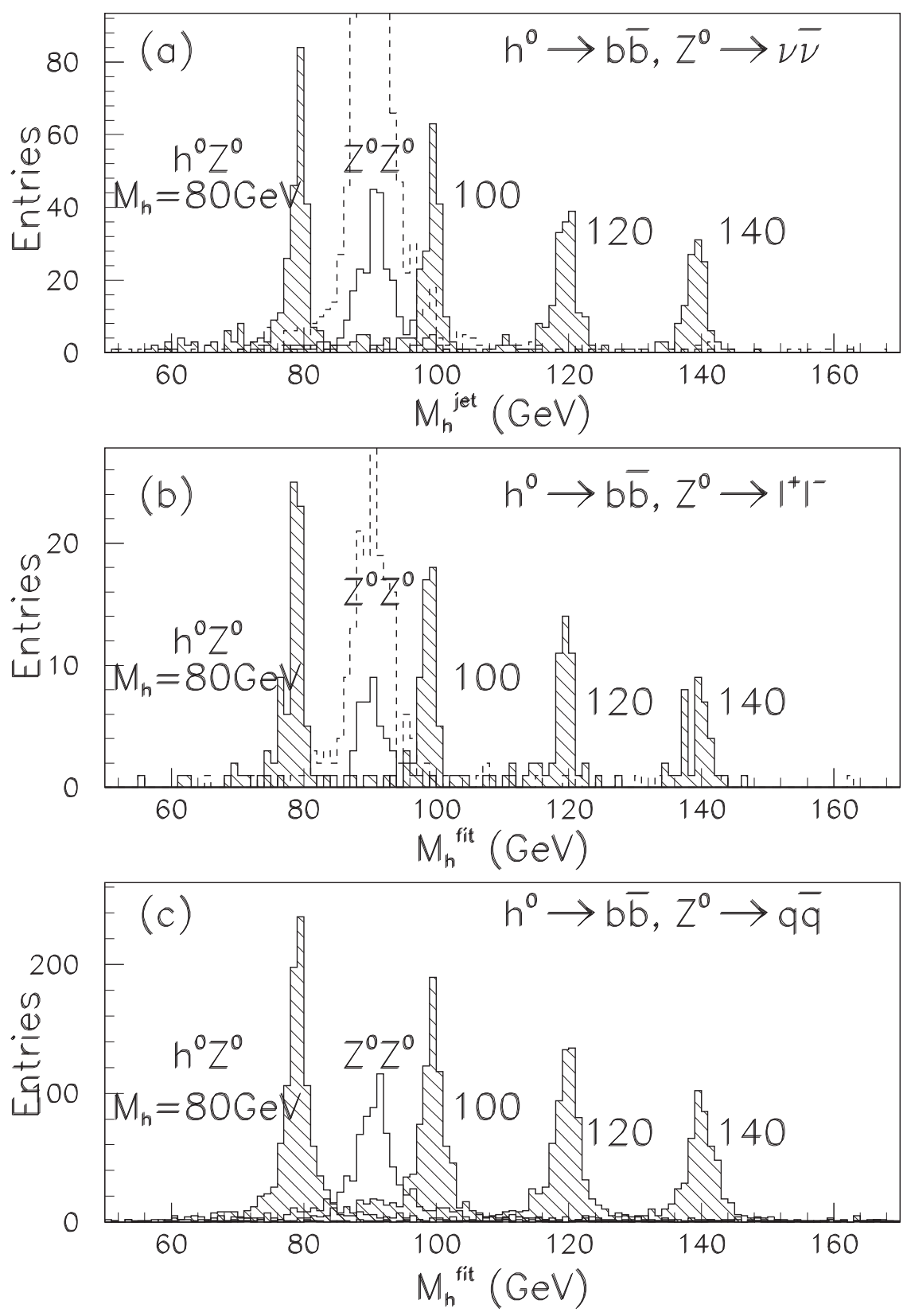

Figure 2.4: Higgs reconstruction in the process $e^{+} e^{-} \rightarrow Z^{0} h^{0}$ for various Higgs boson masses, using $\ell^{+} \ell^{-}, \nu \bar{\nu}$, and hadronic $Z^{0}$ decays, for a $30 \mathrm{fb}^{-1}$ event sample at $300 \mathrm{GeV}$, from [63]. The background is dominated by the process $e^{+} e^{-} \rightarrow Z^{0} Z^{0}$, which produces the missing-mass peak at $m_{Z}$. The unshaded solid histogram gives the background if a $b$-tag is applied to the Higgs candidate. The dashed histograms in (a) and (b) show the background with no $b$-tag. 
"The Case for a $500 \mathrm{GeV}$ Linear Collider"

\begin{tabular}{|cc|r|r|}
\hline \hline & & $200 \mathrm{fb}^{-1}$ & $500 \mathrm{fb}^{-1}$ \\
\hline \hline$\Delta \sigma_{Z H} / \sigma_{Z H}$ & & $4 \%$ & $3 \%$ \\
\hline \hline$\Delta \sigma_{H \nu \nu} B R(b \bar{b}) / \sigma_{H \nu \nu} B R(b \bar{b})$ & & $3 \%$ & $2 \%$ \\
\hline \hline$\Delta B R / B R$ & $b \bar{b}$ & $3 \%$ & $2 \%$ \\
& $W W^{*}$ & $8 \%$ & $5 \%$ \\
& $\tau^{+} \tau^{-}$ & $7 \%$ & $6 \%$ \\
& $c \bar{c}$ & $10 \%$ & $8 \%$ \\
& $g g$ & $8 \%$ & $6 \%$ \\
& $\gamma \gamma$ & $22 \%$ & $14 \%$ \\
\hline \hline
\end{tabular}

Table 2.3: Expected errors in branching ratio and coupling measurements for a Standard Model Higgs boson of mass $120 \mathrm{GeV}$, from measurements at $350 \mathrm{GeV}$.

\subsubsection{Measurement of the Higgs branching ratios}

The Higgs boson branching ratios are crucial indicators of nature of this particle, and of possible extensions beyond the Standard Model. The LHC can only make rough measurements of these, to about the $25 \%$ level, and only for some values of the Higgs boson mass $[62,65]$. Once the mass is known, it is straightforward at the linear collider to measure Higgs boson absolute branching fractions into two fermion or two gauge bosons for any of the production processes of Fig. 2.3 using the energy and momentum constraints. All decay modes of the $Z^{0}$ can be used in this study, even $Z^{0} \rightarrow \nu \bar{\nu}$ (20\% of the $Z^{0}$ total width) [66].

Methods for determining the Higgs cross sections to various decay channels have been studied recently in [66]. It is straightforward that the $b \bar{b}$ decays can be identified by vertex tagging. The studies show that $c \bar{c}$ decays can also be identified by vertex tagging with high efficiency, since the first layer of a vertex detector can be placed at about $1 \mathrm{~cm}$ from the interaction point. Multi-jet decays of the $h^{0}$ are typically $W W^{*}$. Table 3 gives a summary of the precision expected for a large variety of decay modes for the case of a $120 \mathrm{GeV}$ Higgs boson. This case is especially favorable in terms of the number of final states which are accessible, but it is also the value of the Higgs mass which is most probable in the Minimal Supersymmetric Standard Model. Expectations for Higgs branching ratio measurements at other values of the Higgs mass (assuming $500 \mathrm{fb}^{-1}$ at $350 \mathrm{GeV}$ ) are shown in Fig. 2.5 [66]. If the Standard Model Higgs mass approaches $200 \mathrm{GeV}$, the dominance of the $W W$ and $Z Z$ decays will render the fermionic decays progressively more difficult to observe.

The Higgs branching ratios directly address the question of whether the Higgs boson generates the masses of all Standard Model particles. If the vacuum expectation value of $h^{0}$ produces the fermion masses, the couplings of $h^{0}$ to $b, c$, and $\tau$ should be 


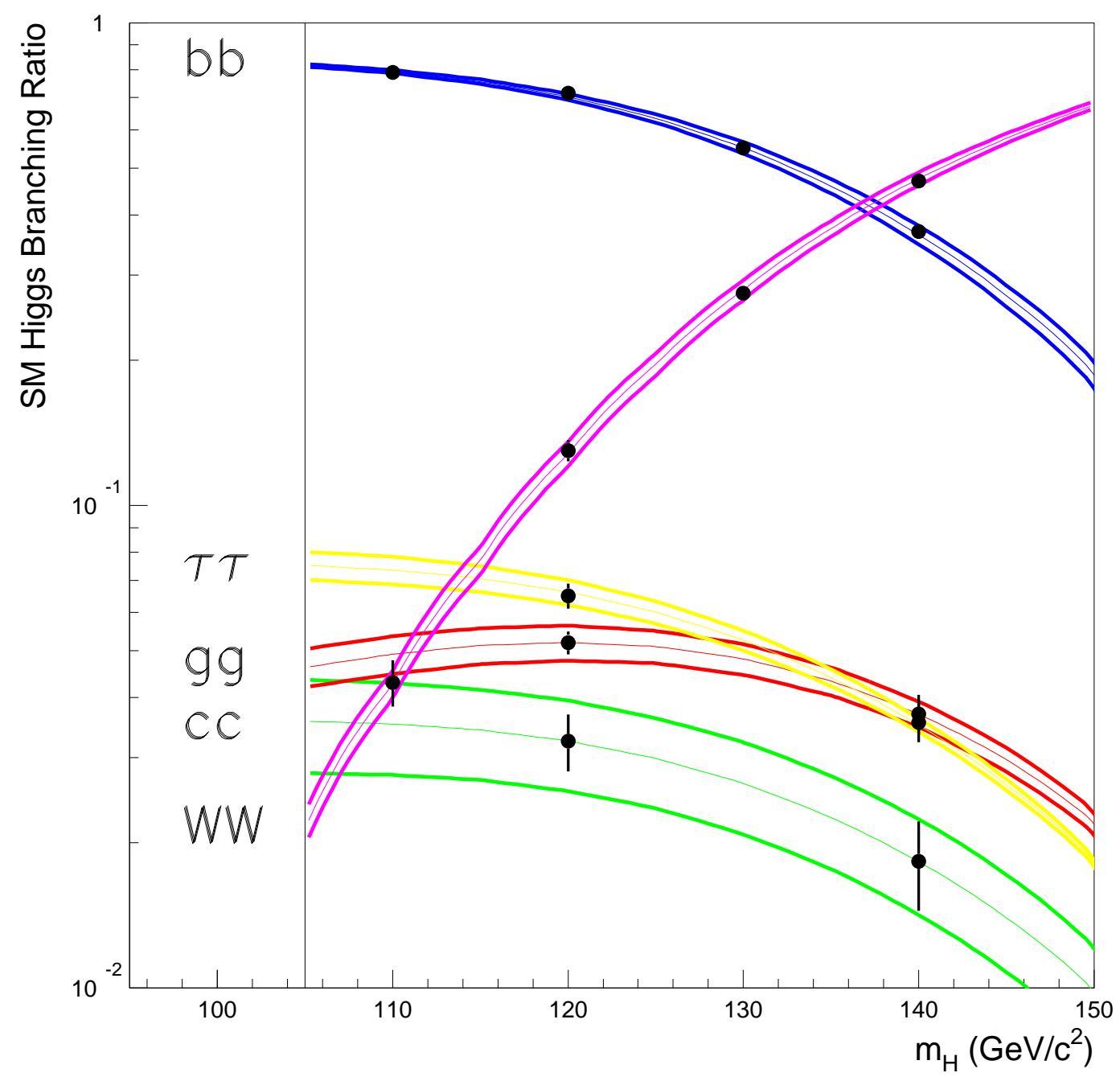

Figure 2.5: Determination of Higgs boson branching ratios in a variety of decay modes, from [66]. The error bars show the expected experimental errors for $500 \mathrm{fb}^{-1}$ at $350 \mathrm{GeV}$. The bands show the theoretical errors in the Standard Model predictions.

simply determined from the ratio of their masses. Similarly, the coupling of the $h^{0}$ to $W W$ or, for the case of a light Higgs, to one on-shell and one off-shell $W$, measures the fraction of the $W$ mass due to the Higgs vacuum expectation value.

The Minimal Supersymmetric Standard Model includes an extended Higgs sector with two $S U(2)$ doublets. For the most general case of a two-Higgs-doublet model, vacuum expectation values of both Higgs fields contribute to the quark, lepton, and 
boson masses and the predictions for branching ratios differ qualitatively from those in the Standard Model. However, in the MSSM with heavy superpartners, one scalar boson $H^{0}$ is typically heavy and the orthogonal boson $h^{0}$, which must be light, tends to resemble the Higgs boson of the Standard Model. For example, the ratio of branching ratios to $b \bar{b}$ and $W W^{*}$ is corrected by the factor

$$
1+2 \cos ^{2} 2 \beta \sin ^{2} 2 \beta \frac{m_{Z}^{2}}{m_{H}^{2}}+\cdots .
$$

Nevertheless, accurate branching ratio measurements can distinguish the MSSM Higgs boson from the Standard Model Higgs boson over a large region of parameter space. From the results of [66], the $500 \mathrm{fb}^{-1}$ experiment discussed above would exclude corrections from the MSSM Higgs structure for $m_{A}$ up to at least $550 \mathrm{GeV}$. The linear collider determination of branching ratios is sufficiently accurate that the theoretical uncertainty in the charm quark mass is actually the dominant source of error. New approaches to the determination of the quark masses in lattice gauge theory should give more accurate values in the next few years [67] and thus improve the power of this measurement.

\subsubsection{Measurement of the Higgs boson width}

It will be critical to know the total width of the Higgs, $\Gamma_{\text {tot }}$, accurately. For a Higgs boson mass below $200 \mathrm{GeV}$, the total width is expected to be below $1 \mathrm{GeV}$, too small to be measured at the LHC or directly at the linear collider. To determine this width, one will need to combine an absolute measurement of a decay rate or coupling constant with the measurement of the branching ratio for the corresponding channel. The most promising method is to use the branching ratio to $W W^{*}$. The absolute size of the $W W h$ coupling can be determined either from the $S U(2) \times U(1)$ relation $g_{W W h}^{2} / g_{Z Z h}^{2}=\cos ^{2} \theta_{w}$ or, in a more model-independent way, from the cross section for $h^{0}$ production by the $W W$ fusion process shown as the second diagram in Fig. 2.3. (The $Z Z$ fusion process is expected to add only a small contribution.) From Table 3, the Higgs branching ratio to $W W^{*}$ gives the dominant source of error in this measurement.

If the $\gamma \gamma$ collider option is realized by backscattering polarized laser light off the $e^{ \pm}$beams, then the process $\gamma \gamma \rightarrow h^{0}$ can be used to measure the absolute partial width $\Gamma\left(h^{0} \rightarrow \gamma \gamma\right)$. This width, which can be determined to about $5 \%$ accuracy with a $200 \mathrm{fb}^{-1}$ dedicated experiment [68], is of great interest in its own right, since it measures a sum of contributions from all heavy charged particles that couple to the $h^{0}$. 


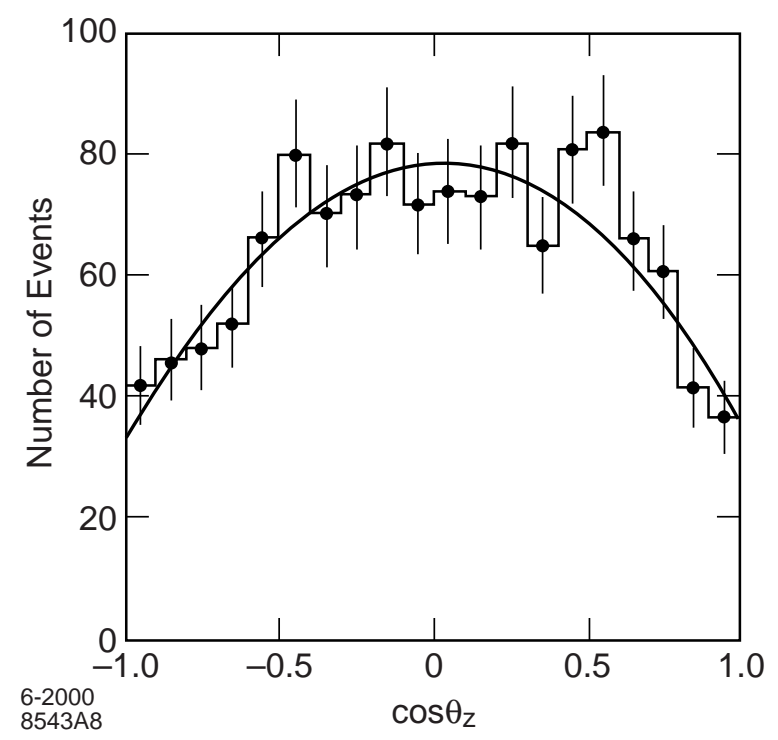

Figure 2.6: Angular distribution of the $Z$ boson in $e^{+} e^{-} \rightarrow Z^{0} h^{0}$, as reconstructed from a $50 \mathrm{fb}^{-1}$ event sample at $300 \mathrm{GeV}$, from [69].

\subsubsection{Measurement of the spin-parity and CP of the Higgs boson}

It will be essential to determine the quantum numbers of an observed Higgs boson unambiguously. The LHC can rule out spin 1 if the decay $H \rightarrow \gamma \gamma$ is observed. If the decay $H \rightarrow Z Z$ is observed, spin 0 and 1 could be distinguished at the LHC, but the $\mathrm{CP}$ quantum numbers will be difficult to determine in any case. The linear collider will thus be needed to determine the Higgs quantum numbers.

If the Higgs field has a vacuum expectation value, it must be a CP-even spin-0 field. Thus, a Higgs boson produced in $e^{+} e^{-} \rightarrow Z^{0} h^{0}$ with a rate comparable to the Standard Model rate must have these quantum numbers. However, there are a number of checks on these properties that are available from the kinematics of Higgs production. In the limit $s \gg m_{Z}^{2}, m_{h}^{2}$, a scalar Higgs boson produced in this reaction has an angular distribution

$$
\frac{d \sigma}{d \cos \theta} \sim \sin ^{2} \theta
$$

and the $Z^{0}$ recoiling against it is dominantly longitudinally polarized, and so that distribution in the decay angle peaks at central values. (For a CP-odd scalar, these distributions differ qualitatively.) If the center of mass energy is not asymptotic, the corrections to these relations are predicted from kinematics. For example, Fig. 2.6 shows a simulation of the angular distribution at $300 \mathrm{GeV}$ and a comparison to the distribution expected for a Higgs scalar.

The production of the Higgs boson in $\gamma \gamma$ collisions goes through a loop diagram 
which can give both scalar and pseudoscalar couplings. Thus, the $\gamma \gamma$ collider option offers a nontrivial test of $\mathrm{CP}$ violation. With longitudinal $\gamma$ polarization, the asymmetry of Higgs production cross sections

$$
A_{\gamma}=\frac{\sigma\left(\gamma_{L} \gamma_{L}\right)-\sigma\left(\gamma_{R} \gamma_{R}\right)}{\sigma\left(\gamma_{L} \gamma_{L}\right)+\sigma\left(\gamma_{R} \gamma_{R}\right)}
$$

vanishes for pure scalar or pseudoscalar coupling to $\gamma \gamma$ but is nonzero if the Higgs is a mixture of CP eigenstates. Models with CP violation in the top sector can give $10 \%$ or larger asymmetries [70]. In models with extended Higgs sectors, this polarization asymmetry can incisively separate the heavy scalar and pseudoscalar Higgs resonances [71].

\subsubsection{Measurement of the Higgs self couplings}

The Higgs self-couplings are uniquely fixed in the Standard Model in terms of the Higgs field expectation value $v$; in the minimal supersymmetric model, they depend on the Higgs field couplings and mixings. Measuring the self-couplings is a crucial step in checking the consistency these models, and it gives added information on the parameters of supersymmetric models. It appears that observation of Higgs pair production at the LHC will be very difficult due to the dominance of gluon fusion production and large QCD backgrounds [72]. In $e^{+} e^{-}$collisions, production of two Higgs bosons in the final state can occur for any of the diagrams of Fig. 2.4 by radiating an additional Higgs from any of the gauge boson legs, or through the trilinear Higgs coupling. The cross sections for production of a pair of Higgs bosons with an associated $Z$ boson have been calculated to be of order $0.5 \mathrm{fb}$ for $m_{h}=110 \mathrm{GeV}$ at $\sqrt{s}=500 \mathrm{GeV}$ in the Standard Model [73]. Cross sections for various supersymmetric Higgs pair-production processes are comparable for much of the supersymmetric parameter space. The final state of $Z h h$, with both Higgs bosons observed as $b \bar{b}$, should provide a detectable signature without large backgrounds, yielding a precision on the trilinear Higgs coupling of roughly $25 \%$ for $600 \mathrm{fb}^{-1}$.

\subsection{Studies of supersymmetry}

In Section 4, we argued that the new physics at the TeV energy scale is likely to be a supersymmetric extension of the Standard Model. If supersymmetric particles appear at the next step in energy, they will provide a rich field for experimental study. This study will address two separate and important issues. First, supersymmetry entails a fundamental modification of the structure of space-time. Supersymmetry can be described as the statement that spinors and fermions are an integral part of space-time geometry, or, alternatively, that there are new space-time dimensions which are fermionic in character. It requires new gravitational equations that include 
a spin- $\frac{3}{2}$ partner of the graviton. If we are to claim that Nature has this structure, we must to prove it experimentally by demonstrating the quantum number assignments and symmetry relations that this structure requires.

Second, phenomenological models with supersymmetry introduce a large number of new physical parameters. The masses of supersymmetric particles, and other parameters associated with spontaneous supersymmetry breaking, are not fixed from currently known principles but, rather, must be determined experimentally. The most general description of supersymmetry breaking even in the 'Minimal' Supersymmetric Standard Model contains 105 parameters. Each explicit model of spontaneous supersymmetry breaking gives predictions for these parameters or relations among them. But there is no 'Standard Model' of supersymmetry breaking. In the literature, one finds at least three general approaches - gravity-, gauge-, and anomaly-mediationeach of which has numerous variants. Each approach is derived from assumptions about new physics at a higher energy scale, which ranges from $10^{5}$ to $10^{19} \mathrm{GeV}$ depending on the model. The various models predict mass spectra and mixing parameters that differ characteristically. These observables provide clues to the nature of physics at extremely short distances, possibly even to the truly fundamental physics at the scale of grand unification or quantum gravity [74].

Supersymmetric particles may well be discovered in Run II of the Tevatron. In any case, if supersymmetry is relevant to electroweak symmetry breaking, supersymmetric particles should surely be found at the LHC. The LHC collaborations have demonstrated that they would be sensitive to quark and gluon superpartners up to masses of at least $2 \mathrm{TeV}$. For the gluino, this reach goes about a factor of 2 beyond the fine-tuning limits given in Table 2. Reactions which produce the squarks and gluinos also produce the lighter supersymmetric particles into which they decay. The ATLAS and CMS collaborations have presented some striking analyses at specific points in the parameter space of mSUGRA models in which 3 to 5 mass parameters can be determined from kinematics. From this information, the four parameters of the mSUGRA model can be determined to $2-10 \%$ accuracy $[62,75]$.

Ultimately, though, hadron colliders are limited in their ability to probe the underlying parameters of supersymmetric models. Because the LHC produces many SUSY particles and observes many of their decay chains simultaneously, it is difficult to isolate parameters and determine them in a model-independent way. It is difficult to determine the spin and electroweak quantum numbers of particles unambiguously. And, only limited information can be obtained about the heavier color-singlet particles, including sleptons and heavier charginos and neutralinos, and about the unobserved lightest neutralino.

It is just for these reasons that one needs a facility that can approach the spectroscopy of supersymmetric particles from an orthogonal direction. An $e^{+} e^{-}$collider can study supersymmetric particles one at a time, beginning with the lightest and working upward to particles with more complex decay patterns. For each particle, 
the measurements go well beyond simple mass determinations. We will give a number of illustrative examples in this section.

To carry out these measurements, it is only necessary that supersymmetric particles can be pair-produced at the energy provided by the $e^{+} e^{-}$collider. In the program that we have presented in Section 2, in which a collider with an initial energy of 500 $\mathrm{GeV}$ evolves to higher center of mass energies, one can eventually create the full set of supersymmetry particles. Here we concentrate on the expectations for $500 \mathrm{GeV}$. In Section 4.4, we have argued that the lightest charginos and neutralinos, the supersymmetric partners of the photon, $W, Z$, and Higgs bosons, should be produced already at the initial $500 \mathrm{GeV}$ stage. The mSUGRA models discussed in Section 4.4 do not place such strong constraints on the masses of lepton superpartners, but in other schemes of supersymmetry breaking, such as gauge-mediation and the noscale limit of gravity-mediation, it is natural for the sleptons to be as light as the charginos. Because the experimental study of sleptons is conceptually very simple, we will present the linear collider experimental program for sleptons in this section along with our discussion of charginos. Other issues for the experimental study of supersymmetry will be discussed in Section 6.2.

Our discussion of the basic supersymmetry measurements in this section will be rather detailed. In reading it, one should keep in mind that the linear collider offers a similar level of detailed information for any other new particles that might appear in its energy range.

\subsubsection{Slepton mass measurement}

The simple kinematics of supersymmetric particle pair production allows direct and accurate mass measurements. The technique may be illustrated with the process of pair production and decay of the $\widetilde{\mu}_{R}^{-}$, the scalar partner of the $\mu_{R}^{-}$. The process $e^{+} e^{-} \rightarrow \widetilde{\mu}_{R}^{-} \widetilde{\mu}_{R}^{+}$produces the sleptons at a fixed energy equal to the beam energy. The $\widetilde{\mu}_{R}^{-}$is expected to decay to the unobserved lightest neutralino via $\widetilde{\mu}_{R}^{-} \rightarrow \mu^{-} \widetilde{\chi}_{1}^{0}$. Then the final muons are distributed in energy between kinematic endpoints determined by the masses in the problem. Since the $\widetilde{\mu}_{R}^{-}$is a scalar, the distribution of muons is isotropic in the $\widetilde{\mu}_{R}^{-}$rest frame and flat in energy in the lab frame. Thus, the observed energy distribution of muons has the shape of a rectangular box, and the masses of both the $\widetilde{\mu}_{R}^{-}$and the $\widetilde{\chi}_{1}^{0}$ can be read off from the positions of the edges.

In measuring slepton pair production in $e^{+} e^{-}$collisions, special attention must be paid to the backgrounds from two-photon processes in which the primary scattered electrons are undetected within the beam pipes. This makes it important for the detector to have good coverage at forward and backward angles. It may be useful for

gaining further control over this process to provide tagging detectors at very small angles [76].

On the left side of Fig. 2.7, we show simulation results for $\widetilde{\mu}_{R}$ pair production 

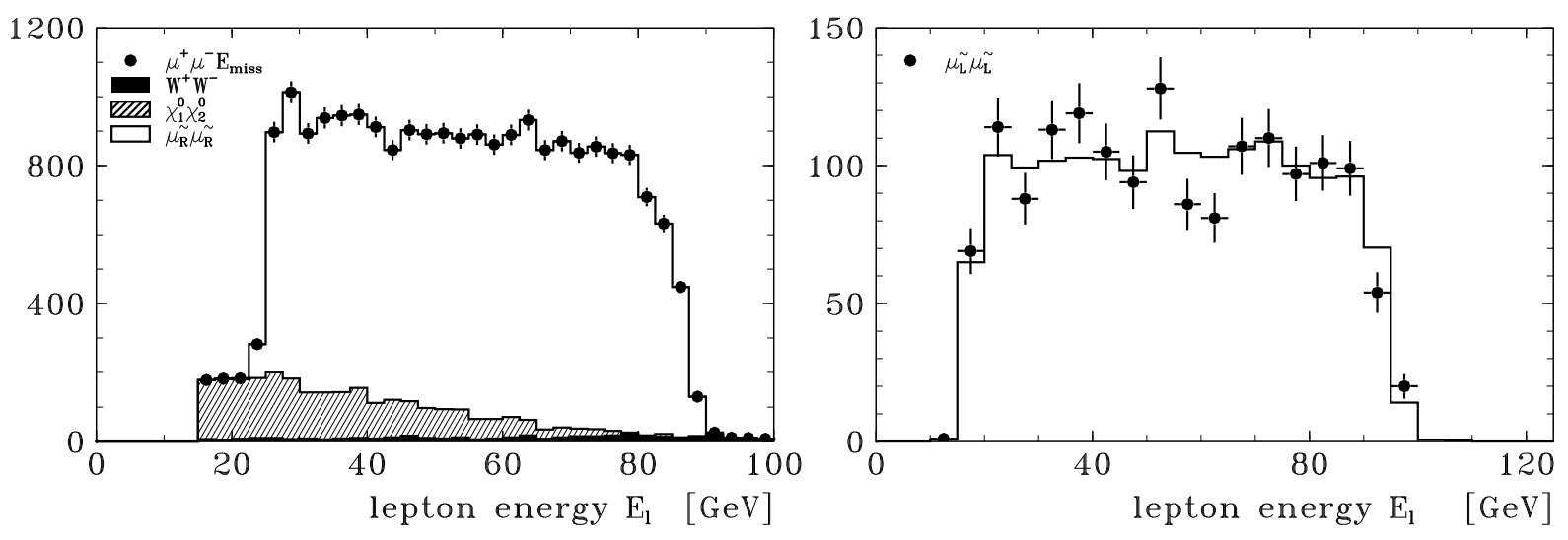

Figure 2.7: Energy distribution of muons resulting from processes $e^{+} e^{-} \rightarrow \widetilde{\mu}^{-} \widetilde{\mu}^{+}$, followed by $\widetilde{\mu}$ decay, from [77]. left: $e^{+} e^{-} \rightarrow \widetilde{\mu}_{R}^{-} \widetilde{\mu}_{R}^{+}$, for a $160 \mathrm{fb}^{-1}$ event sample at $320 \mathrm{GeV}$; right: $e^{+} e^{-} \rightarrow \widetilde{\mu}_{L}^{-} \widetilde{\mu}_{L}^{+}$, with selection of $\widetilde{\mu}_{L} \rightarrow \mu \widetilde{\chi}_{2}^{0}, \widetilde{\chi}_{2}^{0} \rightarrow \ell^{+} \ell^{-} \widetilde{\chi}_{1}^{0}$ decays on both sides, for a $250 \mathrm{fb}^{-1}$ event sample at $500 \mathrm{GeV}$. The electron beam polarization is used to reduce the background from $e^{+} e^{-} \rightarrow W^{+} W^{-}$.

[77]. The dominant background (shaded in the figure) comes from other supersymmetry processes. The rounding of the rectangle on its upper edge is the effect of beamstrahlung and initial state radiation. The simulation predicts a measurement of both the $\widetilde{\mu}_{R}^{-}$and the $\widetilde{\chi}_{1}^{0}$ masses to $0.2 \%$ accuracy. The right side of Fig. 2.7 shows the muon energy distribution from pair production of the $\widetilde{\mu}_{L}^{-}$, the partner of the $\mu_{L}^{-}$. Decays of the form $\widetilde{\mu}_{L} \rightarrow \mu \widetilde{\chi}_{2}^{0}, \widetilde{\chi}_{2}^{0} \rightarrow \ell^{+} \ell^{-} \widetilde{\chi}_{1}^{0}$ are selected on both sides of the event to obtain a very clean 6 lepton signature. Despite the low statistics from the severe event selection, this analysis also gives the $\widetilde{\mu}_{L}^{-}$and the $\widetilde{\chi}_{2}^{0}$ masses to $0.2 \%$ accuracy. At the LHC, the mass of the lightest neutralino $\tilde{\chi}_{1}^{0}$ typically cannot be determined directly, and the masses of heavier superparticles are determined relative to the $\tilde{\chi}_{1}^{0}$ mass. So not only do the $e^{+} e^{-}$measurements provide model-independent slepton masses, they also provide crucial information to make the superpartner mass measurements from the LHC more model-independent.

The same strategy can be applied to determine the masses of other superpartners. Examples with sneutrinos, scalar top, and charginos are shown in [78]. Even higher accuracies can be obtained by scanning the $e^{+} e^{-}$cross section near each pair production threshold. This costs about $100 \mathrm{fb}^{-1}$ per threshold, but it allows particle mass measurements to better than 1 part per mil [77]. 


\subsubsection{Slepton properties}

An $e^{+} e^{-}$collider can not only measure the masses of superparticles but also can determine many more properties of these particles, testing predictions of supersymmetry from the most qualitative to the most detailed.

Before anything else, it is important to verify that particles that seem to be sleptons are spin 0 particles with the Standard Model quantum numbers of leptons. A spin 0 particle has a characteristic angular distribution in $e^{+} e^{-}$annihilation, proportional to $\sin ^{2} \theta$. Even though there are missing neutralinos in the final state of $e^{+} e^{-} \rightarrow \widetilde{\mu}^{-} \widetilde{\mu}^{+}$, there are enough kinematic constraints that the angular distribution can be reconstructed [79]. The magnitude of the cross section can be computed for each electron polarization with typical electroweak precision; it depends only on the Standard Model quantum numbers of the produced particle and thus determines these quantum numbers.

A major issue in supersymmetry is the flavor-dependence of supersymmetry breaking parameters. Using the endpoint technique above, the selectron and smuon masses can be compared at a level below the 1 part per mil level. It is somewhat more difficult to study the superpartners of the $\tau$, but even in this case the masses can be found to percent accuracy by locating the endpoint of the energy distribution of stau decay products [80].

It is typical in supersymmetry scenarios with large $\tan \beta$ that the superpartners of $\tau_{R}^{-}$and $\tau_{L}^{-}$mix, and that the lighter mass eigenstate is actually the lightest slepton. If the mass difference between the lighter stau and the other leptons is significant, this can create a problem for the study of supersymmetry at LHC, since then supersymmetry decay cascades typically end with $\tau$ production. A parameter point studied by the ATLAS supersymmetry group illustrates the problem [62]. We have just noted that there is no difficulty in measuring the stau masses at a linear collider. In addition, since the production cross section depends only on electroweak quantum numbers, it is possible to determine the mixing angle from total cross section and polarization asymmetry measurements. The characteristic dependence of the polarization asymmetry on the stau mixing angle is shown in Fig. 2.8. The final state $\tau$ polarization provides another diagnostic observable which can be used to analyze the composition of the stau or of the neutralino into which it decays [80].

The cross section for production of the electron partners is somewhat more complicated, because this process can proceed both by $e^{+} e^{-}$annihilation and by the exchange of neutralinos, as shown in Fig. 2.9. In typical models, the dominant contribution actually comes from exchange of the lightest neutralino. Thus, the selectron production cross section can give further information on the mass and the properties of this particle. The study of neutralinos is complicated by the fact that the various neutralino species can mix. In the Section 5.2.4, we will discuss this mixing problem and present methods for resolving it experimentally using $e^{+} e^{-}$data on chargino 


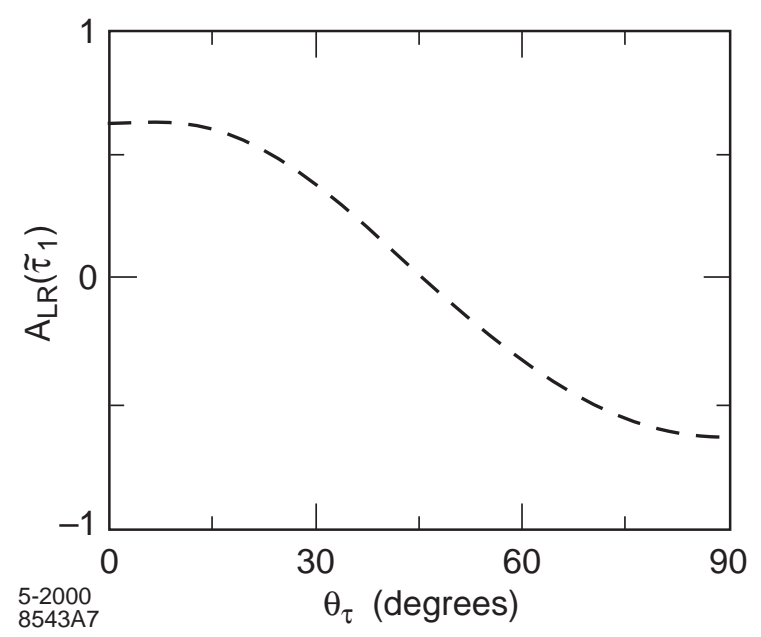

Figure 2.8: Polarization asymmetry of $e^{+} e^{-} \rightarrow \widetilde{\tau}_{1}^{+} \widetilde{\tau}_{1}^{-}$as a function of the stau mixing angle.

(a)

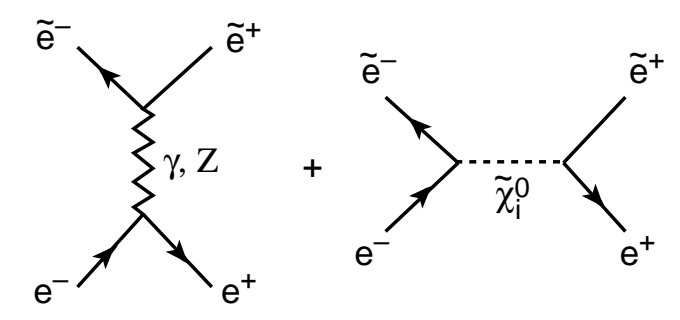

(b)

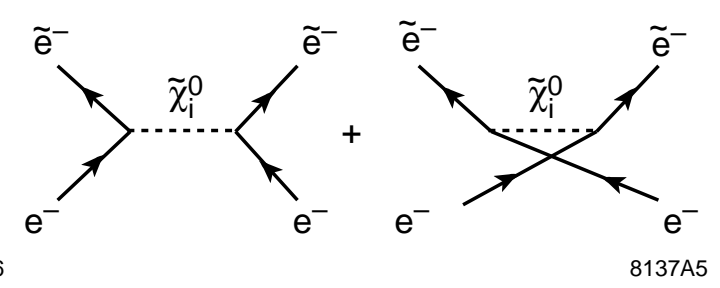

Figure 2.9: Diagrams contributing to selectron pair production: (a) $e^{+} e^{-} \rightarrow \widetilde{e}^{+} \widetilde{e}^{-}$, (b) $e^{-} e^{-} \rightarrow \widetilde{e}^{-} \widetilde{e}^{-}$.

production. Neutralino mixing can also be studied in selectron pair production; an illustrative analysis is given in [79].

Once the mixing of neutralinos is understood, the selectron pair production can test the basic idea of supersymmetry quantitatively, by testing the symmetry relation of coupling constants. For simplicity, consider a model in which the lightest neutralino is the superpartner $\widetilde{b}$ of the $U(1)$ gauge boson of the Standard Model, and imagine comparing the processes of $\widetilde{e}_{R}$ pair production and Bhabha scattering, as illustrated in Fig. 2.10. By supersymmetry, the coupling constant at the $e \widetilde{e} \widetilde{b}$ vertex must be simply related to the $U(1)$ electroweak coupling: $g_{\tilde{b}_{\tilde{e}} e}=\sqrt{2} g^{\prime}$. A measurement of 

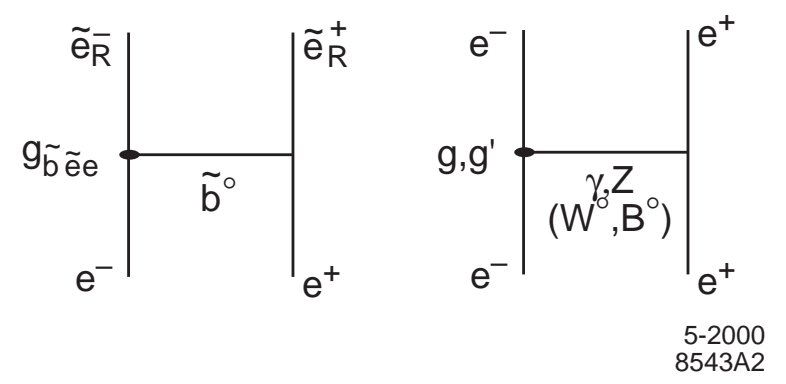

Figure 2.10: Comparison of the weak interaction coupling $g^{\prime}$ and its supersymmetric counterpart $g_{\tilde{b} \tilde{e}_{R}} e^{\text {. }}$

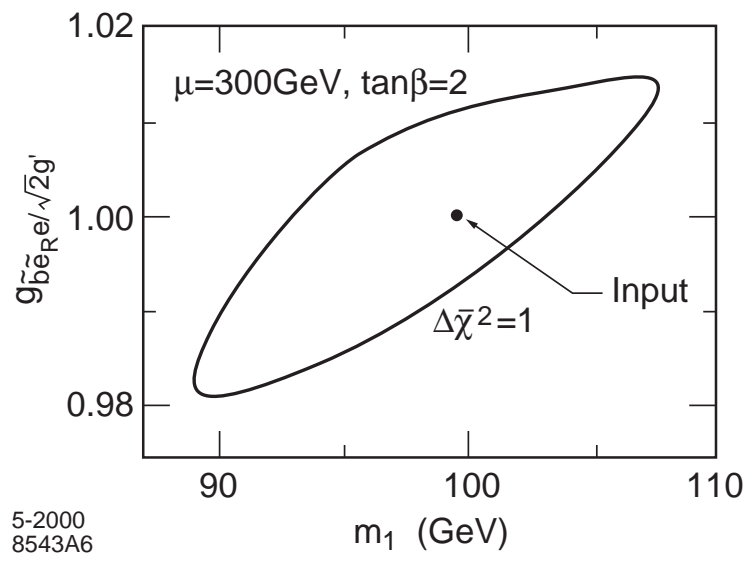

Figure 2.11: Determination of the $g_{\tilde{b} \tilde{e}_{R} e}$ coupling from a $100 \mathrm{fb}^{-1}$ measurement of selectron pair production, from [80].

the forward cross section for $e^{+} e^{-} \rightarrow \widetilde{e}_{R}^{+} \widetilde{e}_{R}^{-}$can give a precision test of this prediction.

Detailed simulation of selectron pair production has shown that the ratio $g_{\tilde{b} \tilde{e} e} / \sqrt{2} g^{\prime}$ can be measured to a precision of about 1\%, as shown in Fig. 2.11 [80]. (This analysis uses data from the same cross section measurement both to fix the parameters of the neutralino mixing and to determine $g_{\tilde{b} \tilde{e} e}$.) Even higher accuracy can be achieved by studying selectron production in $e^{-} e^{-}$collisions. The ratio $g_{\tilde{W} \tilde{\nu} e}$ can also be determined from chargino pair production and compared to its Standard Model counterpart to about $2 \%$ accuracy. At these levels, the measurement would not only provide a stringent test of supersymmetry as a symmetry of Nature, but also it might be sensitive to radiative corrections from heavy squark and slepton species [81-83]. 

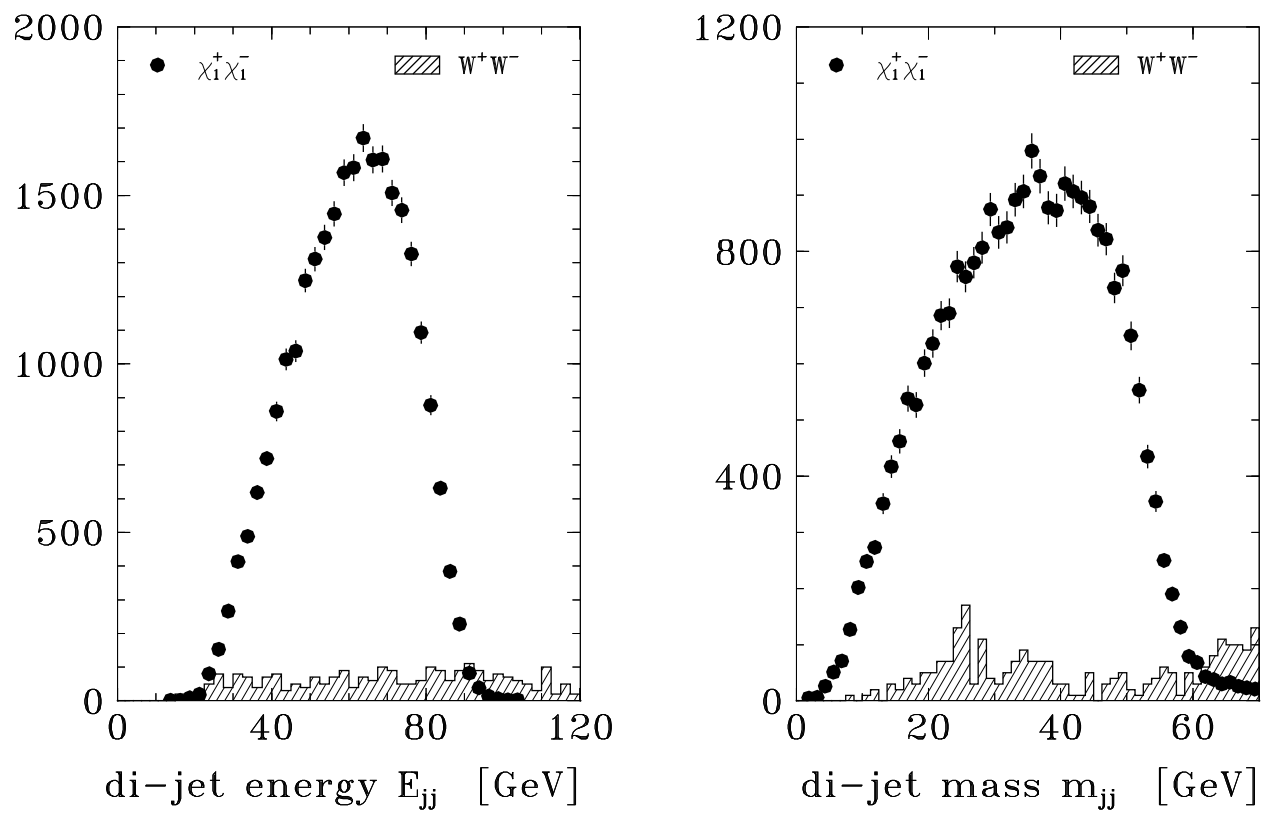

Figure 2.12: Kinematic distributions from a simulation of chargino pair production and decay with $160 \mathrm{fb}^{-1}$ at $320 \mathrm{GeV}$, from [77]. left: dijet energy distribution; right: dijet mass distribution.

\subsubsection{Chargino mass measurement}

The process of chargino pair production in $e^{+} e^{-}$annihilation is somewhat more complicated than slepton pair production, but it also provides more interesting observables. To begin, we discuss the chargino mass measurement. If the chargino is the lightest charged supersymmetric particle, it will decay via $\widetilde{\chi}_{1}^{+} \rightarrow q \bar{q} \widetilde{\chi}_{1}^{0}$ or $\widetilde{\chi}_{1}^{+} \rightarrow \ell^{+} \nu \widetilde{\chi}_{1}^{0}$. The reaction with a hadronic decay on one side and a leptonic decay on the other provides a characteristic sample of events which can be distinguished from $W$ pair production by their large missing energy and momentum. If the lab frame energy of the $q \bar{q}$ system is measured, the kinematic endpoints of this distribution can be used to determine the mass of the $\widetilde{\chi}_{1}^{+}$and of the $\widetilde{\chi}_{1}^{0}$, as in the slepton case. The power of this kinematic fit can be strengthened by segregating events according to the measured value of the $q \bar{q}$ invariant mass. The distributions in the energy and mass of the $q \bar{q}$ system are shown in Fig. 2.12. In the study of [77], one finds mass determinations at the $0.2 \%$ level for event samples of the same size as those used in the slepton case.

At large $\tan \beta$ values, the lighter stau $\left(\tilde{\tau}_{1}\right)$ may be lighter than the lightest chargino $\left(\tilde{\chi}_{1}^{ \pm}\right)$. The decay $\tilde{\chi}_{1}^{ \pm} \rightarrow \tilde{\tau}_{1}^{ \pm} \nu_{\tau}$, followed by $\tilde{\tau}_{1}^{ \pm} \rightarrow \tilde{\chi}_{1}^{0} \tau^{ \pm}$, alters the phenomenology of the chargino production [80]. In this case, one can still measure the mass of a 170 $\mathrm{GeV}$ chargino to better than $5 \mathrm{GeV}$ with $200 \mathrm{fb}^{-1}$ at $\sqrt{s}=400 \mathrm{GeV}$ [84]. 


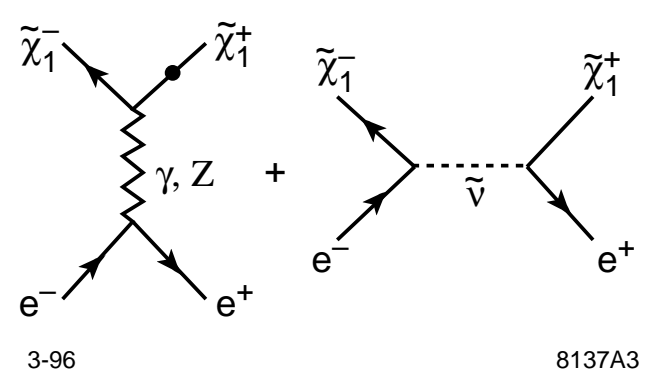

Figure 2.13: Diagrams contributing to chargino pair production.

\subsubsection{Analysis of chargino mixing}

The cross section and angular distribution of chargino pair production is built up from the diagrams shown in Fig. 2.13. This process is intrinsically more complicated than slepton pair production because one must account for chargino mixing. In supersymmetry models, there is always a charged Higgs boson $H^{ \pm}$, and both the $W^{ \pm}$ and the $H^{ \pm}$have spin- $\frac{1}{2}$ partners. These necessarily mix, through a mass matrix of the following form:

$$
\left(\begin{array}{ll}
\widetilde{w}^{-} & i \widetilde{h}_{1}^{-}
\end{array}\right)^{T}\left(\begin{array}{cc}
m_{2} & \sqrt{2} m_{W} \sin \beta \\
\sqrt{2} m_{W} \cos \beta & \mu
\end{array}\right)\left(\begin{array}{c}
\widetilde{w}^{+} \\
i \widetilde{h}_{2}^{+}
\end{array}\right),
$$

where $\widetilde{w}^{ \pm}$are the superpartners of the $W^{ \pm}$and $\widetilde{h}_{1}^{-}$and $\widetilde{h}_{2}^{+}$are the superpartners of the charged components of the two Higgs fields. The matrix depends on the parameters $\mu$, the supersymmetric Higgs mass, $m_{2}$; the supersymmetry breaking mass of the $\widetilde{w}^{ \pm}$; and $\tan \beta$, the ratio of Higgs field vacuum expectation values. The neutralino masses involve a similar mixing problem among four states, the superpartners of the neutral $S U(2)$ and $U(1)$ gauge bosons and the two neutral Higgs fields. The neutralino mass matrix involves the same three parameters $\mu, m_{2}, \tan \beta$, plus $m_{1}$, the supersymmetry breaking mass of the $\widetilde{b}$.

Chargino and neutralino mixing is not an added complication that one may introduce into supersymmetric models if one wishes. It is an intrinsic feature of these models which must be resolved experimentally. Unless this can be done, supersymmetry measurements can only be interpreted in the context of model assumptions. In addition, this measurement is important in resolving the question of whether the lightest neutralino in supersymmetry can provide the cosmological dark matter. In most scenarios of the dark matter, the neutralino must be light and dominantly gaugino rather than Higgsino. In any case, the neutralino mixing must be known to build a quantitative theory of the cosmological neutralino production and relic abundance.

Fortunately, it is possible to measure the chargino and neutralino mixing angles by making use of the special handles that the linear collider offers. To see this, consider 


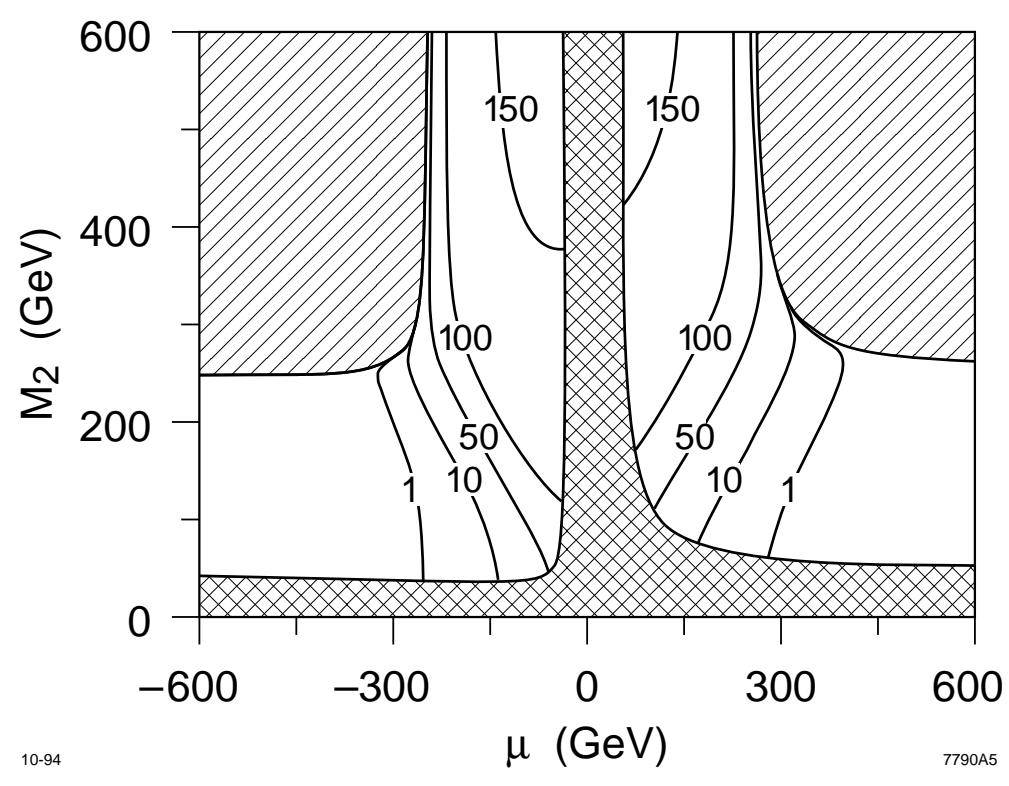

Figure 2.14: Total cross section for $e_{R}^{-} e^{+} \rightarrow \widetilde{\chi}_{1}^{+} \widetilde{\chi}_{1}^{-}$, in fb, as a function of the chargino mass parameters $m_{2}$ and $\mu$.

the diagrams of Fig. 2.13 for a right-handed polarized electron beam. The second diagram, which involves the sneutrino, couples only to left-handed electrons and so vanishes in this case. At high energy, the $\gamma$ and $Z$ exchanged in the first diagram can be traded for the neutral $S U(2)$ and $U(1)$ gauge bosons. The $e_{R}^{-}$does not couple to the $S U(2)$ boson. The $\widetilde{w}^{ \pm}$does not couple to the $U(1)$ boson. Thus, the total cross section for the process $e_{R}^{-} e^{+} \rightarrow \tilde{\chi}_{1}^{+} \tilde{\chi}_{1}^{-}$can be large only if the lighter charginos $\tilde{\chi}_{1}^{+}$ and $\tilde{\chi}_{1}^{-}$are dominantly composed of the Higgs field superpartners. This remarkable feature is evident in the contour map of this cross section against $\mu$ and $m_{2}$ shown in Fig. 2.14. A more detailed analysis shows that, by measuring the angular distribution of chargino pair production, one can determine the separate mixing angles for the positive and negative (left-handed) charginos [85]. Unless the mixing angles are very small, the measurement of the two mixing angles and the $\tilde{\chi}_{1}^{+}$mass allow the complete mass matrix (2.15) to be reconstructed. In an example studied in [85], this analysis gave a $10 \%$ measurement of $\tan \beta$, purely from supersymmetry measurements, in a $100 \mathrm{fb}^{-1}$ experiment at $500 \mathrm{GeV}$.

Having determined the chargino mixing, one can then analyze chargino pair production from left-handed fermions. This brings back the dependence on the sneutrino mass. In fact, it is possible to measure the effect of sneutrino exchange and thus to determine the masses of the left-handed sleptons for slepton masses up to a factor of 2 above the collider center of mass energy. Measurements of the ratio of leptonic to hadronic chargino decays also can give information on the masses of the left-handed 
sleptons [86]. This can provide a consistency test on the supersymmetry parameters or a target for an energy upgrade.

In both the chargino and slepton studies that we have discussed, it is remarkable how the use of polarization and detailed angular distribution measurements can offer new information along a dimension quite orthogonal to that probed by simple mass determinations. The use of beam polarization is particularly incisive in separating complex composite observables into quantities with a direct relation to the parameters in the underlying Lagrangian.

\subsection{Studies of the top quark}

The top quark's special status as the most massive known matter particle, and the only fermion with an unsuppressed coupling to the agents of electroweak symmetry breaking, make it a prime target for all future colliders. The linear collider, operating near the top quark pair-production threshold and at higher energies below $500 \mathrm{GeV}$, can carry out a complete program of top quark physics. This includes the measurement of the top quark mass, width, form factors, and couplings to many species. This broad program of measurements is reviewed in [87]. In this section, we will discuss two particularly important measurements from this collection.

The mass of the top quark is a fundamental parameter in its own right, and it is also an ingredient in precision electroweak analyses and theories of flavor. It is important to measure this parameter as accurately as possible. Future measurements at the Tevatron and the LHC are likely to determine $m_{t}$ to $2-3 \mathrm{GeV}$ precision, dominated by systematic effects $[88,62]$.

At the linear collider, the top quark mass is determined directly by the accelerator energy at which one sees the onset of $t \bar{t}$ production. A simulation of the top quark threshold scan, from [89], is shown in Fig. 2.15. Given a measurement of $\alpha_{s}$ from another source, this scan determines $m_{t}$ to $200 \mathrm{MeV}$ using only $11 \mathrm{fb}^{-1}$ of data. In the part of the cross section described by the top quark threshold, the $t$ and $\bar{t}$ are separated by a distance small compared to the QCD scale. This means that the mass determined from the threshold scan - as opposed to the 'pole mass' determined by the kinematics of high energy production - is a true short-distance quantity which is free of nonperturbative effects. The theoretical error for the conversion of the $e^{+} e^{-}$threshold position to the $\overline{M S}$ top quark mass relevant to grand unified theories is about $300 \mathrm{MeV}$ [90,91]; for the pole mass, it is difficult even to estimate this uncertainty. The expenditure of $100 \mathrm{fb}^{-1}$ at the $t \bar{t}$ threshold allows additional measurements that, for example, determine the top quark width to a few percent precision [92-94].

A second important set of measurements is the study of the top quark couplings to $\gamma, Z, W$. In the reaction $e^{+} e^{-} \rightarrow t \bar{t}$, the final state can be reconstructed as a 6 -jet or 4 -jet plus $\ell \nu$ system. The $b$ jets should be identified with an efficiency greater than $80 \%$. Both the production through $\gamma$ and $Z$ and the decay by $t \rightarrow W^{+} b$ are maximally parity violating. Thus, there are many independent kinematic variables 

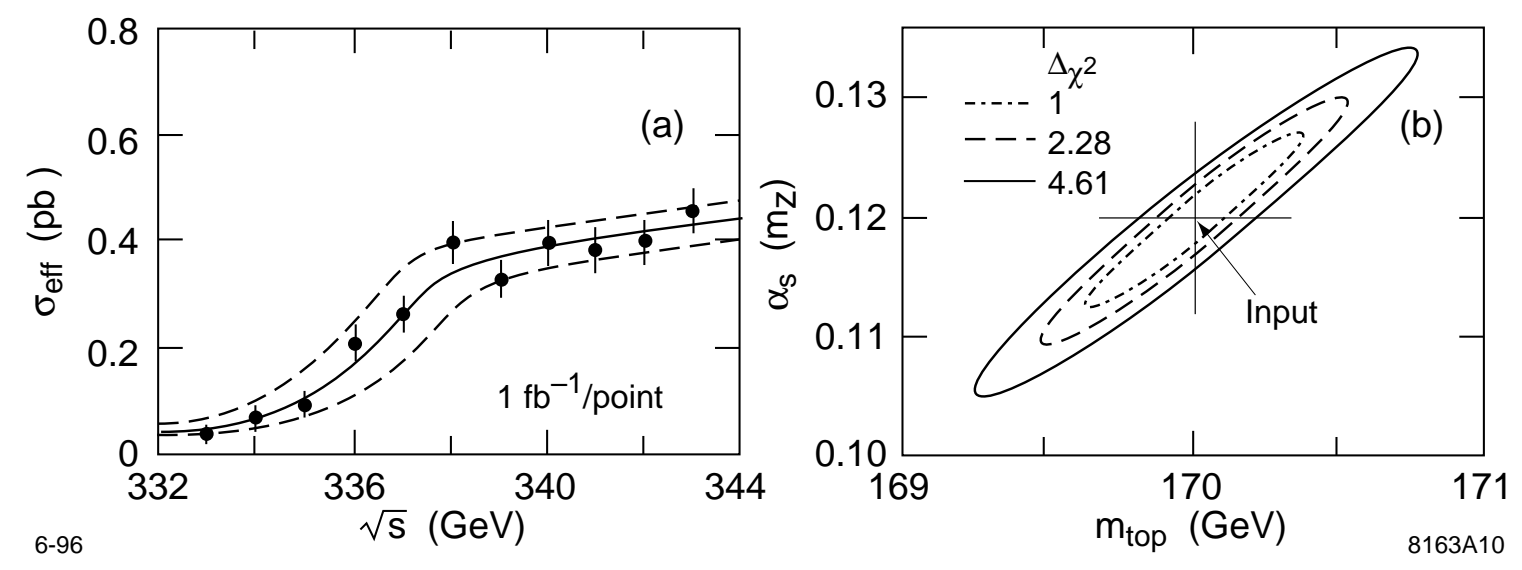

Figure 2.15: Measurement of the top quark mass from the threshold shape, using a threshold scan with a total data sample of $11 \mathrm{fb}^{-1}$. The effects of beamstrahlung, initial state radiation, and accelerator energy spread are included. A top quark mass of $170 \mathrm{GeV}$ was assumed in this study [89].

that can be used to constrain the various possible production and decay form factors. A simulation study using $80 \% e^{-}$beam polarization but only $10 \mathrm{fb}^{-1}$ of luminosity at $500 \mathrm{GeV}$ showed that it is possible to simultaneously constrain the whole set of vector and axial vector $\gamma, Z$, and $W$ form factors of the top quark with errors in the range $5-10 \%$ [87]. This analysis should improve further with high-luminosity data samples [95]. Experiments at the linear collider are sensitive at similar levels to anomalous couplings of $t \bar{t}$ to the gluon [96].

A set of couplings of particular interest are the vector and axial $t \bar{t} Z$ form factors. As we have explained in Section 4.5, these form factors are predicted to receive large contributions in certain models of strong-interaction electroweak symmetry breaking. These contributions result from diagrams in which the $Z$ couples to the new stronglyinteracting species which break electroweak symmetry, and these couple to the top quark through the mechanism which generates the top quark mass [28]. In Fig. 2.16, the $Z$ form factor determinations from the simulation study of [97] are compared to two representative theories [1]. It is interesting that most of the sensitivity in this particular measurement comes from the polarization asymmetry of the total top pair production cross section. The measurement of this quantity is dominated by statistics and can be improved straightforwardly with higher luminosity.

An additional important measurement is the determination of the top quark Higgs Yukawa coupling. At the LHC, the ratio $\lambda_{t \bar{t} h} / \lambda_{W W h}$ can be measured to an accuracy of $25 \%$ for $80<m_{h}<120 \mathrm{GeV}$ [62]. At a linear collider, the top quark Yukawa coupling can be measured by studying the process $e^{+} e^{-} \rightarrow t \bar{t} h^{0}$, relying on the $b \bar{b}$ decay of the $h^{0}$ to produce spectacular events with $4 b$ 's in the final state. This process is difficult to study at $500 \mathrm{GeV}$, but it becomes tractable at higher energy. In 


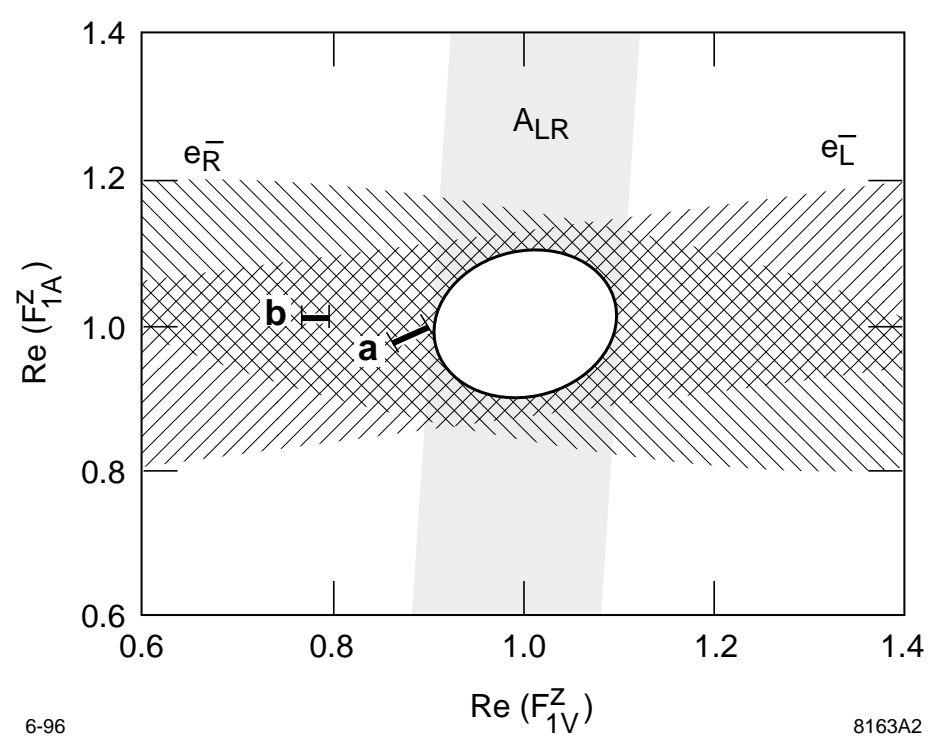

Figure 2.16: Determination of the form factors for the vector and axial vector couplings of the top quark to the $Z$, with $100 \mathrm{fb}^{-1}$ at $400 \mathrm{GeV}$ [97], compared to the predictions of technicolor models, from [1].

simulation studies at $800 \mathrm{GeV}$, where the cross section is about 8 times higher than at $500 \mathrm{GeV}$, a $1000 \mathrm{fb}^{-1}$ sample yields a $6 \%$ uncertainty on $\lambda_{t \bar{t} h}$ for a $120 \mathrm{GeV}$ Higgs boson $[98,99]$.

\subsection{Studies of $W$ boson couplings}

Recent experiments at LEP 2 and the Tevatron have observed weak boson pair production and have verified the general expectations for the cross sections given by the Standard Model $[100,101]$. This is already an important discovery. One of the motivations for building a model of the weak-interaction bosons from a YangMills gauge theory is that the special properties of the Yang-Mills coupling tame the typically bad high energy behavior of massive vector fields. We now know that the behavior of the $W$ and $Z$ production cross sections, at least in the region close to threshold, conforms to the gauge theory predictions.

This discovery sets the stage for the use of $W$ and $Z$ bosons to probe the physics of electroweak symmetry breaking. As we have noted in Section 4.5, new strong interactions that might be responsible for electroweak symmetry breaking can affect the three- and four-particle couplings of the weak vector bosons. The precision measurement of these effects - and the corresponding effects on the top quark couplings discussed in the previous section - can provide a window into the dynamics of electroweak symmetry breaking complementary to that from direct $W$ boson scattering.

Our discussion in Section 4.5 implies that a high level of precision is necessary. We 
estimated there that effects of new strong interactions affect the standard parameters used to describe the $W W \gamma$ and $W W Z$ vertices - $\kappa_{V}, \lambda_{V}$, for $V=\gamma, Z$, and $g_{Z}$-at the level of a few parts in $10^{-3}$. For comparison, the one-loop radiative corrections to these parameters predicted in the Standard Model are of the order of $10^{-3}-10^{-4}$ [102].

In contrast, the current bounds on parameters of the $W$ vertices from LEP 2 and the Tevatron are at the level of $10^{-1}[100,101,103]$. Much improved constraints are expected from the LHC. There one expects to place bounds on the $W W V$ couplings in the range $[62,104]$

$$
\left|\Delta \kappa_{V}\right|<0.01 \text { to } 0.1, \quad\left|\Delta g_{1}^{Z}\right|, \quad\left|\lambda_{V}\right|<0.001 \text { to } 0.01
$$

which might be sensitive to effects of new physics. It should be noted that the LHC analyses integrate over a large range of center-of-mass energies for vector boson pair production. This means that the sensitivity and interpretation of these experiments depend on assumptions about the energy-dependence of the form factors describing the new physics effects.

The linear collider provides an ideal laboratory for the study of the $W W V$ couplings. The process $e^{+} e^{-} \rightarrow W^{+} W^{-}$actually gives the largest single contribution to the $e^{+} e^{-}$annihilation cross section at high energies. The $W$ pair events can be reconstructed in the four-jet final state. More importantly, the events with a leptonic decay on one side and a hadronic decay on the other allow unambiguous reconstruction of the charge and decay angles of the leptonic $W$. Both the production process and the $W$ decay are strongly parity-violating, so both beam polarization and angular distributions can be used to extract the details of the $W$ vertices. The diagrams for $e^{+} e^{-} \rightarrow W^{+} W^{-}$involve both $\gamma$ and $Z$, but these effects can be disentangled by the use of beam polarization. The $W$ pair production cross section is about 30 times larger with left-handed than right-handed polarized beams. The suppression of the right-handed cross section depends on the relation between the $W W \gamma$ and $W W Z$ vertices predicted by the Standard Model and so is a sensitive measure of deviations from this prediction.

Effects from strong-interaction electroweak symmetry breaking, which enter through effective Lagrangian parameters as in (2.9), affect the cross section for longitudinal $W$ pair production through terms proportional to $\left(s / m_{W}^{2}\right)$. At the same time, the fraction of the cross section with longitudinal $W$ pairs grows as $\beta^{2}=\left(1-4 m_{W}^{2} / s\right)$. From these two effects alone, one should expect a factor 15 improvement in the sensitivity to these effects in going from LEP 2 to the linear collider experiments at 500 $\mathrm{GeV}$. The most important advantage, however, is the increase in statistics with high luminosity running. A recent simulation of the $W W V$ coupling measurement at a 500 $\mathrm{GeV}$ collider with $500 \mathrm{fb}^{-1}$ estimates the limits that can be placed on the coupling parameters as [105]

$$
\left|\Delta g_{1}^{Z}\right|<2.5 \times 10^{-3}, \quad\left|\Delta \kappa_{Z}\right|<7.9 \times 10^{-4}, \quad\left|\lambda_{Z}\right|<6.5 \times 10^{-4},
$$




$$
\left|\Delta \kappa_{\gamma}\right|<4.8 \times 10^{-4}, \quad\left|\lambda_{\gamma}\right|<7.2 \times 10^{-4} .
$$

These results qualitatively improve on the LHC sensitivity, to the point where not only effects of new physics but even the Standard Model radiative corrections are visible.

\subsection{Studies of QCD}

In addition to the search for new physics, the linear collider will be able to complete the program of precision tests of the Standard Model with a precise measurement of the QCD coupling constant $\alpha_{s}$. The strong coupling constant is determined in $e^{+} e^{-}$ annihilation from the production rate for 3 -jet events. The reduction in the relative size of hadronization effects at high energy allow a measurement of $\alpha_{s}$ with systematic errors smaller than $1 \%$ [106].

A measurement of $\alpha_{s}$ of similar quality can be obtained from the ratio of hadronic to leptonic decays of the $Z^{0}$, if one can obtain a sample of more than $10^{8} Z^{0}$ decays. This becomes practical in linear collider experiments at the $Z^{0}$, as we will explain in Section 5.6. By comparing the two precision measurements of $\alpha_{s}$ at $Q$ values of $m_{Z}$ and $500 \mathrm{GeV}$, it will be possible to give a precise test of the QCD renormalization group equation.

With confidence in the running of $\alpha_{s}$ from this experiment, one can extrapolate the precise value of $\alpha_{s}$ to the grand unification scale. Current data is consistent with a grand unification with the renormalization group equations of supersymmetry; however, it gives little constraint on the details of unification. With an accurate $\alpha_{s}$, one can anticipate a precise test of grand unification relations. The contributions to be accounted for include next-to-leading order corrections from two-loop beta functions, TeV-scale threshold effects, and GUT-scale threshold effects [36]. The two-loop beta functions are known from the general theoretical scheme. The TeV-scale threshold effects are unknown today, but they will be determined from the new particle masses measured at the LHC and the linear collider. Then a $1 \%$ measurement of $\alpha_{s}$ would allow a $10 \%$ measurement of the GUT-scale threshold correction. This measurement would give an indirect but significant constraint on the spectrum of the massive particles responsible for the GUT level of fundamental symmetry breaking.

The linear collider can also provide the most sensitive experiments on photon structure, including the precise measurement of the photon structure function $F_{2}^{\gamma}$. In addition, with sufficient forward instrumentation, the linear collider could study $\gamma^{*} \gamma^{*}$ scattering at large $s$ and fixed momentum transfer. This is a beautifully clean model system for analyzing a part of QCD that is still very mysterious, the nature of the pomeron and the dynamics of high-energy scattering [107]. 


\begin{tabular}{|l|c|c|}
\hline \hline Parameter & Current Value & LC Measurement \\
\hline \hline $\sin ^{2} \theta_{W}^{\text {eff }}$ & $0.23119 \pm 0.00021$ & \pm 0.00002 \\
$m_{W}$ & $80.419 \pm 0.038 \mathrm{GeV}$ & $\pm 0.006 \mathrm{GeV}$ \\
$\Gamma\left(Z \rightarrow \ell^{+} \ell^{-}\right)$ & $83.96 \pm 0.09 \mathrm{MeV}$ & $\pm 0.04 \mathrm{MeV}$ \\
$R_{b}^{\exp } / R_{b}^{\text {th }}$ & $1.0029 \pm 0.0035$ & \pm 0.0007 \\
$A_{b}^{\exp } / A_{b}^{\text {th }}$ & $0.958 \pm 0.017$ & \pm 0.001 \\
\hline \hline
\end{tabular}

Table 2.4: Current values of some important electroweak parameters, and the potential uncertainty obtainable at a linear collider providing with high statistics (e.g., $10^{9} Z^{0}$ decays).

\subsection{Precision electroweak studies}

In addition to the experimental program at $500 \mathrm{GeV}$ energies, one can envision using the linear collider at the $Z^{0}$ and the $W$ threshold to carry the experimental program of precision electroweak measurements to the next level. Operation of the linear collider at the $Z^{0}$ pole would yield more than $10^{9} Z^{0}$ decays in a $20 \mathrm{fb}^{-1}$ data sample. With more than 100 times LEP 1 statistics and high beam polarization, one could undertake a very ambitious and extensive program of precision measurements. For example [108], employing the left-right polarization asymmetry, leptonic forwardbackward asymmetries, and tau polarization asymmetry (all of which are currently statistics limited) one could improve the determination of $\sin ^{2} \theta_{W}^{\text {eff }}$ at the $Z$ pole by an order of magnitude, bringing it to an unprecedented $\pm 0.01 \%$ level. Other quantities such as the $Z$ line shape parameters, $R_{b}=\Gamma(Z \rightarrow b \bar{b}) / \Gamma(Z \rightarrow$ hadrons $)$, and $A_{b}$ (the polarized $b \bar{b}$ asymmetry) could also be improved. They would be limited only by systematics.

With such a large sample of $Z$ decays, one would have more than $10^{8} b \bar{b}$ and $3 \times 10^{7} \tau^{+} \tau^{-}$pairs. The study of these events could make use of the outstanding vertex resolution and detection efficiency of the linear collider environment. In addition, polarized $e^{+} e^{-}$annihilation at the $Z^{0}$ produces (for a left-handed beam) dominantly forward production of $b$ quarks and backward production of antiquarks, thus eliminating the need for a flavor tag. These features combine to give an ideal environment for studying $\mathrm{CP}$ violating asymmetries and rare decays as well as performing precision measurements [108]. For example, one could improve the current precision on the forward-backward asymmetry parameter $A_{b}$ by more than an order of magnitude.

In Table 2.4, we have listed some improved measurements envisioned at the linear collider. The tiny error on $\sin ^{2} \theta_{W}^{\text {eff }}$ assumes a precise beam polarization measurement that may require polarizing both the electron and positron beams. The importance of refining $\sin ^{2} \theta_{W}^{\text {eff }}$ is well illustrated by the prediction for the Higgs mass that would be obtained by employing these precise values and the improved value of $m_{t}$ from 
Section 5.4 as input. One finds

$$
m_{h}=(140 \pm 5 \mathrm{GeV}) e^{\left[1911\left(\sin ^{2} \theta_{W}^{\mathrm{eff}}-0.23158\right)\right]},
$$

where the dominant error comes from hadronic loop uncertainties in $\alpha$ (assumed here to be reduced by a factor of 3 compared to the current error). Comparison of the indirect loop determination of $m_{h}$ from (2.19) with the direct measurement of $m_{h}$ from the LHC and the linear collider would confront the electroweak prediction at the $5 \%$ level and would provide an accurate sum rule to be satisfied by new heavy particles with electroweak charge. Another way to look at this comparison is that it will probe the $S$ and $T$ parameters to an accuracy of 0.02 , about 8 times better than current constraints. At that level, even the existence of a single heavy chiral fermion doublet (much less an entire dynamical symmetry breaking scenario) would manifest itself. The accurate value of $\sin ^{2} \theta_{W}^{\text {eff }}$ at the $Z$ pole would be a valuable input to the measurements of cross sections and asymmetries at high energy that we will discuss in Sections 6.3 and 6.4, measurements which probe for possible $Z^{\prime}$ bosons, lepton compositeness, or new space dimensions.

A linear collider run near the $W^{+} W^{-}$threshold would also be extremely valuable for improving the determination of $m_{W}$ beyond the capabilities of the LHC [108]. Already at the current uncertainty of $40 \mathrm{MeV}$, the determination of the $m_{W}$ mass from kinematic fitting of $W$ pair production at LEP 2 is affected by systematic uncertainty from the modeling of fragmentation. But the interpretation of the measurement of the $W$ threshold position is almost free of theoretical uncertainty, allowing a $6 \mathrm{MeV}$ measurement to be done with a dedicated $100 \mathrm{fb}^{-1}$ run.

Collectively, the broad program of precision electroweak studies which the high luminosity of the linear collider makes available nicely complements and expands the physics goals at the maximum collider energy.

\section{Further topics from the linear collider physics program}

In the preceding section, we have discussed only those aspects of the linear collider experimental program for which there are strong arguments that the phenomena to be studied will appear at $500 \mathrm{GeV}$. There are many other experiments that can be done at an $e^{+} e^{-}$linear collider which has sufficient energy to reach the required threshold for new particles. In this section, we will describe a number of experiments of this character. All of these experiments will eventually become relevant as components of the long-term program that we have described in Section 2. Measurements at the LHC which estimate the new thresholds could provide specific motivation for upgrading a $500 \mathrm{GeV}$ collider to higher energy. But, one should keep in mind that all of the phenomena we describe in this section could well be present at $500 \mathrm{GeV}$ and provide additional richness to the initial physics program of the linear collider. 
It is well appreciated that an $e^{+} e^{-}$collider provides an excellent environment to search for all varieties of exotic particles with nonzero electroweak quantum numbers. The huge variety of particles which have been searched for at LEP is described, for example, in [109]. In almost all cases, the LEP limits are close to the kinematic limit allowed by the collider. A collider operating above the pair production threshold will be able to accumulate a large sample of events (70,000 events per unit of $R$ in a 200 $\mathrm{fb}^{-1}$ sample at $500 \mathrm{GeV}$ ) and make incisive measurements.

The corresponding discovery reach for exotic particles at the LHC ranges from a few hundred $\mathrm{GeV}$ for new leptons to about $2 \mathrm{TeV}$ for new quarks. So, as a general statement, the locations of the new thresholds are likely to be found at the LHC. Experimenters at a linear collider will measure essential information that is beyond the capability of the LHC. We have seen examples of this in Section 5, and further examples will be discussed in this section.

Rather than summarize all possible measurements of new phenomena at a linear collider, we restrict ourselves in this section to four specific examples that have been worked out in some detail. In Section 6.1, we will discuss the particles of an extended Higgs sector such as that in the Minimal Supersymmetric Standard Model. In Section 6.2, we will discuss studies of supersymmetric particles beyond the lightest chargino, neutralinos, and sleptons. In Section 6.3, we will discuss new and exotic $Z^{\prime}$ bosons. In Section 6.4, we will discuss probes of large extra dimensions and $\mathrm{TeV}$-scale quantum gravity.

Because this paper focuses on the issue of a $500 \mathrm{GeV}$ collider, we do not discuss here the significant capabilities of higher energy $e^{+} e^{-}$collisions to probe $W W$ scattering processes [110]. These include the unique ability to study the reaction $W^{+} W^{-} \rightarrow t \bar{t}$, which directly tests the coupling of the top quark to the particles responsible for strong-interaction electroweak symmetry breaking. These experiments, and the comparison to the LHC capabilities, are reviewed in [15,111].

Although the detailed physics justification for increased $e^{+} e^{-}$collision energy is more difficult to quantify at present than that for the initial $500 \mathrm{GeV}$ step, we fully expect that the experimentation at the LHC and first stage $e^{+} e^{-}$linear collider will reveal phenomena that dictate energy upgrades. It is important to continue the $\mathrm{R} \& \mathrm{D}$ needed for this evolution.

\subsection{Extended Higgs sector}

In Section 5.1, we have discussed the measurement of the properties of the lightest Higgs boson. Many models of new physics allow multiple Higgs fields, leading to additional heavier Higgs particles. In particular, supersymmetry requires the presence of at least two Higgs doublet fields. This produces, in addition to the $h^{0}$, four additional states - the CP-even $H^{0}$, the CP-odd $A^{0}$, and charged states $H^{ \pm}$. The masses of these states should be comparable to the masses of other supersymmetric particles. If the scale of superparticle masses is much greater than $100 \mathrm{GeV}$, then typically the 
four heavy Higgs states are relatively close in mass, and the light $h^{0}$ resembles the Higgs boson of the Standard Model.

The heavy Higgs states are very difficult to find at the LHC. The LHC experiments have studied extensively their sensitivity to the Higgs sector of the MSSM. We have already presented a summary of these analyses in Fig. 2.2. A low mass $H^{ \pm}$can be found at the LHC below about $125 \mathrm{GeV}$ in the decays of the top quark. For $m_{H^{ \pm}}$ above $225 \mathrm{GeV}$, its decay into $t \bar{b}$ can be used to find the charged Higgs if $\tan \beta \gtrsim 25$ or $\tan \beta \lesssim 2$. In the region of intermediate $\tan \beta$ above the LEP limits, only the process $h^{0} \rightarrow \gamma \gamma$ is visible, and the $H$ and $A$ are not seen at all. For larger $\tan \beta$ (>10), the decays $H / A \rightarrow \tau^{+} \tau^{-}$become accessible. Because the technique for detecting $H$ and $A$ involves particles that decay with missing energy, it will be difficult to make a precise mass measurement. ATLAS studies suggest an accuracy on the $H / A$ mass of about $5 \mathrm{GeV}$, for $M_{H / A}=300 \mathrm{GeV}$ and $\tan \beta=10$, only after $300 \mathrm{fb}^{-1}$ has been collected. For comparison, the $H-A$ mass difference is at most a few GeV. For low $\tan \beta, H$ could be detected by $H \rightarrow Z Z^{*}$. This mode, however, applies only to a limited region of parameter space, $\tan \beta<3$ (a region disfavored by the LEP constraint on the mass of $h$ ) and $m_{H}<350 \mathrm{GeV}$.

A crucial aspect of the experimental study of the heavy Higgs states would be to measure the value of $\tan \beta=\left\langle\phi_{2}\right\rangle /\left\langle\phi_{1}\right\rangle$, where $\phi_{1}$ and $\phi_{2}$ are the two Higgs doublets of the MSSM. This quantity is needed to determine the absolute size of the quark and lepton Yukawa couplings. For example, it is possible that the bottom quark Yukawa coupling is large and the lightness of the bottom quark is explained by the fact that the Higgs field responsible for this mass has a small vacuum expectation value. In supersymmetry, $\tan \beta$ also appears in many formulae for the supersymmetry masses and mixings and is a source of theoretical uncertainty unless it can be pinned down. The LHC can measure $\tan \beta$ from the heavy Higgs particles only where $H$ is visible by one of the techniques just listed, to an accuracy of 10-30\%. It should be noted that what is measured is $\sigma \cdot B R$, and so the determination of $\tan \beta$ depends on theoretical assumptions about the total width.

If the masses of $H, A$ are well above that of $h$, these particles are mainly produced at an $e^{+} e^{-}$collider in pairs, through $e^{+} e^{-} \rightarrow H^{0} A^{0}$. The mass determination is straightforward. Kinematic fitting of decays with $b \bar{b}$ on both sides should give an accuracy of $0.3 \%$. The program described earlier for the precision determination of the $h$ branching fractions can be applied also to the $H$ and $A$. The crucial parameter $\tan \beta$ is given by the ratio of the branching ratios to $b \bar{b}$ and $t \bar{t}$. For $A$,

$$
\frac{\Gamma(A \rightarrow t \bar{t})}{\Gamma(A \rightarrow b \bar{b})}=\frac{m_{t}^{2}}{m_{b}^{2}} \cot ^{4} \beta \cdot\left(1-\frac{4 m_{t}^{2}}{m_{A}^{2}}\right)^{1 / 2} .
$$

From this measurement, a completely model-independent determination of $\tan \beta$ to $10 \%$ accuracy is expected. Measurements of other branching fractions of $H, A$, and $H^{ \pm}$will provide cross-checks of this value [112]. 
The ATLAS [62] and CMS [75] analyses of the fitting of LHC data to the minimal supergravity-mediated model gives a remarkable accuracy of $3 \%$ in the determination of $\tan \beta$. However, this determination of $\tan \beta$ is based on the assumption of a specific model of supersymmetry breaking. It uses the precision measurement of the $h^{0}$ mass and thus depends on the detailed theory of the one-loop supersymmetry corrections to this parameter. Linear collider experiments offer a number of methods to determine $\tan \beta$ from supersymmetry observables in a model-independent way. For example, $\tan \beta$ can be extracted from chargino mixing, as we have discussed in Section 5.2.4. In the end, it is a nontrivial test of the theory whether the determinations of $\tan \beta$ from the supersymmetry spectrum agree with the direct determination of this parameter from the Higgs sector.

\subsection{Supersymmetric particle studies}

In Section 4.4, we have argued that, if the new physics at the $\mathrm{TeV}$ scale includes supersymmetry, the lightest supersymmetric particles are likely to appear at a 500 $\mathrm{GeV} e^{+} e^{-}$collider. In Section 5.2, we have discussed the program of detailed measurements on those particles. Of course, nothing precludes a larger set of supersymmetric particles from appearing at $500 \mathrm{GeV}$, though it is likely that increased energy will be needed to produce the full supersymmetry spectrum. In this section, we will discuss what can be learned from a more complete study of the supersymmetry spectrum in $e^{+} e^{-}$annihilation.

For brevity, we focus on two important issues. The first of these is whether supersymmetry does in fact give the dynamics that leads to electroweak symmetry breaking. To verify the mechanism of electroweak symmetry breaking experimentally, we must determine the basic parameters that directly determine the Higgs potential. These include the heavy Higgs boson masses discussed in the previous section. Another essential parameter is $\mu$, the supersymmetric Higgs mass parameter. As we have discussed in Section 5.2, this parameter can already be determined from the study of the lighter chargino if these particles are not almost pure $\widetilde{w}$. In that last case, $\mu$ is determined by measuring the mass of the heavier charginos. We have argued in Section 4.4 that these particles should be found with at most a modest step in energy above $500 \mathrm{GeV}$. A precision mass measurement can be done using the endpoint technique discussed in Section 5.2.

In typical supersymmetric models, the negative Higgs (mass) ${ }^{2}$ which causes electroweak symmetry breaking is due to a mass renormalization involving the top squarks. This same renormalization leads to $\widetilde{t}_{L}-\widetilde{t}_{R}$ mixing and to a downward shift in the top squark masses relative to the masses of the first- and second-generation squarks. The mass shift, at least, might be measured at the LHC. However, in some scenarios with a large mass shift, only the third-generation squark masses can be measured accurately [62]. At the linear collider, flavor-dependent squark masses can be measured to accuracies better than $1 \%$. In addition, the mass differences of the partners of $q_{L}$ 

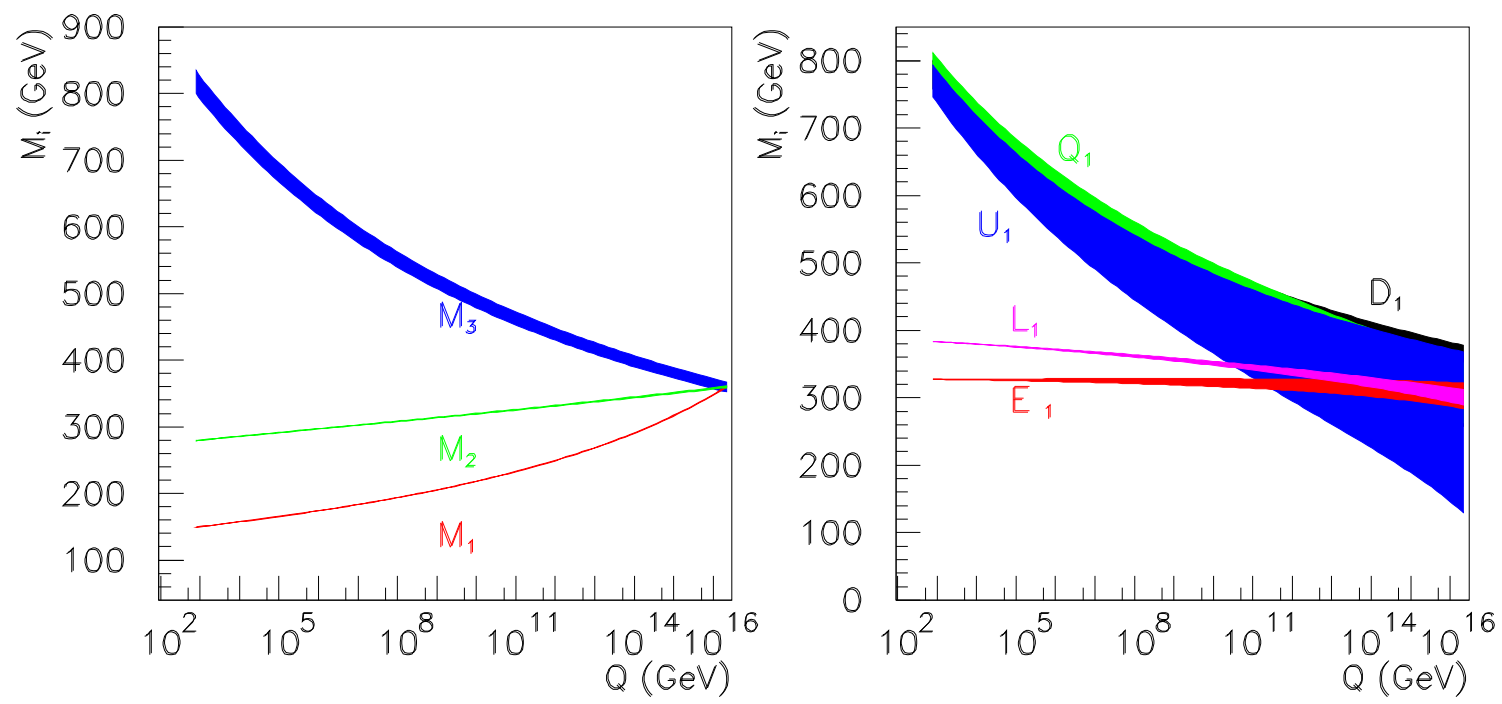

Figure 2.17: Extrapolation of supersymmetry mass parameters determined at a linear collider from the $\mathrm{TeV}$ scale to the grand unification scale, from [115]. The width of each band at the weak scale is the error in the direct parameter determination; these errors are propagated to higher energies using the renormalization group equations.

and $q_{R}$ can be measured to this accuracy using polarization asymmetries [113]. By comparing the pair production cross sections with polarized beams, as described in Section 5.2 for stau mixing, it is possible to measure the top squark mixing angle to better than $1 \%$ accuracy in a $500 \mathrm{fb}^{-1}$ experiment [114].

The second issue is the possibility of the grand unification of supersymmetry breaking parameters. This is the crucial test of whether supersymmetry breaking arises from physics above the grand unification scale or from a different mechanism acting at lower energies. This test requires accurate model-independent determinations of as many supersymmetry mass parameters as possible. Figure 2.17 shows an extrapolation to the grand unification scale at $2 \times 10^{16} \mathrm{GeV}$ of masses determined in a $500 \mathrm{fb}^{-1}$ sample at a linear collider. The most effective tests of grand unification come from the comparison of the gaugino mass parameters $m_{1}$ and $m_{2}$ and from comparison of the masses of the sleptons $\widetilde{e}_{R}$ and $\widetilde{e}_{L}$ (called $E_{1}$ and $L_{1}$ in the figure). Because of QCD threshold corrections, the masses of the gluino $\left(m_{3}\right)$ and the first-generation squarks (labeled $D_{1}, Q_{1}, U_{1}$ ) are less effective in this comparison. It should be noted that the mass ratios which provide the most significant tests of grand unification are just the ones that are most difficult to measure accurately at the LHC. Even for the uncolored states, a $1 \%$ mass error at the weak scale evolves to a $10 \%$ uncertainty at the grand unification scale. So this comparison puts a premium on very precise mass determinations, such as a linear collider will make possible.

These issues are only two slices through the rich phenomenology of supersymmetric 
particles. If supersymmetric particles - or any other family of exotic particles - appear at the $\mathrm{TeV}$ scale, there will be a full program of experiments for both hadron and $e^{+} e^{-}$colliders.

\subsection{New $Z^{\prime}$ bosons}

The new physics at the $\mathrm{TeV}$ scale must have $S U(3) \times S U(2) \times U(1)$ gauge symmetry, but it might have an even larger gauge symmetry with additional heavy vector particles. The simplest extensions are those with extra $U(1)$ gauge symmetries. The corresponding gauge bosons appear as new vector resonances - $Z^{\prime}$ bosons - coupling to lepton and to $q \bar{q}$ pairs.

Extra $U(1)$ factors in the gauge group preserve the predictions of grand unification. In fact, these new symmetries appear naturally in models in which the grand unification group is larger than the minimal choice of $S U(5)$. For example, the grand unification group $E_{6}$ contains the Standard Model gauge group and two additional $U(1)$ factors. This leads to models in which the gauge symmetry at $\mathrm{TeV}$ energies contains an additional $U(1)$ factor which is a linear combination of these [116,117].

In certain grand unified models, the masses of the heavy neutral leptons which give the scale of the neutrino mass seesaw are determined by the scale of breaking of an extra $U(1)$ symmetry. In this case, the extreme lightness of neutrinos puts the mass of the $Z^{\prime}$ beyond the reach of accelerator experiments. But many other motivations for a new $U(1)$ symmetry point to lower masses [118]. In particular, the size of the $\mu$ parameter of supersymmetry may be controlled by the scale of breaking of a $U(1)$ symmetry, in which case the corresponding $Z^{\prime}$ boson must have a mass not far above $1 \mathrm{TeV}$. More generally, the possible richness of gauge symmetries motivates the search for these new states. This is especially true for superstring theories, where explicit model constructions often predict a large number of extra $U(1)$ gauge particles [119].

The abilities of colliders to detect signatures of heavy $Z^{\prime}$ bosons have been studied in great detail. Hadron colliders have impressive sensitivity for searches in which the $Z^{\prime}$ bosons appear as resonances decaying to $\ell^{+} \ell^{-}$. Lepton colliders can be sensitive to $Z^{\prime}$ bosons in a different way, through the precision study of the pair production processes $e^{+} e^{-} \rightarrow \ell^{+} \ell^{-}$and $e^{+} e^{-} \rightarrow q \bar{q}$. Because these reactions can be measured precisely and also predicted theoretically to part per mil accuracy, experiments can be sensitive to interference effects caused by $Z^{\prime}$ bosons of mass a factor of 10 or more above the $e^{+} e^{-}$center of mass energy [120-122]. All of the special handles of the $e^{+} e^{-}$ environment, including polarization asymmetries, flavor tagging, and $\tau$ polarization, can be brought to bear in the search for these interference effects.

Table 5, based on [123], gives a comparison between the sensitivity of $e^{+} e^{-}$linear colliders and that of the LHC. The models listed in the table correspond to particular choices for the quantum number assignments of the $Z^{\prime}$; see the original reference for details. The table shows that the sensitivity of a linear collider operating at $500 \mathrm{GeV}$ is quite comparable to that of the LHC. The sensitivities quoted in the table correspond 


\begin{tabular}{|l|c|c|c|}
\hline \hline Model & $500 \mathrm{GeV}$ & $1000 \mathrm{GeV}$ & LHC \\
\hline \hline$\chi$ & 4.5 & 6.5 & 4.5 \\
$\psi$ & 2.6 & 3.8 & 4.1 \\
$\eta$ & 3.3 & 4.7 & 4.2 \\
I & 4.5 & 6.5 & 4.4 \\
SSM & 5.6 & 8.1 & 4.9 \\
ALRM & 5.4 & 7.9 & 5.2 \\
LRM & 5.2 & 7.5 & 4.5 \\
UUM & 6.7 & 9.8 & 4.6 \\
\hline \hline
\end{tabular}

Table 2.5: Sensitivity of $e^{+} e^{-}$linear colliders and the LHC to effects of a $Z^{\prime}$, after [123]. The table gives the mass reach in $\mathrm{TeV}$ for observability at the $95 \% \mathrm{CL}$. The analysis for linear colliders is based on measurement of indirect effects for an event sample of $200 \mathrm{fb}^{-1}$; it includes the effect of experimental cuts. The analysis for the LHC gives the direct sensitivity to a resonance, assuming an event sample of $100 \mathrm{fb}^{-1}$ and $Z^{\prime}$ decays only to Standard Model fermions.

to different types of measurements, and this point illustrates the complementary relation of the LHC and the linear collider. For a $Z^{\prime}$ at a few $\mathrm{TeV}$, the $\mathrm{LHC}$ will identify a resonance and accurately measure the mass $M$. The linear collider will measure interference effects and thus determine the quantity $g_{e} g_{f} / M^{2}$ which depends on the mass and the coupling strengths to the electron and the flavor $f$. By combining these pieces of information, one may obtain a complete phenomenological profile of the $Z^{\prime}$. Both hadron and lepton collider experiments will thus be needed to understand how the $Z^{\prime}$ fits into the larger picture of unification and symmetry.

This study of $e^{+} e^{-} \rightarrow f \bar{f}$ can also be used to search for composite structure of quarks and leptons. The process most sensitive to compositeness is Bhabha scattering. A $200 \mathrm{fb}^{-1}$ experiment at $500 \mathrm{GeV}$ would be expected to place a limit of $90 \mathrm{TeV}$ on the $\Lambda$ parameters of electron compositeness. Møller scattering $\left(e^{-} e^{-} \rightarrow e^{-} e^{-}\right)$ potentially provides an even more sensitive probe, offering a limit of $130 \mathrm{TeV}$ for a $200 \mathrm{fb}^{-1}$ experiment at $500 \mathrm{GeV}[124]$. Even the $e^{+} e^{-}$limit is a factor of 6 above the expected limit from studies of Drell-Yan production at the LHC [62]. In addition, an effect seen at the LHC could come from any one of a large number of possible operators, while in polarized Bhabha or Møller scattering the operator structure can be determined uniquely.

\subsection{Large extra dimensions}

Among the most remarkable proposals for new physics at the $\mathrm{TeV}$ scale is the idea that new space dimensions play an important role. String theorists have insisted 
for many years that Nature contains more than four dimensions. However, for a long time the extra dimensions were considered to be unobservably small. Recently, new developments in string theory and phenomenology have shaken up this complacent picture and have suggested that new space dimensions may be of the size $\hbar / \mathrm{TeV}$, or even larger [125-127].

There is no space here for a complete review of these new developments. (A brief review can be found in [128].) But we would like to indicate the role that the LHC and the linear collider could play in the elucidation of these models.

Consider first models in which there is a single new dimension of $\mathrm{TeV}$ size. In this model, the basic quantum fields in Nature are five-dimensional. The momentum in the fifth dimension is quantized and can be interpreted as the mass of a fourdimensional field. So, each quantized value of the fifth component of momentum gives a state that we would observe as a new heavy particle. The easiest states to observe are the components of the photon and $Z$ with nonzero momentum in the fifth dimension. These would appear as $Z^{\prime}$ bosons. The sensitivity of the LHC and the linear collider to these states is greater than that to the 'SSM' (Sequential Standard Model) boson listed in Table 5. If several states can be discovered, one can begin to map out the geometry of the extra dimensions. A similar phenenomenology applies to the Randall-Sundrum model [129] in which curvature in the fifth dimension is used to explain the hierarchy between the Planck scale and the weak scale. In this case, the new resonances are actually higher Fourier components of the gravitational field, a fact which can be recognized experimentally by their characteristic spin-2 decay distributions [130].

In another class of models, our apparently four-dimensional world is a membrane in a space of larger dimensionality [127]. This scheme allows the scale at which quantum gravity becomes a strong interaction to be much lower than the apparent Planck scale. In fact, it can be as low as $\mathrm{TeV}$ energies. The authors of [127] emphasized that their theory could be tested by macroscopic gravity experiments. But in fact more stringent tests come from high energy physics, from experiments that look for the effects of gravitational radiation at high energy colliders. These are of two types. First, if the scale $M$ of strong quantum gravity is low, one expects radiation of gravitons $G$ in $e^{+} e^{-}$and $q \bar{q}$ collisions, giving rise to processes such as

$$
e^{+} e^{-} \rightarrow \gamma G \quad q \bar{q} \rightarrow g G
$$

which appear as photons or jets recoiling against an unobserved particle. These effects have been searched for explicitly at LEP and the Tevatron (e.g., [131]), giving lower limits of about $1 \mathrm{TeV}$ on the gravity scale $M$. Second, one can look for the effects of virtual graviton exchange interfering with Standard Model annihilation processes. These interference effects have been searched for both by measurements of $e^{+} e^{-}$annihilation to fermion pairs at LEP 2 (e.g., [132]) and by measurements of Drell-Yan 
and $\gamma \gamma$ pair production at the Tevatron [133]. In both cases, the sensitivity to $M$ reaches above $1 \mathrm{TeV}$.

These experiments will be repeated at the next generation of colliders. The limits on $M$ from missing energy experiments are expected to be about $5 \mathrm{TeV}$ from the high luminosity linear collider at $500 \mathrm{GeV}$, and about $8 \mathrm{TeV}$ from monojet searches at the LHC. Similarly, limits on $M$ from virtual graviton exchange should reach to about 6 $\mathrm{TeV}$ both at the $500 \mathrm{GeV}$ linear collider and in the study of Drell-Yan processes at the LHC [134]. These values are high enough that, if the new dimensions are actually connected to the physics of the TeV scale, their effects should be observed. In that case, the linear collider experiments will take on an added significance. At the linear collider, but not at the LHC, it is possible to determine the parton kinematics of a missing energy event. Then one can determine whether events have a broad mass spectrum, as predicted in ordinary quantum gravity, or whether they are resonant at fixed mass values, as predicted in string theory. For virtual graviton processes, the linear collider can observe the flavor- and helicity-dependence of the interference

effects and determine whether the new couplings are universal, as naively expected for gravity, or are more complex in nature.

If there are more than four dimensions in Nature, the evidence for this will most likely come from high-energy physics. The possibility provides a tremendous opportunity, one which will engage experimenters at both hadron and lepton colliders.

\section{Conclusions}

The beautiful experiments in particle physics over the past 20 years and the tremendous theoretical effort to synthesize the current understanding of electroweak symmetry breaking have brought us to a point of exceptional opportunity for uncovering new laws of physics. The wealth of precision electroweak measurements indicate that a new threshold is close at hand. The precision measurements place strong constraints on models that explain the symmetry breaking and point to new phenomena at the $500 \mathrm{GeV}$ scale.

Later in this decade, we will begin to capitalize on this opportunity with experiments at the LHC. There is no doubt that the LHC will make important discoveries. However, many crucial measurements on the expected new physics are difficult to perform at a hadron collider. In this paper we have argued that a $500 \mathrm{GeV}$ linear collider will provide essential information needed to interpret and to exploit these discoveries.

The LHC should discover a Higgs boson (if LEP 2 or Tevatron experiments have not already done so) in all but rather special circumstances. The linear collider is very well suited to measuring its quantum numbers, total width and couplings. Moreover, if there is an expanded Higgs sector, measurement of the Higgs couplings to fermion 
pairs and to gauge bosons is essential.

If the new physics includes supersymmetry, the LHC experiments should observe supersymmetric particle production. They will measure some fraction of the sparticle masses, but they most likely will not be able to determine their spin and electroweak quantum numbers. Measurement of mixing angles and supersymmetric couplings at the LHC will be very difficult. To the extent that the sparticles are accessible to a linear collider, these measurements are straightforward and precise. We have argued that there is a good probability that some of the crucial sparticles will be within reach of a $500 \mathrm{GeV}$ collider. The measurements of gaugino and sfermion mixings and masses will provide important clues towards understanding how supersymmetry is broken and transmitted to the $\mathrm{TeV}$ scale.

We have reviewed the models in which new strong interactions provide the means by which the Standard Model particles acquire mass, and have found that although such models cannot be ruled out, they have become increasingly constrained by the existing precision data. The LHC has the possibility for observing new strong interactions through modifications to $W W$ scattering. We have argued that analogous modifications to the gauge boson or top quark couplings can be seen with a $500 \mathrm{GeV}$ linear collider. We have also suggested that operation of the linear collider at the $Z$ resonance may be profitable.

In each of these examples, we have argued that the linear collider and the LHC have complementary roles to play. It is likely that neither machine, by itself, will piece together the full picture of electroweak symmetry breaking. The strength of the LHC is its large partonic energy and copious production of many new particles. The linear collider, with its control of partonic energy and beam polarization, and with favorable signal to background ratios, can make crucial measurements that reveal the character of new phenomena. The complementarity of hadron and lepton collisions has been amply demonstrated in the past, and there is every reason to expect that it will continue in the future.

It may be useful to give a few illustrative examples of how the linear collider program might respond to possible outcomes of the LHC experiments:

1. A Higgs-like state is discovered below $150 \mathrm{GeV}$, and strong evidence for supersymmetry is found. In this case, the linear collider program would be based primarily on the exploration of supersymmetry and the extended Higgs sector. It would measure the couplings, quantum numbers, mixing angles and $\mathrm{CP}$ properties of the new states. These precisely measured parameters hold the key for understanding the mechanism of supersymmetry breaking. In this scenario, a premium would be placed on running at sufficiently high energy that the sparticles are produced. This might dictate raising the energy to at least 1 $\mathrm{TeV}$.

2. A Higgs particle is seen, and no evidence for supersymmetry is found. The key 
objective in this scenario would be the thorough investigation of the Higgs particle. Here, precision measurements would be of paramount importance; a linear collider would be able to make precise determinations of the Higgs couplings to all particles (including invisible states), as well as of its total width, quantum numbers and perhaps even the strength of its self coupling. Such measurements would point the way to possible extensions of the Standard Model.

High luminosity operation would be necessary at the optimum energy for Higgs production. In this scenario, revisiting the $Z$ pole might be critical to refine knowledge of electroweak loop corrections. Increased energy would likely be required to search for new phenomena such as strong scattering of $W W$ pairs or evidence for large extra dimensions.

3. No new particles are found. This uncomfortable scenario extends the puzzlement we are in today. In this case the first goal of a linear collider would be to close the loopholes in the LHC measurements (such as the possibility that the Higgs decays dominantly to invisible particles). After that, a detailed study of the top quark or gauge boson couplings would be necessary to reveal evidence for new dynamics. In this scenario, increased energy would be necessary to study $W W$ scattering. One might wish to carry out additional precise measurements at the $Z^{0}$ pole.

4. A wealth of new phenomena is sighted at LEP, Tevatron and LHC. These discoveries would indicate a much richer array of new particles and phenomena than are presently envisioned in any single model. In this case, with multiple sources of new physics, the job of the linear collider is clear. With its unparalleled ability to make detailed measurements of the properties of the new states, a linear collider would be essential to map out the terrain. A long and rich program would be assured.

In each of these representative scenarios, after examination of the many ways that new physics might come into view, we conclude that a linear collider has a decisive role to play. Starting with initial operation at $500 \mathrm{GeV}$, and continuing to higher energies as needed, an $e^{+} e^{-}$linear collider would be at the heart of a rich 20-year program of experimentation and discovery in high energy physics.

There is no guarantee in physics that we can ever predict how Nature chooses to operate in uncharted territory. Over the past two decades, however, through theory and experiment, a remarkable understanding has developed. In this paper we have argued that the data offer a clear picture of how the next step should proceed: We should begin the detailed design and construction of a $500 \mathrm{GeV} e^{+} e^{-}$linear collider. 


\section{ACKNOWLEDGMENTS}

We are grateful to many colleagues in the US, Canada, Europe, and Japan for the insights into linear collider physics which are reflected in this document. This work was supported by grants to the authors from the US Department of Energy and the US National Science Foundation.

\section{References}

[1] H. Murayama and M. E. Peskin, Ann. Rev. Nucl. Part. Sci. 46, 533 (1996), hep-ex/9606003.

[2] E. Accomando et al. [ECFA/DESY LC Physics Working Group Collaboration], Phys. Rept. 299, 1 (1998), hep-ph/9705442.

[3] S. Kuhlman et al. [NLC ZDR Design Group and NLC Physics Working Group Collaboration], Physics and Technology of the Next Linear Collider: A Report Submitted to Snowmass '96, hep-ex/9605011.

[4] Physics and Experiments with Future Linear $e^{+} e^{-}$Colliders, E. Fernández and A. Pacheco, eds. (UAB Publications, Barcelona, 2000).

[5] J. D. Bjorken, Phys. Rev. D19, 335 (1979).

[6] P. Q. Hung and J. J. Sakurai, Ann. Rev. Nucl. Part. Sci. 31, 375 (1981).

[7] M. Claudson, E. Farhi and R. L. Jaffe, Phys. Rev. D34, 873 (1986).

[8] M. Suzuki, Phys. Rev. D37, 210 (1988).

[9] A. Sirlin, in Proceedings of the XIX International Symposium on Lepton and Photon Interactions at High Energies, J. A. Jaros and M. E. Peskin, eds. (World Scientific, 2000). hep-ph/9912227.

[10] N. Bahcall, J. P. Ostriker, S. Perlmutter and P. J. Steinhardt, Science 284, 1481 (1999), astro-ph/9906463.

[11] See Chapter 4 of E. W. Kolb and M. S. Turner, The Early Universe. (AddisonWesley, 1990).

[12] Y. Fukuda et al. [Super-Kamiokande Collaboration], Phys. Rev. Lett. 81, 1562 (1998), hep-ex/9807003.

[13] M. Chanowitz, M. Golden and H. Georgi, Phys. Rev. Lett. 57, 2344 (1986); M. S. Chanowitz, in Proceedings of the 23rd International Conference on High Energy Physcis, S. C. Loken, ed. (World Scientific, 1987).

[14] M. S. Chanowitz and W. Kilgore, Phys. Lett. B322, 147 (1994), hep-ph/9311336.

[15] T. L. Barklow et al., in New Directions for High-Energy Physics: Snowmass 96, D. G. Cassel, L. T. Gennari, and R. H. Siemann, eds. (SLAC, 1997). hepph/9704217. 
[16] P. Raimondi et al., in Proceedings of EPAC 98, S. Myers, L. Liijeby, Ch. PetitJean-Genaz, J. Poole, and K.-G. Rensfelt, eds. (IOP Publishing, 1998), SLACPUB-7847.

[17] P. Chen et al., in New Directions for High-Energy Physics: Snowmass 96, D. G. Cassel, L. T. Gennari, and R. H. Siemann, eds. (SLAC, 1997).

[18] J. P. Delahaye et al., Acta Phys. Polon. B30 (1999) 2029; H. H. Braun et al., CERN-99-06.

[19] J. Irwin and R. Ruth, in preparation.

[20] P. Raimondi and A. Seryi, SLAC-PUB-8460 (2000).

[21] S. Weinberg, Phys. Rev. D19, 1277 (1979).

[22] L. Susskind, Phys. Rev. D20, 2619 (1979).

[23] K. Inoue, A. Kakuto, H. Komatsu and S. Takeshita, Prog. Theor. Phys. 68, 927 (1982).

[24] L. E. Ibáñez, Nucl. Phys. B218, 514 (1983); L. E. Ibáñez and C. López, Phys. Lett. B126, 54 (1983).

[25] J. Ellis, J. S. Hagelin, D. V. Nanopoulos and K. Tamvakis, Phys. Lett. B125, 275 (1983).

[26] L. Alvarez-Gaumé, J. Polchinski and M. B. Wise, Nucl. Phys. B221, 495 (1983).

[27] K. D. Lane and M. E. Peskin, in Proceedings of the 15th Rencontre de Moriond, J. Tran Thanh Van, ed. (Editions Frontieres, 1980).

[28] R. S. Chivukula, S. B. Selipsky and E. H. Simmons, Phys. Rev. Lett. 69, 575 (1992), hep-ph/9204214;

[29] L. Randall, Nucl. Phys. B403, 122 (1993), hep-ph/9210231.

[30] R. Sundrum, Nucl. Phys. B395, 60 (1993), hep-ph/9205203.

[31] T. Appelquist, J. Terning and L. C. Wijewardhana, Phys. Rev. Lett. 79, 2767 (1997), hep-ph/9706238.

[32] B. A. Dobrescu and C. T. Hill, Phys. Rev. Lett. 81, 2634 (1998), hep-ph/9712319; R. S. Chivukula, B. A. Dobrescu, H. Georgi and C. T. Hill, Phys. Rev. D59, 075003 (1999), hep-ph/9809470.

[33] S. Dimopoulos, S. Raby and F. Wilczek, Phys. Rev. D24, 1681 (1981).

[34] M. B. Einhorn and D. R. Jones, Nucl. Phys. B196, 475 (1982).

[35] L. E. Ibanez and G. G. Ross, Phys. Lett. B105, 439 (1981).

[36] P. Langacker and N. Polonsky, Phys. Rev. D47, 4028 (1993), hep-ph/9210235, Phys. Rev. D52, 3081 (1995), hep-ph/9503214.

[37] A more detailed discussion of the Higgs mass limits within the Minimal Standard Model can be found in Chapter 2.5 of J. F. Gunion, H. E. Haber, G. Kane, and S. Dawson, The Higgs Hunter's Guide. (Addison-Wesley, 1990.)

[38] T. Moroi and Y. Okada, Phys. Lett. B295, 73 (1992). 
[39] G. L. Kane, C. Kolda and J. D. Wells, Phys. Rev. Lett. 70, 2686 (1993), hep$\mathrm{ph} / 9210242$.

[40] J. R. Espinosa and M. Quirós, Phys. Rev. Lett. 81, 516 (1998), hep-ph/9804235.

[41] H. E. Haber, in Proceedings of the 4th International Symposium on Radiative Corrections, hep-ph/9901365.

[42] A. Straessner, talk presented at the XXXV Rencontres de Moriond, March 2000.

[43] V. A. Novikov et al. Rept. Prog. Physics 62, 1275 (1999).

[44] B. Holdom and J. Terning, Phys. Lett. B247, 88 (1990).

[45] M. Golden and L. Randall, Nucl. Phys. B361, 3 (1991).

[46] M. E. Peskin and T. Takeuchi, Phys. Rev. Lett. 65, 964 (1990); Phys. Rev. D46, 381 (1992).

[47] M. E. Peskin and J. D. Wells, hep-ph/0101342.

[48] R. Barbieri and G. F. Giudice, Nucl. Phys. B306, 63 (1988).

[49] G. G. Ross and R. G. Roberts, Nucl. Phys. B377, 571 (1992).

[50] B. de Carlos and J. A. Casas, Phys. Lett. B309, 320 (1993), hep-ph/9303291.

[51] G. W. Anderson and D. J. Castaño, Phys. Rev. D52, 1693 (1995), hep$\mathrm{ph} / 9412322$

[52] K. L. Chan, U. Chattopadhyay and P. Nath, Phys. Rev. D58, 096004 (1998), hep-ph/9710473.

[53] L. Giusti, A. Romanino and A. Strumia, Nucl. Phys. B550, 3 (1999), hep$\mathrm{ph} / 9811386$.

[54] J. L. Feng, K. T. Matchev and T. Moroi, Phys. Rev. Lett. 84, 2322 (2000), hep-ph/9908309, Phys. Rev. D61, 075005 (2000), hep-ph/9909334.

[55] J. Gasser and H. Leutwyler, Nucl. Phys. B250, 465 (1985).

[56] R. S. Chivukula, E. H. Simmons and J. Terning, Phys. Lett. B331, 383 (1994), hep-ph/9404209.

[57] K. Hagiwara and N. Kitazawa, Phys. Rev. D52, 5374 (1995), hep-ph/9504332.

[58] U. Mahanta, Phys. Rev. D55, 5848 (1997), hep-ph/9611289; Phys. Rev. D56, 402 (1997).

[59] G. L. Kane, G. D. Kribs, S. P. Martin and J. D. Wells, Phys. Rev. D53, 213 (1996), hep-ph/9508265.

[60] A. Miyamoto, in Physics and Experiments with Linear $e^{+} e^{-}$Colliders, F. A. Harris, S. L. Olsen, S. Pakvasa, and X. Tata, eds. (World Scientific, 1993).

[61] Report of the Tevatron Higgs Working Group, http://fnth37.fnal.gov/susy.html.

[62] ATLAS Detector and Physics Performance Technical Design Report, LHCC 9914/15 (1999).

[63] JLC Group, JLC-I. KEK-Report 92-16 (1992). 
[64] J. F. Gunion, H. E. Haber and J. Wudka, Phys. Rev. D43, 904 (1991).

[65] D. Rainwater and D. Zeppenfeld, Phys. Rev. D60, 113004 (1999), hepph/9906218; T. Plehn, D. Rainwater and D. Zeppenfeld, Phys. Rev. D61, 093005 (2000), hep-ph/9911385.

[66] M. Battaglia, in [4], hep-ph/9910271.

[67] A. S. Kronfeld, Nucl. Phys. Proc. Suppl. 63, 311 (1998), hep-lat/9710007.

[68] D. L. Borden, D. A. Bauer and D. O. Caldwell, Phys. Rev. D48, 4018 (1993).

[69] P. Janot, in Physics and Experiments with Linear $e^{+} e^{-}$Colliders, F. A. Harris, S. L. Olsen, S. Pakvasa, and X. Tata, eds. (World Scientific, 1993).

[70] B. Grzadkowski and J. F. Gunion, Phys. Lett. B294, 361 (1992), hep$\mathrm{ph} / 9206262$.

[71] E. Asakawa, J. Kamoshita, A. Sugamoto and I. Watanabe, Eur. Phys. J. C14, 335 (2000), hep-ph/9912373.

[72] A. Djouadi et al. hep-ph/9904287.

[73] A. Djouadi et al. hep-ph/9903229.

[74] M. E. Peskin, Prog. Theor. Phys. Suppl. 123, 507 (1996), hep-ph/9604339.

[75] S. Abdullin et al. [CMS Collaboration], hep-ph/9806366.

[76] N. Danielson, http://hep-www.colorado.edu/SUSY/danielson_thesis .ps.

[77] H. Martyn and G. A. Blair, in [4], hep-ph/9910416.

[78] M. N. Danielson, et al., in New Directions for High-Energy Physics: Snowmass 96, D. G. Cassel, L. T. Gennari, and R. H. Siemann, eds. (SLAC, 1997).

[79] T. Tsukamoto, K. Fujii, H. Murayama, M. Yamaguchi and Y. Okada, Phys. Rev. D51, 3153 (1995).

[80] M. M. Nojiri, K. Fujii and T. Tsukamoto, Phys. Rev. D54, 6756 (1996), hep$\mathrm{ph} / 9606370$.

[81] H. Cheng, J. L. Feng and N. Polonsky, Phys. Rev. D56, 6875 (1997), hepph/9706438, Phys. Rev. D57, 152 (1998), hep-ph/9706476.

[82] M. M. Nojiri, D. M. Pierce and Y. Yamada, Phys. Rev. D57, 1539 (1998), hepph/9707244; S. Kiyoura, M. M. Nojiri, D. M. Pierce and Y. Yamada, Phys. Rev. D58, 075002 (1998), hep-ph/9803210.

[83] E. Katz, L. Randall and S. Su, Nucl. Phys. B536, 3 (1998), hep-ph/9801416.

[84] Y. Kato, in [4], hep-ph/9910293.

[85] J. L. Feng, M. E. Peskin, H. Murayama and X. Tata, Phys. Rev. D52, 1418 (1995), hep-ph/9502260.

[86] J. L. Feng and M. J. Strassler, Phys. Rev. D51, 4661 (1995), hep-ph/9408359; Phys. Rev. D55, 1326 (1997), hep-ph/9606477. 
[87] R. Frey et al., in New Directions for High-Energy Physics: Snowmass 96, D. G. Cassel, L. T. Gennari, and R. H. Siemann, eds. (SLAC, 1997), hep$\mathrm{ph} / 9704243$.

[88] Future Electroweak Physics at the Fermilab Tevatron, D. Amidei and R. Brock, eds. FERMILAB-PUB-96/082.

[89] Y. Sumino, Acta Phys. Polon. B25, 1837 (1994), hep-ph/9411310.

[90] A. H. Hoang, Nucl. Phys. Proc. Suppl. 86, 512 (2000), hep-ph/9909356.

[91] A. H. Hoang et al., Eur. Phys. J. direct C3, 1 (2000), hep-ph/0001286.

[92] K. Fujii, T. Matsui, and Y. Sumino, Phys. Rev. D50, 4341 (1994).

[93] P. Comas, R. Miquel, M. Martinez, and S. Orteu, in Physics and Experiments with Linear Colliders, A. Miyamoto, Y. Fujii, and T. Matsui, eds. (World Scientific, 1996).

[94] D. Peralta, M. Martinez, and R. Miquel, in [4].

[95] B. Grzadkowski and Z. Hioki, Phys. Lett. B476, 87 (2000), hep-ph/9911505, hep-ph/0004223.

[96] T. G. Rizzo, hep-ph/9605361.

[97] T. L. Barklow and C. R. Schmidt, in The Alburquerque Meeting (DPF94), S. Seidel, ed. (World Scientific, 1995).

[98] H. Baer, S. Dawson and L. Reina, Phys. Rev. D61, 013002 (2000), hep$\mathrm{ph} / 9906419$.

[99] A. Juste and G. Merino, hep-ph/9910301.

[100] J. Ellison and J. Wudka, Ann. Rev. Nucl. Part. Sci. 48, 33 (1998).

[101] G. Bella et al. [LEP TGC Working Group], LEPEWWG/TGC/2000-01, (March 2000).

[102] E.N. Argyres et al., Nucl. Phys. B391, 23 (1993).

[103] B. Abbott et al. (DØ Collaboration), Phys. Rev. D60, 072002 (1999).

[104] S. Haywood et al., hep-ph/0003275, to appear in the CERN Yellow Report on "Standard Model Physics (and More) at the LHC".

[105] C. Burgard, in [4].

[106] P. N. Burrows et al., in New Directions for High-Energy Physics: Snowmass 96, D. G. Cassel, L. T. Gennari, and R. H. Siemann, eds. (SLAC, 1997), hepex/9612012. B. A. Schumm, hep-ex/9612013

[107] S. J. Brodsky, F. Hautmann and D. E. Soper, Phys. Rev. D56, 6957 (1997), hep-ph/9706427.

[108] R. Hawkings and K. Monig, hep-ex/9910022. J. Erler, S. Heinemeyer, W. Hollik, G. Weiglein and P. M. Zerwas, hep-ph/0005024.

[109] V. Ruhlmann-Kleider, in Proceedings of the XIX International Symposium on Lepton and Photon Interactions at High Energies, J. A. Jaros and M. E. Peskin, eds. (World Scientific, 2000). hep-ex/0001061. 
[110] E. Boos, H. J. He, W. Kilian, A. Pukhov, C. P. Yuan and P. M. Zerwas, Phys. Rev. D57, 1553 (1998), hep-ph/9708310; Phys. Rev. D61, 077901 (2000), hep$\mathrm{ph} / 9908409$.

[111] T. Han, Y. J. Kim, A. Likhoded and G. Valencia, hep-ph/0005306.

[112] J. L. Feng and T. Moroi, Phys. Rev. D56, 5962 (1997), hep-ph/9612333.

[113] J. L. Feng and D. E. Finnell, Phys. Rev. D49, 2369 (1994), hep-ph/9310211.

[114] A. Bartl, H. Eberl, S. Kraml, W. Majerotto and W. Porod, hep-ph/0002115.

[115] P. M. Zerwas, hep-ph/0003221; G. A. Blair, W. Porod and P. M. Zerwas, Phys. Rev. D 63, 017703 (2001), hep-ph/0007107.

[116] J. L. Hewett and T. G. Rizzo, Phys. Rept. 183, 193 (1989).

[117] A. Leike, Phys. Rept. 317, 143 (1999), hep-ph/9805494.

[118] M. Cvetič and P. Langacker, hep-ph/9707451.

[119] M. Cvetič and P. Langacker, Mod. Phys. Lett. A11, 1247 (1996), hep$\mathrm{ph} / 9602424$.

[120] A. Leike and S. Riemann, hep-ph/9604321.

[121] M. Cvetic and S. Godfrey, hep-ph/9504216.

[122] F. Del Aguila, M. Cvetič and P. Langacker, Phys. Rev. D52, 37 (1995), hep$\mathrm{ph} / 9501390$.

[123] T. G. Rizzo, Int. J. Mod. Phys. A13, 2245 (1998), hep-ph/9710229.

[124] T. L. Barklow, Int. J. Mod. Phys. A11, 1579 (1996).

[125] I. Antoniadis, Phys. Lett. B246, 377 (1990).

[126] J. D. Lykken, Phys. Rev. D54, 3693 (1996), hep-th/9603133.

[127] N. Arkani-Hamed, S. Dimopoulos and G. Dvali, Phys. Lett. B429, 263 (1998), hep-ph/9803315.

[128] M. E. Peskin, hep-ph/0002041, to appear in the Proceedings of the 1999 European Physical Society High Energy Physics Conference.

[129] L. Randall and R. Sundrum, Phys. Rev. Lett. 83, 3370 (1999), hep-ph/9905221.

[130] H. Davoudiasl, J. L. Hewett and T. G. Rizzo, Phys. Rev. Lett. 84, 2080 (2000), hep-ph/9909255, hep-ph/0006041.

[131] ALEPH Collaboration, ALEPH 99-051, paper contributed to the 1999 European Physical Society High Energy Physics Conference.

[132] M. Acciarri et al. [L3 Collaboration], Phys. Lett. B470, 281 (1999), hepex/9910056.

[133] G. Landsberg [DØ Collaboration], presentation at the April, 2000, APS meeting.

[134] J. L. Hewett, in [4]. 
Sourcebook for Linear Collider Physics 


\section{Chapter 3 Higgs Bosons at the Linear Collider}

\section{Introduction}

This chapter shows how a linear collider (LC) can contribute to our understanding of the Higgs sector through detailed studies of the physical Higgs boson state(s). Although this subject has been reviewed several times in the past [1-5], there are at least two reasons to revisit the subject. First, the completion of the LEP2 Higgs search, together with earlier precise measurements from SLC, LEP, and the Tevatron, gives us a clearer idea of what to expect. The simplest explanations of these results point to a light Higgs boson with (nearly) standard couplings to $W$ and $Z$. The key properties of such a particle can be investigated with a $500 \mathrm{GeV}$ LC. Second, the luminosity expected from the LC is now higher: $200-300 \mathrm{fb}^{-1} \mathrm{yr}^{-1}$ at $\sqrt{s}=500 \mathrm{GeV}$, and $300-500 \mathrm{fb}^{-1} \mathrm{yr}^{-1}$ at $\sqrt{s}=800 \mathrm{GeV}$. Consequently, several tens of thousands of Higgs bosons should be produced in each year of operation. With such samples, several measurements become more feasible, and the precision of the whole body of expected results becomes such as to lend insight not only into the nature of the Higgs boson(s), but also into the dynamics of higher scales.

There is an enormous literature on the Higgs boson and, more generally, on possible mechanisms of electroweak symmetry breaking. It is impossible to discuss all of it here. To provide a manageable, but nevertheless illustrative, survey of LC capabilities, we focus mostly on the Higgs boson of the Standard Model (SM), and on the Higgs bosons of the minimal supersymmetric extension of the SM (MSSM). Although this choice is partly motivated by simplicity, a stronger impetus comes from the precision data collected over the past few years, and some other related considerations.

The SM, which adds to the observed particles a single complex doublet of scalar fields, is economical. It provides an impressive fit to the precision data. Many extended models of electroweak symmetry breaking possess a limit, called the decoupling limit, that is experimentally almost indistinguishable from the SM. These models agree with the data equally well, and even away from the decoupling limit they usually predict a weakly coupled Higgs boson whose mass is at most several hundred GeV. Thus, the SM serves as a basis for discussing the Higgs phenomenology of a wide range of models, all of which are compatible with experimental constraints.

The SM suffers from several theoretical problems, which are either absent or less severe with weak-scale supersymmetry. The Higgs sector of the MSSM is a constrained two Higgs doublet model, consisting of two CP-even Higgs bosons, $h^{0}$ and $H^{0}$, a CP-odd Higgs boson, $A^{0}$, and a charged Higgs pair, $H^{ \pm}$. The MSSM is especially attractive because the superpartners modify the running of the strong, weak, and 
electromagnetic gauge couplings in just the right way as to yield unification at about $10^{16} \mathrm{GeV}[6]$. For this reason, the MSSM is arguably the most compelling extension of the SM. This is directly relevant to Higgs phenomenology, because in the MSSM a theoretical bound requires that the lightest CP-even Higgs boson $h^{0}$ has a mass less than $135 \mathrm{GeV}$. (In non-minimal supersymmetric models, the bound can be relaxed to around $200 \mathrm{GeV}$.) Furthermore, the MSSM offers, in some regions of parameter space, very non-standard Higgs phenomenology, so the full range of possibilities in the MSSM can be used to indicate how well the LC performs in non-standard scenarios. Thus, we use the SM to show how the LC fares when there is only one observable Higgs boson, and the MSSM to illustrate how extra fields can complicate the phenomenology. We also use various other models to illustrate important exceptions to conclusions that would be drawn from these two models alone.

The rest of this chapter is organized as follows. Section 2 gives, in some detail, the argument that one should expect a weakly coupled Higgs boson with a mass that is probably below about $200 \mathrm{GeV}$. In Section 3, we summarize the theory of the Standard Model Higgs boson. In Section 4, we review the expectations for Higgs discovery and the determination of Higgs boson properties at the Tevatron and LHC. In Section 5, we introduce the Higgs sector of the minimal supersymmetric extension of the Standard Model (MSSM) and discuss its theoretical properties. The present direct search limits are reviewed, and expectations for discovery at the Tevatron and LHC are described in Section 6. In Section 7, we treat the theory of the non-minimal Higgs sector more generally. In particular, we focus on the decoupling limit, in which the properties of the lightest Higgs scalar are nearly identical to those of the Standard Model Higgs boson, and discuss how to distinguish the two. We also discuss some non-decoupling exceptions to the usual decoupling scenario.

Finally, we turn to the program of Higgs measurements that can be carried out at the LC, focusing on $e^{+} e^{-}$collisions at higher energy, but also including material on the impact of Giga-Z operation and $\gamma \gamma$ collisions. The measurement of Higgs boson properties in $e^{+} e^{-}$collisions is outlined in Section 8. This includes a survey of the measurements that can be made for a SM-like Higgs boson for all masses up to $500 \mathrm{GeV}$. We also discuss measurements of the extra Higgs bosons that appear in the MSSM. Because the phenomenology of decoupling limit mimics, by definition, the SM Higgs boson, we emphasize how the precision that stems from high luminosity helps to diagnose the underlying dynamics. In Section 9, we outline the impact of Giga-Z operation on constraining and exploring various scenarios. In Section 10, the most important gains from $\gamma \gamma$ collisions are reviewed. Finally, in Section 11, we briefly discuss the case of a Higgs sector containing triplet Higgs representations and also consider the Higgs-like particles that can arise if the underlying assumption of a weakly coupled elementary Higgs sector is not realized in Nature. 
Higgs Bosons at the Linear Collider

\section{Expectations for electroweak symmetry breaking}

With the recent completion of experimentation at the LEP collider, the Standard Model of particle physics appears close to final experimental verification. After more than ten years of precision measurements of electroweak observables at LEP, SLC and the Tevatron, no definitive departures from Standard Model predictions have been found [7]. In some cases, theoretical predictions have been checked with an accuracy of one part in a thousand or better. However, the dynamics responsible for electroweak symmetry breaking has not yet been directly identified. Nevertheless, this dynamics affects predictions for currently observed electroweak processes at the one-loop quantum level. Consequently, the analysis of precision electroweak data can already provide some useful constraints on the nature of electroweak symmetry breaking dynamics.

In the minimal Standard Model, electroweak symmetry breaking dynamics arises via a self-interacting complex doublet of scalar fields, which consists of four real degrees of freedom. Renormalizable interactions are arranged in such a way that the neutral component of the scalar doublet acquires a vacuum expectation value, $v=246 \mathrm{GeV}$, which sets the scale of electroweak symmetry breaking. Hence, three massless Goldstone bosons are generated that are absorbed by the $W^{ \pm}$and $Z$, thereby providing the resulting massive gauge bosons with longitudinal components. The fourth scalar degree of freedom that remains in the physical spectrum is the CP-even neutral Higgs boson of the Standard Model. It is further assumed in the Standard Model that the scalar doublet also couples to fermions through Yukawa interactions. After electroweak symmetry breaking, these interactions are responsible for the generation of quark and charged lepton masses.

The global analysis of electroweak observables provides a superb fit to the Standard Model predictions. Such analyses take the Higgs mass as a free parameter. The electroweak observables depend logarithmically on the Higgs mass through its oneloop effects. The accuracy of the current data (and the reliability of the corresponding theoretical computations) already provides a significant constraint on the value of the Higgs mass. In [8,9], the non-observation of the Higgs boson is combined with the constraints of the global precision electroweak analysis to yield $m_{h_{\mathrm{SM}}} \lesssim 205-230 \mathrm{GeV}$ at $95 \% \mathrm{CL}$ (the quoted range reflects various theoretical choices in the analysis). Meanwhile, direct searches for the Higgs mass at LEP achieved a 95\% CL limit of $m_{h_{\mathrm{SM}}}>113.5 \mathrm{GeV} .^{1}$

One can question the significance of these results. After all, the self-interacting scalar field is only one model of electroweak symmetry breaking; other approaches, based on very different dynamics, are also possible. For example, one can introduce

\footnotetext{
${ }^{1}$ The LEP experiments presented evidence for a Higgs mass signal at a mass of $m_{h_{\mathrm{SM}}}=$ $115.0_{-0.9}^{+1.3} \mathrm{GeV}$, with an assigned significance of $2.9 \sigma[10]$. Although suggestive, the data are not significant enough to warrant a claim of a Higgs discovery.
} 
new fermions and new forces, in which the Goldstone bosons are a consequence of the strong binding of the new fermion fields [11]. Present experimental data are not sufficient to identify with certainty the nature of the dynamics responsible for electroweak symmetry breaking. Nevertheless, one can attempt to classify alternative scenarios and study the constraints of the global precision electroweak fits and the implications for phenomenology at future colliders. Since electroweak symmetry dynamics must affect the one-loop corrections to electroweak observables, the constraints on alternative approaches can be obtained by generalizing the global precision electroweak fits to allow for new contributions at one-loop. These enter primarily through corrections to the self-energies of the gauge bosons (the so-called "oblique" corrections). Under the assumption that any new physics is characterized by a new mass scale $M \gg m_{Z}$, one can parameterize the leading oblique corrections by three constants, $S, T$, and $U$, first introduced by Peskin and Takeuchi [12]. In almost all theories of electroweak symmetry breaking dynamics, $U \ll S, T$, so it is sufficient to consider a global electroweak fit in which $m_{h_{\mathrm{SM}}}, S$ and $T$ are free parameters. (The zero of the $S-T$ plane must be defined relative to some fixed value of the Higgs mass, usually taken to be $100 \mathrm{GeV}$.) New electroweak symmetry breaking dynamics could generate non-zero values of $S$ and $T$, while allowing for a much heavier Higgs mass (or equivalent). Various possibilities have been recently classified by Peskin and Wells [13], who argue that any dynamics that results in a significantly heavier Higgs boson should also generate new experimental signatures at the $\mathrm{TeV}$ scale that can be studied at the $\mathrm{LC}$, either directly by producing new particles or indirectly by improving precision measurements of electroweak observables.

In this chapter, we mainly consider the simplest possible interpretation of the precision electroweak data, namely, that there exists a light weakly coupled Higgs boson. Nevertheless, this still does not fix the theory of electroweak symmetry breaking. It is easy to construct extensions of the scalar boson dynamics and generate non-minimal Higgs sectors. Such theories can contain charged Higgs bosons and neutral Higgs bosons of opposite (or indefinite) CP-quantum numbers. Although some theoretical constraints exist, there is still considerable freedom in constructing models which satisfy all known experimental constraints. Moreover, in most extensions of the Standard Model, there exists a large range of parameter space in which the properties of the lightest Higgs scalar are virtually indistinguishable from those of the Standard Model Higgs boson. One of the challenges of experiments at future colliders, once the Higgs boson is discovered, is to see whether there are any deviations from the properties expected for the Standard Model Higgs boson.

Although the Standard Model provides a remarkably successful description of the properties of the quarks, leptons and spin-1 gauge bosons at energy scales of $\mathcal{O}(100) \mathrm{GeV}$ and below, the Standard Model is not the ultimate theory of the fundamental particles and their interactions. At an energy scale above the Planck scale, $M_{\mathrm{PL}} \simeq 10^{19} \mathrm{GeV}$, quantum gravitational effects become significant and the Standard 
Model must be replaced by a more fundamental theory that incorporates gravity. It is also possible that the Standard Model breaks down at some energy scale, $\Lambda$, below the Planck scale. In this case, the Standard Model degrees of freedom are no longer adequate for describing the physics above $\Lambda$ and new physics must enter. Thus, the Standard Model is not a fundamental theory; at best, it is an effective field theory [14]. At an energy scale below $\Lambda$, the Standard Model (with higher-dimension operators to parameterize the new physics at the scale $\Lambda$ ) provides an extremely good description of all observable phenomena.

An essential question that future experiments must address is: what is the minimum scale $\Lambda$ at which new physics beyond the Standard Model must enter? The answer to this question depends on the value of the Higgs mass, $m_{h_{\mathrm{SM}}}$. If $m_{h_{\mathrm{SM}}}$ is too large, then the Higgs self-coupling blows up at some scale $\Lambda$ below the Planck scale [15]. If $m_{h_{\mathrm{SM}}}$ is too small, then the Higgs potential develops a second (global) minimum at a large value of the scalar field of order $\Lambda$ [16]. Thus, new physics must enter at a scale $\Lambda$ or below in order that the true minimum of the theory correspond to the observed $\mathrm{SU}(2) \times \mathrm{U}(1)$ broken vacuum with $v=246 \mathrm{GeV}$ for scales above $\Lambda$. Thus, given a value of $\Lambda$, one can compute the minimum and maximum Higgs mass allowed. Although the arguments just given are based on perturbation theory, it is possible to repeat the analysis of the Higgs-Yukawa sector non-perturbatively [17]. These results are in agreement with the perturbative estimates. The results of this analysis (with shaded bands indicating the theoretical uncertainty of the result) are illustrated in Fig. 3.1.

Although the Higgs mass range $130 \mathrm{GeV} \lesssim m_{h_{\mathrm{SM}}} \lesssim 180 \mathrm{GeV}$ appears to permit an effective Standard Model that survives all the way to the Planck scale, most theorists consider such a possibility unlikely. This conclusion is based on the "naturalness" [19] argument as follows. In an effective field theory, all parameters of the low-energy theory (i.e., masses and couplings) are calculable in terms of parameters of a more fundamental theory that describes physics at the energy scale $\Lambda$. All low-energy couplings and fermion masses are logarithmically sensitive to $\Lambda$. In contrast, scalar squared-masses are quadratically sensitive to $\Lambda$. The Higgs mass (at one-loop) has the following heuristic form:

$$
m_{h}^{2}=\left(m_{h}^{2}\right)_{0}+\frac{c g^{2}}{16 \pi^{2}} \Lambda^{2},
$$

where $\left(m_{h}^{2}\right)_{0}$ is a parameter of the fundamental theory and $c$ is a constant, presumably of $\mathcal{O}(1)$, that depends on the physics of the low-energy effective theory. The "natural" value for the scalar squared-mass is $g^{2} \Lambda^{2} / 16 \pi^{2}$. Thus, the expectation for $\Lambda$ is

$$
\Lambda \simeq \frac{4 \pi m_{h}}{g} \sim \mathcal{O}(1 \mathrm{TeV}) .
$$

If $\Lambda$ is significantly larger than $1 \mathrm{TeV}$ then the only way for the Higgs mass to be of order the scale of electroweak symmetry breaking is to have an "unnatural" 


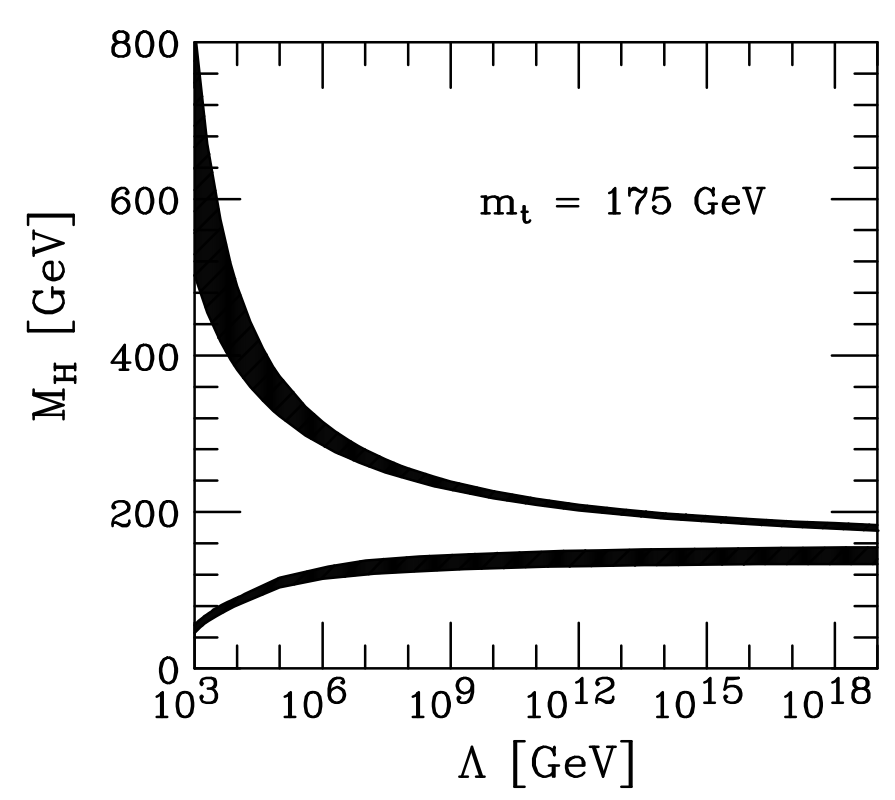

Figure 3.1: The upper [15] and the lower [16] Higgs mass bounds as a function of the energy scale $\Lambda$ at which the Standard Model breaks down, assuming $m_{t}=175 \mathrm{GeV}$ and $\alpha_{s}\left(m_{Z}\right)=$ 0.118. The shaded areas above reflect the theoretical uncertainties in the calculations of the Higgs mass bounds. This figure is taken from [18].

cancellation between the two terms of Eq. (3.1). This seems highly unlikely given that the two terms of Eq. (3.1) have completely different origins.

An attractive theoretical framework that incorporates weakly coupled Higgs bosons and satisfies the constraint of Eq. (3.2) is that of "low-energy" or "weak-scale" supersymmetry [20,21]. In this framework, supersymmetry is used to relate fermion and boson masses and interaction strengths. Since fermion masses are only logarithmically sensitive to $\Lambda$, boson masses will exhibit the same logarithmic sensitivity if supersymmetry is exact. Since no supersymmetric partners of Standard Model particles have yet been found, supersymmetry cannot be an exact symmetry of nature. Thus, $\Lambda$ should be identified with the supersymmetry breaking scale. The naturalness constraint of Eq. (3.2) is still relevant. It implies that the scale of supersymmetry breaking should not be much larger than $1 \mathrm{TeV}$, to preserve the naturalness of scalar masses. The supersymmetric extension of the Standard Model would then replace the Standard Model as the effective field theory of the $\mathrm{TeV}$ scale. One advantage of the supersymmetric approach is that the effective low-energy supersymmetric theory can be valid all the way up to the Planck scale, while still being natural! The unification of the three gauge couplings at an energy scale close to the Planck scale, which does not occur in the Standard Model, is seen to occur in the minimal supersymmetric extension of the Standard Model, and provides an additional motivation 
Higgs Bosons at the Linear Collider

for seriously considering the low-energy supersymmetric framework [6]. However, the fundamental origin of supersymmetry breaking is not known at present. Without a fundamental theory of supersymmetry breaking, one ends up with an effective low-energy theory characterized by over 100 unknown parameters that in principle would have to be measured by experiment. This remains one of the main stumbling blocks for creating a truly predictive model of fundamental particles and their interactions. Nevertheless, the Higgs sectors of the simplest supersymmetric models are quite strongly constrained, and exhibit very specific phenomenological profiles.

\section{The Standard Model Higgs boson-theory}

In the Standard Model, the Higgs mass is given by $m_{h_{S M}}^{2}=\lambda v^{2}$, where $\lambda$ is the Higgs self-coupling. Since $\lambda$ is unknown at present, the value of the Standard Model Higgs mass is not predicted (although other theoretical considerations, discussed in Section 2, place constraints on the Higgs mass, as exhibited in Fig. 3.1). The Higgs couplings to fermions and gauge bosons are proportional to the corresponding particle masses. As a result, Higgs phenomenology is governed primarily by the couplings of the Higgs boson to the $W^{ \pm}$and $Z$ and the third generation quarks and leptons. It should be noted that a $h_{\mathrm{SM}} g g$ coupling, where $g$ is the gluon, is induced by the oneloop graph in which the Higgs boson couples to a virtual $t \bar{t}$ pair. Likewise, a $h_{\mathrm{SM}} \gamma \gamma$ coupling is generated, although in this case the one-loop graph in which the Higgs boson couples to a virtual $W^{+} W^{-}$pair is the dominant contribution. Further details of Standard Higgs boson properties are given in [1].

\subsection{Standard Model Higgs boson decay modes}

The Higgs boson mass is the only unknown parameter in the Standard Model. Thus, one can compute Higgs boson branching ratios and production cross sections as a function of $m_{h_{\mathrm{SM}}}$. The branching ratios for the dominant decay modes of a Standard Model Higgs boson are shown as a function of Higgs boson mass in Fig. 3.2. Note that subdominant channels are important to establish a complete phenomenological profile of the Higgs boson, and to check consistency (or look for departures from) Standard Model predictions. For $115 \mathrm{GeV} \sim m_{h_{\mathrm{SM}}} \lesssim 2 m_{W}$ many decays modes are large enough to measure, as discussed in Section 8.

For $m_{h_{\mathrm{SM}}} \lesssim 135 \mathrm{GeV}$, the main Higgs decay mode is $h_{\mathrm{SM}} \rightarrow b \bar{b}$, while the decays $h_{\mathrm{SM}} \rightarrow \tau^{+} \tau^{-}$and $c \bar{c}$ can also be phenomenologically relevant. In addition, although one-loop suppressed, the decay $h_{\mathrm{SM}} \rightarrow g g$ is competitive with other decays for $m_{h_{\mathrm{SM}}} \lesssim$ $2 m_{W}$ because of the large top Yukawa coupling and the color factor. As the Higgs mass increases above $135 \mathrm{GeV}$, the branching ratio to vector boson pairs becomes dominant. In particular, the main Higgs decay mode is $h_{\mathrm{SM}} \rightarrow W W^{(*)}$, where one

of the $W$ 's must be off-shell (indicated by the star superscript) if $m_{h_{\mathrm{SM}}}<2 m_{W}$. For 
Higgs bosons with $m_{h_{\mathrm{SM}}} \gtrsim 2 m_{t}$, the decay $h_{\mathrm{SM}} \rightarrow t \bar{t}$ begins to increase until it reaches its maximal value of about $20 \%$.

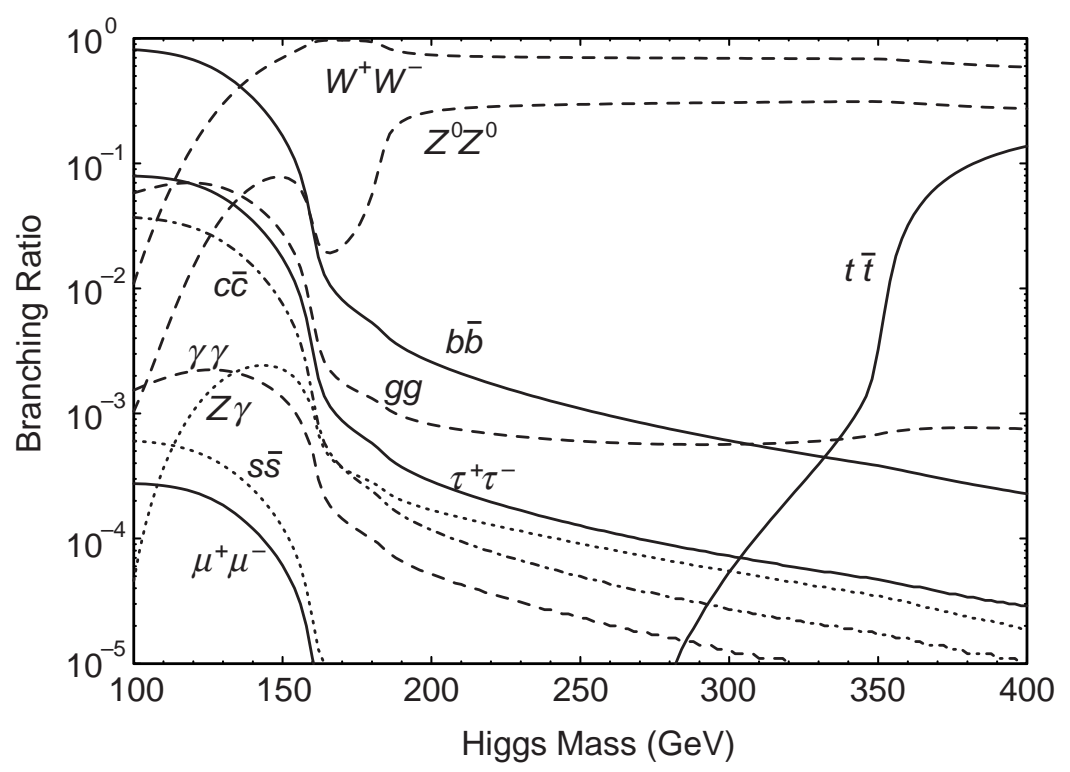

Figure 3.2: Branching ratios of the dominant decay modes of the Standard Model Higgs boson. These results have been obtained with the program HDECAY [22], and include QCD corrections beyond the leading order.

Rare Higgs decay modes can also play an important role. The one-loop decay $h_{\mathrm{SM}} \rightarrow \gamma \gamma$ is a suppressed mode. For $m_{W} \lesssim m_{h_{\mathrm{SM}}} \lesssim 2 m_{W}, \mathrm{BR}\left(h_{\mathrm{SM}} \rightarrow \gamma \gamma\right)$ is above $10^{-3}$. This decay channel provides an important Higgs discovery mode at the LHC for $100 \mathrm{GeV} \lesssim m_{h_{\mathrm{SM}}} \lesssim 150 \mathrm{GeV}$. At the LC, the direct observation of $h_{\mathrm{SM}} \rightarrow \gamma \gamma$ is difficult because of its suppressed branching ratio. Perhaps more relevant is the partial width $\Gamma\left(h^{0} \rightarrow \gamma \gamma\right)$, which controls the Higgs production rate at a $\gamma \gamma$ collider.

\subsection{Standard Model Higgs boson production at the LC}

In the Standard Model there are two main processes to produce the Higgs boson in $e^{+} e^{-}$annihilation. These processes are also relevant in many extensions of the Standard Model, particularly in nearly-decoupled extensions, in which the lightest CP-even Higgs boson possesses properties nearly identical to those of the SM Higgs boson. In the "Higgsstrahlung" process, a virtual $Z$ boson decays to an on-shell $Z$ and the $h_{\mathrm{SM}}$, depicted in Fig. 3.3(a). The cross section for Higgsstrahlung rises sharply at threshold to a maximum a few tens of $\mathrm{GeV}$ above $m_{h}+m_{Z}$, and then falls off as $s^{-1}$, as shown in Fig. 3.4. The associated production of the $Z$ provides an important trigger for Higgsstrahlung events. In particular, in some theories beyond the Standard Model, in which the Higgs boson decays into invisible modes, the Higgs 
(a)

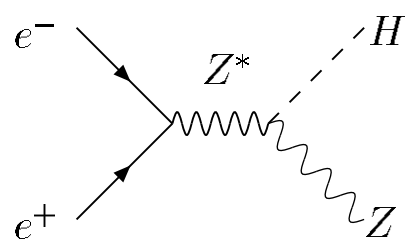

(b)

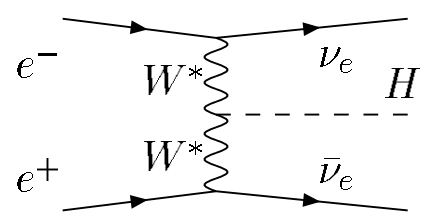

Figure 3.3: Main production processes for Higgs production in $e^{+} e^{-}$annihilation. (a) Higgsstrahlung. (b) $W W$ fusion.

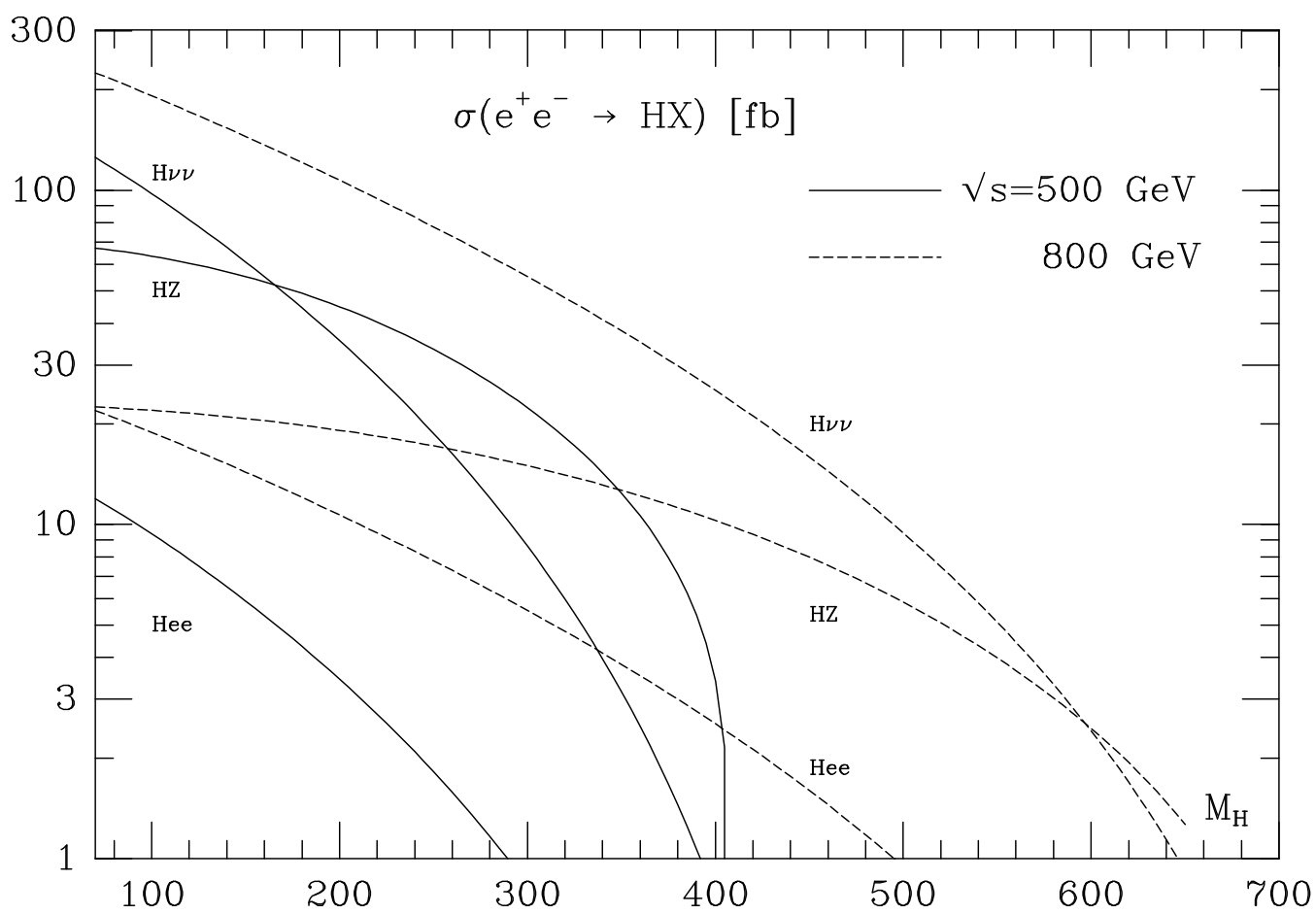

Figure 3.4: Cross sections for Higgsstrahlung $\left(e^{+} e^{-} \rightarrow Z h_{\mathrm{SM}}\right)$ and Higgs production via $W^{+} W^{-}$fusion $\left(e^{+} e^{-} \rightarrow \nu \bar{\nu} h_{\mathrm{SM}}\right)$ and $Z Z$ fusion $\left(e^{+} e^{-} \rightarrow e^{+} e^{-} h_{\mathrm{SM}}\right)$ as a function of $m_{h_{\mathrm{SM}}}$ for two center-of-mass energies, $\sqrt{s}=500$ and $800 \mathrm{GeV}$ [5].

boson mass peak can be reconstructed in the spectrum of the missing mass recoiling against the $Z$. The other production process is called "vector boson fusion", where the incoming $e^{+}$and $e^{-}$each emit a virtual vector boson, followed by vector boson fusion to the $h_{\mathrm{SM}}$. Figure 3.3(b) depicts the $W^{+} W^{-}$fusion process. Similarly, the $Z Z$ fusion process corresponds to $e^{+} e^{-} \rightarrow e^{+} e^{-} h_{\mathrm{SM}}$. In contrast to Higgsstrahlung, the vector boson fusion cross section grows as $\ln s$, and thus is the dominant Higgs production mechanism for $\sqrt{s} \gg m_{h_{\mathrm{SM}}}$. The cross section for $W W$ fusion is about ten times larger than that for $Z Z$ fusion. Nevertheless, the latter provides complementary 
information on the $Z Z h_{\mathrm{SM}}$ vertex. Note that at an $e^{-} e^{-}$collider, the Higgsstrahlung and $W^{+} W^{-}$fusion processes are absent, so that $Z Z$ fusion is the dominant Higgs production process.

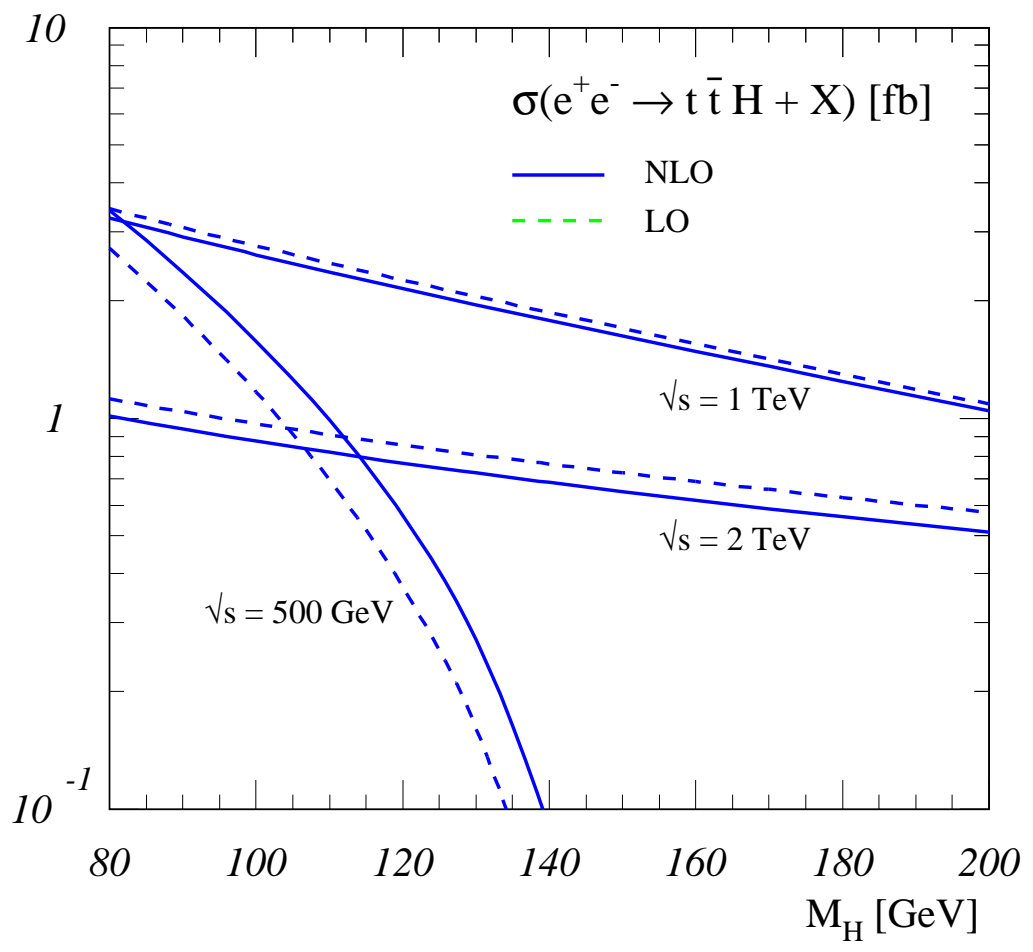

Figure 3.5: Cross-sections for $e^{+} e^{-} \rightarrow t \bar{t} h_{\mathrm{SM}}$ in fb for three choices of center-of-mass energy. The dashed lines correspond to the tree-level result [23], and the solid lines include the nextto-leading order QCD corrections [24].

Other relevant processes for producing Higgs bosons are associated production with a fermion-antifermion pair, and multi-Higgs production. For the former class, only $e^{+} e^{-} \rightarrow t \bar{t} h_{\mathrm{SM}}$ has a significant cross section, around the femtobarn level in the Standard Model, as depicted in Fig. 3.5. As a result, if $m_{h_{\mathrm{SM}}}$ is small enough (or $\sqrt{s}$ is large enough), this process can be used for determining the Higgs-top quark Yukawa coupling. The cross section for double Higgs production $\left(e^{+} e^{-} \rightarrow Z h_{\mathrm{SM}} h_{\mathrm{SM}}\right)$ are even smaller, of order $0.1 \mathrm{fb}$ for $100 \mathrm{GeV} \lesssim m_{h_{\mathrm{SM}}} \lesssim 150 \mathrm{GeV}$ and $\sqrt{s}$ ranging between $500 \mathrm{GeV}$ and $1 \mathrm{TeV}$. With sufficient luminosity, the latter can be used for extracting the triple Higgs self-coupling.

At the $\gamma \gamma$ collider, a Higgs boson is produced as an $s$-channel resonance via the one-loop triangle diagram. Every charged particle whose mass is generated by the Higgs boson contributes to this process. In the Standard Model, the main contributors are the $W^{ \pm}$and the $t$-quark loops. See Section 10 for further discussion. 
Higgs Bosons at the Linear Collider

\section{SM Higgs searches before the linear collider}

\subsection{Direct search limits from LEP}

The LEP collider completed its final run in 2000, and presented tantalizing hints for the possible observation of the Higgs boson. Combining data from all four LEP collaborations [10], one could interpret their observations as corresponding to the production of a Higgs boson with a mass of $m_{h^{0}}=115.0_{-0.9}^{+1.3} \mathrm{GeV}$ with a significance of $2.9 \sigma$. This is clearly not sufficient to announce a discovery or even an "observation". A more conservative interpretation of the data then places a 95\% CL lower limit of $m_{h_{\mathrm{SM}}}>113.5 \mathrm{GeV}$.

\subsection{Implications of precision electroweak measurements}

Indirect constraints on the Higgs boson mass within the SM can be obtained from confronting the SM predictions with results of electroweak precision measurements. In the case of the top quark mass, the indirect determination turned out to be in remarkable agreement with the actual experimental value. In comparison, to obtain constraints on $m_{h_{\mathrm{SM}}}$ of similar precision, much higher accuracy is required for both the experimental results and the theory predictions. This is due to the fact that the leading dependence of the precision observables on $m_{h_{\mathrm{SM}}}$ is only logarithmic, while the dominant effects of the top-quark mass enter quadratically.
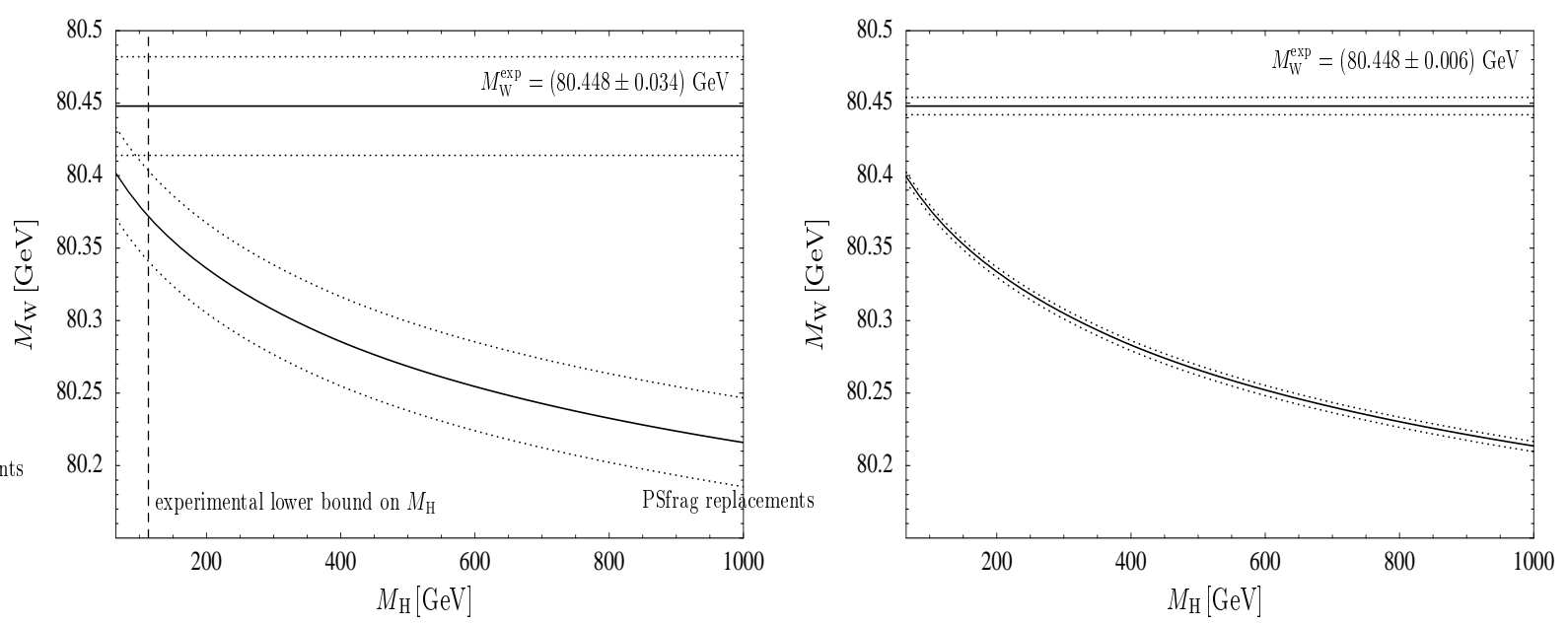

Figure 3.6: The prediction for $m_{W}$ as a function of $m_{h_{\mathrm{SM}}}$ is compared with the experimental value of $m_{W}$ for the current experimental accuracies of $m_{W}$ and $m_{t}$ (left plot) and for the prospective future accuracies at a LC with Giga-Z option (right plot, the present experimental central values are assumed) [25]. In the left plot also the present experimental 95\% CL lower bound on the Higgs-boson mass, $m_{h_{\mathrm{SM}}}=113.5 \mathrm{GeV}$, is indicated. 
The left plot of Fig. 3.6 shows the currently most precise result for $m_{W}$ as function of $m_{h_{\mathrm{SM}}}$ in the SM, and compares it with the present experimental value of $m_{W}$. The calculation incorporates the complete electroweak fermion-loop contributions at $\mathcal{O}\left(\alpha^{2}\right)[25]$. Based on this result, the remaining theoretical uncertainty from unknown higher-order corrections has been estimated to be about $6 \mathrm{MeV}$ [25]. It is about a factor five smaller than the uncertainty induced by the current experimental error on the top-quark mass, $\Delta m_{t}^{\exp }= \pm 5.1 \mathrm{GeV}$, which presently dominates the theoretical uncertainty. The right plot of Fig. 3.6 shows the prospective situation at a future $e^{+} e^{-}$ linear collider after Giga- $Z$ operation and a threshold measurement of the $W$ mass (keeping the present experimental central values for simplicity), which are expected to reduce the experimental errors to $\Delta m_{W}^{\exp }=6 \mathrm{MeV}$ and $\Delta m_{t}^{\exp }=200 \mathrm{MeV}$. This program is described in Chapter 8 . The plot clearly shows the considerable improvement in the sensitivity to $m_{h_{\mathrm{SM}}}$ achievable at the LC via very precise measurements of $m_{W}$ and $m_{t}$. Since furthermore the experimental error of $\sin ^{2} \theta_{w}^{\text {eff }}$ is expected to be reduced by almost a factor of 20 at Giga-Z, the accuracy in the indirect determination of the Higgs-boson mass from all data will improve by about a factor of 10 compared to the present situation [26].

\subsection{Expectations for Tevatron searches}

The upgraded Tevatron began taking data in the spring of 2001. This is the only collider at which the Higgs boson can be produced for the next five years, until the LHC begins operation in 2006. The Tevatron Higgs working group presented a detailed analysis of the Higgs discovery reach at the upgraded Tevatron [27]. Here, we summarize the main results. Two Higgs mass ranges were considered separately: (i) $100 \mathrm{GeV} \lesssim m_{h_{\mathrm{SM}}} \lesssim 135 \mathrm{GeV}$ and (ii) $135 \mathrm{GeV} \lesssim m_{h_{\mathrm{SM}}} \lesssim 190 \mathrm{GeV}$, corresponding to the two different dominant Higgs decay modes: $h_{\mathrm{SM}} \rightarrow b \bar{b}$ for the lighter mass range and $h_{\mathrm{SM}} \rightarrow W W^{(*)}$ for the heavier mass range.

In mass range (i), the relevant production mechanisms are $q_{i} \bar{q}_{j} \rightarrow V h_{\mathrm{SM}}$, where $V=W$ or $Z$. In all cases, the dominant $h_{\mathrm{SM}} \rightarrow b \bar{b}$ decay was employed. The most relevant final-state signatures correspond to events in which the vector boson decays leptonically ( $W \rightarrow \ell \nu, Z \rightarrow \ell^{+} \ell^{-}$and $Z \rightarrow \nu \bar{\nu}$, where $\ell=e$ or $\mu$ ), resulting in $\ell \nu b \bar{b}$, $\nu \bar{\nu} b \bar{b}$ and $\ell^{+} \ell^{-} b \bar{b}$ final states. In mass range (ii), the relevant production mechanisms include $g g \rightarrow h_{\mathrm{SM}}, V^{*} V^{*} \rightarrow h_{\mathrm{SM}}$ and $q_{i} \bar{q}_{j} \rightarrow V h_{\mathrm{SM}}$, with decays $h_{\mathrm{SM}} \rightarrow W W^{(*)}$, $Z Z^{(*)}$. The most relevant phenomenological signals are those in which two of the final-state vector bosons decay leptonically, resulting in $\ell^{+} \ell^{-} \nu \bar{\nu}$ or $\ell^{ \pm} \ell^{ \pm} j j X$, where $j$ is a hadronic jet and $X$ consists of two additional leptons (either charged or neutral). For example, the latter can arise from $W h_{\mathrm{SM}}$ production followed by $h_{\mathrm{SM}} \rightarrow W W^{(*)}$, where the two like-sign $W$ bosons decay leptonically, and the third $W$ decays into hadronic jets. In this case $X$ is a pair of neutrinos.

Figure 3.7 summarizes the Higgs discovery reach versus the total integrated lu- 


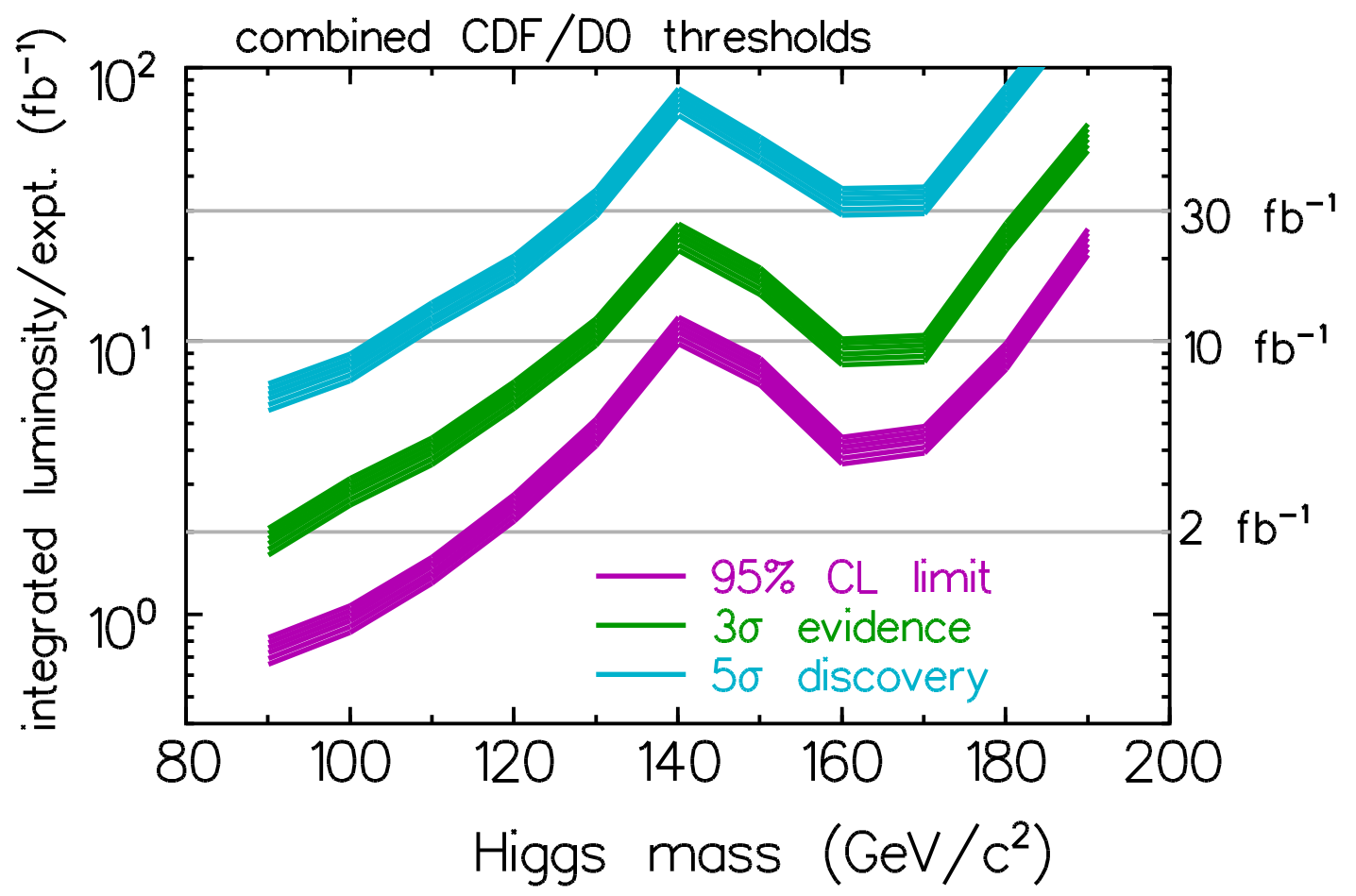

Figure 3.7: The integrated luminosity required per experiment, to either exclude a SM Higgs boson at $95 \%$ CL or discover it at the $3 \sigma$ or $5 \sigma$ level, as a function of the Higgs mass. These results are based on the combined statistical power of both experiments. The curves shown are obtained by combining the $\ell \nu b \bar{b}, \nu \bar{\nu} b \bar{b}$ and $\ell^{+} \ell^{-} b \bar{b}$ channels using the neural network selection in the low-mass Higgs region $\left(90 \mathrm{GeV} \lesssim m_{h_{\mathrm{SM}}} \lesssim 130 \mathrm{GeV}\right)$, and the $\ell^{ \pm} \ell^{ \pm} j j X$ and $\ell^{+} \ell^{-} \nu \bar{\nu}$ channels in the high-mass Higgs region $\left(130 \mathrm{GeV} \lesssim m_{h_{\mathrm{SM}}} \lesssim 190 \mathrm{GeV}\right)$. The lower edge of the bands is the calculated threshold; the bands extend upward from these nominal thresholds by $30 \%$ as an indication of the uncertainties in $b$-tagging efficiency, background rate, mass resolution, and other effects.

minosity delivered to the Tevatron (and by assumption, delivered to each detector). As the plot shows, the required integrated luminosity increases rapidly with Higgs mass to $140 \mathrm{GeV}$, beyond which the high-mass channels play the dominant role. With $2 \mathrm{fb}^{-1}$ per detector (which is expected after one year of running at design luminosity), the 95\% CL limits will barely extend the expected LEP2 limits, but with $10 \mathrm{fb}^{-1}$, the SM Higgs boson can be excluded up to $180 \mathrm{GeV}$ if the Higgs boson does not exist in that mass range.

Current projections envision that the Tevatron, with further machine improvements, will provide an integrated luminosity of $15 \mathrm{fb}^{-1}$ after six years of running. If $m_{h_{\mathrm{SM}}} \simeq 115 \mathrm{GeV}$, as suggested by LEP data, then the Tevatron experiments will be 
able to achieve a $5 \sigma$ discovery of the Higgs boson. If no Higgs events are detected, the LEP limits will be significantly extended, with a 95\% CL exclusion possible up to about $m_{h_{\mathrm{SM}}} \simeq 185 \mathrm{GeV}$. Moreover, evidence for a Higgs boson at the $3 \sigma$ level could be achieved up to about $m_{h_{\mathrm{SM}}} \simeq 175 \mathrm{GeV}$. (The Higgs mass region around $140 \mathrm{GeV}$ might require more luminosity, depending on the magnitude of systematic errors due to uncertainties in $b$-tagging efficiency, background rate, the $b \bar{b}$ mass resolution, etc.) Evidence for or discovery of a Higgs boson at the Tevatron would be a landmark in high energy physics. However, even if a Higgs boson is seen, the Tevatron data would only provide a very rough phenomenological profile. In contrast, the LC, and to a lesser extent, the LHC could measure enough of its properties with sufficient precision to verify that the observed Higgs is truly SM-like. The LHC is also certain to yield $>5 \sigma$ discovery of a SM Higgs boson over the full range of possible masses, up to $1 \mathrm{TeV}$.

\subsection{Expectations for LHC searches}

At the LHC, the ATLAS and CMS detectors have been specifically designed so as to guarantee discovery of a SM Higgs boson, regardless of mass. The most important production processes for the $h_{\mathrm{SM}}$ are the gluon fusion process, $g g \rightarrow h_{\mathrm{SM}}$, and the vector boson fusion process, $W W \rightarrow h_{\mathrm{SM}}$. In particular, for $m_{h_{\mathrm{SM}}} \lesssim 130 \mathrm{GeV}$ the important discovery modes are $g g, W W \rightarrow h_{\mathrm{SM}} \rightarrow \gamma \gamma, \tau^{+} \tau^{-}$. At high luminosity, $q_{i} \bar{q}_{j} \rightarrow W^{ \pm} h_{\mathrm{SM}}$ and $g g \rightarrow t \bar{t} h_{\mathrm{SM}}$ with $h_{\mathrm{SM}} \rightarrow \gamma \gamma$ and $h_{\mathrm{SM}} \rightarrow b \bar{b}$ should also be visible. Once $m_{h_{\mathrm{SM}}}>130 \mathrm{GeV}, g g \rightarrow h_{\mathrm{SM}} \rightarrow Z Z^{(*)} \rightarrow 4 \ell$ is extremely robust except for the small mass region with $m_{h_{\mathrm{SM}}}$ just above $2 m_{W}$ in which $h_{\mathrm{SM}} \rightarrow W W$ is allowed and $B\left(h_{\mathrm{SM}} \rightarrow Z Z^{*}\right)$ drops sharply. In this region, $g g, W W \rightarrow h_{\mathrm{SM}} \rightarrow W W \rightarrow \ell \nu \ell \nu$ provides a strong Higgs signal. Once $m_{h_{\mathrm{SM}}}>300 \mathrm{GeV}(400 \mathrm{GeV})$, the final states $h_{\mathrm{SM}} \rightarrow W W \rightarrow \ell \nu j j$ and $h_{\mathrm{SM}} \rightarrow Z Z \rightarrow \ell \ell \nu \nu$, where the $h_{\mathrm{SM}}$ is produced by a combination of $g g$ and $W W$ fusion, provide excellent discovery channels. These latter allow discovery even for $m_{h_{\mathrm{SM}}} \gtrsim 1 \mathrm{TeV}$, i.e., well beyond the $m_{h_{\mathrm{SM}}} \sim 800 \mathrm{GeV}$ limit of viability for the $h_{\mathrm{SM}} \rightarrow 4 \ell$ mode. These results are summarized in Fig. 3.8, from which we observe that the net statistical significance for the $h_{\mathrm{SM}}$, after combining channels, exceeds $10 \sigma$ for all $m_{h_{\mathrm{SM}}}>80 \mathrm{GeV}$, assuming accumulated luminosity of $L=100 \mathrm{fb}^{-1}$ at the ATLAS detector [29]. Similar results are obtained by the CMS group [30], the $\gamma \gamma$ mode being even stronger in the lower mass region.

Precision measurements for a certain number of quantities will be possible, depending upon the exact value of $m_{h_{\mathrm{SM}}}$. For instance, in [29] it is estimated that $m_{h_{\mathrm{SM}}}$ can be measured to $<0.1 \%$ for $m_{h_{\mathrm{SM}}}<400 \mathrm{GeV}$ and to $0.1-1 \%$ for $400<m_{h_{\mathrm{SM}}}<$ $700 \mathrm{GeV}$. Using the $4 \ell$ final state, $\Gamma_{h_{\mathrm{SM}}}^{T}$ can determined for $m_{h_{\mathrm{SM}}}>250 \mathrm{GeV}$ from the shape of the $4 \ell$ mass peak. Various ratios of branching ratios and a selection of partial widths times branching ratios can be measured in any given mass region. Some early estimates of possibilities and achievable accuracies appear in [2]. A more recent, but probably rather optimistic parton-level theoretical study [31] finds that 


\section{$5 \sigma$ Higgs Signals (statistical errors only)}

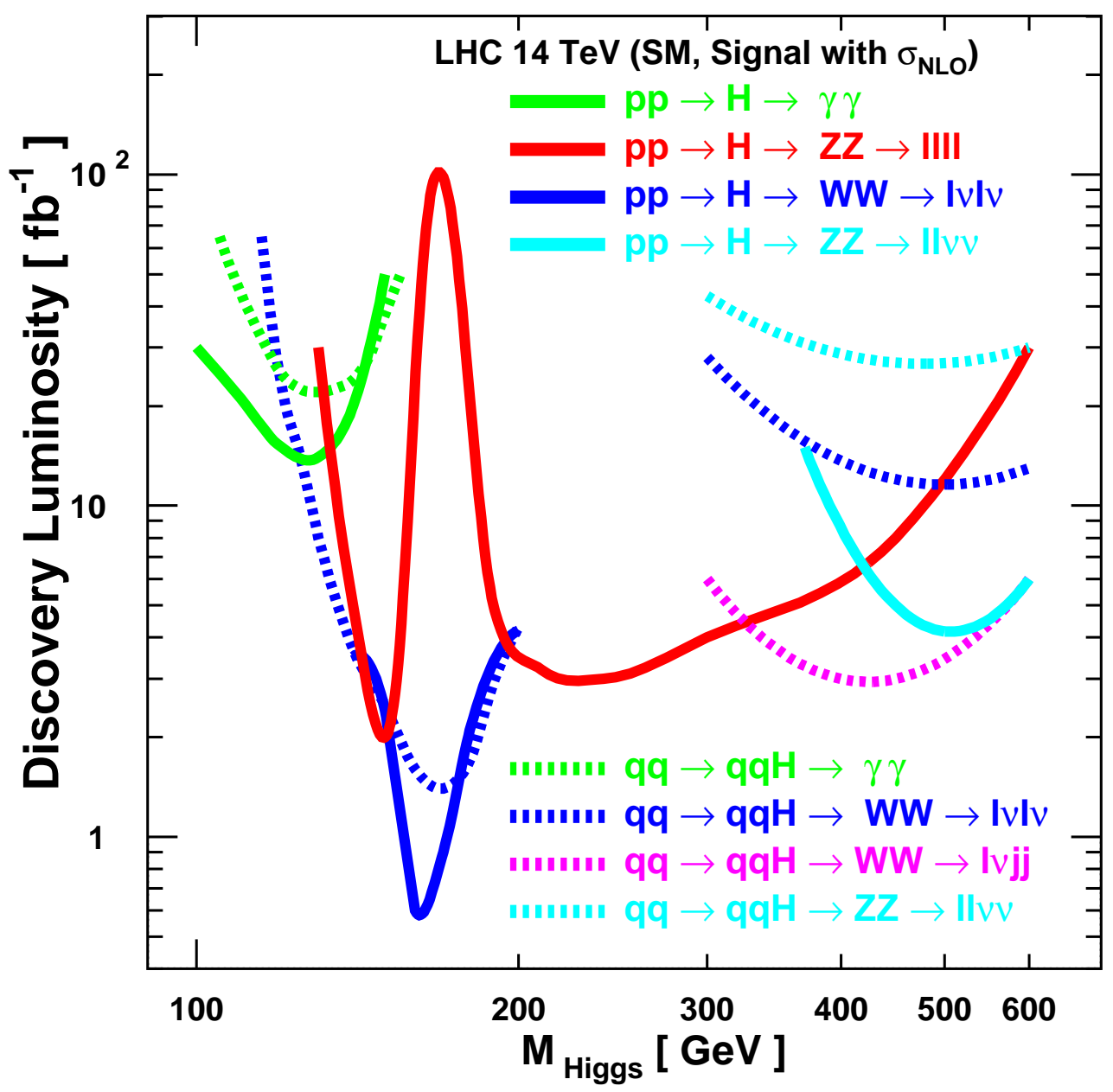

Figure 3.8: Expected $5 \sigma$ SM Higgs discovery luminosity requirements at the LHC, for one experiment, statistical errors only [28]. The study was performed with CMS fast detector simulation.

if $m_{h_{\mathrm{SM}}} \lesssim 200 \mathrm{GeV}$ then good accuracies can be achieved for many absolute partial widths and for the total width provided: (a) $W W$ fusion production can be reliably separated from $g g$ fusion; (b) the $W W / Z Z$ coupling ratio is as expected in the SM from the $\mathrm{SU}(2) \times \mathrm{U}(1)$ symmetry; (c) the $W W^{*}$ final state can be observed in both $g g$ and $W W$ fusion; and (d) there are no unexpected decays of the $h_{\mathrm{SM}}$. Invisible Higgs decays may also be addressed by this technique [32]; CMS simulations show some promise for this channel. The resulting errors estimated for $L=200 \mathrm{fb}^{-1}$ of accumulated data are given in Fig. 3.9. 

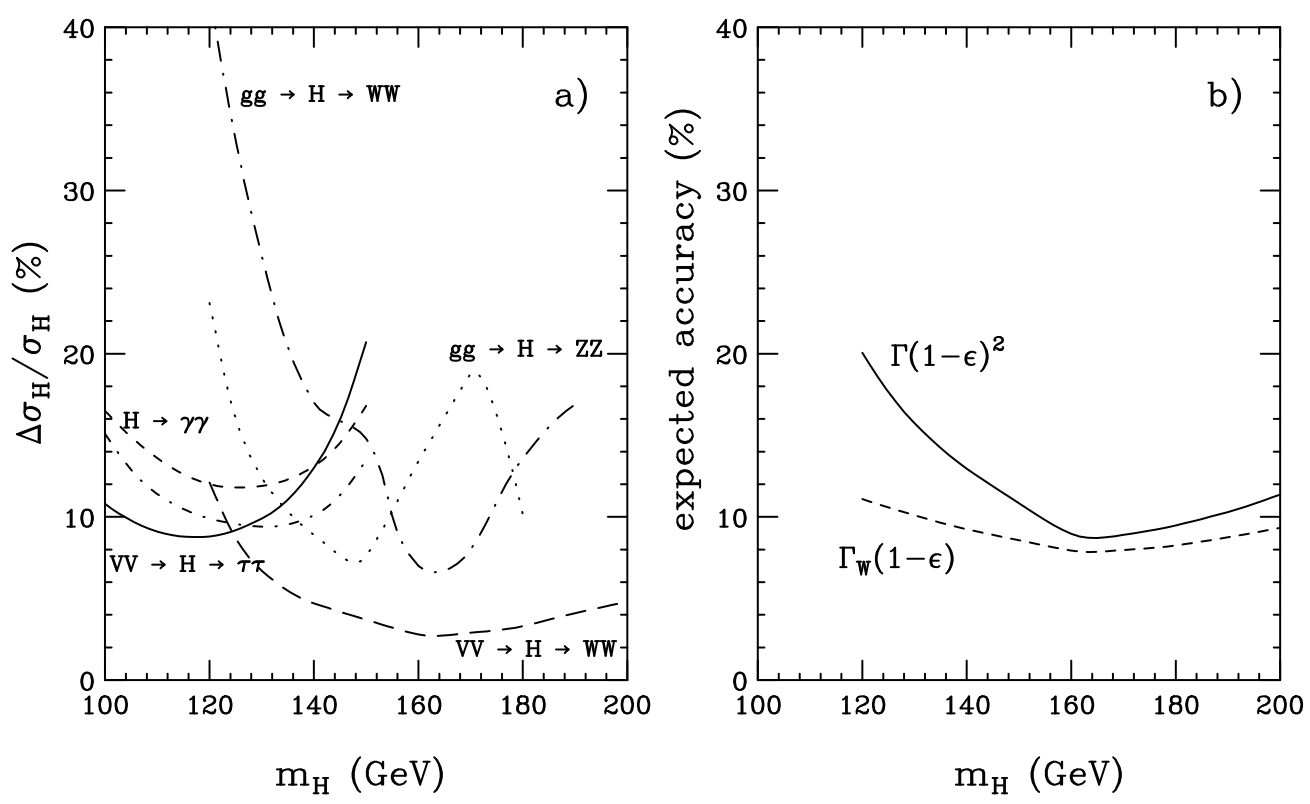

Figure 3.9: Relative accuracy expected at the LHC with $200 \mathrm{fb}^{-1}$ of data. (a) Cross section times branching fraction for several inclusive modes (dotted and dash-dotted lines) and vector boson fusion channels (dashed and solid lines). (b) Extracted total width (solid line) and $H \rightarrow W W$ partial width (dashed line). In the latter, $\epsilon=1-[B(H \rightarrow b \bar{b})+B(H \rightarrow$ $\left.\tau \tau)+B\left(H \rightarrow W W^{(*)}\right)+B\left(H \rightarrow Z Z^{(*)}\right)+B(H \rightarrow g g)+B(H \rightarrow \gamma \gamma)\right]$. To the extent that $\epsilon$ is small, the indicated accuracies can be achieved.

\section{$5 \quad$ Higgs bosons in low-energy supersymmetry}

The simplest realistic model of low-energy supersymmetry is the minimal supersymmetric Standard Model (MSSM), which consists of the two-Higgs-doublet extension of the Standard Model plus the corresponding superpartners [21]. Two Higgs doublets, one with $Y=+1$ and one with $Y=-1$, are needed in order that gauge anomalies due to the higgsino superpartners are exactly canceled. The supersymmetric structure also constrains the Higgs-fermion interactions. In particular, it is the $Y=-1$ Higgs doublet that generates mass for "up"-type quarks and the $Y=+1$ Higgs doublet that generates mass for "down"-type quarks (and charged leptons) $[33,34]$.

After electroweak symmetry breaking, one finds five physical Higgs particles: a charged Higgs pair $\left(H^{ \pm}\right)$, two CP-even neutral Higgs bosons (denoted by $h^{0}$ and $H^{0}$ where $m_{h^{0}} \leq m_{H^{0}}$ ) and one CP-odd neutral Higgs boson $\left(A^{0}\right){ }^{2}$ Two other relevant

\footnotetext{
${ }^{2}$ The tree-level MSSM Higgs sector automatically conserves CP. Hence, the two neutral Higgs vacuum expectation values can be chosen to be real and positive, and the neutral Higgs eigenstates possess definite $\mathrm{CP}$ quantum numbers.
} 
parameters are the ratio of neutral Higgs vacuum expectation values, $\tan \beta$, and an angle $\alpha$ that measures the component of the original $Y= \pm 1$ Higgs doublet states in the physical CP-even neutral scalars.

\subsection{MSSM Higgs sector at tree-level}

The supersymmetric structure of the theory imposes constraints on the Higgs sector of the model [35]. As a result, all Higgs sector parameters at tree-level are determined by two free parameters: $\tan \beta$ and one Higgs mass, conveniently chosen to be $m_{A^{0}}$. There is an upper bound to the tree-level mass of the light CP-even Higgs boson: $m_{h^{0}}^{2} \leq m_{Z}^{2} \cos 2 \beta \leq m_{Z}^{2}$. However, radiative corrections can significantly alter this upper bound as described in Section 5.2.

The limit of $m_{A^{0}} \gg m_{Z}$ is of particular interest, with two key consequences. First, $m_{A^{0}} \simeq m_{H^{0}} \simeq m_{H^{ \pm}}$, up to corrections of $\mathcal{O}\left(m_{Z}^{2} / m_{A^{0}}\right)$. Second, $\cos (\beta-\alpha)=0$ up to corrections of $\mathcal{O}\left(m_{Z}^{2} / m_{A^{0}}^{2}\right)$. This limit is known as the decoupling limit [36] because when $m_{A^{0}}$ is large, the effective low-energy theory below the scale of $m_{A^{0}}$ contains a single CP-even Higgs boson, $h^{0}$, whose properties are nearly identical to those of the Standard Model Higgs boson, $h_{\mathrm{SM}}$.

The phenomenology of the Higgs sector is determined by the various couplings of the Higgs bosons to gauge bosons, Higgs bosons and fermions. The couplings of the two CP-even Higgs bosons to $W$ and $Z$ pairs are given in terms of the angles $\alpha$ and $\beta$ by

$$
\begin{aligned}
g_{h^{0} V V} & =g_{V} m_{V} \sin (\beta-\alpha) \\
g_{H^{0} V V} & =g_{V} m_{V} \cos (\beta-\alpha),
\end{aligned}
$$

where

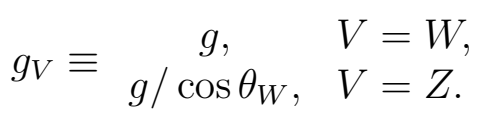

There are no tree-level couplings of $A^{0}$ or $H^{ \pm}$to $V V$. The couplings of one gauge boson to two neutral Higgs bosons are given by:

$$
\begin{aligned}
g_{h^{0} A^{0} Z} & =\frac{g \cos (\beta-\alpha)}{2 \cos \theta_{W}}, \\
g_{H^{0} A^{0} Z} & =\frac{-g \sin (\beta-\alpha)}{2 \cos \theta_{W}} .
\end{aligned}
$$

In the MSSM, the Higgs tree-level couplings to fermions obey the following property: the neutral member of the $Y=-1[Y=+1]$ Higgs doublet couples exclusively to down-type [up-type] fermion pairs. This pattern of Higgs-fermion couplings defines the Type-II two-Higgs-doublet model [37,1]. Consequently, the couplings of the neutral Higgs bosons to $f \bar{f}$ relative to the Standard Model value, $g m_{f} / 2 m_{W}$, are given 
by (using third family notation):

$$
\begin{array}{rll}
h^{0} b \bar{b} \quad\left(\text { or } h^{0} \tau^{+} \tau^{-}\right) & : & -\frac{\sin \alpha}{\cos \beta}=\sin (\beta-\alpha)-\tan \beta \cos (\beta-\alpha), \\
h^{0} t \bar{t} & : & \frac{\cos \alpha}{\sin \beta}=\sin (\beta-\alpha)+\cot \beta \cos (\beta-\alpha), \\
H^{0} b \bar{b} \quad\left(\text { or } H^{0} \tau^{+} \tau^{-}\right) & : & \frac{\cos \alpha}{\cos \beta}=\cos (\beta-\alpha)+\tan \beta \sin (\beta-\alpha), \\
H^{0} t \bar{t} & : & \frac{\sin \alpha}{\sin \beta}=\cos (\beta-\alpha)-\cot \beta \sin (\beta-\alpha), \\
A^{0} b \bar{b} \quad\left(\text { or } A^{0} \tau^{+} \tau^{-}\right) & : & \gamma_{5} \tan \beta, \\
A^{0} t \bar{t} & : & \gamma_{5} \cot \beta .
\end{array}
$$

In these expressions, $\gamma_{5}$ indicates a pseudoscalar coupling.

The neutral Higgs boson couplings to fermion pairs (3.6) have been written in such a way that their behavior can be immediately ascertained in the decoupling limit $\left(m_{A^{0}} \gg m_{Z}\right)$ by setting $\cos (\beta-\alpha)=0$. In particular, in the decoupling limit, the couplings of $h^{0}$ to vector bosons and fermion pairs are equal to the corresponding couplings of the Standard Model Higgs boson.

The region of MSSM Higgs sector parameter space in which the decoupling limit applies is large, because $\sin (\beta-\alpha)$ approaches 1 quite rapidly once $m_{A^{0}}$ is larger than about $200 \mathrm{GeV}$, as shown in Fig. 3.10. As a result, over a significant region of the MSSM parameter space, the search for the lightest CP-even Higgs boson of the MSSM is equivalent to the search for the Standard Model Higgs boson. This result is more general; in many theories of non-minimal Higgs sectors, there is a significant portion of the parameter space that approximates the decoupling limit. Consequently, simulations of the Standard Model Higgs signal are also relevant for exploring the more general Higgs sector.

\subsection{The radiatively corrected MSSM Higgs sector}

When one-loop radiative corrections are incorporated, the Higgs masses and couplings depend on additional parameters of the supersymmetric model that enter via the virtual loops. One of the most striking effects of the radiative corrections to the MSSM Higgs sector is the modification of the upper bound of the light CP-even Higgs mass, as first noted in [38]. When $\tan \beta \gg 1$ and $m_{A^{0}} \gg m_{Z}$, the treelevel prediction for $m_{h^{0}}$ corresponds to its theoretical upper bound, $m_{h}^{\max }=m_{Z}$. Including radiative corrections, the theoretical upper bound is increased, primarily because of an incomplete cancellation of the top-quark and top-squark (stop) loops. (These contributions would cancel if supersymmetry were exact.) The relevant para-

meters that govern the stop sector are the average of the two stop squared-masses: 
Higgs Bosons at the Linear Collider
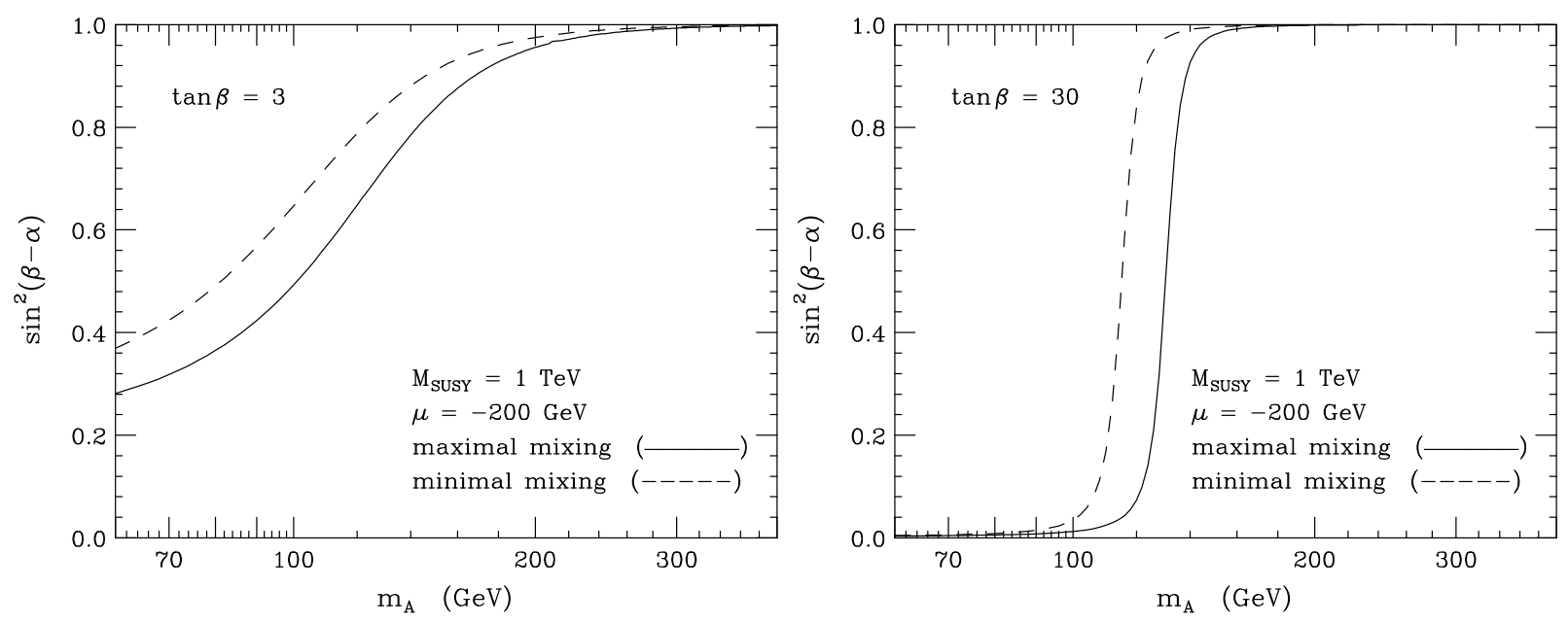

Figure 3.10: The value of $\sin ^{2}(\beta-\alpha)$ is shown as a function of $m_{A^{0}}$ for two choices of $\tan \beta=3$ and $\tan \beta=30$. When radiative corrections are included, one can define an approximate loop-corrected angle $\alpha$ as a function of $m_{A^{0}}, \tan \beta$ and the MSSM parameters. In the figures above, we have incorporated radiative corrections, assuming that $M_{\mathrm{SUSY}}=$ $1 \mathrm{TeV}$. In addition, two extreme cases for the squark mixing parameters are shown (see Section 5.2 for further discussion of the radiative corrections and their dependence on the supersymmetric parameters). The decoupling effect expected when $\sin ^{2}(\beta-\alpha) \simeq 1$ for $m_{A^{0}} \gg m_{Z}$, continues to hold even when radiative corrections are included.

$M_{\mathrm{SUSY}}^{2} \equiv \frac{1}{2}\left(M_{\widetilde{t}_{1}}^{2}+M_{\widetilde{t}_{2}}^{2}\right)$, and the off-diagonal element of the stop squared-mass matrix: $m_{t} X_{t} \equiv m_{t}\left(A_{t}-\mu \cot \beta\right)$, where $A_{t}$ is a soft supersymmetry-breaking trilinear scalar interaction term, and $\mu$ is the supersymmetric Higgs mass parameter. The qualitative behavior of the radiative corrections can be most easily seen in the large top squark mass limit, where, in addition, the splitting of the two diagonal entries and the off-diagonal entry of the stop squared-mass matrix are both small in comparison to $M_{\mathrm{SUSY}}^{2}$. In this case, the upper bound on the lightest CP-even Higgs mass is approximately given by

$$
m_{h^{0}}^{2} \lesssim m_{Z}^{2}+\frac{3 g^{2} m_{t}^{4}}{8 \pi^{2} m_{W}^{2}}\left[\ln \left(\frac{M_{\mathrm{SUSY}}^{2}}{m_{t}^{2}}\right)+\frac{X_{t}^{2}}{M_{\mathrm{SUSY}}^{2}}\left(1-\frac{X_{t}^{2}}{12 M_{\mathrm{SUSY}}^{2}}\right)\right] .
$$

More complete treatments of the radiative corrections include the effects of stop mixing, renormalization group improvement, and the leading two-loop contributions, and imply that these corrections somewhat overestimate the true upper bound of $m_{h^{0}}$ (see [39] for the most recent results). Nevertheless, Eq. (3.7) correctly illustrates some noteworthy features of the more precise result. First, the increase of the light CP-even Higgs mass bound beyond $m_{Z}$ can be significant. This is a consequence of the $m_{t}^{4}$ enhancement of the one-loop radiative correction. Second, the dependence of the light Higgs mass on the stop mixing parameter $X_{t}$ implies that (for a given value 
of $\left.M_{\mathrm{SUSY}}\right)$ the upper bound of the light Higgs mass initially increases with $X_{t}$ and reaches its maximal value at $X_{t} \simeq \sqrt{6} M_{\mathrm{SUSY}}$. This point is referred to as the maximal mixing case (whereas $X_{t}=0$ corresponds to the minimal mixing case).

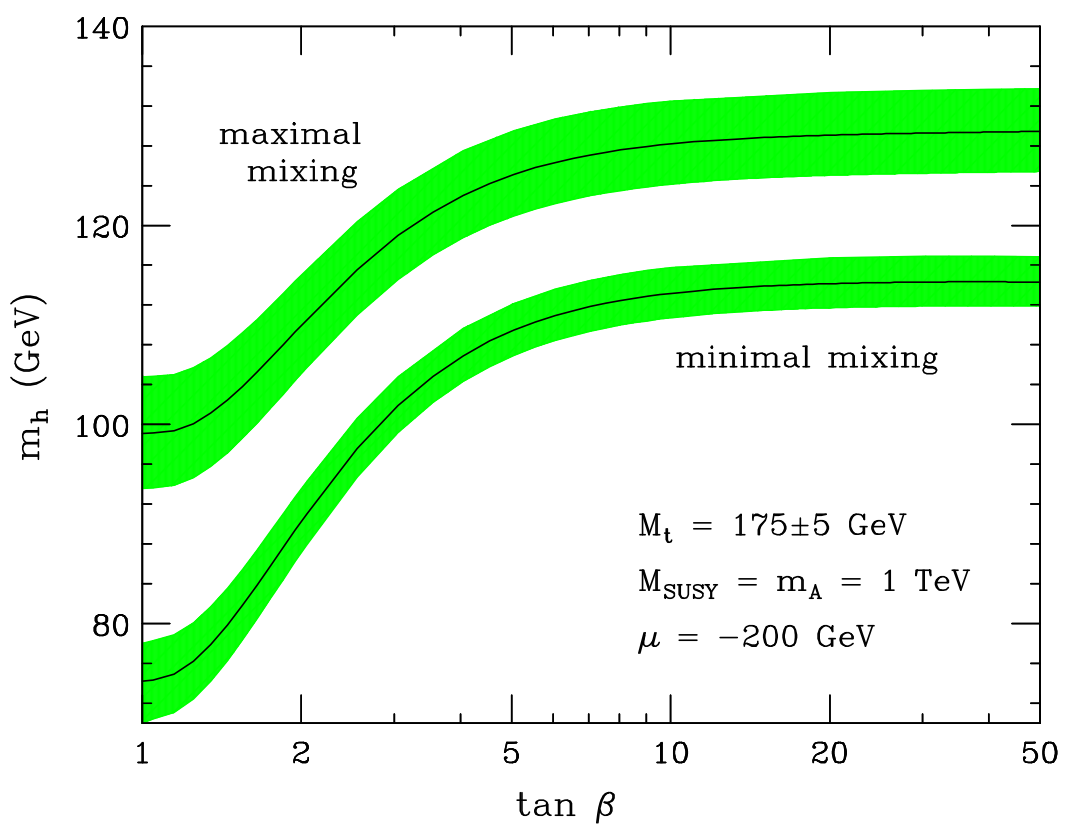

Figure 3.11: The radiatively corrected light CP-even Higgs mass is plotted as a function of $\tan \beta$, for the maximal mixing [upper band] and minimal mixing cases. The impact of the top quark mass is exhibited by the shaded bands; the central value corresponds to $m_{t}=175 \mathrm{GeV}$, while the upper [lower] edge of the bands correspond to increasing [decreasing] $m_{t}$ by $5 \mathrm{GeV}$.

Taking $m_{A^{0}}$ large, Fig. 3.11 illustrates that the maximal value of the lightest CP-even Higgs mass bound is realized at large $\tan \beta$ in the case of maximal mixing. Allowing for the uncertainty in the measured value of $m_{t}$ and the uncertainty inherent in the theoretical analysis, one finds for $M_{\mathrm{SUSY}} \lesssim 2 \mathrm{TeV}$ that $m_{h^{0}} \lesssim m_{h}^{\max }$, where

$$
\begin{aligned}
& m_{h}^{\max } \simeq 122 \mathrm{GeV}, \quad \text { minimal stop mixing, } \\
& m_{h}^{\max } \simeq 135 \mathrm{GeV}, \quad \text { maximal stop mixing. }
\end{aligned}
$$

The $h^{0}$ mass bound in the MSSM quoted above does not apply to non-minimal supersymmetric extensions of the Standard Model. If additional Higgs singlet and/or triplet fields are introduced, then new Higgs self-coupling parameters appear, which are not significantly constrained by present data. For example, in the simplest nonminimal supersymmetric extension of the Standard Model (NMSSM), the addition of a complex Higgs singlet field $S$ adds a new Higgs self-coupling parameter, $\lambda_{S}$ [40]. The mass of the lightest neutral Higgs boson can be raised arbitrarily by increasing 
the value of $\lambda_{S}$, analogous to the behavior of the Higgs mass in the Standard Model. Under the assumption that all couplings stay perturbative up to the Planck scale, one finds in essentially all cases that $m_{h^{0}} \lesssim 200 \mathrm{GeV}$, independent of the details of the low-energy supersymmetric model [41].

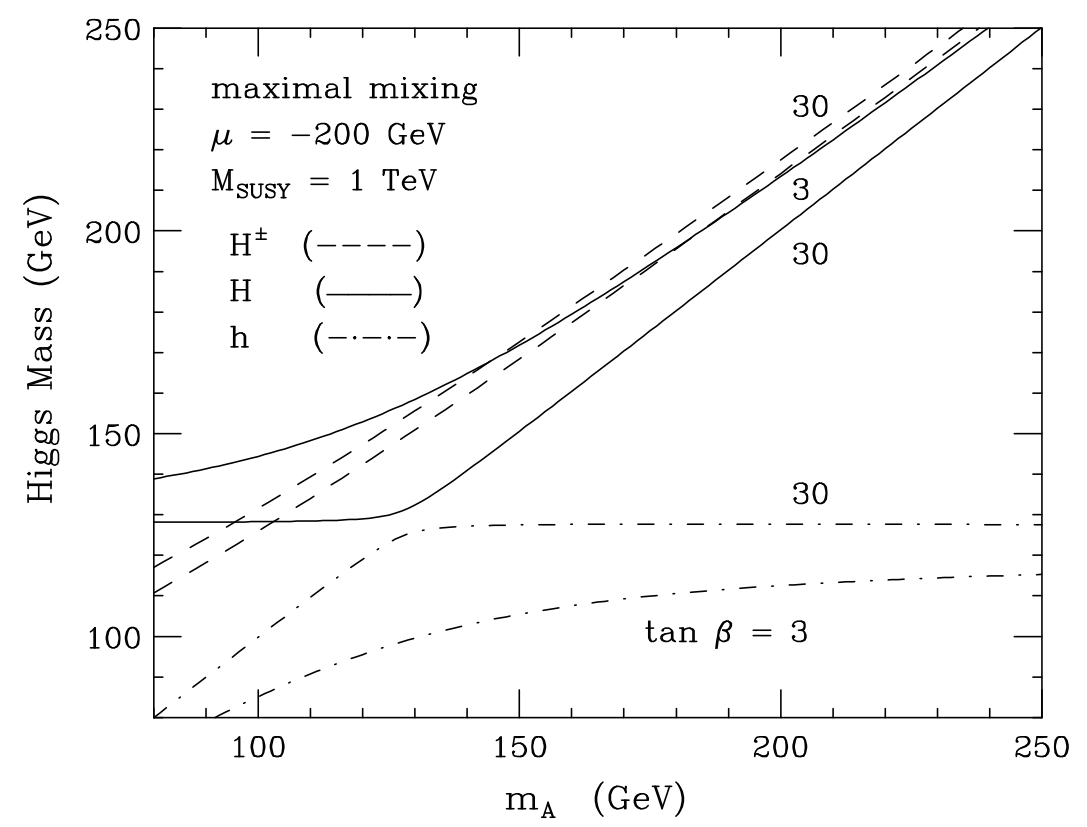

Figure 3.12: Lightest CP-even Higgs mass $\left(m_{h^{0}}\right)$, heaviest CP-even Higgs mass $\left(m_{H^{0}}\right)$ and charged Higgs mass $\left(m_{H^{ \pm}}\right)$as a function of $m_{A^{0}}$ for two choices of $\tan \beta=3$ and $\tan \beta=30$. The slight increase in the charged Higgs mass as $\tan \beta$ is increased from 3 to 30 is a consequence of the radiative corrections.

In Fig. 3.12, we exhibit the masses of the CP-even neutral and the charged Higgs masses as a function of $m_{A^{0}}$. Note that $m_{H^{0}} \geq m_{h}^{\max }$ for all values of $m_{A^{0}}$ and $\tan \beta$, where $m_{h}^{\max }$ is to be evaluated depending on the top-squark mixing, as indicated in Eq. (3.8).

Radiative corrections also significantly modify the tree-level values of the Higgs boson couplings to fermion pairs and to vector boson pairs. As discussed above, the tree-level Higgs couplings depend crucially on the value of $\sin (\beta-\alpha)$. In the first approximation, when radiative corrections of the Higgs squared-mass matrix are computed, the diagonalizing angle $\alpha$ is modified. This provides one important source of the radiative corrections of the Higgs couplings. In Fig. 3.10, we show the effect of radiative corrections on the value of $\sin (\beta-\alpha)$ as a function of $m_{A^{0}}$ for different values of the squark mixing parameters and $\tan \beta$. One can then simply insert the radiatively corrected value of $\alpha$ into eqs. (3.3), (3.5), and (3.6) to obtain radiatively improved couplings of Higgs bosons to vector bosons and to fermions.

At large $\tan \beta$, there is another potentially important class of radiative corrections 
in addition to those that enter through the modified $\alpha$. These corrections arise in the relation between $m_{b}$ and $\tan \beta$ and depend on the details of the MSSM spectrum (which enter via loop-effects). At tree-level, the Higgs couplings to $b \bar{b}$ are proportional to the Higgs-bottom-quark Yukawa coupling. Deviations from the tree-level relation due to radiative corrections are calculable and finite [42-46]. One of the fascinating properties of such corrections is that in certain cases the corrections do not vanish in the limit of large supersymmetric mass parameters. These corrections grow with $\tan \beta$ and therefore can be significant in the large $\tan \beta$ limit. In the supersymmetric limit, $b \bar{b}$ couples only to the neutral component of the $Y=-1$ Higgs doublet. However, when supersymmetry is broken there will be a small coupling of $b \bar{b}$ to the neutral component of the $Y=+1$ Higgs doublet resulting from radiative corrections. From this result, one can compute the couplings of the physical Higgs bosons to $b \bar{b}$ pairs. A useful approximation at large $\tan \beta$ yields the following corrections to Eq. (3.6):

$$
\begin{aligned}
h^{0} b \bar{b}: & -\frac{\sin \alpha}{\cos \beta} \frac{1}{1+\Delta_{b}}\left[1-\frac{\Delta_{b} \cot \alpha}{\tan \beta}\right], \\
H^{0} b \bar{b}: & \frac{\cos \alpha}{\cos \beta} \frac{1}{1+\Delta_{b}}\left[1+\frac{\Delta_{b} \tan \alpha}{\tan \beta}\right], \\
A^{0} b \bar{b}: & \gamma_{5} \frac{\tan \beta}{1+\Delta_{b}},
\end{aligned}
$$

where $\Delta_{b} \propto \tan \beta$. The explicit form of $\Delta_{b}$ at one-loop in the limit of $M_{\mathrm{SUSY}} \gg m_{b}$ is given in [43-45]. The correction $\Delta_{b}$ arises from a bottom-squark-gluino loop, which depends on the gluino mass and the supersymmetric Higgs mass parameter $\mu$, and the top-squark-chargino loop, which depends on the top-squark masses and the topsquark mixing parameters $\mu$ and $A_{t}$. Contributions proportional to the electroweak gauge couplings have been neglected.

Similarly, the neutral Higgs couplings to $\tau^{+} \tau^{-}$are modified by replacing $\Delta_{b}$ in Eq. (3.9) with $\Delta_{\tau}[44,45]$. One can also derive radiatively corrected couplings of the charged Higgs boson to fermion pairs [47,48]. The tree-level couplings of the charged Higgs boson to fermion pairs are modified accordingly by replacing $m_{b} \rightarrow m_{b} /\left(1+\Delta_{b}\right)$ and $m_{\tau} \rightarrow m_{\tau} /\left(1+\Delta_{\tau}\right)$, respectively.

One consequence of the above results is that the neutral Higgs coupling to $b \bar{b}$ (which is expected to be the dominant decay mode over nearly all of the MSSM Higgs parameter space), can be significantly suppressed at large $\tan \beta$ [49-51] if $\Delta_{b} \simeq \mathcal{O}(1)$. Typically $\left|\Delta_{\tau}\right| \ll\left|\Delta_{b}\right|$, since the correction proportional to $\alpha_{s}$ in the latter is absent in the former. For this reason, the $\tau^{+} \tau^{-}$decay mode can be the dominant Higgs decay channel for the CP-even Higgs boson with SM-like couplings to gauge bosons.

In the decoupling limit, one can show that $\cot \alpha \cot \beta=-1+\mathcal{O}\left(m_{Z}^{2} / m_{A^{0}}^{2}\right)$. Inserting this result into Eq. (3.9), one can check that the $h^{0} b \bar{b}$ coupling does indeed approach its Standard Model value. However, because $\Delta_{b} \propto \tan \beta$, the deviation of 
Higgs Bosons at the Linear Collider

the $h^{0} b \bar{b}$ coupling from the corresponding SM result is of $\mathcal{O}\left(m_{Z}^{2} \tan \beta / m_{A^{0}}^{2}\right)$. That is, at large $\tan \beta$, the approach to decoupling may be "delayed" [52], depending on the values of other MSSM parameters that enter the radiative corrections.

\subsection{MSSM Higgs boson decay modes}

In this section, we consider the decay properties of the three neutral Higgs bosons $\left(h^{0}, H^{0}\right.$ and $\left.A^{0}\right)$ and of the charged Higgs pair $\left(H^{ \pm}\right)$. Let us start with the lightest state, $h^{0}$. When $m_{A^{0}} \gg m_{Z}$, the decoupling limit applies, and the couplings of $h^{0}$ to $\mathrm{SM}$ particles are nearly indistinguishable from those of $h_{\mathrm{SM}}$. If some superpartners are light, there may be some additional decay modes, and hence the $h^{0}$ branching ratios would be different from the corresponding Standard Model values, even though the partial widths to Standard Model particles are the same. Furthermore, loops of light charged or colored superpartners could modify the $h^{0}$ coupling to photons and/or gluons, in which case the one-loop $g g$ and $\gamma \gamma$ decay rates would also be different. On the other hand, if all superpartners are heavy, all the decay properties of $h^{0}$ are essentially those of the SM Higgs boson, and the discussion of Section 3.1 applies.

The heavier Higgs states, $H^{0}, A^{0}$ and $H^{ \pm}$, are roughly mass-degenerate and have negligible couplings to vector boson pairs. In particular, $\Gamma\left(H^{0} \rightarrow V V\right) \ll \Gamma\left(h_{\mathrm{SM}} \rightarrow\right.$ $V V$ ), while the couplings of $A^{0}$ and $H^{ \pm}$to the gauge bosons are loop-suppressed. The couplings of $H^{0}, A^{0}$ and $H^{ \pm}$to down-type (up-type) fermions are significantly enhanced (suppressed) relative to those of $h_{\mathrm{SM}}$ if $\tan \beta \gg 1$. Consequently, the decay modes $H^{0}, A^{0} \rightarrow b \bar{b}, \tau^{+} \tau^{-}$dominate the neutral Higgs decay modes for moderate-tolarge values of $\tan \beta$ below the $t \bar{t}$ threshold, while $H^{+} \rightarrow \tau^{+} \nu$ dominates the charged Higgs decay below the $t \bar{b}$ threshold.

For values of $m_{A^{0}}$ of order $m_{Z}$, all Higgs boson states lie below $200 \mathrm{GeV}$ in mass, and would all be accessible at the LC. In this parameter regime, there is a significant area of the parameter space in which none of the neutral Higgs boson decay properties approximates those of $h_{\mathrm{SM}}$. For example, when $\tan \beta$ is large, supersymmetrybreaking effects can significantly modify the $b \bar{b}$ and/or the $\tau^{+} \tau^{-}$decay rates with respect to those of $h_{\mathrm{SM}}$. Additionally, the heavier Higgs bosons can decay into lighter Higgs bosons. Examples of such decay modes are: $H^{0} \rightarrow h^{0} h^{0}, A^{0} A^{0}$, and $Z A^{0}$, and $H^{ \pm} \rightarrow W^{ \pm} h^{0}, W^{ \pm} A^{0}$ (although in the MSSM, the Higgs branching ratio into vector boson-Higgs boson final states, if kinematically allowed, rarely exceeds a few percent). The decay of the heavier Higgs boson into two lighter Higgs bosons can provide information about Higgs self-couplings. For values of $\tan \beta \lesssim 5$, the branching ratio of $H^{0} \rightarrow h^{0} h^{0}$ is dominant for a Higgs mass range of $200 \mathrm{GeV} \lesssim m_{H^{0}} \lesssim 2 m_{t}$. The dominant radiative corrections to this decay arise from the corrections to the self-interaction $\lambda_{H^{0} h^{0} h^{0}}$ in the MSSM and are large [53].

The phenomenology of charged Higgs bosons is less model-dependent, and is governed by the values of $\tan \beta$ and $m_{H^{ \pm}}$. Because charged Higgs couplings are proportional to fermion masses, the decays to third-generation quarks and leptons are 
dominant. In particular, for $m_{H^{ \pm}}<m_{t}+m_{b}$ (so that the channel $H^{+} \rightarrow t \bar{b}$ is closed), $H^{+} \rightarrow \tau^{+} \nu_{\tau}$ is favored if $\tan \beta \gtrsim 1$, while $H^{+} \rightarrow c \bar{s}$ is favored only if $\tan \beta$ is small. Indeed, $\operatorname{BR}\left(H^{+} \rightarrow \tau^{+} \nu_{\tau}\right) \simeq 1$ if $\tan \beta \gtrsim 5$. These results apply generally to Type-II two-Higgs doublet models. For $m_{H^{ \pm}} \gtrsim 180 \mathrm{GeV}$, the decay $H^{+} \rightarrow t \bar{b} \rightarrow W^{+} b \bar{b}$ is the dominant decay mode.

In addition to the above decay modes, there exist new Higgs decay channels that involve supersymmetric final states. Higgs decays into charginos, neutralinos and third-generation squarks and sleptons can become important, once they are kinematically allowed [54]. For Higgs masses below $130 \mathrm{GeV}$, the range of supersymmetric parameter space in which supersymmetric decays are dominant is rather narrow when the current bounds on supersymmetric particle masses are taken into account. One interesting possibility is a significant branching ratio of $h^{0} \rightarrow \widetilde{\chi}^{0} \widetilde{\chi}^{0}$, which could arise for values of $m_{h^{0}}$ near its upper theoretical limit. Such an invisible decay mode could be detected at the LC by searching for the missing mass recoiling against the $Z$ in $e^{+} e^{-} \rightarrow h^{0} Z$.

\subsection{MSSM Higgs boson production at the LC}

For $m_{A^{0}} \gtrsim 150 \mathrm{GeV}$, Fig. 3.10 shows that the MSSM Higgs sector quickly approaches the decoupling limit, where the properties of $h^{0}$ approximately coincide with those of $h_{\mathrm{SM}}$. Thus, the Higgsstrahlung and vector-boson-fusion cross-sections for $h_{\mathrm{SM}}$ production also apply to $h^{0}$ production. In contrast, the $H^{0} V V$ and $A^{0} V V$ couplings are highly suppressed, since $|\cos (\beta-\alpha)| \ll 1$. Equation (3.3) illustrates this for the $H^{0} W$ coupling. Thus, these mechanisms are no longer useful for $H^{0}$ and $A^{0}$ production. The most robust production mechanism is $e^{+} e^{-} \rightarrow Z^{*} \rightarrow H^{0} A^{0}$, which is not suppressed since the $Z H^{0} A^{0}$ coupling is proportional to $\sin (\beta-\alpha)$, as indicated in Eq. (3.5). Radiatively corrected cross-sections for $Z h^{0}, Z H^{0}, H^{0} A^{0}$, and $h^{0} A^{0}$ have been recently obtained in [55]. The charged Higgs boson is also produced in pairs via $s$-channel photon and $Z$ exchange. However, since $m_{H^{0}} \simeq m_{A^{0}} \simeq m_{H^{ \pm}}$ in the decoupling limit, $H^{0} A^{0}$ and $H^{+} H^{-}$production are kinematically allowed only when $m_{A^{0}} \lesssim \sqrt{s} / 2{ }^{3}$ In $\gamma \gamma$ collisions, one can extend the Higgs mass reach for the neutral Higgs bosons. As described in Section 10, the $s$-channel resonant production of $H^{0}$ and $A^{0}$ (due primarily to the top and bottom-quark loops in the one-loop Higgs- $\gamma \gamma$ triangle) can be detected for some choices of $m_{A^{0}}$ and $\tan \beta$ if the heavy Higgs masses are less than about $80 \%$ of the initial $\sqrt{s}$ of the primary $e^{+} e^{-}$system. The corresponding cross sections are a few fb $[56,57]$.

If $m_{A^{0}} \lesssim 150 \mathrm{GeV}$, deviations from the decoupling limit become more apparent, and $H^{0}$ can now be produced via Higgsstrahlung and vector boson fusion at an observable rate. In addition, the factor of $\cos (\beta-\alpha)$ in the $Z h^{0} A^{0}$ coupling no longer

\footnotetext{
${ }^{3}$ The pair production of scalars is $\mathrm{P}$-wave suppressed near threshold, so in practice the corresponding Higgs mass reach is likely to be somewhat lower than $\sqrt{s} / 2$.
} 
significantly suppresses $h^{0} A^{0}$ production. Finally, if $m_{H^{ \pm}} \lesssim 170 \mathrm{GeV}$, the charged Higgs boson will also be produced in $t \rightarrow H^{+} b$. In the non-decoupling regime, all non-minimal Higgs states can be directly produced and studied at the LC.

The associated production of a single Higgs boson and a fermion-antifermion pair can also be considered. Here, the new feature is the possibility of enhanced Higgsfermion Yukawa couplings. Consider the behavior of the Higgs couplings at large $\tan \beta$, where some of the Higgs couplings to down type fermion pairs (denoted generically by $b \bar{b}$ ) can be significantly enhanced. ${ }^{4}$ Let us examine two particular large $\tan \beta$ regions of interest. In the decoupling limit (where $m_{A^{0}} \gg m_{Z}$ and $|\cos (\beta-\alpha)| \ll 1$ ), it follows from Eq. (3.6) that the $b \bar{b} H^{0}$ and $b \bar{b} A^{0}$ couplings have equal strength and are significantly enhanced by a factor of $\tan \beta$ relative to the $b \bar{b} h_{\mathrm{SM}}$ coupling, while the $b \bar{b} h^{0}$ coupling is given by the corresponding Standard Model value. If $m_{A^{0}} \lesssim m_{Z}$ and $\tan \beta \gg 1$, then $|\sin (\beta-\alpha)| \ll 1$, as shown in Fig. 3.10, and $m_{h^{0}} \simeq m_{A^{0}}$. In this case, the $b \bar{b} h^{0}$ and $b \bar{b} A^{0}$ couplings have equal strength and are significantly enhanced (by a factor of $\tan \beta$ ) relative to the $b \bar{b} h_{\mathrm{SM}}$ coupling. ${ }^{5}$ Note that in both cases above, only two of the three neutral Higgs bosons have enhanced couplings to $b \bar{b}$. If $\phi$ is one of the two neutral Higgs bosons with enhanced $b \bar{b} \phi$ couplings, then the cross-section for $e^{+} e^{-} \rightarrow f \bar{f} \phi(f=b$ or $\tau)$ will be significantly enhanced relative to the corresponding Standard Model cross-section by a factor of $\tan ^{2} \beta$. The phase-space suppression is not as severe as in $e^{+} e^{-} \rightarrow t \bar{t} \phi$ (see Fig. 3.5), so this process could extend the mass reach of the heavier neutral Higgs states at the LC given sufficient luminosity. The production of the charged Higgs boson via $e^{+} e^{-} \rightarrow t \bar{b} H^{-}$is also enhanced by $\tan ^{2} \beta$, although this process has a more significant phase-space suppression because of the final state top quark. If any of these processes can be observed, it would provide a direct measurement of the corresponding Higgs-fermion Yukawa coupling.

\section{MSSM Higgs boson searches before the LC}

\subsection{Review of direct search limits}

Although no direct experimental evidence for the Higgs boson yet exists, there are both experimental as well as theoretical constraints on the parameters of the MSSM

\footnotetext{
${ }^{4}$ We do not consider the possibility of $\tan \beta \ll 1$, which would lead to enhanced Higgs couplings to up-type fermions. In models of low-energy supersymmetry, there is some theoretical prejudice that suggests that $1 \lesssim \tan \beta \lesssim m_{t} / m_{b}$, with the fermion masses evaluated at the electroweak scale. For example, $\tan \beta \lesssim 1$ is disfavored since in this case, the Higgs-top quark Yukawa coupling blows up at an energy scale significantly below the Planck scale. The Higgs-bottom quark Yukawa coupling has a similar problem if $\tan \beta \gtrsim m_{t} / m_{b}$. As noted in Section 6.1 , some of the low $\tan \beta$ region is already ruled out by the MSSM Higgs search.

${ }^{5}$ However in this case, the value of the $b \bar{b} H^{0}$ coupling can differ from the corresponding $b \bar{b} h_{\mathrm{SM}}$ coupling when $\tan \beta \gg 1$, since in case (ii), where $|\sin (\beta-\alpha)| \ll 1$, the product $\tan \beta \sin (\beta-\alpha)$ need not be particularly small.
} 
Higgs sector. Experimental limits on the charged and neutral Higgs masses have been obtained at LEP. For the charged Higgs boson, $m_{H^{ \pm}}>78.7 \mathrm{GeV}$ [58]. This is the most model-independent bound. It is valid for more general non-supersymmetric twoHiggs doublet models and assumes only that the $H^{+}$decays dominantly into $\tau^{+} \nu_{\tau}$ and/or $c \bar{s}$. The LEP limits on the masses of $h^{0}$ and $A^{0}$ are obtained by searching simultaneously for $e^{+} e^{-} \rightarrow Z \rightarrow Z h^{0}$ and $e^{+} e^{-} \rightarrow Z \rightarrow h^{0} A^{0}$. Radiative corrections can be significant, as shown in Section 5.2, so the final limits depend on the choice of MSSM parameters that govern the radiative corrections. The third generation squark parameters are the most important of these. The LEP Higgs working group [59] quotes limits for the case of $M_{\mathrm{SUSY}}=1 \mathrm{TeV}$ in the maximal-mixing scenario, which corresponds to the choice of third generation squark parameters that yields the largest corrections to $m_{h^{0}}$. The present LEP 95\% CL lower limits are $m_{A^{0}}>91.9 \mathrm{GeV}$ and $m_{h^{0}}>91.0 \mathrm{GeV}$. The theoretical upper bound on $m_{h^{0}}$ as a function of $\tan \beta$, exhibited in Fig. 3.11, can then be used to exclude a region of $\tan \beta$ in which the predicted value of $m_{h^{0}}$ lies below the experimental bound. Under the same MSSM Higgs parameter assumptions stated above, the LEP Higgs search excludes the region $0.5<\tan \beta<2.4$ at $95 \%$ CL.

In discussing Higgs discovery prospects at the Tevatron and LHC, we shall quote limits based on the assumption of $M_{\mathrm{SUSY}}=1 \mathrm{TeV}$ and maximal squark mixing. This tends to be a conservative assumption; that is, other choices give sensitivity to more of the $m_{A^{0}}$ versus $\tan \beta$ plane. However, there are a number of other parameter regimes in which certain Higgs search strategies become more difficult. While these issues are of vital importance to the Tevatron and LHC Higgs searches, they are much less important at the LC.

\subsection{MSSM Higgs searches at the Tevatron}

At the Tevatron, the SM Higgs search can be reinterpreted in terms of the search for the CP-even Higgs boson of the MSSM. Since the theoretical upper bound was found to be $m_{h^{0}} \lesssim 135 \mathrm{GeV}$ (for $M_{\mathrm{SUSY}}<2 \mathrm{TeV}$ ), only the Higgs search of the low-mass region, $100 \mathrm{GeV} \lesssim m_{h^{0}} \lesssim 135 \mathrm{GeV}$, applies. In the MSSM at large $\tan \beta$, the enhancement of the $A^{0} b \bar{b}$ coupling (and a similar enhancement of either the $h^{0} b \bar{b}$ or $H^{0} b \bar{b}$ coupling) provides a new search channel: $q \bar{q}, g g \rightarrow b \bar{b} \phi$, where $\phi$ is a neutral Higgs boson with enhanced couplings to $b \bar{b}$. Combining both sets of analyses, the Tevatron Higgs Working Group obtained the anticipated 95\% CL exclusion and $5 \sigma$ Higgs discovery contours for the maximal mixing scenario as a function of total integrated luminosity per detector (combining both CDF and D0 data sets) shown in Fig. 3.13 [27].

From these results, one sees that $5 \mathrm{fb}^{-1}$ of integrated luminosity per experiment will allow one to test nearly all of the MSSM Higgs parameter space at 95\% CL. To assure discovery of a CP-even Higgs boson at the $5 \sigma$ level, the luminosity requirement becomes very important. Figure 3.13(b) shows that a total integrated luminosity of 

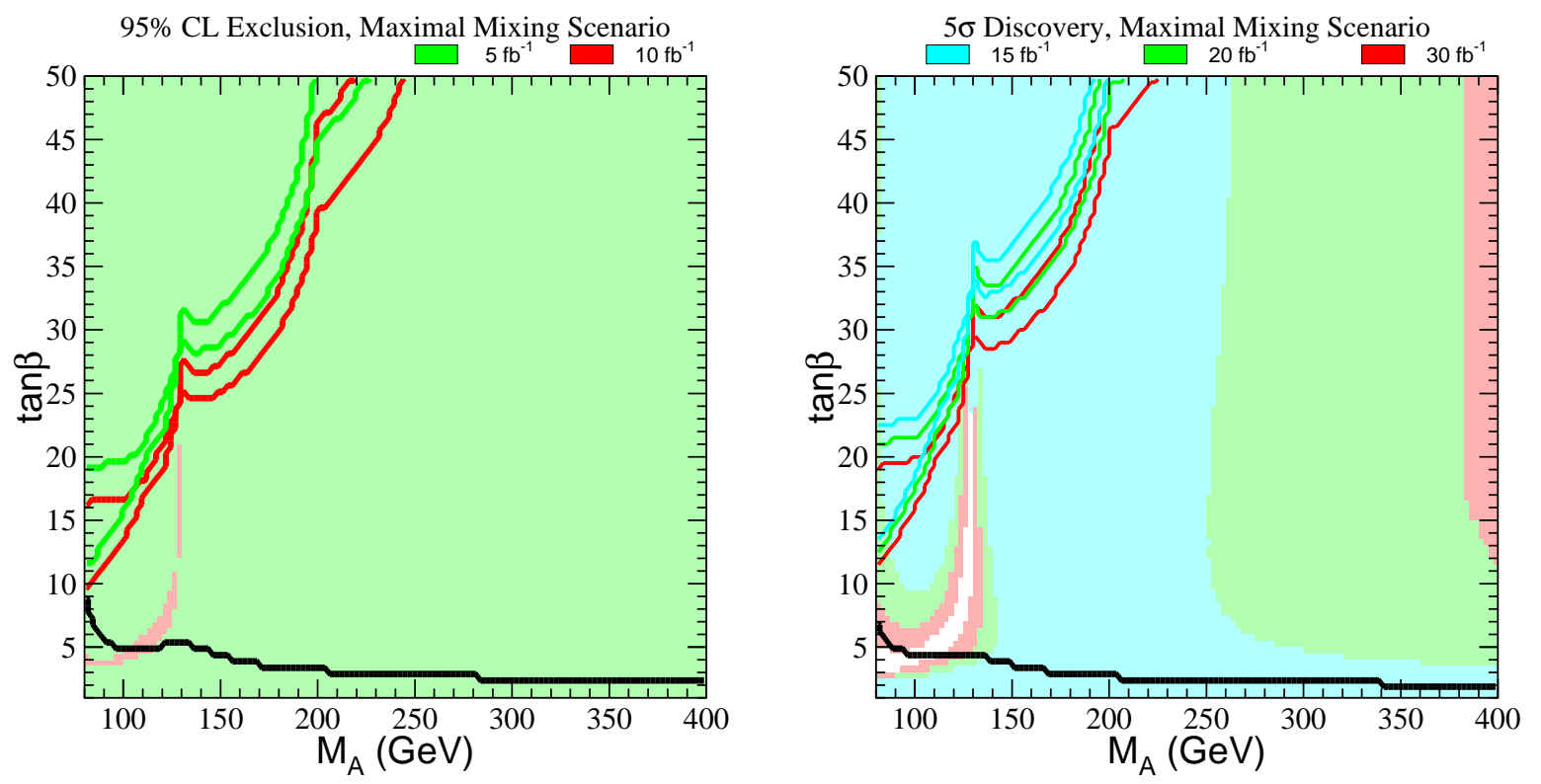

Figure 3.13: (a) 95\% CL exclusion region and (b) $5 \sigma$ discovery region on the $m_{A^{0}}-\tan \beta$ plane, for the maximal mixing scenario and two different search channels: $q \bar{q} \rightarrow V \phi\left(\phi=h^{0}\right.$, $\left.H^{0}\right), \phi \rightarrow b \bar{b}$ (shaded regions) and $g g, q \bar{q} \rightarrow b \bar{b} \phi\left(\phi=h^{0}, H^{0}, A^{0}\right), \phi \rightarrow b \bar{b}$ (region in the upper left-hand corner bounded by the solid lines). Different integrated luminosities are explicitly shown by the color coding. The two sets of lines (for a given color) correspond to the $\mathrm{CDF}$ and $\mathrm{D} \varnothing$ simulations, respectively. The region below the solid black line near the bottom of the plot is excluded by the absence of observed $e^{+} e^{-} \rightarrow Z \phi$ events at LEP2.

about $20 \mathrm{fb}^{-1}$ per experiment is necessary in order to assure a significant, although not exhaustive, coverage of the MSSM parameter space. If the anticipated $15 \mathrm{fb}^{-1}$ integrated luminosity is achieved, the discovery reach will significantly extend beyond that of LEP. A Higgs discovery would be assured if the Higgs interpretation of the Higgs-like LEP events is correct. Nevertheless, the MSSM Higgs boson could still evade capture at the Tevatron. We would then turn to the LHC to try to obtain a definitive Higgs boson discovery.

\subsection{MSSM Higgs searches at the LHC}

The potential of the LHC to discover one or more of the MSSM Higgs bosons has been exhaustively studied for the minimal and maximal mixing scenarios described above. One of the primary goals of these studies has been to demonstrate that at least one of the MSSM Higgs bosons will be observed by ATLAS and CMS for any possible choice of $\tan \beta$ and $m_{A^{0}}$ consistent with bounds coming from current LEP 
data. In order to establish such a 'no-lose' theorem, an important issue is whether or not the Higgs bosons have substantial decays to supersymmetric particle pairs. It is reasonable to suppose that these decays will be absent or relatively insignificant for the light $h^{0}$. Current mass limits on SUSY particles are such that only $h^{0} \rightarrow \widetilde{\chi}_{1}^{0} \widetilde{\chi}_{1}^{0}$ might possibly be kinematically allowed and this possibility arises only in a very limited class of models. For $m_{A^{0}} \gtrsim 200 \mathrm{GeV}$, decays of the $A^{0}, H^{0}, H^{ \pm}$to SUSY pair states (especially pairs of light charginos/neutralinos) are certainly a possibility, but the branching ratios are generally not all that large. The discovery limits we discuss below would be weakened, but not dramatically. Further, at high $\tan \beta$ the enhancement of the $b \bar{b}$ and $\tau^{+} \tau^{-}$couplings of the heavy $A^{0}$ and $H^{0}$ imply that SUSY decay modes will not be important even for quite high $m_{A^{0}} \sim m_{H^{0}} \sim m_{H^{ \pm}}$. We will summarize the LHC discovery prospects for the MSSM Higgs bosons assuming that SUSY decays are not significant.

One of the primary Higgs discovery modes is detection of the relatively SM-like $h^{0}$ using the same modes as employed for a light $h_{\mathrm{SM}}$. Based on Fig. 3.14 (which assumes $L=300 \mathrm{fb}^{-1}$ ) [60], we see that for $m_{A^{0}} \gtrsim 180 \mathrm{GeV}$, the $h^{0}$ will be detected via $g g, W W \rightarrow h^{0}$ and $W h^{0}, t \bar{t} h^{0}$ with $h^{0} \rightarrow \gamma \gamma$, while the $t \bar{t} h^{0}$ with $h^{0} \rightarrow b \bar{b}$ mode is viable down to $m_{A^{0}} \gtrsim 100-120 \mathrm{GeV}$, depending on $\tan \beta$. There are also many possibilities for detecting the other MSSM Higgs bosons. We give a descriptive list. First, there is a small domain in which $m_{A^{0}} \lesssim 130 \mathrm{GeV}$, but yet $m_{A^{0}}$ is still large enough for consistency with LEP limits, in which $t \rightarrow b H^{ \pm}$discovery will be possible. However, the most interesting alternative detection modes are based on $g g \rightarrow A^{0}, H^{0}$ and $g b \rightarrow H^{ \pm} t$ production. We focus first on the former. For low-to-moderate $\tan \beta$ values, the channels $H^{0} \rightarrow Z Z^{(*)} \rightarrow 4 \ell, H^{0} \rightarrow h^{0} h^{0} \rightarrow b \bar{b} \gamma \gamma$ and $A^{0} \rightarrow Z h^{0} \rightarrow \ell \ell b \bar{b}$ are viable when $m_{A^{0}} \lesssim 2 m_{t}$, whereas the $A^{0}, H^{0} \rightarrow t \bar{t}$ modes are viable for $m_{A^{0}}>2 m_{t}$. For large enough $\tan \beta$ the $g g \rightarrow A^{0}, H^{0} \rightarrow \tau^{+} \tau^{-}, \mu^{+} \mu^{-}$discovery modes become viable. For the $g b \rightarrow H^{ \pm} t$ process, the $H^{ \pm} \rightarrow t b$ decays provide a $5 \sigma$ signal both for low-to-moderate $\tan \beta \lesssim 2-3$ and for high $\tan \beta \gtrsim 15-25$, depending upon mass. In addition, the $H^{ \pm} \rightarrow \tau^{ \pm} \nu$ decay mode yields a viable signal for $\tan \beta \gtrsim 7-12$. Of course, if the plot were extended to higher $m_{A^{0}}$, the minimum $\tan \beta$ value required for $H^{0}, A^{0}$ or $H^{ \pm}$detection would gradually increase.

It is important to notice that current LEP constraints exclude all of the low-tomoderate $\tan \beta$ regime in the case of maximal mixing (and, of course, even more in the case of minimal mixing). Thus, it is very likely that $\tan \beta$ and $m_{A^{0}}$ will be in one of two regions: (a) the increasingly large (as $m_{A^{0}}$ increases) wedge of moderate $\tan \beta>3$ in which only the $h^{0}$ will be detected; or, (b) the high $\tan \beta$ region for which the $g g \rightarrow H^{0}, A^{0} \rightarrow \tau^{+} \tau^{-}, \mu^{+} \mu^{-}$and $g b \rightarrow H^{ \pm} t \rightarrow \tau^{ \pm} \nu t$, tbt modes are viable as well. If the $H^{0}, A^{0}, H^{ \pm}$are heavy and cannot be detected either at the LHC (because $\tan \beta$ is not large enough) or at the LC (because they are too heavy to be pair-produced), precision measurements of the $h^{0}$ branching ratios and other properties will be particularly crucial. The precision measurements might provide 
Higgs Bosons at the Linear Collider

the only means for constraining or approximately determining the value of $m_{A^{0}}$ aside from possible direct detection in $\gamma \gamma \rightarrow H^{0}, A^{0}$ production. Expected LC precisions are such that deviations of $h^{0}$ branching ratios from the predicted SM values can be detected for $m_{A^{0}} \lesssim 700 \mathrm{GeV}[2,61]$.

At the LHC there is another important possibility for $h^{0}$ detection. Provided that the mass of the second-lightest neutralino exceeds that of the lightest neutralino (the LSP) by at least $m_{h^{0}}$, gluino and squark production will lead to chain decays in which $\widetilde{\chi}_{2}^{0} \rightarrow h^{0} \widetilde{\chi}_{1}^{0}$ occurs with substantial probability. In this way, an enormous number of $h^{0}$ 's can be produced, and the $h^{0} \rightarrow b \bar{b}$ decay mode will produce a dramatic signal.

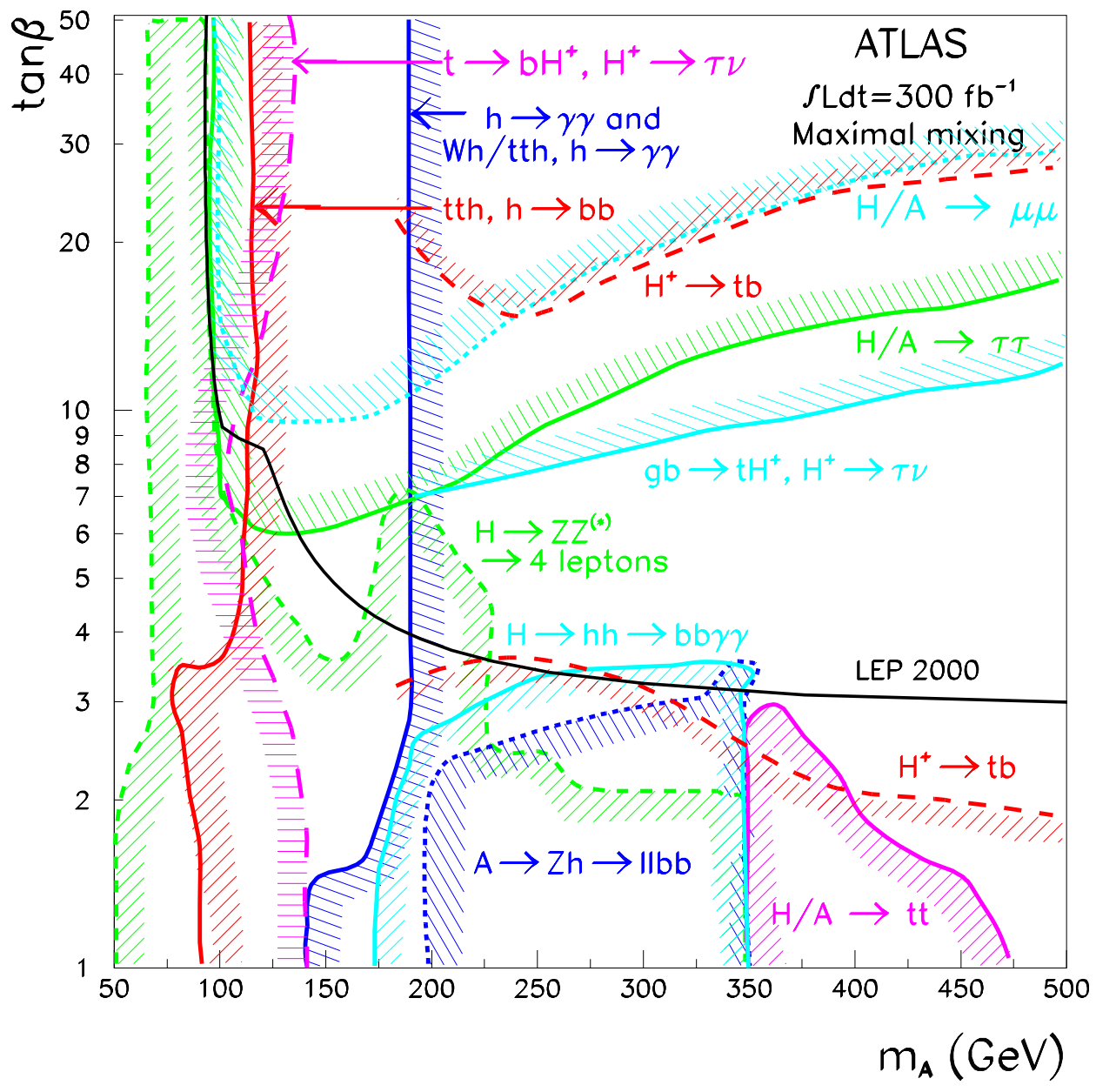

Figure 3.14: $5 \sigma$ discovery contours for MSSM Higgs boson detection in various channels are shown in the $\left[m_{A^{0}}, \tan \beta\right]$ parameter space, assuming maximal mixing and an integrated luminosity of $L=300 \mathrm{fb}^{-1}$ for the ATLAS detector. This figure is preliminary [60]. 


\section{Non-exotic extended Higgs sectors}

In this section, we consider the possibility of extending only the Higgs sector of the SM, leaving unchanged the gauge and fermionic sectors of the SM. We will also consider extensions of the two-doublet Higgs sector of the MSSM.

The simplest extensions of the minimal one-doublet Higgs sector of the SM contain additional doublet and/or singlet Higgs fields. Such extended Higgs sectors will be called non-exotic (to distinguish them from exotic Higgs sectors with higher representations, which will be considered briefly in Section 11). Singlet-only extensions have the advantage of not introducing the possibility of charge violation, since there are no charged Higgs bosons. In models with more than one Higgs doublet, tree-level Higgs-mediated flavor-changing neutral currents are present unless additional symmetries (discrete symmetries or supersymmetry) are introduced to restrict the form of the tree-level Higgs-fermion interactions [62]. Extensions containing additional doublet fields allow for spontaneous and explicit CP violation within the Higgs sector. These could be the source of observed CP-violating phenomena. Such models require that the mass-squared of the charged Higgs boson(s) that are introduced be chosen positive in order to avoid spontaneous breaking of electric charge conservation.

Extensions of the SM Higgs sector containing doublets and singlets can certainly be considered on a purely ad hoc basis. But there are also many dynamical models in which the effective low-energy sector below some scale $\Lambda$ of order 1 to $10 \mathrm{TeV}$, or higher, consists of the SM fermions and gauge bosons plus an extended Higgs sector. Models with an extra doublet of Higgs fields include those related to technicolor, in which the effective Higgs doublet fields are composites containing new heavier fermions. See Chapter 5, Section 3 for further discussion of this case. The heavy fermions should be vector-like to minimize extra contributions to precision electroweak observables. In many of these models, the top quark mixes with the right-handed component of a new vector-like fermion. The top quark could also mix with the right-handed component of a Kaluza-Klein (KK) excitation of a fermion field, so that Higgs bosons would be composites of the top quark and fermionic KK excitations. (For a review and references to the literature, see [63].) Although none of these (non-perturbative) models have been fully developed, they do provide significant motivation for studying the Standard Model with a Higgs sector containing extra doublets and/or singlets if only as the effective low-energy theory below a scale $\Lambda$ in the TeV range.

When considering Higgs representations in the context of a dynamical model with strong couplings at scale $\Lambda$, restrictions on Higgs self-couplings and Yukawa couplings that would arise by requiring perturbativity for such couplings up to some large GUT scale do not apply. At most, one should only demand perturbativity up to the scale $\Lambda$ at which the new (non-perturbative) dynamics enters and the effective theory breaks down. 
The minimal Higgs sector of the MSSM is a Type-II two-doublet model, where one Higgs doublet $\left(H_{d}\right)$ couples at tree-level only to down quarks and leptons while the other $\left(H_{u}\right)$ couples only to up quarks. Non-minimal extended Higgs sectors are also possible in low-energy supersymmetric models. Indeed, string theory realizations of low-energy supersymmetry often contain many extra singlet, doublet and even higher representations, some of which can yield light Higgs bosons (see, e.g., [64]). However, non-singlet Higgs representations spoil gauge coupling unification, unless additional intermediate-scale matter fields are added to restore it. A particularly well-motivated extension is the inclusion of a single extra complex singlet Higgs field, often denoted $S$. Including $S$, the superpotential for the theory can contain the term $\lambda_{S} H_{u} H_{d} S$, which can then provide a natural source of a weak scale value for the $\mu$ parameter appearing in the bilinear superpotential form $\mu H_{u} H_{d}$ required in the MSSM. A weak-scale value for $s \equiv\left\langle S^{0}\right\rangle$, where $S^{0}$ is the scalar component of the superfield $S$, is natural and yields an effective $\mu=\lambda_{S} s$. This extension of the MSSM is referred to as the next-tominimal supersymmetric model, or NMSSM, and has received considerable attention. For an early review and references, see [1].

\subsection{The decoupling limit}

In many extended Higgs sector models, the most natural parameter possibilities correspond to a decoupling limit in which there is only one light Higgs boson, with Yukawa and vector boson couplings close to those of the SM Higgs boson. In contrast, all the other Higgs bosons are substantially heavier (than the $Z$ ) with negligibly small relative mass differences, and with suppressed vector boson couplings (which vanish in the exact limit of decoupling). By assumption, the decoupling limit assumes that all Higgs self-couplings are kept fixed and perturbative in size. ${ }^{6}$ In the MSSM, such a decoupling limit arises for large $m_{A^{0}}$, and quickly becomes a very good approximation for $m_{A^{0}} \gtrsim 150 \mathrm{GeV}$.

The decoupling limit can be evaded in special cases, in which the scalar potential exhibits a special form (e.g., a discrete symmetry can forbid certain terms). In such models, there could exist regions of parameter space in which all but one Higgs boson are significantly heavier than the $Z$, but the light scalar state does not possess SM-like properties [65]. A complete exposition regarding the decoupling limit in the 2HDM, and special cases that evade the limit can be found in [66].

\subsection{Constraints from precision electroweak data and LC implications}

In the minimal SM, precision electroweak constraints require $m_{h_{\mathrm{SM}}} \lesssim 230 \mathrm{GeV}$ at 90\% CL. This is precisely the mass region preferred in the MSSM and its extensions.

\footnotetext{
${ }^{6}$ In the decoupling limit, the heavier Higgs bosons may have enhanced couplings to fermions (e.g., at large $\tan \beta$ in the $2 \mathrm{HDM})$. We assume that these couplings also remain perturbative.
} 
However, in the context of general doublets + singlets extensions of the Higgs sector there are many more complicated possibilities. First, it could be that there are several, or even many, Higgs bosons that couple to vector bosons and it is only their average mass weighted by the square of their $V V$ coupling strength (relative to the SM strength) that must obey this limit. Second, there can be weak isospin violations either within the Higgs sector itself or involving extra dynamics (for example related to the composite Higgs approach) that can compensate for the excessive deviations predicted if there is a SM-like Higgs with mass substantially above $\sim 230 \mathrm{GeV}$.

A particularly simple example of this latter situation arises in the context of the 2HDM [65]. Consider a 2HDM in which one of the CP-even neutral Higgs bosons has SM-like couplings but has mass just above a particular presumed value of $\sqrt{s}$ (500 or $800 \mathrm{GeV}$ ) for the linear collider. In addition, focus on cases in which there is a lighter $A^{0}$ or $h^{0}$ with no $V V$ coupling (for either, we use the notation $\widehat{h}$ ) and in which all other Higgs bosons have mass larger than $\sqrt{s}$. Next, isolate mass and $\tan \beta$ choices for which detection of the $\widehat{h}$ will also be impossible at the LC. Finally, scan over masses of the heavy Higgs bosons so as to achieve the smallest precision electroweak $\Delta \chi^{2}$ relative to that found in the minimal SM for $m_{h_{\mathrm{SM}}}=115 \mathrm{GeV}$. The blobs of overlapping points in Fig. 3.15 indicate the $S, T$ values for the optimal choices and lie well within the current 90\% CL ellipse. The heavy Higgs boson with SM couplings gives a large positive contribution to $S$ and large negative contribution to $T$, and in the absence of the other Higgs bosons would give the $S, T$ location indicated by the star. However, there is an additional positive contribution to $T$ arising from a slight mass non-degeneracy among the heavier Higgs bosons. For instance, for the case of a light $\widehat{h}=A^{0}$, the $h^{0}$ is heavy and SM-like and

$$
\Delta \rho \equiv \alpha \Delta T=\frac{\alpha}{16 \pi m_{W}^{2} c_{W}^{2}}\left\{\frac{c_{W}^{2}}{s_{W}^{2}} \frac{m_{H^{ \pm}}^{2}-m_{H}^{2}}{2}-3 m_{W}^{2}\left[\log \frac{m_{h}^{2}}{m_{W}^{2}}+\frac{1}{6}+\frac{1}{s_{W}^{2}} \log \frac{m_{W}^{2}}{m_{Z}^{2}}\right]\right\}
$$

can be adjusted to place the $S, T$ prediction at the location of the blob in Fig. 3.15 by an appropriate choice of $m_{H^{ \pm}}^{2}-m_{H^{0}}^{2}$. Indeed, even if the "light" decoupled Higgs boson is not so light, but rather has mass equal to $\sqrt{s}$ (and is therefore unobservable), one can still obtain entirely adequate agreement with current precision electroweak data. Fortunately, one can only push this scenario so far. To avoid moving beyond the current $90 \%$ ellipse (and also to maintain perturbativity for the Higgs self-couplings), the Higgs with SM-like $V V$ coupling must have mass $\lesssim 1 \mathrm{TeV}$.

In composite Higgs models with extra fermions, there are similar non-degeneracies of the fermions that can yield a similar positive contribution to $\Delta \rho$ and thence $T$. As reviewed in [13], consistency with current precision electroweak data inevitably constrains parameters so that some type of new physics (including a possible heavy scalar sector) would again have to lie below a TeV or so. Future Giga-Z data could provide much stronger constraints on these types of models, as discussed in Section 9. 

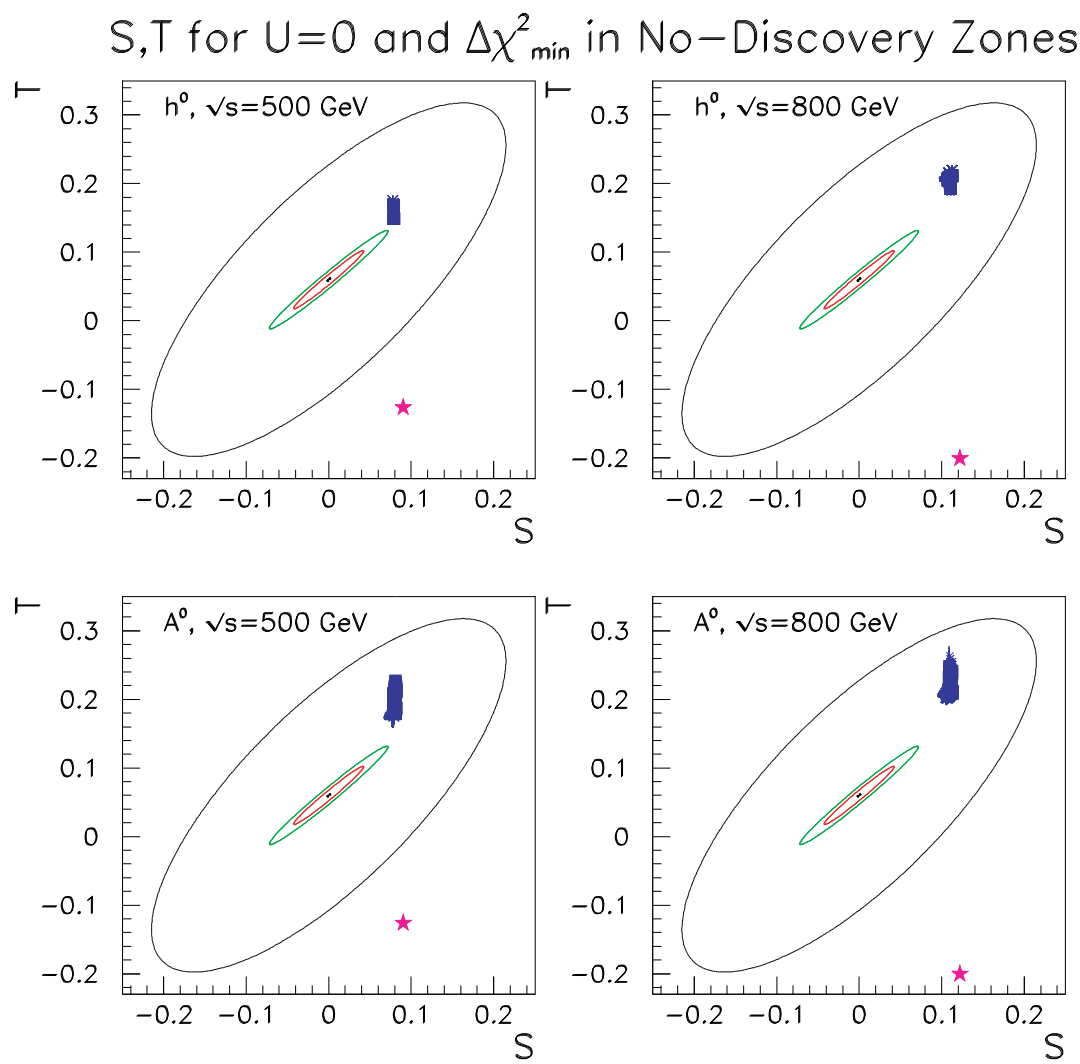

Figure 3.15: The outer ellipse gives the current 90\% CL region for $U=0$ and SM Higgs mass of $115 \mathrm{GeV}$. The blobs show the $S, T$ predictions for the $2 \mathrm{HDM}$ models described in the text that have minimum $\Delta \chi^{2}$ relative to this SM benchmark and for which no Higgs boson of the $2 \mathrm{HDM}$ will be detected at the LC. The innermost (middle) ellipse gives the $90 \%(99.9 \%)$ CL region for $m_{h_{\mathrm{SM}}}=115 \mathrm{GeV}$ obtained after Giga-Z precision measurements and a $\Delta m_{W} \lesssim 6 \mathrm{MeV}$ threshold scan measurement of $m_{W}$. The stars indicate the minimal SM $S, T$ prediction if $m_{h_{\mathrm{SM}}}=\sqrt{s}$.

\subsection{Constraints on Higgs bosons with $V V$ coupling}

In the MSSM, we know that the Higgs boson(s) that carry the $V V$ coupling must be light: if $m_{A^{0}}$ is large (the decoupling limit) then it is the mass-bounded $h^{0}$ that has all the $V V$ coupling strength; if $m_{A^{0}} \lesssim 2 m_{Z}$, then the $H^{0}$ can share the $V V$ coupling with the $h^{0}$, but then $m_{H^{0}}$ cannot be larger than about $2 m_{Z}$. In the NMSSM, assuming Higgs-sector CP conservation, there are 3 neutral CP-even Higgs bosons, $h_{1,2,3}\left(m_{1}<m_{2}<m_{3}\right)$, which can share the $V V$ coupling strength. One can show (see [67] for a recent update) that the masses of the $h_{i}$ with substantial 
$V V$ coupling are strongly bounded from above. This result generalizes to the most general supersymmetric Higgs sector as follows. Labeling the neutral Higgs bosons by $i$ with masses $m_{h_{i}}$ and denoting the $Z Z$ squared-coupling relative to the SM by $K_{i}$, it can be shown that

$$
\sum_{i} K_{i} \geq 1, \quad \sum_{i} K_{i} m_{h_{i}}^{2} \leq(200 \mathrm{GeV})^{2} .
$$

That is, the aggregate strength of the $V V$ coupling-squared of all the neutral Higgs bosons is at least that of the SM, and the masses-squared of the neutral $h_{i}$ weighted by the coupling-squared must lie below a certain bound. The upper bound of $(200 \mathrm{GeV})^{2}$ in Eq. (3.11) is obtained [41] by assuming that the MSSM remains perturbative up to the the GUT scale of order $10^{19} \mathrm{GeV}$. This bound applies for the most general possible Higgs representations (including triplets) in the supersymmetric Higgs sector and for arbitrary numbers of representations. If only doublet and singlet representations are allowed for, the bound would be lower. The $(200 \mathrm{GeV})^{2}$ bound also applies to general Higgs-sector-only extensions of the SM by requiring consistency with precision electroweak constraints and assuming the absence of a large contribution to $T$ from the Higgs sector itself or from new physics, such as discussed in Section 7.2.

\subsection{Detection of non-exotic extended Higgs sector scalars at the Tevatron and LHC}

In the case of extended Higgs sectors, all of the same processes as discussed for the SM and MSSM will again be relevant. However, we can no longer guarantee Higgs discovery at the Tevatron and/or LHC. In particular, if there are many Higgs bosons sharing the $W W, Z Z$ coupling, Higgs boson discovery based on processes that rely on the $V V$ coupling could be much more difficult than in models with just a few light Higgs bosons with substantial $V V$ coupling. This is true even if the sum rule of Eq. (3.11) applies. For example, at the LHC even the NMSSM addition of a single singlet to the minimal two-doublet structure in the perturbative supersymmetric context allows for parameter choices such that no Higgs boson can be discovered [68] using any of the processes considered for SM Higgs and MSSM Higgs detection. The $\gamma \gamma$ decay channel signals are all weak (because of decreased $W$-loop contribution to the coupling). Further, if a moderate value of $\tan \beta$ is chosen then $t \bar{t}+$ Higgs processes are small and $b \bar{b}+$ Higgs processes are insufficiently enhanced. In short, the equivalent to the wedge of Fig. 3.14 enlarges. The $h^{0}$ signal is divided among the three light neutral CP-even Higgs bosons and diluted to too low a statistical significance.

However, in other cases, the Tevatron and LHC could observe signals not expected in an approximate decoupling limit. For example, in the 2HDM model discussed earlier the light $\widehat{h}$ with no $V V$ couplings decays via $\widehat{h} \rightarrow b \bar{b}, \tau^{+} \tau^{-}$and discovery in $t \widehat{t} \widehat{h}, b \bar{b} \widehat{h}$ and even $g g \rightarrow \widehat{h}[69]$ is possible, though certainly not guaranteed. Further, in these models there is a heavy neutral Higgs boson having the bulk of the $V V$ 
Higgs Bosons at the Linear Collider

coupling and (for consistency with current precision electroweak constraints or with perturbativity) mass $\lesssim 1 \mathrm{TeV}$. This latter Higgs boson would be detected at the LHC using $g g, W W$ fusion production and $Z Z \rightarrow 4 \ell, W W \rightarrow 2 j \ell \nu, \ldots$ decay modes, just like a heavy minimal SM Higgs boson.

\subsection{LC production mechanisms for non-exotic extended Higgs sector scalars}

Any physical Higgs eigenstate with substantial $W W$ and $Z Z$ coupling will be produced in Higgsstrahlung and $W W$ fusion at the LC. Although there could be considerable cross section dilution and/or resonance peak overlap, the LC will nonetheless always detect a signal. This has been discussed for the MSSM in Section 5.4. In the NMSSM, if one of the heavier CP-even $h_{i}$ has most of the $V V$ coupling, the strong bound on its mass [67] noted earlier implies that it will be detected at any LC with $\sqrt{s}>350 \mathrm{GeV}$ within a small fraction of a year when running at planned luminosities. The worst possible case is that in which there are many Higgs bosons with $V V$ coupling with masses spread out over a large interval with separation smaller than the mass resolution. In this case, the Higgs signal becomes a kind of continuum distribution. Still, in [70] it is shown that the sum rule of Eq. (3.11) guarantees that the Higgs continuum signal will still be detectable for sufficient integrated luminosity, $L \gtrsim 200 \mathrm{fb}^{-1}$, as a broad excess in the recoil mass spectrum of the $e^{+} e^{-} \rightarrow Z X$ process. (In this case, $W W$ fusion events do not allow for the reconstruction of Higgs events independently of the final state Higgs decay channel.) As already noted, the value of $200 \mathrm{GeV}$ appearing in Eq. (3.11) can be derived from perturbative RGE constraints for the most general Higgs sector in supersymmetric theories and is also required by precision electroweak data for general SM Higgs sector extensions, at least in theories that do not have a large positive contribution to $T$ from a non-decoupling structure in the Higgs sector or from new physics not associated with the Higgs sector.

Other production modes of relevance include Higgs pair production, $t \bar{t}+$ Higgs, and $b \bar{b}+$ Higgs. In multi-doublet models, $t \bar{b} H^{-}$and $b \bar{t} H^{+}$reactions are present. However, none of these are guaranteed to be either kinematically accessible or, if accessible, to have a sufficiently high event rate to be observed.

Regardless of the production process, relevant decay channels could include cases where heavier Higgs bosons decay to lighter ones. If observed, such decays would provide vital information regarding Higgs self-couplings.

We should particularly consider what production processes are most relevant for those Higgs bosons (denoted $\widehat{h}$ ) that do not have substantial $V V$ coupling. Such processes have particular relevance in the non-decoupling scenario for the general $2 \mathrm{HDM}$ model discussed earlier. There, such a $\widehat{h}$ is the only Higgs boson light enough to be produced at an LC with $\sqrt{s} \lesssim 1 \mathrm{TeV}$ and it cannot be produced and detected in $W W$ fusion or Higgsstrahlung. Since the other Higgs bosons are heavy, the $\widehat{h}$ also 


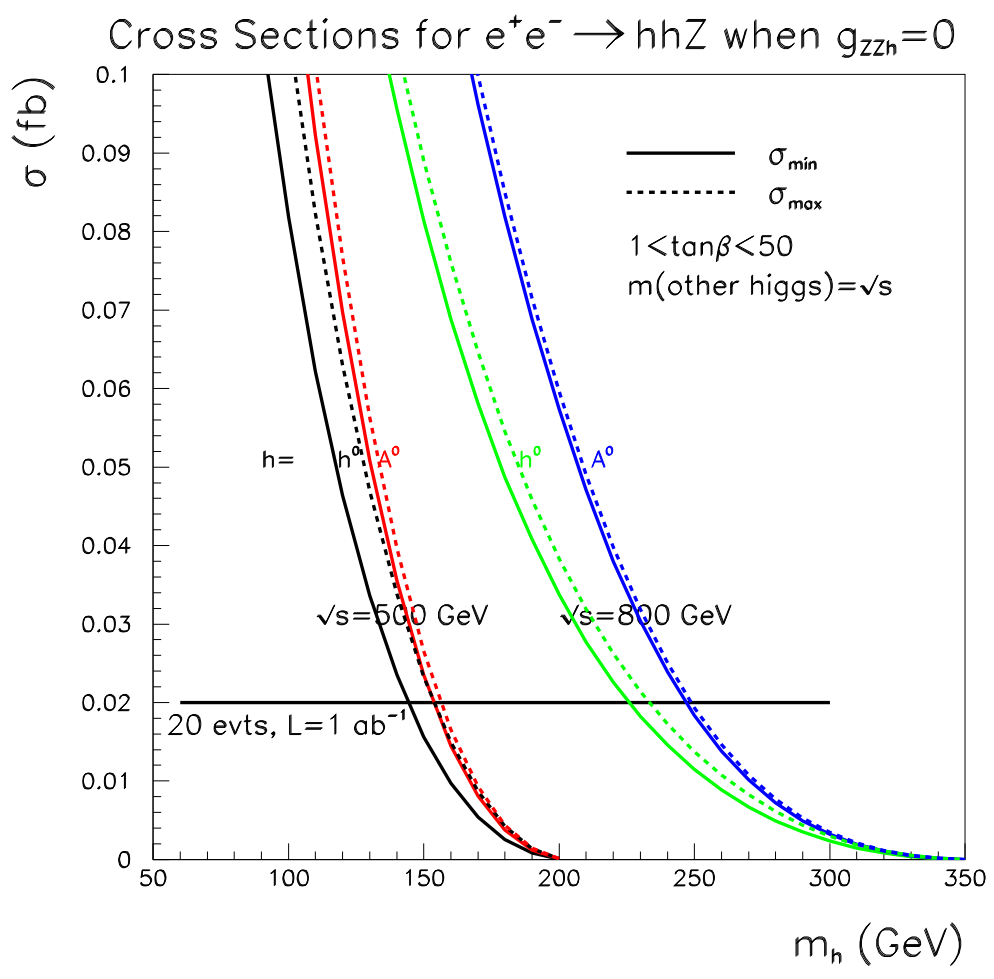

Figure 3.16: For $\sqrt{s}=500 \mathrm{GeV}$ and $800 \mathrm{GeV}$ and for $\widehat{h}=h^{0}$ and $\widehat{h}=A^{0}$, we plot as a function of $m_{\widehat{h}}$ the maximum and minimum values of $\sigma\left(e^{+} e^{-} \rightarrow \widehat{h} \widehat{h} Z\right)$ found after scanning $1<\tan \beta<50$ taking all other higgs masses equal to $\sqrt{s}$. For $\widehat{h}=h^{0}$, we require $\sin (\beta-\alpha)=$ 0 during the scan. The 20 event level for $L=1000 \mathrm{fb}^{-1}$ is indicated.

cannot be produced in association with another Higgs boson. As shown in [71,65], the $b \bar{b} \widehat{h}$ and $t \widehat{t} \widehat{h}$ processes will also not be detectable at the LC if $\tan \beta$ is moderate in value. The most interesting tree-level processes are then those based on the quartic couplings $W W \widehat{h} \hat{h}$ and $Z Z \widehat{h} \widehat{h}$ required by gauge invariance [72,73]. These couplings allow for $W W \rightarrow \widehat{h} \hat{h}$ fusion and $Z^{*} \rightarrow Z \widehat{h} \widehat{h}$ production, respectively. The exact cross sections for these processes are only mildly sensitive to the masses of the other heavier Higgs bosons via 2HDM Higgs self-couplings. Of course, phase space restrictions imply an upper limit on the $\widehat{h}$ masses that can be probed in this way. Cross sections in the case of $Z^{*} \rightarrow Z \widehat{h} \widehat{h}$ are plotted in Fig. 3.16 for both $\widehat{h}=A^{0}$ and $\widehat{h}=h^{0}$ taking $\sqrt{s}=500$ [74]. Assuming optimistically that 20 events in $L=1000 \mathrm{fb}^{-1}$ could be detected, $Z^{*} \rightarrow Z \widehat{h} \hat{h}$ could be detected for $m_{\widehat{h}}$ as large as $150 \mathrm{GeV}$. At $\sqrt{s}=800 \mathrm{GeV}$, this limit increases to $250 \mathrm{GeV}$. Similar results are obtained for $W W \rightarrow \widehat{h} \widehat{h}$ fusion production. 
Higgs Bosons at the Linear Collider

\section{Measurements of Higgs boson properties at the LC}

The strength of the LC physics program is that it cannot only observe one or more Higgs boson(s), but also precisely determine the Higgs boson mass, width, couplings, and quantum numbers, and parameters of the Higgs potential. These measurements are crucial to establish the nature of the Higgs and thus to illuminate the mechanism of electroweak symmetry breaking. Measurements of the Higgs couplings can demonstrate that a Higgs boson generates the masses of vector bosons, charged leptons, and up- and down-type quarks. If the measured couplings are not simply proportional to mass, this will require a Higgs sector more complex than a single complex Higgs doublet. Accurate measurements are needed to distinguish the SM Higgs and $h^{0}$ of the MSSM near the decoupling limit. Couplings are determined through measurements of Higgs branching ratios and cross sections. Higgs bosons are also expected to couple to themselves, and this self-coupling $\lambda$ can only be explored through the direct production of two or more Higgs bosons. The measurement of direct and model independent absolute Higgs couplings is a major cornerstone of the LC program.

Details of some of the studies of Higgs coupling measurements can be found in [75]. A comprehensive description of European studies using the simulated TESLA detector can be found in [76]. North American studies consider simulations of detectors with capabilities described in Chapter 15. The program of measurements of Higgs boson properties strongly impacts detector design. Measurement of branching ratios into fermions requires sophisticated vertex detectors to separate $b$ from $c$ (and gluon) jets. Precise recoil mass measurements need excellent momentum resolution (particularly for $\mu^{+} \mu^{-}$) from charged particle tracking. The performance of the combined tracking and calorimetry systems needs to result in precise jet-jet invariant masses, missing mass measurements, and the ability to separate hadronic $W$ from hadronic $Z$ decays.

The specific measurements used to determine the Higgs couplings to vector bosons, fermions and scalars are significantly different depending on the mass of the Higgs boson. A generic neutral CP-even Higgs boson will be denoted by $h$ in this section. We treat three cases separately: a light Higgs boson $\left(m_{h}<2 m_{W}\right)$, an intermediate mass Higgs boson $\left(2 m_{W} \leq m_{h}<2 m_{t}\right)$, and a heavy Higgs boson $\left(m_{h} \geq 2 m_{t}\right)$.

\subsection{Mass}

In the Standard Model, the Higgs mass determines all its other properties. Thus, the precision of the mass measurement affects the comparison of theory and experiment, for example, in a global fit of cross sections, branching ratios, and precision electroweak data. Similarly, in the MSSM or other models with extended Higgs sec-

tors, the masses of all the Higgs bosons are an important input in determining the underlying model parameters.

For this fundamental mass measurement, a LC can reconstruct the system recoiling against a $Z$ (independent of Higgs decay). Full event reconstruction, plus 
kinematic constraints, can improve resolution and clean up mass tails. For a light or intermediate mass Higgs boson, the optimal running conditions would have a smaller center-of-mass energy such as $\sqrt{s}=350 \mathrm{GeV}$, to allow better momentum resolution and to minimize the beamstrahlung. Under such conditions, one can precisely measure the recoil mass in $e^{+} e^{-} \rightarrow Z h$ events opposite to the reconstructed leptonic decay $Z \rightarrow e^{+} e^{-}$or $\mu^{+} \mu^{-}$. This measurement is independent of the Higgs decay mode. Accuracy can be improved by reconstructing specific decay modes, leading, for example, to a four-jet topology where effective (5-C) kinematic constrained fits can be employed.

Figure 3.17 shows the distribution of the recoil mass,

$$
M_{\text {recoil }}=\sqrt{s-2 \sqrt{s} \cdot E_{\ell^{+} \ell^{-}}+M_{\ell^{+} \ell^{-}}^{2}},
$$

in a simulation of the L linear collider detector [77] described in Chapter 15 for Higgs masses between 115 and $160 \mathrm{GeV}$ [78]. Using Monte Carlo shape templates and an integrated luminosity of $500 \mathrm{fb}^{-1}$, precisions of $\Delta m_{h_{\mathrm{SM}}} \simeq 80 \mathrm{MeV}$ at $\sqrt{s}=350 \mathrm{GeV}$ and $\Delta m_{h_{\mathrm{SM}}} \simeq 140 \mathrm{MeV}$ at $\sqrt{s}=500 \mathrm{GeV}$ have been estimated for either the $e^{+} e^{-}$ or $\mu^{+} \mu^{-}$mode.
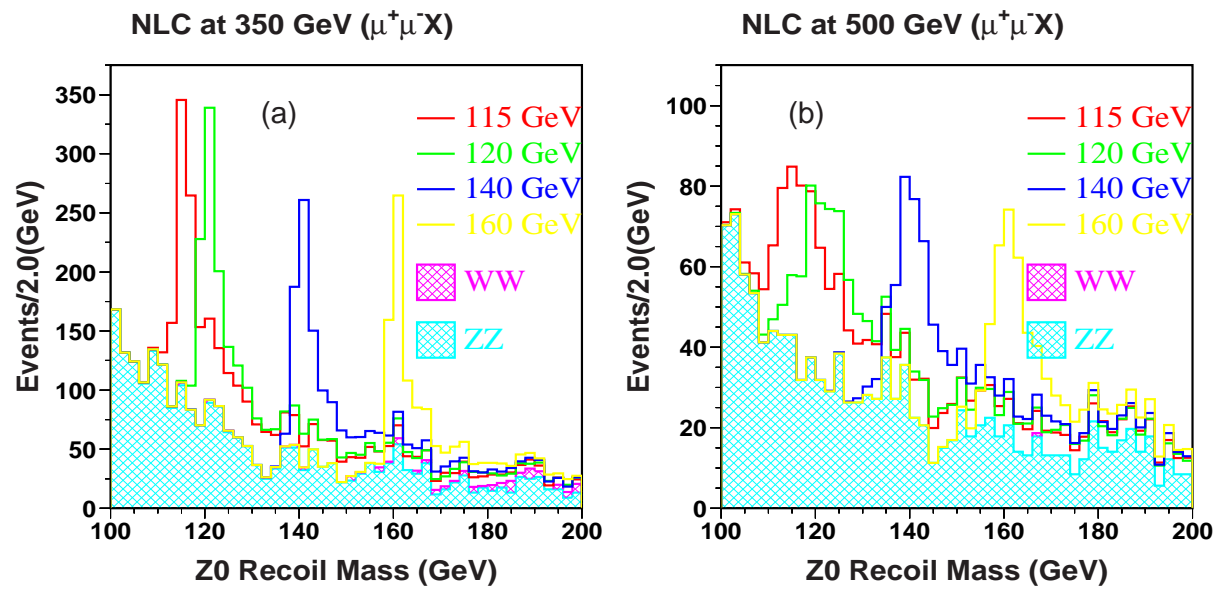

Figure 3.17: Recoil mass from a pair of leptons for different Higgs masses at (a) $\sqrt{s}=$ $350 \mathrm{GeV}$ and (b) $500 \mathrm{GeV}$ simulated in the L detector described in Chapter 15.

Realistic simulations have also been made with the L detector for the process $Z h \rightarrow q \bar{q} h$ resulting in four jets. Figure 3.18(a) shows the jet-jet invariant mass distribution for pairs of jets for Higgs with $m_{h_{\mathrm{SM}}}=115 \mathrm{GeV}$ recoiling against a $Z$ reconstructed from its hadronic decay mode [79]. A clean Higgs signal with a mass resolution of approximately $2 \mathrm{GeV}$ is observed. The central Higgs mass is shifted down by the loss of low-energy charged and neutral particles in the simulated event 
reconstruction. A low-mass tail of the Higgs signal arises from missing neutrinos in semi-leptonic $b$ and $c$ quark decays. Using neural net tags and full kinematic fitting [80], the mass peak shown in Fig. 3.18(b) is obtained for $m_{h_{\mathrm{SM}}}=120 \mathrm{GeV}$, $\sqrt{s}=500 \mathrm{GeV}$, and $500 \mathrm{fb}^{-1}$ resulting in $\Delta m_{h_{\mathrm{SM}}} \simeq 50 \mathrm{MeV}$. If a second lower-energy IR is available, it might be attractive to perform a scan across the $Z h$ threshold. With a total integrated luminosity of $100 \mathrm{fb}^{-1}, \Delta m_{h_{\mathrm{SM}}} \simeq 100 \mathrm{MeV}$ at $m_{h_{\mathrm{SM}}}=150 \mathrm{GeV}$ is achievable [81], competitive with the methods above.
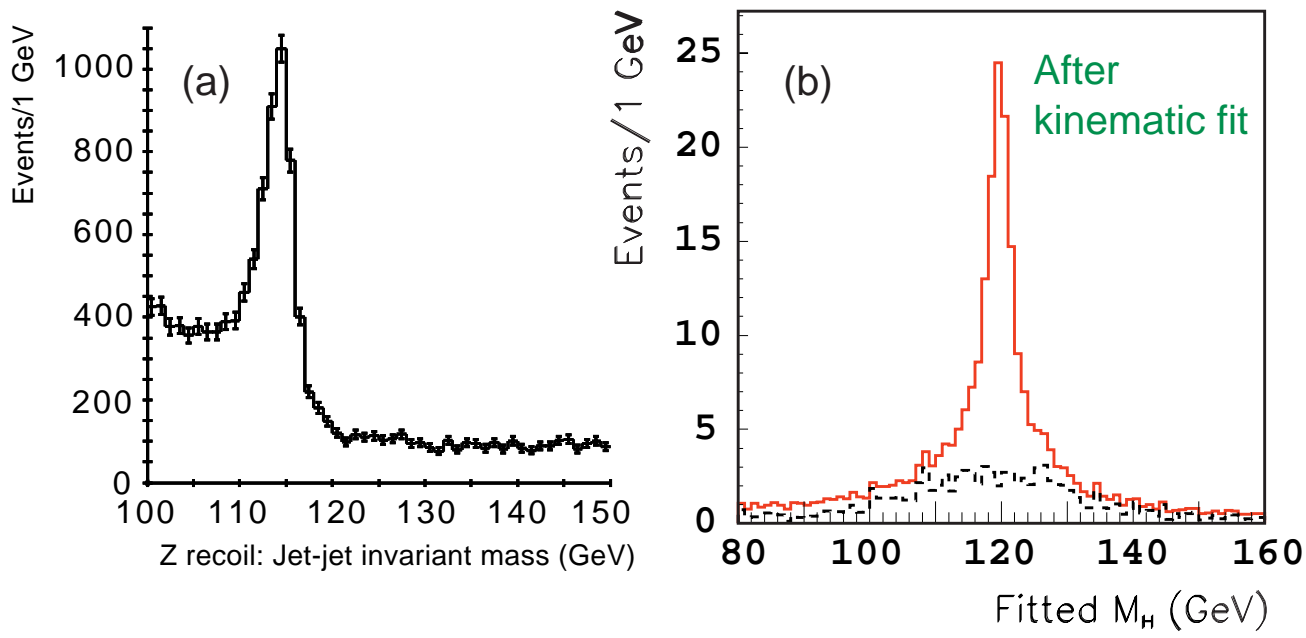

Figure 3.18: (a) Jet-jet invariant mass of the jets recoiling from a $Z$ reconstructed hadronically simulated in the LCD Large detector, $m_{h_{\mathrm{SM}}}=115 \mathrm{GeV}$. (b) Direct reconstruction of the four-jet $q \bar{q} h_{\mathrm{SM}}$ state simulated in the L detector after fitting with full kinematic constraints, $m_{h_{\mathrm{SM}}}=120 \mathrm{GeV}$.

Further work is necessary to confirm analogous precisions for heavier Higgs bosons and MSSM Higgs bosons with different decay modes and possible close mass-degeneracies. The number of $Z h$ events with $Z \rightarrow \ell^{+} \ell^{-}$for an intermediate-mass $\left(m_{h}>2 m_{W}\right)$ or heavy Higgs $\left(m_{h}>2 m_{t}\right)$ with SM coupling falls quickly [82]. In this case, and for the decays $h \rightarrow Z Z$, hadronic decays of the $Z$ would have to be considered to gain sufficient statistics. For the heavier MSSM Higgs boson states, European studies [83] have shown typical mass precisions of $\Delta m_{H^{ \pm}}$and $\Delta m_{A^{0}, H^{0}}$ of around $1 \mathrm{GeV}$ for $500 \mathrm{fb}^{-1}$, but at $\sqrt{s}=800 \mathrm{GeV}$. The MSSM $H^{0}$ and $A$ may be studied separately using $\gamma \gamma \rightarrow H / A$ with different states of $\gamma$ linear polarization, thus helping to refine mass determinations in the nearly degenerate case. 


\subsection{Coupling determinations - light Higgs bosons}

\subsubsection{Cross sections}

For Higgs masses below $2 m_{W}$, the couplings $g_{h Z Z}$ and $g_{h W W}$ are best measured through measurements of the Higgsstrahlung and $W W$ fusion cross sections, respectively. These cross sections are also critical in the extraction of branching ratios since the experimental measurement will be a product of cross section and branching ratio.

Measurement of the cross section $\sigma\left(Z^{*} \rightarrow Z h\right)$ is best addressed via the recoil mass method outlined above [78]. Again, in this case, to reduce the contribution from the $W W$ fusion process, it may be preferrable to run at a lower energy, i.e., $\sqrt{s}=$ $350 \mathrm{GeV}$, and to examine recoil against $\mu^{+} \mu^{-}$to avoid large Bhabha backgrounds. The study with the $\mathrm{L}$ detector described above finds $\Delta \sigma / \sigma \simeq 4 \%$ at $\sqrt{s}=350 \mathrm{GeV}$ and $\simeq 6.5 \%$ at $500 \mathrm{GeV}$ with $500 \mathrm{fb}^{-1}$ as shown in Fig. 3.19(a). These agree roughly with estimates from European studies [84].
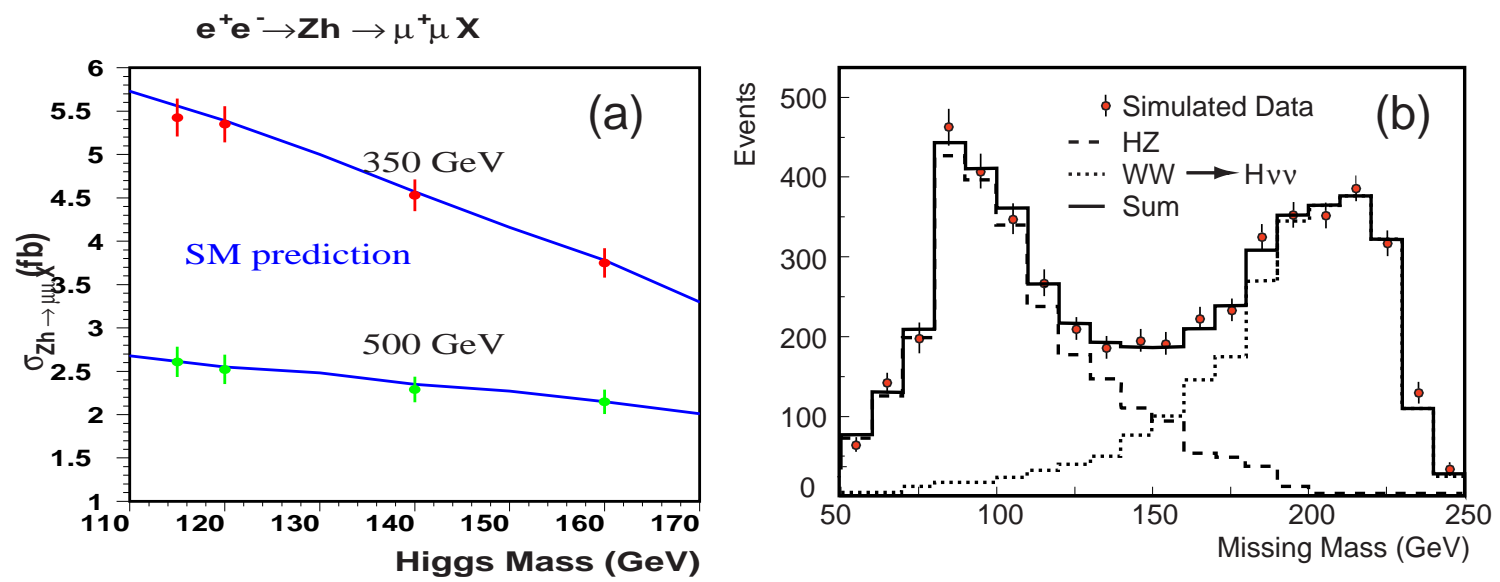

Figure 3.19: (a) Cross section measurement for $500 \mathrm{fb}^{-1}$ and (b) separation of Higgsstrahlung and $W W$ fusion $(\sqrt{s}=350 \mathrm{GeV})$ through a fit (after background subtraction), both simulated in the $\mathrm{L}$ detector.

With efficient and pure $b$-jet tagging, events due to $e^{+} e^{-} \rightarrow W^{+} W^{-} \nu \bar{\nu} \rightarrow \nu \bar{\nu} h \rightarrow$ $\nu \bar{\nu} b \bar{b}$ can be separated from those due to Higgsstrahlung, $Z h \rightarrow \nu \bar{\nu} h \rightarrow \nu \bar{\nu} b \bar{b}$ by examining the missing mass distribution and fitting to the expected shapes of a peak at $m_{Z}$ from Higgsstrahlung and the higher missing masses from $W W$ fusion. This technique has been confirmed with simulations of the L detector as shown in Fig. 3.19(b) [85]. With $500 \mathrm{fb}^{-1}$ and a precision $\mathrm{BR}\left(h_{\mathrm{SM}} \rightarrow b \bar{b}\right) \simeq 3 \%$ (see below), the fusion-process cross section with this analysis can be found with a precision $\Delta \sigma / \sigma=$ $3.5 \%$ for $m_{h_{\mathrm{SM}}}=120 \mathrm{GeV}$. 
Higgs Bosons at the Linear Collider

\begin{tabular}{l||c|c||c|c}
\hline \hline \multicolumn{1}{c||}{} & \multicolumn{2}{c||}{$m_{h_{\mathrm{SM}}}=120 \mathrm{GeV}$} & \multicolumn{2}{c}{$m_{h_{\mathrm{SM}}}=140 \mathrm{GeV}$} \\
\hline & $\mathrm{BR}$ & $\delta \mathrm{BR} / \mathrm{BR}$ & $\mathrm{BR}$ & $\delta \mathrm{BR} / \mathrm{BR}$ \\
\hline$h_{\mathrm{SM}} \rightarrow b \bar{b}$ & $(69 \pm 2.0) \%$ & $2.9 \%$ & $(34 \pm 1.3) \%$ & $4.1 \%$ \\
$h_{\mathrm{SM}} \rightarrow W W^{*}$ & $(14 \pm 1.3) \%$ & $9.3 \%$ & $(51 \pm 1.8) \%$ & $3.7 \%$ \\
$h_{\mathrm{SM}} \rightarrow c \bar{c}$ & $(2.8 \pm 1.1) \%$ & $39 \%$ & $(1.4 \pm 0.64) \%$ & $45 \%$ \\
$h_{\mathrm{SM}} \rightarrow g g$ & $(5.2 \pm 0.93) \%$ & $18 \%$ & $(3.5 \pm 0.79) \%$ & $23 \%$ \\
$h_{\mathrm{SM}} \rightarrow \tau^{+} \tau^{-}$ & $(7.1 \pm 0.56) \%$ & $7.9 \%$ & $(3.6 \pm 0.38) \%$ & $10 \%$ \\
\hline \hline
\end{tabular}

Table 3.1: Predicted branching ratio precisions in the L detector and typical vertex detector configuration for $500 \mathrm{fb}^{-1}$ and $\sqrt{s}=500 \mathrm{GeV}$.

\subsubsection{Branching ratios}

A key advantage of the linear collider in Higgs studies is the identification of Higgsstrahlung $Z h$ events through the tag of the $Z$ decays. This selection is essentially independent of the decay mode of the $h$ and simplifies the measurement of Higgs boson branching ratios.

Small beam sizes, the possibility of a first track measurement as close as $1 \mathrm{~cm}$ from the beam axis, and sophisticated pixel vertex detectors allow for efficient and clean separation of quark flavors. Separate tagging of $b, c$ and $g$ jets is possible.

In a study [86] of vertexing using a CCD vertex detector in a standard LC detector configuration (C1 in [87]), topological vertexing [88] with neural net selection was used for flavor (or anti-flavor, i.e., $W W^{*}$ ) tagging. The separation of $b \bar{b}$ and $c \bar{c}$ events by this method is illustrated in Fig. 3.20(a). Assuming $500 \mathrm{fb}^{-1}$ and $80 \%$ polarization, the results shown in Table 3.1 were obtained.

These results scale approximately as $\left(\sigma \int \mathcal{L} d t\right)^{-1 / 2}$ when taken together with other studies [89-91], but the results of [91] (shown in Fig. 3.20(b)) are noticeably more precise for the $c \bar{c}$ and $g g$ modes. These branching ratio measurements can then be used to either distinguish a SM Higgs boson from an MSSM Higgs boson, or to probe higher-mass states and extract MSSM parameters such as $m_{A^{0}}$ even if the CP-odd $A^{0}$ is not accessible. That analysis is described in more detail below.

An accessible decay mode for lighter Higgs bosons is $h \rightarrow \gamma \gamma$, which requires excellent electromagnetic calorimetry. As shown in Fig. 3.21, for a SM Higgs boson in a typical LC detector, this is a difficult measurement requiring a large luminosity, which is best optimized for masses around $120 \mathrm{GeV}$ [92]. A higher-luminosity study [93] with $1000 \mathrm{fb}^{-1}$ and $m_{h_{\mathrm{SM}}}=120 \mathrm{GeV}$ for the TESLA detector finds $\delta \mathrm{BR} / \mathrm{BR}=14 \%$. A $\gamma \gamma$ collider, discussed in Section 10, would be a more powerful tool for determining the Higgs coupling to photons.

For light Higgs bosons, the coupling to top quarks is still accessible via the radiative process $t \bar{t} h$ described below, or indirectly through $\mathrm{BR}(h \rightarrow g g)$.

A set of difficult decay channels for the LHC is invisible decays of the Higgs boson 

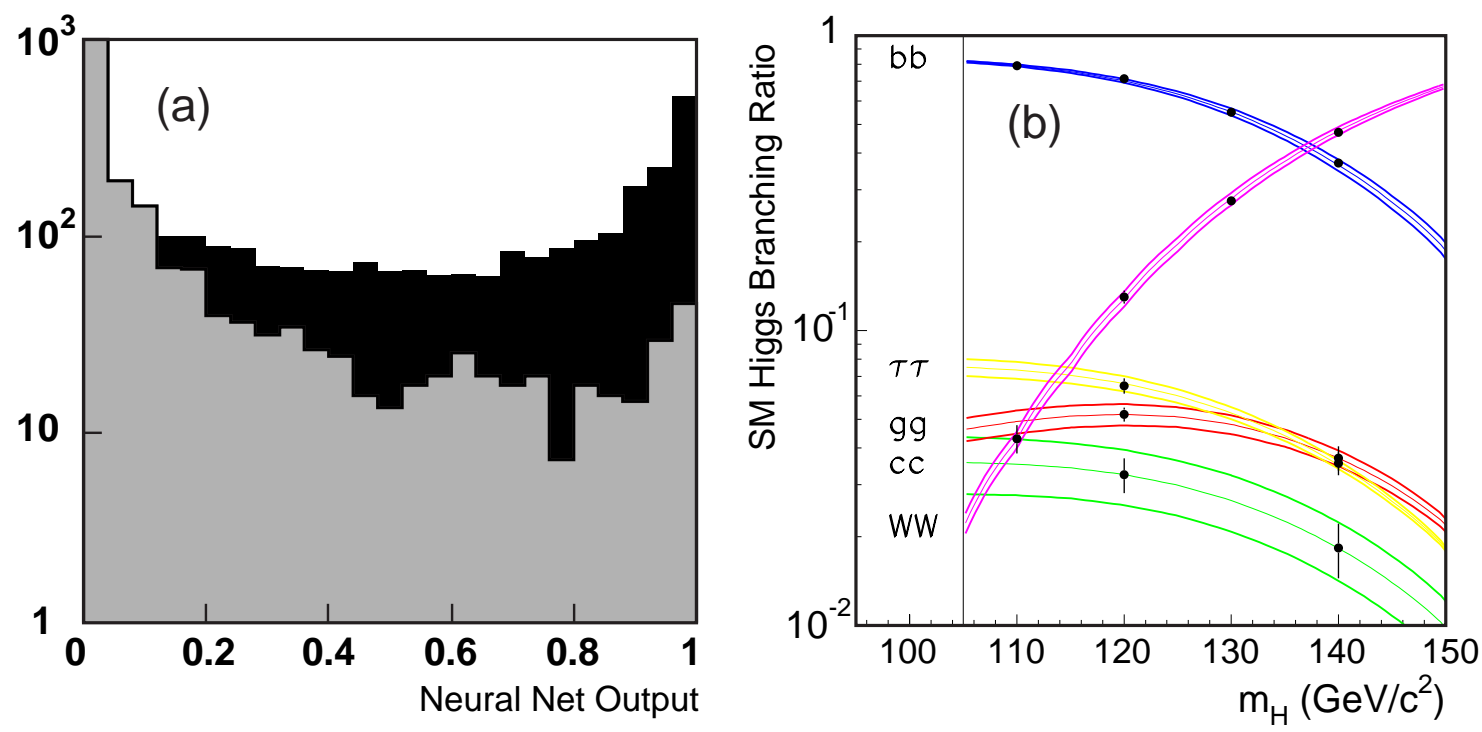

Figure 3.20: (a) For the simulated L detector with CCD vertex detector, neural net $h_{\mathrm{SM}} \rightarrow$ $c \bar{c}$ output for $h_{\mathrm{SM}} \rightarrow c \bar{c}$ events (dark) compared to output for $h_{\mathrm{SM}} \rightarrow b \bar{b}$ events (gray). (b) Variation of branching ratios with SM Higgs mass (bands are $1 \sigma$ uncertainties on the theoretical predictions) and measurement precisions in the TESLA detector (points with error bars).

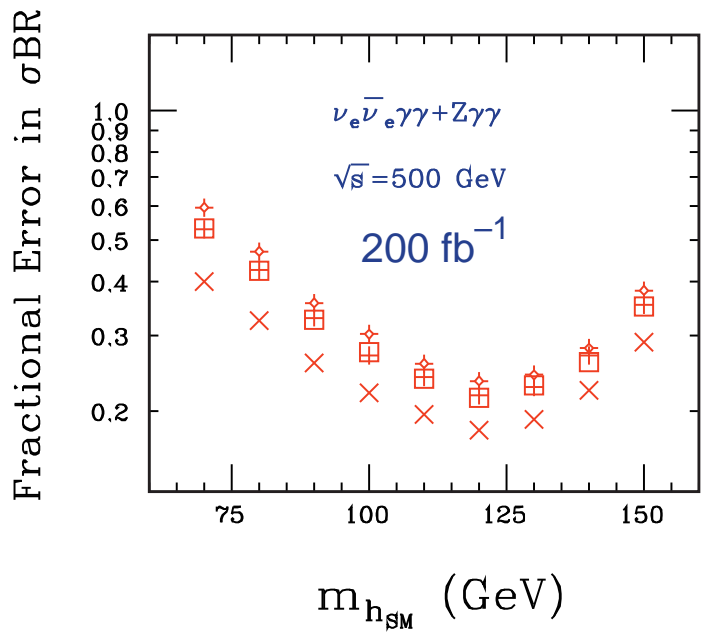

Figure 3.21: Fractional error on the branching ratio $\mathrm{BR}\left(h_{\mathrm{SM}} \rightarrow \gamma \gamma\right)$. The open squares are for a typical LC detector electromagnetic energy resolution of $\Delta E / E=10 \% / \sqrt{E} \oplus 1.0 \%$. 
Higgs Bosons at the Linear Collider

into, e.g., neutralinos, majorans or heavy neutrinos. The LC can close this loophole and measure the branching ratio easily, even for branching ratios as small as $5 \%$ for a relatively narrow Higgs state, by using the recoil mass method and demanding no detector activity opposite the $Z$, or by comparing the number of events tagged with $Z \rightarrow \ell^{+} \ell^{-}$with the total number of observed Higgs decays into known states.

\subsubsection{Radiative production and tt̄h coupling}

For a light Higgs boson, production through radiation off a top quark is feasible, resulting in a final state of $t \bar{t} h$. This allows a determination of the Yukawa top quark coupling $g_{h t t}[23,24]$. For a SM-like Higgs boson with $m_{h}=120 \mathrm{GeV}$, the $t \bar{t} h$ cross section is roughly 10 times larger at $\sqrt{s}=700-800 \mathrm{GeV}$ than at $500 \mathrm{GeV}$. At $\sqrt{s}=$ $800 \mathrm{GeV}$, a statistical error of $\delta g_{h t t} / g_{h t t} \sim 5 \%$ was estimated [94] for $L=500 \mathrm{fb}^{-1}$ on the basis of an optimal observable analysis. At $\sqrt{s}=500 \mathrm{GeV}$, a statistical error of $\delta g_{h t t} / g_{h t t} \simeq 21 \%$ is estimated [95] using $1000 \mathrm{fb}^{-1}$. A more sophisticated analysis using neural net selections, full simulation, and the same integrated luminosity at $\sqrt{s}=800 \mathrm{GeV}$ finds a total error of $6 \%$ on the coupling [96]. More details on this process can be found in Chapter 6, Section 3.1.

\subsubsection{Higgs self-coupling}

To delineate the Higgs sector fully, it is essential to measure the shape of the Higgs potential. The cross section for double Higgs production (e.g., Zhh) is related to the triple Higgs coupling $g_{h h h}$, which in turn is related to the spontaneous symmetry breaking shape of the Higgs potential. The Higgs mass, $m_{h}^{2}=4 \lambda v^{2}$, also measures the potential shape parameter $\lambda$, so independent determinations through $h h$ production give a cross-check. In the MSSM, a variety of double Higgs production processes would be required to determine $g_{h^{0} h^{0} h^{0}}, g_{A^{0} h^{0} h^{0}}$, etc. [73].

These cross sections are low, and high integrated luminosity is needed, bolstered by polarization and neural net selections. Experimental studies [97,98] indicate that for a SM-like Higgs boson with $m_{h}=120 \mathrm{GeV}$ at $\sqrt{s}=500 \mathrm{GeV}$ and $1000 \mathrm{fb}^{-1}$, a precision of $\delta g_{h h h} / g_{h h h}=23 \%$ is possible. Regions of accessibility in MSSM parameters for MSSM Higgs self-couplings have also been determined [99,100].

The cross section for SM triple Higgs production is very low, $\sigma(Z h h)<10^{-3} \mathrm{fb}$, so measurement of the quartic coupling $g_{h h h h}$ is hopeless with currently envisioned luminosities.

\subsubsection{Implications for the MSSM Higgs sector}

The discussion of light Higgs coupling determinations has been based on the assumption that the actual Higgs couplings to fermions, vector bosons and scalars are close 
to the corresponding Standard Model expectations. In Section 7.1, it was argued that such an expectation is rather generic, and applies to the decoupling limit of models of Higgs physics beyond the Standard Model. In particular, the decoupling limit of the MSSM Higgs sector sets in rather rapidly once $m_{A^{0}} \gtrsim 150 \mathrm{GeV}$ [see Section 5.1]. Since $m_{h^{0}} \lesssim 135 \mathrm{GeV}$ in the MSSM [Eq. (3.8)], the precision study of $h^{0}$ using the techniques discussed above can distinguish between $h^{0}$ and $h_{\mathrm{SM}}$ with a significance that depends on how close the model is to the decoupling limit. Said another way, the detection of deviations in the Higgs couplings from their Standard Model predictions would yield evidence for the existence of the non-minimal Higgs sector, and in the context of the MSSM would provide constraints on the value of $m_{A^{0}}$ (with some dependence on $\tan \beta$ and other MSSM parameters that enter in the Higgs radiative corrections).

In [101], the potential impact of precision Higgs measurements at the LC on distinguishing $h^{0}$ from $h_{\mathrm{SM}}$ was examined. The fractional deviation of the $h^{0}$ branching ratios into a given final state from the corresponding result for $h_{\mathrm{SM}}$ (assuming the same Higgs mass in both cases) is defined as:

$$
\delta \mathrm{BR}=\frac{\mathrm{BR}_{\mathrm{MSSM}}-\mathrm{BR}_{\mathrm{SM}}}{\mathrm{BR}_{\mathrm{SM}}} .
$$

For the MSSM Higgs boson decay, both $m_{h^{0}}$ and the corresponding branching ratios were computed including the radiative corrections due to the virtual exchange of Standard Model and supersymmetric particles, as described in Section 5.2. Thus, the $h^{0}$ branching ratios depend on $m_{A^{0}}$ and $\tan \beta$ (which fix the tree-level MSSM Higgs sector properties) and a variety of MSSM parameters that govern the loop corrections. Four scenarios were considered: the minimal and maximal top-squark mixing cases [see Eq. (3.8) and surrounding text], and two additional cases with large $|\mu|=\left|A_{t}\right|$ (for $\mu A_{t}<0$ and two possible sign choices of $\mu$ ), where $\mu$ and $A_{t}$ control the top-squark mixing. In the latter two scenarios, significant renormalization of the CP-even Higgs mixing angle $\alpha$ and $\Delta_{b}$ [see Eq. (3.9)] can arise.

In Fig. 3.22, contours of $\delta \mathrm{BR}$ are plotted for three $h^{0}$ decay modes: $b \bar{b}, W W^{*}$ and $g g$. The contours shown correspond roughly to the $1 \sigma$ and $2 \sigma$ measurements claimed by [91], rescaled for the LC at $\sqrt{s}=500 \mathrm{GeV}$ (see also the $b \bar{b}$ and $W W^{*}$ branching ratio precisions given in Table 3.1). In the minimal and maximal scenarios, the dependence on $m_{A^{0}}$ is nearly independent of $\tan \beta$, and demonstrates that one can achieve sensitivity to values of $m_{A^{0}}$ that lie significantly beyond $\sqrt{s} / 2$ where direct production at the $\mathrm{LC}$ via $e^{+} e^{-} \rightarrow H^{0} A^{0}$ is kinematically forbidden. However, the cases with large $|\mu|=\left|A_{t}\right|$ exhibit the possibility of "premature" decoupling, that is, relatively low values of $m_{A^{0}}$ (at a particular large value of $\tan \beta$ ) at which the properties of $h^{0}$ and $h_{\mathrm{SM}}$ cannot be distinguished by the decay modes considered above. ${ }^{7}$ Thus, a measured deviation of Higgs branching ratios that distinguishes $h^{0}$

\footnotetext{
${ }^{7}$ The premature decoupling is a consequence of the renormalization of the mixing angle $\alpha$ which
} 
Higgs Bosons at the Linear Collider

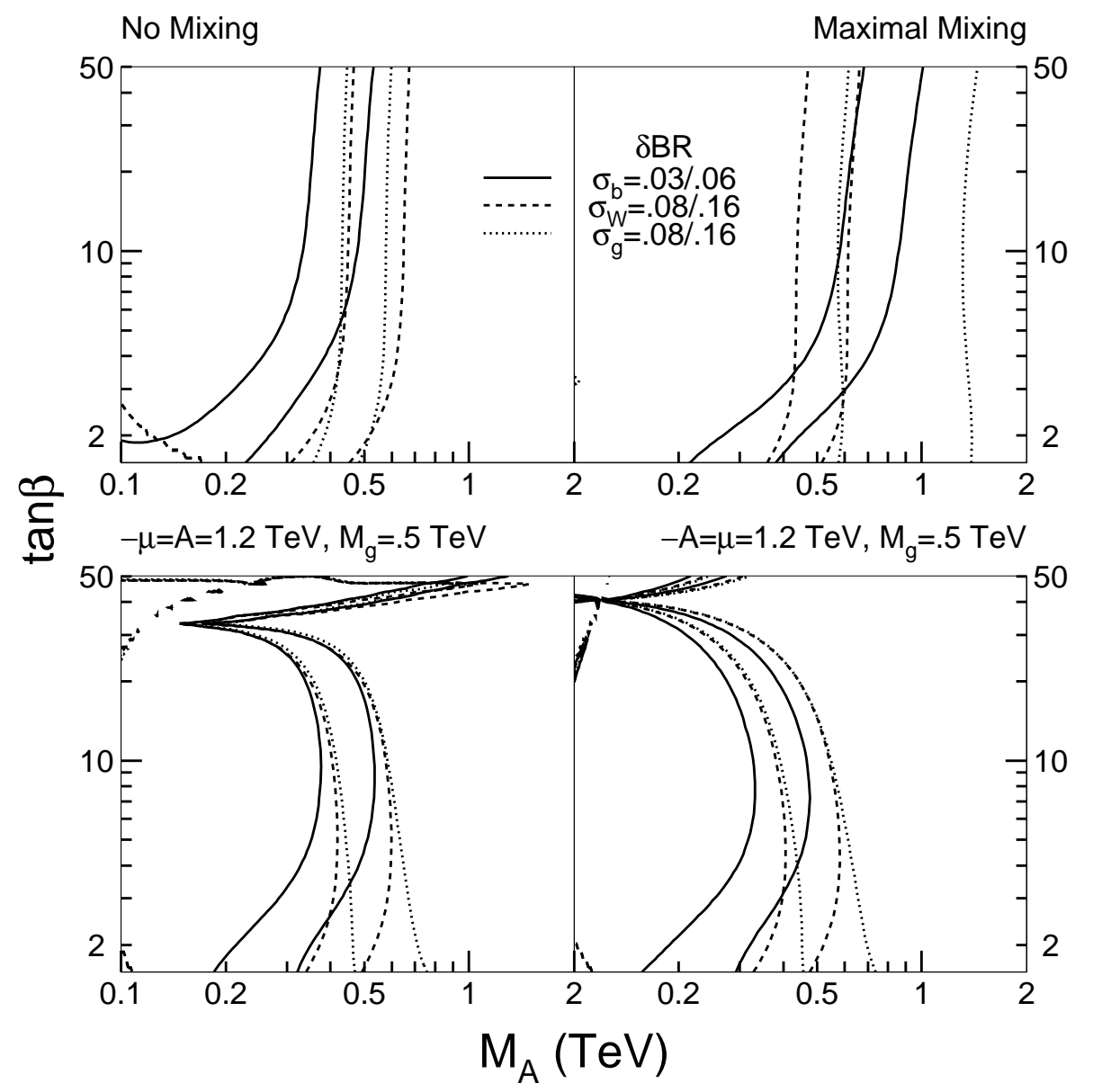

Figure 3.22: Contours of $\delta \mathrm{BR}(b \bar{b})=3$ and $6 \%$ (solid), $\delta \mathrm{BR}\left(W W^{*}\right)=8$ and $16 \%$ (dashed) and $\delta \operatorname{BR}(g g)=8$ and $16 \%$ (dotted) [BR deviations defined in Eq. (3.13)] in the no (i.e. minimal) mixing scenario (top left), the maximal mixing scenario (top right), and the large $\mu$ and $A_{t}$ scenario with $\mu=-A_{t}=1.2 \mathrm{TeV}$ (bottom left) and $\mu=-A_{t}=-1.2 \mathrm{TeV}$ (bottom right). Taken from [101].

from $h_{\mathrm{SM}}$ can place significant constraints on the heavier non-minimal Higgs states, although the resulting constraints can depend in a nontrivial way on the value of the MSSM parameters that control the Higgs radiative corrections.

\subsection{Coupling determinations - intermediate mass Higgs bosons}

For $m_{h}<2 m_{W}$, the measurement of branching ratios is extremely rich, yielding couplings to both many of the fermions and bosons. For larger masses, decays to $f \bar{f}$ just happens to yield $\cos (\beta-\alpha)=0$, in which case the $h^{0}$ couplings reduce to those of $h_{\mathrm{SM}}$ as shown in Section 5.1. 
become rarer until the threshold for decays into top is crossed. In this intermediate mass range, a LC can measure the $W$ and $Z$ couplings more precisely than the LHC both through Higgs production rates and via branching ratios for decays into these bosons. Whether the observed Higgs boson fully generates the $W$ and $Z$ mass can then be checked.

Precision electroweak measurements in the framework of the Standard Model indirectly predict $[8,9] m_{h_{\mathrm{SM}}} \lesssim 205-230 \mathrm{GeV}$ at $95 \% \mathrm{CL}$, and a Higgs observed with mass much greater than this would imply new physics. At this point, measurements from a Giga-Z dataset would be particularly useful to probe this new sector.

\subsubsection{Cross sections}

Techniques described earlier $[78,85]$ for cross section measurements of both the Higgsstrahlung and $W$-fusion processes, with subsequent Higgs decays into $b \bar{b}$, can still be used for the lower portion of the intermediate mass range, i.e., $m_{h} \sim 160 \mathrm{GeV}$. Even in this intermediate mass range, it is beneficial to run at the peak of the cross section at roughly $m_{h}+m_{Z}+50 \mathrm{GeV}$. The typical precisions that can be obtained are $\Delta \sigma\left(Z h_{\mathrm{SM}}\right) / \sigma\left(Z h_{\mathrm{SM}}\right) \simeq 5 \%$ and $\Delta \sigma\left(\nu \bar{\nu} h_{\mathrm{SM}}\right) / \sigma\left(\nu \bar{\nu} h_{\mathrm{SM}}\right) \simeq 17 \%$ for $m_{h_{\mathrm{SM}}}=160 \mathrm{GeV}$, at $\sqrt{s}=350 \mathrm{GeV}$ with $500 \mathrm{fb}^{-1}$.

For heavier Higgs bosons in this mass range, cross sections for both Higgsstrahlung and $W$-fusion will need to be extracted from using the decay $h \rightarrow W W^{*}$, for example, as described in [90]. Couplings determined from $t \bar{t} h$ and $Z h h$ production would clearly need higher $\sqrt{s}$.

\subsubsection{Branching ratios}

Using Higgsstrahlung events at an optimal $\sqrt{s}$, the statistical error on $\mathrm{BR}\left(h_{\mathrm{SM}} \rightarrow\right.$ $b \bar{b})$ is still only $6.5 \%$ at $m_{h_{\mathrm{SM}}}=160 \mathrm{GeV}$ [91]. At $\sqrt{s}=500 \mathrm{GeV}$, with leptonic decays of the $Z$ only, the statistical error on this branching ratio reaches $25 \%$ at $m_{h_{\mathrm{SM}}} \simeq 165 \mathrm{GeV}$ with $250 \mathrm{fb}^{-1}$ and remains below $30 \%$ for $m_{h_{\mathrm{SM}}}<200 \mathrm{GeV}$ with $2000 \mathrm{fb}^{-1}$ [82]. However, in addition to the leptonic decays of the $Z$, hadronic decays can also be used to tag the associated $Z$. Extrapolating from full LCD detector simulations, it is conservatively estimated that including the hadronic decays of the $Z$ results in an increase in signal statistics above background by a factor of four. With these assumptions and $500 \mathrm{fb}^{-1}$, again with the optimal $\sqrt{s} \simeq 350 \mathrm{GeV}$, the error on the $b \bar{b}$ branching ratio can then be estimated to reach $25 \%$ at $m_{h_{\mathrm{SM}}} \simeq 200 \mathrm{GeV}$. Measurement of branching ratios to $c \bar{c}, \tau^{+} \tau^{-}, g g$, and $\gamma \gamma$ does not seem feasible in this mass range.

Branching ratios into vector bosons can be measured with good precision in the intermediate mass range. For $m_{h_{\mathrm{SM}}}=160 \mathrm{GeV}$ and $500 \mathrm{fb}^{-1}$, a predicted excellent 
Higgs Bosons at the Linear Collider

precision of $2.1 \%$ on $\mathrm{BR}\left(h_{\mathrm{SM}} \rightarrow W W\right)$, has been reported [90], with extrapolated estimated precision of better than $7 \%$ over the mass range of 150 to $200 \mathrm{GeV}$ [82].

To measure $\operatorname{BR}(h \rightarrow Z Z)$, it will be necessary to distinguish hadronic $Z$ decays from hadronic $W$ decays. This serves as an important benchmark for electromagnetic and hadronic calorimetry. With $500 \mathrm{fb}^{-1}$, and assuming that this separation allows one to identify one of the two $Z$ 's in the Higgs decays (through leptons or $b \bar{b}$ ) $40 \%$ of the time, the statistical uncertainty of this branching ratio would be approximately $8 \%$ for $m_{h_{\mathrm{SM}}} \simeq 210 \mathrm{GeV}$ [82], degrading to $17 \%$ for $m_{h_{\mathrm{SM}}}=160 \mathrm{GeV}$ [76] where the branching ratio into $Z$ 's is still small.

\subsection{Coupling determinations - heavy Higgs bosons}

If the Higgs boson is heavy, i.e., $m_{h}>2 m_{t}$, and if this Higgs boson possesses couplings close to those expected in the SM, then consistency with the precision electroweak data (which implies $m_{h_{\mathrm{SM}}} \lesssim 230 \mathrm{GeV}$ at $95 \% \mathrm{CL}$ ) would require the existence of new physics beyond the SM. A high statistics measurements at the $Z$ peak could be useful to elucidate the non-SM effects. In addition, with high center of mass energy and large integrated luminosity, an experiment at the LC could directly observe heavy Higgs decay and make measurements of the Higgs couplings. These measurements could reveal departures from the SM Higgs properties and provide indirect evidence for the nature of the new physics, which would modify the SM Higgs couplings through loop effects.

\subsubsection{Cross sections}

As a specific case, for $m_{h}=500 \mathrm{GeV}$, a SM-like Higgs boson would have a width of $70 \mathrm{GeV}$ and dominant decay modes into $W^{+} W^{-}$(55\%), $Z Z(25 \%)$, and $t \bar{t}(20 \%)$. The production cross section at $\sqrt{s}=800 \mathrm{GeV}$ for $Z h$ would be $6 \mathrm{fb}$, but Higgs production would be dominated by the $W$-fusion process, whose cross section would be $10 \mathrm{fb}$. With $1000 \mathrm{fb}^{-1}$, one would expect $400 Z h$ events where the $Z$ decays to electrons or muons. With reasonable selection and acceptance cuts, a measurement of $\sigma(Z h)$ to better than $7 \%$ should be feasible.

\subsubsection{Branching ratios}

The LHC will have great difficulty distinguishing $h \rightarrow t \bar{t}$ decays from the huge QCD $t \bar{t}$ backgrounds. On the other hand, this mode should be observable at a LC. In the SM, the important coupling $g_{t t h_{S M}}^{2} \simeq 0.5$ can be compared to $g_{b b h_{S M}}^{2} \simeq 4 \times$ $10^{-4}$. If the Higgs boson is heavier than $350 \mathrm{GeV}$, it will be possible obtain a good determination of the top-Higgs Yukawa coupling. Full simulations are needed for heavy Higgs decays into top, but with reasonable assumptions, one can expect a 
statistical error of $\delta \mathrm{BR} / \mathrm{BR} \simeq 14 \%$ with $500 \mathrm{fb}^{-1}$ [82]. Simulations using the TESLA detector of the $W^{+} W^{-} \rightarrow h_{\mathrm{SM}} \rightarrow t \bar{t}$ process with $1000 \mathrm{fb}^{-1}$ and 6 -jet final states show impressive signal significance for $\sqrt{s}=1000 \mathrm{GeV}$ and reasonably good significance at $\sqrt{s}=800 \mathrm{GeV}$ [102]. These studies find that a relative error of better than $10 \%$ in the top quark Yukawa coupling measurement can be achieved for Higgs masses in the $350-500 \mathrm{GeV}$ and $350-650 \mathrm{GeV}$ ranges at $\sqrt{s}=800 \mathrm{GeV}$ and $1000 \mathrm{GeV}$, respectively.

Assuming that detector performance allows separation of hadronic $W$ and $Z$ decays, and using production through $W$-fusion, the $W W$ and $Z Z$ coupling of the Higgs boson can be studied by using methods similar to those for $t \bar{t}$. This gives the estimates on $\mathrm{BR}\left(h_{\mathrm{SM}} \rightarrow W^{+} W^{-}\right)$and $\mathrm{BR}\left(h_{\mathrm{SM}} \rightarrow Z Z\right)$ shown in Table 3.2.

\subsection{Summary of couplings}

The relative measurement errors for a SM Higgs at various masses are summarized in Table 3.2. As much as possible, the entries have been collected from simulations with the $\mathrm{L}$ detector described in Chapter 15. For uniformity, the entries have been scaled to $500 \mathrm{fb}^{-1}$, except where otherwise noted. The significant measurements of many branching ratios and couplings demonstrate the strength of the LC Higgs program.

Just as the computer program ZFITTER [103] is used with $Z$ mass, widths, asymme-

\begin{tabular}{|c|c|c|c|c|c|}
\hline$\overline{\Delta m_{h}}$ & \multicolumn{5}{|c|}{$\begin{array}{l}\simeq 140 \mathrm{MeV} \text { (recoil against leptons from } Z \text { ) } \\
\simeq 50 \mathrm{MeV} \text { (direct reconstruction) }\end{array}$} \\
\hline$m_{h}(\mathrm{GeV})$ & 120 & 140 & 160 & 200 & $400-500$ \\
\hline$\sqrt{s}(\mathrm{GeV})$ & \multicolumn{4}{|c|}{500} & 800 \\
\hline$\overline{\Delta \Delta \sigma(Z h) / \sigma(Z h)}$ & $\overline{6.5 \%}$ & $6.5 \%$ & $6 \%$ & $\overline{7 \%}$ & $10 \%$ \\
\hline$\Delta \sigma(\nu \bar{\nu} h) \mathrm{BR}(b \bar{b}) / \sigma \mathrm{BR}$ & $3.5 \%$ & $6 \%$ & $17 \%$ & - & - \\
\hline \multicolumn{6}{|l|}{ 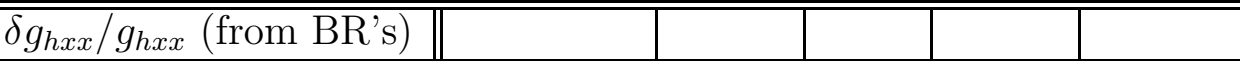 } \\
\hline$t \bar{t}$ & $\overline{77-20 \% \dagger}$ & - & - & - & $10 \%$ \\
\hline$b \bar{b}$ & $1.5 \%$ & $2 \%$ & $3.5 \%$ & $12.5 \%$ & - \\
\hline$c \bar{c}$ & $20 \%$ & $22.5 \%$ & - & - & - \\
\hline$\tau^{+} \tau^{-}$ & $4 \%$ & $5 \%$ & - & - & - \\
\hline$W W^{(*)}$ & $4.5 \%$ & $2 \%$ & $1.5 \%$ & $3.5 \%$ & $8.5 \%$ \\
\hline$Z Z^{(*)}$ & - & - & $8.5 \%$ & $4 \%$ & $10 \%$ \\
\hline$g g$ & $10 \%$ & $12.5 \%$ & - & - & - \\
\hline$\gamma \gamma$ & $7 \%$ & $10 \%$ & - & - & - \\
\hline$\frac{g_{h h h}}{2}$ & $23 \% \S$ & - & - & - & - \\
\hline
\end{tabular}

Table 3.2: Summary of measurement precisions for the properties of a SM-like Higgs boson, $h$, and couplings for a range of Higgs boson masses for $500 \mathrm{fb}^{-1}$, unless otherwise indicated. $\dagger$ radiative $t \bar{t} h$ production, $1000 \mathrm{fb}^{-1}, \sqrt{s}=800-1000 \mathrm{GeV} ; \S 1000 \mathrm{fb}^{-1}$. 
Higgs Bosons at the Linear Collider

tries and branching ratios to make global fits for $Z$ couplings, a program HFITTER [104] is now available that performs a global fit taking into account correlations between measurements of Higgs boson properties. Individual couplings of the Higgs boson can then be extracted optimally, for example through the correct combination of cross section and branching ratio measurements for such couplings as $g_{h W W}$ and $g_{h Z Z}$. Such precision fits can be used to probe for indirect evidence of higher-mass states.

\subsection{Total width}

Determination that a Higgs boson total width is anomalously large would indicate new non-SM effects. For light Higgs bosons, the predicted SM width is too small to be measured directly, but a combination of branching ratios and coupling measurements allows the indirect and model-independent measurement of the total width through

$$
\Gamma_{t o t}=\Gamma(h \rightarrow X) / \operatorname{BR}(h \rightarrow X) .
$$

For $m_{h_{\mathrm{SM}}}<115 \mathrm{GeV}$, the total width measurement would very likely require a $\gamma \gamma$ collider, an $e^{+} e^{-}$LC, and input from the LHC [2]. However, limits from LEP2 indicate $m_{h_{\mathrm{SM}}} \gtrsim 115 \mathrm{GeV}$ and therefore a significant branching ratio to $W W^{*}$. This gives the attractive prospect of a model-independent measurement of the total width using LC measurements alone.

First, measurements of $\sigma(h \nu \nu) \cdot \mathrm{BR}(h \rightarrow b \bar{b})$ and $\mathrm{BR}(h \rightarrow b \bar{b})$, through recoil Higgsstrahlung measurements, give $\Gamma\left(h \rightarrow W W^{*}\right)$. Then, a similar independent measurement of $\mathrm{BR}\left(h \rightarrow W W^{*}\right)$ gives the total width, through the relation $\Gamma_{\text {tot }}=$ $\Gamma\left(h \rightarrow W W^{*}\right) / \operatorname{BR}\left(h \rightarrow W W^{*}\right)$. For example, from Table 3.2, even with as little as $200 \mathrm{fb}^{-1}, \Gamma_{\text {tot }}$ can be found to approximately $10 \%$ for $m_{h_{\mathrm{SM}}}=120 \mathrm{GeV}$, improving to a few percent for $m_{h_{\mathrm{SM}}}=150 \mathrm{GeV}$. Even better precision can be attained with the introduction of some model assumptions in the value used for $\Gamma\left(h_{\mathrm{SM}} \rightarrow W W^{*}\right)$, e.g., assuming the $\mathrm{SU}(2)$ relation between $W$ and $Z$ couplings along with $\sigma_{\text {meas }}\left(Z h_{\mathrm{SM}}\right)$.

For $m_{h_{\mathrm{SM}}} \gtrsim 205 \mathrm{GeV}, \Gamma_{\text {tot }}\left(h_{\mathrm{SM}}\right)$ exceeds $2 \mathrm{GeV}$, and the physical width would be directly resolvable with typical LC detector resolutions. References $[2,105]$ track these variations of precision for indirect and direct measurements for different values of $m_{h_{\mathrm{SM}}}$ and inputs from different machines. The jet-jet mass resolution assumed in [2] has been verified by full simulations [79] in the L detector with $200 \mathrm{fb}^{-1}$ of data, resulting in estimated direct measurements of the total width whose accuracy reaches a minimum value of $6 \%$ in the mass range of $240-280 \mathrm{GeV}$. The indirect determination described above can also be pursued, and the combination would allow even better precision.

\subsection{Quantum numbers}

The spin, parity, and charge conjugation quantum numbers $J^{P C}$ of a Higgs boson, generically denoted by $\phi$ in this subsection, can potentially be determined in a modelindependent way. Useful ingredients include the following: 
- A Higgs boson produced in $\gamma \gamma$ collisions cannot have $J=1$ and must have positive $C$ [106].

- The behavior of the $Z \phi$ Higgsstrahlung cross section at threshold constrains the possible values of $J^{P C}$ of the state. If the spin of the $\phi$ is 2 or less, a cross section growing as $\beta$ indicates a $\mathrm{CP}$-even object, whereas a cross section growing as $\beta^{3}$ signals a CP-odd state [107], as shown in Fig. 3.23(a).
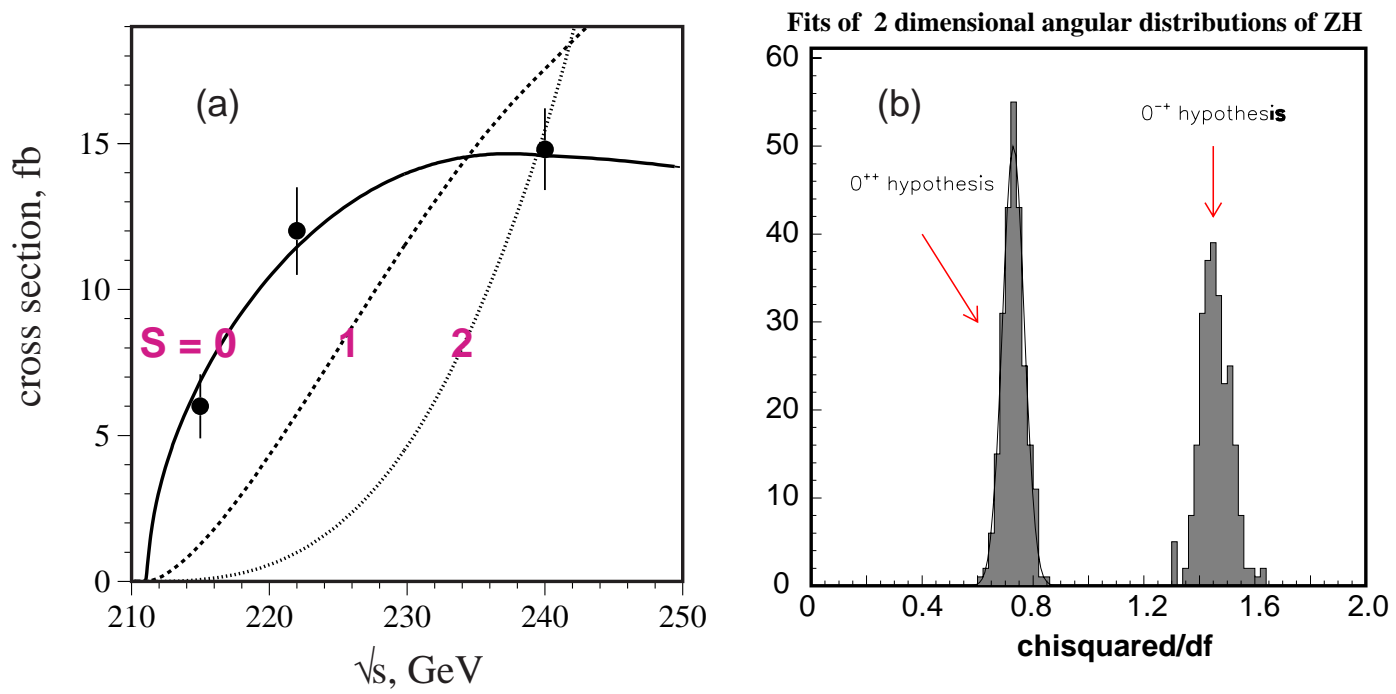

Figure 3.23: (a) Behavior of Higgsstrahlung threshold for various spin states along with typical measurement precisions on the cross section. (b) Fit to the double-differential angular distribution in $Z \phi$ events (see text) to distinguish CP-even and CP-odd states.

- The angular dependence of the $e^{+} e^{-} \rightarrow Z \phi$ cross section depends upon whether the $\phi$ is CP-even, CP-odd, or a mixture [107-110]. Following [110] we parameterize the $Z Z \phi$ vertex as

$$
\Gamma_{\mu \nu}\left(k_{1}, k_{2}\right)=a g_{\mu \nu}+b \frac{k_{1 \mu} k_{2 \nu}-g_{\mu \nu} k_{1} \cdot k_{2}}{m_{Z}^{2}}+\tilde{b} \frac{\epsilon_{\mu \nu \alpha \beta} k_{1}^{\alpha} k_{2}^{\beta}}{m_{Z}^{2}},
$$

where $k_{1}$ and $k_{2}$ are the momenta of the two $Z \mathrm{~s}$. The first term arises from a Standard-Model-like $Z Z \phi$ coupling, and the last two from effective interactions that could be induced by high-mass virtual particles. With this vertex the Higgsstrahlung cross section becomes

$$
\frac{d \sigma}{d \cos \theta_{Z}} \propto 1+\frac{p_{Z}^{2}}{m_{Z}^{2}} \sin ^{2} \theta_{Z}-4 \operatorname{Im}\left[\frac{\tilde{b}}{\tilde{a}}\right] \frac{v_{e} a_{e}}{v_{e}^{2}+a_{e}^{2}} \frac{p_{z} \sqrt{s}}{m_{Z}^{2}} \cos \theta_{Z}+\left|\frac{\tilde{b}}{\tilde{a}}\right|^{2} \frac{p_{z}^{2} s}{2 m_{Z}^{4}}\left(1+\cos ^{2} \theta_{Z}\right),
$$


where $\theta_{Z}, p_{Z}$, and $E_{Z}$ are the scattering angle, momentum, and energy of the final-state $Z$ boson; $v_{e}$ and $a_{e}$ are the vector and axial-vector couplings at the $e^{+} e^{-} Z$ vertex; and $\tilde{a} \equiv a-b E_{Z} \sqrt{s} / m_{Z}^{2}$. The term in Eq. (3.16) proportional to $\cos \theta_{Z}$ arises from interference between the CP-even and CP-odd couplings in Eq. (3.15). If the CP-odd coupling $\tilde{b}$ is large enough, it can be extracted from the forward-backward asymmetry. Even upper limits on this asymmetry would be interesting. Note that the CP-even component of a Higgs boson will typically couple at tree-level whereas the CP-odd component will only couple via one-loop diagrams (typically dominated by the $t$ quark loop). As a result the coupling strength $\tilde{b}$ is typically proportional to $m_{Z}^{2} / s$ times a loop suppression factor. Thus, an asymmetry measurement may be able to provide a crude determination of the $\tilde{b} / a$ term. If $\phi$ is a purely CP-odd state with one-loop coupling, the resulting $Z A^{0}$ cross section will simply be too small to provide a useful measurement of the asymmetry.

- The angular distribution of the fermions in the $Z \rightarrow f \bar{f}$ decays in $Z \phi$ production also reflects the $\mathrm{CP}$ nature of the state $\phi[108,109]$. For the decay $Z \rightarrow e^{+} e^{-}$or $\mu^{+} \mu^{-}$, the following angles can be defined: the angle between the initial $e^{-}$and the $Z$; the angle between the final state $e^{-}$or $\mu^{-}$and the direction of motion of the $Z$, in the rest frame of the $Z$; and the angle between the $Z$ production plane and $Z$ decay plane. Correlations between these angles can be exploited, e.g., a fit to the double-differential angular distribution of the first two of these angles results in a $14 \sigma$ separation between the $0^{++}\left(\mathrm{CP}\right.$-even, scalar) and the $0^{-+}$ (CP-odd, pseudoscalar) [82], assuming that the $Z \phi$ cross section is independent of the CP nature of $\phi$ (see Fig. 3.23(b)). Even more powerful are fits to the triple-differential angular distribution, where sufficient luminosity can uncover non-standard $Z Z \phi$ couplings. However, this technique again suffers from the difficulty described in the previous item; namely, the CP-odd part of the state $\phi$ is typically so weakly coupled to $Z Z$ that there is little sensitivity to the $\mathrm{CP}$-odd component if there is any significant CP-even component in $\phi$ ), or a very small cross section, if $\phi$ is almost purely CP-odd.

- If $\phi$ has significant branching ratios to either $\tau^{+} \tau^{-}$or $t \bar{t}$, the polarization of the decay fermions can be measured. This can provide a direct determination of the ratio $b_{f} / a_{f}$ in the $y_{f} \bar{f}\left(a_{f}+i b_{f} \gamma_{5}\right) f \phi(f=\tau$ or $t)$ Yukawa coupling structure of $\phi$ [111-113].

- The angular distributions in the $t \bar{t} \phi$ final state, which has adequate cross section for $\sqrt{s} \gtrsim 800 \mathrm{GeV}$ for modest values of $m_{\phi} \lesssim 200 \mathrm{GeV}$, assuming Yukawa coupling $y_{t} \bar{t}\left(a_{t}+i b_{t} \gamma_{5}\right) t \phi$ comparable to SM values, appear to provide an excellent means for determining the $\mathrm{CP}$ nature of $\phi$ by allowing one to probe the ratio $b_{t} / a_{t}[114,94]$. 
- It is likely that the $\mathrm{CP}$ properties of the $\phi$ can be well determined using photon polarization asymmetries in $\gamma \gamma \rightarrow \phi$ collisions $[115,116,113]$. This is discussed in Section 10.

- If the $\phi$ has substantial $Z Z$ coupling, then $e^{-} e^{-} \rightarrow Z Z e^{-} e^{-} \rightarrow \phi e^{-} e^{-}$can be used to probe its CP nature [117] via the energy distributions of the $\phi$ and the final electrons, which are much harder in the case of a CP-odd state than for a $\mathrm{CP}$-even state. Certain correlations are also useful probes of the CP properties of the $\phi$. However, if the CP-odd portion of $\phi$ couples at one-loop (as expected for a Higgs boson), there will be either little sensitivity to this component or little cross section.

\subsection{Precision studies of non-SM-like Higgs bosons}

We confine our remarks to a two-doublet Higgs model (either the MSSM Higgs sector or a more general $2 \mathrm{HDM}$ ). In the MSSM, we noted in Section 5.4 that for $m_{A^{0}} \lesssim \sqrt{s} / 2$, as long as one is not too close to threshold, it is possible to observe all Higgs scalars of the non-minimal Higgs sector. In particular, in parameter regions away from the decoupling limit, none of the CP-even Higgs scalars may resemble the SM Higgs boson. Precision studies of all the Higgs bosons will provide a detailed profile of the non-minimal Higgs sector. Once $m_{A^{0}} \gtrsim \sqrt{s} / 2$, only the $h^{0}$ will be visible at the LC. There may still be some possibilities for observing the heavier Higgs states produced singly, either in association with a $b \bar{b}$ pair at large $\tan \beta$ where the coupling to $b \bar{b}$ is enhanced, or by $s$-channel resonance production at a $\gamma \gamma$ collider.

Masses $m_{A^{0}}$ and $m_{H^{0}}$ in excess of $500 \mathrm{GeV}$ to $1 \mathrm{TeV}$ are certainly possible. In such cases, very substantial energy for the LC will be required to observe these states directly, either in association with $b \bar{b}$ (at large $\tan \beta$ ) or via $H^{0} A^{0}$ production. Measuring the former will provide a crucial determination of the $b \bar{b}$ couplings, which in the given model context will provide a determination of $\tan \beta$, with accuracy determined by the production rates. Moreover, if the $H^{0}$ and $A^{0}$ can be produced at a high rate (by whatever process), a detailed study of their branching ratios has the potential for providing very vital information regarding model parameters. In the supersymmetric context, the heavy $H^{0}, A^{0}$ and $H^{ \pm}$would generally decay to various pairs of supersymmetric particles as well as to $b$ 's and $t$ 's. A study of the relative branching ratios would provide powerful determinations of $\tan \beta$ and many of the soft-SUSY-breaking parameters [118-120].

\section{The Giga-Z option-implications for the Higgs sector}

Measurements of the effective leptonic mixing angle and the $W$ boson mass to precisions of $\delta \sin ^{2} \theta_{w}^{\text {eff }} \simeq 10^{-5}$ and $\delta m_{W} \approx 6 \mathrm{MeV}$ at Giga-Z can be exploited in many 
Higgs Bosons at the Linear Collider

ways. The size of the Giga-Z 90\% CL ellipses is illustrated in Fig. 3.15. Potential implications include the following.

- Within the SM context, the Higgs boson mass can be determined indirectly to a precision of about $7 \%$. Deviation between the directly observed value and the value implied by Giga- $Z$ data would require new physics beyond the SM.

- In the MSSM context it will be possible to obtain information about new high mass scales beyond the direct reach of the collider. This would be of particular importance if the heavier scalar top quark, $\tilde{t}_{2}$, and the heavy Higgs bosons $A^{0}, H^{0}$ and $H^{ \pm}$were beyond the kinematical reach of the LC and background problems precluded their observation at the LHC.

- In the context of a non-minimal Higgs sector, such as the general 2HDM extension of the minimal SM, constraints on the Higgs sector and/or new physics can be obtained. These would be particularly important in those cases where none of the Higgs bosons or new particles could be observed at the LC without higher $\sqrt{s}$ or at the LHC because of backgrounds.

\subsection{The MSSM context}

In the case of the MSSM, the relation between $m_{W}$ and $\sin ^{2} \theta_{w}^{\text {eff }}$ is affected by the parameters of the supersymmetric sector, especially the $\tilde{t}$ sector. At a LC, the mass of the light $\tilde{t}, m_{\tilde{t}_{1}}$, and the $\tilde{t}$ mixing angle, $\theta_{\tilde{t}}$, should be measurable very well if the process $e^{+} e^{-} \rightarrow \tilde{t}_{1} \bar{t}_{1}$ is accessible [121].

In Fig. 3.24 (from [26]), it is demonstrated how upper bounds on $m_{A^{0}}$ and $m_{\tilde{t}_{2}}$ can be derived from measurements of $m_{h^{0}}, m_{W}$ and $\sin ^{2} \theta_{w}^{\text {eff }}$, supplemented by precise determinations of $m_{\tilde{t}_{1}}$ and $\theta_{\tilde{t}}$. The analysis assumes a lower bound, $\tan \beta \geq 10$, which can be expected from measurements in the gaugino sector (see, e.g., [122]). The other parameters values are assumed to have the uncertainties as expected from LHC [123] and a LC [76].

For low $\tan \beta$ (where the prediction for $m_{h^{0}}$ depends sensitively on $\tan \beta$ ) the heavier $\tilde{t}$ mass, $m_{\tilde{t}_{2}}$, can be restricted to $760 \mathrm{GeV} \lesssim m_{\tilde{t}_{2}} \lesssim 930 \mathrm{GeV}$ from the $m_{h^{0}}$, $m_{W}$ and $\sin ^{2} \theta_{w}^{\text {eff }}$ precision measurements. The mass $m_{A^{0}}$ varies between $200 \mathrm{GeV}$ and $1600 \mathrm{GeV}$. If $\tan \beta \geq 10$ (where $m_{h^{0}}$ has only a mild dependence on $\tan \beta$ ), the allowed region for the $\tilde{t}_{2}$ turns out to be much smaller, $660 \mathrm{GeV} \lesssim m_{\tilde{t}_{2}} \lesssim 680 \mathrm{GeV}$, and the mass $m_{A^{0}}$ is restricted to $m_{A^{0}} \lesssim 800 \mathrm{GeV}$.

In deriving the bounds on the heavier $\tilde{t}$ mass, $m_{\tilde{t}_{2}}$, the constraints from $m_{h^{0}}$ and from $\sin ^{2} \theta_{w}^{\text {eff }}$ and $m_{W}$ play an important role. For the bounds on $m_{A^{0}}$, the main effect comes from $\sin ^{2} \theta_{w}^{\text {eff }}$. The assumed value of $\sin ^{2} \theta_{w}^{\text {eff }}=0.23140$ differs slightly from the corresponding value obtained in the SM limit. For this value the (logarithmic) dependence on $m_{A^{0}}$ is still large enough (see [124]) so that from the high precision 


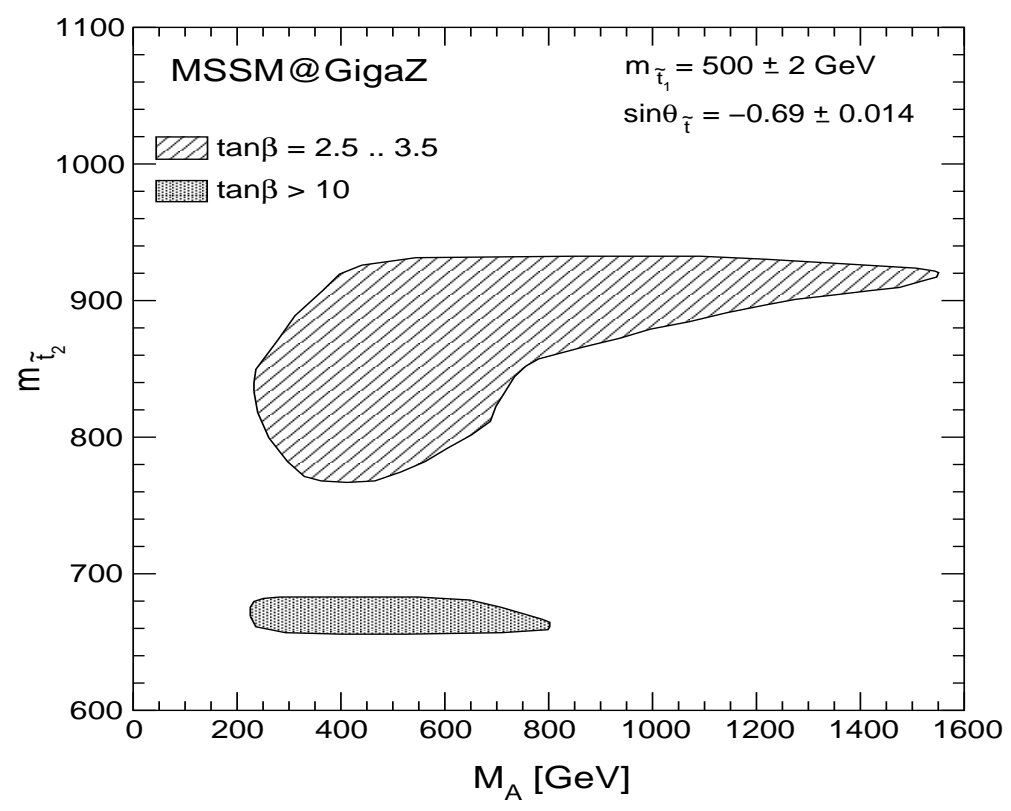

Figure 3.24: The region in the $m_{A^{0}}-m_{\tilde{t}_{2}}$ plane, allowed by $1 \sigma$ errors obtained from the Giga-Z measurements of $m_{W}$ and $\sin ^{2} \theta_{w}^{\text {eff }}: m_{W}=80.400 \pm 0.006 \mathrm{GeV}, \sin ^{2} \theta_{w}^{\text {eff }}=0.23140 \pm$ 0.00001 , and from the LC measurement of $m_{h^{0}}: m_{h^{0}}=115 \pm 0.05$ (exp.) \pm 0.5 (theo.) $\mathrm{GeV}$. $\tan \beta$ is assumed to be $\tan \beta=3 \pm 0.5$ or $\tan \beta>10$. The other parameters are given by $m_{\tilde{t}_{1}}=500 \pm 2 \mathrm{GeV}, \sin \theta_{\tilde{t}}=-0.69 \pm 0.014, A_{b}=A_{t} \pm 10 \%, m_{\tilde{g}}=500 \pm 10 \mathrm{GeV}$, $\mu=-200 \pm 1 \mathrm{GeV}$ and $M_{2}=400 \pm 2 \mathrm{GeV}$.

in $\sin ^{2} \theta_{w}^{\text {eff }}$ at Giga-Z an upper limit on $m_{A^{0}}$ can be set. For the error of $\sin ^{2} \theta_{w}^{\text {eff }}$ that could be obtained at an LC without the Giga-Z mode (which is at least ten times larger), no bound on $m_{A^{0}}$ could be inferred.

\subsection{Non-exotic extended Higgs sector context}

Building on the discussion of the general 2HDM given earlier, one can imagine many situations for which the very small Giga-Z 90\% CL ellipses illustrated in Fig. 3.15 would provide crucial (perhaps the only) constraints. For example, suppose the LHC observes a $1 \mathrm{TeV}$ Higgs boson with very SM-like properties and no other new physics below the few-TeV scale. We have seen that this is possible in the $2 \mathrm{HDM}$ scenarios consistent with current precision electroweak constraints. Suppose further that it is not immediately possible to increase $\sqrt{s}$ sufficiently so that $h^{0} A^{0}$ production is allowed (typically requiring $\sqrt{s}>1.5 \mathrm{TeV}$ in these models). Giga-Z measurements would provide strong guidance as to the probable masses of the non-SM-like Higgs bosons of any given non-minimal Higgs sector. However, it must be accepted that a particular Giga-Z result for $S, T$ might have other non-Higgs interpretations as well. 
Higgs Bosons at the Linear Collider

\section{The $\gamma \gamma$ collider option}

Higgs production in $\gamma \gamma$ collisions offers a unique capability to measure the twophoton width of the Higgs and to determine its CP composition through control of the photon polarization. A brief discussion of photon collider technology can be found in Chapter 13.

The $\gamma \gamma$ coupling of a SM-like Higgs boson $h_{\mathrm{SM}}$ of relatively light mass receives contributions from loops containing any particle whose mass arises in whole or part from the vacuum expectation value of the corresponding neutral Higgs field. A measurement of $\Gamma\left(h_{\mathrm{SM}} \rightarrow \gamma \gamma\right)$ provides the possibility of revealing the presence of arbitrarily heavy particles that acquire mass via the Higgs mechanism. ${ }^{8}$ However, since such masses are basically proportional to some coupling times $v$, if the coupling is perturbative the masses of these heavy particles are unlikely to be much larger than $0.5-1 \mathrm{TeV}$. Since $B\left(h_{\mathrm{SM}} \rightarrow X\right)$ is entirely determined by the spectrum of light particles, and is thus not affected by heavy states, $N\left(\gamma \gamma \rightarrow h_{\mathrm{SM}} \rightarrow X\right) \propto \Gamma\left(h_{\mathrm{SM}} \rightarrow \gamma \gamma\right) B\left(h_{\mathrm{SM}} \rightarrow X\right)$ will provide an extraordinary probe for such heavy states. Even if there are no new particles that acquire mass via the Higgs mechanism, a precision measurement of $N(\gamma \gamma \rightarrow \widehat{h} \rightarrow X)$ for specific final states $X\left(X=b \bar{b}, W W^{*}, \ldots\right)$ can allow one to distinguish between a $\widehat{h}$ that is part of a larger Higgs sector and the SM $h_{\mathrm{SM}}$. The deviations from the SM predictions typically exceed $5 \%$ if the other heavier Higgs bosons have masses below about $400 \mathrm{GeV}$.

The predicted rate for Higgs boson production followed by decay to final state $X$ can be found in [56]. This rate depends strongly on $d \mathcal{L}_{\gamma \gamma} / d y$, the differential $\gamma \gamma$ collider luminosity, where $y=m_{\widehat{h}} / \sqrt{s}$ and $\sqrt{s}$ is the ee collider center-of-mass energy. An important parameter to maximize peak luminosity is $\left\langle\lambda \lambda^{\prime}\right\rangle$, the average value of the product of the helicities of the two colliding photons after integration over their momentum fractions $z$ and $z^{\prime}$. Larger values of this parameter also suppress the dominant $J_{z}= \pm 2, \gamma \gamma \rightarrow b \bar{b} g$ background, which is proportional to $\left(1-\left\langle\lambda \lambda^{\prime}\right\rangle\right)$. The computation of $d \mathcal{L}_{\gamma \gamma} / d y$ was first considered in $[125,126]$. More realistic determinations [127] including beamstrahlung, secondary collisions between scattered electrons and photons from the laser beam, and other non-linear effects result in a substantial enhancement of the luminosity in the low- $E_{\gamma \gamma}$ region as shown in Fig. 3.25.

The choice of parameters that gives a peaked spectrum is well suited for light Higgs studies. Using the spectrum of Fig. 3.25 as an example, the di-jet invariant mass distributions for the Higgs signal and for the $b \bar{b}(g)$ background for $m_{h_{\mathrm{SM}}}=120 \mathrm{GeV}$ are shown in Fig. 3.26 [128]. After a year of operation, $\Gamma\left(h_{\mathrm{SM}} \rightarrow \gamma \gamma\right) B\left(h_{\mathrm{SM}} \rightarrow b \bar{b}\right)$ could be measured with an accuracy of about 5\%. (A much more optimistic error of close to $2 \%$ is quoted in [129] for $m_{h_{\mathrm{SM}}}=120 \mathrm{GeV}$, based upon a substantially higher peak luminosity.) The error for this measurement increases to about $20 \%$ for

\footnotetext{
${ }^{8}$ Loop contributions from particles that acquire a large mass from some other mechanism will decouple as $(\text { mass })^{-2}$ and $\Gamma\left(h_{\mathrm{SM}} \rightarrow \gamma \gamma\right)$ will not be sensitive to their presence.
} 
$m_{h_{\mathrm{SM}}}=160 \mathrm{GeV}$, primarily due to the decrease of the Higgs di-jet branching fraction by a factor of 18 .

In many scenarios, it is possible that by combining this result with other types of precision measurements for the SM-like Higgs boson, small deviations can be observed indicating the possible presence of heavier Higgs bosons. For a 2HDM (either the MSSM or a two-Higgs-doublet model with partial decoupling), if $m_{H^{0}} \sim m_{A^{0}}>\sqrt{s} / 2$ then $e^{+} e^{-} \rightarrow H^{0} A^{0}$ is not possible and $\gamma \gamma \rightarrow H^{0}, A^{0}$ may be the only option allowing their discovery (other than implementing higher $\sqrt{s}$ ). The alternatives of $b \bar{b} H$ and $b \bar{b} A$ production will only allow $H$ and $A$ detection if $\tan \beta$ is large [71]. A LC for which the maximum energy is $\sqrt{s}=630 \mathrm{GeV}$ can potentially probe Higgs masses as high as $500 \mathrm{GeV}$. If $m_{H^{0}}$ and $m_{A^{0}}$ are known to within roughly $50 \mathrm{GeV}$ on the basis of precision $h^{0}$ data, then there is an excellent chance of detecting them by scanning, i.e. stepping in $\sqrt{s}$, using a peaked $\gamma \gamma$ spectrum $[57,128]$. If no constraints have been placed on the $H^{0}, A^{0}$ masses (other than $m_{A^{0}} \sim m_{H^{0}}>\sqrt{s} / 2$ ), it is best to employ a broad $\gamma \gamma$ spectrum, which would yield a visible signal for $H^{0}, A^{0}$ production for only some parameter choices of $m_{A^{0}}$ and $\tan \beta$ [128].

In the non-decoupling $2 \mathrm{HDM}$ model with a light decoupled $\widehat{h}$ and all other Higgs bosons heavier than $\sqrt{s}, \gamma \gamma \rightarrow \widehat{h} \rightarrow b \bar{b}$ might allow detection of the $\widehat{h}$ for some of the $\tan \beta$ values in the wedge where the $b \bar{b} \widehat{h}$ and $t \bar{t} \widehat{h}$ production processes both yield fewer than 20 events for an integrated luminosity of $1000 \mathrm{fb}^{-1}$ [128].

Once one or several Higgs bosons have been detected, precision studies can be performed including: determination of $\mathrm{CP}$ properties; a detailed scan to separate the $H^{0}$ and $A^{0}$ in the decoupling limit of a $2 \mathrm{HDM}$; and branching ratios measurements. The branching ratios to supersymmetric final states are especially important for determining the basic supersymmetry breaking parameters $[130,118,120,57]$.

The CP properties can be determined for any spin- 0 Higgs $\widehat{h}$ produced in $\gamma \gamma$ collisions. Since $\gamma \gamma \rightarrow \widehat{h}$ is of one-loop order, whether $\widehat{h}$ is CP-even, CP-odd or a mixture, the CP-even and CP-odd parts of $\widehat{h}$ have $\gamma \gamma$ couplings of similar size. However, the structure of the couplings is very different:

$$
\mathcal{A}_{\mathrm{CP}=+} \propto \vec{\epsilon}_{1} \cdot \vec{\epsilon}_{2}, \quad \mathcal{A}_{\mathrm{CP}=-} \propto\left(\vec{\epsilon}_{1} \times \vec{\epsilon}_{2}\right) \cdot \widehat{p}_{\mathrm{beam}} .
$$

By adjusting the orientation of the initial laser photon polarization vectors with respect to one another, it is possible to determine the relative amounts of CP-even and CP-odd content in the resonance $\widehat{h}[115]$. If $\widehat{h}$ is a mixture, one can use helicity asymmetries for this purpose $[115,113]$. However, if $\widehat{h}$ is either purely CP-even or purely CP-odd, then one must employ transverse linear polarizations [116,113]. Substantial luminosity with transverse polarization can be obtained, although the spectrum is not peaked, as shown in Fig. 3.25.

One measure of the CP nature of a Higgs is the asymmetry for parallel vs. per- 


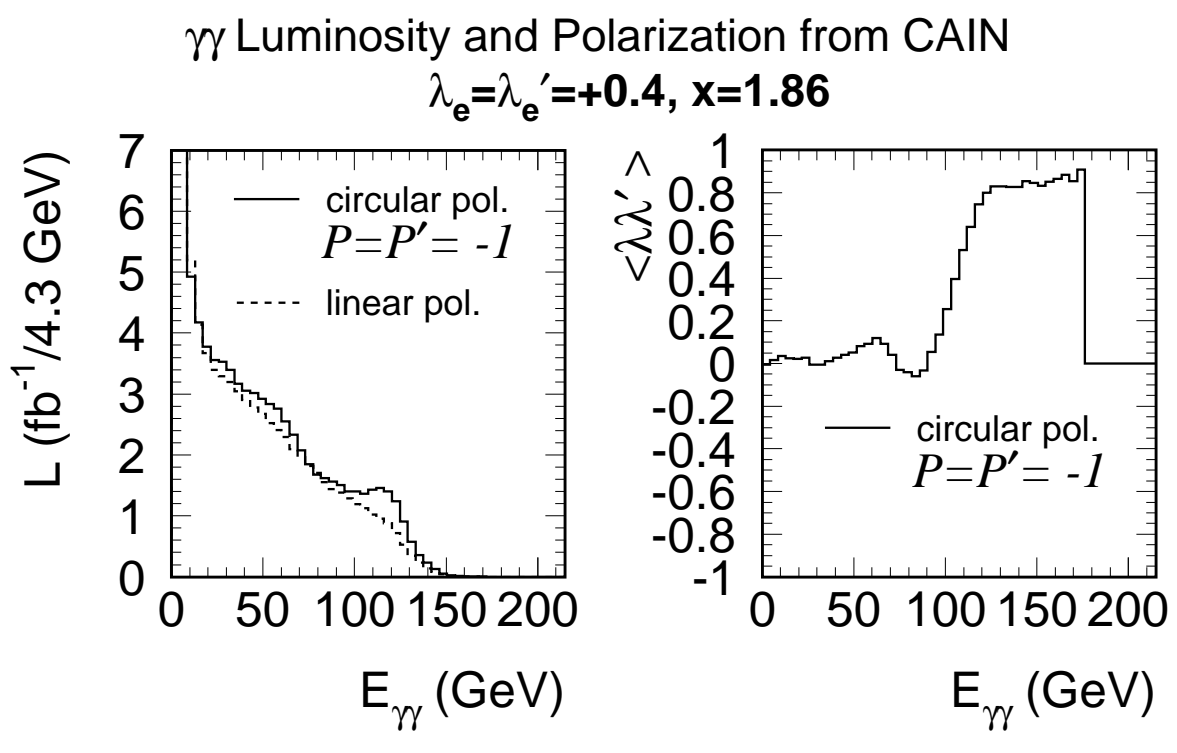

Figure 3.25: Left: CAIN [127] predictions for the $\gamma \gamma$ luminosity distribution for circularly polarized $\left(\lambda_{e}=\lambda_{e}^{\prime}=0.4, P=P^{\prime}=-1\right)$ and linearly polarized photons assuming $10^{7} \mathrm{sec} /$ year, $\sqrt{s}=206 \mathrm{GeV}, 80 \%$ electron beam polarization, and a 1.054 micron laser wavelength, after including beamstrahlung and other effects, from [128]. Right: The corresponding value of $\left\langle\lambda \lambda^{\prime}\right\rangle$, for circular polarization.

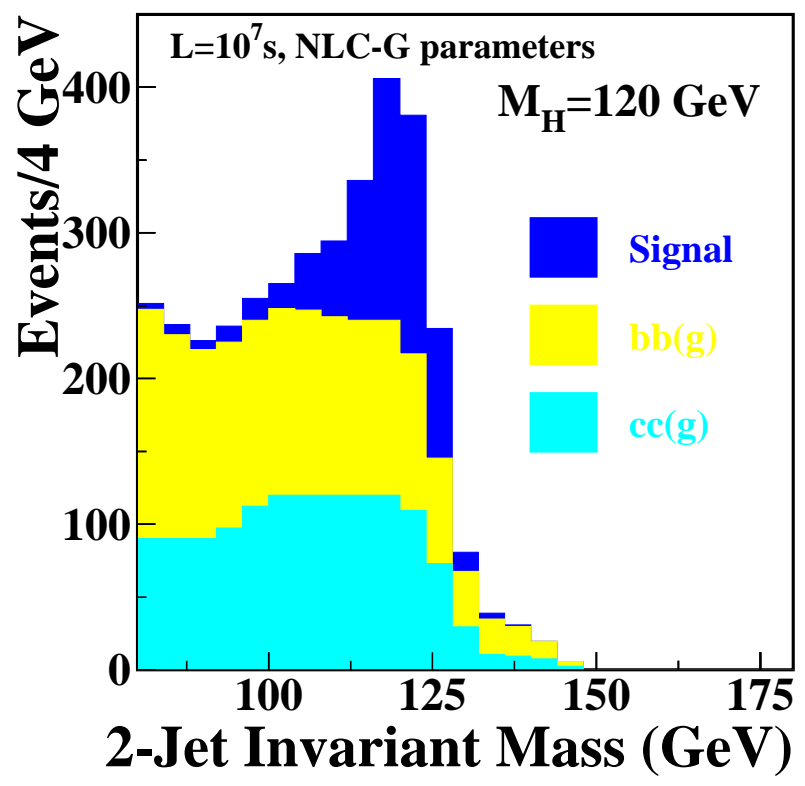

Figure 3.26: Higgs signal and heavy quark background in $\gamma \gamma \rightarrow h$ for a Higgs mass of $120 \mathrm{GeV}[128]$. 
pendicular orientation of the linear polarizations of the initial laser beams,

$$
\mathcal{A} \equiv \frac{N_{\|}-N_{\perp}}{N_{\|}+N_{\perp}},
$$

which is positive (negative) for a CP-even (odd) state. Since $100 \%$ linear polarization for the laser beams translates into only partial linear polarization for the colliding photons, both $N_{\|}$and $N_{\perp}$ will be non-zero for the signal. In addition, the heavy quark background contributes to both. The expected value of $\mathcal{A}$ must be carefully computed for a given model. For the SM Higgs with $m_{h_{\mathrm{SM}}}=120 \mathrm{GeV}$, it is estimated [128] that $\mathcal{A}$ can be measured with an accuracy of about $20 \%$ in one year of operation, assuming the linear polarization spectrum of Fig. 3.25, 60\% linear polarization of the colliding photons, and $S / B$ comparable to that shown in Fig. 3.26. This measurement would thus provide a moderately strong test of the $\mathrm{CP}=+$ nature of the $h_{\mathrm{SM}}$.

We end by noting that the $e^{-} \gamma$ and $e^{-} e^{-}$collider options are most relevant to exotic Higgs scenarios, as discussed in Section 11.

\section{Exotic Higgs sectors and other possibilities}

As we have seen, there are many scenarios and models in which the Higgs sector is more complicated than the one-Higgs-doublet of the minimal SM. Supersymmetry requires at least two Higgs doublets. Even in the absence of supersymmetry, a twodoublet Higgs sector allows for CP-violating phenomena. Singlets can also be added without altering the tree-level prediction of $\rho=1$. However, the possibility of Higgs representations with still higher weak (left handed, denoted $L$ ) isospin should not be ignored. The primary negative is that, for triplets and most higher representations, if the vacuum expectation value of the neutral Higgs field member of the representation is non-zero $\left(v_{L} \neq 0\right)$ then $\rho$ becomes infinitely renormalized and can no longer be computed [131]; instead it becomes a parameter that must be input as part of the renormalization program. Triplets have received the most attention, as they arise naturally in left-right symmetric extensions of the Standard Model gauge group [132]. (These and other models that utilize Higgs triplets are reviewed in [1].) In this section we will also briefly consider the Higgs-like pseudo-Nambu-Goldstone bosons that arise in generic technicolor theories.

\subsection{A triplet Higgs sector}

Including a single complex $\mathrm{SU}(2)$-triplet Higgs representation, in addition to some number of doublets and singlets, results in six additional physical Higgs eigenstates: $H^{--,++}, H^{-,+}, H^{0}$ and $H^{0 \prime}$. All but the doubly-charged states can mix with the doublet/singlet Higgs states under some circumstances. Even if $v_{L} \neq 0$ for the neutral field, $\rho=1$ can be preserved at tree-level if, in addition, a real triplet field is 
Higgs Bosons at the Linear Collider

also included $[133,134]$. However, $\rho$ will still be infinitely renormalized at one-loop unless $v_{L}=0$ is chosen. Left-right symmetric models capable of yielding the see-saw mechanism for neutrino mass generation require two triplet Higgs representations (an L-triplet and an R-triplet). The large see-saw mass entry, $M$, arises from a leptonnumber-violating Majorana coupling (which L-R symmetry requires to be present for both the L-triplet and R-triplet representations). Again, $\rho$ will not be altered if $v_{L}=0$, but $v_{R}$ must be non-zero and large for large $M$. We will briefly discuss the phenomenology of an L-triplet. That for the R-triplet of the L-R symmetric model is quite different. (See [1] for a review.)

The resulting Higgs sector phenomenology can be very complex. We focus on the most unequivocal signal for a triplet representation, namely observation of a doublycharged Higgs boson. Pair production, $Z^{*} \rightarrow H^{++} H^{--}$, has limited mass reach, $m_{H^{++}}<\sqrt{s} / 2$. Fortunately, single production is also generally possible. Most interestingly, the generically-allowed lepton-number-violating Majorana coupling leads to an $e^{-} e^{-} \rightarrow H^{--}$coupling and the possibility of $s$-channel resonance production of the $H^{--}$in $e^{-} e^{-}$collisions. Observation of this process would provide a dramatic confirmation of the presence of the Majorana coupling and, in many cases, the ability to actually measure its magnitude. For a discussion and review, see [135] (and also $[136,137]$ ). If the $H^{--}$is heavy and has significant $W^{-} W^{-}$coupling (requiring $v_{L} \neq 0$ ), then it can become broad and the $s$-channel resonant production cross section is suppressed (see, e.g., [138]) and might not be observable. Another production mechanism sensitive to the $e^{-} e^{-} \rightarrow H^{--}$coupling that might be useful in such an instance is $e^{-} e^{-} \rightarrow H^{--} Z$, and $e^{-} e^{-} \rightarrow H^{-} W^{-}$will be sensitive to the $e^{-} \nu_{e} \rightarrow H^{-}$ coupling that would be present for the $H^{-}$member of the triplet representation [139]. Using just the Majorana coupling, doubly-charged Higgs bosons can also be produced via $e^{-} \gamma \rightarrow e^{+} H^{--}$and $e^{+} e^{-} \rightarrow e^{+} e^{+} H^{--}[140]$ and the singly-charged members of the same representation can be produced in $e^{-} e^{-} \rightarrow H^{-} W^{-}$[139].

Despite loss of $\rho$ predictivity, it could be that non-zero $v_{L}$ is Nature's choice. In this case, the $e^{-} e^{-}$collider option again has some unique advantages. The neutral, singly-charged and doubly-charged Higgs bosons of the triplet representation can all be produced (via $Z Z$ fusion, $W^{-} Z$ fusion and $W^{-} W^{-}$fusion, respectively). For example, [141] studies $W^{-} W^{-} \rightarrow H^{--}$fusion.

\subsection{Pseudo Nambu Goldstone bosons}

In the context of technicolor and related theories, the lowest-mass states are typically a collection of pseudo-Nambu-Goldstone bosons, of which the lightest is very possibly a state $P^{0}$ which can have mass below $200 \mathrm{GeV}$ and couplings and other properties not unlike those of a light SM-like Higgs boson. Typically, its $W W, Z Z$ coupling is very small (arising via loops or anomalies), while its $b \bar{b}$ coupling can be larger. The phenomenology of such a $P^{0}$ was studied in [142]. The best modes for

detection of the $P^{0}$ at an LC are $e^{+} e^{-} \rightarrow \gamma P^{0} \rightarrow \gamma b \bar{b}$ and $\gamma \gamma \rightarrow P^{0} \rightarrow b \bar{b}$. Since the 
$P^{0}$ is likely to be discovered at the LHC in the $\gamma \gamma$ final state, we will know ahead of time of its existence, and precision measurements of its properties would be a primary goal of the LC.

\section{References}

[1] J. F. Gunion, H. E. Haber, G. Kane and S. Dawson, The Higgs Hunter's Guide (Perseus Publishing, Reading, MA, 2000).

[2] J. F. Gunion, L. Poggioli, R. Van Kooten, C. Kao, P. Rowson et al., "Higgs boson discovery and properties," in New Directions for High Energy Physics, Proceedings of the 1996 DPF/DPB Summer Study on High Energy Physics, Snowmass '96, edited by D. G. Cassel, L. T. Gennari and R. H. Siemann (Stanford Linear Accelerator Center, Stanford, CA, 1997) pp. 541-587 [hep$\mathrm{ph} / 9703330]$.

[3] H. E. Haber, T. Han, F. S. Merritt, J. Womersley et al., "Weakly coupled Higgs bosons and precision electroweak physics," in New Directions for High Energy Physics, Proceedings of the 1996 DPF/DPB Summer Study on High Energy Physics, Snowmass '96, edited by D. G. Cassel, L. T. Gennari and R. H. Siemann (Stanford Linear Accelerator Center, Stanford, CA, 1997) pp. 482-498 [hep$\mathrm{ph} / 9703391]$.

[4] H. Murayama and M. E. Peskin, Ann. Rev. Nucl. Part. Sci. 46, 533 (1996) [hep-ex/9606003].

[5] E. Accomando et al. [ECFA/DESY LC Physics Working Group], Phys. Rep. 299, 1 (1998) [hep-ph/9705442].

[6] For a recent review, see R. N. Mohapatra, "Supersymmetric grand unification: An update," in Supersymmetry, Supersymmetry and Supercolliders, edited by J. Bagger (World Scientific, 1998) [hep-ph/9911272].

[7] D. Abbaneo et al. [ALEPH, DELPHI, L3 and OPAL Collaborations, LEP Electroweak Working Group, and SLD Heavy Flavor and Electroweak Groups], CERN-EP-2000-016.

[8] J. Erler, hep-ph/0102143.

[9] G. Degrassi, hep-ph/0102137.

[10] R. Barate et al. [ALEPH Collaboration], Phys. Lett. B495, 1 (2000) [hepex/0011045]; P. Abreu et al. [DELPHI Collaboration], Phys. Lett. B499, 23 (2001) [hep-ex/0102036]; M. Acciarri et al. [L3 Collaboration], hep-ex/0012019; G. Abbiendi et al. [OPAL Collaboration], Phys. Lett. B499, 38 (2001) [hepex/0101014]. 
Higgs Bosons at the Linear Collider

[11] E. Farhi and L. Susskind, Phys. Rep. 74, 277 (1981); R.K. Kaul, Rev. Mod. Phys. 55, 449 (1983); K. Lane, in The Building Blocks of Creation-From Microfermis to Megaparsecs, Proceedings of the Theoretical Advanced Study Institute (TASI 93) in Elementary Particle Physics, Boulder, CO, 6 June - 2 July 1993, edited by S. Raby and T. Walker (World Scientific, Singapore, 1994) [hep-ph/9401324].

[12] M. E. Peskin and T. Takeuchi, Phys. Rev. Lett. 65, 964 (1990); Phys. Rev. D46, 381 (1992).

[13] M. E. Peskin and J. D. Wells, hep-ph/0101342.

[14] A. G. Cohen, in The Building Blocks of Creation-From Microfermis to Megaparsecs, Proceedings of the Theoretical Advanced Study Institute (TASI 93) in Elementary Particle Physics, Boulder, CO, 6 June - 2 July 1993, edited by S. Raby and T. Walker (World Scientific, Singapore, 1994).

[15] The most recent analysis of this type can be found in: T. Hambye and K. Riesselmann, Phys. Rev. D55, 7255 (1997) [hep-ph/9610272].

[16] The most recent analyses of this type can be found in: G. Altarelli and G. Isidori, Phys. Lett. B337, 141 (1994); J. A. Casas, J. R. Espinosa and M. Quirós, Phys. Lett. B342, 171 (1995) [hep-ph/9409458]; B382, 374 (1996) [hep-ph/9603227].

[17] R. Dashen and H. Neuberger, Phys. Rev. Lett. 50, 1897 (1983); M. Lüscher and P. Weisz, Phys. Lett. B212, 472 (1988), Nucl. Phys. B318, 705 (1989); U. M. Heller, M. Klomfass, H. Neuberger and P. Vranas, Nucl. Phys. B405, 555 (1993).

[18] K. Riesselmann, hep-ph/9711456.

[19] G. 't Hooft, in Recent Developments in Gauge Theories, Proceedings of the NATO Advanced Summer Institute, Cargese, 1979, edited by G. 't Hooft et al. (Plenum, New York, 1980) p. 135; E. Witten, Nucl. Phys. B188, 513 (1981); L. Susskind, Phys. Rep. 104, 181 (1984).

[20] H. P. Nilles, Phys. Rep. 110, 1 (1984).

[21] H. E. Haber and G. L. Kane, Phys. Rep. 117, 75 (1985); S. P. Martin, hep$\mathrm{ph} / 9709356$.

[22] A. Djouadi, J. Kalinowski and M. Spira, Comput. Phys. Commun. 108, 56 (1998) [hep-ph/9704448]. The HDECAY program is available at http://home.cern.ch/ mspira/proglist.html.

[23] A. Djouadi, J. Kalinowski and P. M. Zerwas, Z. Phys. C54, 155 (1992).

[24] S. Dittmaier, M. Kramer, Y. Liao, M. Spira and P. M. Zerwas, Phys. Lett. B441, 383 (1998) [hep-ph/9808433].

[25] A. Freitas, W. Hollik, W. Walter and G. Weiglein, Phys. Lett. B495, 338 (2000) [hep-ph/0007091]; A. Freitas, S. Heinemeyer, W. Hollik, W. Walter and 
G. Weiglein, Nucl. Phys. Proc. Suppl. 89, 82 (2000) [hep-ph/0007129]; hep$\mathrm{ph} / 0101260$.

[26] J. Erler, S. Heinemeyer, W. Hollik, G. Weiglein and P. M. Zerwas, Phys. Lett. B486, 125 (2000) [hep-ph/0005024].

[27] M. Carena, J. S. Conway, H. E. Haber and J. D. Hobbs et al., FERMILABCONF-00/270-T [hep-ph/0010338].

[28] M. Dittmar, "LHC luminosity requirements to observe H0 and measure its couplings," presented at Workshop on the Future of Higgs Physics, Fermilab, Chicago, May 3-5 2001.

[29] For a recent review, see V. A. Mitsou, ATLAS-CONF-2000-002.

[30] K. Lassila-Perini, ETH Dissertation thesis No. 12961 (1998).

[31] D. Zeppenfeld, R. Kinnunen, A. Nikitenko and E. Richter-Wąs, Phys. Rev. D62, 013009 (2000) [hep-ph/0002036].

[32] O. J. Eboli and D. Zeppenfeld, Phys. Lett. B495, 147 (2000) [hep-ph/0009158].

[33] K. Inoue, A. Kakuto, H. Komatsu, and S. Takeshita, Prog. Theor. Phys. 68, 927 (1982) [E: 70, 330 (1983)]; 71, 413 (1984); R. Flores and M. Sher, Ann. Phys. (NY) 148, 95 (1983).

[34] J. F. Gunion and H. E. Haber, Nucl. Phys. B272, 1 (1986); B278, 449 (1986) [E: B402, 567 (1993)].

[35] For a review of the two-Higgs-doublet model (both non-supersymmetric and supersymmetric), see Chapter 4 of [1].

[36] H. E. Haber and Y. Nir, Phys. Lett. B306, 327 (1993) [hep-ph/9302228]; H. E. Haber, in Physics From the Planck Scale to the Electroweak Scale, Proceedings of the US-Polish Workshop, Warsaw, Poland, September 21-24, 1994, edited by P. Nath, T. Taylor, and S. Pokorski (World Scientific, Singapore, 1995) pp. 49-63 [hep-ph/9501320].

[37] L. J. Hall and M. B. Wise, Nucl. Phys. B187, 397 (1981).

[38] H. E. Haber and R. Hempfling, Phys. Rev. Lett. 66, 1815 (1991); Y. Okada, M. Yamaguchi and T. Yanagida, Prog. Theor. Phys. 85, 1 (1991); J. Ellis, G. Ridolfi and F. Zwirner, Phys. Lett. B257, 83 (1991).

[39] S. Heinemeyer, W. Hollik and G. Weiglein, Phys. Rev. D58, 091701 (1998) [hep-ph/9803277]; Phys. Lett. B440, 296 (1998) [hep-ph/9807423]; Eur. Phys. J. C9, 343 (1999) [hep-ph/9812472]; M. Carena, H. E. Haber, S. Heinemeyer, W. Hollik, C. E. M. Wagner and G. Weiglein, Nucl. Phys. B580, 29 (2000) [hep-ph/0001002]; R.-J. Zhang, Phys. Lett. B447, 89 (1999) [hep-ph/9808299]; J. R. Espinosa and R.-J. Zhang, JHEP 0003, 026 (2000) [hep-ph/9912236]; Nucl. Phys. B586, 3 (2000) [hep-ph/0003246]. The computer programs FeynHiggs and FeynHiggsFast are described respectively in S. Heinemeyer, W. Hollik and G. Weiglein, Comput. Phys. Commun. 124, 76 (2000) [hep-ph/9812320]; CERN-TH-2000-055 [hep-ph/0002213]. 
Higgs Bosons at the Linear Collider

[40] J. Ellis, J. F. Gunion, H. E. Haber, L. Roszkowski and F. Zwirner, Phys. Rev. D39, 844 (1989). For a recent treatment and guide to the literature see: C. Panagiotakopoulos and A. Pilaftsis, Phys. Rev. D 63, 055003 (2001) [hep$\mathrm{ph} / 0008268]$. A detailed discussion of NMSSM phenomenology can be found in U. Ellwanger, M. Rausch de Traubenberg and C. A. Savoy, Nucl. Phys. B492, 21 (1997) [hep-ph/9611251].

[41] J. R. Espinosa and M. Quirós, Phys. Rev. Lett. 81, 516 (1998) [hep$\mathrm{ph} / 9804235]$.

[42] J. A. Coarasa, R. A. Jiménez and J. Solà, Phys. Lett. B389, 312 (1996) [hepph/9511402]; R. A. Jiménez and J. Solà, Phys. Lett. B389, 53 (1996) [hepph/9511292]; A. Bartl et al., Phys. Lett. B378, 167 (1996) [hep-ph/9511385].

[43] L. Hall, R. Rattazzi and U. Sarid, Phys. Rev. D50, 7048 (1994) [hepph/9306309]; R. Hempfling, Phys. Rev. D49, 6168 (1994).

[44] M. Carena, M. Olechowski, S. Pokorski and C. E. M. Wagner, Nucl. Phys. B426, 269 (1994) [hep-ph/9402253].

[45] D. Pierce, J. Bagger, K. Matchev, and R. Zhang, Nucl. Phys. B491, 3 (1997) [hep-ph/9606211].

[46] S. Heinemeyer, W. Hollik and G. Weiglein, Eur. Phys. J. C16, 139 (2000) [hep-ph/0003022].

[47] M. Carena, D. Garcia, U. Nierste and C. E. M. Wagner, Nucl. Phys. B577, 88 (2000) [hep-ph/9912516].

[48] H. Eberl, K. Hidaka, S. Kraml, W. Majerotto and Y. Yamada, Phys. Rev. D62, 055006 (2000) [hep-ph/9912463].

[49] M. Carena, S. Mrenna and C.E.M. Wagner, Phys. Rev. D60, 075010 (1999) [hep-ph/9808312]; Phys. Rev. D62, 055008 (2000) [hep-ph/9907422].

[50] H. Baer and J. D. Wells, Phys. Rev. D57, 4446 (1998) [hep-ph/9710368]; W. Loinaz and J. D. Wells, Phys. Lett. B445, 178 (1998) [hep-ph/9808287].

[51] J. L. Diaz-Cruz, H.-J. He, T. Tait and C. P. Yuan, Phys. Rev. Lett. 80, 4641 (1998) [hep-ph/9802294]; C. Balázs, J. L. Diaz-Cruz, H.-J. He, T. Tait and C. P. Yuan, Phys. Rev. D59, 055016 (1999) [hep-ph/9807349].

[52] H. E. Haber et al., Phys. Rev. D63, 055004 (2001) [hep-ph/0007006].

[53] P. H. Chankowski, S. Pokorski and J. Rosiek, Nucl. Phys. B423, 497 (1994).

[54] A. Djouadi, P. Janot, J. Kalinowski and P. M. Zerwas, Phys. Lett. B376, 220 (1996) [hep-ph/9603368]; A. Djouadi, J. Kalinowski, P. Ohmann and P. M. Zerwas, Z. Phys. C74, 93 (1997) [hep-ph/9605339].

[55] S. Heinemeyer, W. Hollik, J. Rosiek and G. Weiglein, Eur. Phys. J. C19, 535 (2001) [hep-ph/0102081].

[56] J. F. Gunion and H. E. Haber, Phys. Rev. D48, 5109 (1993).

[57] M. M. Mühlleitner, M. Krämer, M. Spira and P. M. Zerwas, hep-ph/0101083. 
[58] The LEP working group for Higgs boson searches, LHWG note 2001-1 (3 April 2001).

[59] The ALEPH, DELPHI, L3 and OPAL Collaborations and the LEP Higgs Working Group, LHWG note 2001-2 (26 March 2001).

[60] The results of Fig. 3.14 were provided by F. Gianotti on behalf of the ATLAS collaboration. They are the preliminary results available as of March 3, 2001.

[61] See, for example, J. F. Gunion, hep-ph/9705282.

[62] S. L. Glashow and S. Weinberg, Phys. Rev. D15, 1958 (1977); E. A. Paschos, Phys. Rev. D15, 1966 (1977).

[63] B. A. Dobrescu, hep-ph/9903407 and hep-ph/0103038.

[64] M. Cvetic, H. Lu, C. N. Pope, A. Sadrzadeh and T. A. Tran, Nucl. Phys. B586, 275 (2000) [hep-th/0003103].

[65] P. Chankowski et al., Phys. Lett. B496, 195 (2000) [hep-ph/0009271].

[66] J. F. Gunion and H. E. Haber, in preparation.

[67] U. Ellwanger and C. Hugonie, hep-ph/9909260.

[68] J. F. Gunion, H. E. Haber and T. Moroi, "Will at least one of the Higgs bosons of the next-to-minimal supersymmetric extension of the Standard Model be observable at LEP2 or the LHC?" in New Directions for High Energy Physics, Proceedings of the 1996 DPF/DPB Summer Study on High Energy Physics, Snowmass '96, edited by D. G. Cassel, L. T. Gennari and R. H. Siemann (Stanford Linear Accelerator Center, Stanford, CA, 1997) [hep-ph/9610337].

[69] J. F. Gunion and B. Grzadkowski, work in progress.

[70] J. R. Espinosa and J. F. Gunion, Phys. Rev. Lett. 82, 1084 (1999) [hep$\mathrm{ph} / 9807275]$.

[71] B. Grzadkowski, J. F. Gunion and J. Kalinowski, Phys. Lett. B480, 287 (2000) [hep-ph/0001093].

[72] H. E. Haber and Y. Nir, Phys. Lett. B306, 327 (1993) [hep-ph/9302228].

[73] A. Djouadi, P. M. Zerwas and H. E. Haber, Phys. Lett. B375, 203 (1996) [hep-ph/9602234].

[74] J. F. Gunion and T. Farris, LC note in preparation.

[75] M. Battaglia and K. Desch, hep-ph/0101165.

[76] TESLA Technical Design Report, R. Heuer, D. Miller, F. Richard, A. Wagner and P. M. Zerwas (editors), obtainable from www.desy.de/ lcnotes/tdr/ .

[77] See also http://www-sldnt.slac.stanford.edu/nld/.

[78] H. Yang and K. Riles, "Measurement of Higgs Mass and Cross Section at NLC", presented at Workshop on Physics and Detectors for Future $e^{+} e^{-}$Linear Colliders, Johns Hopkins University, Baltimore, 19-21 March 2001. 
Higgs Bosons at the Linear Collider

[79] M. Ronan, "Jet-Jet Reconstruction in Full Detector Simulations", presented at Workshop on Physics and Detectors for Future $e^{+} e^{-}$Linear Colliders, Johns Hopkins University, Baltimore, 19-21 March 2001.

[80] A. Juste, hep-ex/9912041.

[81] V. Barger, M. S. Berger, J. F. Gunion and T. Han, Phys. Rev. Lett. 78, 3991 (1997) [hep-ph/9612279].

[82] P. F. Derwent et al., FERMILAB-FN-701, report in preparation.

[83] A. Küskinen, M. Battaglia, P. Pöyhoönen, "Study of $e^{+} e^{-} \rightarrow H^{+} H^{-}$at a 800 GeV Linear Collider", to appear in the Proc. of the Fifth Int. Workshop on Linear Colliders - LCSW2000, Fermilab, October 2000, LC-PHSM-2001-041; A. Andreazza, C. Troncon, "Study of $H A$ Production in $e^{+} e-$ Collisions at $\sqrt{s}=800 \mathrm{GeV}$, DESY-123-E, p. 417.

[84] P. Garcia-Abia and W. Lohmann, hep-ex/9908065.

[85] R. Van Kooten, "Separation of the Higgsstrahlung and $W W$-fusion process", presented at Workshop on Physics and Detectors for Future $e^{+} e^{-}$Linear Colliders, Johns Hopkins University, Baltimore, 19-21 March 2001.

[86] J. Brau, C. Potter, and M. Iwasaki, "Higgs Branching Ratio Measurements at a Future Linear Collider", presented at Workshop on Physics and Detectors for Future $e^{+} e^{-}$Linear Colliders, Johns Hopkins University, Baltimore, 19-21 March 2001.

[87] J. Brau, C. Potter, and M. Iwasaki, "Linear Collider Vertex Detector Optimization for Higgs Branching Ratio Measurements", LCWS 2000 Proceedings, session D3.

[88] D. J. Jackson, Nucl. Instrum. Meth. A388, 247 (1997).

[89] M. D. Hildreth, T. L. Barklow, and D. L. Burke, Phys. Rev. D49, 3441 (1994); K. Kawagoe, UT-ICEPP-93-10, contributed to the 2nd International Workshop on Physics and Experiments with Linear $e^{+} e^{-}$Colliders, Waikoloa, HI, 26-30 April 1993.

[90] G. Borisov and F. Richard, hep-ph/9905413.

[91] M. Battaglia, hep-ex/0012021; hep-ph/9910271.

[92] J. F. Gunion and P. C. Martin, hep-ph/9610417; J. F. Gunion and P. C. Martin, Phys. Rev. Lett. 78, 4541 (1997) [hep-ph/9607360].

[93] E. Boos, J. C. Brient, D. W. Reid, H. J. Schreiber and R. Shanidze, hep$\mathrm{ph} / 0011366$.

[94] J. F. Gunion, B. Grzadkowski and X. He, Phys. Rev. Lett. 77, 5172 (1996) [hep-ph/9605326].

[95] S. Dawson and L. Reina, Phys. Rev. D57, 5851 (1998); D59, 054012 (1999); D60, 015003 (1999); H. Baer, S. Dawson, and L. Reina, Phys. Rev. D61, $0113002(2000)$. 
[96] A. Juste and G. Merino, hep-ph/9910301.

[97] D. J. Miller and S. Moretti, hep-ph/0001194.

[98] C. Castanier, P. Gay, P. Lutz and J. Orloff, hep-ex/0101028.

[99] R. Lafaye, D. J. Miller, M. Mühlleitner and S. Moretti, hep-ph/0002238.

[100] A. Djouadi, W. Kilian, M. Mühlleitner and P. M. Zerwas, hep-ph/0001169.

[101] M. Carena, H. E. Haber, H. E. Logan and S. Mrenna, FERMILAB-Pub-00/334$\mathrm{T}$, in preparation.

[102] J. Alcaraz and E. Ruiz Morales, hep-ph/0012109.

[103] D. Bardin, P. Christova, M. Jack, L. Kalinovskaya, A. Olchevski, S. Riemann and T. Riemann, Comput. Phys. Commun. 133, 229 (2001) [hep-ph/9908433].

[104] M. Battaglia and K. Desch, hep-ph/0101165.

[105] V. Drollinger and A. Sopczak, hep-ph/0102342.

[106] L. D. Landau, Dokl. Akad. Nauk Ser. Fiz. 60, 207 (1948); C. N. Yang, Phys. Rev. 77, 242 (1950); J.J. Sakurai, Invariance Principles and Elementary Particles (Princeton University Press, 1964).

[107] D. J. Miller, S. Y. Choi, B. Eberle, M. M. Muhlleitner and P. M. Zerwas, Phys. Lett. B 505, 149 (2001) [hep-ph/0102023].

[108] K. Hagiwara and M. L. Stong, Z. Phys. C62, 99 (1994) [hep-ph/9309248].

[109] V. Barger, K. Cheung, A. Djouadi, B. A. Kniehl and P. M. Zerwas, Phys. Rev. D49, 79 (1994) [hep-ph/9306270].

[110] T. Han and J. Jiang, Phys. Rev. D63, 096007 (2001) [hep-ph/0011271].

[111] B. Grzadkowski and J. F. Gunion, Phys. Lett. B350, 218 (1995) [hep$\mathrm{ph} / 9501339]$.

[112] B. Grzadkowski and J. F. Gunion, hep-ph/9503409.

[113] M. Krämer, J. Kühn, M. L. Stong and P. M. Zerwas, Z. Phys. C64, 21 (1994) [hep-ph/9404280].

[114] J. F. Gunion and X. G. He, hep-ph/9609453.

[115] B. Grzadkowski and J. F. Gunion, Phys. Lett. B294, 361 (1992) [hep$\mathrm{ph} / 9206262]$.

[116] J. F. Gunion and J. G. Kelly, Phys. Lett. B333, 110 (1994) [hep-ph/9404343].

[117] C. A. Boe, O. M. Ogreid, P. Osland and J. Zhang, Eur. Phys. J. C9, 413 (1999) [hep-ph/9811505].

[118] J. F. Gunion and J. Kelly, Phys. Rev. D56, 1730 (1997) [hep-ph/9610495].

[119] J. F. Gunion and J. Kelly, hep-ph/9610421.

[120] J. L. Feng and T. Moroi, Phys. Rev. D56, 5962 (1997) [hep-ph/9612333].

[121] A. Bartl et al., Z. Phys. C76, 549 (1997) [hep-ph/9701336]; hep-ph/9909378; M. Berggren, R. Keränen, H. Nowak and A. Sopczak, hep-ph/9911345. 
Higgs Bosons at the Linear Collider

[122] S. Y. Choi, A. Djouadi, M. Guchait, J. Kalinowski and P. M. Zerwas, Eur. Phys. J. C14, 535 (2000) [hep-ph/0002033].

[123] ATLAS Collaboration, "Detector and Physics Performance Technical Design Report", CERN/LHCC/99-15 (1999); CMS Collaboration, Technical Design Reports, CMS TDR 1-5 (1997/98).

[124] J. Erler and S. Heinemeyer, hep-ph/0102083.

[125] I. F. Ginzburg, G. L. Kotkin, V. G. Serbo and V. I. Telnov, Nucl. Instrum. Meth. A205, 47 (1983).

[126] I. F. Ginzburg, G. L. Kotkin, S. L. Panfil, V. G. Serbo and V. I. Telnov, Nucl. Instrum. Meth. A219, 5 (1984).

[127] P. Chen, G. Horton-Smith, T Ohgaki, A. W. Weidemann and K. Yokoya, Nucl. Instrum. Meth. A355, 107 (1995). See http://wwwacc-theory.kek.jp/members/cain/cain21b.manual/main.html.

[128] D. Asner, J. Gronberg, J. Gunion and T. Hill, UCRL-ID-143967.

[129] S. Söldner-Rembold and G. Jikia, hep-ex/0101056.

[130] J. F. Gunion, J. G. Kelly and J. Ohnemus, Phys. Rev. D51, 2101 (1995) [hep$\mathrm{ph} / 9409357]$.

[131] J. F. Gunion, R. Vega and J. Wudka, Phys. Rev. D43, 2322 (1991).

[132] R. N. Mohapatra, Fortsch. Phys. 31, 185 (1983); J. F. Gunion, J. Grifols, A. Mendez, B. Kayser and F. Olness, Phys. Rev. D40, 1546 (1989); N. G. Deshpande, J. F. Gunion, B. Kayser and F. Olness, Phys. Rev. D44, 837 (1991).

[133] H. Georgi and M. Machacek, Nucl. Phys. B262, 463 (1985).

[134] M. S. Chanowitz and M. Golden, Phys. Lett. B165, 105 (1985).

[135] J. F. Gunion, Int. J. Mod. Phys. A11, 1551 (1996) [hep-ph/9510350]; A13, 2277 (1998) [hep-ph/9803222].

[136] P. H. Frampton, Int. J. Mod. Phys. A15, 2455 (2000) [hep-ph/0002017].

[137] F. Cuypers and M. Raidal, Nucl. Phys. B501, 3 (1997) [hep-ph/9704224].

[138] J. Gluza, Phys. Lett. B403, 304 (1997) [hep-ph/9704202].

[139] R. A. Alanakian, Phys. Lett. B436, 139 (1998) [hep-ph/9706383].

[140] G. Barenboim, K. Huitu, J. Maalampi and M. Raidal, Phys. Lett. B394, 132 (1997) [hep-ph/9611362].

[141] V. Barger, J. F. Beacom, K. Cheung and T. Han, Phys. Rev. D50, 6704 (1994) [hep-ph/9404335].

[142] R. Casalbuoni, A. Deandrea, S. De Curtis, D. Dominici, R. Gatto and J. F. Gunion, Nucl. Phys. B555, 3 (1999) [hep-ph/9809523]. 


\section{Chapter 4 Supersymmetry Studies at the Linear Collider}

\section{Introduction}

The Standard Model (SM) has been tested by a spectacularly large and diverse set of experiments. The resulting body of data is consistent with the matter content and gauge interactions of the SM with a Higgs boson of mass $m_{h} \lesssim 250 \mathrm{GeV}$ [1]. If a fundamental Higgs boson exists, it fits much more naturally into supersymmetric extensions of the SM than into the SM itself [2-5]. Thus, the study of supersymmetry (SUSY) is among the highest priorities for future accelerators.

If SUSY exists, many of its most important motivations suggest that at least some superpartners have masses below about $1 \mathrm{TeV}$. These motivations, ranging from gauge coupling unification [6-10] to the existence of an excellent dark matter candidate [11], are discussed in previous chapters and also below. While none of these is a guarantee of SUSY, they all provide motivation for the presence of SUSY at the weak-interaction scale.

In the supersymmetric extension of the SM with minimal field content, hundreds of additional parameters enter the Lagrangian. If SUSY is discovered, this discovery will open new questions - to understand the pattern of the SUSY parameters, to determine from them the mechanism of SUSY breaking, and to infer from them the nature of physics at the very highest energy scales. Such grand goals may be contemplated only if precise and model-independent measurements of superpartner properties are possible.

In this chapter, we describe the prospects for such measurements at a $0.5-1.0 \mathrm{TeV}$

$e^{+} e^{-}$linear collider (LC) with longitudinally polarized electron beams. The potential of linear colliders for detailed studies of supersymmetry has been discussed previously in numerous reports [12-18]. In this chapter, many well-established results are reviewed, including the potential for model-independent measurements of superpartner masses. In addition, several less well-appreciated topics are discussed. These include loop-level effects in supersymmetry, CP violation, and supersymmetric flavor violation. This discussion serves both to illustrate the rich program of supersymmetric studies available at linear colliders, and to highlight areas that merit further study. This chapter concludes with a review of the important complementarity of the LC and the Large Hadron Collider (LHC) with respect to supersymmetry studies.

The signatures of supersymmetry are many, ranging from the well-known missing energy in supergravity with R-parity conservation $[19,20]$ to exotic signatures appearing in models with gauge-mediated [21] and anomaly-mediated [22,23] supersymmetry breaking. Space constraints prevent a complete review of the considerable work done 
in each of these, and other, frameworks. Instead, this review focuses on supergravity frameworks leading to the conventional signature of missing energy. R-parity violation and alternative supersymmetry-breaking mechanisms are treated as variations, and are discussed where they are especially pertinent.

\section{The scale of supersymmetry}

The cleanliness of the linear collider environment implies that precise, modelindependent measurements in supersymmetry are possible, but only if supersymmetric final states are kinematically accessible. The mass scale of supersymmetric particles is therefore of paramount importance. In this section we review bounds on superpartner masses from naturalness criteria, dark matter constraints, Higgs boson searches, and precision electroweak data. We also consider the potential of experimental evidence for new physics to constrain the supersymmetric mass scale; we discuss the muon anomalous magnetic moment as an example.

\subsection{Naturalness}

In supersymmetric extensions of the SM, quadratically divergent quantum corrections to the masses of fundamental scalars are of the order of the superpartner mass scale. Given a mechanism for producing sufficiently light superpartners, the observed weak scale is obtained without unnaturally large cancellations in the electroweak potential. While no analysis of naturalness can claim quantitative rigor, the importance of naturalness as a fundamental motivation for supersymmetry has prompted many studies [24-46], with important qualitative implications for the superparticle spectrum.

To study naturalness one must first assume a certain supersymmetric framework. Models in this framework are specified by a set of input parameters, typically defined at some high energy scale. Together with experimental constraints and renormalization group equations, these parameters determine the entire weak-scale Langrangian, including the $Z$ boson mass, which at tree level is

$$
\frac{1}{2} m_{Z}^{2}=\frac{m_{H_{d}}^{2}-m_{H_{u}}^{2} \tan ^{2} \beta}{\tan ^{2} \beta-1}-\mu^{2},
$$

where $m_{H_{d}}^{2}, m_{H_{u}}^{2}$ are the mass parameters of the two Higgs doublets of the model and $\tan \beta=\left\langle H_{u}\right\rangle /\left\langle H_{d}\right\rangle$. Naturalness is then often imposed by demanding that the weak scale be insensitive to variations in some set of parameters $a_{i}$, which are assumed to be continuously variable, independent, and fundamental. The $a_{i}$ may be scalar masses, gaugino masses, and other parameters, but are not necessarily input parameters. The sensitivity is typically quantified by defining coefficients $[24,25]$ 
$c_{i} \equiv\left|\left(a_{i} / m_{Z}\right)\left(\partial m_{Z} / \partial a_{i}\right)\right|$ for each parameter $a_{i}$ and taking some simple combination of the $c_{i}$, often $c=\max \left\{c_{i}\right\}$, as an overall measure of naturalness. A naturalness criterion $c<c_{\max }$ then implies upper bounds on supersymmetry parameters and superpartner masses.

Following the early studies [24,25], the authors of [27] stressed the importance of including one-loop corrections to Eq. (4.1). They also noted that it is possible in principle for a given $c_{i}$ to be large for all possible choices of $a_{i}$. In the latter case, the authors of [28-30] argued that, to avoid misleading results, only unusually large sensitivity should be considered unnatural and proposed replacing $c$ by $\tilde{\gamma} \equiv \max \left\{c_{i} / \bar{c}_{i}\right\}$, with $\bar{c}_{i}$ an average sensitivity. More recently, another alternative prescription has been proposed [34-38] in which the sensitivity coefficients are replaced by $\left|\left(\Delta a_{i} / m_{Z}\right)\left(\partial m_{Z} / \partial a_{i}\right)\right|$, where $\Delta a_{i}$ is the experimentally allowed range of $a_{i}$. This definition implies that arbitrarily large but well-measured supersymmetry parameters are natural, and has been argued to differ sharply from conventional notions of naturalness [46].

The results of naturalness studies are strongly dependent on the choice of framework, the choice of fundamental parameters $a_{i}$, and, of course, the choice of $c_{\max }$ (or the equivalent $\tilde{\gamma}$ parameter). The dependence on framework assumptions is inescapable. In other studies of supersymmetry there exists, at least in principle, the possibility of a model-independent study, where no correlations among parameters are assumed. In studies of naturalness, however, the correlations determine the results, and there is no possibility, even in principle, of an all-inclusive framework. We describe here only some of the qualitatively distinct possibilities. For alternative analyses, readers are referred to the original literature [24-46].

In minimal supergravity, one assumes both scalar and gaugino universality at a high scale. If one requires insensitivity of the weak scale with respect to both supersymmetry breaking and Standard Model parameters, none of the superpartner masses can naturally be far above the weak scale. Examples of the resulting naturalness bounds are given in Fig. 4.1. The bounds for non-strongly interacting superpartners are typically more stringent than those for colored superpartners. Similar results are found in other frameworks where all scalar and gaugino masses are comparable at some high scale.

Naturalness bounds may be very different in other frameworks, however, especially for scalars. For squark and slepton masses, if no correlations are assumed, the bounds are highly generation-dependent. At one-loop, the weak scale is sensitive to sfermion masses only through renormalization group terms proportional to Yukawa couplings. Thus, while the scalar masses of the third generation are still usefully constrained by naturalness criteria, first- and second-generation scalars may have masses above $10 \mathrm{TeV}$ without requiring large fine-tuning [31,32], putting them far beyond the kinematic reaches of both the LHC and future linear colliders. 'Superheavy' first and second generation scalars ameliorate the supersymmetric flavor and CP problems and 


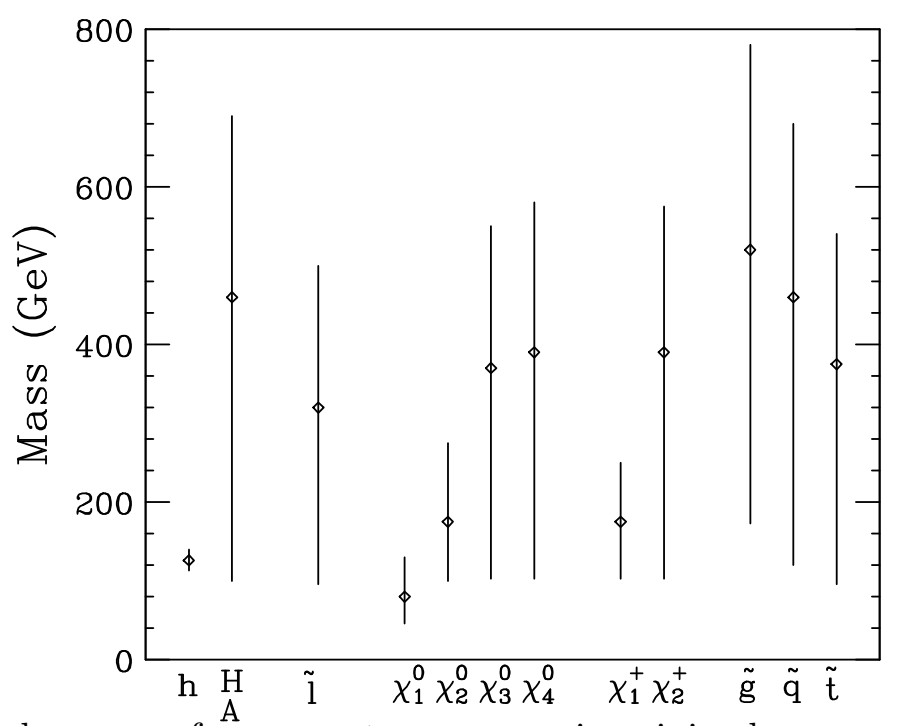

Figure 4.1: Natural ranges of superpartner masses in minimal supergravity. The upper limits are set by the requirement $\tilde{\gamma}<10$ and the diamonds indicate upper bounds corresponding to $\tilde{\gamma}<5$. The lower limits are roughly those from current collider constraints. Updated from [29].

are found in many models [47-63].

Alternatively, given the possibility that SM couplings are fixed in sectors separate from supersymmetry breaking, one may reasonably require only that the weak scale be insensitive to variations in parameters related to supersymmetry breaking [4446]. With this less stringent criterion, in many simple models, including minimal supergravity, all scalar partners may be naturally in the $2-3 \mathrm{TeV}$ range, as a result of focusing behavior in renormalization group trajectories [44-46,64-68]. Such "focus point supersymmetry" models also have significant virtues with respect to low-energy constraints, and predict that even third-generation scalars may have masses well above $1 \mathrm{TeV}$ and be beyond the reach of linear colliders.

Bounds on the masses of fermionic superpartners are less framework-dependent. If the gaugino masses are uncorrelated, the gluino mass is typically stringently bounded by its indirect influence on the weak scale through the top squarks. In this general context, the electroweak gaugino masses may be significantly larger [42,43]. However, in most well-motivated models, the gluino is much heavier than the electroweak gauginos, and so naturalness implies stringent limits on Bino and Wino masses. While the scale of the $\mu$ parameter may be determined [69], a quantitative theory for the $\mu$ term is lacking. The $\mu$ parameter is therefore usually determined through Eq. (4.1) and is otherwise assumed to be uncorrelated with other parameters. Large $\mu$ then necessarily leads to large fine-tuning, and so heavy Higgsinos are disfavored. As a result, given our present understanding, naturalness criteria typically imply relatively stringent bounds on the masses of all six chargino and neutralino states, and they 
Supersymmetry Studies at the Linear Collider

encourage the expectation that all of these particles will be available for study at linear colliders.

\subsection{Neutralino relic abundance}

An important virtue of many supersymmetric theories is the existence of a nonbaryonic dark matter candidate. The most straightforward possibility is the lightest neutralino $\chi[11,70]$, which is often the lightest supersymmetric particle (LSP) and so is stable in models with conserved R-parity. Current cosmological and astrophysical measurements prefer $0.1 \lesssim \Omega_{m} h^{2} \lesssim 0.3$ [71], where $\Omega_{m}$ is the ratio of dark matter density to critical density, and $h \approx 0.65$ is the Hubble parameter in units of $100 \mathrm{~km} \mathrm{~s}^{-1} \mathrm{Mpc}^{-1}$. The superpartner spectrum is then constrained by the requirement that the thermal relic density of the lightest neutralino satisfy $\Omega_{\chi} h^{2} \lesssim 0.3$.

The neutralino relic density is determined by the neutralino pair annihilation cross section and has been the subject of many analyses [72-100]. These include refined treatments of poles [72-74], annihilation thresholds [72,73], and co-annihilation among Higgsinos [75] and with staus [76,77]. The S- and P-wave contributions to all tree-level processes with two-body final states are given in [78].

In general, neutralinos may annihilate through $t$-channel sfermions to $f \bar{f}$, through $s$-channel $Z$ and Higgs bosons to $f \bar{f}$, and through $t$-channel charginos and neutralinos to $W W$ and $Z Z$. For Bino dark matter, only the sfermion-mediated amplitudes are non-vanishing. An upper bound on $\Omega_{\chi} h^{2}$ then leads to an upper bound on at least one sfermion mass. This, together with the requirement that $\chi$ be the LSP, implies an upper bound on $m_{\chi}$. Such reasoning has led to claims of cosmological upper bounds on superpartner masses with optimistic implications for supersymmetry at linear colliders [79-89].

These claims must be viewed cautiously, however, as they are true only in the $\chi \approx \tilde{B}$ limit and are violated even in the simplest scenarios. In minimal supergravity, for example, multi-TeV LSPs are possible for large $m_{0}$ [94], where the LSP has a significant Higgsino admixture, leading to large annihilation cross sections to gauge bosons. Useful upper bounds are also absent in minimal supergravity at large $\tan \beta$ [94-97], where the importance of a small Higgsino admixture in $\chi$ is amplified and leads to large Higgs boson-mediated annihilation. More generally, no guarantee of light superpartners is possible for Wino- [98-100] and Higgsino-like [75,90] LSPs, which annihilate very efficiently to negligible relic densities. Finally, it is worth recalling that these upper bounds are also inapplicable in theories with low-energy supersymmetry breaking or R-parity violation, where the lightest neutralino is no longer stable. 


\subsection{Higgs mass and precision electroweak constraints}

As is well known, supersymmetry places severe constraints on the mass of the lightest Higgs boson. In the Minimal Supersymmetric Standard Model (MSSM), one-loop calculations [101-110] have now been supplemented with leading two-loop corrections in the Feynman diagrammatic [111-115], renormalization group [116119], and effective potential [120-122] approaches, leading to an upper bound of $m_{h} \lesssim 135 \mathrm{GeV}$ [113]. The consistency of this bound with precision electroweak fits is a considerable success of supersymmetry. At the same time, though, one might expect that the current lower bound $m_{h}>113.5 \mathrm{GeV}$ from direct Higgs searches [123-126] and the success of precision electroweak fits to the SM disfavors the possibility of light superpartners.

However, closer analysis shows that light superpartners are consistent with the current Higgs mass bound. For example, in general scenarios, the current Higgs mass limit may be satisfied with large masses only for the top and bottom squarks. Even for these, the constraints are not severe. Charginos, neutralinos, and sleptons may be light and within the reach of linear colliders. In simpler frameworks, the Higgs limit is more constraining. Even in minimal supergravity, however, the current Higgs mass bound, along with the requirement of a suitable dark matter candidate, may be satisfied either for chargino masses above $200 \mathrm{GeV}$ [127] or for large $m_{0}$ [46,128]. In the latter case, charginos may be as light as their current LEP bound. The Higgs mass bound can also be made consistent with light superpartners if there are large CP-violating phases, which must necessarily cancel to high accuracy in electric dipole moments, or new singlets [129]. Thus, the current Higgs mass constraint, although already rather stringent, does not exclude the possibility of light superpartners.

The supersymmetric spectrum is also constrained by precision electroweak measurements. The effects of supersymmetry have been studied in numerous recent works (see, e.g., [130-135]). While there are at present no strong indications for supersymmetry from these considerations, light superparticles cannot be excluded either. This issue is discussed further in Chapter 8, Section 3.2.

\subsection{Evidence for new physics}

Finally, weak-scale supersymmetry has implications for a broad range of experiments in particle physics and astrophysics. If deviations from SM predictions are found, these deviations may also constrain the scale of superpartner masses.

As an example, we consider the recently reported $2.6 \sigma$ deviation in the anomalous magnetic moment of the muon [136]: $a_{\mu}^{\exp }-a_{\mu}^{\mathrm{SM}}=(43 \pm 16) \times 10^{-10}$. Supersymmetric contributions to $a_{\mu}$ are well known [137-141], and the measured deviation is naturally explained by supersymmetry [142-153]. If a supersymmetric interpretation is adopted, the result restricts the masses of some superpartners. Highly modelindependent upper bounds on the mass of the lightest observable supersymmetric 
Supersymmetry Studies at the Linear Collider
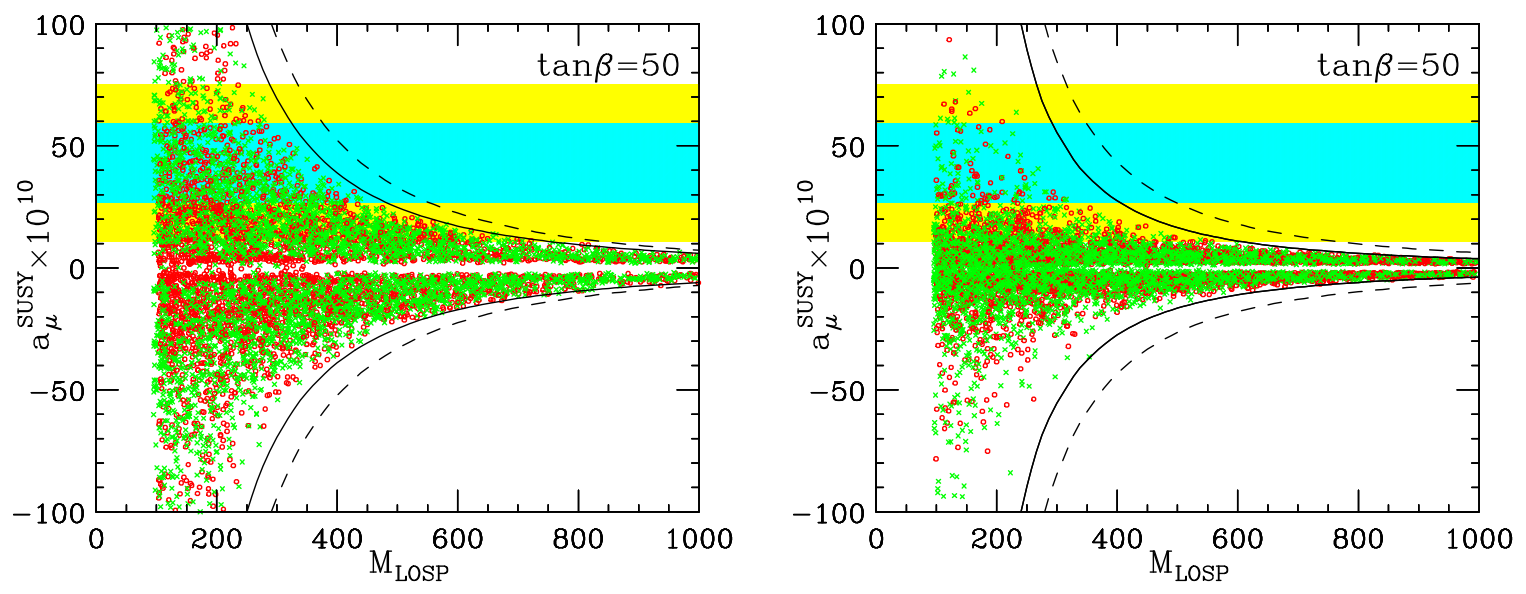

Figure 4.2: Possible values of the mass of the lightest observable supersymmetric particle, $M_{\mathrm{LOSP}}$, and the supersymmetric contribution to the muon's anomalous magnetic moment, $a_{\mu}^{\text {SUSY }}$, assuming a stable LSP (left) and a visibly decaying LSP (right). Crosses (circles) have smuon (chargino/neutralino) LOSPs and satisfy the parameter constraints $M_{2}=2 M_{1}$, $A_{\mu}=0$, and $\tan \beta=50$. Relaxing the gaugino unification assumption leads to the solid envelope curve, and further allowing arbitrary $A_{\mu}$ leads to the dashed curve. The envelope contours scale linearly with $\tan \beta$. The shaded regions are the $1 \sigma$ and $2 \sigma$ experimentally preferred regions. From [144].

particle are given in Fig. 4.2. If theory and experiment are required to agree within $1 \sigma$, at least one observable superpartner must be lighter than $490 \mathrm{GeV}$ if the LSP is stable, and lighter than $410 \mathrm{GeV}$ if the LSP decays visibly in the detector. If agreement only within $2 \sigma$ is required, these limits weaken to $800 \mathrm{GeV}$ and $640 \mathrm{GeV}$, respectively. The bounds are for the case $\tan \beta \leq 50$ and scale linearly with $\tan \beta$.

These results illustrate the power of evidence for new physics to constrain the scale of supersymmetry. Of course, many other experiments may also see supersymmetric effects. Among the areas in which great experimental progress is expected in the next few years are searches for new physics at the Tevatron, $B$ physics (CP violation, rare decays), lepton flavor violation ( $\mu$-e conversion, $\mu \rightarrow e \gamma$, etc.), electric dipole moments, searches for dark matter (both direct and indirect), and cosmic ray physics. Pre-LHC evidence for supersymmetry is not guaranteed, but, in simple frameworks like minimal supergravity where systematic and comprehensive analyses are possible, it is very likely [95]. Strong evidence for new physics, even if indirect, will provide important additional constraints on the mass scale of supersymmetric particles. 


\section{Determination of masses and couplings}

The usefulness of a linear collider in the study of SUSY particles lies both in the simplicity of the production process and in the fact that the electron can have a large longitudinal polarization. These features allow one to carry out accurate measurements of the masses and the quantum numbers of the particles being produced, and also to determine their gauge coupling constants in a model-independent manner $[154,155]$. Such measurements are crucial in understanding the nature of the processes being uncovered.

\subsection{Measurement of superpartner masses}

We begin our review of mass measurements by considering one particular process that illustrates the essential simplicity of the analyses. The process we will consider is selectron production,

$$
e^{+} e^{-} \rightarrow \widetilde{e}_{L, R}^{+} \widetilde{e}_{L, R}^{-}
$$

where $\widetilde{e}_{R}^{-}, \widetilde{e}_{L}^{-}$are the supersymmetry partners of the right- and left-handed electron. We assume that both selectrons decay by $\widetilde{e}_{L, R} \rightarrow e \widetilde{\chi}_{1}^{0}$. The process has a number of interesting features. The masses of the $\widetilde{e}_{R}$ and $\widetilde{e}_{L}$ can differ substantially. The combinations $\widetilde{e}_{R}^{+} \widetilde{e}_{R}^{-}$and $\widetilde{e}_{L}^{+} \widetilde{e}_{L}^{-}$are produced by $s$-channel photon and $Z^{0}$ exchange, but all four possible selectron combinations are produced by $t$-channel neutralino exchange. Thus, the study of this process can give information on SUSY masses, quantum numbers, and coupling constants.

In the reaction (4.2), the selectrons are produced at a fixed energy. Since they are scalars, they decay isotropically in their own frames. These distributions of the decay electrons and positrons boost to distributions in the lab that are flat in energy between the kinematic endpoints. The electrons and positrons then show box-like distributions. The maximum and minimum energies which form the edges of the box determine the masses of the $\widetilde{e}$ and the $\widetilde{\chi}_{1}^{0}$ through the relations

$$
\begin{aligned}
M_{\widetilde{e}}^{2} & =E_{\mathrm{cm}}^{2}\left\{\frac{E_{e, \max } E_{e, \min }}{\left(E_{e, \max }+E_{e, \min }\right)^{2}}\right\} \\
M_{\widetilde{\chi}_{1}^{0}}^{2} & =M_{\widetilde{e}}^{2}\left\{1-2 \frac{E_{e, \max }+E_{e, \min }}{E_{\mathrm{cm}}}\right\} .
\end{aligned}
$$

If several different combinations of selectrons are produced, the electron and positron energy spectra will show a superposition of several box-like distributions. Each set of endpoints gives the associated selectron masses and an independent determination of the $\tilde{\chi}_{1}^{0}$ mass.

Figure 4.3 shows the electron and positron spectra for a particular set of MSUGRA parameters constructed for the Snowmass ' 96 summer study [156], assuming $50 \mathrm{fb}^{-1}$ of data at $\sqrt{s}=500 \mathrm{GeV}$ [157]. The simulations use the event generator ISAJET [158]. 
Supersymmetry Studies at the Linear Collider
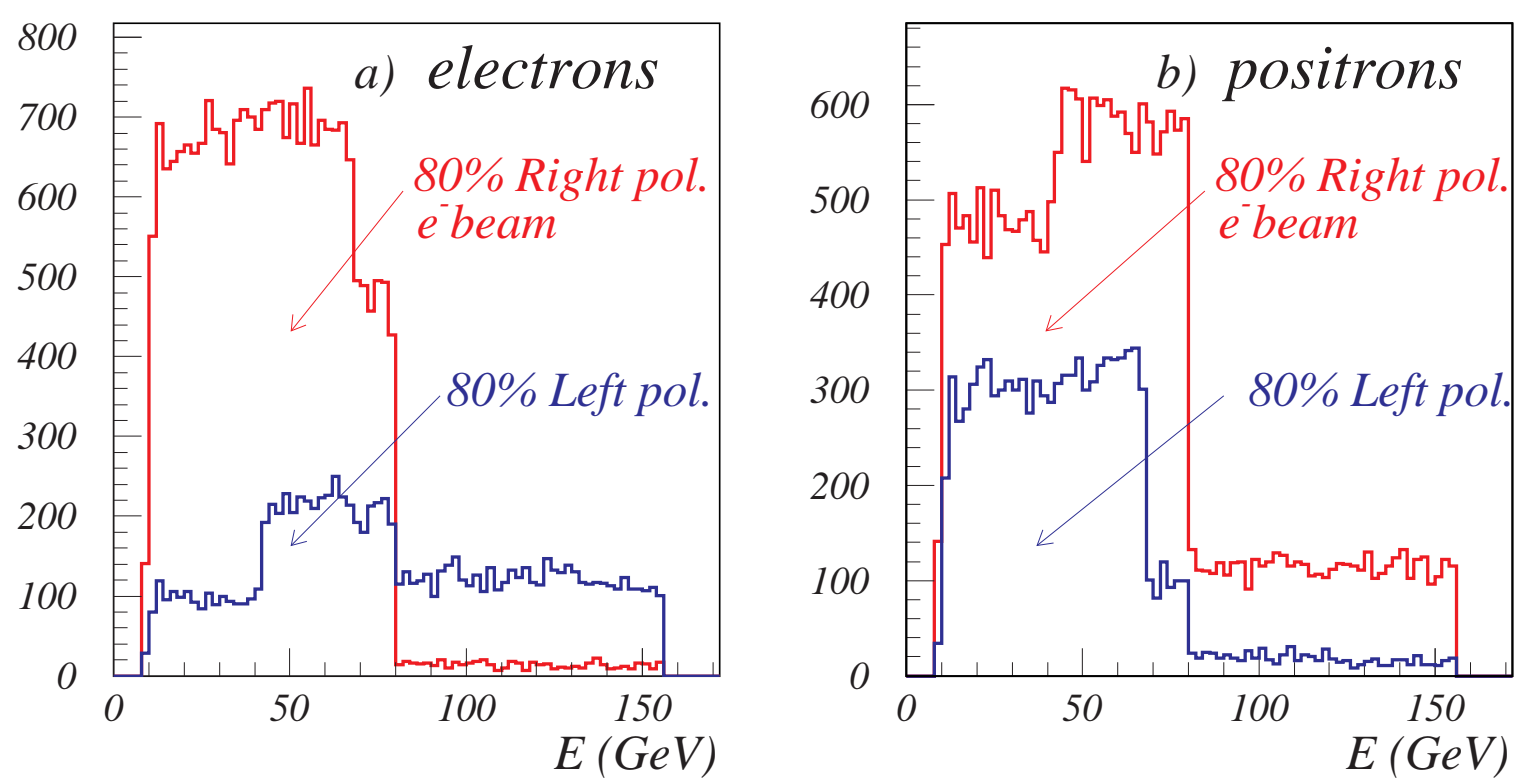

Figure 4.3: Electron and positron energy distributions for selectron pair production, with the indicated beam polarizations and integrated luminosity $50 \mathrm{fb}^{-1}[157]$.

The expected box-like spectra appear clearly, with sharp endpoints. Both the electron and positron spectra have a strong dependence on polarization, and this allows us to recognize which components are associated with $\widetilde{e}_{L}$ and which with $\widetilde{e}_{R}$. The electron and positron spectra also differ from each other, reflecting the different production of $\widetilde{e}_{R}^{-} \widetilde{e}_{L}^{+}$versus $\widetilde{e}_{L}^{-} \widetilde{e}_{R}^{+}$from polarized beams.

Figure 4.4 compares the generated electron and positron distributions to those reconstructed using energy measurements from the electromagnetic calorimeter of the $\mathrm{L}$ detector described in Chapter 15. The study uses full GEANT simulation of the calorimeter [159]. The effect of resolution is clearly observed in the upper edge of the energy distribution. This analysis does not include beamstrahlung and initial state radiation, but these effects are not expected to affect significantly the determination of the edges in the energy spectra [156].

Many similar analyses of the determination of slepton masses have been carried out using fast Monte Carlo techniques [160-163]. Some of the results are summarized in Table 4.1. One can see from the table that we expect to be able to measure these masses with an accuracy of a few percent or less in most cases. The determination of the mass of the lighter chargino $\tilde{\chi}_{1}^{ \pm}$has been studied by many groups. Measurements based on an analysis using background cuts $[154,163,164]$ indicate that this mass can be measured with accuracies of $1 \%$ or less by this method. An interesting signal thast may be background-free is the case where one $\tilde{\chi}_{1}^{ \pm}$decays into a lepton and a 


\section{Selectron Calorimetry (Left Beam Pol.) Selectron Calorimetry (Right Beam Pol.)}
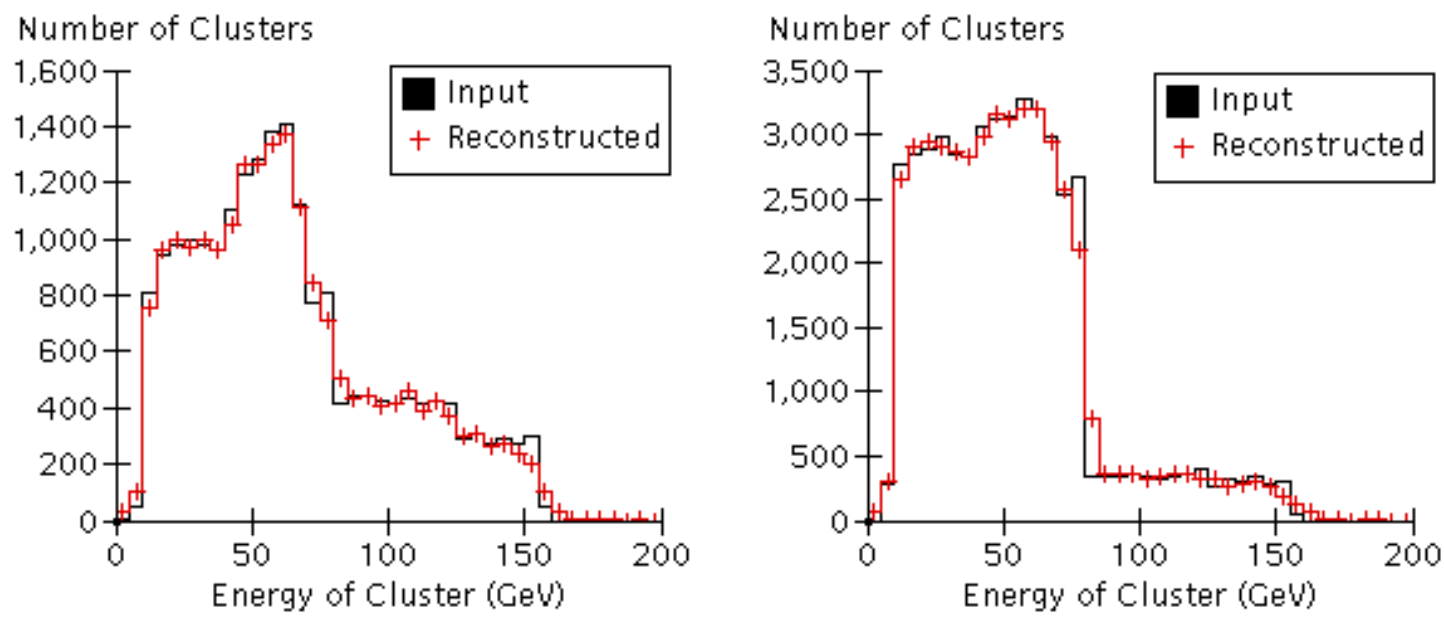

Figure 4.4: Input and calorimeter-reconstructed $e^{ \pm}$energy distributions from selectron pair production for $80 \%$ left-polarized (left) and $80 \%$ right-polarized (right) electron beams [159]. The effect of calorimeter resolution is evident at high cluster energies.

\begin{tabular}{c|c|cc|c|rc}
\hline \hline Reference & Particle & Input & Measured & Particle & Input & Measured \\
\hline$[157]$ & $\widetilde{e}_{L}^{ \pm}$ & 238.2 & 239.4 & $\widetilde{\chi}_{1}^{0}$ & 128.7 & 129.0 \\
{$[157]$} & $\widetilde{e}_{R}^{ \pm}$ & 157.0 & 158.0 & $\widetilde{\chi}_{1}^{0}$ & 128.7 & 129.0 \\
{$[173]$} & $\widetilde{\mu}_{R}^{ \pm}$ & 157.1 & 143.2 & $\widetilde{\chi}_{1}^{0}$ & 128.7 & 117.3 \\
{$[162]$} & $\widetilde{\nu}_{e}$ & 206.6 & 199.4 & $\widetilde{\chi}_{1}^{ \pm}$ & 96.4 & 96.5 \\
{$[154]$} & $\widetilde{\chi}_{1}^{ \pm}$ & 219.0 & 212.0 & $\widetilde{\chi}_{1}^{0}$ & 118.0 & 116.5 \\
{$[165]$} & $\widetilde{\chi}_{1}^{ \pm}$ & 238.0 & 239.8 & $\widetilde{\widetilde{\nu}}_{\ell}$ & 220.0 & 221.2 \\
{$[163]$} & $\widetilde{\chi}_{2}^{ \pm}$ & 175.2 & 176.5 & $\widetilde{\chi}_{1}^{ \pm}$ & 85.9 & 86.1 \\
{$[166]$} & $\widetilde{\chi}_{2}^{ \pm}$ & 290.4 & 282.7 & $\widetilde{\chi}_{1}^{ \pm}$ & 96.0 & 97.9 \\
\hline \hline
\end{tabular}

Table 4.1: Comparison of the input and measured masses (in $\mathrm{GeV}$ ) for a few supersymmetric particles as determined from the end-point spectrum of the observed particles smeared via fast MC techniques. Most of the results are based on a $50 \mathrm{fb}^{-1}$ data sample. The pair of masses in each row are determined from the end-point measurement in pair-production of the first particle listed.

$\overline{\widetilde{\nu}}_{\ell}$, with the $\overline{\tilde{\nu}}_{\ell}$ decaying to a $\nu \widetilde{\chi}_{1}^{0}$, while the other $\widetilde{\chi}_{1}^{ \pm}$decays into $q \bar{q} \widetilde{\chi}_{1}^{0}$. In this case, it should be possible to remove the $W W$ background completely without affecting the signal [165]. The mass measurement for the heavier chargino $\widetilde{\chi}_{2}^{ \pm}$has also been studied, assuming a CM energy of $750 \mathrm{GeV}$. By using the decay of the $\widetilde{\chi}_{2}^{ \pm}$into $\widetilde{\chi}_{1}^{ \pm} Z^{0}$, where the $Z$ decays into leptons and the $\widetilde{\chi}_{1}^{ \pm}$decays into hadrons, one is able to get 


\section{Muon Energy from Right Handed SMuons at $80 \%$ Left $\left(L=50 \mathrm{fb}^{\wedge}\{-1\}\right)$}

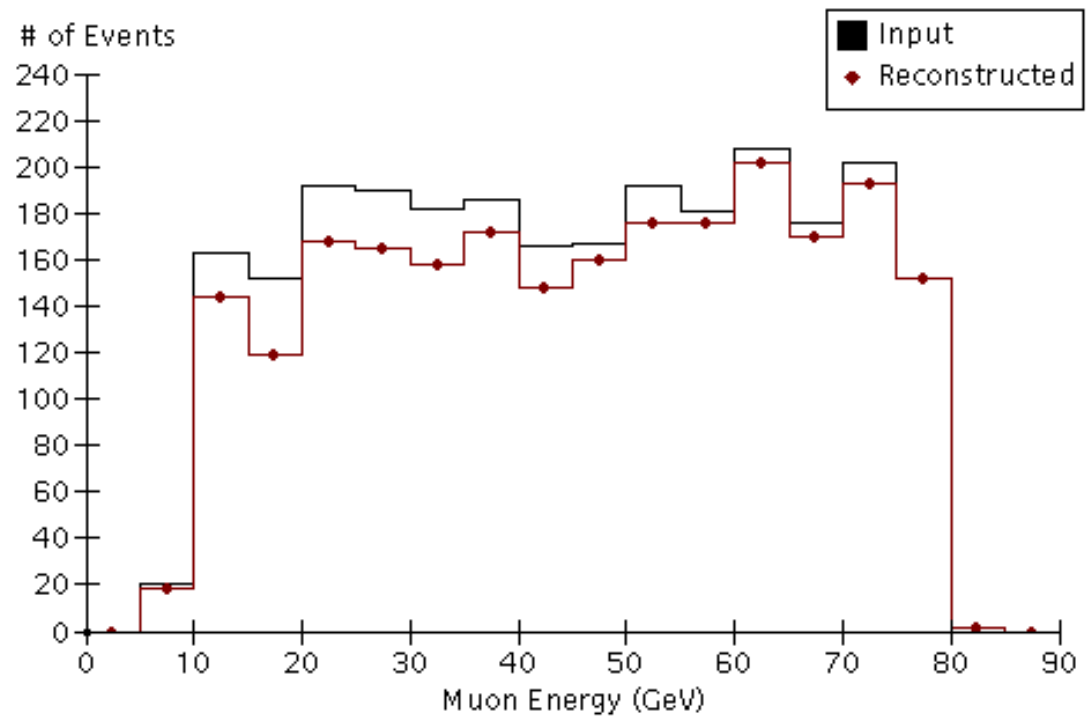

Figure 4.5: Input and tracker-reconstructed muon energy spectra from smuon pair production with an $80 \%$ left-polarized electron beam [173].

quite accurate results [166]. The conclusions of all these analyses are also shown in Table 4.1.

It is worth reviewing some of the experimental issues that arise in these measurements. We have already given an example in which the calorimeter resolution affects the mass measurements for selectrons decaying to $e^{-}$and $e^{+}$. For the case of smuons decaying to $\mu^{ \pm}$, the corresponding issue is tracking resolution. In Fig. 4.5, we show a comparison of generator-level and reconstructed muon energy in $\tilde{\mu}$ pair production. It is clear that the tracking reconstruction does not significantly affect the energy edge resolution, and hence it does not affect our ability to determine supersymmetric masses accurately. For chargino decays, both calorimeter and tracking resolution enter the determination of kinematic endpoints [154].

To examine the supersymmetry signals, it is necessary to remove backgrounds events efficiently. The major sources of SM backgrounds are the two-photon $\left(\gamma^{\star} \gamma^{\star}\right)$ process, which gives rise to lepton and quark pairs in the detector, $e^{+} e^{-}$annihilation to the $W^{+} W^{-}, Z^{0} Z^{0}$, and $Z^{0} h^{0}$, and single- $W$ production $\left(e \gamma^{*} \rightarrow \nu W\right)$. Methods for removing the annihilation and single $W$ backgrounds from the supersymmetry sample are explained in $[154,167,168]$. The two-photon background is a problem in reactions whose signatures involve missing energy, but it can be controlled by also requiring missing transverse momentum. Methods for measuring the two-photon background 
have been studied in $[169,168,170-172]$. There may also be backgrounds from the decays of other supersymmetric particles but, in most cases, these are either small or have distinctive signals that allow one to identify them.
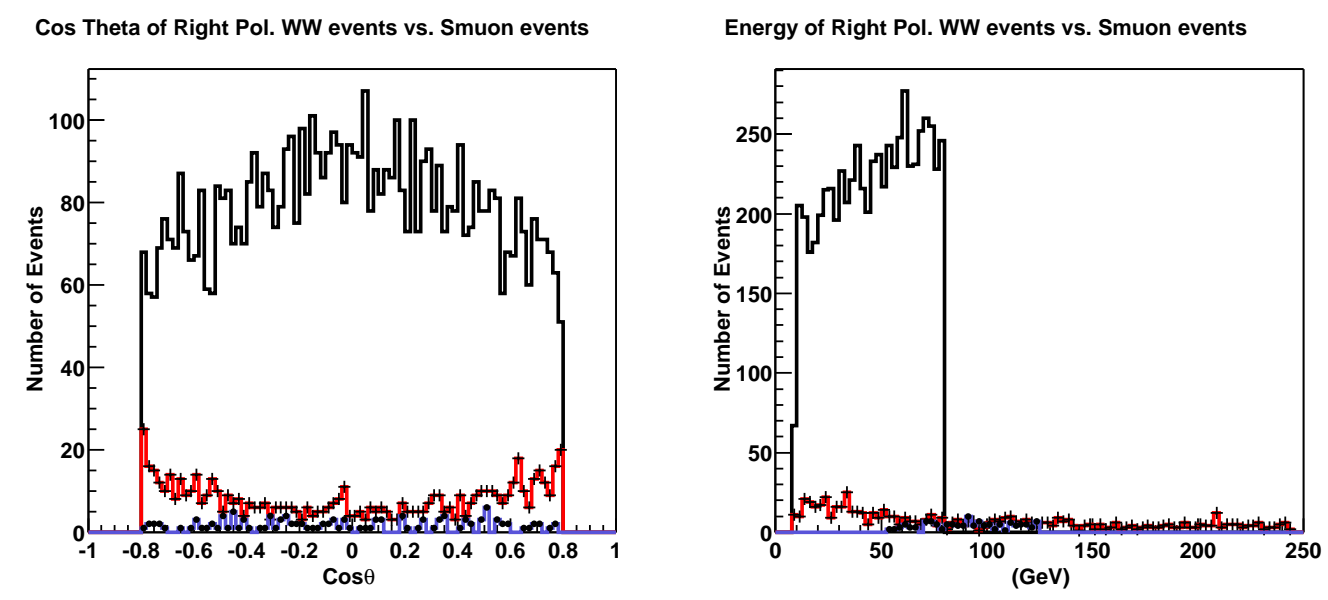

Cos Theta of Left Pol. WW events vs. Smuon events

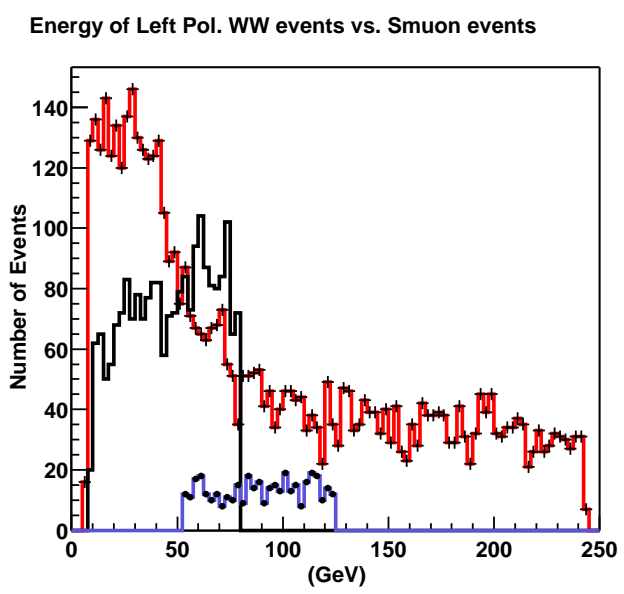

Figure 4.6: Kinematic distributions of muons from $\widetilde{\mu}_{R}$ pair production (solid), $\widetilde{\mu}_{L}$ pair production (dotted), and $W^{+} W^{-}$background (crossed) [173]. An electron beam polarization of $80 \%$ is assumed.

One case in which $W$ pair production is a serious background is the study of the muon energy spectrum $\widetilde{\mu}_{R, L}^{ \pm}$. The cross section for $\widetilde{\mu}$ pair production is small, and the $W$ pair production process leads to muon pairs with missing transverse momentum from neutrinos. Figure 4.6 shows the effect of the $W$ pair background after appropriate cuts [173]. The figure also shows that electron polarization can be used to remove this background. The $\widetilde{\mu}_{R}$ signal is most clearly seen with a right-handed polarized electron beam, since the $W^{+} W^{-}$production is strongly reduced in this case. Observing the signal for $\widetilde{\mu}_{L}$ is difficult with either polarization. If the model parameters are 
such that the $\widetilde{\ell}_{R, L}$ is heavier than the $\tilde{\chi}_{2}^{0}$, this problem can be avoided by studying the decay $\widetilde{\ell}_{R, L}^{ \pm} \rightarrow \ell^{ \pm}+\widetilde{\chi}_{2}^{0}$, with the $\widetilde{\chi}_{2}^{0}$ decaying to a lepton pair and $\widetilde{\chi}_{1}^{0}$. Then, because of the large lepton multiplicity, there are no important SM backgrounds [174].

Another kinematic method for determining the masses of supersymmetric particles is to exploit the correlations between the products of the two decaying sparticles in a given event [175]. This technique is especially useful in cases where low- $p_{T}$ backgrounds tend to overwhelm the signal. Some experimental analyses have been carried out using this method [176,177], and it should receive more attention.

One can also carry out mass measurements using threshold scans [174,164], though in some cases this requires $100 \mathrm{fb}^{-1}$ of luminosity per threshold. The method has the potential to measure masses with accuracies of $0.1 \%$. The effect of backgrounds from SM processes and other SUSY signals and the effects of beamstrahlung and bremsstrahlung need to be understood to determine the systematics limits of this method [178].

A special case of spectrum parameters for which SUSY detection and mass measurement are especially difficult is that of an almost-degenerate chargino and neutralino. This situation can occur in the Higgsino limit of gaugino-Higgsino mixing, and in anomaly-mediated supersymmetry breaking (AMSB). A recent analysis [179] shows how to extract the chargino signal in this limit using the reaction $\mathrm{e}^{+} \mathrm{e}^{-} \rightarrow \gamma \widetilde{\chi}_{1}^{+} \widetilde{\chi}_{1}^{-}$. In some cases, in particular, those from AMSB, the $\tilde{\chi}_{1}^{ \pm}$has a long enough lifetime that, at the linear collider, one can see the chargino's track in the vertex detector before it decays. One then observes a stiff track turning into a very soft track, which would be a dramatic signal.

Table 4.1 makes clear that it is possible to measure the first-generation slepton masses with a precision of about $1 \%$. This would allow experiments at linear colliders to probe the underlying GUT-scale universality of intra-generation slepton masses, with enough sensitivity to discriminate the MSUGRA framework from other models (e.g., gaugino-mediation) where small GUT-scale splittings of sleptons are expected [180]. Another important observation from Table 4.1 is that the linear collider measurements of SUSY particles will provide multiple high-accuracy measurements of the mass of the lightest neutralino $\widetilde{\chi}_{1}^{0}$. As we will discuss in Section 7, this information will directly complement supersymmetry measurements at the LHC, since this key parameter will not be well determined there.

\subsection{Measurement of supersymmetry parameters}

Once superpartners are identified and their masses are measured, it is important to convert the mass and cross section information into determinations of the parameters of the SUSY theory. For the example of the MSSM with R-parity conservation, studies have been done to determine how well one can measure the fundamental parameters. By studying the production and subsequent decays of $\tilde{\chi}_{1}^{ \pm}$and $\tilde{\chi}_{2}^{ \pm}$, the masses and 
the gaugino-higgsino mixing angles of these states can be measured and hence the values of the MSSM parameters $M_{2}, \mu$, and $\tan \beta$ can be determined to about $1 \%$ accuracy $[155,181,182]$. This is illustrated in Fig. 4.7, where it is shown that the value of the chargino production cross section from a right-handed polarized beam allows one to map out whether the lighter chargino is mainly gaugino or Higgsino. A measurement of both the cross section and the angular distribution allows one to measure all of the terms in the chargino mass matrix. It should be noted that the figure shows the tree-level cross section. A true determination of parameters to $1 \%$ accuracy should take account of electroweak and SUSY radiative corrections.

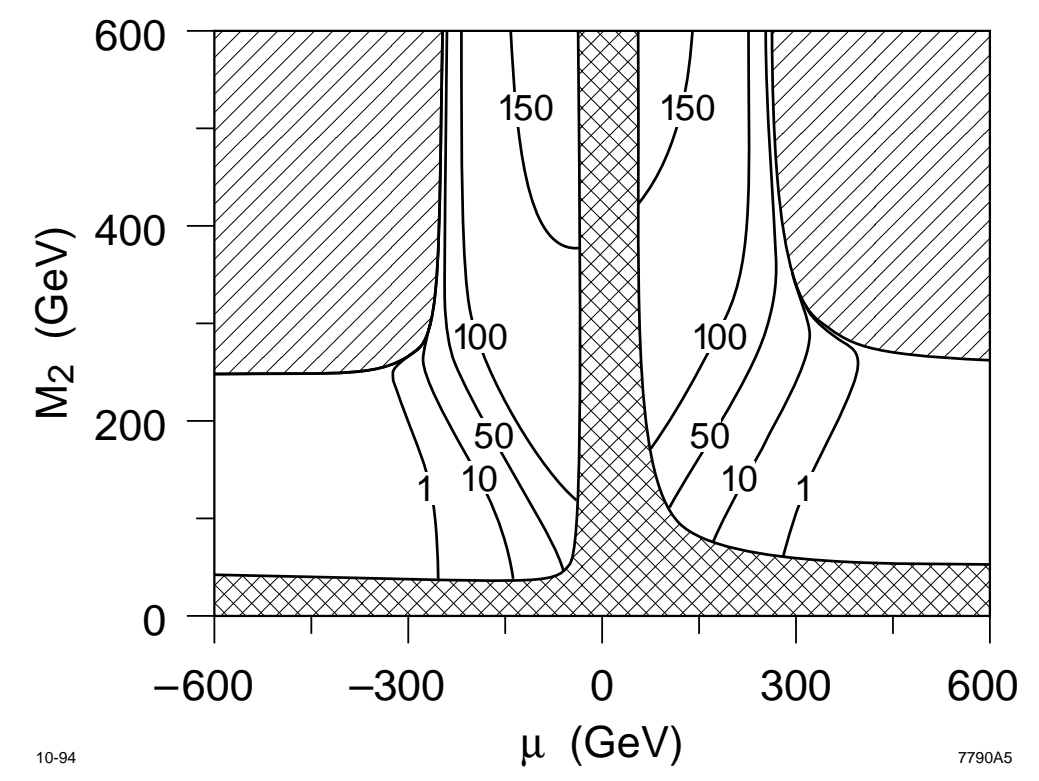

Figure 4.7: The dependence of the chargino production cross section $\sigma\left(e_{R}^{-} e^{+} \rightarrow \widetilde{\chi}_{1}^{+} \tilde{\chi}_{1}^{-}\right)$, in $\mathrm{fb}$, on $\mathrm{M}_{2}$ and $\mu$ [155]. The value $\tan \beta=4$ is used for this plot, but the result is only weakly dependent on this parameter.

Another method for determining whether the lightest neutralinos and chargino are mostly gaugino or Higgsino is to study slepton pair production with left-handed and right-handed beam polarization. This is done by measuring the magnitude of the cross section and the shape of the production angular distribution [154]. Similarly, measuring the cross sections of $\widetilde{t}_{1}, \widetilde{t}_{2}, \widetilde{\tau}_{1}^{ \pm}$and $\widetilde{\tau}_{2}^{ \pm}$and $\bar{\nu}_{\tau}$ with polarized beams allows one to determine their mixing angles [183-185]. Additional measurements associated with polarization in $\widetilde{\tau}$ reactions are discussed in $[154,186]$.

By looking at the angular distributions of supersymmetric particles that have a $t$-channel exchange involving another supersymmetric particle, the mass of the exchanged particle can be determined. Similarly, if the decays of the charginos have 
Supersymmetry Studies at the Linear Collider

three-body decays because the two-body decay to $W \widetilde{\chi}_{1}^{0}$ is not allowed kinematically, decays via $W^{*}$ can interfere with decays involving a virtual slepton or squark. This could give useful indirect signals for these particles in the cases where they cannot be produced because they are too heavy [187].

We should recall that the parameter $\tan \beta$ can be determined not only from supersymmetry reactions but also by direct experimental studies of the extended Higgs sector. For $\tan \beta<30$, one can obtain an accurate value of this parameter by measuring the branching ratios for the various possible decays of the SUSY Higgs particles: $H^{-}$into $\tau \nu, b \bar{t}$, and $W^{-} h$, and $A^{0}$ and $H^{0}$ into $\tau^{+} \tau^{-}, b \bar{b}, t \bar{t}$, and $Z h[188,189]$. If the Higgs sector is heavy enough, one can include decays into lighter supersymmetric particles. These can provide quite sensitive measurements in the high-tan $\beta$ region [189].

Finally, it is important to verify the spin of each supersymmetric partner experimentally. This can be done at a linear collider, because the simplicity of the production reactions often makes the spin obvious from the angular distributions. For example, the $\widetilde{\mu}_{R}$ signal in Fig. 4.6 exhibits a $\sin ^{2} \theta$ distribution that is a clear indication that the spin of the $\widetilde{\mu}_{R}$ is 0 . The spin of supersymmetric particles can also be determined by measuring the pair-production cross section near threshold, which rises as $\beta$ and $\beta^{3}$, where $\beta$ is the particle velocity, for spin- $\frac{1}{2}$ and spin- 0 particles, respectively.

\section{Tests of supersymmetry}

If new particles are discovered with quantum numbers expected in supersymmetry, it is desirable to determine whether they are in fact superparticles. Linear colliders can verify supersymmetry through highly model-indepedent tests accurate at the percent level. In addition, since these tests are sensitive to loop-level effects, they may yield a wealth of additional information.

Supersymmetry may be tested in many ways. For example, confirmation that some of the newly discovered particles are scalars, as discussed at the end of Section 3 , constitutes an important, if weak, test of supersymmetry. More quantitatively, verification of the consistency of direct discoveries with the expected indirect supersymmetric effects in SM processes, as discussed in Chapter 8, Section 3, also provides a test of supersymmetric interpretations of new physics. Measurements of the mass differences between scalar partners in the same $\mathrm{SU}(2)$ doublet may also provide quantitative and rather model-independent checks.

In this section we focus on investigations of supersymmetric coupling relations, which are among the most incisive and model-independent tests. In addition to providing precise quantitative confirmation of supersymmetry, such tests may also shed light on otherwise inaccessible superpartners, much as current precision electroweak measurements bound the Higgs boson mass and constrain new physics. 


\subsection{Confirming supersymmetry}

If supersymmetry were an exact symmetry of nature, the properties of supersymmetric particles would be completely determined by the properties of their SM partners. Of course, relations between masses are broken by soft supersymmetry breaking parameters. However, supersymmetry also predicts the equivalence of dimensionless couplings. For example, supersymmetry implies

$$
g_{i}=h_{i},
$$

where $g_{i}$ are the SM gauge couplings, $h_{i}$ are their supersymmetric analogues, the gaugino-fermion-sfermion couplings, and the subscript $i=1,2,3$ refers to the $\mathrm{U}(1)$, $\mathrm{SU}(2)$, and SU(3) gauge groups, respectively. These identities are not broken by soft supersymmetry-breaking parameters at tree level and are therefore known as "hard supersymmetry relations" [190]. They are valid in all supersymmetric theories, in contrast to other predictions such as the universality of scalar or gaugino masses. Hard supersymmetry relations therefore provide, in principle, a model-independent method of quantitatively confirming that newly-discovered particles are indeed superpartners $[155,183]$.

\subsection{Super-oblique corrections}

At the loop-level, however, even hard supersymmetry relations receive corrections that would vanish in the supersymmetric limit [191]. These corrections are analogous to the oblique corrections [192] of the Standard Model. In the SM, SU(2) multiplets with custodial $\mathrm{SU}(2)$-breaking masses, such as the $(t, b)$ multiplet, induce splittings in the couplings of the $(W, Z)$ vector multiplet at the quantum level. Similarly, in supersymmetric models, supermultiplets with soft supersymmetry-breaking masses, such as the $(\tilde{f}, f)$ supermultiplets, induce splittings in the couplings of the (gauge boson, gaugino) vector supermultiplet at the quantum level. This analogy can be made very precise [193-196]. Corrections to hard supersymmetry relations are therefore called 'super-oblique corrections', and the splittings are typically written in terms of 'super-oblique parameters.'

If some scalar superpartners $\tilde{f}$ have masses at a high scale $M$, and all others are light with mass $m \sim M_{\text {weak }}$, the super-oblique parameters are given by

$$
\widetilde{U}_{i} \equiv \frac{h_{i}(m)}{g_{i}(m)}-1 \approx \frac{g_{i}^{2}(m)}{16 \pi^{2}} \Delta b_{i} \ln \frac{M}{m},
$$

where $\Delta b_{i}$ is the one-loop $\beta$-function coefficient contribution from all light particles whose superpartners are heavy. Equation (4.4) is the leading logarithm contribution to $\widetilde{U}_{i}$. The super-oblique parameters for some representative models are given in Table 4.2. The super-oblique parameters may also receive contributions from split exotic supermultiplets, such as the messengers of gauge-mediation $[193,196]$. 
Supersymmetry Studies at the Linear Collider

\begin{tabular}{cccc}
\hline \hline & $\tilde{U}_{1}$ & $\tilde{U}_{2}$ & $\tilde{U}_{3}$ \\
\hline 2-1 Models & $0.35 \% \times \ln (M / m)$ & $0.71 \% \times \ln (M / m)$ & $2.5 \% \times \ln (M / m)$ \\
Heavy QCD Models & $0.29 \% \times \ln (M / m)$ & $0.80 \% \times \ln (M / m)$ & - \\
\hline \hline
\end{tabular}

Table 4.2: The super-oblique parameters $\widetilde{U}_{i}$ in two representative models: ' $2-1$ Models,' with all first and second generation sfermions at the heavy scale $M$, and 'Heavy QCD Models,' with all squarks and gluinos at the heavy scale.

From Eq. (4.4) we see that, although super-oblique parameters are one-loop effects, they may be greatly enhanced if many states are heavy (large $\Delta b_{i}$ ). They also grow logarithmically with $M / m$ : super-oblique parameters are non-decoupling, and so are sensitive to particles with arbitrarily high mass. As noted in Section 2, the squarks and sleptons of the first and second generations are only loosely bounded by finetuning arguments. They may have masses far beyond the reach of the LHC, and in fact, such massive squarks and sleptons considerably ameliorate many supersymmetric flavor and $\mathrm{CP}$ problems. In these cases, the super-oblique parameters are large and provide a rare window on these heavy scalars.

\subsection{Measurements at linear colliders}

With respect to super-oblique parameters, the program at a linear collider consists of two parts: First, one would like to verify as many hard supersymmetry relations as possible to determine that newly-discovered particles are in fact superparticles. Second, if new particles are determined to be supersymmetric, small violations of hard supersymmetry relations may provide the first evidence for as-yet-undiscovered superparticles. Precise measurements of the super-oblique parameters may constrain the mass scales of these superparticles.

The experimental observables that are dependent on super-oblique parameters have been exhaustively categorized in [194] for both lepton and hadron colliders. The most promising observables at colliders are cross sections and branching ratios involving gauginos, and several of these possibilities have been examined in detailed studies. The potential of linear colliders is, of course, highly dependent on the supersymmetry scenario realized in nature, but we present a brief synopsis below.

To date, all studies have used tree-level formulae in which the gaugino couplings are allowed to vary. Constraints on these gaugino couplings are then interpreted as measurements of super-oblique parameters. At the level of precision required, however, it will ultimately be necessary to make a detailed comparison of cross sections and other observables with full one-loop predictions. In chargino pair production, for example, studies of triangle [197-199] and box [200] contributions have been shown to be important. In addition, beam polarization may enhance the effect of quantum 
corrections [198]. To extract the non-decoupling effects of very heavy superpartners, one must therefore control many other effects, including all other virtual effects, either by including data from direct detection, or by verifying that such effects are sufficiently suppressed to be negligible. The study of super-oblique parameters should be viewed as the first step in the complete program of one-loop SUSY studies that will be possible at a linear collider.

Potential super-oblique parameter measurements at a linear collider should include:

- Measurements of $\widetilde{U}_{1}$. Selectron pair production at electron colliders includes a contribution from $t$-channel gaugino exchange. In particular, in the reaction $e^{+} e^{-} \rightarrow \tilde{e}_{R}^{+} \tilde{e}_{R}^{-}$, its dependence upon the $\tilde{B} e \tilde{e}$ coupling $h_{1}$ has been studied in [183]. Under the assumption that the selectrons decay through $\tilde{e} \rightarrow e \tilde{B}$, the selectron and gaugino masses may be measured through kinematic endpoints. Combining this information with measurements of the differential cross section, $\widetilde{U}_{1}$ may be determined to $\sim 1 \%$ with $20 \mathrm{fb}^{-1}$ of data at $\sqrt{s}=500 \mathrm{GeV}$.

This high-precision measurement may be further improved by considering the process $e^{-} e^{-} \rightarrow \tilde{e}_{R}^{-} \tilde{e}_{R}^{-}$. This process is made possible by the Majorana nature of gauginos. Relative to the $e^{+} e^{-}$process, this reaction benefits from large statistics for typical supersymmetry parameters and extremely low backgrounds, especially if the electron beams are right-polarized. Depending on experimental systematic errors, determinations of $\widetilde{U}_{1}$ at the level of $0.3 \%$ may be possible with integrated luminosities of $50 \mathrm{fb}^{-1}$ [194].

- Measurements of $\widetilde{U}_{2}$. Chargino pair production has a dependence on $\widetilde{U}_{2}$ at lepton colliders through the $\tilde{\nu}$ exchange amplitude. This process was first studied as a way to verify hard supersymmetry relations [155]. In [194], estimates of 2-3\% uncertainties for $\widetilde{U}_{2}$ were obtained from pair production of $172 \mathrm{GeV}$ charginos with $\sqrt{s}=400-500 \mathrm{GeV}$. These results are conservative, and are improved in most other regions of parameter space [197]. Dramatic improvements may also be possible if both charginos are within kinematic reach and large luminosities with polarized beams are available, a scenario studied in [201].

The process $e^{+} e^{-} \rightarrow \tilde{\nu}_{e} \tilde{\nu}_{e}$ also depends on $\widetilde{U}_{2}$ through the $t$-channel chargino exchange amplitude. With a data sample of $100 \mathrm{fb}^{-1}, \widetilde{U}_{2}$ may be determined to $\sim 0.6 \%$ [195].

- Measurements of $\widetilde{U}_{3}$. The strong super-oblique parameter may be measured through processes involving squarks. The squark pair-production cross sections at lepton colliders are independent of super-oblique corrections, but the three-body production processes, such as $\tilde{t} t \tilde{g}$ and $\tilde{b} b \tilde{g}$, have been suggested as a probe $[194,196]$. 
Supersymmetry Studies at the Linear Collider

Squark branching ratios are also sensitive to super-oblique corrections if there are two or more competing modes [190]. In [194], parameters were studied in which the two decays $\tilde{b}_{L} \rightarrow b \tilde{g}$ and $\tilde{b}_{L} \rightarrow b \tilde{W}$ were open. For parameters where the gluino decay is suppressed by phase space, these modes may be competitive, and measurements of the branching ratios yield constraints on $\widetilde{U}_{3}$. For example, for $m_{\tilde{b}_{L}}=300 \mathrm{GeV}, \tilde{b}_{L}$ pair production at a $\sqrt{s}=1 \mathrm{TeV}$ collider with integrated luminosity $200 \mathrm{fb}^{-1}$ yields measurements of $\widetilde{U}_{3}$ at or below the $5 \%$ level for $10 \mathrm{GeV} \lesssim m_{\tilde{b}_{L}}-m_{\tilde{g}} \lesssim 100 \mathrm{GeV}$. These measurements are typically numerically less stringent than those discussed above, but the $\mathrm{SU}(3)$ super-oblique correction is also larger by a factor $\alpha_{s} / \alpha_{w}$.

- Measurements of Wino-Higgsino mixing. The presence of the $W$ boson mass in the tree-level chargino mixing matrix is also a consequence of supersymmetry (relating the $W W h$ and $\tilde{W} \tilde{h} h$ couplings). Wino-Higgsino mixing receives non-decoupling corrections, and may be constrained through chargino pair production $[155,197]$.

- Measurements of trilinear gaugino/gauge boson couplings. Finally, the supersymmetric equivalence of triple gauge boson and gaugino couplings may also be broken. In [202], splittings of the $W W \gamma$ and $W \tilde{W} \tilde{\gamma}$ couplings were calculated and found to be present at the few-percent level. Such splittings could be probed in $\tilde{W} \rightarrow W \tilde{\gamma}$ decays.

These studies demonstrate the promise of linear colliders for loop-level studies of supersymmetry. If charginos or sleptons are produced at linear colliders, precision tests will be able to verify that their couplings are as predicted by supersymmetry to the percent level. In addition, small corrections to these relations are sensitive to arbitrarily heavy superpartners, and, if some superpartners are kinematically inaccessible, precise determination of the super-oblique parameters may provide a target mass range for future searches.

\section{$5 \quad$ Symmetry violating phenomena}

\section{$5.1 \quad \mathrm{R}$-parity violation}

Up to this point we have considered only R-parity $\left(\mathrm{R}_{\mathrm{p}}\right)$-conserving supersymmetric theories. $\mathrm{R}_{\mathrm{p}}$ is a multiplicative discrete symmetry [203-206] defined for each particle to be

$$
\mathrm{R}_{\mathrm{p}}=(-1)^{3 B+L+2 S}
$$

where $B$ is baryon number, $L$ is lepton number, and $S$ is the particle's spin. This symmetry is not automatic in the MSSM as it is in the SM. We now consider the possibility that the symmetry is not respected [207]. 
Without $R_{p}$ conservation, the most general gauge-invariant and Lorentz-invariant superpotential is

$$
\begin{aligned}
W= & \mu H_{u} H_{d}+y_{i j}^{e} H_{d} L_{j} e_{k}^{c}+y_{j k}^{d} H_{d} Q_{j} d_{k}^{c}+y_{j k}^{u} H_{u} Q_{j} u_{k}^{c} \\
& +\lambda_{i j k} L_{i} L_{j} e_{k}^{c}+\lambda_{i j k}^{\prime} L_{i} Q_{j} d_{k}^{c}+\lambda_{i j k}^{\prime \prime} u_{i}^{c} d_{j}^{c} d_{k}^{c}+\mu_{i} H_{u} L_{i} .
\end{aligned}
$$

The $\lambda$ - and $\lambda^{\prime}$ - terms do not respect lepton number and the $\lambda^{\prime \prime}$-terms do not respect baryon number. Proton decay is unacceptably rapid if all terms are allowed without extreme suppressions; this requires $\lambda^{\prime} \lambda^{\prime \prime} \lesssim 10^{-36}$. But, since proton decay requires both lepton and baryon number violation, it is possible to escape this constraint by forbidding one or the other of lepton number violation or baryon number violation. That is, the constraint on $\lambda^{\prime} \lambda^{\prime \prime}$ can be accomodated by setting $\lambda^{\prime}=0$ (lepton number conservation) or $\lambda^{\prime \prime}=0$ (baryon number conservation). The $\mu_{i}$ terms also violate lepton number conservation, although these terms can be defined away at tree level.

In the next few paragraphs, we will describe the signals expected at a $500 \mathrm{GeV}$ linear collider for a theory with non-zero $\lambda$ as the only $\mathrm{R}_{\mathrm{p}}$-violating couplings. We will then reanalyze the same theory but this time with only non-zero $\lambda^{\prime}$ couplings, and finally with only non-zero $\lambda^{\prime \prime}$ couplings. We further assume that the $\mathrm{R}_{\mathrm{p}}$-violating couplings are too weak to participate in observables in any way except to allow the lightest neutralino to decay promptly in the detector. Making the couplings stronger usually implies even more phenomena by which to discover supersymmetry (additional production modes via $R_{p}$ violation). Making the couplings very weak will cause the phenomenology to asymptotically approach that of the MSSM with $\mathrm{R}_{\mathrm{p}}$ conservation.

When applicable, we will illustrate phenomena with model $\mathrm{E}$ of [208], which is the heaviest superpartner model considered in this paper. This model assumes $M_{2}=2 M_{1}=200 \mathrm{GeV}, \mu=-250 \mathrm{GeV}, \tan \beta=20$, and $m_{\tilde{e}_{L}}=m_{\tilde{e}_{R}}=200 \mathrm{GeV}$. The chargino masses are then 173.4 and $292.1 \mathrm{GeV}$, and the neutralino masses are 97.7, 173.6, 260.8, and $290.1 \mathrm{GeV}$.

\subsection{1 $\lambda_{L L e^{c}} \neq 0$}

In these theories the LSP always decays into two charged leptons and a neutrino (missing energy):

$$
\tilde{\chi}_{1}^{0} \rightarrow \ell^{+}+\ell^{-}+\not \mathbb{E} .
$$

When superpartners are produced in pairs, they will cascade-decay down to two LSPs (plus SM jets or leptons), and the LSPs will then decay into two leptons plus missing energy. Therefore, the signal always includes at least four leptons plus missing energy, and quite often contains more leptons and additional jets from the cascades. This is a spectacular signature that will not go unnoticed. For example, the cross section for the $4 l+E_{T}$ signature for our considered example model is approximately $274 \mathrm{fb}$, much higher than the expected $0.4 \mathrm{fb}$ background rate [208]. 


\subsection{2 $\lambda_{L Q d^{c}}^{\prime} \neq 0$}

In these theories the LSP always decays into two jets with an accompanying charged lepton or neutrino:

$$
\tilde{\chi}_{1}^{0} \rightarrow l^{ \pm} q \bar{q}^{\prime} \quad \text { or } \quad \nu q \bar{q} .
$$

All supersymmetry signals must pass through $\tilde{\chi}_{1}^{0} \tilde{\chi}_{1}^{0}+X_{\mathrm{SM}}$, where $X_{\mathrm{SM}}$ represents SM states (jets, leptons, or neutrinos) arising from the cascade decays of the produced parent superpartners. In this case the final-state signatures of all superpartner production processes will be

$$
(0,1 \text {, or } 2 \text { leptons })+4 \text { jets }+X_{S M} .
$$

Furthermore, all events that do not have 2 leptons will have some missing energy in them from escaping neutrinos.

Many of the signal events of this type of $R_{p}$ violation will be swamped by backgrounds. The two most promising modes to search are $3 l$ and $4 l$ final states, where at least one additional lepton comes from the cascade products in $X_{\mathrm{SM}}$. Another intriguing possibility is to search for like-sign dilepton events. This signature is made possible by each independent $\tilde{\chi}_{1}^{0}$ decaying into a lepton of either positive or negative charge. Approximately one-eighth of the $\tilde{\chi}_{1}^{0} \tilde{\chi}_{1}^{0}$ decays end in like-sign dileptons. The background in this case is very small whether $X_{\mathrm{SM}}$ contains leptons or not. Furthermore, it appears that the LSP mass may be obtainable by analyzing the invariant mass distribution of the hardest lepton combined with all hadronic jets in the same hemisphere [208].

\subsection{3 $\quad \lambda_{u^{c} d^{c} d^{c}}^{\prime \prime} \neq 0$}

In these theories the LSP always decays into three jets:

$$
\tilde{\chi}_{1}^{0} \rightarrow q^{\prime} q \bar{q} .
$$

All supersymmetry events will then have at least six jets from LSP decays in the final state plus the cascade decay products of the parent sparticles. Although jet reconstruction algorithms will generally not resolve all six jets, they will usually register at least three in the event [209].

Perhaps the most important signature for discovery in these theories comes from chargino pair production, where each chargino decays as $\tilde{\chi}_{1}^{ \pm} \rightarrow l^{ \pm} \nu \tilde{\chi}_{1}^{0}$. The final state will then be 2 leptons plus many jets. Unfortunately the lepton often finds itself inside one of the many hadronic jets and fails the isolation requirements. Nevertheless, the rate is sufficiently large that it is a viable signal for our example model. According to [208], the signal in this mode - including also the smaller contribution from $\tilde{\chi}_{i}^{0} \tilde{\chi}_{j}^{0}$ 
production - is approximately $40 \mathrm{fb}$ compared to a background of $243 \mathrm{fb}$. A moderate luminosity of $10 \mathrm{fb}^{-1}$ would produce a $S / \sqrt{B}$ significance greater than 8 .

To determine the LSP mass, one can use strategies similar to ALEPH's four-jet analysis [210] to combine jets within same hemispheres to look for matching invariant mass peaks. Careful comparisons with background have not yet been performed to see how accurately the LSP mass can be extracted with this technique.

\subsection{4 $\mu_{i} \neq 0$}

The parameter space with just $\mu_{i} \neq 0$ is often called Bilinear R-Parity Violation $\left(\mathrm{BR}_{\mathrm{p}} \mathrm{V}\right)$. It has special theoretical motivations in supersymmetry [211-214]. One interesting phenomenological feature of the model is its ability to predict the three neutrino masses and the three mixing angles by adding to the MSSM only one or two extra parameters. This is done in a SUGRA context with radiative electroweak symmetry breaking and universality of soft parameters at the GUT scale [215]. At tree level, one neutrino acquires a mass from neutrino-neutralino mixing. The masslessness and degeneracy of the other two neutrinos is lifted at one loop, giving masses and mixings that account for the solar and atmospheric neutrino anomalies [216-219]. The parameters of the model can be measured from the leptonic branching fractions of the lightest neutralino $[219,220]$. Thus, in this model, crucial information needed to understand neutrino physics comes from experiments at the linear collider.

\subsection{Lepton flavor violation}

A linear collider enables the careful study of flavor physics in supersymmetry. With the apparent confirmation of neutrino masses, non-trivial lepton-slepton flavor angles are assumed to exist. There are constraints on the magnitude of these angles from $B(\mu \rightarrow e \gamma)$ bounds, for example. However, the constraints are weaker if the sleptons are nearly degenerate in mass. We will make this assumption here, thereby invoking a super-GIM suppression to suppress the radiative flavor-violating lepton decays.

Direct production of sleptons and close scrutiny of their decays allow probing of these flavor angles at more sensitive levels [221-226]. The nearly degenerate sleptons will undergo flavor oscillation after being produced and then decay quickly. Analogous to neutrino oscillations, the detectability of slepton oscillations is best characterized in the $\left(\sin 2 \theta, \Delta m^{2}\right)$ plane, where $\theta$ is the angle between the weak eigenstates $|\tilde{e}\rangle,|\tilde{\mu}\rangle$ and the mass eigenstates $|1\rangle,|2\rangle$ :

$$
\begin{array}{r}
|\tilde{e}\rangle=+\cos \theta|1\rangle+\sin \theta|2\rangle \\
|\tilde{\mu}\rangle=-\sin \theta|1\rangle+\cos \theta|2\rangle .
\end{array}
$$




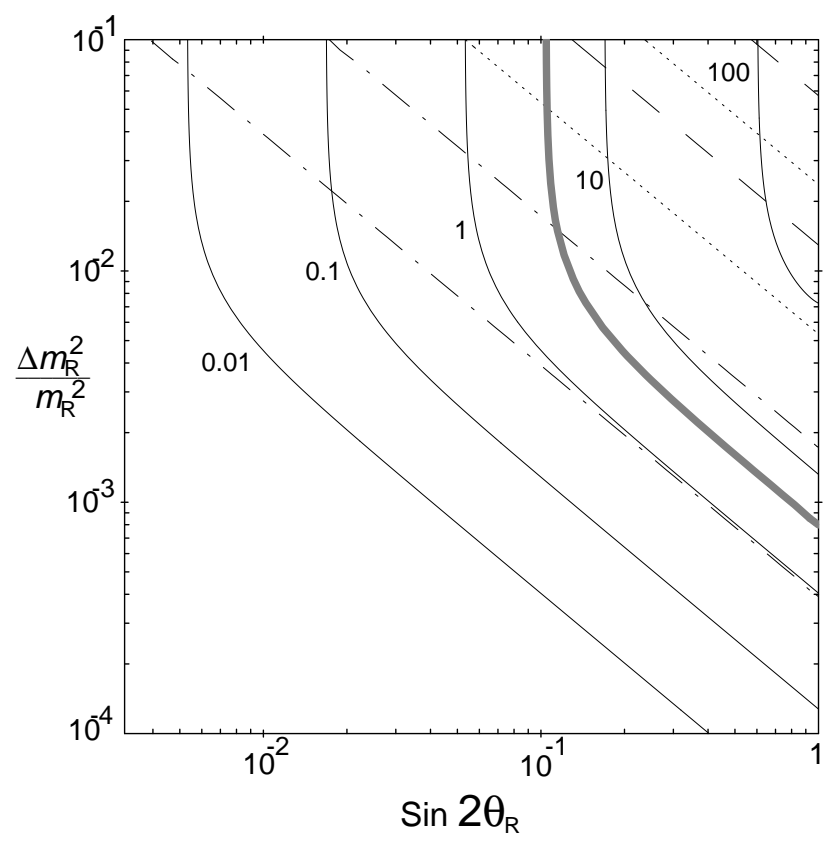

Figure 4.8: Contours of constant $\sigma\left(e^{+} e^{-} \rightarrow e^{ \pm} \mu^{\mp} \tilde{\chi}_{1}^{0} \tilde{\chi}_{1}^{0}\right)$ in fb at a $\sqrt{s}=500 \mathrm{GeV} e^{+} e_{R}^{-}$ collider. The signal arises from right-slepton production and subsequent decay to lepton plus lightest neutralino. The $\tilde{\ell}_{R}$ masses are approximately $200 \mathrm{GeV}$ and the lightest neutralino is a Bino with mass $100 \mathrm{GeV}$. The thick gray contour represents optimal experimental reach with $50 \mathrm{fb}^{-1}$ integrated luminosity. The straight lines (dotted and dashed) represent contours of constant $B(\mu \rightarrow e \gamma)$. These depend on additional parameters such as the $\tilde{\ell}_{L}$ mass and the off-diagonal entries of the slepton mass matrix. See [221] for more details.

Figure 4.8 shows contours of constant $\sigma\left(e^{+} e^{-} \rightarrow e^{ \pm} \mu^{\mp} \tilde{\chi}_{1}^{0} \tilde{\chi}_{1}^{0}\right)$, in $\mathrm{fb}$, at a $\sqrt{s}=500$ $\mathrm{GeV}$ collider with $e^{+} e_{R}^{-}$collisions. The signal arises from $\widetilde{\ell}_{R}$ production and subsequent decay to a lepton plus the lightest neutralino. The $\tilde{\ell}_{R}$ masses are approximately $200 \mathrm{GeV}$, and the lightest neutralino is a Bino with mass $100 \mathrm{GeV}$. From this figure we can see that careful measurement of the cross section enables probing of flavorviolating couplings to very small mass splitting and mixing angle.

\subsection{CP violation}

The new mass parameters associated with supersymmetry may not all be real, and could lead to CP violation effects [227] at high-energy colliders. The parameters $\mu, M_{1}$ and $M_{2}$ can in general be complex. By rotating the phases of the gauginos we are free to choose $M_{2}$ real, leaving us with

$$
\mu=|\mu| e^{i \phi_{\mu}} \quad \text { and } \quad M_{1} \rightarrow\left|M_{1}\right| e^{i \phi_{1}}
$$


In addition to these phases, each of the tri-scalar $A$ terms connecting the Higgs bosons with left and right scalar superpartners of the fermions can in principle have its own independent phase.

Generic $\mathcal{O}(1)$ phases associated with superpartner masses near the weak scale are ruled out by the electric dipole moments (EDMs) of the neutron and electron if superpartners are light enough to be accessible at a $1 \mathrm{TeV}$ linear collider. Therefore, we assume here that the phases must be small, $\mathcal{O}(0.1)$. We remark that tuned cancellations [228,229] may allow $\mathcal{O}(1)$ phases for light superpartners, thereby leading to effects much larger than the estimates given below.

Supersymmetric CP-violating phases have two important effects: they disrupt the relations among CP-conserving observables, and they give birth to non-zero CPviolating observables. Much work has gone into both types of analyses. For example, CP-violating observables in $e^{+} e^{-} \rightarrow t \bar{t}$ may be the most promising way to find actual $\mathrm{CP}$ violation effects at the linear collider. We refer the reader to [230-232] for a comprehensive review of this subject, and a description of the challenges facing experiment to confirm CP-violating effects. Here, we briefly focus on the effects that small phases have on CP-conserving observables.

Recently several groups have shown how CP-violating phases affect almost all interesting MSSM observables at a linear collider [233-235,181,236]. For example, the chargino mass eigenstates depend non-trivially on the phase of $\mu$ :

$$
m_{\tilde{\chi}_{1,2}^{ \pm}}^{2}=\frac{1}{2}\left[M_{2}^{2}+|\mu|^{2}+2 m_{W}^{2} \mp \Delta_{C}\right],
$$

where

$$
\begin{aligned}
\Delta_{C}= & {\left[\left(M_{2}^{2}-|\mu|^{2}\right)^{2}+4 m_{W}^{4} \cos ^{2} 2 \beta+4 m_{W}^{2}\left(M_{2}^{2}+|\mu|^{2}\right)\right.} \\
& \left.+8 m_{W}^{2} M_{2}|\mu| \sin 2 \beta \cos \Phi_{\mu}\right]^{1 / 2} .
\end{aligned}
$$

The effects of phases on observables have been illustrated in [236] with a reference model corresponding to an mSUGRA point with $m_{1 / 2}=200 \mathrm{GeV}, m_{0}=100 \mathrm{GeV}$, $A_{0}=0, \tan \beta=4$, and $\mu>0$. This parameter choice corresponds to the mass values $\left|M_{1}\right|=83 \mathrm{GeV}, M_{2}=165 \mathrm{GeV}, \mu=310 \mathrm{GeV}, m_{\tilde{e}_{L}}=180 \mathrm{GeV}, m_{\tilde{\nu}}=166 \mathrm{GeV}$, and $m_{\tilde{e}_{R}}=132 \mathrm{GeV}$. In Fig. 4.9, the effects of varying the phases $\phi_{1}$ and $\phi_{\mu}$ are demonstrated for several observables.

Motivated by the EDM constraints on the phases of supersymmetric mass parameters, the authors of [236] set $\phi_{\mu}=0$ and simulated how evidence for a small but non-zero $\phi_{1}$ phase would be extracted at a linear collider. They generated 10000 data sets, smeared with respect to the true values by experimental resolution. The input data included three cross sections $\left(\tilde{\chi}_{1}^{0} \tilde{\chi}_{2}^{0}, \tilde{\chi}_{2}^{0} \tilde{\chi}_{2}^{0}\right.$, and $\left.\tilde{\chi}_{1}^{ \pm} \tilde{\chi}_{1}^{\mp}\right)$ and three masses $\left(m_{\tilde{\chi}_{1}^{0}}, m_{\tilde{\chi}_{2}^{0}}\right.$, and $\left.m_{\tilde{\chi}_{1}^{ \pm}}\right)$. Figure 4.10 demonstrates the extraction of several different parameters, and their interdependence. For example, the bottom figures show the 

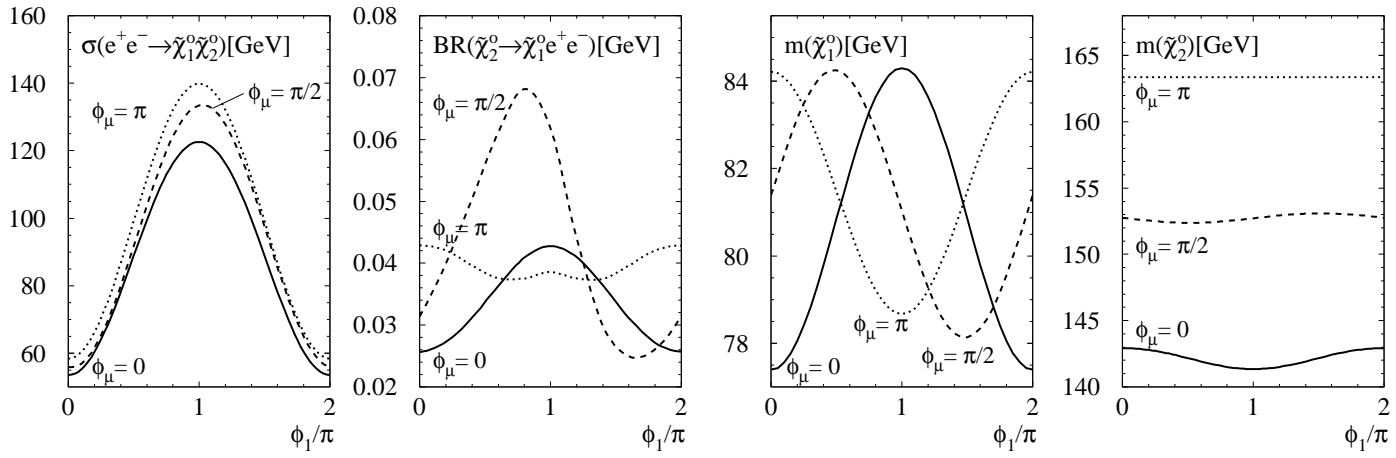

Figure 4.9: The effects on supersymmetry observables obtained by varying the phases $\phi_{1}$ and $\phi_{\mu}$ in the example model discussed in the text [236].
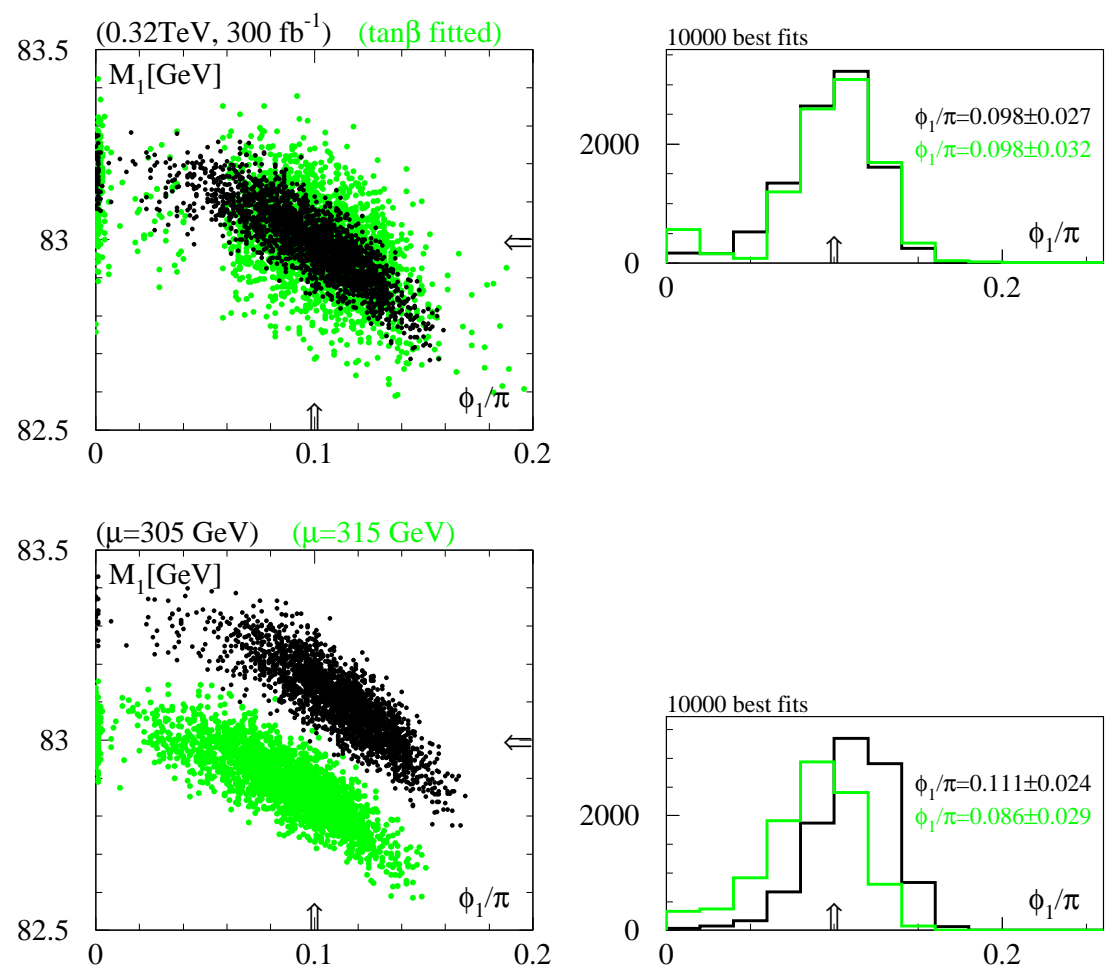

Figure 4.10: Demonstration of the interdependence of parameters in the extraction of CPviolating phases from linear collider SUSY observables [236]. 
systematic error one would encounter by having a wrong input for $|\mu|$ given a known $\tan \beta$. Perhaps the most interesting conclusion one can draw from this exercise is that $\phi_{1}=0$ is strongly disfavored, incicating that the linear collider measurements of $\mathrm{CP}$-conserving observables can give a strong signal for nonzero $\mathrm{CP}$-violating phases if they are present.

\section{Supersymmetry and $e^{-} e^{-}, e^{-} \gamma$, and $\gamma \gamma$ colliders}

\subsection{Supersymmetry and $e^{-} e^{-}$colliders}

The features of $e^{-} e^{-}$colliders are reviewed in Chapter 14. The unique quantum numbers of the $e^{-} e^{-}$initial state forbid the production of most superpartners. However, slepton pair production through $t$-channel neutralino exchange is always possible [237]. The opportunities at $e^{-} e^{-}$colliders for measurements of slepton masses, mixings, and couplings are unparalleled, and exploit many of the unique properties of $e^{-} e^{-}$colliders.

\subsubsection{Masses}

As reviewed in Section 2, masses at linear colliders are most accurately determined through kinematic endpoints and threshold scans. In $e^{+} e^{-}$mode, the threshold cross section for pair production of identical scalars rises as $\beta^{3}$, where $\beta$ is the velocity of the produced particles. Threshold studies for identical scalars are therefore far less effective than for fermions, and consequently require large investments of integrated luminosity [174].

At $e^{-} e^{-}$colliders, however, the same-helicity selectron pair production cross section has a $\beta$ dependence at threshold [238]. This is easily understood: the initial state in $e_{R}^{-} e_{R}^{-} \rightarrow \tilde{e}_{R}^{-} \tilde{e}_{R}^{-}$has angular momentum $J=0$, and so the selectrons may be produced in the $\mathrm{S}$ wave state. Cross sections for $\tilde{e}_{R}$ pair production in $e^{-} e^{-}$and $e^{+} e^{-}$ modes are compared in Fig. 4.11. For round beams, the increased beamstrahlung and decreased luminosity of the $e^{-} e^{-}$mode compromise this advantage. However, beamstrahlung is reduced for flat beams [239], and mass measurements of order 100 $\mathrm{MeV}$ can be achieved with two orders of magnitude less luminosity than required in $e^{+} e^{-}$collisions [240,241]. Incidentally, the full arsenal of linear collider modes allows one to extend this mass measurement to the rest of the first-generation sleptons through a series of $\beta$ threshold scans: $e^{-} e^{-} \rightarrow \tilde{e}_{R}^{-} \tilde{e}_{R}^{-}$yields $m_{\tilde{e}_{R}} ; e^{+} e^{-} \rightarrow \tilde{e}_{R}^{ \pm} \tilde{e}_{L}^{\mp}$ yields $m_{\tilde{e}_{L}} ; e^{+} e^{-} \rightarrow \tilde{\chi}_{1}^{+} \tilde{\chi}_{1}^{-}$yields $m_{\tilde{\chi}_{1}^{ \pm}}$; and $e^{-} \gamma \rightarrow \tilde{\nu}_{e} \tilde{\chi}_{1}^{-}$yields $m_{\tilde{\nu}_{e}}[242]$. The process $e^{-} e^{-} \rightarrow \tilde{e}_{R}^{-} \tilde{e}_{R}^{-}$may also be used to determine the Bino mass $M_{1}$ with high accuracy even for very large $M_{1}[238,241]$. 


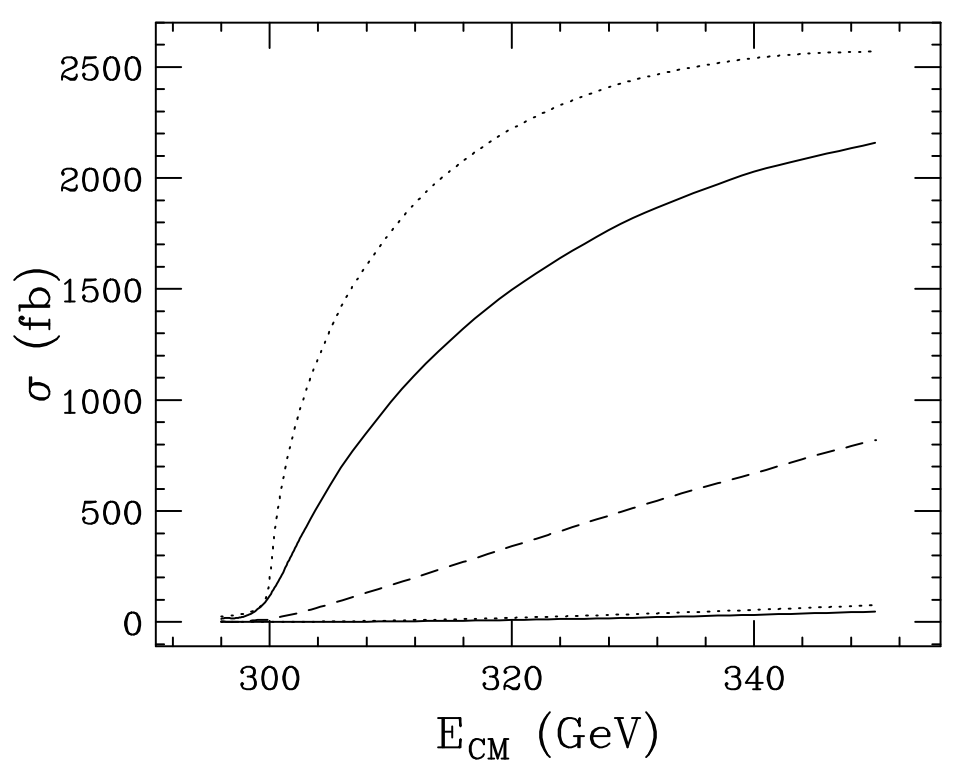

Figure 4.11: Threshold behavior for $\sigma\left(e^{-} e^{-} \rightarrow \tilde{e}_{R}^{-} \tilde{e}_{R}^{-}\right.$) (upper two contours) and $\sigma\left(e^{+} e^{-} \rightarrow\right.$ $\tilde{e}_{R}^{+} \tilde{e}_{R}^{-}$) (lower two contours) for $m_{\tilde{e}_{R}}=150 \mathrm{GeV}$ and $M_{1}=100 \mathrm{GeV}$ [240]. In each pair, the dotted curve neglects all beam effects, and the solid curve includes the initial state radiation, beamstrahlung, and beam energy spread for flat beams. Results for $e^{-} e^{-}$round beams (dashed) are also shown. The selectron width is included, and beam polarizations $P_{e^{-}}=0.8$ and $P_{e^{+}}=0$ are assumed.

\subsubsection{Mixings}

Now that neutrinos are known to mix, lepton flavor is no longer a perfect symmetry. Sleptons may also have inter-generational mixings. Such mixing leads to decays $\tilde{e} \rightarrow$ $\mu \tilde{\chi}_{1}^{0}, \tau \tilde{\chi}_{1}^{0}$ and may be searched for at either $e^{+} e^{-}$or $e^{-} e^{-}$colliders.

At $e^{+} e^{-}$colliders, the signal is $e^{+} e^{-} \rightarrow e^{ \pm} \mu^{\mp} \tilde{\chi}_{1}^{0} \tilde{\chi}_{1}^{0}, e^{ \pm} \tau^{\mp} \tilde{\chi}_{1}^{0} \tilde{\chi}_{1}^{0}$. The backgrounds are $e^{+} e^{-} \rightarrow W^{+} W^{-}, e^{+} e^{-} \rightarrow \nu \nu W^{+} W^{-}, e^{+} e^{-} \rightarrow e^{ \pm} \nu W^{\mp}$, and $\gamma \gamma \rightarrow W^{+} W^{-}$. The first two backgrounds may be reduced by $e_{R}^{-}$beam polarization; however, the last two are irreducible.

In the $e^{-} e^{-}$case, the signal is $e^{-} e^{-} \rightarrow e^{-} \mu^{-} \tilde{\chi}_{1}^{0} \tilde{\chi}_{1}^{0}, e^{-} \tau^{-} \tilde{\chi}_{1}^{0} \tilde{\chi}_{1}^{0}$. Among potential backgrounds, $e^{-} e^{-} \rightarrow W^{-} W^{-}$is forbidden by total lepton number conservation, $e^{-} e^{-} \rightarrow \nu \nu W^{-} W^{-}$and $e^{-} e^{-} \rightarrow e^{-} \nu W^{-}$may be suppressed by right-polarizing both $e^{-}$beams, and $\gamma \gamma \rightarrow W^{+} W^{-}$does not yield two like-sign leptons. As a result, the sensitivity of $e^{-} e^{-}$colliders to slepton flavor violation is much greater than at $e^{+} e^{-}$colliders, and probes regions of parameter space beyond current and near-future low-energy experiments searching for $\mu$-e and $\tau-e$ transitions [221,222]. 


\subsubsection{Couplings}

The excellent properties of $e^{-} e^{-}$colliders are also ideal for exploring selectron gauge

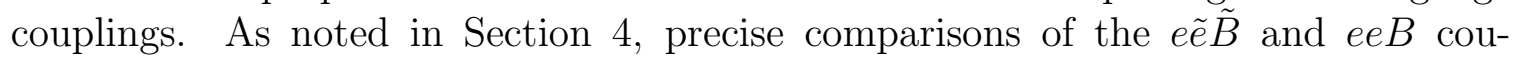
plings provide a model-independent test of supersymmetry. The eẽ $\tilde{B}$ coupling is a non-decoupling observable sensitive to arbitrarily heavy superpartners. The nearly background-free environment of $e^{-} e^{-}$colliders makes possible extremely precise measurements of selectron couplings, surpassing those available at $e^{+} e^{-}$colliders [194], and may help set the scale for far-future colliders in scenarios where some superpartners are extremely heavy.

\subsection{Supersymmetry and $e^{-} \gamma$ colliders}

Even if several neutralinos and charginos have light masses such that they can be produced in pairs at the LC, the sleptons might be above threshold for pair production in $e^{+} e^{-}$collisions. In this case, the sleptons may be accessible in the $e^{-} \gamma$ colliding option in the single-slepton plus lighter-neutralino final state $\tilde{\chi}_{i}^{0} \tilde{e}_{L, R}$.

This reaction was studied in $[243,244,242]$. For example, the parameters chosen in [242] lead to the masses: $m_{\tilde{\chi}_{1}^{0}}=65 \mathrm{GeV}, m_{\tilde{\chi}_{1}^{ \pm}}=136 \mathrm{GeV}, m_{\tilde{e}_{L}}=320 \mathrm{GeV}$, $m_{\tilde{e}_{R}}=307 \mathrm{GeV}$, and $m_{\tilde{\nu}_{e}}=315 \mathrm{GeV}$. With these values, pair production of charginos is accessible at a $500 \mathrm{GeV}$ linear collider but slepton pair production is not.

Figure 4.12 shows the cross sections for slepton-neutralino production as a function of the $e^{-} \gamma$ center-of-mass energy for the four different helicity combinations of the incoming electron and photon. The cross section for $\tilde{e}_{R} \tilde{\chi}_{1}^{0}$ in the $(+,+)$ helicity combination is sharply peaked at center-of-mass energies not far from the threshold. The signal for this process is $e^{-}$plus missing energy. The background [243,242] has a cross section of a few picobarns and mainly arises from $W^{-} \nu \rightarrow e^{-} \nu \nu$. This background can be reduced dramatically by using a polarized $e_{R}^{-}$beam. With the above parameters, using polarization and a few judicious kinematic cuts on the final state particles, the slepton can be discovered and studied. It has been estimated that both the slepton and sneutrino masses can be measured to about $1 \%$ accuracy.

\subsection{Supersymmetry at $\gamma \gamma$ colliders}

One of the main motivations for the $\gamma \gamma$ collider option is to study direct single Higgs production through the $\gamma \gamma h$ coupling. This motivation is especially powerful in supersymmetry since most versions of the theory predict a Higgs boson below about $135 \mathrm{GeV}$. The motivation is further strengthened by the realization that additional Higgs states exist in supersymmetry that may not be accessible at the LHC or $e^{+} e^{-}$annihilation but may be visible in single production from $\gamma \gamma$. These issues are discussed in more detail in Chapters 3 and 13.

For direct superpartner pair production, $\gamma \gamma$ collisions also have an important advantage: the unambiguous production mode for superpartners through photons 
Supersymmetry Studies at the Linear Collider
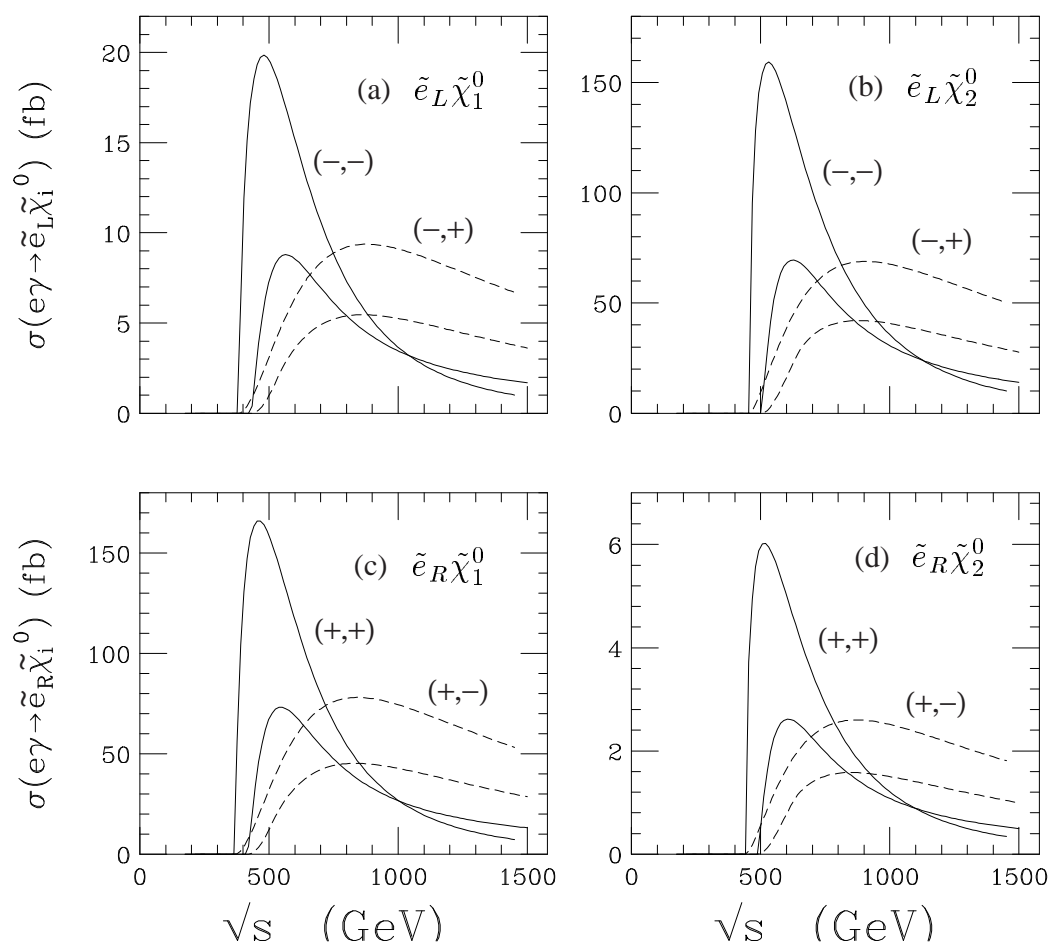

Figure 4.12: Cross sections for $e^{-} \gamma \rightarrow \widetilde{e} \widetilde{\chi}^{0}$ processes, from [242]. The upper two curves show the total cross section (in fb) for $e^{-} \gamma \rightarrow \tilde{e} \tilde{\chi}^{0}$ versus $\sqrt{s_{e \gamma}}$ (in $\mathrm{GeV}$ ) for the SUSY and machine parameters given in the text: (a) $\tilde{e}_{L} \tilde{\chi}_{1}^{0}$; (b) $\tilde{e}_{L} \tilde{\chi}_{2}^{0}$; (c) $\tilde{e}_{R} \tilde{\chi}_{1}^{0}$; (d) $\tilde{e}_{R} \tilde{\chi}_{2}^{0}$. The solid curves represent $e, \gamma$ helicities $(-,-)$ for $(\mathrm{a}),(\mathrm{b})$ and $(+,+)$ for $(\mathrm{c}),(\mathrm{d})$. The dashed curves represent helicities $(-,+)$ for $(a),(b)$ and $(+,-)$ for $(c),(d)$. The lower two curves are corresponding results, convoluted with the backscattered photon spectrum, versus $\sqrt{s_{e e}}$.

coupled to charge. Knowing exactly how a particle is produced reaps great benefits when analyzing the actual data recorded by the detectors. Production cross sections of superpartners have been calculated most recently by $[245,246]$. It has been argued [246] that some observables derived from $\gamma \gamma \rightarrow \chi_{1}^{ \pm} \chi_{1}^{\mp}$ production are very useful in extracting fundamental parameters of the supersymmetric Lagrangian. The special advantages $\gamma \gamma$ collisions offer supersymmetry deserve additional careful study.

\section{Comparison with LHC}

If SUSY is relevant to electroweak symmetry breaking, then the arguments summarized in Section 2 suggest that in many models the gluino and some squark masses are less than $\mathcal{O}(1 \mathrm{TeV})$. This is also true in most models with SUSY particles visible at a $500 \mathrm{GeV}$ LC. Gluinos and squarks then dominate the LHC SUSY cross section, 

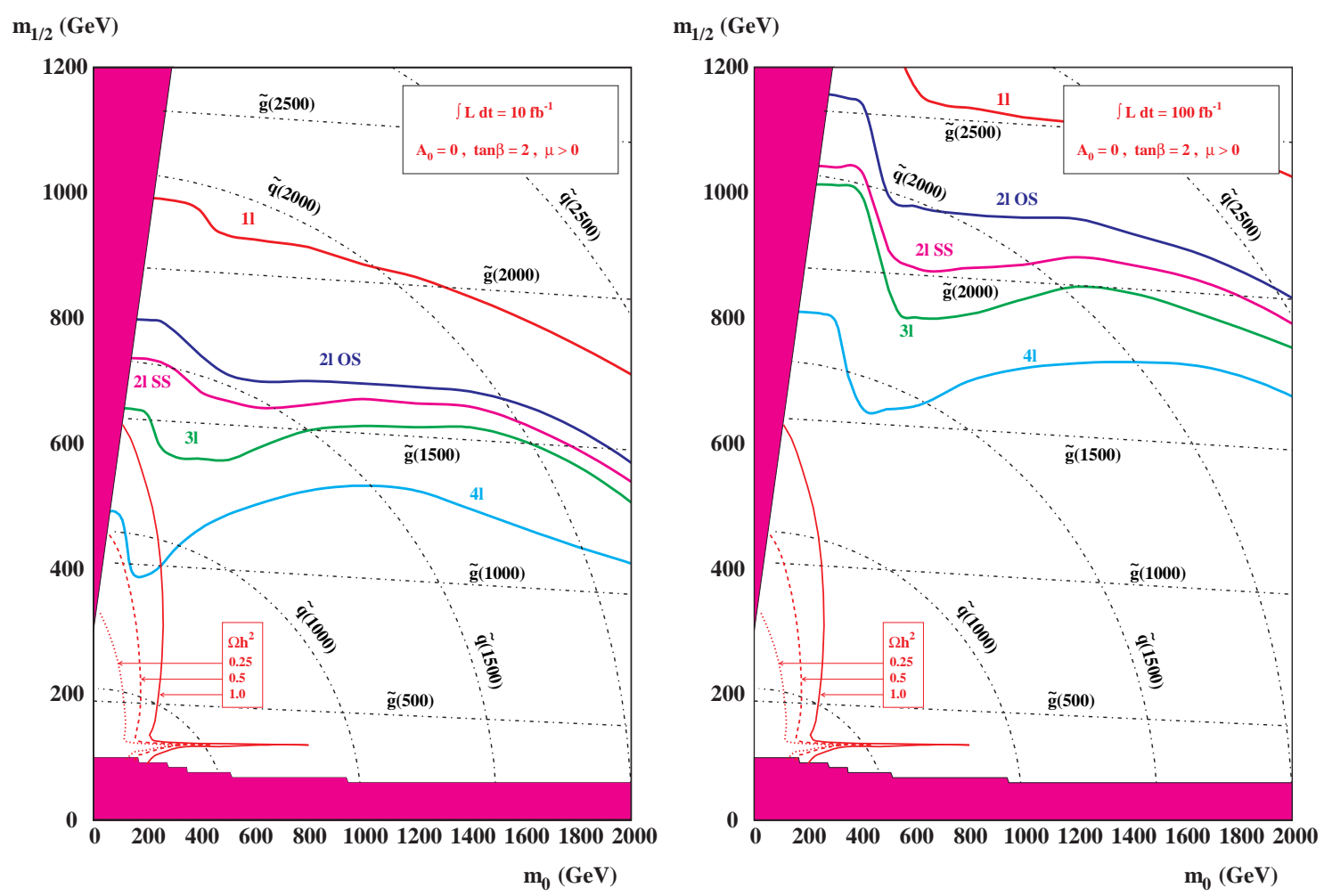

Figure 4.13: Plot of $5 \sigma$ reach with multiple jets plus $\mathbb{E}_{T}$ plus leptons in minimal SUGRA model at LHC for $10 \mathrm{fb}^{-1}$ (left) and $100 \mathrm{fb}^{-1}$ (right) [248]. Also shown are contours of the squark and gluino masses and of the cold dark matter density $\Omega h^{2}$.

which is of order $10 \mathrm{pb}$. Since they are strongly produced, it is easy to separate SUSY from SM backgrounds provided only that the SUSY decays are distinctive. In the MSUGRA model, these decays produce multiple jets and $\mathbb{E}_{T}$ plus varying numbers of leptons [247]. Figure 4.13 shows the $5 \sigma$ reach in this model at the LHC for an integrated luminosity of $10 \mathrm{fb}^{-1}$ and $100 \mathrm{fb}^{-1}$ [248]. The reach is comfortably more than the expected mass range.

While the reach in Fig. 4.13 has been calculated for a specific SUSY model, the multiple jet plus $\mathbb{E}_{T}$ signature is generic in most R-parity-conserving models. GMSB models can give additional photons or leptons or long-lived sleptons with high $p_{T}$ but $\beta<1$, making the search easier [249,250]. R-parity-violating models with leptonic $\widetilde{\chi}_{1}^{0}$ decays also give extra leptons and very likely violate $e-\mu$ universality. R-parityviolating models with $\tilde{\chi}_{1}^{0} \rightarrow q q q$ give signals at the LHC with very large jet multiplicity, for which the SM background is not well known. For such models, it may be necessary to rely on leptons produced in the cascade decay of the gluinos and squarks. In AMSB models, cascade decays of gluinos and squarks again lead to a substantial reach for SUSY by the LHC [251]. In all cases, it seems likely that SUSY can be 
discovered at the LHC if the masses are in the expected range [252-254].
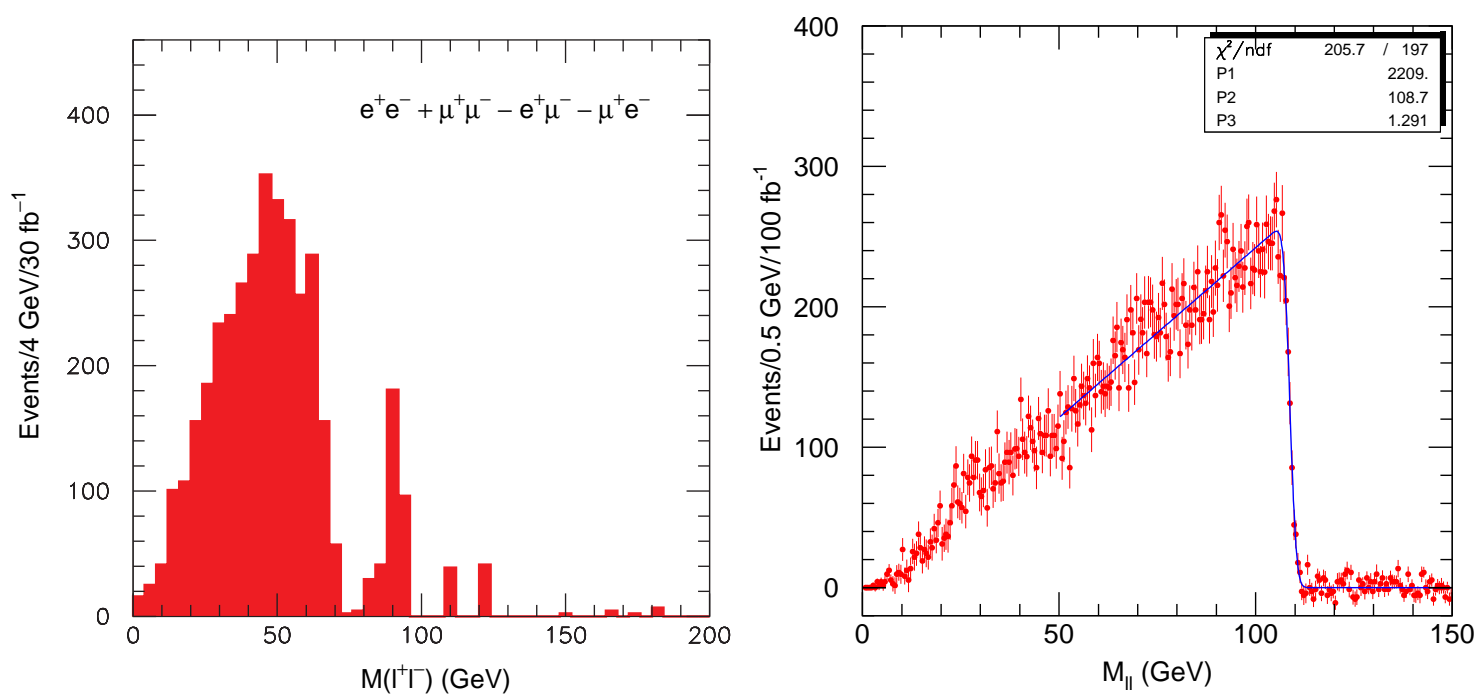

Figure 4.14: Plot of the $e^{+} e^{-}+\mu^{+} \mu^{-}-e^{ \pm} \mu^{\mp}$ mass distribution for LHC SUGRA Point 4 with direct $\widetilde{\chi}_{2}^{0} \rightarrow \widetilde{\chi}_{1}^{0} \ell \ell$ decay (left) and for LHC SUGRA Point 5 with $\widetilde{\chi}_{2}^{0} \rightarrow \widetilde{\ell}^{ \pm} \ell^{\mp} \rightarrow \widetilde{\chi}_{1}^{0} \ell^{+} \ell^{-}$ (right) [252]. The event generator ISAJET is used. The shape of the peak on the left plot below $70 \mathrm{GeV}$ should be compared to the shape of the peak in the right plot. The left plot also contains a $Z \rightarrow \ell^{+} \ell^{-}$signal that comes from heavier gauginos.

The main problem at the LHC is not to observe a signal that deviates from the SM but to separate the many different channels produced by all the SUSY cascade decays from the produced squarks [255] and gluinos. One promising approach is to try to identify particular decay chains and to measure kinematic endpoints for combinations of the visible particles in these [256]. For example, the $\ell^{+} \ell^{-}$mass distribution from $\tilde{\chi}_{2}^{0} \rightarrow \widetilde{\chi}_{1}^{0} \ell^{+} \ell^{-}$has an endpoint that measures $M_{\widetilde{\chi}_{2}^{0}}-M_{\widetilde{\chi}_{1}^{0}}[257]$, while the distribution from $\tilde{\chi}_{2}^{0} \rightarrow \tilde{\ell}^{ \pm} \ell^{\mp} \rightarrow \widetilde{\chi}_{1}^{0} \ell^{+} \ell^{-}$has a different shape and measures

$$
M_{\ell \ell}^{\max }=\sqrt{\frac{\left(M_{\widetilde{\chi}_{2}^{0}}^{2}-M_{\widetilde{\ell}}^{2}\right)\left(M_{\widetilde{\ell}}^{2}-M_{\widetilde{\chi}_{1}^{0}}^{2}\right)}{M_{\widetilde{\ell}}^{2}}} .
$$

The flavor-subtraction combination $e^{+} e^{-}+\mu^{+} \mu^{-}-e^{ \pm} \mu^{\mp}$ removes backgrounds from two independent decays. Dilepton mass distributions [252] after cuts for an example of each decay are shown in Fig. 4.14.

If a longer decay chain can be identified, then more combinations of masses can be measured. Consider, for example, the decay chain

$$
\widetilde{q}_{L} \rightarrow \widetilde{\chi}_{2}^{0} q \rightarrow \widetilde{\ell}_{R}^{ \pm} \ell^{\mp} q \rightarrow \widetilde{\chi}_{1}^{0} \ell^{+} \ell^{-} q
$$


For this decay chain, kinematics gives $\ell^{+} \ell^{-}, \ell^{+} \ell q$, and two $\ell q$ endpoints in terms of the masses. If a lower limit is imposed on the $\ell^{+} \ell^{-}$mass, there is also a $\ell^{+} \ell^{-} q$ lower edge. With suitable cuts all of these can be measured $[252,258]$ for the cases considered. The statistical errors on the measured endpoints are typically comparable to the systematic limits, $\mathcal{O}(0.1 \%)$ for leptons and $\mathcal{O}(1 \%)$ for jets. Figure 4.15 shows a scatter plot of the resulting $\widetilde{\ell}_{R}$ and $\widetilde{\chi}_{1}^{0}$ masses for LHC SUGRA Point 5 and for a similar point in another SUSY model with this decay chain [259]. The relations between masses are determined with good precision, so these two models are easily distinguished. However, the LSP mass is only measured to $\mathcal{O}(10 \%)$.
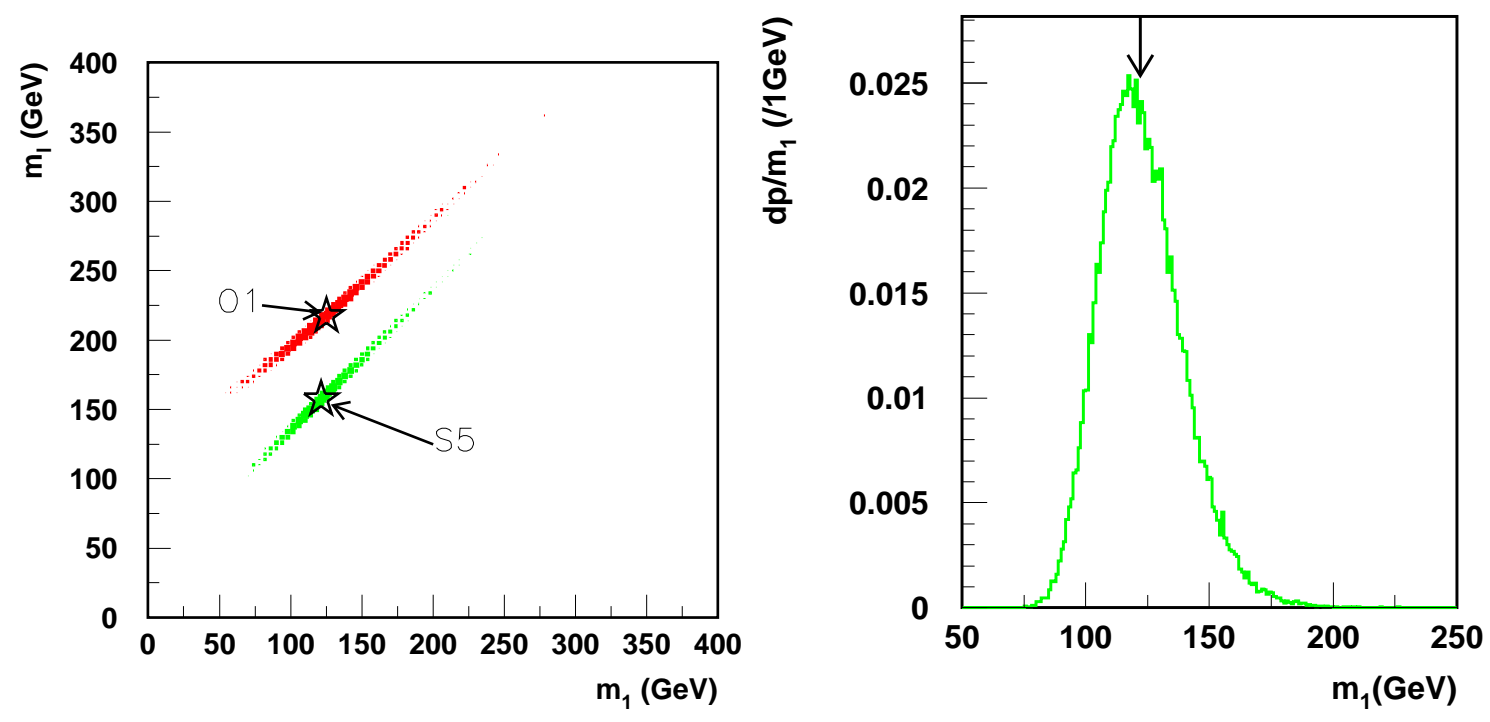

Figure 4.15: Left: Scatter plot of reconstructed values of the $\widetilde{\ell}_{R}$ and $\widetilde{\chi}_{1}^{0}$ masses for LHC Point 5 (S5) and for a different model (O1) using the decay chain $\widetilde{q}_{L} \rightarrow \widetilde{\chi}_{2}^{0} q \rightarrow \widetilde{\ell}_{R} \ell q \rightarrow \widetilde{\chi}_{1}^{0} \ell \ell q$. Right: Projection of $M_{\widetilde{\chi}_{1}^{0}}$ for LHC Point 5 [259].

Analyses such as these have proved useful for a number of SUSY points in a variety of SUSY models [252]. The method seems fairly general: there is usually at least one distinctive mode - typically $\widetilde{\chi}_{2}^{0} \rightarrow \widetilde{\chi}_{1}^{0} \ell^{+} \ell^{-}, \widetilde{\chi}_{2}^{0} \rightarrow \widetilde{\ell}_{R}^{ \pm} \ell^{\mp}$, or $\widetilde{\chi}_{2}^{0} \rightarrow \widetilde{\chi}_{1}^{0} h \rightarrow \widetilde{\chi}_{1}^{0} b \bar{b}-$ from which to start. But some points are much more difficult than others. For example, in MSUGRA with $\tan \beta \gg 1$ it is possible to choose parameters such that the only allowed 2-body decays of $\widetilde{\chi}_{2}^{0}$ and $\widetilde{\chi}_{1}^{ \pm}$are $\widetilde{\tau}_{1}^{ \pm} \tau^{\mp}$ and $\widetilde{\tau}_{1}^{ \pm} \nu_{\tau}[260]$ respectively. ${ }^{1}$ These modes then have branching ratios in excess of $99 \%$. While it is possible to identify and to measure hadronic $\tau$ decays [252], the measurements are much less precise than those involving leptons. Even if $\tau$ decays are not dominant, they may be important, since they can provide information on $\widetilde{\tau}_{L}-\widetilde{\tau}_{R}$ and gaugino-Higgsino mixing.

\footnotetext{
${ }^{1}$ The simple class of such models considered in [252], however, gives an excessively large contribution to $g_{\mu}-2[136]$.
} 
Supersymmetry Studies at the Linear Collider

If SUSY is found at the LHC, the SUSY events will contain much more information than just endpoints like those described above. For example, while it is not possible to reconstruct $\tilde{\chi}_{1}^{ \pm}$decays in the same way because of the missing neutrino, one can get information about the chargino mass by studying $M_{\ell q}$ and other distributions for 1-lepton events. Cross sections and branching ratios can also be measured; interpretation of these will be limited by the theoretical errors on the calculation of cross sections and acceptances. Without real experimental data, it is difficult to assess such theoretical systematic errors.

SUSY signatures at the LHC typically come from a combination of many SUSY particles, so the analysis is considerably more complicated than that at a LC. However, the initial steps at the LHC are fairly clear. First, one will look for a deviation from the SM in inclusive distributions such as multiple jets plus $\mathbb{E}_{T}$, perhaps accompanied by leptons and/or photons. If a signal consistent with SUSY is found, it should determine both the mass scale $[252,261]$ and the qualitative nature of the signal. (As a simple example, in a GMSB model with a long-lived slepton NLSP, SUSY events would contain two high- $p_{T}$ particles with $\beta<1$.) Next, one will look for various kinematic endpoints like those described above and use them further to constrain the SUSY masses. After this, one will look at more model-dependent quantities such as kinematic distributions, cross sections, and branching ratios. These seem difficult to assess without real data.

This program is likely to provide considerable information about gluinos, squarks, and their primary decay products, including $\widetilde{\chi}_{1}^{0}, \widetilde{\chi}_{2}^{0}, \widetilde{\chi}^{ \pm}$, and any sleptons that occur in their decays. It is more dangerous to predict what cannot be done, but there are measurements that appear difficult at the LHC and that could be done at a $500 \mathrm{GeV}$ LC. For example:

- While it is possible to measure the $\tilde{\chi}_{1}^{0}$ mass at the LHC in favorable cases, it seems difficult to reduce the error below $\mathcal{O}(10 \%)$. If any visible SUSY particle is produced at a LC, the error on $M_{\widetilde{\chi}_{1}^{0}}$ should be $\mathcal{O}(1 \%)$.

- Sleptons that are not produced in $\tilde{\chi}_{2}^{0}$ or $\tilde{\chi}_{1}^{ \pm}$decays are difficult to study at the LHC: both the Drell-Yan process and decays of heavier gauginos typically give very small rates [262]. They can be precisely measured at a LC.

- Distinguishing $\tilde{\ell}_{L}$ from $\tilde{\ell}_{R}$ appears very difficult at the LHC except perhaps for $\widetilde{\tau}$ 's, but this is straightforward at a LC using the polarized beam.

- Hadronic $\tau$ decays are easier to identify and to measure at a LC because there is no underlying hadronic event.

- Branching ratios currently seem difficult to measure with high precision at the LHC: both the production cross sections and the acceptance have theoretical 
uncertainties of $\mathcal{O}(10 \%)$. In particular, it seems difficult to make precise tests of SUSY relations among couplings.

More generally, while the LHC seems sure to discover SUSY at the TeV scale if it exists, the measurements of SUSY that can be made there depend on the SUSY model. A LC can provide precise, detailed measurements of any kinematically accessible SUSY particles. Ultimately, one will want such measurements for the entire SUSY spectrum.

\section{References}

[1] The LEP Collaborations, the LEP Electroweak Working Group, and the SLD Heavy Flavour and Electroweak Working Groups, hep-ex/0103048, CERNEP/2001-021 (February 28, 2001).

[2] L. Maiani, in Proc. Gif-sur-Yvette Summer School (Paris, 1980), p. 3.

[3] M. Veltman, Acta Phys. Polon. B12, 437 (1981).

[4] E. Witten, Nucl. Phys. B188, 513 (1981).

[5] R. K. Kaul, Phys. Lett. B109, 19 (1982).

[6] S. Dimopoulos, S. Raby and F. Wilczek, Phys. Rev. D24, 1681 (1981).

[7] U. Amaldi et al., Phys. Rev. D36, 1385 (1987).

[8] P. Langacker and M. Luo, Phys. Rev. D44, 817 (1991).

[9] J. Bagger, K. Matchev and D. Pierce, Phys. Lett. B348, 443 (1995) [hep-ph/9501277].

[10] P. Langacker and N. Polonsky, Phys. Rev. D52, 3081 (1995) [hep-ph/9503214].

[11] H. Goldberg, Phys. Rev. Lett. 50, 1419 (1983).

[12] C. Ahn et al., High-Energy E+ E- Collider," SLAC-0329.

[13] P. M. Zerwas, editor, "E+ E- Collisions At 500-Gev: The Physics Potential. Proceedings, Workshop, Munich, Germany, February 4, 1991, Annecy, France, June 10-11, 1991, Hamburg, Germany, September 2-3, 1991," DESY-92-123A-B.

[14] JLC Group Collaboration, KEK-92-16.

[15] S. Kuhlman et al. [NLC ZDR Design Group and NLC Physics Working Group Collaboration], hep-ex/9605011.

[16] M. Danielson et al., in New Directions in High Energy Physics, Proc. 1996 Snowmass Summer Study, editors D. Cassel, L. Trindle Gennari and R. H. Siemann (SLAC, 1997).

[17] S. Abdullin et al., hep-ph/0005142.

[18] R. D. Heuer, D. Miller, F. Richard and P. Zerwas, DESY-01-011C.

[19] H. P. Nilles, Phys. Rept. 110 (1984) 1. 
Supersymmetry Studies at the Linear Collider

[20] H. E. Haber and G. L. Kane, Phys. Rept. 117 (1985) 75.

[21] G. F. Giudice and R. Rattazzi, Phys. Rept. 322, 419 (1999) [hep-ph/9801271].

[22] L. Randall and R. Sundrum, Nucl. Phys. B557, 79 (1999) [hep-th/9810155].

[23] G. F. Giudice, M. A. Luty, H. Murayama and R. Rattazzi, JHEP 9812, 027 (1998) [hep-ph/9810442].

[24] J. Ellis, K. Enqvist, D. V. Nanopoulos and F. Zwirner, Mod. Phys. Lett. A1, 57 (1986).

[25] R. Barbieri and G. F. Giudice, Nucl. Phys. B306, 63 (1988).

[26] G. G. Ross and R. G. Roberts, Nucl. Phys. B377, 571 (1992).

[27] B. de Carlos and J. A. Casas, Phys. Lett. B309, 320 (1993) [hep-ph/9303291].

[28] G. W. Anderson and D. J. Castaño, Phys. Lett. B347, 300 (1995) [hep-ph/9409419].

[29] G. W. Anderson and D. J. Castaño, Phys. Rev. D52, 1693 (1995) [hep-ph/9412322].

[30] G. W. Anderson and D. J. Castaño, Phys. Rev. D53, 2403 (1996) [hep-ph/9509212].

[31] S. Dimopoulos and G. F. Giudice, Phys. Lett. B357, 573 (1995) [hep-ph/9507282].

[32] A. Pomarol and D. Tommasini, Nucl. Phys. B466, 3 (1996) [hep-ph/9507462].

[33] K. Agashe and M. Graesser, Nucl. Phys. B507, 3 (1997) [hep-ph/9704206].

[34] P. Ciafaloni and A. Strumia, Nucl. Phys. B494, 41 (1997) [hep-ph/9611204].

[35] G. Bhattacharyya and A. Romanino, Phys. Rev. D55, 7015 (1997) [hep-ph/9611243].

[36] R. Barbieri and A. Strumia, Phys. Lett. B433, 63 (1998) [hep-ph/9801353].

[37] L. Giusti, A. Romanino and A. Strumia, Nucl. Phys. B550, 3 (1999) [hep-ph/9811386].

[38] A. Romanino and A. Strumia, Phys. Lett. B487, 165 (2000) [hep-ph/9912301].

[39] K. L. Chan, U. Chattopadhyay and P. Nath, Phys. Rev. D58, 096004 (1998) [hep-ph/9710473].

[40] P. H. Chankowski, J. Ellis and S. Pokorski, Phys. Lett. B423, 327 (1998) [hep-ph/9712234].

[41] P. H. Chankowski, J. Ellis, M. Olechowski and S. Pokorski, Nucl. Phys. B544, 39 (1999) [hep-ph/9808275].

[42] G. L. Kane and S. F. King, Phys. Lett. B451, 113 (1999) [hep-ph/9810374].

[43] M. Bastero-Gil, G. L. Kane and S. F. King, Phys. Lett. B474, 103 (2000) [hep-ph/9910506].

[44] J. L. Feng, K. T. Matchev and T. Moroi, Phys. Rev. Lett. 84, 2322 (2000) [hep-ph/9908309]. 
[45] J. L. Feng, K. T. Matchev and T. Moroi, Phys. Rev. D61, 075005 (2000) [hep-ph/9909334].

[46] J. L. Feng and K. T. Matchev, Phys. Rev. D63, 095003 (2001) [hep-ph/0011356].

[47] M. Drees, Phys. Rev. D33, 1468 (1986).

[48] M. Dine, A. Kagan and S. Samuel, Phys. Lett. B243, 250 (1990).

[49] G. Dvali and A. Pomarol, Phys. Rev. Lett. 77, 3728 (1996) [hep-ph/9607383].

[50] G. Dvali and A. Pomarol, Nucl. Phys. B522, 3 (1998) [hep-ph/9708364].

[51] A. G. Cohen, D. B. Kaplan and A. E. Nelson, Phys. Lett. B388, 588 (1996) [hep-ph/9607394].

[52] R. Zhang, Phys. Lett. B402, 101 (1997) [hep-ph/9702333].

[53] H. P. Nilles and N. Polonsky, Phys. Lett. B412, 69 (1997) [hep-ph/9707249].

[54] J. L. Feng, C. Kolda and N. Polonsky, Nucl. Phys. B546, 3 (1999) [hep-ph/9810500].

[55] J. Bagger, J. L. Feng and N. Polonsky, Nucl. Phys. B563, 3 (1999) [hep-ph/9905292].

[56] J. A. Bagger, J. L. Feng, N. Polonsky and R. Zhang, Phys. Lett. B473, 264 (2000) [hep-ph/9911255].

[57] Q. Shafi and Z. Tavartkiladze, Phys. Lett. B473, 272 (2000) [hep-ph/9911264].

[58] H. Baer, M. A. Díaz, P. Quintana and X. Tata, JHEP 0004, 016 (2000) [hep-ph/0002245].

[59] H. Baer, C. Balazs, P. Mercadante, X. Tata and Y. Wang, Phys. Rev. D63, 015011 (2001) [hep-ph/0008061].

[60] H. Baer, C. Balazs, M. Brhlik, P. Mercadante, X. Tata and Y. Wang, hep$\mathrm{ph} / 0102156$.

[61] J. Hisano, K. Kurosawa and Y. Nomura, Phys. Lett. B445, 316 (1999) [hep-ph/9810411].

[62] J. Hisano, K. Kurosawa and Y. Nomura, Nucl. Phys. B584, 3 (2000) [hep-ph/0002286].

[63] D. E. Kaplan and G. D. Kribs, Phys. Rev. D61, 075011 (2000) [hep-ph/9906341].

[64] J. L. Feng and T. Moroi, Phys. Rev. D61, 095004 (2000) [hep-ph/9907319].

[65] K. Agashe, Phys. Rev. D61, 115006 (2000) [hep-ph/9910497].

[66] U. Chattopadhyay, A. Datta, A. Datta, A. Datta and D. P. Roy, Phys. Lett. B493, 127 (2000) [hep-ph/0008228].

[67] B. C. Allanach, J. P. Hetherington, M. A. Parker and B. R. Webber, JHEP 0008, 017 (2000) [hep-ph/0005186].

[68] U. Chattopadhyay, T. Ibrahim and D. P. Roy, hep-ph/0012337.

[69] G. F. Giudice and A. Masiero, Phys. Lett. B206, 480 (1988). 
Supersymmetry Studies at the Linear Collider

[70] J. Ellis, J. S. Hagelin, D. V. Nanopoulos and M. Srednicki, Phys. Lett. B127, 233 (1983).

[71] A. H. Jaffe et al., astro-ph/0007333.

[72] K. Griest and D. Seckel, Phys. Rev. D43, 3191 (1991).

[73] P. Gondolo and G. Gelmini, Nucl. Phys. B360, 145 (1991).

[74] P. Nath and R. Arnowitt, Phys. Rev. Lett. 70, 3696 (1993) [hep-ph/9302318].

[75] S. Mizuta and M. Yamaguchi, Phys. Lett. B298, 120 (1993) [hep-ph/9208251].

[76] J. Ellis, T. Falk and K. A. Olive, Phys. Lett. B444, 367 (1998) [hep-ph/9810360].

[77] M. E. Gomez, G. Lazarides and C. Pallis, Phys. Rev. D61, 123512 (2000) [hep-ph/9907261].

[78] M. Drees and M. M. Nojiri, Phys. Rev. D47, 376 (1993) [hep-ph/9207234].

[79] K. A. Olive and M. Srednicki, Phys. Lett. B230, 78 (1989).

[80] K. Griest, M. Kamionkowski and M. S. Turner, Phys. Rev. D41, 3565 (1990).

[81] K. A. Olive and M. Srednicki, Nucl. Phys. B355, 208 (1991).

[82] J. L. Lopez, K. Yuan and D. V. Nanopoulos, Phys. Lett. B267, 219 (1991).

[83] S. Kelley, J. L. Lopez, D. V. Nanopoulos, H. Pois and K. Yuan, Phys. Rev. D47, 2461 (1993) [hep-ph/9207253].

[84] R. G. Roberts and L. Roszkowski, Phys. Lett. B309, 329 (1993) [hep-ph/9301267].

[85] G. L. Kane, C. Kolda, L. Roszkowski and J. D. Wells, Phys. Rev. D49, 6173 (1994) [hep-ph/9312272].

[86] H. Baer and M. Brhlik, Phys. Rev. D53, 597 (1996) [hep-ph/9508321].

[87] R. Arnowitt and P. Nath, Phys. Lett. B437, 344 (1998) [hep-ph/9801246].

[88] S. Abdullin and F. Charles, Nucl. Phys. B547, 60 (1999) [hep-ph/9811402].

[89] J. Ellis, G. Ganis and K. A. Olive, Phys. Lett. B474, 314 (2000) [hep-ph/9912324].

[90] M. Drees, M. M. Nojiri, D. P. Roy and Y. Yamada, Phys. Rev. D56, 276 (1997) [hep-ph/9701219].

[91] V. Barger and C. Kao, Phys. Rev. D57, 3131 (1998) [hep-ph/9704403].

[92] C. Boehm, A. Djouadi and M. Drees, Phys. Rev. D62, 035012 (2000) [hep-ph/9911496].

[93] A. Bottino, F. Donato, N. Fornengo and S. Scopel, Phys. Rev. D62, 056006 (2000) [hep-ph/0001309].

[94] J. L. Feng, K. T. Matchev and F. Wilczek, Phys. Lett. B482, 388 (2000) [hep-ph/0004043].

[95] J. L. Feng, K. T. Matchev and F. Wilczek, Phys. Rev. D63, 045024 (2001) [astro-ph/0008115]. 
[96] H. Baer, M. Brhlik, M. A. Díaz, J. Ferrandis, P. Mercadante, P. Quintana and X. Tata, Phys. Rev. D63, 015007 (2001) [hep-ph/0005027].

[97] J. Ellis, T. Falk, G. Ganis, K. A. Olive and M. Srednicki, hep-ph/0102098.

[98] T. Moroi and L. Randall, Nucl. Phys. B570, 455 (2000) [hep-ph/9906527].

[99] B. Murakami and J. D. Wells, hep-ph/0011082.

[100] A. Birkedal-Hansen and B. D. Nelson, hep-ph/0102075.

[101] H. E. Haber and R. Hempfling, Phys. Rev. Lett. 66, 1815 (1991).

[102] M. S. Berger, Phys. Rev. D41, 225 (1990).

[103] Y. Okada, M. Yamaguchi and T. Yanagida, Prog. Theor. Phys. 85, 1 (1991).

[104] J. Ellis, G. Ridolfi and F. Zwirner, Phys. Lett. B257, 83 (1991).

[105] R. Barbieri and M. Frigeni, Phys. Lett. B258, 395 (1991).

[106] H. E. Haber and R. Hempfling, Phys. Rev. D48, 4280 (1993) [hep-ph/9307201].

[107] P. Chankowski, S. Pokorski and J. Rosiek, Nucl. Phys. B423, 437 (1994) [hep-ph/9303309].

[108] A. Dabelstein, Z. Phys. C67, 495 (1995) [hep-ph/9409375].

[109] A. Dabelstein, Nucl. Phys. B456, 25 (1995) [hep-ph/9503443].

[110] D. M. Pierce, J. A. Bagger, K. Matchev and R. Zhang, Nucl. Phys. B491, 3 (1997) [hep-ph/9606211].

[111] S. Heinemeyer, W. Hollik and G. Weiglein, Phys. Rev. D58, 091701 (1998) [hep-ph/9803277].

[112] S. Heinemeyer, W. Hollik and G. Weiglein, Phys. Lett. B440, 296 (1998) [hep-ph/9807423].

[113] S. Heinemeyer, W. Hollik and G. Weiglein, Eur. Phys. J. C9, 343 (1999) [hep-ph/9812472].

[114] S. Heinemeyer, W. Hollik and G. Weiglein, Phys. Lett. B455, 179 (1999) [hep-ph/9903404].

[115] M. Carena, H. E. Haber, S. Heinemeyer, W. Hollik, C. E. Wagner and G. Weiglein, Nucl. Phys. B580, 29 (2000) [hep-ph/0001002].

[116] J. A. Casas, J. R. Espinosa, M. Quiros and A. Riotto, Nucl. Phys. B436, 3 (1995) [hep-ph/9407389].

[117] M. Carena, J. R. Espinosa, M. Quiros and C. E. Wagner, Phys. Lett. B355, 209 (1995) [hep-ph/9504316].

[118] M. Carena, M. Quiros and C. E. Wagner, Nucl. Phys. B461, 407 (1996) [hep-ph/9508343].

[119] H. E. Haber, R. Hempfling and A. H. Hoang, Z. Phys. C75, 539 (1997) [hep-ph/9609331].

[120] R. Hempfling and A. H. Hoang, Phys. Lett. B331, 99 (1994) [hep-ph/9401219].

[121] R. Zhang, Phys. Lett. B447, 89 (1999) [hep-ph/9808299]. 
Supersymmetry Studies at the Linear Collider

[122] J. R. Espinosa and R. Zhang, Nucl. Phys. B586, 3 (2000) [hep-ph/0003246].

[123] M. Acciarri et al. [L3 Collaboration], Phys. Lett. B495, 18 (2000)

[hep-ex/0011043].

[124] R. Barate et al. [ALEPH Collaboration], Phys. Lett. B495, 1 (2000) [hep-ex/0011045].

[125] G. Abbiendi et al. [OPAL Collaboration], Phys. Lett. B499, 38 (2001) [hep-ex/0101014].

[126] P. Abreu et al. [DELPHI Collaboration], Phys. Lett. B499, 23 (2001) [hep-ex/0102036].

[127] J. Ellis, G. Ganis, D. V. Nanopoulos and K. A. Olive, hep-ph/0009355.

[128] S. Su, Nucl. Phys. B573, 87 (2000) [hep-ph/9910481].

[129] G. L. Kane, S. F. King and L. Wang, hep-ph/0010312.

[130] A. Djouadi, P. Gambino, S. Heinemeyer, W. Hollik, C. Junger and G. Weiglein, Phys. Rev. Lett. 78, 3626 (1997) [hep-ph/9612363].

[131] A. Djouadi, P. Gambino, S. Heinemeyer, W. Hollik, C. Junger and G. Weiglein, Phys. Rev. D57, 4179 (1998) [hep-ph/9710438].

[132] J. Erler and D. M. Pierce, Nucl. Phys. B526, 53 (1998) [hep-ph/9801238].

[133] G. Cho and K. Hagiwara, Nucl. Phys. B574, 623 (2000) [hep-ph/9912260].

[134] G. Cho, K. Hagiwara and M. Hayakawa, Phys. Lett. B478, 231 (2000) [hep-ph/0001229].

[135] J. Erler, S. Heinemeyer, W. Hollik, G. Weiglein and P. M. Zerwas, Phys. Lett. B486, 125 (2000) [hep-ph/0005024].

[136] H. N. Brown et al. [Muon g-2 Collaboration], Phys. Rev. Lett. 86, 2227 (2001) [hep-ex/0102017].

[137] J. A. Grifols and A. Mendez, Phys. Rev. D26, 1809 (1982).

[138] J. Ellis, J. Hagelin and D. V. Nanopoulos, Phys. Lett. B116, 283 (1982).

[139] R. Barbieri and L. Maiani, Phys. Lett. B117, 203 (1982).

[140] D. A. Kosower, L. M. Krauss and N. Sakai, Phys. Lett. B133, 305 (1983).

[141] T. C. Yuan, R. Arnowitt, A. H. Chamseddine and P. Nath, Z. Phys. C26, 407 (1984).

[142] A. Czarnecki and W. J. Marciano, hep-ph/0102122.

[143] L. Everett, G. L. Kane, S. Rigolin and L. Wang, hep-ph/0102145.

[144] J. L. Feng and K. T. Matchev, Phys. Rev. Lett. 86, 3480 (2001)

[hep-ph/0102146].

[145] E. A. Baltz and P. Gondolo, hep-ph/0102147.

[146] U. Chattopadhyay and P. Nath, hep-ph/0102157.

[147] S. Komine, T. Moroi and M. Yamaguchi, hep-ph/0102204. 
[148] H. Baer, C. Balazs, J. Ferrandis and X. Tata, hep-ph/0103280.

[149] J. Hisano and K. Tobe, hep-ph/0102315.

[150] T. Ibrahim, U. Chattopadhyay and P. Nath, hep-ph/0102324.

[151] J. Ellis, D. V. Nanopoulos and K. A. Olive, hep-ph/0102331.

[152] K. Choi, K. Hwang, S. K. Kang, K. Y. Lee and W. Y. Song, hep-ph/0103048.

[153] S. P. Martin and J. D. Wells, hep-ph/0103067.

[154] T. Tsukamoto, K. Fujii, H. Murayama, M. Yamaguchi, and Y. Okada, Phys. Rev. D51, 3153 (1995).

[155] J. L. Feng, M. E. Peskin, H. Murayama and X. Tata, Phys. Rev. D52, 1418 (1995) [hep-ph/9502260].

[156] D. G. Cassel, L. Trindle Gennari, R. H. Siemann, editors "DPF/DPB Summer Study on New Directions for High-Energy Physics (Snowmass 96), Snowmass, CO, 25 Jun - 12 Jul 1996. NEW DIRECTIONS FOR HIGH-ENERGY PHYSICS: Proceedings."

[157] E. Goodman, COLO-HEP-398, http://hep-www . colorado.edu/SUSY.

[158] H. Baer, F. E. Paige, S. D. Protopopescu and X. Tata, hep-ph/0001086.

[159] J. Dunn, "Study of the Detector Response to a Selectron Signal at the NLC," COLO-HEP-461, in preparation.

[160] E. Goodman, COLO-HEP-455, http://hep-www . colorado.edu/SUSY.

[161] B. Williams, COLO-HEP-402, http://hep-www. colorado.edu/SUSY.

[162] N. Danielson, COLO-HEP-404, http://hep-www. colorado.edu/SUSY.

[163] H. Baer, R. Munroe and X. Tata, Phys. Rev. D54, 6735 (1996) [hep-ph/9606325].

[164] "Supersymmetry, Chapter 3 of TESLA TDR", DESY-2001-011, ECFA-2001209.

[165] J. Barron "Finding the Mass of the Chargino and the Sneutrino at the NLC," COLO-HEP-459, in preparation.

[166] C. Veeneman "Study of the Decay $\tilde{\chi}_{2}^{ \pm} \rightarrow \tilde{\chi}_{1}^{ \pm} Z^{0}$ with decays $\rightarrow \mathrm{q} \overline{\mathrm{q}} \mathrm{l}^{+} \mathrm{l}^{-}$at the NLC," COLO-HEP-463, in preparation.

[167] M. Dima, "Susy $\widetilde{e}_{R, L}^{ \pm} / \widetilde{\mu}_{R, L}^{ \pm}$Studies, COLO-HEP-454," Talk presented at LCWS 2000, Fermilab, October, 2000; http://hep-www. colorado.edu/SUSY.

[168] N. Danielson and E. Goodman, COLO-HEP-422, http://hep-www. colorado.edu/SUSY.

[169] T. W. Markiewicz, T. Maruyama, "Interaction region issues at the NLC," Proceedings of the Worldwide Study on Physics and Experiments with Future Linear $\mathrm{e}^{+} \mathrm{e}^{-}$Linear Colliders, Sitges, Barcelona, Spain, April 1999.

[170] N. Danielson and B. Newman, COLO-HEP-428, http://hep-www. colorado.edu/SUSY. 
Supersymmetry Studies at the Linear Collider

[171] R. Kelly and C. Takeuchi, COLO-HEP-450, http://hep-www. colorado.edu/SUSY.

[172] J. Barron, COLO-HEP-449, http://hep-www.colorado.edu/SUSY.

[173] D. Staszak, "Studies of a Smuon Signal in a NLC Detector," COLO-HEP-460, in preparation.

[174] H. Martyn and G. A. Blair, hep-ph/9910416.

[175] J. L. Feng and D. E. Finnell, Phys. Rev. D49, 2369 (1994) [hep-ph/9310211].

[176] David Wagner, COLO-HEP-400, http://hep-www. colorado.edu/SUSY.

[177] David Wagner, COLO-HEP-401, http://hep-www. colorado.edu/SUSY.

[178] H. Baer, J. K. Mizukoshi, and X. Tata, private communication.

[179] J. F. Gunion and S. Mrenna, hep-ph/0103167.

[180] H. Baer, C. Balazs, S. Hesselbach, J. K. Mizukoshi and X. Tata, hep$\mathrm{ph} / 0012205$.

[181] S. Y. Choi, A. Djouadi, M. Guchait, J. Kalinowski, H. S. Song and P. M. Zerwas, Eur. Phys. J. C14, 535 (2000) [hep-ph/0002033].

[182] G. Moortgat-Pick, A. Bartl, H. Fraas and W. Majerotto, Eur. Phys. J. C18, 379 (2000) [hep-ph/0007222].

[183] M. M. Nojiri, K. Fujii and T. Tsukamoto, Phys. Rev. D54, 6756 (1996) [hep-ph/9606370].

[184] A. Bartl, H. Eberl, S. Kraml, W. Majerotto, W. Porod and A. Sopczak, Z. Phys. C76, 549 (1997) [hep-ph/9701336].

[185] H. Baer, C. Balazs, J. K. Mizukoshi and X. Tata, Phys. Rev. D63, 055011 (2001) [hep-ph/0010068].

[186] M. M. Nojiri, K. Fujii and T. Tsukamoto, Prepared for 6th Workshop on the Japan Linear Collider (JLC), Tokyo, Japan, 4-5 Dec 1996, Phys. Rev. D54, 6756 (1886).

[187] K. Hikasa and T. Nagano, Phys. Lett. B435, 67 (1998) [hep-ph/9805246].

[188] J. L. Feng and T. Moroi, Phys. Rev. D56, 5962 (1997) [hep-ph/9612333].

[189] V. Barger, T. Han and J. Jiang, hep-ph/0006223.

[190] K. Hikasa and Y. Nakamura, Z. Phys. C70, 139 (1996) [hep-ph/9501382];

erratum, Z. Phys. C71, 356 (1996).

[191] P. H. Chankowski, Phys. Rev. D41, 2877 (1990).

[192] M. E. Peskin and T. Takeuchi, Phys. Rev. Lett. 65, 964 (1990).

[193] H. Cheng, J. L. Feng and N. Polonsky, Phys. Rev. D56, 6875 (1997) [hep-ph/9706438].

[194] H. Cheng, J. L. Feng and N. Polonsky, Phys. Rev. D57, 152 (1998) [hep-ph/9706476]. 
[195] M. M. Nojiri, D. M. Pierce and Y. Yamada, Phys. Rev. D57, 1539 (1998) [hep-ph/9707244].

[196] E. Katz, L. Randall and S. Su, Nucl. Phys. B536, 3 (1998) [hep-ph/9801416].

[197] S. Kiyoura, M. M. Nojiri, D. M. Pierce and Y. Yamada, Phys. Rev. D58, 075002 (1998) [hep-ph/9803210].

[198] M. A. Díaz, S. F. King and D. A. Ross, Nucl. Phys. B529, 23 (1998) [hep-ph/9711307].

[199] M. A. Díaz, S. F. King and D. A. Ross, hep-ph/0008117.

[200] T. Blank and W. Hollik, hep-ph/0011092.

[201] S. Y. Choi, M. Guchait, J. Kalinowski and P. M. Zerwas, Phys. Lett. B479, 235 (2000) [hep-ph/0001175].

[202] U. Mahanta, Phys. Rev. D59, 015017 (1999) [hep-ph/9810344].

[203] H. Dreiner, hep-ph/9707435.

[204] G. Bhattacharyya, hep-ph/9709395.

[205] G. Bhattacharyya, Nucl. Phys. Proc. Suppl. 52A, 83 (1997) [hep-ph/9608415].

[206] M. Bisset, O. C. Kong, C. Macesanu and L. H. Orr, Phys. Lett. B430, 274 (1998) [hep-ph/9804282].

[207] L. J. Hall and M. Suzuki, Nucl. Phys. B231, 419 (1984).

[208] D. K. Ghosh, R. M. Godbole and S. Raychaudhuri, hep-ph/9904233.

[209] D. K. Ghosh, R. M. Godbole and S. Raychaudhuri, Z. Phys. C75, 357 (1997) [hep-ph/9605460].

[210] R. Barate et al. [ALEPH Collaboration], Phys. Lett. B420, 196 (1998).

[211] F. de Campos, M. A. Garcia-Jareno, A. S. Joshipura, J. Rosiek and J. W. Valle, Nucl. Phys. B451, 3 (1995) [hep-ph/9502237].

[212] T. Banks, Y. Grossman, E. Nardi and Y. Nir, Phys. Rev. D52, 5319 (1995) [hep-ph/9505248].

[213] A. Y. Smirnov and F. Vissani, Nucl. Phys. B460, 37 (1996) [hep-ph/9506416].

[214] H. Nilles and N. Polonsky, Nucl. Phys. B484, 33 (1997) [hep-ph/9606388].

[215] M. A. Diaz, J. C. Romao and J. W. Valle, Nucl. Phys. B524, 23 (1998) [hep-ph/9706315].

[216] R. Hempfling, Nucl. Phys. B478, 3 (1996) [hep-ph/9511288].

[217] E. J. Chun and S. K. Kang, Phys. Rev. D61, 075012 (2000) [hep-ph/9909429];

E. J. Chun, Phys. Lett. B505, 155 (2001) [hep-ph/0101170].

[218] M. Hirsch, M. A. Diaz, W. Porod, J. C. Romao and J. W. Valle, Phys. Rev. D62, 113008 (2000) [hep-ph/0004115].

[219] J. C. Romao, M. A. Diaz, M. Hirsch, W. Porod and J. W. Valle, Phys. Rev. D61, 071703 (2000) [hep-ph/9907499].

[220] W. Porod, M. Hirsch, J. Romao and J. W. Valle, hep-ph/0011248. 
Supersymmetry Studies at the Linear Collider

[221] N. Arkani-Hamed, H. Cheng, J. L. Feng and L. J. Hall, Phys. Rev. Lett. 77, 1937 (1996) [hep-ph/9603431].

[222] N. Arkani-Hamed, J. L. Feng, L. J. Hall and H. Cheng, Nucl. Phys. B505, 3 (1997) [hep-ph/9704205].

[223] J. Hisano, M. M. Nojiri, Y. Shimizu and M. Tanaka, Phys. Rev. D60, 055008 (1999) [hep-ph/9808410].

[224] J. J. Cao, T. Han, X. Zhang and G. R. Lu, Phys. Rev. D59, 095001 (1999) [hep-ph/9808466].

[225] D. Nomura, hep-ph/0004256.

[226] M. Guchait, J. Kalinowski and P. Roy, hep-ph/0103161.

[227] M. Dugan, B. Grinstein and L. Hall, Nucl. Phys. B255, 413 (1985).

[228] T. Ibrahim and P. Nath, Phys. Rev. D58, 111301 (1998) [hep-ph/9807501].

[229] M. Brhlik, G. J. Good and G. L. Kane, Phys. Rev. D59, 115004 (1999) [hep-ph/9810457].

[230] D. Atwood, S. Bar-Shalom, G. Eilam and A. Soni, hep-ph/0006032.

[231] B. Grzadkowski, Phys. Lett. B305, 384 (1993) [hep-ph/9303204].

[232] A. Bartl, E. Christova, T. Gajdosik and W. Majerotto, Nucl. Phys. B507, 35 (1997) [hep-ph/9705245].

[233] S. Y. Choi, A. Djouadi, H. S. Song and P. M. Zerwas, Eur. Phys. J. C8, 669 (1999) [hep-ph/9812236].

[234] J. L. Kneur and G. Moultaka, Phys. Rev. D61, 095003 (2000) [hep-ph/9907360].

[235] V. Barger, T. Han, T. Li and T. Plehn, Phys. Lett. B475, 342 (2000) [hep-ph/9907425].

[236] V. Barger, T. Falk, T. Han, J. Jiang, T. Li and T. Plehn, hep-ph/0101106.

[237] W. Y. Keung and L. Littenberg, Phys. Rev. D28, 1067 (1983).

[238] J. L. Feng, Int. J. Mod. Phys. A13, 2319 (1998) [hep-ph/9803319].

[239] K. A. Thompson, Int. J. Mod. Phys. A15, 2485 (2000).

[240] J. L. Feng and M. E. Peskin, hep-ph/0105100.

[241] C. Bloechinger and W. Porod, in preparation.

[242] V. Barger, T. Han and J. Kelly, Phys. Lett. B419, 233 (1998) [hep-ph/9709366].

[243] D. Choudhury and F. Cuypers, Nucl. Phys. B451, 16 (1995) [hep-ph/9412245].

[244] K. Kiers, J. N. Ng and G. Wu, Phys. Lett. B381, 177 (1996) [hep-ph/9604338].

[245] S. Berge, M. Klasen and Y. Umeda, Phys. Rev. D63, 035003 (2001)

[hep-ph/0008081].

[246] T. Mayer and H. Fraas, hep-ph/0009048.

[247] H. Baer, V. Barger, D. Karatas and X. Tata, Phys. Rev. D36, 96 (1987).

[248] S. Abdullin et al. [CMS Collaboration], hep-ph/9806366. 
[249] H. Baer, P. G. Mercadante, F. Paige, X. Tata and Y. Wang, Phys. Lett. B435, 109 (1998) [hep-ph/9806290].

[250] H. Baer, P. G. Mercadante, X. Tata and Y. Wang, Phys. Rev. D62, 095007 (2000) [hep-ph/0004001].

[251] H. Baer, J. K. Mizukoshi and X. Tata, Phys. Lett. B488, 367 (2000) [hep-ph/0007073].

[252] ATLAS Collaboration, ATLAS Detector and Physics Performance Technical Design Report, CERN/LHCC/99-14, http://atlasinfo.cern.ch/Atlas/GROUPS/PHYSICS/TDR/access.html.

[253] H. Baer, C. Chen, F. Paige and X. Tata, Phys. Rev. D52, 2746 (1995) [hep-ph/9503271].

[254] H. Baer, C. Chen, F. Paige and X. Tata, Phys. Rev. D53, 6241 (1996) [hep-ph/9512383].

[255] H. Baer, C. Chen and X. Tata, Phys. Rev. D55, 1466 (1997) [hep-ph/9608221].

[256] I. Hinchliffe, F. E. Paige, M. D. Shapiro, J. Soderqvist and W. Yao, Phys. Rev. D55, 5520 (1997) [hep-ph/9610544].

[257] H. Baer, C. Chen, F. Paige and X. Tata, Phys. Rev. D50, 4508 (1994) [hep-ph/9404212].

[258] H. Bachacou, I. Hinchliffe and F. E. Paige, Phys. Rev. D62, 015009 (2000) [hep-ph/9907518].

[259] B. C. Allanach, C. G. Lester, M. A. Parker and B. R. Webber, JHEP 0009, 004 (2000) [hep-ph/0007009].

[260] H. Baer, C. Chen, M. Drees, F. Paige and X. Tata, Phys. Rev. D59, 055014 (1999) [hep-ph/9809223].

[261] D. R. Tovey, hep-ph/0006276.

[262] H. Baer, C. Chen, F. Paige and X. Tata, Phys. Rev. D49, 3283 (1994) [hep-ph/9311248]. 


\section{Chapter 5 New Physics at the TeV Scale and Beyond}

\section{Introduction}

The impressive amount of data collected in the past several decades in particle physics experiments is well accommodated by the Standard Model. This model provides an accurate description of Nature up to energies of order $100 \mathrm{GeV}$. Nonetheless, the Standard Model is an incomplete theory, since many key elements are left unexplained: (i) the origin of electroweak symmetry breaking, (ii) the generation and stabilization of the hierarchy, i.e., the large disparity between the electroweak and the Planck scale, (iii) the connection of elementary particle forces with gravity, and (iv) the generation of fermion masses and mixings. These deficiencies imply that there is physics beyond the Standard Model and point toward the principal goal of particle physics during the next decade: the elucidation of the electroweak symmetry breaking mechanism and the new physics that must necessarily accompany it. Electroweak symmetry is broken at the $\mathrm{TeV}$ scale. In the absence of highly unnatural fine-tuning of the parameters in the underlying theory, the energy scales of the associated new phenomena should also lie in the TeV range or below.

Numerous theories have been proposed to address these outstanding issues and embed the Standard Model in a larger framework. In this chapter, we demonstrate the ability of a linear collider operating at $500 \mathrm{GeV}$ and above to make fundamental progress in the illumination of new phenomena over the broadest possible range. The essential role played by $e^{+} e^{-}$machines in this endeavor has a strong history. First, $e^{+} e^{-}$colliders are discovery machines and are complementary to hadron colliders operating at similar energy regions. The discoveries of the gluon, charm, and tau sustain this assertion. Here, we show that $500-1000 \mathrm{GeV}$ is a discovery energy region and that $e^{+} e^{-}$experiments there add to the search capability of the LHC in many scenarios. Second, $e^{+} e^{-}$collisions offer excellent tools for the intensive study of new phenomena, to precisely determine the properties of new particles and interactions, and to unravel the underlying theory. This claim is chronicled by the successful program at the $Z$ pole carried out at LEP and the SLC. The diagnostic tests of new physics scenarios provided by a 500-1000 GeV linear collider are detailed in this chapter. For the new physics discovered at the LHC or at the LC, the linear collider will provide further information on what it is and how it relates to higher energy scales.

Chapter 9 of this book gives a survey of the various possible mechanisms for electroweak symmetry breaking that motivate the search for new physics beyond the Standard Model at energies below $1 \mathrm{TeV}$. Among these models, supersymmetry has 
been the most intensively studied in the past few years. We have devoted Chapter 4 of this document to a discussion of how supersymmetry can be studied at a linear collider. But supersymmetry is only one of many proposals that have been made for the nature of the new physics that will appear at the $\mathrm{TeV}$ scale. In this chapter, we will discuss how several other classes of models can be tested at the linear collider. We will also discuss the general experimental probes of new physics that the linear collider makes available.

The first few sections of this chapter present the tools that linear collider experiments bring to models in which electroweak symmetry breaking is the result of new strong interactions at the $\mathrm{TeV}$ energy scale. We begin this study in Section 2 with a discussion of precision measurements of the $W$ and $Z$ boson couplings. New physics at the $\mathrm{TeV}$ scale typically modifies the couplings of the weak gauge bosons, generating, in particular, anomalous contributions to the triple gauge couplings (TGCs). These effects appear both in models with strong interactions in the Higgs sector, where they are essentially nonperturbative, and in models with new particles, including supersymmetry, where they arise as perturbative loop corrections. We document the special power of the linear collider to observe these effects.

In Section 3, we discuss the role of linear collider experiments in studying models in which electroweak symmetry breaking arises from new strong interactions. These include both models with no Higgs boson and models in which the Higgs boson is a composite of more fundamental fermions. The general methods from Section 2 play an important role in this study, but there are also new features specific to each class of model.

In Section 4, we discuss the related notion that quarks and leptons are composite states built of more fundamental constituents. The best tests for composite structure of quarks and leptons involve the sort of precision measurements that are a special strength of the linear collider.

In Section 5, we discuss the ability of linear collider experiments to discover new gauge bosons. New $Z$ and $W$ bosons arise in many extensions of the Standard Model. They may result, for example, from extended gauge groups of grand unification or from new interactions associated with a strongly coupled Higgs sector. The linear collider offers many different experimental probes for these particles, involving their couplings to all Standard Model species that are pair-produced in $e^{+} e^{-}$annihilation. This experimental program neatly complements the capability of the LHC to discover new gauge bosons as resonances in dilepton production. We describe how the LHC and linear collider results can be put together to obtain a complete phenomenological profile of a $Z^{\prime}$. Grand unified models that lead to $Z^{\prime}$ bosons often also lead to exotic fermions, so we also discuss the experiments that probe for these particles at a linear collider.

It is possible that the new physics at the $\mathrm{TeV}$ scale includes the appearance of new dimensions of space. In fact, models with extra spatial dimensions have recently been 
New Physics at the TeV Scale and Beyond

introduced to address the outstanding problems of the Standard Model, including the origin of electroweak symmetry breaking. In Section 6, we review these models and explain how they can be tested at a linear collider.

Further new and distinctive ideas about physics beyond the Standard Model are likely to appear in the future. We attempt to explore this unchartered territory in Section 7 by discussing collider tests of some unconventional possibilities arising from string theory. More generally, our limited imagination cannot span the whole range of alternatives for new physics allowed by the current data. We must prepare to discover the unexpected!

Finally, we devote Section 8 to a discussion of the determination of the origin of new physics effects. Many investigations of new phenomena at colliders focus only on defining the search reach. But once a discovery is made, the next step is to elucidate the characteristics of the new phenomena. At the linear collider, general methods such as the precision study of $W$ pair production and fermion-antifermion production can give signals in many different scenarios for new physics. However, the specific signals expected in each class of models are characteristic and can be used to distinguish the possibilities. We give an example of this and review the tools that the linear collider provides to distinguish between possible new physics sources.

We shall see in this chapter that the reach of the linear collider to discover new physics and the ability of the linear collider to perform detailed diagnostic tests combine to provide a facility with very strong capabilities to study the unknown new phenomena that we will meet at the next step in energy.

\section{Gauge boson self-couplings}

The measurement of gauge boson self-couplings at a linear collider can provide insight into new physics processes in the presence or absence of new particle production. In the absence of particle resonances, and in particular in the absence of a Higgs boson resonance, the measurement of gauge boson self-couplings will provide a window to the new physics responsible for electroweak symmetry breaking. If there are many new particles being produced - if, for example, supersymmetric particles abound - then the measurement of gauge boson self-couplings will prove valuable since the gauge boson self-couplings will reflect the properties of the new particles through radiative corrections.

\subsection{Triple gauge boson coupling overview}

Gauge boson self-couplings include the triple gauge couplings (TGCs) and quartic gauge couplings (QGCs) of the photon, $W$ and $Z$. Of special importance at a linear collider are the $W W \gamma$ and $W W Z$ TGCs since a large sample of fully reconstructed $e^{+} e^{-} \rightarrow W^{+} W^{-}$events will be available to measure these couplings. 
The effective Lagrangian for the general $W^{+} W^{-} V$ vertex $(V=\gamma, Z)$ contains 7 complex TGCs, denoted by $g_{1}^{V}, \kappa_{V}, \lambda_{V}, g_{4}^{V}, g_{5}^{V}, \tilde{\kappa}_{V}$, and $\tilde{\lambda}_{V}$ [1]. The magnetic dipole and electric quadrupole moments of the $W$ are linear combinations of $\kappa_{\gamma}$ and $\lambda_{\gamma}$ while the magnetic quadrupole and electric dipole moments are linear combinations of $\tilde{\kappa}_{\gamma}$ and $\tilde{\lambda}_{\gamma}$. The TGCs $g_{1}^{V}, \kappa_{V}$, and $\lambda_{V}$ are C- and P-conserving, $g_{5}^{V}$ is $\mathrm{C}$ - and P-violating but conserves CP, and $g_{4}^{V}, \tilde{\kappa}_{V}$, and $\tilde{\lambda}_{V}$ are CP-violating. In the $\mathrm{SM}$ at tree-level all the TGCs are zero except $g_{1}^{V}=\kappa_{V}=1$.

If there is no Higgs boson resonance below about $800 \mathrm{GeV}$, the interactions of the $W$ and $Z$ gauge bosons become strong above $1 \mathrm{TeV}$ in the $W W, W Z$ or $Z Z$ center-of-mass system. In analogy with $\pi \pi$ scattering below the $\rho$ resonance, the interactions of the $W$ and $Z$ bosons below the strong symmetry breaking resonances can be described by an effective chiral Lagrangian [2]. These interactions induce anomalous TGC's at tree-level:

$$
\begin{aligned}
\kappa_{\gamma} & =1+\frac{e^{2}}{32 \pi^{2} s_{w}^{2}}\left(L_{9 L}+L_{9 R}\right) \\
\kappa_{Z} & =1+\frac{e^{2}}{32 \pi^{2} s_{w}^{2}}\left(L_{9 L}-\frac{s_{w}^{2}}{c_{w}^{2}} L_{9 R}\right) \\
g_{1}^{Z} & =1+\frac{e^{2}}{32 \pi^{2} s_{w}^{2}} \frac{L_{9 L}}{c_{w}^{2}}
\end{aligned}
$$

where $s_{w}^{2}=\sin ^{2} \theta_{w}, c_{w}^{2}=\cos ^{2} \theta_{w}$, and $L_{9 L}$ and $L_{9 R}$ are chiral Lagrangian parameters. If we replace $L_{9 L}$ and $L_{9 R}$ by the values of these parameters in QCD, $\kappa_{\gamma}$ is shifted by $\Delta \kappa_{\gamma} \sim-3 \times 10^{-3}$.

Standard Model radiative corrections [3] cause shifts in the TGCs of $\mathcal{O}\left(10^{-4}-\right.$ $10^{-3}$ ) for CP-conserving couplings and of $\mathcal{O}\left(10^{-10}-10^{-8}\right)$ for CP-violating TGC's. Radiative corrections in the MSSM can cause shifts of $\mathcal{O}\left(10^{-4}-10^{-2}\right)$ in both the CP-conserving [4] and CP-violating TGC's [5].

\section{$2.2 \quad$ Triple gauge boson measurements}

The methods used at LEP2 to measure TGCs provide a useful guide to the measurement of TGCs at a linear collider. When measuring TGCs the kinematics of an $e^{+} e^{-} \rightarrow W^{+} W^{-}$event can be conveniently expressed in terms of the $W^{+} W^{-}$centerof-mass energy following initial-state radiation (ISR), the masses of the $W^{+}$and $W^{-}$, and five angles: the angle between the $W^{-}$and initial $e^{-}$in the $W^{+} W^{-}$rest frame, the polar and azimuthal angles of the fermion in the rest frame of its parent $W^{-}$, and the polar and azimuthal angles of the anti-fermion in the rest frame of its parent $W^{+}$.

In practice not all of these variables can be reconstructed unambiguously. For example, in events with hadronic decays it is often difficult to measure the flavor of the quark jet, and so there is usually a two-fold ambiguity for quark jet directions. Also, 
New Physics at the TeV Scale and Beyond

it can be difficult to measure ISR and consequently the measured $W^{+} W^{-}$center-ofmass energy is often just the nominal $\sqrt{s}$. Monte Carlo simulation is used to account for detector resolution, quark hadronization, initial- and final-state radiation, and other effects.

The TGC measurement error at a linear collider can be estimated to a good approximation by considering $e \nu q \bar{q}$ and $\mu \nu q \bar{q}$ channels only, and by ignoring all detector and radiation effects except for the requirement that the $W^{+} W^{-}$fiducial volume be restricted to $\left|\cos \theta_{W}\right|<0.9$. Such an approach correctly predicts the TGC sensitivity of LEP2 experiments and of detailed linear collider simulations [6]. This rule-ofthumb approximation works because LEP2 experiments and detailed linear collider simulations also use the $\tau \nu q \bar{q}, \ell \nu \ell \nu$ and $q \bar{q} q \bar{q}$ channels, and the increased sensitivity from these extra channels makes up for the lost sensitivity due to detector resolution, initial- and final-state radiation, and systematic errors.

Table 5.1 contains the estimates of the TGC precision that can be obtained at $\sqrt{s}=500$ and $1000 \mathrm{GeV}$ for the CP-conserving couplings $g_{1}^{V}, \kappa_{V}$, and $\lambda_{V}$. These estimates are derived from one-parameter fits in which all other TGC parameters are kept fixed at their tree-level SM values. Table 5.2 contains the corresponding estimates for the C- and P-violating couplings $\tilde{\kappa}_{V}, \tilde{\lambda}_{V}, g_{4}^{V}$, and $g_{5}^{V}$. An alternative method of measuring the $W W \gamma$ couplings is provided by the channel $e^{+} e^{-} \rightarrow \nu \bar{\nu} \gamma$ [7].

The difference in TGC precision between the LHC and a linear collider depends on the TGC, but typically the TGC precision at the linear collider will be substantially better, even at $\sqrt{s}=500 \mathrm{GeV}$. Figure 5.1 shows the measurement precision expected for the LHC [8] and for linear colliders of three different energies for four different TGCs.

If the goal of a TGC measurement program is to search for the first sign of deviation from the SM, one-parameter fits in which all other TGCs are kept fixed at their tree-level SM values are certainly appropriate. But what if the goal is to survey a large number TGCs, all of which seem to deviate from their SM value? Is a 28-parameter fit required? The answer is probably no, as illustrated in Fig. 5.2.

Figure 5.2 shows the histogram of the correlation coefficients for all 171 pairs of TGCs when 19 different TGCs are measured at LEP2 using one-parameter fits. The entries in Fig. 5.2 with large positive correlations are pairs of TGCs that are related to each other by the interchange of $\gamma$ and $Z$. The correlation between the two TGCs of each pair can be removed using the dependence on electron beam polarization. The entries in Fig. 5.2 with large negative correlations are TGC pairs of the type $\operatorname{Re}\left(\tilde{\kappa}_{\gamma}\right) / \operatorname{Re}\left(\tilde{\lambda}_{\gamma}\right), \operatorname{Re}\left(\tilde{\kappa}_{Z}\right) / \operatorname{Re}\left(\tilde{\lambda}_{Z}\right)$, etc. Half of the TGC pairs with large negative correlations will become uncorrelated once polarized electron beams are used, leaving only a small number of TGC pairs with large negative or positive correlation coefficients. 

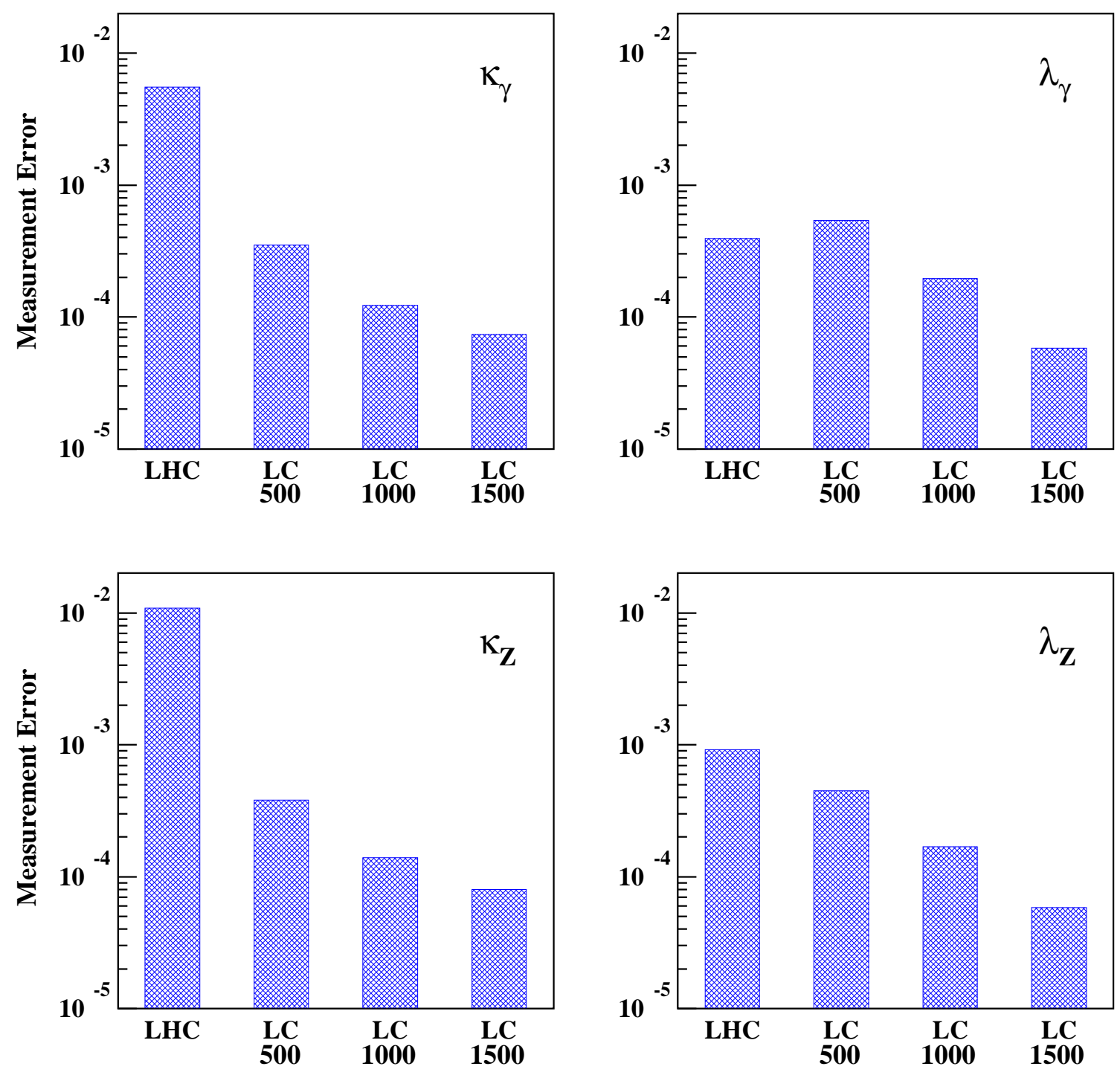

Figure 5.1: Expected measurement error for the real part of four different TGCs. The numbers below the "LC" labels refer to the center-of-mass energy of the linear collider in $\mathrm{GeV}$. The luminosity of the LHC is assumed to be $300 \mathrm{fb}^{-1}$, while the luminosities of the linear colliders are assumed to be 500,1000, and $1000 \mathrm{fb}^{-1}$ for $\sqrt{s}=500,1000$, and $1500 \mathrm{GeV}$ respectively. 
New Physics at the TeV Scale and Beyond

\begin{tabular}{|c|c|c|c|c|}
\hline & \multicolumn{4}{|c|}{ error $\times 10^{-4}$} \\
\hline \multirow[b]{2}{*}{ TGC } & \multicolumn{2}{|c|}{$\sqrt{s}=500 \mathrm{GeV}$} & \multicolumn{2}{|c|}{$\sqrt{s}=1000 \mathrm{GeV}$} \\
\hline & $\operatorname{Re}$ & $\operatorname{Im}$ & $\mathrm{Re}$ & $\mathrm{Im}$ \\
\hline$g_{1}^{\gamma}$ & 15.5 & 18.9 & 12.8 & 12.5 \\
\hline$\kappa_{\gamma}$ & 3.5 & 9.8 & 1.2 & 4.9 \\
\hline$\lambda_{\gamma}$ & 5.4 & 4.1 & 2.0 & 1.4 \\
\hline$g_{1}^{Z}$ & 14.1 & 15.6 & 11.0 & 10.7 \\
\hline$\kappa_{Z}$ & 3.8 & 8.1 & 1.4 & 4.2 \\
\hline$\lambda_{Z}$ & 4.5 & 3.5 & 1.7 & 1.2 \\
\hline
\end{tabular}

Table 5.1: Expected errors for the real and imaginary parts of CP-conserving TGCs assuming $\sqrt{s}=500 \mathrm{GeV}, \mathcal{L}=500 \mathrm{fb}^{-1}$ and $\sqrt{s}=1000 \mathrm{GeV}, \mathcal{L}=1000 \mathrm{fb}^{-1}$. The results are for one-parameter fits in which all other TGCs are kept fixed at their SM values.

\begin{tabular}{|l|rr|rr|}
\hline \hline & \multicolumn{4}{|c|}{ error $\times 10^{-4}$} \\
\hline & \multicolumn{2}{|c|}{$\sqrt{s}=500 \mathrm{GeV}$} & \multicolumn{2}{c|}{$\sqrt{s}=1000 \mathrm{GeV}$} \\
TGC & Re & \multicolumn{1}{|c|}{ Im } & \multicolumn{1}{c|}{ Re } & \multicolumn{1}{c|}{ Im } \\
\hline & & & & \\
$\tilde{\kappa}_{\gamma}$ & 22.5 & 16.4 & 14.9 & 12.0 \\
$\tilde{\lambda}_{\gamma}$ & 5.8 & 4.0 & 2.0 & 1.4 \\
$\tilde{\kappa}_{Z}$ & 17.3 & 13.8 & 11.8 & 10.3 \\
$\tilde{\lambda}_{Z}$ & 4.6 & 3.4 & 1.7 & 1.2 \\
$g_{4}^{\gamma}$ & 21.3 & 18.8 & 13.9 & 12.8 \\
$g_{5}^{\gamma}$ & 19.3 & 21.6 & 13.3 & 13.4 \\
$g_{4}^{Z}$ & 17.9 & 15.2 & 12.0 & 10.4 \\
$g_{5}^{Z}$ & 16.0 & 16.7 & 11.4 & 10.7 \\
\hline \hline
\end{tabular}

Table 5.2: Expected errors for the real and imaginary parts of C- and P-violating TGCs assuming $\sqrt{s}=500 \mathrm{GeV}, \mathcal{L}=500 \mathrm{fb}^{-1}$ and $\sqrt{s}=1000 \mathrm{GeV}, \mathcal{L}=1000 \mathrm{fb}^{-1}$. The results are for one-parameter fits in which all other TGCs are kept fixed at their SM values.

\subsection{Electroweak radiative corrections to $e^{+} e^{-} \rightarrow 4$ fermions}

We have seen that the experimental accuracy at a linear collider for the basic electroweak cross section measurements is expected to be at the level of $0.1-0.01 \%$, requiring the inclusion of electroweak radiative corrections to the predictions for the underlying production processes such as $e^{+} e^{-} \rightarrow W W \rightarrow 4 f$.

The full treatment of the processes $e^{+} e^{-} \rightarrow 4 f$ at the one-loop level is of enormous 


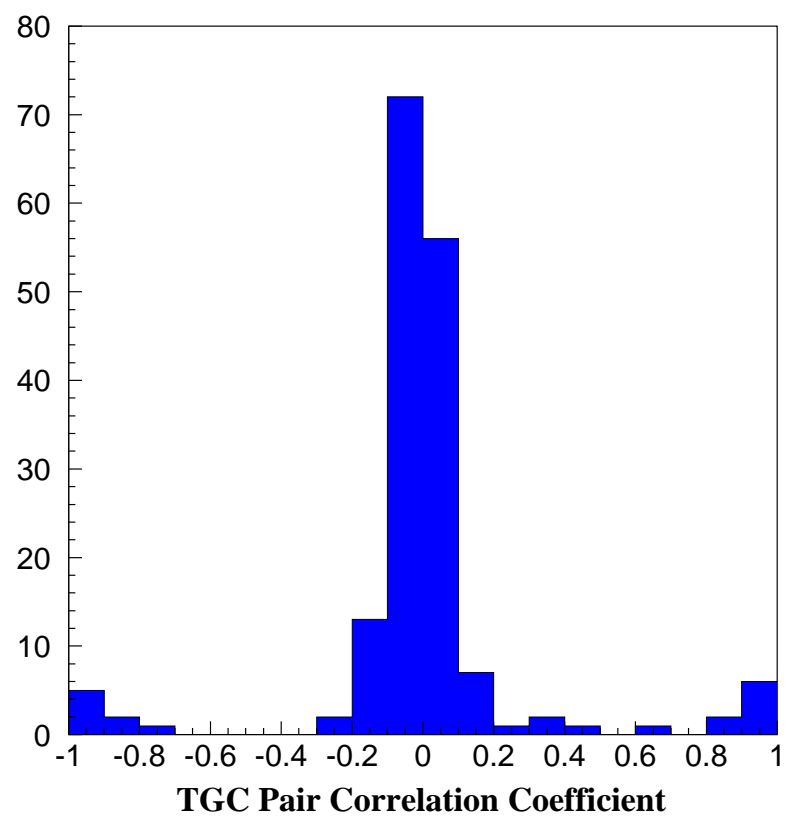

Figure 5.2: Histogram of correlation coefficients for all 171 pairs of TGCs when 19 different TGCs are measured using one-parameter fits at LEP2 (unpolarized beams). The 19 TGCs are made up of the real and imaginary parts of the $8 \mathrm{C}$ - and P-violating couplings along with the real parts of the three CP-conserving couplings $g_{1}^{Z}, \kappa_{\gamma}, \lambda_{\gamma}$.

complexity. Nevertheless there is ongoing work in this direction [9]. While the real bremsstrahlung contribution is known exactly, there are severe theoretical problems with the virtual order- $\alpha$ corrections. A detailed description of the status of predictions for $e^{+} e^{-} \rightarrow 4 f(\gamma)$ processes can be found in [10]. A suitable approach to include order$\alpha$ corrections to gauge-boson pair production is a double-pole approximation (DPA), keeping only those terms in an expansion about the gauge-boson resonance poles that are enhanced by two resonant gauge bosons. All present calculations of order- $\alpha$ corrections to $e^{+} e^{-} \rightarrow W W \rightarrow 4 f$ rely on a DPA [11-14]. Different versions of a DPA have been implemented in the Monte Carlo (MC) generators RacoonWW [12] and YFSWW3 [13]. The intrinsic DPA error is estimated to be $\alpha \Gamma_{W} /\left(\pi M_{W}\right) \sim 0.5 \%$ whenever the cross section is dominated by doubly resonant contributions. This is the case at LEP2 energies sufficiently above threshold. The DPA is not a valid approximation close to the $W$-pair production threshold. At higher energies diagrams without two resonant $W$ bosons become sizable, especially single $W$ production, and appropriate cuts must be applied to extract the $W W$ signal.

The theoretical uncertainty of present predictions for the total $W$-pair production 
New Physics at the TeV Scale and Beyond

cross section, $\sigma_{W W}$, is of the order of $0.5 \%$ for energies between $170 \mathrm{GeV}$ and $500 \mathrm{GeV}$ [10], which is within the expected DPA uncertainty. This is a result of a tuned numerical comparison between the state-of-the-art MC generators RacoonWW and YFSWW3, supported by a comparison with a semi-analytical calculation [11] and a study of the intrinsic DPA ambiguity with RacoonWW [10,12]. In the threshold region $\sigma_{W W}$ is known only to about $2 \%$, since predictions are based on an improved Born approximation [10] that neglects non-universal electroweak corrections. Further improvements of the theoretical uncertainty on $\sigma_{W W}$ are anticipated only when the full order- $\alpha$ calculation becomes available. Above $500 \mathrm{GeV}$, large electroweak logarithms of Sudakov type become increasingly important and contributions of higher orders need to be taken into account.

A tuned comparison has also been performed of RacoonWW and YFSWW3 predictions for the $W$ invariant mass and the $W$ production angle distributions, as well as for several photon observables such as photon energy and production angle distributions, at $200 \mathrm{GeV}[10,15]$ and $500 \mathrm{GeV}[15]$. Taking the observed differences between the RacoonWW and YFSWW3 predictions as a guideline, a theoretical uncertainty of the order of $1 \%$ can be assigned to the $W$ production angle distribution and the $W$ invariant mass distribution in the $W$ resonance region. A recent comparison of RacoonWW predictions for photon observables including leading higher-order initialstate radiation [15] with YFSWW3 predictions yields relative differences of less than $5 \%$ at $200 \mathrm{GeV}$ and about $10 \%$ at $500 \mathrm{GeV}$. These differences might be attributed to the different treatment of visible photons in the two MC generators: in RacoonWW the real order- $\alpha$ corrections are based on the full $4 f+\gamma$ matrix element, while in YFSWW3 multi-photon radiation in $W$-pair production is combined with order- $\alpha^{2}$ LL photon radiation in $W$ decays.

\subsection{Quartic gauge boson couplings}

The potential for directly probing anomalous quartic gauge boson couplings (AQGCs) via triple gauge-boson production at LEP2, at a future high-energy LC, and at hadron colliders has been investigated in [15-19], [15,16,21-23] and [18,24,25], respectively. The AQGCs under study arise from genuine 4- and 6-dimensional operators, i.e., they have no connection to the parametrization of the anomalous TGCs. It is conceivable that there are extensions of the SM that leave the SM TGCs unchanged but modify quartic self-interactions of the electroweak gauge bosons [21]. The possible number of operators is considerably reduced by imposing a global custodial $\mathrm{SU}(2)$ symmetry to protect the $\rho$ parameter from large contributions, i.e., to keep $\rho$ close to 1 , and by the local $\mathrm{U}(1)_{\mathrm{QED}}$ symmetry whenever a photon is involved.

The sensitivity of triple-gauge-boson cross sections to dimension- 4 operators, which only involve massive gauge bosons, has been studied for a high-energy LC and the LHC in $[21,23]$ and [24], respectively. Only weak constraints are expected from $W W W, W W Z, W Z Z$ and $Z Z Z$ productions at the LHC [24], but these processes 
may provide complementary information if non-zero AQGCs are found. The genuine dimension-4 AQGCs may be best probed in a multi-TeV LC. The sensitivity to the

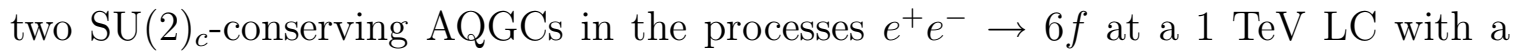
luminosity of $1000 \mathrm{fb}^{-1}$ can be expected to be between $10^{-3}$ and $10^{-2}$ [23].

The following discussion is restricted to AQGCs involving at least one photon, which can be probed in $W W \gamma, Z Z \gamma, Z \gamma \gamma$ and $W \gamma \gamma$ production. The lowest-dimension operators that lead to the photonic AQGCs $a_{0}, a_{\mathrm{c}}, a_{\mathrm{n}}, \tilde{a}_{0}$, and $\tilde{a}_{\mathrm{n}}$ are of dimension- 6 $[15,21,22,25]$ and yield anomalous contributions to the SM $W W \gamma \gamma, W W Z \gamma$ vertices, and a non-standard $Z Z \gamma \gamma$ interaction at the tree level. Most studies of AQGCs consider the separately $\mathrm{P}$ - and C-conserving couplings $a_{0}, a_{\mathrm{c}}$ and the CP-violating coupling $a_{\mathrm{n}}$. Recently the P-violating AQGCs $\tilde{a}_{0}$, and $\tilde{a}_{\mathrm{n}}$ have also been considered [15]. More general AQGCs that have been embedded in manifestly $\mathrm{SU}(2)_{\mathrm{L}} \times \mathrm{U}(1)_{\mathrm{Y}}$ gauge invariant operators are discussed in $[17,19]$. The AQGCs depend on a mass scale $\Lambda$ characterizing the scale of new physics. The choice for $\Lambda$ is arbitrary as long as no underlying model is specified which gives rise to the AQGCs. For instance, anomalous quartic interactions may be interpreted as contact interactions, which might be the manifestation of the exchange of heavy particles with a mass scale $\Lambda$.

Recently, at LEP2, the first direct bounds on the AQGCs $a_{0}, a_{\mathrm{c}}, a_{\mathrm{n}}$ have been imposed by investigating the total cross sections and photon energy distributions for the processes $e^{+} e^{-} \rightarrow W W \gamma, Z \gamma \gamma, Z \nu \bar{\nu}[20]$. The results, in units of $\mathrm{GeV}^{-2}$, are

$$
-0.037<\frac{a_{0}}{\Lambda^{2}}<0.036 \quad-0.077<\frac{a_{\mathrm{c}}}{\Lambda^{2}}<0.095 \quad-0.45<\frac{a_{\mathrm{n}}}{\Lambda^{2}}<0.41,
$$

for $95 \%$ CL intervals. These limits are expected to improve considerably as the energy increases. It has been found that a $500 \mathrm{GeV} \mathrm{LC}$ with a total integrated luminosity of $500 \mathrm{fb}^{-1}$ can improve the LEP2 limits by as much as three orders of magnitude [17].

At hadron colliders the search for AQGCs is complicated by an arbitrary form factor that is introduced to suppress unitarity-violating contributions at large parton center-of-mass energies. At the LHC, however, the dependence of a measurement of AQGCs on the form-factor parametrization may be avoided by measuring energydependent AQGCs [24]. At Run II of the Tevatron at $2 \mathrm{TeV}$, with $2 \mathrm{fb}^{-1}$, AQGC limits comparable to the LEP2 limits are expected [18,25].

Numerical studies of AQGCs are not yet as sophisticated as the ones for TGCs. For instance, most studies of AQGCs have not yet included gauge boson decays, and MC generators for the process $e^{+} e^{-} \rightarrow 4 f+\gamma$ including photon AQGCs have only recently become available $[15,19]$. To illustrate the typical size of the limits that can be obtained for the AQGCs at a $500 \mathrm{GeV}$ LC with $50 \mathrm{fb}^{-1}$, the following $1 \sigma$ bounds have been extracted from the total cross section measurement of $e^{+} e^{-} \rightarrow u \bar{d} \mu^{-} \bar{\nu}_{\mu}+\gamma$, with all bounds in units of $10^{-3} \mathrm{GeV}^{-2}$ [15]:

$$
-0.12<\frac{a_{0}}{\Lambda^{2}}<0.14 \quad-0.31<\frac{a_{\mathrm{c}}}{\Lambda^{2}}<0.16 \quad-0.82<\frac{a_{\mathrm{n}}}{\Lambda^{2}}<0.79
$$


New Physics at the TeV Scale and Beyond

$$
-0.10<\frac{\tilde{a}_{0}}{\Lambda^{2}}<0.10 \quad-0.69<\frac{\tilde{a}_{\mathrm{n}}}{\Lambda^{2}}<0.90 .
$$

The availability of MC programs $[15,19,23]$ will allow more detailed studies to be performed. For example, longitudinally polarized gauge bosons have the greatest effect on AQGCs, and gauge bosons with this polarization can be isolated through an analysis of gauge boson production and decay angles [21].

\section{$3 \quad$ Strongly coupled theories}

The Standard Model with a light Higgs boson provides a good fit to the electroweak data. Nevertheless, the electroweak observables depend only logarithmically on the Higgs mass, so that the effects of the light Higgs could be mimicked by new particles with masses as large as several TeV. A recent review of such scenarios is given in [26]. One can even imagine that no Higgs boson exists. In that case, the electroweak symmetry should be broken by some other interactions, and gauge boson scattering should become strong at a scale of order $1 \mathrm{TeV}$. An often discussed class of theories of this kind is called technicolor [27], which is discussed in the next subsection.

Electroweak symmetry is often assumed to be either connected to supersymmetry or driven by some strong dynamics, such as technicolor, without a Higgs boson. There is, however, a distinctive alternative where a strong interaction gives rise to bound states that include a Higgs boson. The latter could be light and weakly coupled at the electroweak scale. At sub-TeV energies these scenarios are described by a (possibly extended) Higgs sector, while the strong dynamics manifests itself only above a $\mathrm{TeV}$ or so.

\subsection{Strong $W W$ scattering and technicolor}

The generic idea of technicolor theories is that a new gauge interaction, which is asymptotically free, becomes strong at a scale of order $1 \mathrm{TeV}$, such that the new fermions ("technifermions") that feel this interaction form condensates that break the electroweak symmetry. This idea is based on the observed dynamics of QCD, but arguments involving the fits to the electroweak data and the generation of quark masses suggest that the technicolor interactions should be described by a strongly coupled gauge theory that has a different behavior from QCD (see, e.g., [28]).

A generic prediction of technicolor theories is that there is a vector resonance with mass below about $2 \mathrm{TeV}$ which unitarizes the $W W$ scattering cross section. In what follows we will concentrate on the capability of a linear $e^{+} e^{-}$collider of studying $W W$ scattering, but first we briefly mention other potential signatures associated

with various technicolor models. The chiral symmetry of the technifermions may be 
large enough that its dynamical breaking leads to pseudo-Goldstone bosons, which are pseudoscalar bound states that can be light enough to be produced at a linear $e^{+} e^{-}$ collider (for a recent study, see [29]). The large top-quark mass typically requires a special dynamics associated with the third generation. A thoroughly studied model along these lines is called Topcolor Assisted Technicolor [30], and leads to a rich phenomenology. This model predicts the existence of spinless bound states with large couplings to the top quark, called top-pions and top-Higgs, which may be studied at a linear $e^{+} e^{-}$collider [31].

Strong $W^{+} W^{-}$scattering is an essential test not only of technicolor theories, but in fact of any model that does not include a Higgs boson with large couplings to gauge boson pairs. It can be studied at a linear collider with the reactions $e^{+} e^{-} \rightarrow$ $\nu \bar{\nu} W^{+} W^{-}, \nu \bar{\nu} Z Z, \nu \bar{\nu} t \bar{t}$, and $W^{+} W^{-}$[32]. The final states $\nu \bar{\nu} W^{+} W^{-}, \nu \bar{\nu} Z Z$ are used to study the $\mathrm{I}=\mathrm{J}=0$ channel in $W^{+} W^{-}$scattering, while the final state $W^{+} W^{-}$ is best suited for studying the $\mathrm{I}=\mathrm{J}=1$ channel. The $\nu \bar{\nu} t \bar{t}$ final state can be used to investigate strong electroweak symmetry breaking in the fermion sector through the process $W^{+} W^{-} \rightarrow t \bar{t}$.

The first step in studying strong $W^{+} W^{-}$scattering is to separate the scattering of a pair of longitudinally polarized $W^{\prime}$ 's, denoted by $W_{\mathrm{L}} W_{\mathrm{L}}$, from transversely polarized $W^{\prime}$ 's, and from background such as $e^{+} e^{-} \rightarrow e^{+} e^{-} W^{+} W^{-}$and $e^{-} \bar{\nu} W^{+} Z$. Studies have shown that simple cuts can be used to achieve this separation in $e^{+} e^{-} \rightarrow \nu \bar{\nu} W^{+} W^{-}$, $\nu \bar{\nu} Z Z$ at $\sqrt{s}=1000 \mathrm{GeV}$, and that the signals are comparable to those obtained at the LHC [33]. Furthermore, by analyzing the gauge boson production and decay angles it is possible to use these reactions to measure chiral Lagrangian parameters with an accuracy greater than that which can be achieved at the LHC [34].

The reaction $e^{+} e^{-} \rightarrow \nu \bar{\nu} t \bar{t}$ provides unique access to $W^{+} W^{-} \rightarrow t \bar{t}$, since this process is overwhelmed by the background $g g \rightarrow t \bar{t}$ at the LHC. Techniques similar to those employed to isolate $W_{\mathrm{L}} W_{\mathrm{L}} \rightarrow W^{+} W^{-}, Z Z$ can be used to measure the enhancement in $W_{\mathrm{L}} W_{\mathrm{L}} \rightarrow t \bar{t}$ production [35]. Even in the absence of a resonance it will be possible to establish a clear signal. The ratio $S / \sqrt{B}$ is expected to be 12 for a linear collider with $\sqrt{s}=1 \mathrm{TeV}$ and $1000 \mathrm{fb}^{-1}$ and $80 \% / 0 \%$ electron/positron beam polarization, increasing to 28 for the same data sample at $1500 \mathrm{GeV}$.

There are two approaches to studying strong $W^{+} W^{-}$scattering with the process $e^{+} e^{-} \rightarrow W^{+} W^{-}$. The first approach was discussed in Section 2: a strongly coupled gauge boson sector induces anomalous TGCs that could be measured in $e^{+} e^{-} \rightarrow$ $W^{+} W^{-}$. The precision of $4 \times 10^{-4}$ for the TGCs $\kappa_{\gamma}$ and $\kappa_{Z}$ at $\sqrt{s}=500 \mathrm{GeV}$ can be interpreted as a precision of 0.26 for the chiral Lagrangian parameters $L_{9 L}$ and $L_{9 R}$. Assuming naive dimensional analysis [36], such a measurement would provide a $8 \sigma$ $(5 \sigma)$ signal for $L_{9 L}$ and $L_{9 R}$ if the strong symmetry breaking energy scale were $3 \mathrm{TeV}$ $(4 \mathrm{TeV})$. The only drawback to this approach is that the detection of anomalous TGCs does not by itself provide unambiguous proof of strong electroweak symmetry breaking. 
New Physics at the TeV Scale and Beyond

The second approach involves an effect unique to strong $W^{+} W^{-}$scattering. When $W^{+} W^{-}$scattering becomes strong the amplitude for $e^{+} e^{-} \rightarrow W_{\mathrm{L}} W_{\mathrm{L}}$ develops a complex form factor $F_{T}$ in analogy with the pion form factor in $e^{+} e^{-} \rightarrow \pi^{+} \pi^{-}$[37]. To evaluate the size of this effect the following expression for $F_{T}$ has been suggested:

$$
F_{T}=\exp \left[\frac{1}{\pi} \int_{0}^{\infty} d s^{\prime} \delta\left(s^{\prime}, M_{\rho}, \Gamma_{\rho}\right)\left\{\frac{1}{s^{\prime}-s-i \epsilon}-\frac{1}{s^{\prime}}\right\}\right]
$$

where

$$
\delta\left(s, M_{\rho}, \Gamma_{\rho}\right)=\frac{1}{96 \pi} \frac{s}{v^{2}}+\frac{3 \pi}{8}\left[\tanh \left(\frac{s-M_{\rho}^{2}}{M_{\rho} \Gamma_{\rho}}\right)+1\right] .
$$

Here $M_{\rho}, \Gamma_{\rho}$ are the mass and width, respectively, of a vector resonance in $W_{\mathrm{L}} W_{\mathrm{L}}$ scattering. The term

$$
\delta(s)=\frac{1}{96 \pi} \frac{s}{v^{2}}
$$

is the Low Energy Theorem (LET) amplitude for $W_{\mathrm{L}} W_{\mathrm{L}}$ scattering at energies below a resonance. Below the resonance, the real part of $F_{T}$ is proportional to $L_{9 L}+L_{9 R}$ and can therefore be interpreted as a TGC. The imaginary part, however, is a distinct new effect.

The real and imaginary parts of $F_{T}$ are measured [38] in the same manner as the TGCs. The $W^{+} W^{-}$production and decay angles are analyzed, and an electron beam polarization of $80 \%$ is assumed. In contrast to TGCs, the analysis of $F_{T}$ seems to benefit from even small amounts of jet flavor tagging. We therefore assume that charm jets can be tagged with a purity/efficiency of $100 / 33 \%$. These purity/efficiency numbers are based on research [39] that indicates that it may be possible to tag charm jets with a purity/efficiency as high as $100 / 65 \%$, given that $b$-jet contamination is not a significant factor in $W^{+} W^{-}$pair production and decay.

The expected $95 \%$ confidence level limits for $F_{T}$ for $\sqrt{s}=500 \mathrm{GeV}$ and a luminosity of $500 \mathrm{fb}^{-1}$ are shown in Fig. 5.3, along with the predicted values of $F_{T}$ for various masses $M_{\rho}$ of a vector resonance in $W_{\mathrm{L}} W_{\mathrm{L}}$ scattering. The masses and widths of the vector resonances are chosen to coincide with those used in the ATLAS TDR [8]. The technipion form factor $F_{T}$ affects only the amplitude for $e^{+} e^{-} \rightarrow W_{\mathrm{L}} W_{\mathrm{L}}$, whereas TGCs affect all amplitudes. Through the use of electron beam polarization and the rich angular information in $W^{+} W^{-}$production and decay, it will be possible to disentangle anomalous values of $F_{T}$ from other anomalous TGC values and to deduce the mass of a strong vector resonance well below threshold, as suggested by Fig. 5.3.

The signal significances obtained by combining the results for $e^{+} e^{-} \rightarrow \nu \bar{\nu} W^{+} W^{-}$, $\nu \bar{\nu} Z Z$ [33] with the $F_{T}$ analysis of $W^{+} W^{-}$[38] are displayed in Fig. 5.4 along with the results expected from the LHC [8]. The LHC signal is a mass bump in $W^{+} W^{-}$; the LC signal is less direct. Nevertheless, the signals at the LC are strong, particularly in $e^{+} e^{-} \rightarrow W^{+} W^{-}$, where the technirho effect gives a large enhancement of a very well-understood Standard Model process. Since the technipion form factor includes 


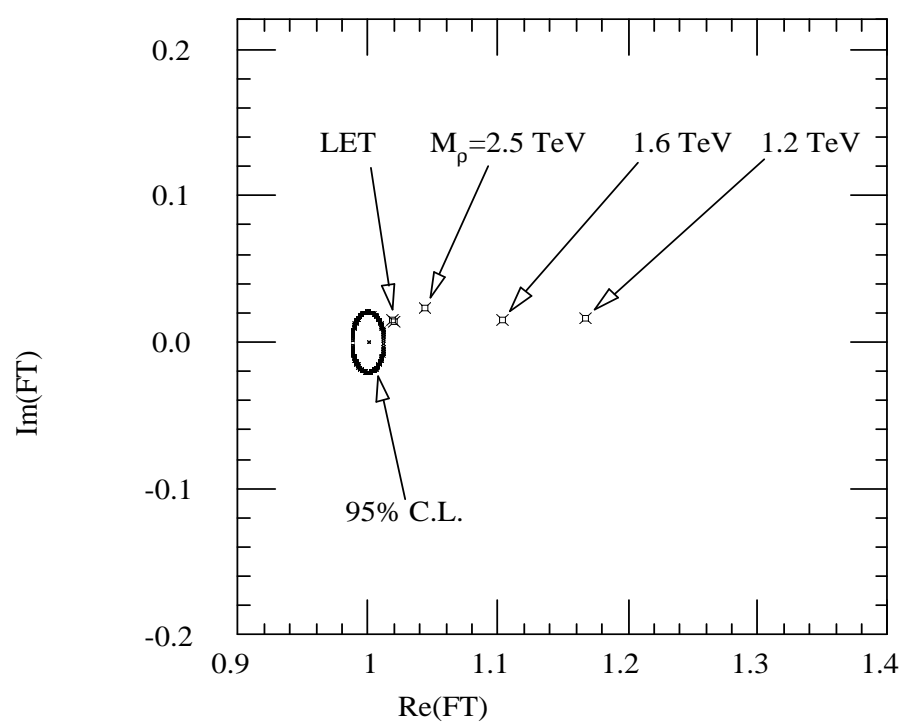

Figure 5.3: $95 \%$ C.L. contour for $F_{T}$ for $\sqrt{s}=500 \mathrm{GeV}$ and $500 \mathrm{fb}^{-1}$. Values of $F_{T}$ for various masses $M_{\rho}$ of a vector resonance in $W_{\mathrm{L}} W_{\mathrm{L}}$ scattering are also shown. The $F_{T}$ point "LET" refers to the case where no vector resonance exists at any mass in strong $W_{\mathrm{L}} W_{\mathrm{L}}$ scattering.

an integral over the technirho resonance region, the linear collider signal significance is relatively insensitive to the technirho width. (The real part of $F_{T}$ remains fixed as the width is varied, while the imaginary part grows as the width grows.) The LHC signal significance will drop as the technirho width increases. The large linear collider signals can be utilized to study a vector resonance in detail; for example, the evolution of $F_{T}$ with $\hat{s}$ can be determined by measuring the initial-state radiation in $e^{+} e^{-} \rightarrow W^{+} W^{-}$.

Only when the vector resonance disappears altogether (the LET case in the lower right-hand panel in Fig. 5.4 ) does the direct strong symmetry breaking signal from the $\sqrt{s}=500 \mathrm{GeV}$ linear collider drop below the LHC signal. At higher $e^{+} e^{-}$centerof-mass energies the linear collider signal exceeds the LHC signal.

\subsection{Composite Higgs models}

The good fit of the Standard Model to the electroweak data suggests that the new physics has a decoupling limit in which the new particles carrying $S U(2)_{W} \times U(1)_{Y}$ charges can be much heavier than the electroweak scale without affecting the Standard Model. This is the reason why the Minimal Supersymmetric Standard Model is viable: all the superpartners and the states associated with a second Higgs doublet can be taken to be heavier than the electroweak scale, leaving a low-energy theory given by 
New Physics at the TeV Scale and Beyond
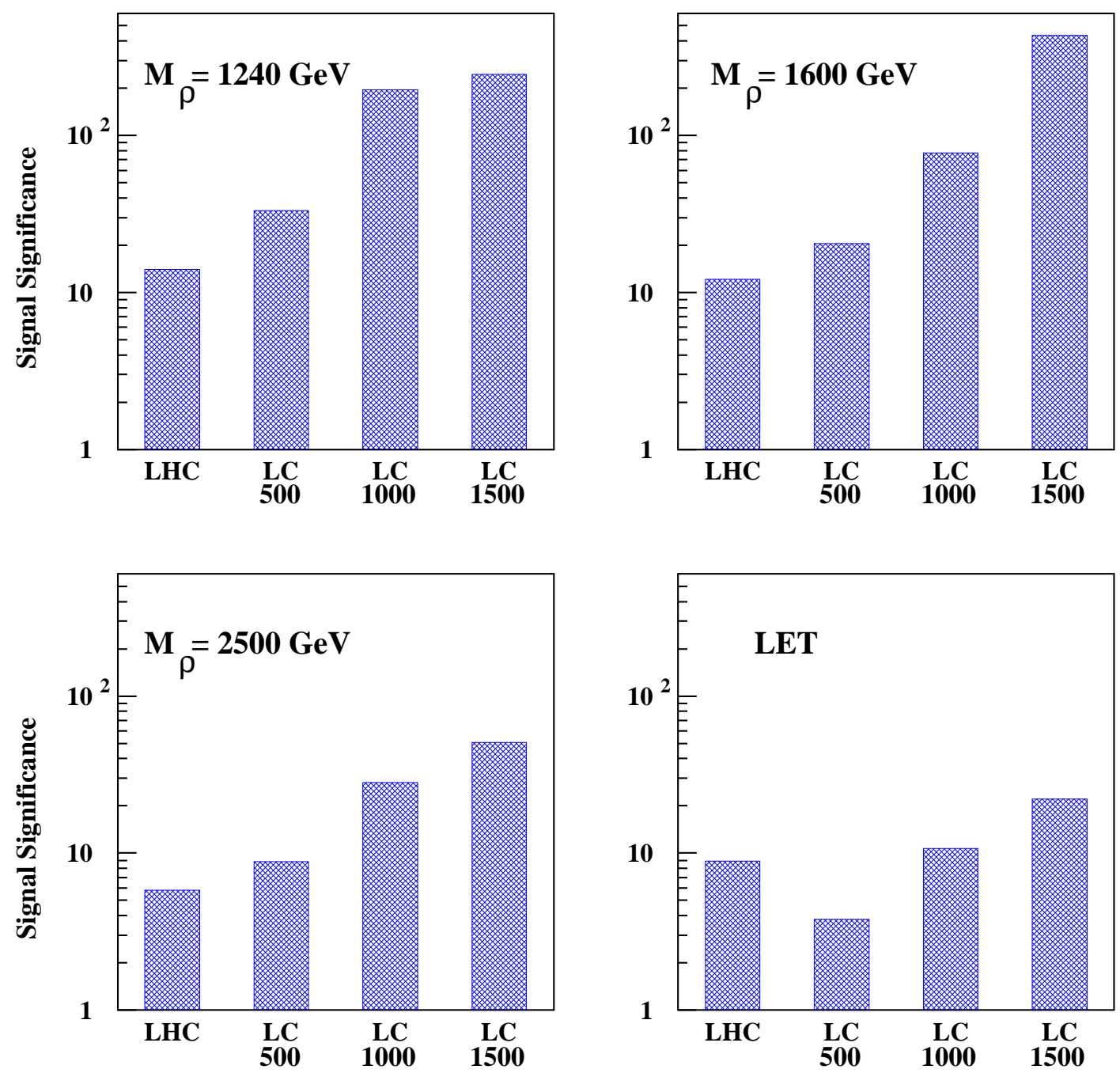

Figure 5.4: Direct strong symmetry breaking signal significance in $\sigma$ 's for various masses $M_{\rho}$ of a vector resonance in $W_{\mathrm{L}} W_{\mathrm{L}}$ scattering. In the first three plots the signal at the LHC is a bump in the $W W$ cross section; in the LET plot, the LHC signal is an enhancement over the SM cross section. The various LC signals are for enhancements of the amplitude for pair production of longitudinally polarized $W$ bosons. The numbers below the "LC" labels refer to the center-of-mass energy of the linear collider in GeV. The luminosity of the LHC is assumed to be $300 \mathrm{fb}^{-1}$, while the luminosities of the linear colliders are assumed to be 500,1000 , and $1000 \mathrm{fb}^{-1}$ for $\sqrt{s}=500,1000$, and $1500 \mathrm{GeV}$ respectively. The lower right hand plot "LET" refers to the case where no vector resonance exists at any mass in strong $W_{\mathrm{L}} W_{\mathrm{L}}$ scattering. 
the Standard Model. At the same time, it is hard to construct viable technicolor models because they do not have a decoupling limit: the new fermions that condense and give the $W$ and $Z$ masses are chiral, i.e., their masses break the electroweak symmetry.

There is a class of models of electroweak symmetry breaking that have a decoupling limit given by the Standard Model, so they are phenomenologically viable, and yet the Higgs field arises as a bound state due to some strong interactions. An example of such a composite Higgs model is the Top Quark Seesaw Theory, in which a Higgs field appears as a bound state of the top quark with a new heavy quark. This has proven phenomenologically viable and free of excessive fine-tuning [40]. Furthermore, the top quark is naturally the heaviest Standard Model fermion in this framework, because it participates directly in the breaking of the electroweak symmetry.

The interaction responsible for binding the Higgs field is provided by a spontaneously broken gauge symmetry, such as topcolor [41], or some flavor or family symmetry [42]. Such an interaction is asymptotically free, allowing for a solution to the hierarchy problem. At the same time the interaction is non-confining, and therefore has a very different behavior from the technicolor interaction discussed in the first part of this section.

Typically, in the top quark seesaw theory, the Higgs boson is heavy, with a mass of order $500 \mathrm{GeV}$ [43]. However, the effective theory below the compositeness scale may include an extended Higgs sector, in which case the mixing between the CPeven scalars could bring the mass of the Standard Model-like Higgs boson down to the current LEP limit $[40,44]$. One interesting possibility in this context is that there is a light Higgs boson with nearly standard couplings to fermions and gauge bosons, but whose decay modes are completely non-standard. This happens whenever a CP-odd scalar has a mass less than half the Higgs mass and the coupling of the Higgs to a pair of CP-odd scalars is not suppressed. The Higgs boson decays in this case into a pair of CP-odd scalars, each of them subsequently decaying into a pair of Standard Model particles with model-dependent branching fractions [45]. If the Higgs boson has Standard Model branching fractions, then the capability of an $e^{+} e^{-}$linear collider depends on $M_{H}$, as discussed in [46]. On the other hand, if the Higgs boson has non-standard decays, an $e^{+} e^{-}$collider may prove very useful in disentangling the composite nature of the Higgs boson, by measuring its width and branching fractions.

The heavy-quark constituent of the Higgs has a mass of a few $\mathrm{TeV}$, and the gauge bosons associated with the strong interactions that bind the Higgs are expected to be even heavier. Above the compositeness scale there must be some additional physics that leads to the spontaneous breaking of the gauge symmetry responsible for binding the Higgs. This may involve new gauge dynamics [47], or fundamental scalars and supersymmetry. For studying these interesting strongly interacting particles, the $e^{+} e^{-}$ collider should operate at the highest energy achievable.

Other models of Higgs compositeness have been proposed recently [48], and more 
New Physics at the TeV Scale and Beyond

are likely to be constructed in the future. Another framework in which a composite Higgs boson arises from a strong interaction is provided by extra spatial dimensions accessible to the Standard Model particles; this is discussed in Section 6 .

\section{Contact interactions and compositeness}

There is a strong historical basis for the consideration of composite models that is currently mirrored in the proliferation of fundamental particles. If the fermions have substructure, then their constituents are bound by a confining force at the mass scale $\Lambda$, which characterizes the radius of the bound states. At energies above $\Lambda$, the composite nature of fermions would be revealed by the break-up of the bound states in hard scattering processes. At lower energies, deviations from the Standard Model may be observed via form factors or residual effective interactions induced by the binding force. These composite remnants are usually parameterized by the introduction of contact terms in the low-energy Lagrangian. More generally, four-fermion contact interactions represent a useful parametrization of many types of new physics originating at high energy scales, and specific cases will be discussed throughout this chapter.

The lowest-order four-fermion contact terms are of dimension 6. A general helicityconserving, flavor-diagonal, Standard Model-invariant parameterization can be written as [49]

$$
\mathcal{L}=\frac{g_{\text {eff }}^{2} \eta}{\Lambda^{2}}\left(\bar{q} \gamma^{\mu} q+\mathcal{F}_{\ell} \bar{\ell} \gamma^{\mu} \ell\right)_{L / R}\left(\bar{q} \gamma_{\mu} q+\mathcal{F}_{\ell} \bar{\ell} \gamma_{\mu} \ell\right)_{L / R},
$$

where the generation and color indices have been suppressed, $\eta= \pm 1$, and $\mathcal{F}_{\ell}$ is inserted to allow for different quark and lepton couplings but is anticipated to be $\mathcal{O}(1)$. Since the binding force is expected to be strong when $Q^{2}$ approaches $\Lambda^{2}$, it is conventional to define $g_{\text {eff }}^{2}=4 \pi$.

Interference between the contact terms and the usual gauge interactions can lead to observable deviations from Standard Model predictions at energies lower than $\Lambda$. Currents limits from various processes at the Tevatron and LEP II place $\Lambda$ above the few-TeV range. At the LHC [8], $\Lambda_{\ell q}$ terms can be probed to $\sim 20-30 \mathrm{TeV}$ for integrated luminosities of $10-100 \mathrm{fb}^{-1}$, while the $\Lambda_{q q}$ case is more problematic because of uncertainties in the parton distributions and the extrapolation of the calorimeter energy calibration to very high values of the jet $p_{T}$.

At a LC, the use of polarized beams, combined with angular distributions, allows for a clear determination of the helicity of the contact term. An examination of contact effects in $e^{+} e^{-} \rightarrow f \bar{f}$, where $f=\mu, c, b$ was performed for LC energies in [50]. This study concentrated on tagged final states, since contact effects are diluted when all quark flavors are summed because of cancellations between the up- and down-type quarks. Here, both polarized and unpolarized angular distributions were 


\begin{tabular}{|l|c|c|c|c|}
\hline \hline & $\Lambda_{L L}$ & $\Lambda_{L R}$ & $\Lambda_{R L}$ & $\Lambda_{R R}$ \\
\hline$\sqrt{s}=0.5 \mathrm{TeV}$ & & & & \\
\hline$e_{L}^{-} e^{+} \rightarrow \mu^{+} \mu^{-}$ & 57 & 52 & 18 & 18 \\
$e_{R}^{-} e^{+} \rightarrow \mu^{+} \mu^{-}$ & 20 & 18 & 52 & 55 \\
$e_{L}^{-} e^{+} \rightarrow c \bar{c}$ & 59 & 50 & 9 & 15 \\
$e_{R}^{-} e^{+} \rightarrow c \bar{c}$ & 21 & 20 & 43 & 57 \\
$e_{L}^{-} e^{+} \rightarrow b \bar{b}$ & 68 & 53 & 9 & 16 \\
$e_{R}^{-} e^{+} \rightarrow b \bar{b}$ & 30 & 21 & 59 & 59 \\
\hline$\sqrt{s}=1.0 \mathrm{TeV}$ & & & & \\
\hline$e_{L}^{-} e^{+} \rightarrow \mu^{+} \mu^{-}$ & 79 & 72 & 25 & 26 \\
$e_{R}^{-} e^{+} \rightarrow \mu^{+} \mu^{-}$ & 28 & 25 & 73 & 78 \\
$e_{L}^{-} e^{+} \rightarrow c \bar{c}$ & 82 & 72 & 12 & 21 \\
$e_{R}^{-} e^{+} \rightarrow c \bar{c}$ & 30 & 28 & 62 & 78 \\
$e_{L}^{-} e^{+} \rightarrow b \bar{b}$ & 94 & 77 & 14 & 23 \\
$e_{R}^{-} e^{+} \rightarrow b \bar{b}$ & 43 & 30 & 82 & 84 \\
\hline \hline
\end{tabular}

Table 5.3: 95\% CL search reach in $\mathrm{TeV}$ for contact interaction scales with various helicities.

examined with tagging efficiencies of $60 \%$ and $35 \%$ for $b$ - and $c$-quarks, respectively, and the detector acceptance was taken to be $|\cos \theta|<0.985$. The resulting $95 \% \mathrm{CL}$ sensitivity for $\mathcal{L}=500 \mathrm{fb}^{-1}$ to $\Lambda$ from the polarized distributions with $90 \%$ electron beam polarization is listed in Table 5.3.

Compositeness limits for $\Lambda_{\mathrm{LL}}^{+}$from Møller and Bhabha scattering [51] are summarized in Fig. 5.5. For equal luminosities the limits from Møller scattering are significantly better than those from Bhabha scattering. This is due not only to the polarization of both beams, but also to the Møller/Bhabha crossing relation in the central region of the detector. Limits on $\Lambda_{\mathrm{LL}}^{+}$for different energies and luminosities can be calculated under the assumption that the compositeness limit scales as $\mathcal{L}^{1 / 4} s^{1 / 2}$.

\section{New particles in extended gauge sectors and GUTs}

\subsection{Extended gauge sectors}

New gauge bosons are a feature of many extensions of the Standard Model. They arise naturally in grand unified theories, such as $S O(10)$ and $E_{6}$, where the GUT group gives rise to extra $U(1)$ or $S U(2)$ subgroups after decomposition. There are also numerous non-unified extensions, such as the Left-Right Symmetric model and Topcolor. More recently, there has been renewed interest in Kaluza-Klein excitations of the SM gauge bosons, which are realized in theories of extra space dimensions at semi-macroscopic scales. All of these extensions of the SM predict the existence of 
New Physics at the TeV Scale and Beyond

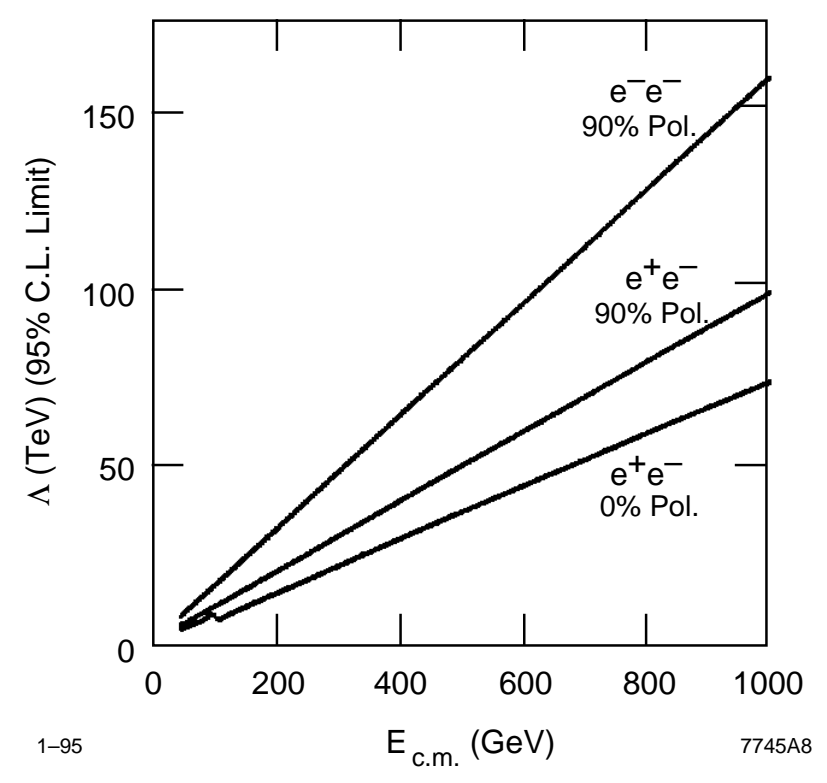

Figure 5.5: The 95\% confidence level limits for the compositeness scale $\Lambda_{\mathrm{LL}}^{+}$from Møller and Bhabha scattering as a function of the $e^{-} e^{-}$or $e^{+} e^{-}$center-of-mass energy. The luminosity is given by $\mathcal{L}=680 \mathrm{pb}^{-1} \cdot s / M_{Z}^{2}$. The polarization of the electron beam(s) is indicated in the figure.

new gauge bosons, generically denoted as $Z^{\prime}$ or $W^{\prime}$. The search for extra gauge bosons thus provides a common coin in the quest for new physics at high-energy colliders. Here, we concentrate on the most recent developments on the subject, and refer the interested to recent reviews [52].

\subsubsection{Z' discovery limits and identification}

The signal for the existence of a new neutral gauge boson at linear collider energies arises through the indirect effects of $s$-channel $Z^{\prime}$ exchange. Through its interference with the SM $\gamma$ and $Z$ exchange in $e^{+} e^{-} \rightarrow f \bar{f}$, significant deviations from SM predictions can occur even when $M_{Z^{\prime}}$ is much larger than $\sqrt{s}$. This sensitivity to the $Z^{\prime}$ nicely complements the ability of the LHC to discover a $Z^{\prime}$ as a resonance in lepton pair production. The combination of many LC observables such as the cross sections for $f \bar{f}$ final states, forward-backward asymmetries, $A_{F B}^{f}$, and left-right asymmetries, $A_{L R}^{f}$, where $f=\mu, \tau, c, b$, and light quarks, can fill in the detailed picture of the $Z^{\prime}$ couplings.

The combined sensitivity of the LC measurements for various $Z^{\prime}$ models is shown in Fig. 5.6 [52]. We see that if a $Z^{\prime}$ is detected at the LHC, precision measurements at the $\mathrm{LC}$ could be used to measure its properties and determine the underlying theory. 


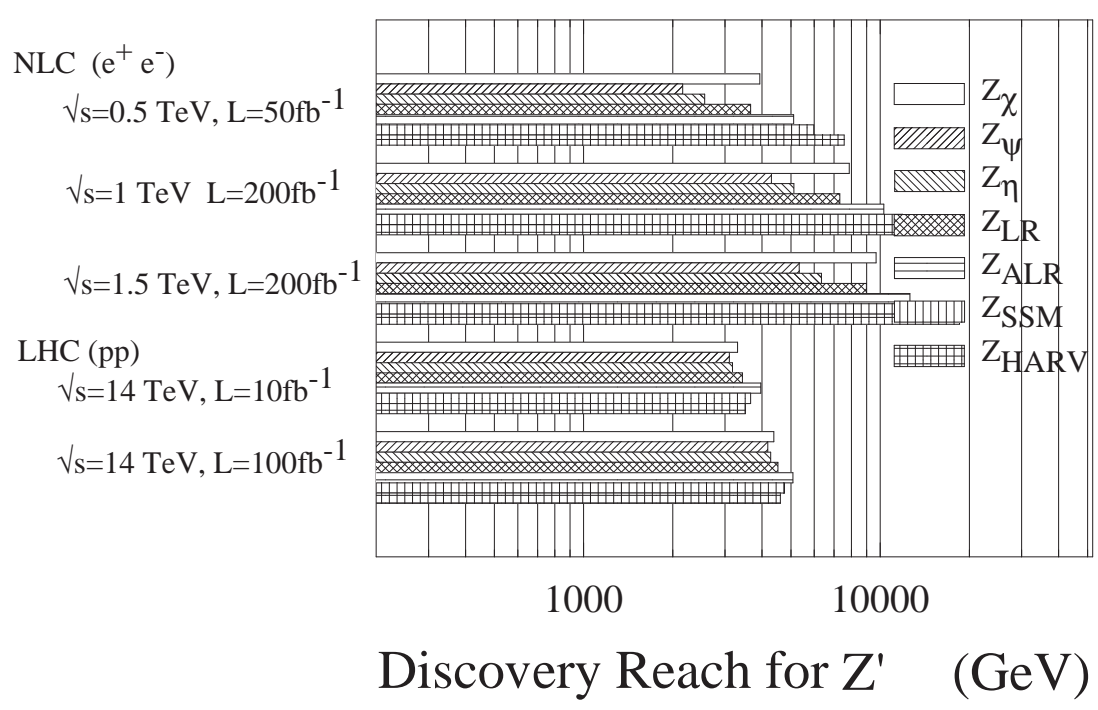

Figure 5.6: 95\% CL search limits for extra neutral gauge bosons, for various models, at high-energy linear colliders, by observation of corrections to $e^{+} e^{-} \rightarrow f \bar{f}$ processes, and at the LHC, by observation of a peak in dilepton pairs.

Figure 5.7 displays the resolving power between $Z^{\prime}$ models assuming that the mass of the $Z^{\prime}$ was measured previously at the LHC. This study only considers leptonic final states and assumes lepton universality. If $M_{Z^{\prime}}$ were beyond the LHC discovery reach or if the $Z^{\prime}$ does not couple to quarks then no prior knowledge of it would be obtained before the LC turns on. However, in this case, the LC can still yield some information on the $Z^{\prime}$ couplings and mass. Instead of extracting $Z^{\prime}$ couplings directly, "normalized" couplings, defined by

$$
a_{f}^{N}=a_{f}^{\prime} \sqrt{\frac{s}{M_{Z^{\prime}}^{2}-s}} ; \quad v_{f}^{N}=v_{f}^{\prime} \sqrt{\frac{s}{M_{Z^{\prime}}^{2}-s}}
$$

could be measured. For a demonstration of this case, the diagnostic power of a $1 \mathrm{TeV}$ LC for a $Z^{\prime}$ with couplings of the $E_{6}$ model $\chi$ and mass $M_{Z^{\prime}}=5 \mathrm{TeV}$ is displayed in Fig. 5.7 for $f=\ell$. An additional determination of the $Z^{\prime}$ mass and couplings could be performed [52] in this case from cross section and asymmetry measurements at several different values of $\sqrt{s}$.

A recent study of the process $e^{+} e^{-} \rightarrow \nu \bar{\nu} \gamma$ has demonstrated that the process can also be used to obtain information on $Z^{\prime}-\nu \bar{\nu}$ couplings [53].

\subsection{2 $W^{\prime}$ discovery limits and identification}

While considerable effort has been devoted to the study of $Z^{\prime}$ bosons at $e^{+} e^{-}$colliders, a corresponding endeavor for the $W^{\prime}$ sector has only recently been undertaken. A 
New Physics at the TeV Scale and Beyond
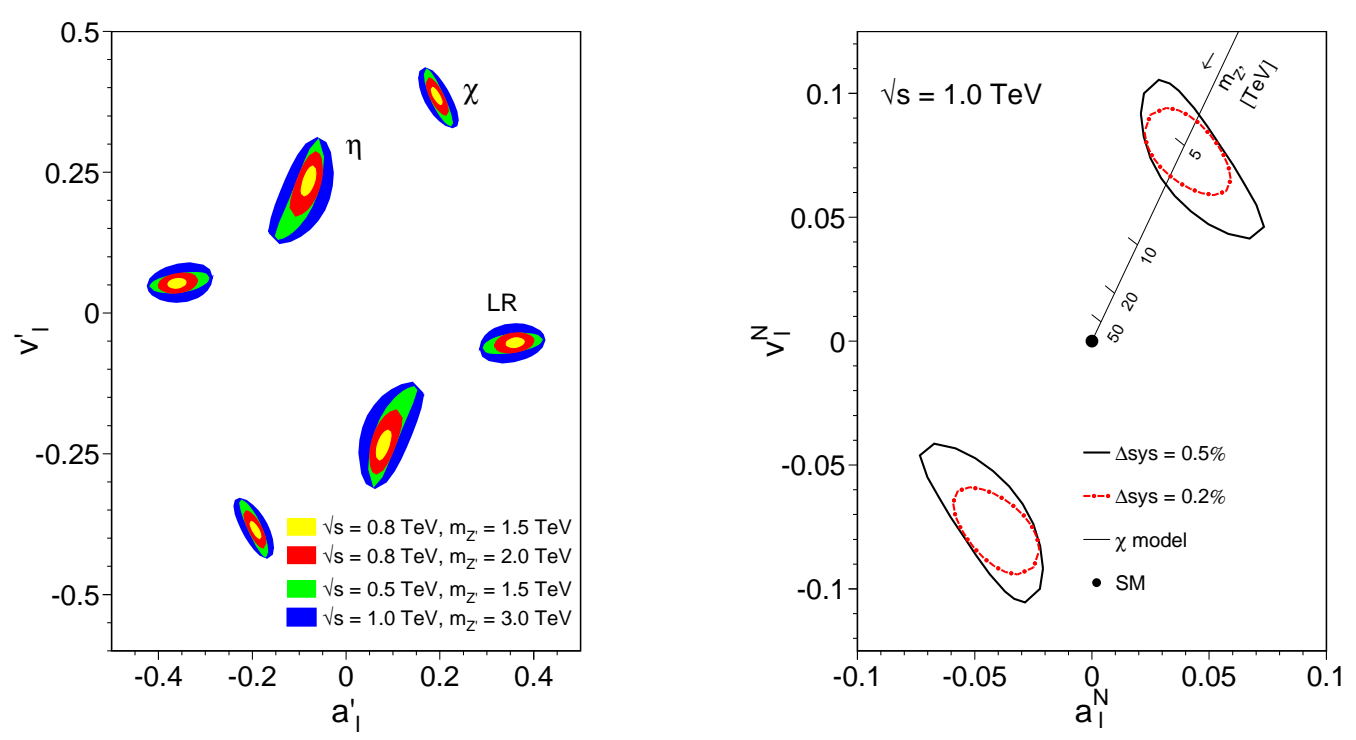

Figure 5.7: Left Panel: Resolution power (95\% C.L.) for different $M_{Z_{\chi}^{\prime}}$ based on measurements of leptonic observables at $\sqrt{s}=1 \mathrm{TeV}$ with a luminosity of $L_{\mathrm{int}}=1 \mathrm{ab}^{-1}$ [56]. Right Panel: Resolution power (95\% C.L.) for different $M_{Z^{\prime}}$ based on measurements of leptonic observables at $\sqrt{s}=500 \mathrm{GeV}, 800 \mathrm{GeV}, 1 \mathrm{TeV}$ with a luminosity of $L_{\text {int }}=1 \mathrm{ab}^{-1}$. The leptonic couplings of the $Z^{\prime}$ correspond to the $\chi, \eta$, or $L R$ model [56].

preliminary investigation [54] of the sensitivity of $e^{+} e^{-} \rightarrow \nu \bar{\nu} \gamma$ to $W^{\prime}$ bosons was performed at Snowmass 1996, and more detailed examinations [53,55] have recently been performed. The models with extra $S U(2)$ factors considered in these studies are the Left-Right symmetric model (LRM) based on the gauge group $S U(2)_{L} \times$ $S U(2)_{R} \times U(1)_{B-L}$, the Un-Unified model $(\mathrm{UUM})$ based on $S U(2)_{q} \times S U(2)_{l} \times U(1)_{Y}$ where the quarks and leptons each transform under their own $S U(2)$, a Third Family Model (3FM) based on the group $S U(2)_{h} \times S U(2)_{l} \times U(1)_{Y}$ where the quarks and leptons of the third (heavy) family transform under a separate group, and the KK model which contains the Kaluza-Klein excitations of the SM gauge bosons that are a possible consequence of theories with large extra dimensions.

In the process $e^{+} e^{-} \rightarrow \nu \bar{\nu} \gamma$, both charged and neutral extra gauge bosons can contribute. In the analysis of [53], the photon energy and angle with respect to the beam axis are restricted to $E_{\gamma} \geq 10 \mathrm{GeV}$ and $10^{\circ} \leq \theta_{\gamma} \leq 170^{\circ}$, to take into account detector acceptance. The most serious background, radiative Bhabha scattering in which the scattered $e^{+}$and $e^{-}$go undetected down the beam pipe, is suppressed by restricting the photon's transverse momentum to $p_{T}^{\gamma}>\sqrt{s} \sin \theta_{\gamma} \sin \theta_{v} /\left(\sin \theta_{\gamma}+\sin \theta_{v}\right)$, where $\theta_{v}$ is the minimum angle at which the veto detectors may observe electrons or positrons; here, $\theta_{v}=25 \mathrm{mrad}$. The observable $d \sigma / d E_{\gamma}$ was found to provide the most statistically significant search reach. The $95 \%$ CL reach is displayed graphically in Fig. 5.8 and in Table 5.4, which shows the degradation when a $2 \%$ systematic error 
is added in quadrature with the statistical error. The corresponding $W^{\prime}$ search reach at the $\mathrm{LHC}$ is in the range $5-6 \mathrm{TeV}[52]$.

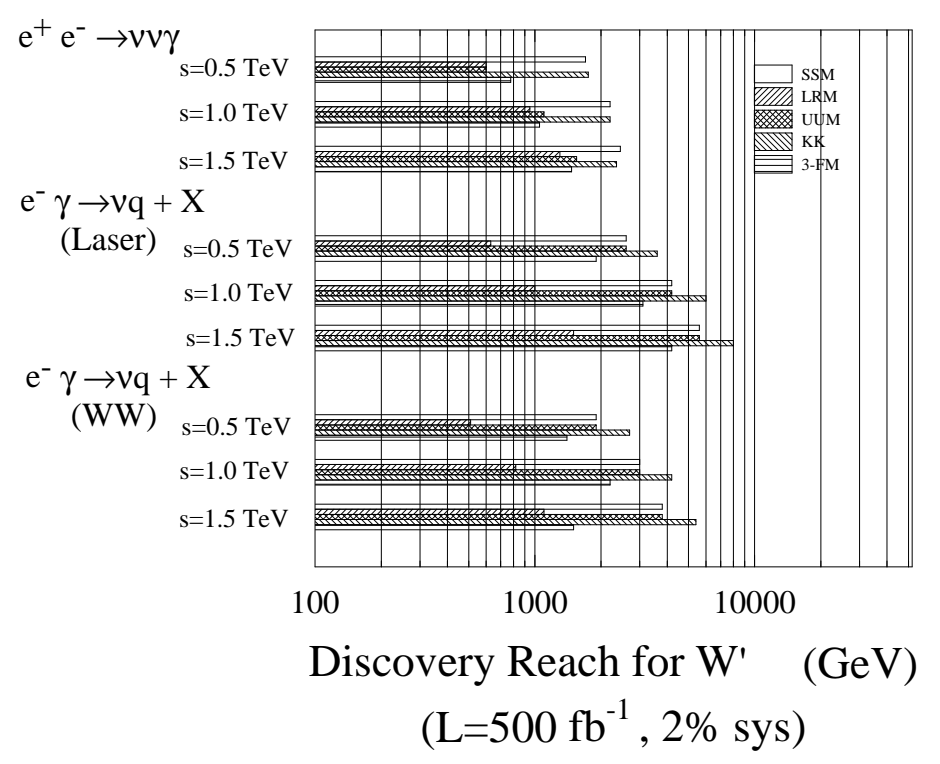

Figure 5.8: 95\% CL search limits for $W^{\prime}$ bosons at the LC.

The $95 \%$ CL constraints that can be placed on the right- and left-handed couplings of a $W^{\prime}$ to fermions, assuming that the $W^{\prime}$ has Standard Model-like couplings, and that there is no corresponding $Z^{\prime}$ contribution to $e^{+} e^{-} \rightarrow \nu \bar{\nu} \gamma$, are shown in Fig. 5.9. Here, the total cross section $\sigma$ and the left-right asymmetry $A_{L R}$ are used as observables, with the systematic errors for $\sigma\left(A_{L R}\right)$ taken as $2 \%(1 \%)$ and $80 \%$ electron and $60 \%$ positron polarization are assumed. The axes in this figure correspond to couplings normalized as $L_{f}(W)=C_{L}^{W^{\prime}} g /(2 \sqrt{2})$ and similarly for $R_{f}(W)$. It is found that $2 \%$ systematic errors dominate the coupling determination. In addition, we note that the $W^{\prime}$ couplings can only be constrained up to a two-fold ambiguity, which could be resolved by reactions in which the $W^{\prime}$ couples to a triple gauge vertex.

Additional sensitivity to the existence of a $W^{\prime}$ can be gained from $e \gamma \rightarrow \nu q+X$ [55]. This process receives contributions only from charged and not from neutral gauge bosons. The $W^{\prime}$ contribution can be isolated by imposing a kinematic cut requiring either the $q$ or $\bar{q}$ to be collinear to the beam axis. In order to take into account detector acceptance, the angle $\theta_{q}$ of the detected quark relative to the beam axis is restricted to $10^{\circ} \leq \theta_{q} \leq 170^{\circ}$. The kinematic variable that is most sensitive to a $W^{\prime}$ is the $p_{T_{q}}$ distribution. The quark's transverse momentum relative to the beam is restricted to $p_{T}^{q}>40(75) \mathrm{GeV}$ for $\sqrt{s}=0.5(1.0) \mathrm{TeV}$, to suppress various Standard Model backgrounds. Figure 5.9 and Table 5.4 show the resulting $95 \%$ CL constraints on the $W^{\prime}$ fermionic couplings for the case of backscattered laser photons. As seen above, the assumed systematic error of $2 \%$ again dominates the statistical 
New Physics at the TeV Scale and Beyond
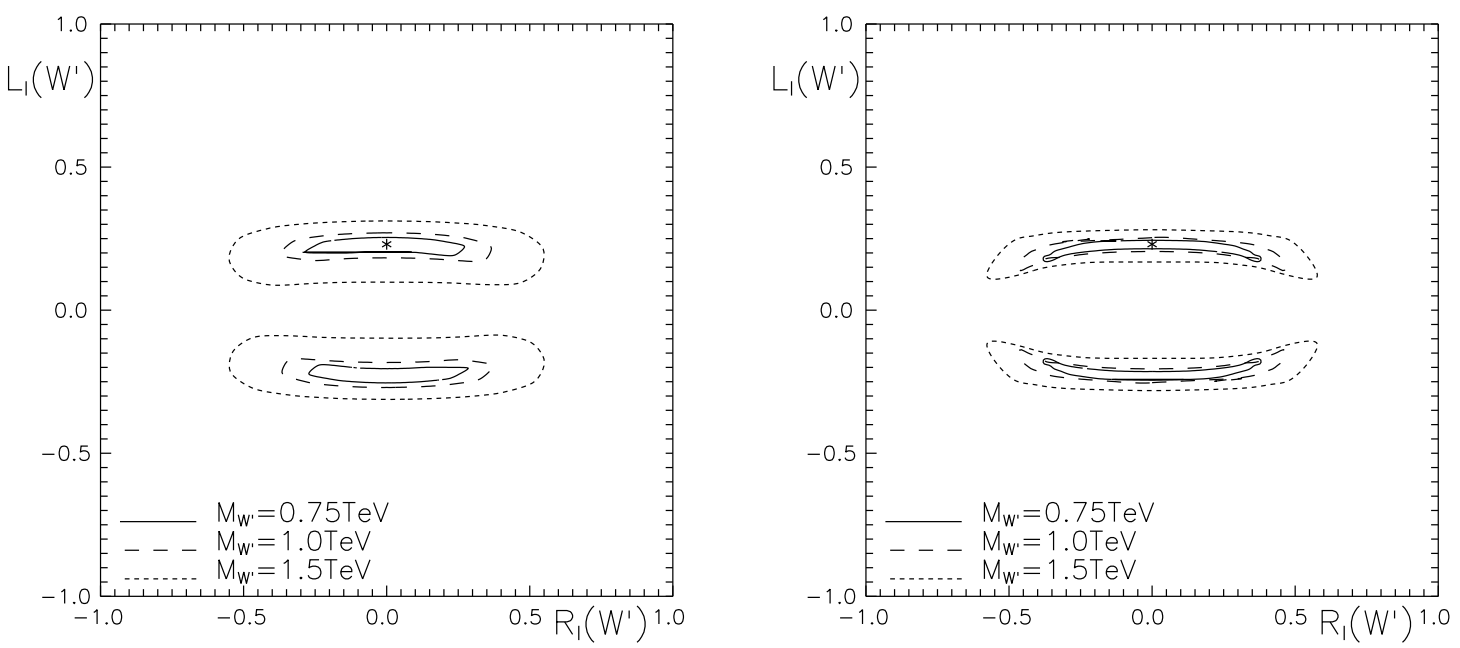

Figure 5.9: Left Panel: 95\% CL constraints from $e^{+} e^{-} \rightarrow \nu \bar{\nu} \gamma$ on couplings of the SSM $W^{\prime}$ indicated by a star for $\sqrt{s}=0.5 \mathrm{TeV}$ and $L_{\mathrm{int}}=1000 \mathrm{fb}^{-1}$ with a systematic error of $0.5 \%(0.25 \%)$ for $\sigma\left(A_{L R}\right)$ for different $W^{\prime}$ masses. Right Panel: $95 \%$ C.L. constraints from $e \gamma \rightarrow \bar{\nu} q+X$ on couplings of the SSM $W^{\prime}$ for $\sqrt{s}=0.5 \mathrm{TeV}$ and $L_{\text {int }}=1000 \mathrm{fb}^{-1}$ with a $2 \%$ systematic error for different $W^{\prime}$ masses.

\begin{tabular}{|l|ll|ll|ll|ll|}
\hline \hline & \multicolumn{3}{|c|}{$\sqrt{s}=0.5 \mathrm{TeV}, L_{\text {int }}=500 \mathrm{fb}^{-1}$} & \multicolumn{3}{c|}{$\sqrt{s}=1 \mathrm{TeV}, L_{\text {int }}=500 \mathrm{fb}^{-1}$} \\
\hline \multirow{2}{*}{ Model } & \multicolumn{2}{|c|}{$e^{+} e^{-} \rightarrow \nu \bar{\nu} \gamma$} & \multicolumn{2}{|c|}{$e \gamma \rightarrow \nu q+X$} & \multicolumn{2}{c|}{$e^{+} e^{-} \rightarrow \nu \bar{\nu} \gamma$} & \multicolumn{2}{c|}{$e \gamma \rightarrow \nu q+X$} \\
no syst. & syst. & no syst & syst. & no syst. & syst. & no syst. & syst. \\
\hline SSM $W^{\prime}$ & 4.3 & 1.7 & 4.1 & 2.6 & 5.3 & 2.2 & 5.8 & 4.2 \\
LRM & 1.2 & 0.6 & 0.8 & 0.6 & 1.6 & 1.1 & 1.2 & 1.1 \\
UUM & 2.1 & 0.6 & 4.1 & 2.6 & 2.5 & 1.1 & 5.8 & 4.2 \\
KK & 4.6 & 1.8 & 5.7 & 3.6 & 5.8 & 2.2 & 8.3 & 6.0 \\
$3 \mathrm{FM}$ & 2.3 & 0.8 & 3.1 & 1.9 & 2.7 & 1.1 & 4.4 & 3.1 \\
\hline \hline
\end{tabular}

Table 5.4: 95\% CL search limits for $W^{\prime}$ bosons, in $\mathrm{TeV}$, for various reactions.

error, thus eliminating the potential gain from high luminosities. $W^{\prime}$ coupling determination from backscattered laser photons are considerably better than those from Weizsäcker-Williams photons or from $e^{+} e^{-}$collisions. Polarized beams give only a minor improvement to these results after the inclusion of systematic errors.

If a $W^{\prime}$ were discovered elsewhere, measurements of its couplings in both $e^{+} e^{-} \rightarrow$ $\nu \bar{\nu} \gamma$ and $e \gamma \rightarrow \nu q+X$ could provide valuable information regarding the underlying model, with the latter process serving to isolate the $W^{\prime}$ couplings from those of the $Z^{\prime}$. 


\section{$5.2 \quad$ Leptoquarks}

Leptoquarks are natural in theories that relate leptons and quarks at a more fundamental level. These spin-0 or -1 particles carry both baryon and lepton number and are color triplets under $\mathrm{SU}(3)_{C}$. They can be present at the electroweak scale in models where baryon and lepton number are separately conserved, thus avoiding conflicts with rapid proton decay. Their remaining properties depend on the model in which they appear, and would need to be determined in order to ascertain the framework of the underlying theory. Given the structure of the Standard Model fermions, there are 14 different possible types of leptoquarks; their classification can be found in [57]. Their fermionic couplings proceed through a Yukawa interaction of unknown strength, while their gauge couplings are specified for a particular leptoquark. Lowenergy data place tight constraints on intergenerational leptoquark Yukawa couplings and also require that these couplings be chiral. A summary of the current state of experimental searches for leptoquarks is given in [58].

At a linear collider, leptoquarks may be produced in pairs or as single particles, while virtual leptoquark exchange may be present in $e^{+} e^{-} \rightarrow$ hadrons. Pair production receives a $t$-channel quark-exchange contribution whose magnitude depends on the size of the Yukawa coupling. This only competes with the usual $s$-channel exchange, which depends on the leptoquark's gauge couplings, if the Yukawa coupling is of order electromagnetic strength. The possible signatures are $e^{+} e^{-}, e^{ \pm}$plus missing $E_{T}$, or missing $E_{T}$ alone, combined with two jets. The observation is straightforward essentially up to the kinematic limit. A thorough study of the background and resulting search reach for each type of leptoquark can be found in [59]. Single leptoquark production is most easily studied in terms of the quark content of the photon [60]. In this case a lepton fuses with a quark from a Weiszäcker-Williams photon (in $e^{+} e^{-}$ mode) or a laser-backscattered photon (in $e \gamma$ mode) to produce a leptoquark. The cross section is a convolution of the parton-level process with distribution functions for the photon in the electron and the quark in the photon, and is directly proportional to the eqLQ Yukawa coupling. The kinematic advantage of single production is lost if the Yukawa coupling is too small. For Yukawa couplings of electromagnetic strength, leptoquarks with mass up to about $90 \%$ of $\sqrt{s}$ can be discovered at a LC [60]. If the Yukawa couplings are sizable enough, then virtual leptoquark exchange [61] will lead to observable deviations in the hadronic production cross section for leptoquark masses in excess of $\sqrt{s}$. A summary of the search reach from these three processes is shown in Fig. 5.10 from [59] in the leptoquark mass-coupling plane. In comparison, leptoquarks are produced strongly at the LHC, with search reaches in the $1.5 \mathrm{TeV}$ range [62] independent of the Yukawa couplings.

The strength of the LC is in the determination of the leptoquark's electroweak quantum numbers and the strength of its Yukawa couplings once it is discovered. Together, the production rate and polarized left-right asymmetry can completely de- 
New Physics at the TeV Scale and Beyond

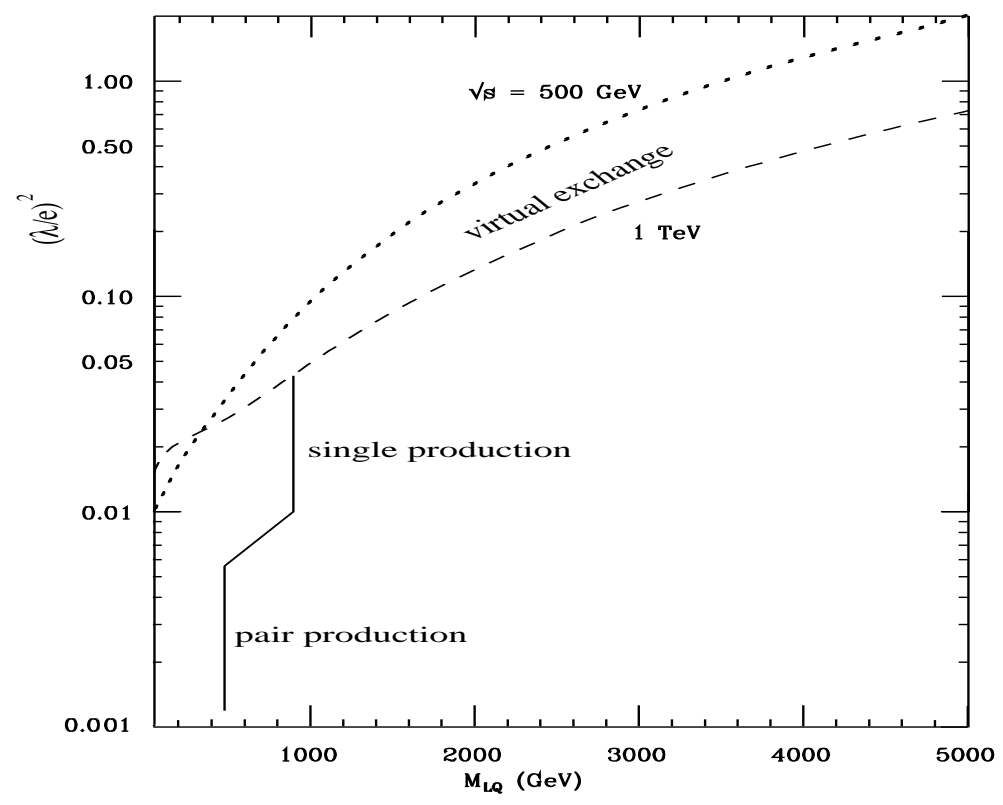

Figure 5.10: Leptoquark search limits at a LC from the three processes discussed in the text. The Yukawa coupling is scaled to $e$. The pair- and single-production reaches are shown for $\sqrt{s}=1 \mathrm{TeV}$, while the indirect reach is displayed for $\sqrt{s}=0.5$ and $1 \mathrm{TeV}$.

termine the leptoquark's electroweak properties and identify its type [63] in both the pair and single production channels, up to the kinematic limit. In addition, the Yukawa coupling strength can be measured via the forward-backward asymmetry in leptoquark pair production (which is non-vanishing for significant Yukawa couplings), deviations in the hadronic cross sections, and the comparison of pair and single production rates.

\subsection{Exotic fermions}

Fermions beyond the ordinary Standard Model content arise in many extensions of the Standard Model, notably in grand unified theories. They are referred to as exotic fermions if they do not have the usual $\mathrm{SU}(2)_{L} \times \mathrm{U}(1)_{Y}$ quantum numbers. For a review, we refer the reader to [64]. Examples of new fermions are the following: (i) The sequential repetition of a Standard Model generation (of course, in this case the fermions maintain their usual $\mathrm{SU}(2)_{L} \times \mathrm{U}(1)_{Y}$ assignments). (ii) Mirror fermions, which have chiral properties opposite to those of their Standard Model counterparts [65]. The restoration of left-right symmetry is a motivating factor for this possibility. (iii) Vector-like fermions that arise when a particular weak isospin representation is present for both left and right handed components. For instance, in $\mathrm{E}_{6}$ grand unified theories, with each fermion generation in the representation of dimension 27, there are two additional isodoublets of leptons, one sequential (left-handed) and one mirror 
(right-handed). This sort of additional content is referred to as a vector doublet model (VDM) [66], whereas the addition of weak isosinglets in both chiralities is referred to as a vector singlet model (VSM) [67].

Exotic fermions can mix with the Standard Model fermions; in principle, the mixing pattern may be complicated and is model-independent. One simplifying factor is that intergenerational mixing is severely limited by the constraints on flavor-changing neutral currents, as such mixing is induced at the tree level [66]. Thus most analyses neglect intergenerational mixing. Global fits of low-energy electroweak data and the high-precision measurements of the $Z$ properties provide upper limits for the remaining mixing angles of the order of $\sin ^{2} \theta_{\text {mix }} \leq 10^{-2}-10^{-3}[68]$.

Exotic fermions may be produced in $e^{+} e^{-}$collisions either in pairs or singly in association with their Standard Model partners as a result of mixing. The cross section for pair production of exotic quarks via gluon fusion and the Drell-Yan process at the LHC is large enough that the reach of the LC is unlikely to be competitive [69]. On the other hand, the backgrounds to exotic lepton production are large in $p p$ collisions, with production in $e^{+} e^{-}$collisions providing a promising alternative. Generally, the search reach for exotic leptons is up to the kinematic limit of the $e^{+} e^{-}$ machine, for allowed mixings [70]. The experimental signature requires knowledge of the $L^{ \pm}$decay mode, which is model-dependent and also depends on the mass difference of the charged and neutral exotic leptons. Studies indicate that the signals for exotic lepton production are clear and easy to separate from Standard Model backgrounds $[64,70,71]$, and that the use of polarized beams is important in determining the electroweak quantum numbers [71].

Almeida et al. have recently presented a detailed study of neutral heavy lepton production at high-energy $e^{+} e^{-}$colliders [72]. They find single heavy neutrino production to be more important than pair production and have calculated the process $e^{+} e^{-} \rightarrow \nu e^{ \pm} W^{\mp}$ including on-shell and off-shell heavy neutrinos. They conclude that $e^{+} e^{-}$colliders can test the existence of heavy Dirac and Majorana neutrinos up to

$\sqrt{s}$ in the $\nu e^{ \pm}+$hadrons channel. Single heavy neutrino production can be clearly separated from Standard Model backgrounds, particularly with the application of angular cuts on the final-state particle distributions. Figure 5.11 shows the on-shell approximation cross sections for various pair- and single-production processes, with all mixing angles such that $\sin ^{2} \theta_{\text {mix }}=0.0052$ [68].

\section{Extra dimensions}

The possibility has recently been proposed of utilizing the geometry of extra spatial dimensions to address the hierarchy problem, i.e., the disparity between the electroweak and Planck scales [73,74]. This idea exploits the fact that gravity has yet to be probed at energy scales much above $10^{-3} \mathrm{eV}$ in laboratory experiments, imply- 
New Physics at the TeV Scale and Beyond

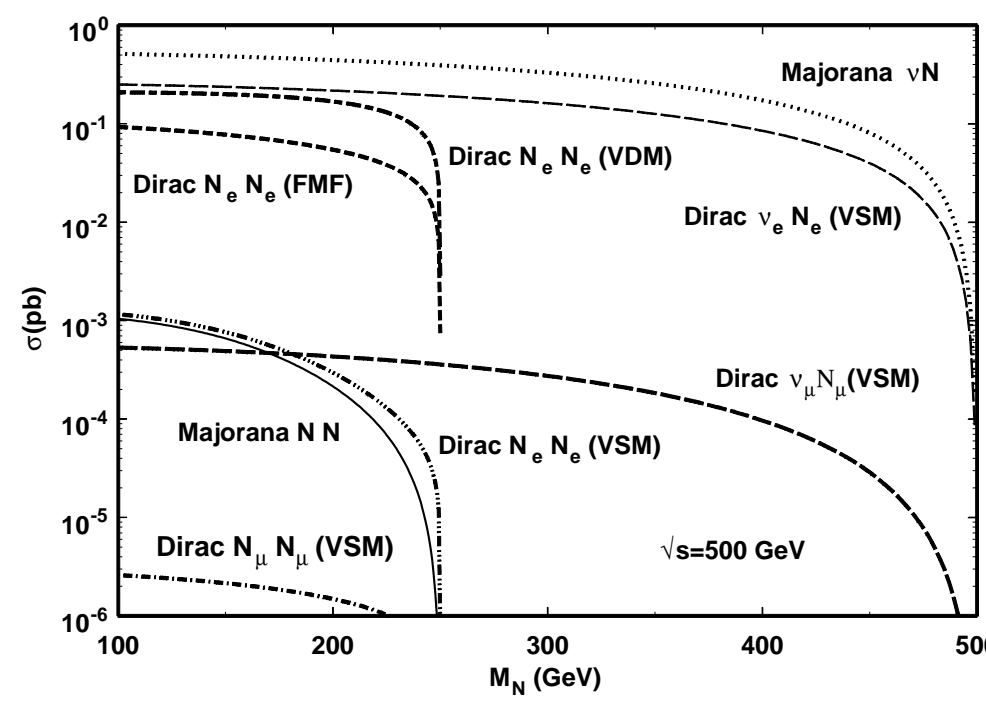

Figure 5.11: Single and pair production cross sections of on-shell heavy Dirac and Majorana neutrinos at $\sqrt{s}=500 \mathrm{GeV}$ for $e^{+} e^{-}$colliders [72].

ing that the Planck scale (of order $10^{19} \mathrm{GeV}$ ), where gravity becomes strong, may not be fundamental but simply an artifact of the properties of the higher-dimensional space. In one such scenario [73], the apparent hierarchy is generated by a large volume for the extra dimensions, while in a second case [74], the observed hierarchy is created by an exponential function of the compactification radius of the extra dimension. An exciting feature of these theories is that they afford concrete and distinctive experimental tests both in high energy physics and in astrophysics. Furthermore, if they truly describe the source of the observed hierarchy, then their signatures should appear in high-energy experiments at the TeV scale.

Another possibility is the existence of $\mathrm{TeV}^{-1}$-sized extra dimensions accessible to Standard Model fields. Although these theories do not explicitly address the hierarchy between the Electroweak and Planck scales, they are not ruled out experimentally and may arise naturally from string theory [75]. Furthermore, they serve as a mechanism for suppressing proton decay and generating the hierarchical pattern of fermion masses [76]. Models with TeV-scale extra dimensions provide a context for new approaches to the problem of explaining electroweak symmetry breaking $[77,78]$ and the existence of three generations of quarks and leptons [79]. These theories also give rise to interesting phenomenology at the TeV scale. 
We first describe some common features of these theories. In all the above scenarios, our universe lies on a 3+1-dimensional brane (sometimes called a wall) that is embedded in the higher $4+\delta$-dimensional space, known as the bulk. The field content that is allowed to propagate in the bulk varies between the different models. Upon compactification of the additional dimensions, all bulk fields expand into a Kaluza-Klein (KK) tower of states on the $3+1$-dimensional brane, where the masses of the KK states are related to the $\delta$-dimensional kinetic motion of the bulk field. It is the direct observation or indirect effects of the KK states that signal the existence of extra dimensions at colliders.

\subsection{Large extra dimensions}

In this scenario [73], gravitational fields propagate in the $\delta$ new large spatial dimensions, as well as in the usual $3+1$ dimensions. It is postulated that their interactions become strong at the $\mathrm{TeV}$ scale. The volume of the compactified dimensions, $V_{\delta}$, relates the scale where gravity becomes strong in the $4+\delta$-dimensional spaces to the apparent Planck scale via Gauss' Law

$$
M_{P l}^{2}=V_{\delta} M_{*}^{2+\delta},
$$

where $M_{*}$ denotes the fundamental Planck scale in the higher-dimensional space. Setting $M_{*}$ to be of order $1 \sim \mathrm{TeV}$ thus determines the compactification radius $r_{c}$ $\left(V_{\delta} \sim r_{c}^{\delta}\right)$ of the extra dimensions, which ranges from a sub-millimeter to a few fermi for $\delta=2-6$, assuming that all radii are of equal size. The compactification scale $\left(M_{c}=1 / r_{c}\right)$ associated with these parameters then ranges from $10^{-4} \mathrm{eV}$ to a few $\mathrm{MeV}$. The case of $\delta=1$ (which yields $r_{c} \approx 10^{11} \mathrm{~m}$ ) is immediately excluded by astronomical data. Cavendish-type experiments, which search for departures from the inversesquare law gravitational force, exclude [80] $r_{c}>190 \mu \mathrm{m}$ for $\delta=2$, which translates to the bound $M_{*}>1.6 \mathrm{TeV}$ using the convention in [81]. In addition, astrophysical and cosmological considerations [82], such as the rate of supernova cooling and the diffuse $\gamma$ ray spectrum, disfavor a value of $M_{*}$ near the TeV scale for $\delta=2$. Precision electroweak data [83] do not allow the Standard Model fields to propagate in extra dimensions with $M_{c}<$ a few $\mathrm{TeV}$, and hence they are constrained to the $3+1$ dimensional brane in this model.

The Feynman rules for this scenario $[81,84]$ are obtained by considering a linearized theory of gravity in the bulk. The bulk field strength tensor can be decomposed into spin- 0,1 , and 2 states, each of which expands into KK towers upon compactification. These KK states are equally spaced and have masses of $n / r_{c}$ where $n$ labels the KK excitation level. Taking $M_{*}=1 \mathrm{TeV}$, we see that the KK state mass splittings are equal to $5 \times 10^{-4} \mathrm{eV}, 20 \mathrm{keV}$, and $7 \mathrm{MeV}$ for $\delta=2,4$, and 6 , respectively. The interactions of the KK gravitons with the Standard Model fields on the wall are governed by the conserved stress-energy tensor of the wall fields. The spin- 1 KK 
New Physics at the TeV Scale and Beyond

states do not interact with the wall fields because of the form of the wall stress-energy tensor. The non-decoupling scalar KK states couple to the trace of the stress-energy tensor, and are phenomenologically irrelevant for most collider processes. Each state in the spin-2 KK tower, $G_{n}$, couples identically to the Standard Model wall fields via their stress-energy tensor with the strength proportional to the inverse 4-dimensional Planck scale, $M_{P l}^{-1}$. It is important to note that this description is an effective 4dimensional theory, valid only for energies below $M_{*}$. The full theory above $M_{*}$ is unknown.

Two classes of collider signatures arise in this model. The first is emission of the graviton KK tower states in scattering processes $[81,85]$. The relevant process at a linear collider is $e^{+} e^{-} \rightarrow \gamma / Z+G_{n}$, where the graviton appears as missing energy in the detector, behaving as if it were a massive, non-interacting, stable particle. The cross section is computed for the production of a single massive graviton excitation and then summed over the full tower of KK states. Since the mass splittings of the KK excitations are quite small compared to the collider center-of-mass energy, this sum can be replaced by an integral weighted by the density of KK states which is cut off by the specific process kinematics. The cross section for this process scales as simple powers of $\sqrt{s} / M_{*}$. It is important to note that because of the integral over the effective density of states, the emitted graviton appears to have a continuous mass distribution. This corresponds to the probability of emitting gravitons with different extra-dimensional momenta. The observables for graviton production, such as the $\gamma / Z$ angular and energy distributions, are thus distinct from those of other new physics processes, such as supersymmetric particle production, since the latter corresponds to a fixed invisible particle mass. The Standard Model background transition $e^{+} e^{-} \rightarrow \nu \bar{\nu} \gamma$ also has different characteristics, since it is a three-body process.

The cross section for $e^{+} e^{-} \rightarrow \gamma G_{n}$ as a function of the fundamental Planck scale is presented in Fig. 5.12 for $\sqrt{s}=1 \mathrm{TeV}$. The level of Standard Model background is also shown, with and without electron beam polarization set at $90 \%$. We note that the signal (background) increases (decreases) with increasing $\sqrt{s}$. Details of the various distributions associated with this process can be found in Cheung and Keung [85]. The discovery reach from this process has been computed in [86], with $\sqrt{s}=800$ $\mathrm{GeV}, 1000 \mathrm{fb}^{-1}$ of integrated luminosity, including various beam polarizations and kinematic acceptance cuts, ISR, and beamstrahlung. The results are displayed in Table 5.5. In this table, we have also included the 95\% CL bounds obtained [87] at LEP for $\sqrt{s}>200 \mathrm{GeV}$.

The associated emission process at hadron colliders, $q \bar{q} \rightarrow g+G_{n}$, results in a mono-jet signal. In this case, the effective low-energy theory breaks down for some regions of the parameter space, as the parton-level center-of-mass energy can exceed the value of $M_{*}$. The experiment is then sensitive to the new physics appearing above $M_{*}$. An ATLAS simulation [88] of the missing transverse energy in signal and background events at the $\mathrm{LHC}$ with $100 \mathrm{fb}^{-1}$ results in the discovery range for the 


\begin{tabular}{|c|l|c|c|c|}
\hline \hline$e^{+} e^{-} \rightarrow \gamma+G_{n}$ & & 2 & 4 & 6 \\
\hline $\mathrm{LC}$ & $P_{-,+}=0$ & 5.9 & 3.5 & 2.5 \\
$\mathrm{LC}$ & $P_{-}=0.8$ & 8.3 & 4.4 & 2.9 \\
$\mathrm{LC}$ & $P_{-}=0.8, P_{+}=0.6$ & 10.4 & 5.1 & 3.3 \\
$\mathrm{LEP} \mathrm{II}$ & & 1.45 & 0.87 & 0.61 \\
\hline \hline$p p \rightarrow g+G_{n}$ & & 2 & 3 & 4 \\
\hline $\mathrm{LHC}$ & & $4.0-8.9$ & $4.5-6.8$ & $5.0-5.8$ \\
\hline \hline
\end{tabular}

Table 5.5: $95 \%$ CL sensitivity to the fundamental Planck scale $M_{*}$ in $\mathrm{TeV}$ for different values of $\delta$, from the emission process for various polarization configurations and different colliders as discussed in the text. $\sqrt{s}=800 \mathrm{GeV}$ and $1 \mathrm{ab}^{-1}$ has been assumed for the LC and $100 \mathrm{fb}^{-1}$ for the LHC.

effective theory displayed in Table 5.5. The lower end of the range corresponds to values at which the ultraviolet physics sets in and the effective theory fails, while the upper end represents the boundary where the signal is no longer observable above background.

If an emission signal is observed, one would like to determine the values of the fundamental parameters, $M_{*}$ and $\delta$. In this case, measurement of the cross section at a linear collider at two different values of $\sqrt{s}$ can be used to determine $\delta$ [86] and test the consistency of the data with the hypothesis of large extra dimensions. This is displayed for a LC in Fig. 5.13.

The second class of collider signals for large extra dimensions is that of graviton exchange $[81,84,89]$ in $2 \rightarrow 2$ scattering. This leads to deviations in cross sections and asymmetries in Standard Model processes such as $e^{+} e^{-} \rightarrow f \bar{f}$, and may also give rise to new production processes that are not otherwise present at tree-level, such as $e^{+} e^{-} \rightarrow h h$, or $\tilde{g} \tilde{g}$. The exchange amplitude is proportional to the sum over the propagators for the graviton KK tower states which, as before, may be converted to an integral over the density of states. However, in this case the integral is divergent for $\delta>1$ and thus introduces a sensitivity to the unknown ultraviolet physics. Several approaches have been proposed to regulate this integral: (i) a naive cut-off scheme [81,84,89], (ii) an exponential damping due to the brane tension [90], (iii) restrictions from unitarity [91], or (iv) the inclusion of full weakly coupled TeV-scale string theory in the scattering process [92]. Here, we adopt the most model-independent approach, that of a naive cut-off, and set the cut-off equal to $M_{*} / \lambda^{1 / 4}$, where $\lambda$ accounts for the effects of the unknown ultraviolet physics. Assuming that the integral is dominated by the lowest-dimensional local operator, which is dimension-8, this results in a contacttype interaction limit for graviton exchange, which can be described via

$$
i \frac{4 \lambda}{M_{*}^{4}} T^{\mu \nu} T_{\mu \nu}
$$




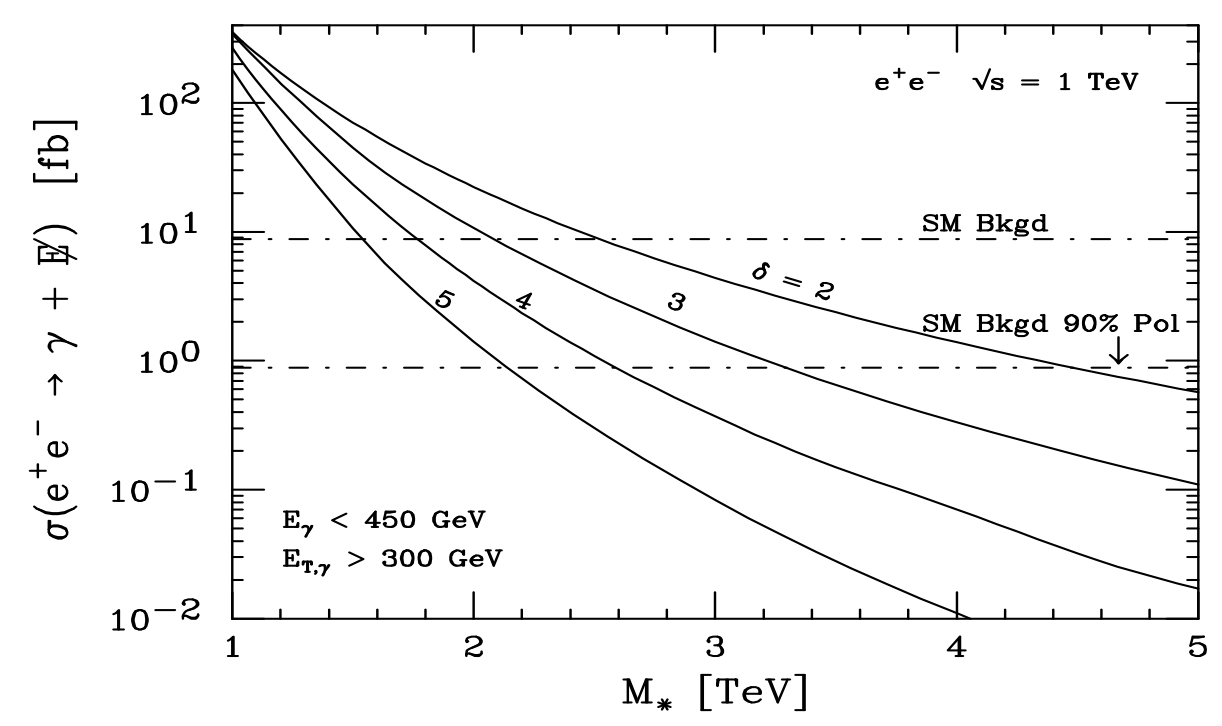

Figure 5.12: The cross section for $e^{+} e^{-} \rightarrow \gamma G_{n}$ for $\sqrt{s}=1 \mathrm{TeV}$ as a function of the fundamental Planck scale for various values of $\delta$ as indicated. The cross sections for the Standard Model background, with and without $90 \%$ beam polarization, correspond to the horizontal lines as labeled. The signal and background are computed with the requirement $E_{\gamma}<450 \mathrm{GeV}$ in order to eliminate the $\gamma Z \rightarrow \nu \bar{\nu} \gamma$ contribution to the background. From [81].

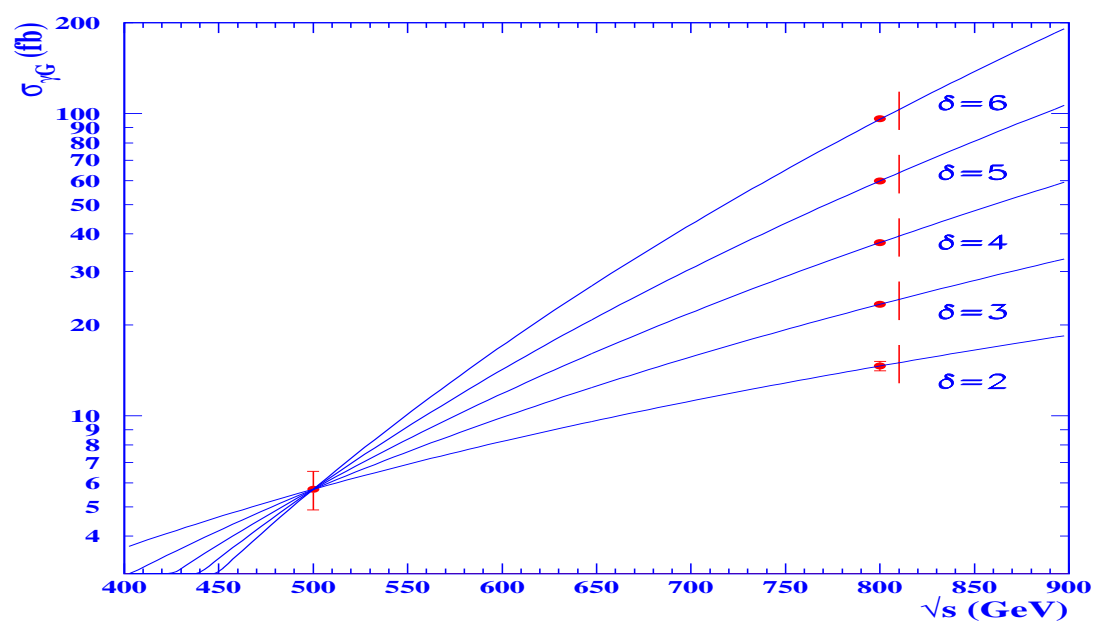

Figure 5.13: The determination of $\delta$ from cross section measurements of $e^{+} e^{-} \rightarrow \gamma G_{n}$ at $\sqrt{s}=500$ and $800 \mathrm{GeV}$ with $500 \mathrm{fb}^{-1}$ and $1 \mathrm{ab}^{-1}$, respectively, taking $P_{-}=80 \%$ and $P_{+}=60 \%$. The $500 \mathrm{GeV}$ cross section has been normalized for the case $M_{*}=5 \mathrm{TeV}$ and $\delta=2$. From [82]. 
where $T^{\mu \nu}$ is the stress-energy tensor. This is described in the matrix element for $s$-channel $2 \rightarrow 2$ scattering by the replacement

$$
\frac{i^{2} \pi}{M_{P l}^{2}} \sum_{n=1}^{\infty} \frac{1}{s-m_{n}^{2}} \rightarrow \frac{\lambda}{M_{*}^{4}}
$$

with corresponding substitutions for $t$ - and $u$-channel scattering. Here $m_{n}$ represents the mass of $G_{n}$, the $n^{\text {th }}$ graviton KK excitation. This substitution is universal for any $2 \rightarrow 2$ process. The resulting angular distributions for fermion pair production are quartic in $\cos \theta$ and thus provide a signal for spin-2 exchange. An illustration of this is given in Fig. 5.14 from [89], which displays the unpolarized angular distribution as well as the angular dependence of the left-right asymmetry in $e^{+} e^{-} \rightarrow b \bar{b}$, taking $M_{*}=3 \sqrt{s}=1.5 \mathrm{TeV}$ and $\lambda= \pm 1$. The two sets of data points correspond to the two choices of sign for $\lambda$, and the error bars represent the statistics in each bin for an integrated luminosity of $75 \mathrm{fb}^{-1}$. Here, a $60 \%$-tagging efficiency, $90 \%$ electron beam polarization, $10^{\circ}$ angular cut, and ISR have been included. The resulting 95\% CL search reach with $500 \mathrm{fb}^{-1}$ of integrated luminosity is given in Table 5.6 from summing over the unpolarized and $A_{L R}$ angular distributions for fermion $(e, \mu, \tau, c, b$, and $t)$ final states. For comparison, we also present the current bounds [87] from LEP II, HERA, and the D $\varnothing$ Collaboration at the Tevatron, as well as estimates for the LHC with $100 \mathrm{fb}^{-1}[89,93]$ and $\gamma \gamma$ colliders [94]. Note that the $\gamma \gamma \rightarrow W W$ process has the highest sensitivity to graviton exchange. This is due to the large $W$ pair cross section and the multitude of observables that can be formed utilizing polarized beams and $W$ decays.

The ability of the LC to determine that a spin-2 exchange has taken place in $e^{+} e^{-} \rightarrow f \bar{f}$ is demonstrated in Fig. 5.15 from [89]. Here, the confidence level of a fit of spin-2 exchange data to a spin- 1 exchange hypothesis is displayed; the quality of such a fit is quite poor almost up to the $M_{*}$ discovery limit, indicating that the spin-2 nature is discernable.

The scenario with large extra dimensions resolves the hierarchy problem without invoking supersymmetry. However, if this mechanism is embedded in a string theory, then supersymmetry may also be present at the weak scale. A supersymmetric bulk then results in a KK tower of gravitinos, in addition to the KK gravitons. In supersymmetric models that expect a light gravitino, such as gauge-mediated supersymmetry breaking, the gravitino KK tower can yield interesting phenomenological effects. An example of this is in the process $e^{+} e^{-} \rightarrow \tilde{e}^{+} \tilde{e}^{-}$, which would now also receive contributions from $t$-channel KK gravitino exchange and $s$-channel KK graviton exchange. This has been studied in [95], which considered an $N=2$ supersymmetry in the bulk, and after compactifying the gravitino sector, derived the KK gravitino couplings to $N=1$ supersymmetric matter on the brane. The resulting dramatic effect on selectron pair production is highlighted by the ability to select various production channels via the use of electron beam polarization. This is displayed in Fig. 

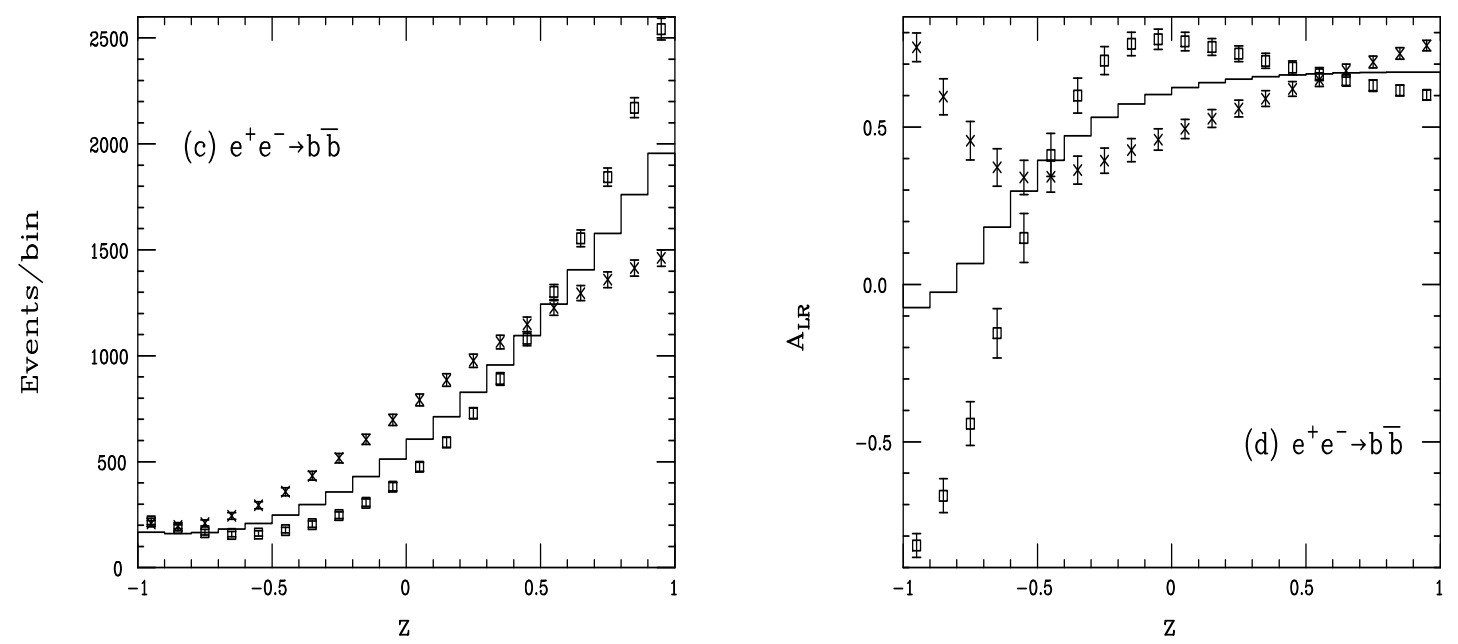

Figure 5.14: Bin-integrated angular distribution and $z$-dependent $(z=\cos \theta)$ left-right asymmetry for $e^{+} e^{-} \rightarrow b \bar{b}$ at $\sqrt{s}=500 \mathrm{GeV}$. The solid histogram represents the Standard Model while the 'data' points are for $M_{*}=1.5$ with $\lambda= \pm 1$. The error bars indicate the statistics in each bin.

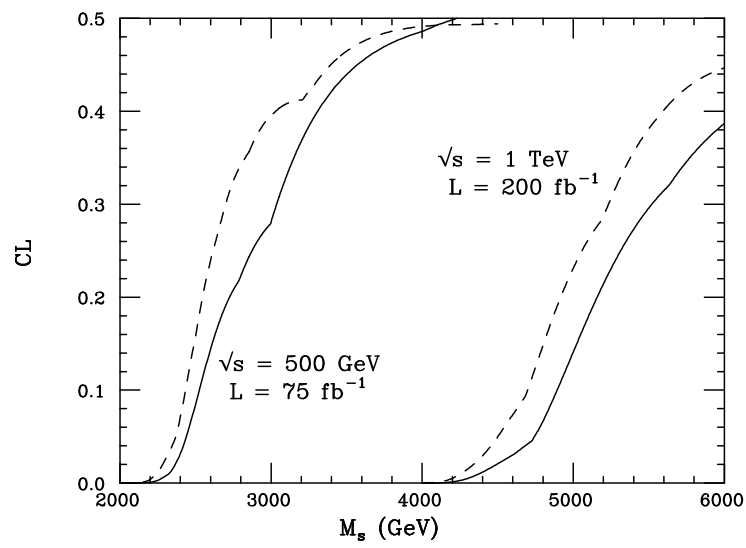

Figure 5.15: The percentage confidence level as a function of $M_{*}$ for a fit of spin-2 data under a spin-1 hypothesis. The dashed and solid curves correspond to the choice $\lambda= \pm 1$. 


\begin{tabular}{|c|c|c|c|}
\hline \hline & & $\sqrt{s}(\mathrm{TeV})$ & $M_{*}(\mathrm{TeV})$ \\
\hline LEPII & $e^{+} e^{-} \rightarrow \ell^{+} \ell^{-}, \gamma \gamma, Z Z$ & 0.2 & $1.03-1.17$ \\
LC & $e^{+} e^{-} \rightarrow f \bar{f}$ & 0.5 & 4.1 \\
LC & $e^{+} e^{-} \rightarrow f \bar{f}$ & 1.0 & 7.2 \\
LC & $\gamma \gamma \rightarrow W W$ & 1.0 & 13.0 \\
LC & $\gamma \gamma \rightarrow \gamma \gamma$ & 1.0 & 3.5 \\
LC & $e \gamma \rightarrow e \gamma$ & 1.0 & 8 \\
HERA & $e p \rightarrow e+$ jet & 0.314 & $0.81-0.93$ \\
Tevatron Run I & $p \bar{p} \rightarrow \ell^{+} \ell^{-}, \gamma \gamma$ & 1.8 & $1.01-1.08$ \\
LHC & $p p \rightarrow \ell^{+} \ell^{-}$ & 14.0 & 7.5 \\
LHC & $p p \rightarrow \gamma \gamma$ & 14.0 & 7.1 \\
\hline \hline
\end{tabular}

Table 5.6: 95\% CL search reach for $M_{*}$ from graviton exchange in various processes as indicated and discussed in the text. In the bounds from present data, a range is indicated to account for $\lambda= \pm 1$.

5.16, which shows the binned angular distribution for $e_{L, R}^{-} e^{+} \rightarrow \tilde{e}_{L}^{\mp} e_{R}^{ \pm}$for various values of $M_{*}$; this choice of polarization isolates the $t$-channel neutralino and $\mathrm{KK}$ gravitino contributions. The search reach for this process at $\sqrt{s}=500 \mathrm{GeV}$ with $80 \%$ beam polarization and $500 \mathrm{fb}^{-1}$ of integrated luminosity is $M_{*} \sim 12 \mathrm{TeV}$ for the case $\delta=6$.

\subsection{Localized gravity}

We now turn to the scenario where the hierarchy is generated by an exponential function of the compactification radius. In its simplest form, gravity propagates in the bulk, while the Standard Model fields are constrained to a 3-brane. This model contains a non-factorizable geometry embedded in a slice of 5-dimensional Anti-de Sitter space $\left(\mathrm{AdS}_{5}\right)$, which is a space of negative curvature. Two 3-branes reside rigidly at fixed points at the boundaries of the $\operatorname{AdS}_{5}$ slice, located at $|\phi|=0, \pi$ where $\phi$ parameterizes the fifth dimension. The 5-dimensional Einstein equations permit a solution that preserves 4-d Poincaré invariance with the metric

$$
d s^{2}=e^{-2 k r_{c}|\phi|} \eta_{\mu \nu} d x^{\mu} d x^{\nu}-r_{c}^{2} d \phi^{2},
$$

where $\pi r_{c}$ is the length of the fifth dimenion. The exponential function, or warp factor, multiplying the usual 4-d Minkowski term curves space away from the branes. The constant $k$ is the $\mathrm{AdS}_{5}$ curvature scale, which is of order the Planck scale and is determined by the bulk cosmological constant. The scale of physical phenomena as realized by the 4-d flat metric transverse to the fifth dimension is specified by the exponential warp factor. If the gravitational wavefunction is localized on the brane at $\phi=0$ (called the 'Planck brane'), then TeV scales can naturally be attained [74] 
New Physics at the TeV Scale and Beyond

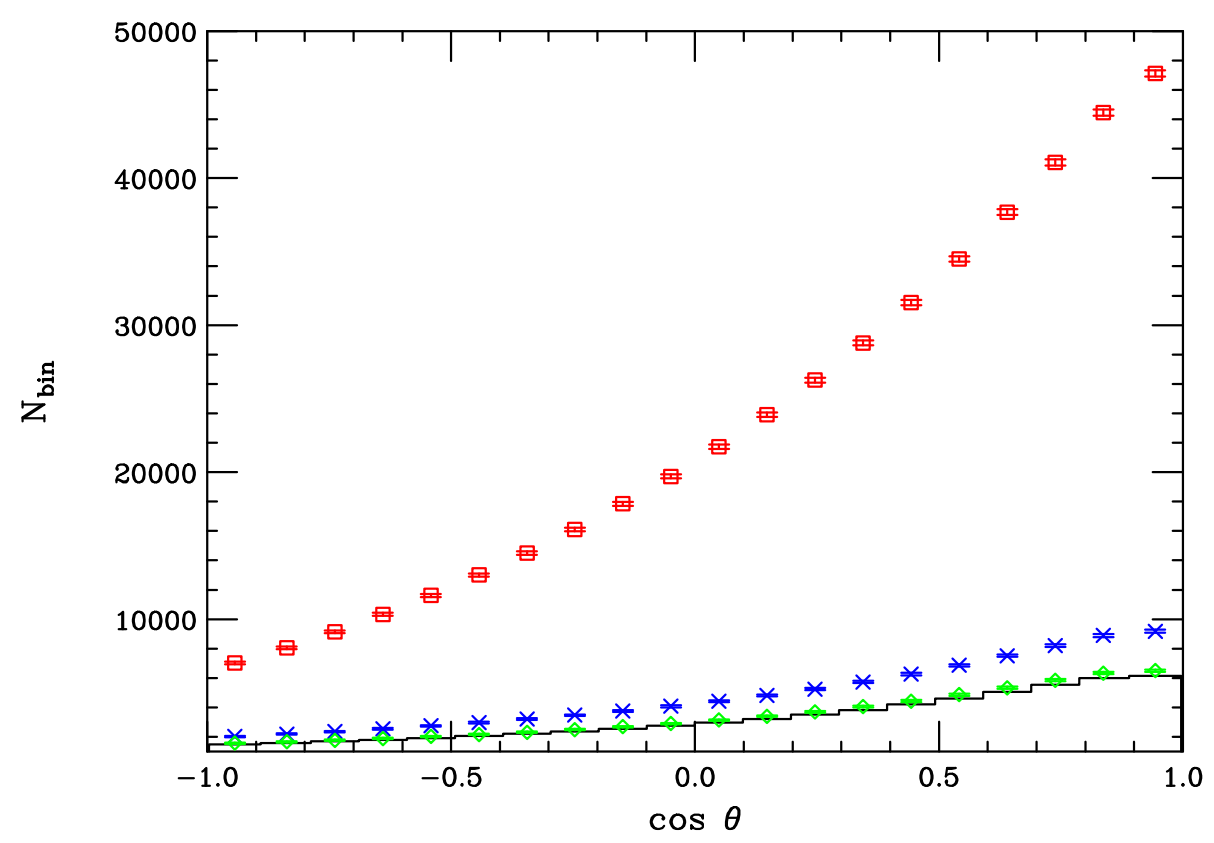

Figure 5.16: The number of events per bin in $e_{L, R}^{-} e^{+} \rightarrow \tilde{e}_{L}^{\mp} \tilde{e}_{R}^{ \pm}$for $\sqrt{s}=500 \mathrm{GeV}$ with 500 $\mathrm{fb}^{-1}$ of integrated luminosity and $P_{-}=80 \%$. The curves correspond to $M_{*}=1.5,3,6$ $\mathrm{TeV}$ from top to bottom with the solid histogram representing the minimal supersymmetric case. The error bars correspond to the statistics in each bin. Here the values $m_{\tilde{e}_{L}}=220$ $\mathrm{GeV}$ and $m_{\tilde{e}_{R}}=117 \mathrm{GeV}$ are assumed.

on the 3-brane at $\phi=\pi$ (the ' $\mathrm{TeV}$ brane', where the Standard Model fields reside) if $k r_{c} \simeq 11-12$. The scale $\Lambda_{\pi} \equiv \bar{M}_{P l} e^{-k r_{c} \pi} \sim 1 \mathrm{TeV}$, where $\bar{M}_{P l}=M_{P l} / \sqrt{8} \pi$ is the reduced Planck scale, then describes the scale of all physical processes on the TeV-brane. We note that it has been demonstrated [96] that this value of $k r_{c}$ can be stabilized without fine tuning of parameters.

Two parameters govern the 4-d phenomenology of this model, $\Lambda_{\pi}$ and the ratio $k / \bar{M}_{P l}$. Constraints on the curvature of the $\mathrm{AdS}_{5}$ space suggest that $k / \bar{M}_{P l} \lesssim 0.1$. The Feynman rules are obtained by a linear expansion of the flat metric, including the warp factor. After compactification, a KK tower of gravitons appears on the TeVbrane and has masses $m_{n}=x_{n} k e^{-k r_{c} \pi}=x_{n} \Lambda_{\pi} k / \bar{M}_{P l}$ with the $x_{n}$ being the roots of the first-order Bessel function, i.e., $J_{1}\left(x_{n}\right)=0$. Note that the first excitation is naturally of order a few hundred $\mathrm{GeV}$ and that the KK states are not evenly spaced. The interactions of the graviton KK tower with the Standard Model fields on the TeV brane are [97]

$$
\mathcal{L}=-\frac{1}{\bar{M}_{P l}} T^{\mu \nu}(x) h_{\mu \nu}^{(0)}(x)-\frac{1}{\Lambda_{\pi}} T^{\mu \nu}(x) \sum_{n=1}^{\infty} h_{\mu \nu}^{(n)}(x),
$$

where $T^{\mu \nu}$ is the stress-energy tensor. Note that the zero-mode decouples and that the 


\begin{tabular}{|c|c|c|c|}
\hline \hline & \multicolumn{3}{|c|}{$k / \overline{M_{P l}}$} \\
\hline & 0.01 & 0.1 & 1.0 \\
\hline $\mathrm{LC} \sqrt{s}=0.5 \mathrm{TeV}$ & 20.0 & 5.0 & 1.5 \\
$\mathrm{LC} \sqrt{s}=1.0 \mathrm{TeV}$ & 40.0 & 10.0 & 3.0 \\
LEP II & 4.0 & 1.5 & 0.4 \\
Tevatron Run II & 5.0 & 1.5 & 0.5 \\
LHC & 20.0 & 7.0 & 3.0 \\
\hline \hline
\end{tabular}

Table 5.7: 95\% CL search reach for $\Lambda_{\pi}$ in $\mathrm{TeV}$ in the contact interaction regime taking $500,2.5,2$, and $100 \mathrm{fb}^{-1}$ of integrated luminosity at the LC, LEP II, Tevatron, and LHC, respectively. From [97].

couplings of the higher states have inverse-TeV strength. This results in a strikingly different phenomenology from the case of large extra dimensions. Here, the graviton KK tower states are directly produced as single resonances if kinematically allowed.

If the KK gravitons are too massive to be produced directly, their contributions to fermion pair production may still be felt via virtual exchange. In this case, the uncertainties associated with the introduction of a cut-off are avoided, since there is only one additional dimension and the KK states may be neatly summed. The sensitivity [97] to $\Lambda_{\pi}$ at a linear collider for various values of $k / \bar{M}_{P l}$ is listed in Table 5.7 for $500 \mathrm{fb}^{-1}$ of integrated luminosity. For purposes of comparison, the corresponding reach at LEP II, Tevatron Run II, and the LHC is also displayed.

With sufficient center-of-mass energy the graviton KK states may be produced as resonances. To exhibit how this may appear at a linear collider, Fig. 5.17 displays the cross section for $e^{+} e^{-} \rightarrow \mu^{+} \mu^{-}$as a function of $\sqrt{s}$, assuming $m_{1}=500 \mathrm{GeV}$ and taking $k / \bar{M}_{P l}=0.01-0.05$. The height of the third resonance is somewhat reduced, because the higher KK excitations decay to the lighter graviton states once it is kinematically allowed [98]. In this case one can study graviton self-couplings, and higher-energy $e^{+} e^{-}$colliders may become graviton factories!

Searches for the first graviton KK resonance in Drell-Yan and di-jet data at the Tevatron already place non-trivial restrictions [97] on the parameter space of this model, given roughly by $m_{1} \gtrsim 175,550,1100 \mathrm{GeV}$ for $k / \bar{M}_{P l}=0.01,0.1,1.0$. Precision electroweak data extend [99] this search reach for smaller values of $k$. These constraints, taken together with the theoretical prejudices that (i) $\Lambda_{\pi} \lesssim 10 \mathrm{TeV}$, i.e., the scale of physics on the $\mathrm{TeV}$ brane is not far above the electroweak scale and (ii) $k / \bar{M}_{P l} \lesssim 0.1$ from the above-mentioned $\mathrm{AdS}_{5}$ curvature considerations, result in a closed allowed region in the 2-dimensional parameter space, which can be completely explored at the LHC $[99,100]$ via the Drell-Yan mechanism.

Lastly, we note that if the Standard Model fields are also allowed to propagate in the bulk $[99,101]$, the phenomenology can be markedly different, and is highly dependent on the value of the 5-dimensional fermion mass. For various phenomenological 
New Physics at the TeV Scale and Beyond

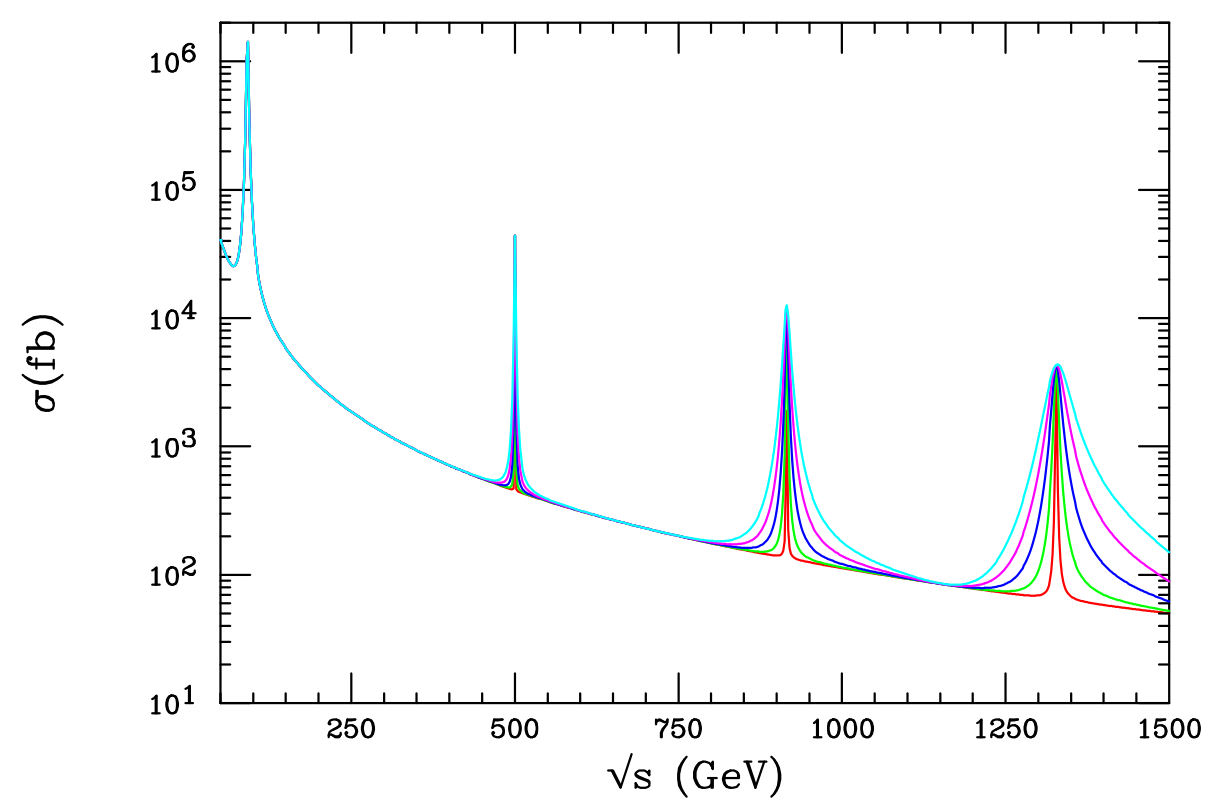

Figure 5.17: The cross section for $e^{+} e^{-} \rightarrow \mu^{+} \mu^{-}$including the exchange of a KK tower of gravitons with $m_{1}=500 \mathrm{GeV}$. The curves correspond to $k / \bar{m}_{P l}=$ in the range $0.01-0.05$.

reasons, it is least problematic to keep the Higgs field on the TeV brane [101]. As a first step, one can study the effect of placing the Standard Model gauge fields in the bulk and keeping the fermions on the TeV-brane. In this case, the fermions on the wall couple to the KK gauge fields a factor of $\sqrt{2 k r_{c} \pi} \sim 9$ times more strongly than they couple to the $\left(\gamma, g, W^{ \pm}, Z\right)$. In this case, precision electroweak data place strong constraints, requiring that the lightest KK gauge boson have a mass greater than about $25 \mathrm{TeV}$. This value pushes the scale on the TeV-brane above $100 \mathrm{TeV}$, making this scenario disfavored in the context of the hierarchy problem.

This bound can be relaxed if the fermions also reside in the bulk [101]. By introducing bulk fermion 5 -d masses $m_{5}$, the couplings of the fermion zero modes (i.e., the Standard Model fermions) to various KK fields become a function of the bulk mass parameter $\nu \equiv m_{5} / k$. The parameter $\nu$ controls the shape of the fermion zero-mode wavefunction, with negative (positive) values of $\nu$ serving to localize the wavefunction near the Planck brane ( $\mathrm{TeV}$ brane). Constraints from avoiding flavor-changing neutral currents, Yukawa coupling blow-up, and the generation of a new hierarchy result in a rather narrow allowed range of $\nu$. For some values of $\nu$ in this range, the fermionic couplings of the KK graviton states essentially vanish, and hence the graviton production mechanisms discussed above are no longer viable. In this case, the gravitons retain a small coupling to the Standard Model gauge bosons, and the most promising production mechanism [99] is at a photon collider via $\gamma \gamma \rightarrow G_{n} \rightarrow h h$, with $h$ being the Higgs boson. 


\subsection{TeV-scale extra dimensions}

$\mathrm{TeV}^{-1}$-sized extra dimensions can naturally arise in some string theory models [75], and in this case, the Standard Model fields may feel their effects. The physics of models with KK excitations of the Standard Model gauge bosons arising from TeVscale extra dimensions has been discussed for some time [102]. The various models in this class of theories differ in detail in two regards: $(i)$ the placement of the Higgs field(s) in the bulk or on the wall(s), and (ii) the treatment of the fermion fields.

If Higgs fields propagate in the bulk, the expectation value of the zero-mode field generates electroweak symmetry breaking. In this case, there is no mixing among the various gauge boson KK modes. Thus the KK mass matrix is diagonal, with the masses of the excitations given by $\left[M_{0}^{2}+\vec{n} \cdot \vec{n} M_{c}^{2}\right]^{1 / 2}$, where $M_{0}$ is the zero-mode mass, $M_{c}$ is the compactification mass scale and $\vec{n}$ is a set of integers labeling the excitation state. However, if the Higgs is a wall field, its expectation value induces off-diagonal elements in the mass matrix and thus a mixing among the gauge KK states. In this case the mass matrix needs to be diagonalized to determine the masses and couplings of the gauge KK states. It is also possible to imagine a more generalized mixed scenario with two Higgs fields, one residing in the bulk and one on the wall, that share the SM symmetry breaking. Clearly, the detailed phenomenology of these possibilities will be quite different. For example, a small mixing of the gauge KK states may show up in precision measurements when $W$ and $Z$ properties are compared with Standard Model expectations.

An even more diverse situation arises when one considers the placement of the Standard Model fermions within the extra dimensions. There are essentially three possibilities:

(a) The fermions are constrained to 3-branes located at fixed points. This is the most common situation discussed in the literature [83] and in this case the fermions are not directly affected by the extra dimensions. For models in this class, global fits to precision electroweak data place strong lower bounds on the value of $M_{c}$, which corresponds to the mass of the first gauge KK excitation. Following the analysis of Rizzo and Wells [83] and employing the most recent data [103], one finds that $M_{c}>4.4 \mathrm{TeV}$ when the Higgs field is on the wall; the bound is $4.6 \mathrm{TeV}$ when the Higgs field is in the bulk. Such a large mass for gauge KK states is beyond the direct reach of a LC, but the KK states can be directly produced as resonances at the LHC in the Drell-Yan channel provided that $M_{c} \lesssim 6 \mathrm{TeV}$. This reach at the LHC may be extended by a $\mathrm{TeV}$ or so [104] by examination of the Drell-Yan line shape at high lepton-pair invariant mass. However, the LC can indirectly observe the existence of heavy gauge KK states via their $s$-channel exchanges in the contact interaction limit. Combining the results from various fermion final states in $e^{+} e^{-} \rightarrow f \bar{f}$ gives the $95 \%$ CL search reach displayed in Table 5.8.

If a $\gamma^{(1)} / Z^{(1)} \mathrm{KK}$ resonance is observed at the LHC, a $\sqrt{s}=500 \mathrm{GeV}$ linear 
New Physics at the TeV Scale and Beyond

\begin{tabular}{|c|c|}
\hline \hline & $M_{c}$ Reach $(\mathrm{TeV})$ \\
\hline Tevatron Run II $2 \mathrm{fb}^{-1}$ & 1.1 \\
LHC $100 \mathrm{fb}^{-1}$ & $6.3(\sim 7.5)$ \\
LEP II & 3.1 \\
$\mathrm{LC} \sqrt{s}=0.5 \mathrm{TeV} 500 \mathrm{fb}^{-1}$ & 13.0 \\
$\mathrm{LC} \sqrt{s}=1.0 \mathrm{TeV} 500 \mathrm{fb}^{-1}$ & 23.0 \\
$\mathrm{LC} \sqrt{s}=1.5 \mathrm{TeV} 500 \mathrm{fb}^{-1}$ & 31.0 \\
\hline \hline
\end{tabular}

Table 5.8: 95\% CL search reach for the mass of the first KK gauge boson excitation. From Rizzo and Wells [83]. The LHC reach is via direct observation of a resonance, while the LC sensitivities are from indirect effects as in the case of a search for a new neutral gauge boson. The number in parentheses for the LHC is an estimate of the extension of the complete search reach including indirect effects from contact interactions.

collider can distinguish this state from a new neutral gauge boson arising from an extended gauge sector by using the Bhabha scattering channel. If one attempts to fit the induced deviations in the Bhabha cross section and polarized asymmetry by varying the vector and axial-vector couplings of a hypothetical non-KK $Z^{\prime}$, one finds [105] that the CL of the fit is quite poor $\left(\lesssim 10^{-3}\right)$. This demonstrates that the assumption that the $\mathrm{KK}$ state is a $Z^{\prime}$ is incorrect. A separate fit assuming that the resonance is a KK state yields a good fit. At the LHC, it is currently unclear whether the $\gamma^{(1)} / Z^{(1)} \mathrm{KK}$ resonance can be distinguished from a $Z^{\prime}$ in a model-independent manner.

(b) The Standard Model fermions are localized at specific points, $x_{i}$, in the extra $\mathrm{TeV}$ dimension, which are not necessarily at the orbifold fixed points. Here, the zero- and excited-mode fermions obtain narrow Gaussian-like wave functions in the extra dimensions with a width $\sigma$ much smaller than the compactification scale, i.e., $\left(\sigma / \pi r_{c}\right)^{2} \ll 1$. The placement of SM fermions at different locations and the narrowness of their wavefunctions can then suppress [76] the rates for a number of dangerous processes such as proton decay. For the lighter gauge KK modes (small values of $n$ ), the width of the fermion wavefunction centered at a given point cannot be resolved, so that the wavefunction appears similar to a delta function. Thus the coupling of the fermion to these gauge KK states is determined by the value of the gauge KK wavefunction evaluated at that point. However, when $n \sigma / \pi r_{c}$ grows to order unity or larger, the KK gauge field can resolve the finite size of the fermion wavefunction and the coupling of the fermion becomes exponentially damped. This decouples the heavy gauge KK states, providing a means of rendering sums over KK towers of gauge bosons finite in the case of two or more extra dimensions [106]. An analysis of precision electroweak data in this case shows that $M_{c}$ is typically found to be $\geq 3-4$ $\mathrm{TeV}$. Depending upon the properties of the compactification manifold, measurements at colliders may probe the distance in the extra dimensions between two fermions, 
$\left|x_{i}-x_{j}\right|$, in $2 \rightarrow 2$ scattering. For example, in this case Bhabha scattering can probe the distance between the left- and right-handed electrons, as illustrated in Fig. 5.18. A study of the cartography of the localized fermions at linear colliders has been performed in [107]. At very large energies, the cross section for the polarized version of this process will tend rapidly to zero since the two particles completely miss each other in the extra dimension [108].

(c) The fermions are fields in the bulk. This possibility is known as the "universal extra dimensions' scenario [109]. This case is different in that walls or branes are not present and hence momentum is conserved in the additional dimensions. The consequence of this is that KK number is conserved at all interaction vertices, hence only pairs of KK gauge bosons couple to the zero-mode fermions. In this case, electroweak precision data as well as direct searches for KK states lead to a reduced lower bound of $M_{c} \simeq 0.4 \mathrm{TeV}$. Without further ingredients, this model may have trouble satisfying cosmological constraints, since the lightest KK excitations are absolutely stable. This may be avoided if there is any small breaking of translation invariance in the extra dimensions. Alternatively, one can imagine the gauge and fermion KK

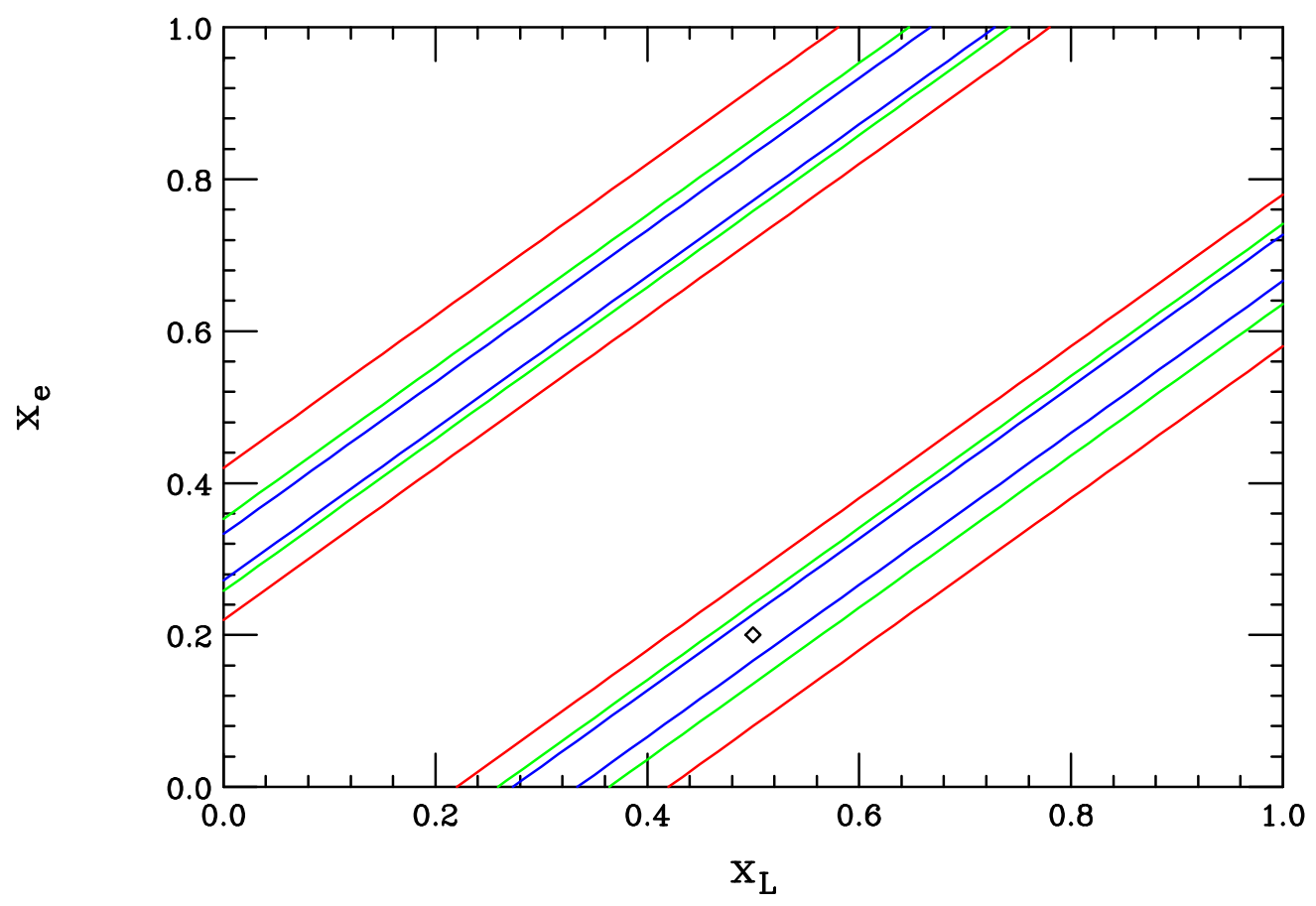

Figure 5.18: The ability of a LC to determine the separation in the extra dimension of right- and left-handed electrons from Bhabha scattering. The red, green, and blue (outer, middle, and inner) set of curves correspond to $\sqrt{s}=500,1000,1500 \mathrm{GeV}$, respectively, with $500 \mathrm{fb}^{-1}$ assumed for each energy. This case assumes $M_{c}=4 \mathrm{TeV}$ and that the location of the right- (left-)-handed electron, $x_{e(L)}$, is given by a Gaussian centered at $0.2(0.5) \cdot 2 \pi r_{c}$. 
New Physics at the TeV Scale and Beyond

fields as confined to a brane of thickness $\mathrm{TeV}^{-1}$, i.e., a thick brane, embedded in a highe-dimensional space that includes gravity. In this case the higher-level KK modes can decay down to the zero modes via graviton emission, but at a rate determined by the 'form factor' of the brane [110]. In either case an interesting phenomenology results. The KK states are produced in pairs at colliders and then either decay via one of these two mechanisms or are long-lived and appear as tracks in a detector.

\section{Highly non-conventional theories and possible surprises}

So far in this chapter, we have delineated the potential of a linear collider to explore the new physics that is present in set classes of established models. However, as likely as not, when Nature finally reveals her mysteries they will be full of surprises that lie outside the realm of our limited imaginations.

Along these lines, we note that some of the most striking recent developments have occured in string theory. While it is currently difficult to relate these theories to experiment, some of their ingredients, when considered on their own, have interesting phenomenological consequences. Here, we consider two such examples of this top-down approach, as a demonstration of the potential of the LC to discover the unforseen.

\subsection{String resonances}

If the scenario with large extra dimensions discussed in a previous section is embedded in a string theory, then stringy effects must also appear at the $\mathrm{TeV}$ scale. Hence, not only the gravitons, but also the Standard Model fields must have an extended structure. The exchange of string Regge excitations of Standard Model particles in $2 \rightarrow 2$ scattering may appear as contact-like interactions with a strength that overwhelms the corresponding graviton exchange. This is deduced from simple coupling-counting arguments. Yang-Mills bosons live at the end of open strings, while gravitons correspond to closed string states, which require an additional coupling constant factor at the amplitude level. Hence the exchange of KK graviton states is suppressed by a factor of $g^{2}$ compared to the exchange of string Regge excitations.

This has been examined in [111], where an illustrative string model was assumed. This model makes use of scattering amplitudes on the 3-brane of weakly coupled type IIB string theory to describe a string version of QED. Electrons and photons then correspond to massless states of open strings ending on the 3-brane and are characterized by the quantum theory of fluctuations of an open string with specified boundary conditions. Within the context of this model, Bhabha scattering and pair annihilation receive contributions from the string Regge exchanges. The differential 
cross section for these processes is modified by a form factor,

$$
\frac{d \sigma}{d \cos \theta}=\left(\frac{d \sigma}{d \cos \theta}\right)_{S M}\left|\frac{\Gamma\left(1-s / M_{s t r}^{2}\right) \Gamma\left(1-t / M_{s t r}^{2}\right)}{\Gamma\left(1-s / M_{s t r}^{2}-t / M_{s t r}^{2}\right)}\right|^{2}
$$

which essentially mirrors the original Veneziano result [112]. Here, $M_{\text {str }}$ represents the string scale and can be related to the fundamental Planck scale in the large extra dimension scenario via $M_{*} / M_{s t r}=\pi^{-1 / 8} \alpha^{-1 / 4}$. Figure 5.19 displays the deviation from Standard Model expectations to Bhabha scattering from these string exchanges, and compares their effect to those arising from other types of contact interactions. The $95 \%$ CL exclusion limits for $\sqrt{s}=1 \mathrm{TeV}$ and $200 \mathrm{fb}^{-1}$ is $M_{\text {str }}>3.1 \mathrm{TeV}$, which corresponds to $M_{*} / \lambda^{1 / 4}>9.3 \mathrm{TeV}$.

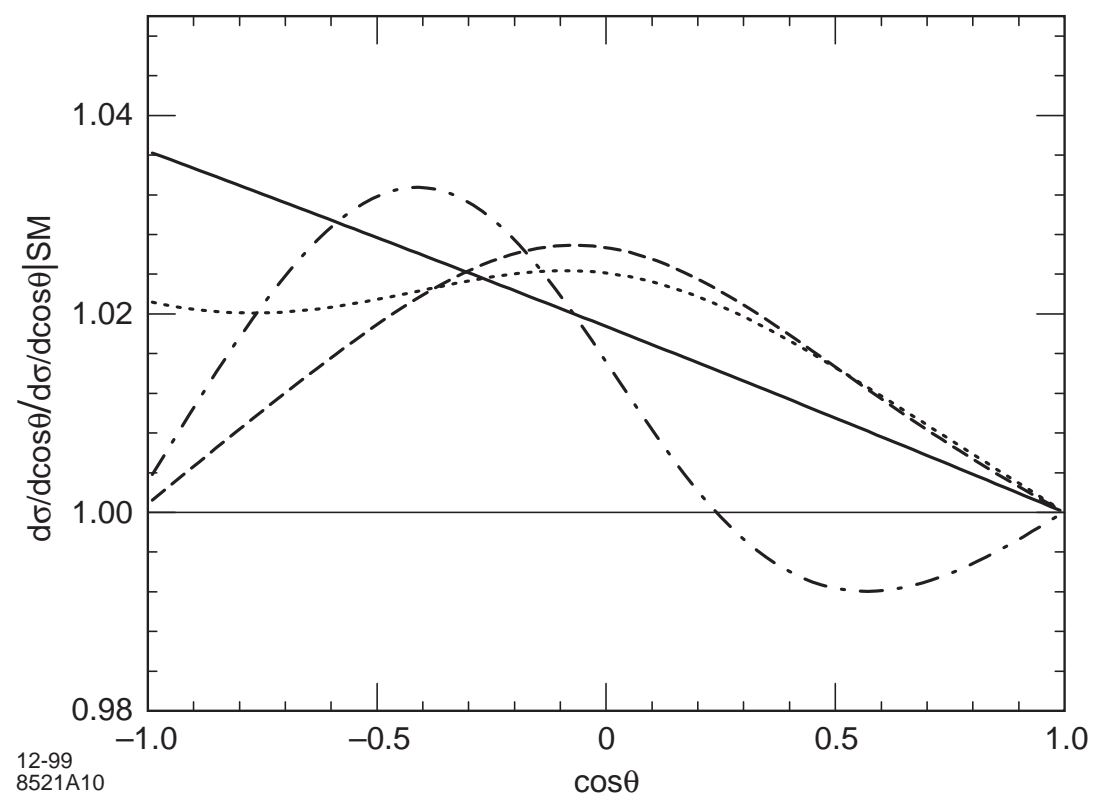

Figure 5.19: Comparison of deviations from the Standard Model prediction for Bhabha scattering at $1 \mathrm{TeV}$ due to corrections from higher-dimension operators [111]. The curves correspond to: string model with $M_{\text {str }}=3.1 \mathrm{TeV}$ (solid), KK graviton exchange with $M_{*} / \lambda^{1 / 4}=6.2 \mathrm{TeV}$ (dotted), $\mathrm{VV}$ contact interactions with $\Lambda=88 \mathrm{TeV}$ (dashed), and AA contact interactions with $\Lambda=62 \mathrm{TeV}$ (dot-dashed).

\subsection{Non-commutative field theories}

Recent theoretical results have demonstrated that non-commutative quantum field theories (NCQFT) naturally appear within the context of string theory and M-theory [113]. In this case, the usual $\delta$-dimensional space associated with commuting space-time coordinates is generalized to one that is non-commuting. In such 
New Physics at the TeV Scale and Beyond

a space, the conventional coordinates are represented by operators that no longer commute,

$$
\left[\hat{X}_{\mu}, \hat{X}_{\nu}\right]=i \theta_{\mu \nu} \equiv \frac{i}{\Lambda_{N C}^{2}} c_{\mu \nu}
$$

Here, the effect has been parameterized in terms of an overall scale $\Lambda_{N C}$, which characterizes the threshold where non-commutative (NC) effects become important, and a real antisymmetric matrix $c_{\mu \nu}$, whose dimensionless elements are presumably of order unity. The most likely value of $\Lambda_{N C}$ is near the string scale or the true Planck scale, which could be as low as the $\mathrm{TeV}$ scale. The matrix $c_{\mu \nu}$ is related to the Maxwell field-strength tensor $F_{\mu \nu}$ in a straightforward fashion, since NCQFT arises in string theory in the presence of background electromagnetic fields. The matrix $c_{\mu \nu}$ is identical in all reference frames, defining a preferred $\mathrm{NC}$ direction in space, and hence Lorentz invariance is violated at energies of order $\Lambda_{N C}$. The usual description of Lorentz violation needs to be modified in order to apply to NCQFT; present experiments only constrain such effects at the few-TeV level [114].

Caution must be exercised to preserve orderings of the products of fields when formulating NCQFT. This is accomplished with the introduction of the star product, $\phi(\hat{X}) \phi(\hat{X})=\phi(x) * \phi(x)=\phi(x) e^{\left[i \theta^{\mu \nu} \partial_{\mu} \partial_{\nu} / 2\right]} \phi(x)$, which absorbs the effect of the commutation relation via a series of Fourier transforms. The NC action for a quantum field theory is thus obtained from the ordinary one by replacing the products of fields by star products. A striking consequence of this is that the $\mathrm{NC}$ version of QED takes on a non-Abelian nature in that both 3-point and 4-point photon couplings are generated. In addition, all QED vertices pick up additional phase factors that are dependent upon the momenta flowing through the vertex. We note that propagators, however, are not modified since quadratic forms remain unchanged under the properties of the star product. NC effects thus produce striking signatures in QED processes at a linear collider. The modifications to pair annihilation, Bhabha and Møller scattering, as well as $\gamma \gamma \rightarrow \gamma \gamma$ have been studied in [115]. Pair annihilation and $\gamma \gamma$ scattering both receive new diagrammatic contributions due to the non-Abelian couplings, and all four processes acquire a phase dependence due to the relative interference of the vertex kinematic phases. The lowest-order correction to the Standard Model in these processes occurs at dimension 8. The most striking result is that a $\phi$ dependence is induced in $2 \rightarrow 2$ scattering processes because of the existence of the NC preferred direction in space-time. This azimuthal dependence in pair annihilation is illustrated in Fig. 5.20 for the case where the NC direction is perpendicular to the beam axis. The results of [115] are summarized in Table 5.9, which displays the $95 \% \mathrm{CL}$ search reach for the $\mathrm{NC}$ scale in these four reactions. We see that these processes are complementary in their ability to probe different structures of non-commuting space-time. 


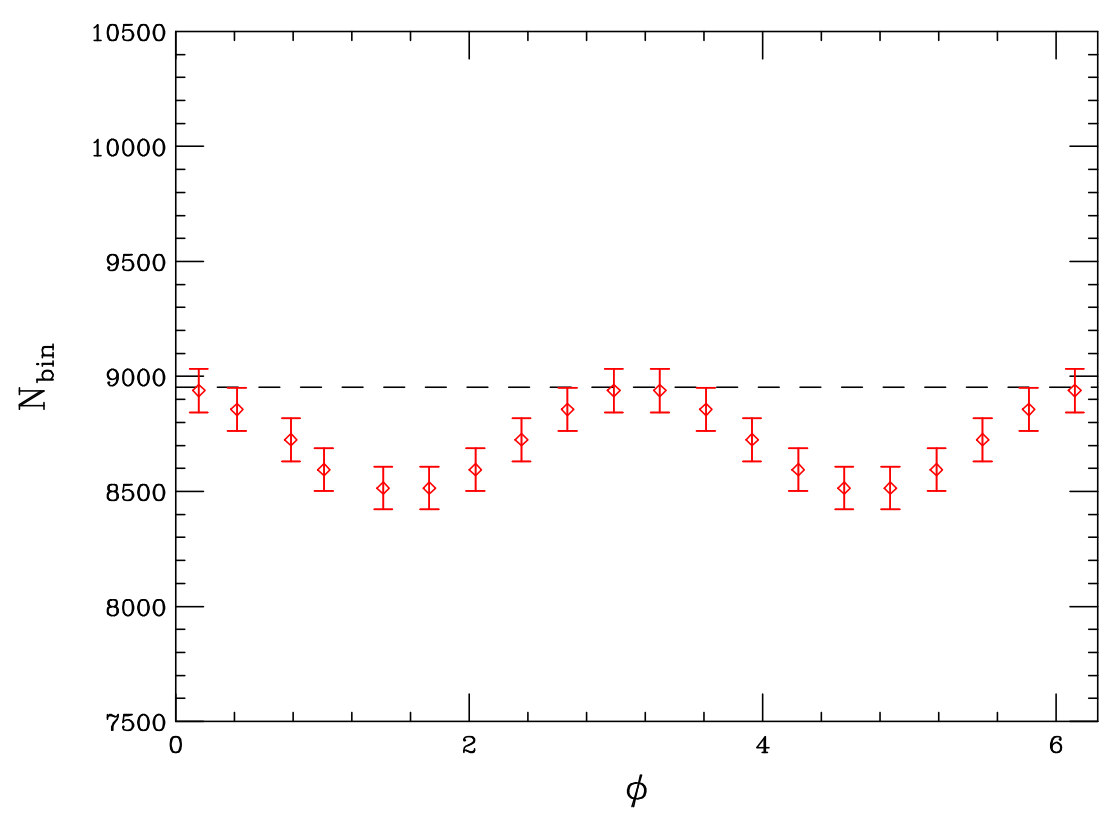

Figure 5.20: $\phi$ dependence of the $e^{+} e^{-} \rightarrow \gamma \gamma$ cross section, taking $\Lambda_{N C}=\sqrt{s}=500$ $\mathrm{GeV}$ a luminosity of $500 \mathrm{fb}^{-1}$. A cut of $|\cos \theta|<0.5$ has been employed. The dashed line corresponds to the SM expectations and the 'data' points represent the NCQED results.

\begin{tabular}{|c|c|c|}
\hline \hline Process & Structure Probed & Bound on $\Lambda_{N C}$ \\
\hline$e^{+} e^{-} \rightarrow \gamma \gamma$ & Space-Time & $740-840 \mathrm{GeV}$ \\
Møller Scattering & Space-Space & $1700 \mathrm{GeV}$ \\
Bhabha Scattering & Space-Time & $1050 \mathrm{GeV}$ \\
$\gamma \gamma \rightarrow \gamma \gamma$ & Space-Time & $700-800 \mathrm{GeV}$ \\
& Space-Space & $500 \mathrm{GeV}$ \\
\hline \hline
\end{tabular}

Table 5.9: Summary of the 95\% CL search limits on the NC scale $\Lambda_{N C}$ from the various processes considered above at a $500 \mathrm{GeV}$ linear collider with an integrated luminosity of $500 \mathrm{fb}^{-1}$.

\section{Determining the origin of new physics}

As demonstrated in this chapter, some reactions at linear colliders may receive contributions from many different models. An example of this is $e^{+} e^{-} \rightarrow f \bar{f}$, in which indirect effects of compositeness, extended gauge sectors, extra dimensions, string resonances, or supersymmetry may be revealed. Once a signal for new physics is found, the next step is to unravel the properties associated with the new phenomena. If the mass spectrum of the new particles in these theories is kinematically accessible, then their properties may be directly measured. However, if these states are too heavy, then we must explore their characteristics indirectly. This is feasible at a linear 
New Physics at the TeV Scale and Beyond

collider because of the precision at which measurements can be performed. Here, we give a single example to illustrate our point, namely, the ability of $e^{+} e^{-}$colliders to provide unique information about the spin structure of new objects. The angular distributions and polarization asymmetries associated with $e^{+} e^{-} \rightarrow f \bar{f}$ are sensitive probes of the spin of new particles. An illustration of this was presented in Fig. 5.15, which showed the extent to which spin-2 exchange in $e^{+} e^{-} \rightarrow f \bar{f}$ is distinguishable from other new physics sources. This figure showed that deviations induced by spin-2 graviton exchanges can be distinguished from those due to lower spins, such as new vector bosons $Z^{\prime}$ or a scalar neutrino in R-parity-violating models, up to the discovery limit. In addition, discrimination between spin- 1 and spin-0 particles at a LC was demonstrated [116] by studying the angular distributions induced by the exchange of a $Z^{\prime}$ and of a scalar neutrino, $\tilde{\nu}$ in $e^{+} e^{-} \rightarrow f \bar{f}$. A two-parameter fit of a trial distribution of the form $\sim A(1+z)^{2}+B(1-z)^{2}$ was performed to the observables, with $A, B$ being parameters determined by the fit. In the case of the Standard Model and $Z^{\prime}$, the fitted parameters $A, B$ are constant, while, in the case of $\tilde{\nu}$, the parameter $B$ depends on $z$. The results of the fit are displayed in Fig. 5.21. The Standard Model values of $A$ and $B$ are shown in the center of the figure and are assumed to be known precisely. The $Z^{\prime}$ mass was set to $3 \mathrm{TeV}$ and four different $Z^{\prime}$ coupling values were considered. The $\tilde{\nu}$ was allowed to mediate the reaction in both $s$ - and $t$-channels. All

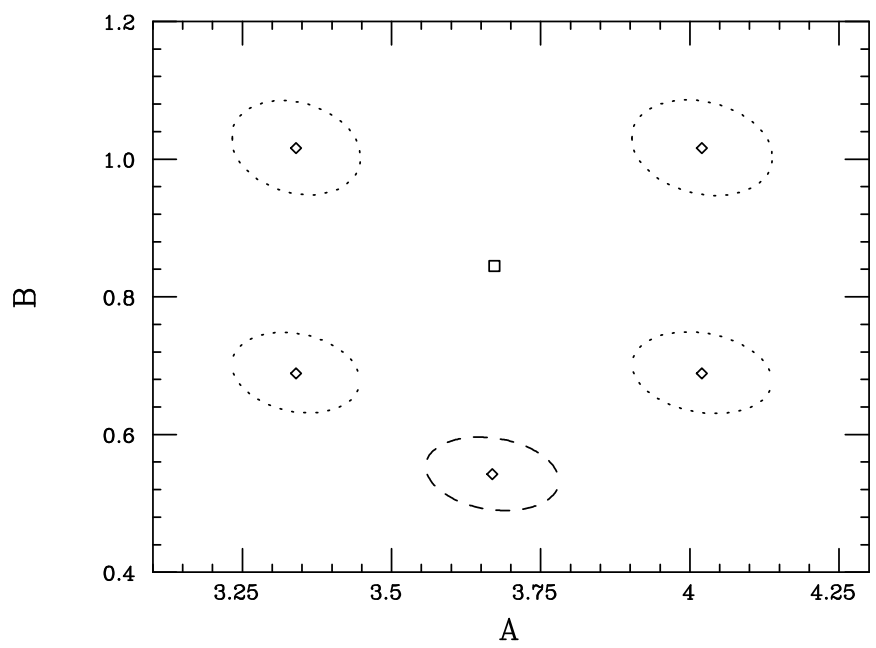

Figure 5.21: Results of the fit with 95\% CL contours circled around the fitted values. The box in the center corresponds to the Standard Model, the dotted ellipses represent the fit to the four $Z^{\prime}$ cases considered, and the dashed ellipse is for the case of sneutrino exchange. The fit was performed taking $\sqrt{s}=1 \mathrm{TeV}$ with $150 \mathrm{fb}^{-1}$. 
five regions are statistically well separated from each other, and clearly distant from the Standard Model solution.

\section{Conclusions}

In this chapter, we have discussed several classes of motivated models that contain new phenomena, and we have delineated the ability of a linear collider to explore them. We have seen that the LHC and the linear collider have a comparable and complementary discovery potential. In many cases, a signal for new physics will first be observed at the LHC, and the linear collider will precisely determine its properties. While a $500 \mathrm{GeV}$ linear collider has a large discovery reach and potential to elucidate the underlying physics, every physics scenario we have also explored benefits from an upgrade to higher energy.

However, our limited imagination does not span the full range of alternatives allowed by present data. We thus must be prepared to discover the unexpected, which is best accomplished by exploration of the energy frontier by both $e^{+} e^{-}$and hadron colliders.

\section{References}

[1] K. Hagiwara, R. D. Peccei, D. Zeppenfeld and K. Hikasa, Nucl. Phys. B282, 253 (1987).

[2] J. Bagger, S. Dawson, and G. Valencia, Nucl. Phys. B399, 264 (1993).

[3] C. Ahn, M. E. Peskin, B. W. Lynn and S. B. Selipsky, Nucl. Phys. B309, 221 (1988); J. Fleischer, J. L. Kneur, K. Kolodziej, M. Kuroda and D. Schildknecht, Nucl. Phys. B378, 443 (1992) [Erratum-ibid. B426, 443 (1992)].

[4] A. Arhrib, J. L. Kneur and G. Moultaka, Phys. Lett. B376, 127 (1996); E. N. Argyres, A. B. Lahanas, C. G. Papadopoulos and V. C. Spanos, Phys. Lett. B383, 63 (1996); G. Couture, J. N. Ng, J. L. Hewett, and T. G. Rizzo, Phys. Rev. D38, 860 (1988).

[5] M. Kitahara, M. Marui, N. Oshimo, T. Saito and A. Sugamoto, Eur. Phys. J. C4, 661 (1998), [hep-ph/9710220].

[6] C. Burgard, in Physics and Experiments with Future $e^{+} e^{-}$Linear Colliders, E. Fernández and A. Pacheco, eds. (UAB Publications, Barcelona, 2000); W. Menges, "A Study of Charge Current Triple Gauge Couplings at TESLA." LC-PHSM-2001-022. http://www.desy.de/ ${ }^{\sim} 1$ cnotes.

[7] D. Choudhury, J. Kalinowski, Nucl. Phys. B491, 129 (1997); D. Choudhury, J. Kalinowski, A. Kulesza, Phys. Lett. B457, 193 (1999). 
New Physics at the TeV Scale and Beyond

[8] ATLAS Detector and Physics Performance Technical Design Report, LHCC 9914/15 (1999).

[9] A. Vicini, Acta Phys. Polon. B29, 2847 (1998).

[10] M. W. Grünewald et al., in Reports of the Working Groups on Precision Calculations for LEP2 Physics, S. Jadach, G. Passarino, and R. Pittau, eds. (CERN 2000-009, Geneva, 2000) [hep-ph/0005309].

[11] W. Beenakker, F. A. Berends and A. P. Chapovsky, Nucl. Phys. B548, 3 (1999).

[12] A. Denner, S. Dittmaier, M. Roth and D. Wackeroth, Nucl. Phys. B587, 67 (2000); Phys. Lett. B475, 127 (2000).

[13] S. Jadach, W. Placzek, M. Skrzypek, B. F. Ward and Z. Was, Phys. Lett. B417, 326 (1998); Phys. Rev. D61, 113010 (2000); hep-ph/0007012; hep-ph/0103163; hep-ph/0104049.

[14] Y. Kurihara, M. Kuroda and D. Schildknecht, Nucl. Phys. B565, 49 (2000).

[15] A. Denner, S. Dittmaier, M. Roth and D. Wackeroth, hep-ph/0104057; A. Denner, S. Dittmaier, M. Roth and D. Wackeroth, in Proceedings of the 22nd Annual MRST Meeting, C.R. Hagen, ed. (Melville, NY, 2000) [hep-ph/0007245].

[16] W. J. Stirling and A. Werthenbach, Phys. Lett. B466, 369 (1999); Eur. Phys. J. C14, 103 (2000).

[17] G. Belanger, F. Boudjema, Y. Kurihara, D. Perret-Gallix and A. Semenov, Eur. Phys. J. C13, 283 (2000).

[18] P. J. Dervan, A. Signer, W. J. Stirling and A. Werthenbach, J. Phys. G26, 607 (2000).

[19] G. Montagna, M. Moretti, O. Nicrosini, M. Osmo and F. Piccinini, hep$\mathrm{ph} / 0103155$.

[20] The LEP Collaborations ALEPH, DELPHI, L3, OPAL, the LEP Electroweak Working Group, and the SLD Heavy and Flavor and Electroweak Groups, CERN-EP/2001-021, hep-ex/0103048.

[21] G. Belanger and F. Boudjema, Phys. Lett. B288, 201 (1992). F. Boudjema et al., in Proceedings of the workshop on $e^{+} e^{-}$collisions at $500 \mathrm{GeV}$, P. Zerwas, ed. (Munich/Annecy/Hamburg, 1991), ENSLAPP-A-365-92. S. Godfrey, in Proceedings of the International Symposium On Vector Boson Self-interactions, U. Baur, S. Errede, T. Muller, eds. (Woodbury, NY, American Inst. Phys., 1996) [hep-ph/9505252].

[22] G. Abu Leil and W. J. Stirling, J. Phys. G21, 517 (1995).

[23] S. Dawson, A. Likhoded, G. Valencia and O. Yushchenko, in Proceedings of the 1996 DPF/DPB Summer Study On New Directions For High-Energy Physics (Snowmass 96), D. G. Cassel, L. T. Gennari, R. H. Siemann, eds. (Stanford, CA, 1997) [hep-ph/9610299]; T. Han, H. He and C. P. Yuan, Phys. Lett. B422, 
294 (1998); O. J. Eboli, M. C. Gonzalez-Garcia and J. K. Mizukoshi, Phys. Rev. D58, 034008 (1998) [hep-ph/9711499]; F. Gangemi, hep-ph/0002142.

[24] A. S. Belyaev, O. J. Eboli, M. C. Gonzalez-Garcia, J. K. Mizukoshi, S. F. Novaes and I. Zacharov, Phys. Rev. D59, 015022 (1999); S. Haywood et al., in Proceedings of the CERN-TH workshop on Standard Model Physics (and more) at the LHC, G. Altarelli and M. Mangano, eds. (Geneva, CERN, 2000); W. J. Stirling and A. Werthenbach, Eur. Phys. J. C12, 441 (2000).

[25] O. J. Eboli, M. C. Gonzalez-Garcia, S. M. Lietti and S. F. Novaes, Phys. Rev. D63, 075008 (2001); O. J. Eboli, M. C. Gonzalez-Garcia and S. F. Novaes, Nucl. Phys. B411, 381 (1994) [hep-ph/9306306].

[26] M. E. Peskin and J. D. Wells, hep-ph/0101342.

[27] For recent reviews, see K. Lane, "Technicolor 2000," hep-ph/0007304; R. S. Chivukula and J. Womersley, in "Review of Particle Physics," D. E. Groom et al., Eur. Phys. J. C15, 1 (2000).

[28] T. Appelquist, J. Terning and L. C. Wijewardhana, Phys. Rev. Lett. 79, 2767 (1997).

[29] R. Casalbuoni, A. Deandrea, S. De Curtis, D. Dominici, R. Gatto and J. F. Gunion, Nucl. Phys. B555, 3 (1999).

[30] C. T. Hill, Phys. Lett. B345, 483 (1995); K. Lane and E. Eichten, Phys. Lett. B352, 382 (1995) [hep-ph/9503433].

[31] C. Yue, G. Lu, J. Cao, J. Li and G. Liu, Phys. Lett. B496, 93 (2000).

[32] T. L. Barklow et al., in Proceedings of the 1996 DPF/DPB Summer Study On New Directions For High-Energy Physics (Snowmass 96), D. G. Cassel, L. T. Gennari, and R. H. Siemann, eds. (SLAC,1997) [hep-ph/9704217].

[33] V. Barger, K. Cheung, T. Han and R. J. Phillips, Phys. Rev. D52, 3815 (1995); E. Boos, H. J. He, W. Kilian, A. Pukhov, C. P. Yuan and P. M. Zerwas, Phys. Rev. D57, 1553 (1998); Phys. Rev. D61, 077901 (2000).

[34] R. Chierici, S. Rosati, M. Kobel, "Strong electroweak symmetry breaking signals in WW scattering at TESLA," LC-PHSM-2001-038, http://www.desy.de/ Icnotes.

[35] T. L. Barklow, in Proceedings of the 1996 DPF/DPB Summer Study On New Directions For High-Energy Physics (Snowmass 96), D. G. Cassel, L. T. Gennari, and R. H. Siemann, eds. (SLAC,1997); E. Ruiz Morales and M. E. Peskin, hepph/9909383; T. Han, Y. J. Kim, A. Likhoded and G. Valencia, Nucl. Phys. B593, 415 (2001); F. Larios, T. M. Tait and C. P. Yuan, hep-ph/0101253.

[36] A. Manohar and H. Georgi, Nucl. Phys. B 234, 189 (1984).

[37] M. Peskin, in Physics in Collision IV, A. Seiden, ed. (Éditions Frontières, GifSur-Yvette, France, 1984); F. Iddir, A. Le Yaouanc, L. Oliver, O. Pene and J. C. Raynal, Phys. Rev. D41, 22 (1990). 
New Physics at the TeV Scale and Beyond

[38] T. L. Barklow, "LET Signals in $e^{+} e^{-} \rightarrow W^{+} W^{-}$at $\sqrt{s}=800 \mathrm{GeV"} \mathrm{in} \mathrm{Proceed-}$ ings of 5th International Linear Collider Workshop (LCWS2000).

[39] C. J. S. Damerell and D. J. Jackson, in Proceedings of the 1996 DPF/DPB Summer Study On New Directions For High-Energy Physics (Snowmass 96), D. G. Cassel, L. T. Gennari, and R. H. Siemann, eds. (SLAC,1997).

[40] B. A. Dobrescu and C. T. Hill, Phys. Rev. Lett. 81, 2634 (1998); R. S. Chivukula, B. A. Dobrescu, H. Georgi and C. T. Hill, Phys. Rev. D59, 075003 (1999).

[41] C. T. Hill, Phys. Lett. B266, 419 (1991).

[42] G. Burdman and N. Evans, Phys. Rev. D59, 115005 (1999).

[43] R. S. Chivukula, C. Hölbling and N. Evans, Phys. Rev. Lett. 85, 511 (2000).

[44] B. A. Dobrescu, Phys. Rev. D63, 015004 (2001).

[45] B. A. Dobrescu, G. Landsberg and K. T. Matchev, Phys. Rev. D63, 075003 (2001)

[46] P. F. Derwent et al., "Linear Collider Physics", FERMILAB-FN-701, (2000).

[47] H. Collins, A. Grant and H. Georgi, Phys. Rev. D61, 055002 (2000).

[48] H. Georgi and A. K. Grant, Phys. Rev. D63, 015001 (2001).

[49] E. Eichten, K. Lane, and M. Peskin, Phys. Rev. Lett. 50, 811 (1983).

[50] K. Cheung, S. Godfrey, and J. L. Hewett, in Proceedings of the 1996 DPF/DPB Summer Study On New Directions For High-Energy Physics (Snowmass 96), D. G. Cassel, L. T. Gennari, R. H. Siemann, eds. (Stanford, CA, 1997) [hep$\mathrm{ph} / 9612257]$.

[51] T. L. Barklow, Int. J. Mod. Phys A11, 1579 (1996).

[52] A. Leike, Phys. Reports 317 (1999) 143; M. Cvetič, S. Godfrey, in "Electroweak Symmetry Breaking and Beyond the Standard Model", T. Barklow, S. Dawson, H. Haber, J. Siegrist eds., World Scientific 1995; S. Godfrey, Phys. Rev. D51, 1402 (1995); S. Godfrey, J. Hewett, and L. Price, in Proceedings of the 1996 DPF/DPB Summer Study On New Directions For High-Energy Physics (Snowmass 96), D. G. Cassel, L. T. Gennari, R. H. Siemann, eds. (Stanford, CA, 1997) [hep-ph/9704291]; T. Rizzo, ibid., [hep-ph/9612440].

[53] S. Godfrey, P. Kalyniak, B. Kamal, A. Leike, Phys. Rev. D61, 113009 (2000).

[54] J. Hewett, in Proceedings of the 1996 DPF/DPB Summer Study On New Directions For High-Energy Physics (Snowmass 96), D. G. Cassel, L. T. Gennari, R. H. Siemann, eds. (Stanford, CA, 1997) [hep-ph/9704292].

[55] M. Doncheski, S. Godfrey, P. Kalyniak, B. Kamal, A. Leike, Phys. Rev. D63, 053005 (2001).

[56] S. Riemann, LC-TH-2001-007 and in Proceedings of the 5th International Linear Collider Workshop (LCSW2000).

[57] W. Buchmüller, R. Rückl and D. Wyler, Phys. Lett. B191, 442 (1987). 
[58] A. F. Żarnecki, Eur. Phys. J. C17, 695 (2000).

[59] M. Heyssler, R. Rückl, and H. Spiesberger, in International Workshop on Linear Colliders, Sitges, Spain, 1999, hep-ph/9908319.

[60] M. A. Doncheski and S. Godfrey, Phys. Rev. D49, 6220 (1994); Phys. Rev. D51, 1040 (1995); Phys. Lett. B393, 355 (1997); J. L. Hewett and S. Pakvasa, Phys. Lett. B227, 178 (1989).

[61] J. L. Hewett and T. G. Rizzo, Phys. Rev. D36, 3367 (1987); J. Blümlein and R. Rückl, Phys, Lett. B304, 337 (1993); J. Hewett in 2nd International Workshop on Physics and Experimentation with $e^{+} e^{-}$Linear Colliders, Waikoloa, HI, 1993, hep-ph/9308321.

[62] S. Abdullin and F. Charles, Phys. Lett. B464, 223 (1999).

[63] J. L. Hewett and T. G. Rizzo, Phys. Rev. D56, 5709 (1997).

[64] A. Djouadi, J. Ng, and T. G. Rizzo, in Electroweak Symmetry Breaking and New Physics at the TeV Scale, ed. T. Barklow et al., (World Scientific, Singapore), 416 (1996).

[65] J. Maalampi, K. Mursula and M. Roos, Nucl. Phys. 207B, 233 (1982).

[66] J. L. Hewett and T. G. Rizzo, Phys. Rep. 183, 193 (1989).

[67] J. W. F. Valle, Nucl. Phys. Proc. Suppl. 11, 119 (1989).

[68] P. Langacker and D. London, Phys. Rev. D38, 886 (1988). E. Nardi, E. Roulet and D. Tommasini, Nucl. Phys. 386B, 239 (1992), Phys. Lett. 327B, 319 (1994). F. M. L. Almeida Jr. et al., Phys. Rev. D62, 075004 (2000).

[69] V. Barger, T. Han, and J. Ohnemus, Phys. Rev. D37, 1174 (1988); V. Barger and R. Phillips, Collider Physics, (New York, Addison Wesley, 1987).

[70] A. Djouadi, Z. Phys. C63, 317 (1994); G. Azuelos and A. Djouadi, Z. Phys. C63, 327 (1994).

[71] F. M. L. Almeida Jr. et al., Phys. Rev. D51, 5990 (1995).

[72] F. M. L. Almeida Jr. et al., Phys. Rev. D63, 075005 (2001).

[73] N. Arkani-Hamed, S. Dimopoulos, and G. Dvali, Phys. Lett. B429, 263 (1998), and Phys. Rev. D59, 086004 (1999); I. Antoniadis, N. Arkani-Hamed, S. Dimopoulos, and G. Dvali, Phys. Lett. B436, 257 (1998).

[74] L. Randall and R. Sundrum, Phys. Rev. Lett. 83, 3370 (1999), and ibid., 4690, (1999).

[75] I. Antoniadis, Phys. Lett. B246, 377 (1990); J. Lykken, Phys. Rev. D54, 3693 (1996); E. Witten, Nucl. Phys. B471, 135 (1996); P. Horava and E. Witten, Nucl. Phys. B460, 506 (1996), ibid., B475, 94 (1996).

[76] N. Arkani-Hamed and M. Schmaltz, Phys. Rev. D61, 033005 (2000); N. ArkaniHamed, Y. Grossman and M. Schmaltz, Phys. Rev. D61, 115004 (2000); E. A. Mirabelli and M. Schmaltz, Phys. Rev. D61, 113001 (2000); G. C. Branco, A. De Gouvea and M. N. Rebolo, hep-ph/0012289; T. G. Rizzo, hep-ph/0101278. 
New Physics at the TeV Scale and Beyond

[77] H. Cheng, B. A. Dobrescu and C. T. Hill, Nucl. Phys. B589, 249 (2000) [hepph/9912343]; B. A. Dobrescu, Phys. Lett. B461, 99 (1999) [hep-ph/9812349].

[78] N. Arkani-Hamed, H. Cheng, B. A. Dobrescu and L. J. Hall, Phys. Rev. D62, 096006 (2000) [hep-ph/0006238].

[79] B. A. Dobrescu and E. Poppitz, hep-ph/0102010.

[80] C. D. Hoyle, U. Schmidt, B. R. Heckel, E. G. Adelberger, J. H. Gundlach, D. J. Kapner and H. E. Swanson, Phys. Rev. Lett. 86, 1418 (2001) [hep$\mathrm{ph} / 0011014]$.

[81] G. F. Giudice, R. Rattazzi, and J. D. Wells, Nucl. Phys. B544, 3 (1999).

[82] S. Cullen and M. Perelstein, Phys. Rev. Lett. 83, 268 (1999); L. Hall and D. Smith, Phys. Rev. D60, 085008 (1999); V. Barger, T. Han, C. Kao, and R. J. Zhang, Phys. Lett. B461, 34 (1999); C. Hanhart, D. R. Phillips, S. Reddy, and M. J. Savage nucl-th/0007016.

[83] See, for example, T. G. Rizzo and J. D. Wells, Phys. Rev. D61, 016007 (2000); P. Nath and M. Yamaguchi, Phys. Rev. D60, 116006 (1999); M. Masip and A. Pomarol, Phys. Rev. D60, 096005 (1999); W. J. Marciano, Phys. Rev. D60, 093006 (1999); L. Hall and C. Kolda, Phys. Lett. B459, 213 (1999); R. Casalbuoni, S. DeCurtis and D. Dominici, Phys. Lett. B460, 135 (1999); R. Casalbuoni, S. DeCurtis, D. Dominici and R. Gatto, Phys. Lett. B462, 48 (1999); A. Strumia, Phys. Lett. B466, 107 (1999); F. Cornet, M. Relano and J. Rico, Phys. Rev. D61, 037701 (2000); C. D. Carone, Phys. Rev. D61, 015008 (2000);

A. Delgado, A. Pomarol and M. Quiros, JHEP 0001, 030 (2000).

[84] T. Han, J. Lykken, and R. J. Zhang, Phys. Rev. D59, 105006 (1999) .

[85] E. A. Mirabelli, M. Perelstein, and M. Peskin, Phys. Rev. Lett. 82, 2236 (1999); K. Cheung and W. Y. Keung Phys. Rev. D60, 112003 (1999).

[86] G.W. Wilson, LC-PHSM-2001-010; A. Vest, LC-TH-2000-058, http://www. desy.de/ l lcnotes.

[87] M. Gataullin, talk presented at $36^{\text {th }}$ Rencontres de Moriond on Electroweak Interactions and Unified Theories, Les Arcs, France, 10-17 March 2001; G. Landsberg, ibid., A. Schoning, ibid., D. Bourilkov, hep-ex/0103039; B. Abbott et al., (DØ Collaboration), Phys. Rev. Lett. 86, 1156 (2001).

[88] L. Vacavant and I. Hinchliffe, SN-ATLAS-2001-005, to appear in J. Phys. G.

[89] J. L. Hewett Phys. Rev. Lett. 82, 4765 (1999); T. G. Rizzo, Phys. Rev. D59, 115010 (1999).

[90] M. Bando, T. Kugo, T. Noguchi, and K. Yoshioka Phys. Rev. Lett. 83, 3601 (1999); J. Hisano, and N. Okada Phys. Rev. D61, 106003 (2000).

[91] O. J. P. Eboli, T. Han, M. B. Magro, and P. C. Mercadante, Phys. Rev. D61, 094007 (2000). 
[92] E. Dudas, J. Mourad, Nucl. Phys. B575, 3 (2000); E. Accomando, I. Antoniadis, K. Benakli, Nucl. Phys. B579, 3 (2000); S. Cullen, M. Perelstein, M. E. Peskin, Phys. Rev. D62, 055012 (2000).

[93] A. Miagkov, talk presented at Workshop on Higgs and Supersymmetry, Orsay, France, 11-22 March, 2001.

[94] T. G. Rizzo, Phys. Rev. D60, 115010 (1999); H. Davoudiasl, Phys. Rev. D60, 084022 (1999); Phys. Rev. D61, 044018 (2000).

[95] D. Sadri and J. L. Hewett, SLAC-PUB-8782 (2001).

[96] W. D. Goldberger and M. B. Wise Phys. Rev. Lett. 83, 4922 (1999); O. DeWolfe, D. Z. Freedman, S. S. Gubser, and A. Karch, Phys. Rev. D62, 046008 (2000); M. A. Luty and R. Sundrum, Phys. Rev. D62, 035008 (2000).

[97] H. Davoudiasl, J. L. Hewett, and T. G. Rizzo, Phys. Rev. Lett. 84, 2080 (2000).

[98] H. Davoudiasl, T. G. Rizzo, hep-ph/0104199.

[99] H. Davoudiasl, J. L. Hewett, and T. G. Rizzo, Phys. Rev. D63, 075004 (2001).

[100] B. C. Allanach, K. Odagiri, M. A. Parker, and B. R. Webber, JHEP 0009, 019 (2000).

[101] H. Davoudiasl, J. L. Hewett, and T. G. Rizzo, Phys. Lett. B473, 43 (2000) and hep-ph/0006097; A. Pomarol, Phys. Lett. B486, 153 (2000); Y. Grossman and M. Neubert, Nucl. Phys. B474, 361 (2000); R. Kitano, Phys. Lett. B481, 39 (2000); S. Chang et al., Phys. Rev. D62, 084025 (2000); T. Ghergetta and A. Pomarol, Nucl. Phys. B586, 141 (2000).

[102] It will be assumed in the discussion below that all of the SM gauge fields are in the bulk. See, for example, I. Antoniadis, C. Munoz and M. Quiros, Nucl. Phys. B397, 515 (1993); I. Antoniadis and K. Benalki, Phys. Lett. B326, 69 (1994), and Int. J. Mod. Phys. A15, 4237 (2000); I. Antoniadis, K. Benalki and M. Quiros, Phys. Lett. B331, 313 (1994); K. Benalki, Phys. Lett. B386, 106 (1996); T. G. Rizzo, Phys. Rev. D61, 055005 (2000).

[103] E. Tourneifer, talk presented at the $36^{\text {th }}$ Rencontres de Moriond on Electroweak Interactions and Unified Theories, Les Arcs, France, 10-17 March 2001.

[104] G. Azuelos, and G. Polesello, in preparation.

[105] T. G. Rizzo, Phys. Rev. D61, 055005 (2000).

[106] Finite KK sums can also arise from other kinds of new physics; see for example, M. Bando, T. Kugo, T. Noguchi and K. Yoshioka, hep-ph/9906549; I. Antoniadis, K. Benalki and M. Quiros, hep-ph/9905311.

[107] T.G. Rizzo, hep-ph/0101278.

[108] N. Arkani-Hamed, Y. Grossman, and M. Schmaltz, Phys. Rev. D61, 115004 (2000).

[109] T. Appelquist, H.-C. Cheng and B. A. Dobrescu, hep-ph/0012100; R. Barbieri, L. J. Hall and Y. Nomura, hep-ph/0011311. 
[110] A. De Rujula, A. Donini, M. B. Gavela, S. Rigolin, Phys. Lett. B482, 195 (2000).

[111] S. Cullen, M. Perelstein, and M. E. Peskin Phys. Rev. D62, 055012 (2000).

[112] G. Veneziano, Nuovo Cim. A57, 190 (1968).

[113] For a review of non-commutative theories, see, N. Seiberg and E. Witten, JHEP 9909, 032 (1999).

[114] S. M. Carroll et al., hep-th/0105082.

[115] J. L. Hewett, F. J. Petriello, T. G. Rizzo, hep-ph/0010354.

[116] T. G. Rizzo, Phys. Rev. D59, 113004 (1999). 


\section{Chapter 6 Top Quark Physics}

\section{Introduction}

The linear collider, operating near the $t \bar{t}$ production threshold and at higher energies, can carry out a comprehensive program of top quark physics. Measurements at the threshold include the determination of the top quark mass, $m_{t}$, and width, $\Gamma_{t}$, as well as the top quark Yukawa coupling, $g_{t t h}$. The quantities $m_{t}$ and $g_{t t h}$ can also be measured at higher energies, together with the couplings of the top quark to the electroweak gauge bosons. In this chapter we present a brief summary of our current understanding of top quark physics at a linear collider.

The top is unique among the quarks in that it decays before nonperturbative strong interaction effects can influence it. Its large mass gives it stronger coupling to many proposed new physics effects that try to explain electroweak symmetry breaking and/or the origin of particle masses. Thus, precise measurement of the parameters of the top quark would provide important insights into physics beyond the Standard Model.

\section{Physics in the threshold region}

\section{$2.1 \quad$ Introduction}

One of the primary goals of a high-energy $e^{+} e^{-}$linear collider is the study of sharp features in the cross section for $e^{+} e^{-}$annihilation to hadrons. The $t \bar{t}$ threshold is an excellent example of such a structure. The cross section for $e^{+} e^{-} \rightarrow t \bar{t}$ is expected to rise by an order of magnitude with only a $5 \mathrm{GeV}$ change in center-ofmass energy around $350 \mathrm{GeV}$. Careful study of this $t \bar{t}$ threshold structure can precisely measure many parameters of the top quark, including its mass and width, and the top quark Yukawa coupling. In this section we briefly summarize the current status of $t \bar{t}$ threshold studies. More comprehensive discussions can be found in [1-3].

\subsection{QCD dynamics and cross section}

It is well known that, because of the large top quark width $\left(\Gamma_{t} \approx 1.4 \mathrm{GeV} \gg\right.$ $\left.\Lambda_{Q C D}\right)$, a top-antitop pair cannot form narrow toponium resonances. Instead, the cross section is expected to have a smooth line-shape showing only a moderate $1 S$ peak. The dynamics of the top quark in the threshold region is described by perturbative QCD. The top quark width serves as an infrared cutoff. As a result, nonperturbative QCD effects (as measured, for example, by the influence of the gluon 

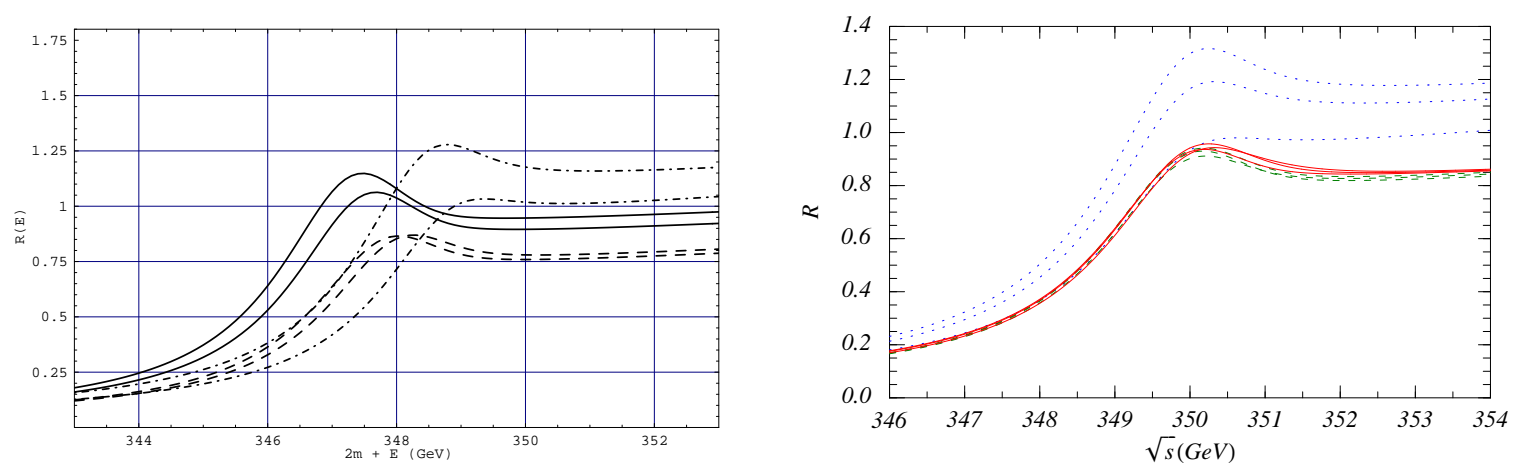

Figure 6.1: The normalized cross section $R_{t}=\sigma\left(e^{+} e^{-} \rightarrow t \bar{t}\right) / \sigma\left(e^{+} e^{-} \rightarrow \mu^{+} \mu^{-}\right)$as a function of $\sqrt{s}$, computed in QCD perturbation theory at various levels. These are theoretical curves that do not include initial state radiation, beamstrahlung, or beam energy spread. (Left:) The normalized cross section computed with the pole mass $m_{t}^{\text {pole }}=175 \mathrm{GeV}$, at LO (dashed-dotted lines), NLO (dashed lines), and NNLO (solid lines). Each pair of the curves corresponds to the two different soft normalization scales $\mu=30 \mathrm{GeV}$ (upper curve) and $\mu=60 \mathrm{GeV}$ (lower curve). (Right:) The normalized cross section computed with the $1 S$ mass $m_{t}^{1 S}=175 \mathrm{GeV}$, at LL order (dotted), NLL order (dashed) and NNLL order in QCD (solid). The calculation includes the summation of logarithms of the top quark velocity, and at each order curves are shown for $\nu=0.15,0.2,0.4$, where $\nu$ is the so-called subtraction velocity.

condensate) are small [4], allowing us, in principle at least, to calculate the cross section from QCD with high accuracy.

The convergence of QCD perturbation theory in the threshold region depends on the quark mass definition used. The simplest definition of $m_{t}$ is the position of the pole in the top quark propagator. This 'pole mass' is similar to the kinematic mass observed in top quark pair production above threshold, and similar to the mass definition used by the CDF and D $\varnothing$ experiments in the original papers on the top quark discovery [5,6]. Unfortunately, with this choice of the mass definition, the NNLO corrections are uncomfortably large [1] and shift the $1 S$ peak by about $0.5 \mathrm{GeV}$, spoiling the possibility to extract the top quark mass with high accuracy. The threshold cross sections computed at successive order in QCD are shown in the left-hand graph in Fig. 6.1. The instability of this perturbation series is caused by the fact that the pole mass has a renormalon ambiguity, that is, it obtains an additive correction from nonperturbative QCD effects.

To remove this difficulty, one can use a different mass definition that refers only to short-distance QCD physics. For example, a possible definition of the mass, called the $1 S$ mass, is one-half of the mass of the lowest toponium bound state computed in the hypothetical limit of zero top quark width [7]. Three other mass definitions have been considered in the literature. The $\overline{\mathrm{PS}}$ mass [8] is defined via the top quark self-energy. The $L S$ ('low scale') mass is given in terms of perturbative evaluations 
of matrix elements of operators in the heavy quark effective theory that describe the difference between the pole mass and a fictitious $T$ meson mass [9]. Finally, the PS ('potential-subtracted') mass is defined by

$$
m_{t}^{P S}(\mu)=m_{t}^{\text {pole }}+\frac{1}{2} \int_{|k|<\mu} \frac{d^{3} k}{(2 \pi)^{3}} V_{C}(k)=m_{t}^{\text {pole }}-\frac{4}{3} \frac{\alpha_{s}}{\pi} \mu+\ldots
$$

where $\mu$ is the soft renormalization scale. All of these mass definitions, collectively called 'threshold masses' have the property that they are free of the $\mathcal{O}\left(\Lambda_{Q C D}\right)$ renormalon ambiguity $[10,11]$. These masses also have the property that they are connected to the $\overline{M S}$ top quark mass by a convergent QCD perturbation series.

The position of the $1 S$ peak becomes much more stable at higher orders of QCD if threshold masses are used. The shifts from order to order are less than $100 \mathrm{MeV}$. However, a large theoretical normalization uncertainty of about $10 \%$ remains. The normalization uncertainty can be reduced to a few percent by resumming terms logarithmic in the top velocity. The convergence for the $1 S$ mass definition is shown in the right-hand graph of Fig. 6.1 [12]. Simultaneous accurate measurements of the top mass and other quantities thus appear feasible, as discussed further below.

\subsection{Top width}

The scan of the $t \bar{t}$ threshold will allow a direct measurement of the top quark width, $\Gamma_{t}$. The cross section at the $1 S$ quarkonium bound state energy is proportional to $1 / \Gamma_{t}$. Realistic studies, which include initial state radiation and other effects, show that $\Gamma_{t}$ can be measured with an experimental precision of a few percent [2], now that higher-order QCD corrections appear to be under control [12].

$\Gamma_{t}$ can also be measured using the forward-backward asymmetry [13]. The $t \bar{t}$ vector coupling to $\gamma$ and $Z$ produces mainly $\mathrm{S}$-wave states, while the axial-vector coupling from the $Z t \bar{t}$ vertex produces $t \bar{t}$ in a $\mathrm{P}$ state. The top quark width causes the $\mathrm{S}$ and $\mathrm{P}$ states to overlap and allows these states to interfere in the final angular distribution. This produces a forward-backward asymmetry. Since the top quark width controls the amount of S-P overlap, the asymmetry is sensitive to $\Gamma_{t}$. Realistic studies are needed to better quantify the experimental sensitivity.

\subsection{Top quark Yukawa coupling}

In addition to the QCD potential, the $t \bar{t}$ pair interacts via a Yukawa potential associated with Higgs boson exchange

$$
V_{t t h}=-\frac{g_{t t h}^{2}}{4 \pi} \frac{e^{-m_{h} r}}{r}
$$

where $m_{h}$ is the Higgs boson mass and $g_{t t h}$ is the Yukawa coupling. Therefore, top threshold measurements can also be used to determine $g_{t t h}$ if the Higgs boson is light. 
A SM Higgs boson with a mass of $115 \mathrm{GeV}$ enhances the normalization of the cross section by $5-8 \%$ at energies near the threshold. The theoretical uncertainty of the cross section in this region is $2-3 \%$ when the summation of logarithms of the top quark velocity is taken into account [12]. A precision measurement of the $t \bar{t}$ threshold cross section thus will be sensitive to the top Yukawa coupling. If we fix all other parameters and assume $m_{h}=115 \mathrm{GeV}$, then varying the SM Yukawa coupling by $\pm 14 \%$ gives a $\pm 2 \%$ variation in the normalization of the cross section near the $1 S$ peak [14]. For larger values of $m_{h}$, the sensitivity to $g_{t t h}$ is expected to decrease. Again, realistic experimental studies that make use of recent theoretical advances in understanding the threshold cross section are needed.

\subsection{Experimental issues}

The experimental situation of the $t \bar{t}$ threshold is fairly well understood, and there has not been much progress since the experimental methods were reviewed at the 1999 Sitges meeting [15]. It is expected that the top mass can be measured with a statistical uncertainty of $40 \mathrm{MeV}$ in a modest scan of $10 \mathrm{fb}^{-1}$, a small fraction of a year at typical design luminosities. A longer scan of about $100 \mathrm{fb}^{-1}$ can determine the top width to $2 \%$. A key experimental issue for the threshold study is the measurement of the $\mathrm{d} \mathcal{L} / \mathrm{dE}$ spectrum, but many complementary methods have been proposed. The issues are similar to and less severe than the measurement of the $\mathrm{d} \mathcal{L} / \mathrm{dE}$ spectrum needed for a precision $W$ mass measurement from the $W^{+} W^{-}$threshold, discussed in Chapter 8, Section 2. The limitations are likely to come from the uncertainty in machine-generated backgrounds and from the theoretical understanding of the Bhabha cross section. The impact of a precision top quark mass measurement can be seen in [16] and [17], which show how the current knowledge of the top mass and precision electroweak measurements limit the range of the Higgs mass and anomalous $W$ and $Z$ couplings caused by new physics.

\section{Physics above the top threshold}

\subsection{Determination of the top quark-Higgs Yukawa coupling}

\subsubsection{Introduction}

If there is a light Higgs boson, this particle is likely to be discovered at the Tevatron or the LHC. The role of a high-energy $e^{+} e^{-}$linear collider is then to test the connection of this particle to the physics of mass generation by accurately measuring its mass, width, and couplings to bosons and fermions. The top quark provides a unique opportunity to measure the Higgs Yukawa coupling to fermions through the process

$e^{+} e^{-} \rightarrow t \bar{t} h$. For a light Higgs boson, the Higgs decays dominantly to $b \bar{b}$. Assuming 
Top Quark Physics

$\mathrm{BR}(t \rightarrow W b)=100 \%$, this leads to multi-jet event topologies involving $4 b$-jets in the final state. Therefore, one of the crucial experimental aspects will be flavor tagging.

\subsubsection{Basic scenario}

The rate for $e^{+} e^{-} \rightarrow t \bar{t} h$ has been calculated to $\mathcal{O}\left(\alpha_{s}\right)$ and is less than $1 \mathrm{fb}$ at $\sqrt{s}=500 \mathrm{GeV}$. The total cross section decreases at low $\sqrt{s}$ because of limited phase space and approaches a constant at high $\sqrt{s}$. The maximum of the cross section (for a 100-150 GeV Higgs boson) occurs around $\sqrt{s} \simeq 700-800 \mathrm{GeV}$.

Since the Yukawa coupling is determined from the cross section measurement, it is straightforward to estimate the statistical and some systematic uncertainties on $g_{t t h}$ for a selection with efficiency $\epsilon$ and purity $\rho$, with an integrated luminosity $L$ :

$$
\begin{aligned}
& \left(\frac{\Delta g_{\text {th }}}{g_{\text {tth }}}\right)_{\text {stat }}=\frac{1}{S_{\text {stat }}\left(g_{t t h}^{2}\right) \sqrt{\epsilon \rho L}}, \\
& \left(\frac{\Delta g_{t t h}}{g_{t t h}}\right)_{\text {syst }}=\frac{1}{S_{\text {syst }}\left(g_{t t h}^{2}\right)}\left[\frac{1-\rho}{\rho} \frac{\Delta \sigma_{B}^{\text {eff }}}{\sigma_{B}^{\text {eff }}} \oplus \frac{1}{\rho} \frac{\Delta L}{L} \oplus \frac{\Delta \epsilon}{\epsilon}\right],
\end{aligned}
$$

where $\left(\Delta g_{t t h} / g_{t t h}\right)_{\text {syst }}$ accounts for the uncertainties in the effective background crosssection (after selection), the integrated luminosity and the selection signal efficiency. $S_{\text {stat }}\left(g_{t t h}^{2}\right)$ and $S_{\text {syst }}\left(g_{t t h}^{2}\right)$ are defined as:

$$
S_{\text {stat }}\left(g_{t t h}^{2}\right)=\frac{1}{\sqrt{\sigma_{t t h}}}\left|\frac{d \sigma_{t t h}}{d g_{t t h}^{2}}\right|, \quad S_{\mathrm{syst}}\left(g_{t t h}^{2}\right)=\frac{1}{\sigma_{t t h}}\left|\frac{d \sigma_{t t h}}{d g_{t t h}^{2}}\right| .
$$

$S_{\text {stat }}$ reaches a 'plateau' for $\sqrt{s} \geq 700 \mathrm{GeV}$, whereas $S_{\text {syst }}$ is essentially independent of $\sqrt{s}$. At $\sqrt{s}=800 \mathrm{GeV}, S_{\text {stat }} \simeq 3.09 \mathrm{fb}^{1 / 2}$ and $S_{\text {syst }} \simeq 1.92$. Therefore, assuming $\epsilon=5 \%$ and $\rho=50 \%$, a statistical precision of around $6.5 \%$ could be achieved in $g_{\text {tth }}$ for $\sqrt{s} \geq 700 \mathrm{GeV}$ and $L=1000 \mathrm{fb}^{-1}$. The case is considerably worse at $\sqrt{s}=500 \mathrm{GeV}$ where $S_{\text {stat }}=0.9 \mathrm{fb}^{1 / 2}$, leading to a statistical uncertainty of $22 \%$ on the Yukawa coupling measurement (with $\epsilon=5 \%$ and $\rho=50 \%$ ). The systematic uncertainty is dominated by the uncertainty in the background normalization, if one assumes that both the signal selection efficiency and integrated luminosity can be known at the $1 \%$ level or better [18].

\subsubsection{Analysis}

We consider the process $e^{+} e^{-} \rightarrow t \bar{t} h \rightarrow W^{+} W^{-} b \bar{b} b \bar{b}$ in both semileptonic and fully hadronic $W$ decay channels. In spite of the apparently clean signature of both channels $(\geq 6$ jets in the final state, with $\geq 4 b$-jets and multi-jet invariant mass constraints), the measurement has many difficulties. Among these are the tiny signal

with backgrounds about 3 orders of magnitude larger, the limitations of jet-clustering 


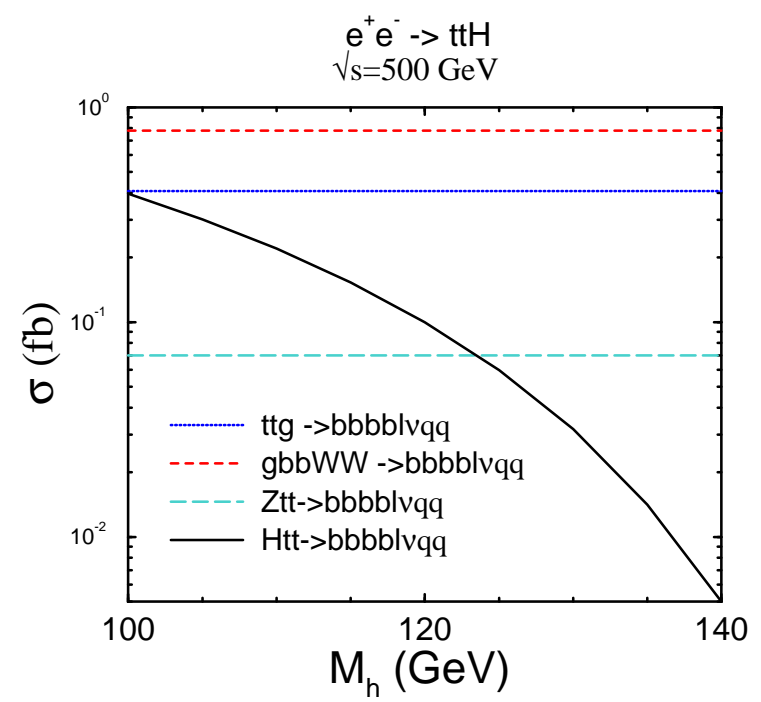

Figure 6.2: Parton level signal and backgrounds to $e^{+} e^{-} \rightarrow t \bar{t} h$ at $\sqrt{s}=500 \mathrm{GeV}$.

algorithms in properly reconstructing multi-jets in the final state, and the degradation of $b$-tagging performance due to hard gluon radiation and jet mixing.

The dominant electroweak background to the semi-leptonic decay is [18-20]:

$$
e^{+} e^{-} \rightarrow t \bar{t} Z \rightarrow Z W^{+} W^{-} b \bar{b} \rightarrow b \bar{b} b \bar{b} \ell^{ \pm} \nu q \bar{q}^{\prime} .
$$

The largest background is from radiative top quark decays:

$$
e^{+} e^{-} \rightarrow t \bar{t} \rightarrow g W^{+} W^{-} b \bar{b} \rightarrow b \bar{b} b \bar{b} \ell^{ \pm} \nu q \bar{q}^{\prime} .
$$

This background has been calculated at the parton level [20] and is shown in Fig. 6.2. Since the $b$ jets resulting from the gluon splitting are logarithmically enhanced at low energy, cuts on the jet energy are effective at eliminating this background. A preliminary study of $e^{+} e^{-} \rightarrow t \bar{t} h$ at $\sqrt{s}=500 \mathrm{GeV}$ included statistical, but not systematic errors and found that the top quark-Higgs Yukawa coupling could be measured with $\sim 21 \%$ accuracy with perfect $b$-tagging and $L=1000 \mathrm{fb}^{-1}[19]$.

The case for a $120 \mathrm{GeV}$ Higgs boson and $\sqrt{s}=800 \mathrm{GeV}$ with $L=1000 \mathrm{fb}^{-1}$ has been considered in [18], with events processed through a simulation of a detector for TESLA. In this analysis, the $b$ jets are defined as those four jets with the lowest probability to originate from the primary vertex. The analysis applies a standard preselection in order to remove as much background as possible while keeping a high efficiency for the signal. Then, in order to improve the statistical sensitivity further, a multivariate analysis using a Neural Network (NN) is performed. After preselection, the overall effective cross section for the background is $17.60 \mathrm{fb}$, while for the signal it is only $0.61 \mathrm{fb}$. This translates into such a poor sample purity $(\rho \sim 3.3 \%)$, that any uncertainty in the background normalization completely erases the significance in the 
Top Quark Physics

signal. After the NN analysis [18], the statistical error is reduced to 5.1\%, and the systematic error to $3.8 \%$, leading to an overall uncertainty of $6.3 \%$ for the Yukawa coupling measurement in the semi-leptonic channel. Combining this with the analysis for the hadronic channel gives a total uncertainty of $5.5 \%$.

\subsubsection{Conclusion}

The reaction $e^{+} e^{-} \rightarrow t \bar{t} h$ allows a direct determination of the top quark-Higgs Yukawa coupling. For $m_{h}=120 \mathrm{GeV}$ and $\mathcal{L}=1000 \mathrm{fb}^{-1}$, a total uncertainty of roughly $5.5 \%$ on the top-Higgs Yukawa coupling at $\sqrt{s}=800 \mathrm{GeV}$ can be obtained. Preliminary studies show that the anticipated precision is about a factor of 4 worse at $\sqrt{s}=500 \mathrm{GeV}$. The dominant systematic uncertainty is from the overall background normalization, pointing to the importance of a complete $2 \rightarrow 8$ background calculation.

\subsection{Top mass reconstruction}

The top quark mass in $e^{+} e^{-}$collisions can not only be measured in a threshold scan, but also at center-of-mass energies above the $t \bar{t}$ threshold. A recent study [21] has shown that a statistical precision of $200 \mathrm{MeV}$ or better may be reached for the top mass from a full kinematical reconstruction of $e^{+} e^{-} \rightarrow t \bar{t} \rightarrow W^{+} b W^{-} \bar{b} \rightarrow \ell^{+} \nu b \ell^{-} \bar{\nu} \bar{b}$ events. It should be noted that the mass measured from final-state shape variables is the pole mass, which is subject to a theoretical uncertainty of $\mathcal{O}\left(\Lambda_{Q C D}\right)$; this point was explained in Section 2.2. Here we give a brief status report of a new study that focuses on extracting the top quark mass from the the $b-\ell$ invariant mass distribution $d \sigma / d m_{b \ell}$, where $\ell$ is the lepton from the $W$ decay, and the $b$-quark energy spectrum, $d \sigma / d E_{b}$.

The extraction of the top mass from final-state shape variables is best done using templates, using a method similar to that described in [22]. It depends crucially on the modeling of the multiparton radiation that is associated with the top production and decay stages. Standard Monte Carlo event generators simulate multiple emission in the soft or collinear approximation and leave empty regions of the phase space corresponding to hard and large-angle gluon radiation ("dead zones"), which can be populated using the exact matrix element ("matrix-element corrections"). Matrixelement corrections to top decays $t \rightarrow b W(g)$ [23] have been implemented in the most recent version of the HERWIG event generator, HERWIG 6.2 [24], which is used in the following. These corrections were found to have a significant effect on jet observables and on the top mass measurement at lepton and hadron colliders [23,25].

The $m_{b \ell}$ distribution, within the precision of the Monte Carlo integration, is independent of the hard-scattering process and of the center-of-mass energy. $m_{b \ell}$ is a Lorentz-invariant observable and is therefore insensitive to the boost from the top 

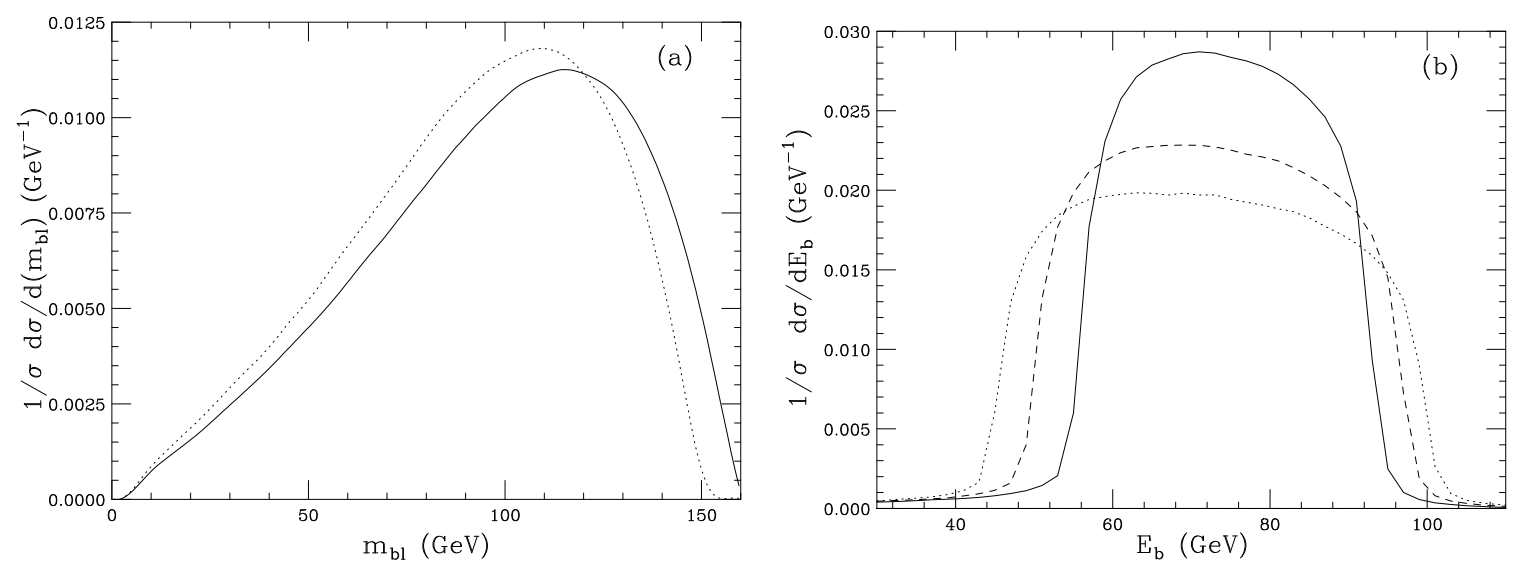

Figure 6.3: a) Invariant mass $m_{b \ell}$ distributions for $m_{t}=171 \mathrm{GeV}$ (dotted line) and $m_{t}=$ $179 \mathrm{GeV}$ (solid line). b) b-quark energy distribution at $\sqrt{s}=370 \mathrm{GeV}$, for $m_{t}=179 \mathrm{GeV}$ (solid), $175 \mathrm{GeV}$ (dashed) and $171 \mathrm{GeV}$ (dotted).

quark rest frame to the laboratory frame. In Fig. 6.3a we plot the $m_{b \ell}$ distribution for $m_{t}=171 \mathrm{GeV}$ and $179 \mathrm{GeV}$. As $m_{t}$ increases, the peak position of the $m_{b \ell}$ distribution is shifted towards larger values. The average value $\left\langle m_{b \ell}\right\rangle$ is proportional to the top quark mass. The best fit is:

$$
\left\langle m_{b \ell}\right\rangle=0.756 m_{t}-37.761 \mathrm{GeV}, \epsilon=0.002 \mathrm{GeV}
$$

where $\epsilon$ is the mean square deviation in the fit. Solving Eq. (6.6), one finds $\Delta m_{t} \approx$ $1.32 \Delta\left\langle m_{b \ell}\right\rangle$, where $\Delta\left\langle m_{b \ell}\right\rangle$ is the uncertainty on the measurement of $\left\langle m_{b \ell}\right\rangle$. No detailed study of the precision that can be achieved with this method has been carried out yet.

In contrast to $m_{b \ell}$, the $b$-quark energy $E_{b}$ is not a Lorentz-invariant observable. One therefore expects that the $E_{b}$ distribution does depend on the boost from the top rest frame to the laboratory frame, and hence on the center-of-mass energy. Since the $t \bar{t}$ pair is produced almost at rest at the $t \bar{t}$ threshold, the dependence of $E_{b}$ on the top mass is maximized in this region. The $E_{b}$ distribution for $\sqrt{s}=370 \mathrm{GeV}$ and several values of $m_{t}$ is shown in Fig. 6.3b. For $m_{t}$ approaching the threshold value of $\sqrt{s} / 2$, the $E_{b}$ distribution becomes very narrow. The half-maximum width $\sigma_{b}$ therefore shows a strong dependence on the top mass. The best polynomial fit to express $\sigma_{b}$ in terms of $m_{t}$ for $\sqrt{s}=370 \mathrm{GeV}$ is found to be:

$$
\sigma_{b}=-0.081 m_{t}^{2}+26.137 m_{t}-2048.968 \mathrm{GeV}, \epsilon=0.393 \mathrm{GeV} .
$$

For a top quark mass in the range $171 \mathrm{GeV} \lesssim m_{t} \lesssim 179 \mathrm{GeV}$, the induced uncertainty on $m_{t}$ is $\Delta m_{t} \approx 0.35-0.65 \Delta \sigma_{b}$, where $\Delta \sigma_{b}$ is the uncertainty on the half-maximum width. $E_{b}$ thus may be an interesting observable to reconstruct the top mass at energies slightly above the $t \bar{t}$ threshold. It is probably less useful at higher energies. 
Top Quark Physics

\subsection{Anomalous couplings}

At present, the couplings of the top quark to gluons and the electroweak gauge bosons are largely untested. A linear collider provides an ideal tool to probe the couplings of the top quark to the electroweak gauge bosons. It is important to note that the neutral electroweak couplings are accessible only at lepton colliders, because top quarks at hadron colliders are pair-produced via gluon exchange. Since the charged electroweak current is involved in the top decay, $t \bar{t}$ production in $e^{+} e^{-}$collisions is sensitive to both the neutral and charged gauge boson couplings of the top quark. Because the top quark width, $\Gamma_{t}$, is much larger than $\Lambda_{\mathrm{QCD}}$, the decay process is not influenced by fragmentation effects and decay products will provide useful information.

The most general $(\gamma, Z) t \bar{t}$ couplings can be written as $[26,27]$

$$
\Gamma_{t \bar{t} \gamma, Z}^{\mu}=i e\left\{\gamma^{\mu}\left[F_{1 V}^{\gamma, Z}+F_{1 A}^{\gamma, Z} \gamma^{5}\right]+\frac{\left(p_{t}-p_{\bar{t}}\right)^{\mu}}{2 m_{t}}\left[F_{2 V}^{\gamma, Z}+F_{2 A}^{\gamma, Z} \gamma^{5}\right]\right\}
$$

where the only form factors different from zero in the SM are

$$
F_{1 V}^{\gamma}=\frac{2}{3}, F_{1 V}^{Z}=\frac{1}{4 \sin \theta_{W} \cos \theta_{W}}\left(1-\frac{8}{3} \sin ^{2} \theta_{W}\right), F_{1 A}^{Z}=-\frac{1}{4 \sin \theta_{W} \cos \theta_{W}} .
$$

$\left(e / m_{t}\right) \cdot F_{2 A}^{\gamma}$ is the CP-violating electric dipole moment (EDM) form factor of the top quark and $\left(e / m_{t}\right) \cdot F_{2 A}^{Z}$ is the weak electric dipole moment (WDM). $\left(e / m_{t}\right) \cdot F_{2 V}^{\gamma, Z}$ are the electric and weak magnetic dipole moments (MDM).

In the SM, the EDM and WDM terms violate $\mathrm{CP}$ and receive contributions only at the three-loop level and beyond. The CP-conserving form factors are zero at tree level but receive non-zero $\mathcal{O}\left(\alpha_{s}\right)$ QCD corrections.

The most general $W t b$ couplings can be parametrized in the form [27]

$$
\Gamma_{t b W}^{\mu}=-\frac{g}{\sqrt{2}} V_{t b}\left\{\gamma^{\mu}\left[f_{1}^{L} P_{L}+f_{1}^{R} P_{R}\right]-\frac{i \sigma^{\mu \nu}}{M_{W}}\left(p_{t}-p_{b}\right)_{\nu}\left[f_{2}^{L} P_{L}+f_{2}^{R} P_{R}\right]\right\}
$$

where $P_{R, L}=\left(1 \pm \gamma_{5}\right) / 2$. In the limit $m_{b} \rightarrow 0, f_{1}^{R}$ and $f_{2}^{L}$ vanish. In the SM, at tree level, $f_{1}^{L}=1$, and all other form factors are zero. Similarly, the $W \bar{t} \bar{b}$ vertex function can be parametrized in terms of form factors $\bar{f}_{1,2}^{L, R}$. If CP is conserved, $\bar{f}_{1,2}^{L, R}=f_{1,2}^{L, R}$.

In Table 6.1, we present the $1 \sigma$ sensitivity limits for the real parts of the $(\gamma, Z) t \bar{t}$ form factors obtained from a recent analysis of the process $e^{+} e^{-} \rightarrow t \bar{t} \rightarrow \ell^{ \pm}+$jets at $\sqrt{s}=500 \mathrm{GeV}$. Only one coupling at a time is varied. Top quarks are selected and reconstructed, and $b$ quarks are tagged using the LCD fast simulation package for the L detector configuration. The combined efficiency is $20 \%$, and the purity after selection is $88 \%$. To extract limits on $F_{1 V}^{\gamma, Z}$ and $F_{1 A}^{\gamma, Z}$, the angular distribution of the reconstructed top quark is used. $F_{1 V}^{\gamma, Z}$ and $F_{2 V}^{\gamma, Z}$ are derived from the left-right 


\begin{tabular}{|c|c|c|c|c|}
\hline \hline Coupling & LO SM Value & $\mathcal{P}\left(e^{-}\right)$ & $\int \mathcal{L} d t\left(\mathrm{fb}^{-1}\right)$ & $1 \sigma$ sensitivity \\
\hline$F_{1 A}^{\gamma}$ & 0 & \pm 0.8 & 100 & 0.011 \\
$F_{1 A}^{Z}$ & -0.6 & -0.8 & 100 & 0.013 \\
$F_{1 V}^{\gamma}$ & $2 / 3$ & \pm 0.8 & 200 & 0.047 \\
$F_{1 V}^{Z}$ & 0.2 & \pm 0.8 & 200 & 0.012 \\
$F_{2 A}^{\gamma}$ & 0 & +0.8 & 100 & 0.014 \\
$F_{2 A}^{Z}$ & 0 & +0.8 & 100 & 0.052 \\
$F_{2 V}^{\gamma}$ & 0 & \pm 0.8 & 200 & 0.038 \\
$F_{2 V}^{Z}$ & 0 & \pm 0.8 & 200 & 0.009 \\
\hline \hline
\end{tabular}

Table 6.1: The $1 \sigma$ statistical uncertainties for the real parts of the $(\gamma, Z) t \bar{t}$ form factors obtained from an analysis of the process $e^{+} e^{-} \rightarrow t \bar{t} \rightarrow \ell^{ \pm}+$jets for $\sqrt{s}=500 \mathrm{GeV}$. Only one coupling at a time is varied.

polarization asymmetry, and $F_{2 A}^{\gamma, Z}$ from the angular distribution of the reconstructed top quark and the decay angles of the $t$ and $\bar{t}$.

The limits shown in Table 6.1 could be strengthened if positron beam polarization becomes available, mostly from the increased $t \bar{t}$ cross section. If $\mathcal{P}\left(e^{+}\right)=0.5$, the $t \bar{t}$ cross section is about a factor 1.45 larger than that obtained with $\mathcal{P}\left(e^{+}\right)=0$. This improves the bounds by up to $25 \%$. Increasing the CM energy to $\sqrt{s}=800 \mathrm{GeV}$ improves the limits by a factor 1.3-1.5 [28].

The decay form factor $f_{2}^{R}$, corresponding to a $(V+A)$ top decay, can be measured with a precision of about 0.01 for $\sqrt{s}=500 \mathrm{GeV}$ and $\int \mathcal{L} d t=500 \mathrm{fb}^{-1}$ if electron and positron beam polarization are available [27]. This quantity can also be measured at the LHC, though the expected limit is a factor three to eight weaker than the limit we project for a linear collider [29].

Many models predict anomalous top quark couplings. In technicolor models and other models with a strongly-coupled Higgs sector, the CP-conserving couplings may be induced at the 5-10\% level [30-32]. In supersymmetric and multi-Higgs models, the CP-violating couplings $F_{2 V, A}^{\gamma, Z}$ may be induced at the one-loop level, with predictions in the range $F_{2 V, A}^{\gamma, Z}=\mathcal{O}\left(10^{-3}-10^{-2}\right)[17]$. A measurement of the $(\gamma, Z) t \bar{t}$ couplings at a linear collider will thus be sensitive to interesting sources of non-SM physics.

\subsection{QCD and electroweak radiative corrections}

For $\sqrt{s}=500 \mathrm{GeV}$ and an integrated luminosity of $500 \mathrm{fb}^{-1}$, the statistical error of the $e^{+} e^{-} \rightarrow t \bar{t} \rightarrow \ell \nu j j \bar{b} b$ cross section is well below $1 \%$. In order to match this experimental accuracy with robust theoretical predictions, precision calculations beyond tree level are required. Such theoretical accuracy is needed both when top itself is the subject of study and when top is a background to other physics of interest. 
Top Quark Physics

QCD corrections can have important effects in top events. Jets from radiated gluons can be indistinguishable from quark jets, complicating identification of top quark events from the reconstruction of the top decay products. In addition, real emission may occur either in the top production or decay processes, so that radiated gluons may or may not themselves be products of the decay. Subsequent mass measurements can be degraded, not only from misidentification of jets but also from subtle effects such as jet broadening when gluons are emitted near other partons. Virtual corrections must also be included to predict correct overall rates.

Most calculations of QCD corrections in $e^{+} e^{-} \rightarrow t \bar{t}$ to date have been performed for on-shell top quarks. In this approximation, corrections to the production and decay processes can be computed separately. A calculation of the QCD corrections to the production process $e^{+} e^{-} \rightarrow t \bar{t}$, which includes real gluon emission from the $t$ and $\bar{t}$ and virtual gluon exchange between the $t$ and $\bar{t}$ has been presented in [33]. A discussion of the QCD corrections to the decay $t \rightarrow W b$ can be found in [34]; QCD corrections are found to reduce the tree-level width of $1.55 \mathrm{GeV}$ to $\Gamma_{t}^{\mathcal{O}\left(\alpha_{s}\right)}=1.42 \mathrm{GeV}$ after all the known QCD and EW corrections are taken into account.

Because of the large width of the top quark and the fact that it does not hadronize before decaying [35], it is necessary to compute corrections to the entire production and decay process, including off-shell effects. In the soft gluon approximation, real gluon corrections for the process $e^{+} e^{-} \rightarrow t \bar{t} \rightarrow W W b b$ with the top allowed to be offshell were calculated in [36]. Interference effects of gluons radiated in the production and decay stages were found to be sensitive to the top width $\Gamma_{t}$, with the effects being largest for gluon energies comparable to $\Gamma_{t}$. Similarly, real gluon radiation in top production and decay is sensitive to top width effects [37].

Since the process observed experimentally is

$$
e^{+} e^{-} \rightarrow b W^{+} \bar{b} W^{-},
$$

it is desirable to take into account all Feynman diagrams that contribute to (6.11). This has not been done yet. At next-to-leading order, it is sufficient to take into account only the QCD corrections to the diagrams containing an intermediate top and antitop quark, as has been done in the computations discussed here. This approach uses the double pole approximation (DPA), in which only the double resonant terms (due to top and antitop propagators) are kept. Work done in this area follows closely the treatment of the $W$ pair production process at LEP II [38].

Radiative corrections to $e^{+} e^{-} \rightarrow t \bar{t} \rightarrow b W^{+} \bar{b} W^{-}$are usually split into two classes: corrections to particular subprocesses (production and decay), also called factorizable corrections, and corrections involving interference between these subprocesses (nonfactorizable corrections). In most approaches, the factorizable corrections are computed using the on-shell approximation for the top quarks; either using the on-shell phase space, or making an on-shell projection from the exact phase space $[39,40]$. In the latter the on-shell projection restricts the effect of the off-shell particles to the 


\begin{tabular}{|c|c|c|c|}
\hline \hline $2 E_{\text {beam }}$ & $360 \mathrm{GeV}$ & $500 \mathrm{GeV}$ & $1000 \mathrm{GeV}$ \\
\hline$\sigma_{0}$ & $0.386 \mathrm{pb}$ & $0.565 \mathrm{pb}$ & $0.172 \mathrm{pb}$ \\
$\sigma_{1}^{\text {on-shell }}$ & $0.737 \mathrm{pb}$ & $0.666 \mathrm{pb}$ & $0.186 \mathrm{pb}$ \\
$\sigma_{1}^{D P A}$ & $0.644 \mathrm{pb}$ & $0.652 \mathrm{pb}$ & $0.191 \mathrm{pb}$ \\
\hline \hline
\end{tabular}

Table 6.2: Cross sections (tree level, on-shell NLO and DPA NLO) for top production and decay at a linear collider [41]; results do not include ISR, beamstrahlung or beam energy spread.

interference terms. These interference terms are computed in DPA, for virtual as well as for real gluons. As a consequence, interference terms do not contribute to the total cross section.

In [41], a different approach is used. Instead of starting with the on-shell computation and adding the nonfactorizable corrections, the starting point is the exact amplitudes for the off-shell process from which terms that are not doubly resonant are dropped. Also, the real gluon contributions are treated exactly (as in [37]); as a consequence, the cancellation between virtual gluon and real gluon interference is no longer complete. Table 6.2 summarizes the total cross section results. The QCD corrections are found to increase the $t \bar{t}$ production cross section by up to a factor two near the threshold, and by about $11-13 \%$ in the continuum.

Electroweak $\mathcal{O}(\alpha)$ corrections for top processes at linear colliders have also been computed so far only to on-shell $t \bar{t}$ production and top decay. The electroweak $\mathcal{O}(\alpha)$ corrections can be naturally subdivided into two gauge-invariant subclasses, QED and weak corrections. The QED corrections depend on the cuts imposed on the photon phase space and thus on the experimental setup. As discussed in [42], initial-state $\mathcal{O}(\alpha)$ QED corrections can significantly reduce the cross section because of large logarithms of the form $\alpha / \pi \ln \left(s / m_{e}^{2}\right)$ with $s \gg m_{e}^{2}$. These terms arise when photons are radiated off in the direction of the incoming electrons. Thus, the inclusion of higher-order initial-state radiation (ISR) has to be considered. The leading-log initialstate QED corrections are universal and can be calculated using the so-called structure function approach [43].

The model-dependent contributions to corrections to top pair production are contained in the weak corrections. The numerical impact of the weak one-loop corrections is discussed in detail in [42]. Close to the $t \bar{t}$ threshold, the weak corrections to $\sigma_{t \bar{t}}$ are found to be quite sensitive to the Higgs boson mass. An updated analysis of the weak corrections to $\sigma_{t \bar{t}}$, using the current value of the top-quark mass, is presented in [44]. The weak corrections are found to reduce the Born cross section (expressed in terms of $G_{\mu}$ ) near threshold by about $7 \%$, which is mainly due to the box diagrams.

The complete electroweak $\mathcal{O}(\alpha)$ corrections to $\Gamma_{t}$ are calculated in [45]. When using $G_{\mu}$ and $M_{W}$ to parametrize the lowest-order top decay width, the electroweak 
Top Quark Physics

\begin{tabular}{ccccc}
\hline \hline Observable & Precision & $\int \mathcal{L} d t\left(\mathrm{fb}^{-1}\right)$ & $\sqrt{s}(\mathrm{GeV})$ & Comment \\
\hline$m_{t}$ & $<100 \mathrm{MeV}$ & 10 & 350 & theory dominated \\
$m_{t}$ & $200 \mathrm{MeV}$ & 50 & 500 & not fully explored \\
$\Gamma_{t}$ & $\mathcal{O}(30 \mathrm{MeV})$ & 100 & 350 & not fully explored \\
$g_{t t h}$ & $\mathcal{O}(10 \%)$ & 100 & 350 & need realistic study \\
$g_{t t h}$ & $21 \%$ & 1000 & 500 & stat. uncert. only \\
$g_{t t h}$ & $5.5 \%$ & 1000 & 800 & need improved bgd. estimate \\
$F_{i V, A}^{\gamma, Z}, f_{2}^{R}$ & $0.01-0.2$ & 500 & 500 & polarized beams essential \\
\hline \hline
\end{tabular}

Table 6.3: Summary of top quark-related measurements at a linear $e^{+} e^{-}$collider.

corrections amount to typically 1-2 \% with no significant dependence on $m_{h}$.

Ultimately it will be necessary to combine the QCD and electroweak corrections to top processes. This has been done for $e^{+} e^{-} \rightarrow t \bar{t}$ in [46], and work is in progress to combine both types of correction for the entire production and decay process [47].

\section{Conclusions}

Remarkable progress has been made in the last two years in our theoretical understanding of $t \bar{t}$ production in $e^{+} e^{-}$collisions at the threshold. Problems associated with defining the top quark mass in a way that removes QCD ambiguities have been solved. The remaining theoretical uncertainties are sufficiently small to allow a simultaneous measurement of $m_{t}$ (to $100 \mathrm{MeV}$ ), $\Gamma_{t}$ (to a few percent) and $g_{t t h}$. The top quark mass can also be measured with a precision of $200 \mathrm{MeV}$ or better at higher energies, using a variety of kinematic variables. Not all interesting variables have been fully explored yet. An ideal process to determine the top quark Yukawa coupling at energies above the $t \bar{t}$ threshold is $t \bar{t} h$ production in $e^{+} e^{-}$collisions. However, to fully exploit this process, energies significantly larger than $\sqrt{s}=500 \mathrm{GeV}$ are necessary. On the other hand, a center-of-mass energy of $500 \mathrm{GeV}$ is sufficient to measure the top quark couplings to the electroweak gauge bosons with a precision of $\mathcal{O}(1-10 \%)$. Polarized electron and positron beams are essential to disentangle the various couplings. We have summarized the estimated precision on the various quantities in Table 6.3. Finally, we have given a brief overview of the status of calculations of the QCD and electroweak corrections to $e^{+} e^{-} \rightarrow t \bar{t}$. The potential for precision studies of top quark physics at a linear collider requires a detailed understanding of these corrections. 


\section{References}

[1] A. H. Hoang et al., Eur. Phys. J. direct C3, 1 (2000).

[2] K. Fujii, T. Matsui and Y. Sumino, Phys. Rev. D50, 4341 (1994).

[3] S. Kuhlman et al., hep-ex/9605011; J. Bagger et al., hep-ex/0007022; E. Accomando et al., Phys. Rep. 299, 1 (1998).

[4] V. S. Fadin and O. Yakovlev, Sov. J. Nucl. Phys. 53, 688 (1991); Sov. J. Nucl. Phys. 53, 1053 (1991); M. J. Strassler and M. E. Peskin, Phys. Rev. D43, 1500 (1991).

[5] F. Abe et al. [CDF Collaboration], Phys. Rev. Lett. 74, 2626 (1995) [hepex/9503002].

[6] S. Abachi et al. [DØ Collaboration], Phys. Rev. Lett. 74, 2632 (1995) [hepex/9503003].

[7] A. Hoang and T. Teubner, Phys. Rev. D60, 114027 (1999).

[8] O. Yakovlev and S. Groote, Phys. Rev. D 63, 074012 (2001) [hep-ph/0008156].

[9] I. I. Bigi et al., Phys. Rev. D56, 4017 (1997).

[10] M. Beneke, Phys. Lett. B434, 115 (1998).

[11] A. H. Hoang et al., Phys. Rev. D59, 114014 (1999); N. Uraltsev, in "Varenna 1997, Heavy flavor physics", hep-ph/9804275.

[12] A. Hoang et al., Phys. Rev. Lett. 86, 1951 (2001).

[13] H. Murayama and Y. Sumino, Phys. Rev. D47, 82 (1993).

[14] A. Hoang, A. Manohar, I. Stewart, and T. Teubner, in preparation.

[15] M. Martinez in Physics and Experiments with Future Linear $e^{+} e^{-}$Colliders, eds. E. Fernandez and A. Pacheco (1999) Barcelona and references therein.

[16] M. E. Peskin and J. D. Wells, hep-ph/0101342.

[17] M. Jeżabek, T. Nagano, and Y. Sumino, Phys. Rev. D62, 014034 (2000) and references therein.

[18] A. Juste and G. Merino, hep-ph/9910301.

[19] H. Baer, S. Dawson and L. Reina, Phys. Rev. D61, 013002 (2000).

[20] S. Moretti, hep-ph/9911501.

[21] J. Antõs and G.P. Yeh, FERMILAB-Conf-99/260.

[22] G. Corcella, E. K. Irish and M. H. Seymour, hep-ph/0012319.

[23] G. Corcella and M. H. Seymour, Phys. Lett. B442, 417 (1998).

[24] G. Corcella et al., JHEP 01, 010 (2000).

[25] G. Corcella, M. L. Mangano and M. H. Seymour, JHEP 07, 004 (2000).

[26] W. Hollik et al., Nucl. Phys. B551, 3 (1999).

[27] B. Grzadkowski and Z. Hioki, Nucl. Phys. B585, 3 (2000). 
[28] W. Bernreuther, talk given at the ECFA/DESY Linear Collider Workshop, Oxford, UK, March 1999.

[29] M. Beneke et al., hep-ph/0003033, Proceedings of the Workshop on Standard Model Physics (and more) at the LHC, CERN 2000-004, p. 419.

[30] R. S. Chivukula, S. B. Selipsky and E. H. Simmons, Phys. Rev. Lett. 69, 575 (1992), hep-ph/9204214; R. S. Chivukula, E. H. Simmons and J. Terning, Phys. Lett. B331, 383 (1994), hep-ph/9404209.

[31] K. Hagiwara and N. Kitazawa, Phys. Rev. D52, 5374 (1995), hep-ph/9504332.

[32] U. Mahanta, Phys. Rev. D55, 5848 (1997), hep-ph/9611289; Phys. Rev. D56, 402 (1997).

[33] J. Jersak, E. Laerman, and P. Zerwas, Phys. Rev. D25, 1218 (1982); Yu. L. Dokshitzer, V. A. Khoze, and W. J. Stirling, Nucl. Phys. B428, 3 (1994); A. Brandenburg, Eur. Phys. J. C11, 127 (1999).

[34] M. Jeżabek and J. H. Kühn, Nucl. Phys. B314, 1 (1989); A. Czarnecki, Phys. Lett. B252, 467 (1990); C. S. Li et al., Phys. Rev. D43, 3759 (1991).

[35] Y. L. Dokshitzer et al., Phys. Lett. B181, 157 (1986); L. H. Orr and J. L. Rosner, Phys. Lett. B246, 221 (1990), B248, 474(E) (1990).

[36] G. Jikia, Phys. Lett. B257, 196 (1991); V. A. Khoze et al., Nucl. Phys. B378, 413 (1992); Y. L. Dokshitzer et al., Nucl. Phys. B403, 65 (1993).

[37] C. Macesanu and L. H. Orr, hep-ph/0012177.

[38] A. Denner et al., Nucl. Phys. B587, 67 (2000).

[39] K. Melnikov and O. Yakovlev, Nucl. Phys. B 471, 90 (1996) [hep-ph/9501358].

[40] W. Beenakker et al., Phys. Lett. B454, 129 (1999).

[41] C. Macesanu, in Physics and Experiments with Future Linear $e^{+} e^{-}$Colliders (LCWS 2000), ed. A. Para. (AIP Conference Proceedings, 2001).

[42] W. Beenakker, S. C. van der Marck and W. Hollik, Nucl. Phys. B365, 24 (1991).

[43] W. Beenakker et al., hep-ph/9602351.

[44] W. Hollik and C. Schappacher, Nucl. Phys. B545, 98 (1999).

[45] A. Denner and T. Sack, Nucl. Phys. B358, 46 (1991).

[46] J. Kühn, T. Hahn and R. Harlander, hep-ph/9912262.

[47] C. Macesanu, L.H. Orr, and D. Wackeroth, in progress. 


\section{Chapter 7 QCD and Two-Photon Physics}

\section{Introduction}

A relatively clean environment and well-understood initial-state parton content render $e^{+} e^{-}$colliding beam experiments ideal for both the qualitative confirmation and quantitative testing of Quantum Chromodynamics (QCD). Through the years, a number of seminal discoveries and measurements performed at $e^{+} e^{-}$colliding beam facilities have served to establish the $\mathrm{SU}(3)$ color gauge theory QCD as the accepted dynamical model of the strong nuclear interaction. Highlights unique to the $e^{+} e^{-}$ QCD program include the discovery of the gluon at PETRA in 1979, the confirmation of the SU(3) gauge structure of quark-gluon and gluon-gluon vertices at LEP in the early 1990s, and the precise measurement of the strong coupling constant $\alpha_{s}$ from hadronic observables and from the $Z$ and $\tau$ decay widths.

The study of QCD, and the dynamics of the strong force in general, is expected to provide a significant contribution to the physics program at a high-energy $e^{+} e^{-}$ colliding beam facility. The highlights of this program include

- the precise determination of the strong coupling constant $\alpha_{s}$;

- the search for anomalous strong couplings of the top quark;

- the study of photon structure; and

- the study of strong-interaction dynamics at high $\sqrt{s}$ and fixed $t$.

Together, these measurements probe some of the most important topics in the study of strong force dynamics, in ways that are often superior to measurements at hadron colliders.

\section{QCD from annihilation processes}

\subsection{The precise determination of $\alpha_{s}$}

As the single free parameter of the $\mathrm{SU}(3)$ gauge theory of the strong interaction, the strong coupling constant $\alpha_{s}$ should be measured to the highest available precision. Renormalization group extrapolations of the $\mathrm{U}(1), \mathrm{SU}(2)$ and $\mathrm{SU}(3)$ coupling strengths constrain physics scenarios at the GUT scale. The current constraints are limited by the few-percent relative precision [1] of the value of $\alpha_{s}\left(m_{Z}^{2}\right)$. The value of $\alpha_{s}$ should also be determined with comparable accuracy over as large a range of scales as possible in order to measure the renormalization-group running of $\alpha_{s}$ and to reveal potential anomalous running in the strength of the strong interaction. In this article, as a matter of convention, measurements of $\alpha_{s}$ performed at other scales 
will be evolved to the scale $Q^{2}=M_{Z}^{2}$ according to Standard Model renormalization group equations and quoted in terms of their implied value of $\alpha_{s}\left(m_{Z}^{2}\right)$.

\subsubsection{Event observables in $e^{+} e^{-}$annihilation}

The determination of $\alpha_{s}\left(m_{Z}^{2}\right)$ from the process $e^{+} e^{-} \rightarrow Z / \gamma \rightarrow q \bar{q}(g)$, using 'shape' observables that are sensitive to the underlying parton content, has been pursued for two decades and is generally well understood [2]. In this method one usually forms a differential distribution, makes corrections for detector and hadronization effects, and fits a perturbative QCD prediction to the data, allowing $\alpha_{s}\left(m_{Z}^{2}\right)$ to vary. Examples of such observables are thrust, jet masses and jet rates.

The latest generation of such $\alpha_{s}\left(m_{Z}^{2}\right)$ measurements, from SLC and LEP, has shown that statistical errors below the $1 \%$ level can be obtained with samples of a few tens of thousands of hadronic events. With the current linear collider design luminosity of $2.2 \times 10^{34} \mathrm{~cm}^{-2} \mathrm{~s}^{-1}$, at $\sqrt{s}=500 \mathrm{GeV}$, hundreds of thousands of $e^{+} e^{-} \rightarrow q \bar{q}$ events would be produced each year, and a statistical error on $\alpha_{s}\left(m_{Z}^{2}\right)$ below $0.5 \%$ would be achieved.

At energies far above the $Z$ pole, the electron-positron collision cross section is dominated by $t$-channel processes such as $Z Z$ and $W^{+} W^{-}$production. In addition, because of the substantial mass of the $t$ quark, the inclusive characteristics of $e^{+} e^{-} \rightarrow t \bar{t}$ events tend to mimic those of lighter quark events with hard gluon radiation. A prescription for the elimination of these backgrounds was developed for the 1996 Snowmass workshop [3,4]. This prescription makes use of electron beam polarization and precise tracking to reduce the effects of these backgrounds on the measured three-jet rate to less than $5 \%$, with the corresponding systematic uncertainty on the extraction of $\alpha_{s}\left(m_{Z}^{2}\right)$ expected to be substantially less than $1 \%$. The sizable initial-state and beamstrahlung radiation associated with linear collider energies will act to smear the CM energy of the $e^{+} e^{-}$annihilation process, as well as to boost the particle flow into the forward regions of the detector. A PYTHIA study [5], including the full effects of ISR, has shown that these considerations can be accurately taken into account in the measurement of $\alpha_{s}\left(m_{Z}^{2}\right)$.

Hadronization effects, which lead to corrections of order $10 \%$ at the $Z^{0}$ pole, are expected to fall at least as fast as $1 / \sqrt{s}$, leading to corrections of order $1 \%$ at $\sqrt{s} \geq$ $500 \mathrm{GeV}$ [6]. The corresponding systematic error on the extraction of $\alpha_{s}\left(m_{Z}^{2}\right)$ is thus expected to be substantially below $1 \%$. Detector systematics, due primarily to limited acceptance and resolution smearing, and which are observable-dependent, are found to contribute at the level of $\delta \alpha_{s}\left(m_{Z}^{2}\right)= \pm 1-4 \%$ at LEP-II [7]. The greater hermeticity and $\cos \theta$ coverage anticipated for linear collider detectors are again expected to reduce this substantially.

Currently, perturbative calculations of event shapes are complete only up to $O\left(\alpha_{s}^{2}\right)$, although resummed calculations are available for some observables [8]. One must 
QCD and Two-Photon Physics

therefore estimate the possible bias inherent in measuring $\alpha_{s}\left(m_{Z}^{2}\right)$ using the truncated QCD series. Though not universally accepted, it is customary to estimate this from the dependence of the fitted $\alpha_{s}\left(m_{Z}^{2}\right)$ value on the QCD renormalization scale, yielding a large and dominant uncertainty of about $\Delta \alpha_{s}\left(m_{Z}^{2}\right) \simeq \pm 6 \%$ [2]. Therefore, although a $\pm 1 \%$-level $\alpha_{s}\left(m_{Z}^{2}\right)$ measurement is possible experimentally, it will not be realized until $O\left(\alpha_{s}^{3}\right)$ contributions are completed. There is a reasonable expectation that this will be achieved within the next three years $[9,10]$.

\subsubsection{The $t \bar{t}(g)$ system}

The dependence of the $e^{+} e^{-} \rightarrow t \bar{t}$ cross section on $m_{t}$ and $\alpha_{s}\left(m_{Z}^{2}\right)$ is presented in Chapter 6, Section 2. As discussed there, next-to-next-to-leading-order calculations of the $t \bar{t}$ cross section in the resonance region show convergence to the few-percent level for an appropriate definition of $m_{t}$, if logarithms of the top quark velocity are resummed. This is good news for the extraction of $m_{t}$; however, we will probably not obtain a competitive value of $\alpha_{s}\left(m_{Z}^{2}\right)$ from this system.

\subsubsection{A high-luminosity run at the $Z^{0}$ resonance}

A sample of $10^{9} Z^{0}$ decays offers two additional options for the determination of $\alpha_{s}\left(m_{Z}^{2}\right)$ via measurements of the inclusive ratios $\Gamma_{Z}^{\text {had }} / \Gamma_{Z}^{\text {lept }}$ and $\Gamma_{\tau}^{\text {had }} / \Gamma_{\tau}^{\text {lept }}$. In both cases, $\alpha_{s}$ enters in through the QCD radiative correction; thus, both observables require a very large event sample for a precise measurement. For example, the current LEP data sample of $16 \mathrm{M} Z^{0}$ decays yields an error of $\pm 2.5 \%$ on $\alpha_{s}\left(m_{Z}^{2}\right)$ from $\Gamma_{Z}^{\text {had }} / \Gamma_{Z}^{\text {lept }}$, with an experimental systematic of $\pm 1 \%$. With a Giga-Z sample, the statistical error would be pushed to below $\Delta \alpha_{s}\left(m_{Z}^{2}\right)=0.4 \%$. Even with no improvement in experimental systematics, this would be a precise and reliable measurement. In the case of $\Gamma_{\tau}^{\text {had }} / \Gamma_{\tau}^{\text {lept }}$ the experimental precision from LEP and CLEO is already at the $1 \%$ level on $\alpha_{s}\left(m_{Z}^{2}\right)$. However, there has been considerable debate about the size of the theoretical uncertainties, with estimates as large as $5 \%$ [11]. If this situation is clarified, and the theoretical uncertainty is small, $\Gamma_{\tau}^{\text {had }} / \Gamma_{\tau}^{\text {lept }}$ may offer a further 1\%-level $\alpha_{s}\left(m_{Z}^{2}\right)$ measurement.

\section{$2.2 \quad Q^{2}$ evolution of $\alpha_{s}$}

In the preceding sections we discussed the expected precision on the measurement of the benchmark parameter $\alpha_{s}\left(m_{Z}^{2}\right)$. Translation of the measurements of $\alpha_{s}\left(Q^{2}\right)$ $\left(Q^{2} \neq M_{Z}^{2}\right)$ to $\alpha_{s}\left(m_{Z}^{2}\right)$ requires the assumption that the 'running' of the coupling is determined by the QCD $\beta$ function. However, since the logarithmic decrease of $\alpha_{s}$ with $Q^{2}$ is a telling prediction of QCD, reflecting the underlying non-Abelian dynamics, it is essential to test this $Q^{2}$ dependence explicitly. In particular, such a 
test would be sensitive to new colored degrees of freedom with mass below the limit for pair production at the highest explored scale. For this measurement of the $Q^{2}$ dependence of $\alpha_{s}$, rather than its overall magnitude, many common systematic effects would be expected to cancel. Hence it would be desirable to measure $\alpha_{s}$ in the same detector, with the same technique, and by applying the same treatment to the data at a series of different $Q^{2}$ scales, so as to maximize the lever-arm for constraining the running.

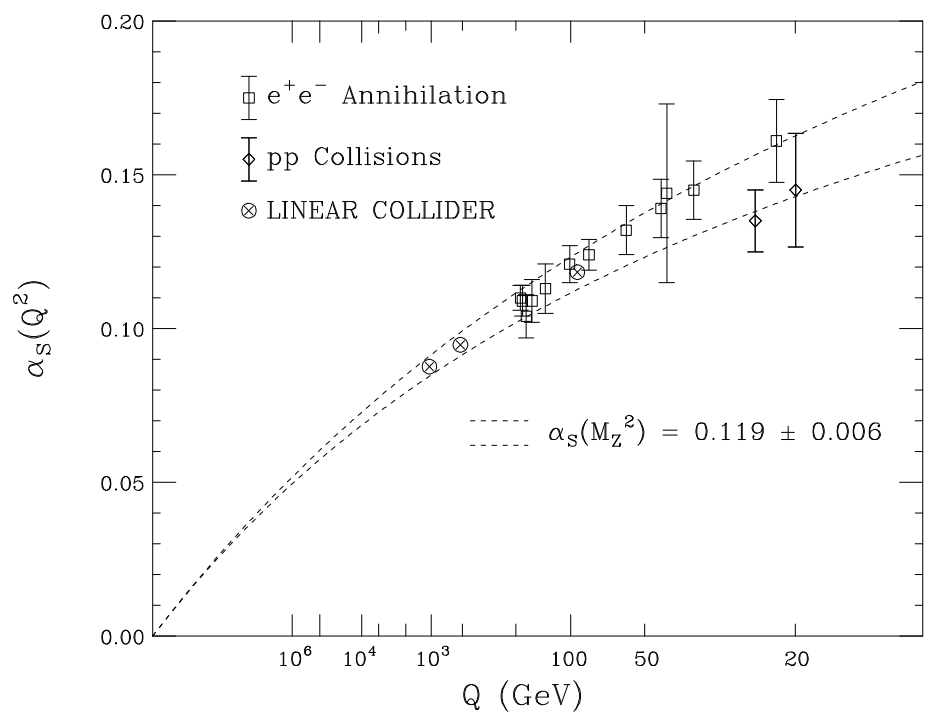

Figure 7.1: Linear collider measurements of $\alpha_{s}\left(m_{Z}^{2}\right)$, in comparison to existing measurements from $e^{+} e^{-}$and $p \bar{p}$ collisions, as a function of interaction scale.

Proposed linear collider measurements of $\alpha_{s}\left(Q^{2}\right)$ at $\sqrt{s}=91,500$ and $1000 \mathrm{GeV}$ are shown in Fig. 7.1, together with existing measurements which span the range $20 \leq \sqrt{s} \leq 200 \mathrm{GeV}$. The linear collider point at $\sqrt{s}=91 \mathrm{GeV}$ can be obtained either from jet rates or from the $\Gamma_{Z}^{\text {had }} / \Gamma_{Z}^{\text {lept }}$ technique, while those at 500 and 1000 $\mathrm{GeV}$ are based on jet rates. A theoretical uncertainty of $\pm 1 \%$ is assumed for all LC points.

The linear collider data would add significantly to the lever-arm in $Q^{2}$, and would allow a substantially improved extrapolation to the GUT scale. Consider, for example, making a simultaneous fit for $\alpha_{s}\left(m_{Z}^{2}\right)$ and for $\beta_{0}$, the leading term in the expansion of the QCD $\beta$-function which establishes the rate at which the strong coupling constant runs. (This term is expected to be about 0.61 in the SM.) The linear collider data alone would give a precision on these quantities of \pm 0.0018 and \pm 0.034 , respectively. Including accurate measurements at low $Q^{2}$ (particularly from $e$ and $\mu$ deep inelastic scattering), the existing constraints are \pm 0.0030 and \pm 0.042 , respectively. Combining existing data with that available from the LC would yield constraints of \pm 0.0009 and 
\pm 0.016 , providing a substantial improvement on the measurement of the running of $\alpha_{s}\left(m_{Z}^{2}\right)$, as well as the extrapolation to the GUT scale (see Fig. 7.2). Note that, unlike the determination of $\beta_{0}$, the accuracy of the GUT-scale extrapolation is not dependent upon future running at the $Z^{0}$.

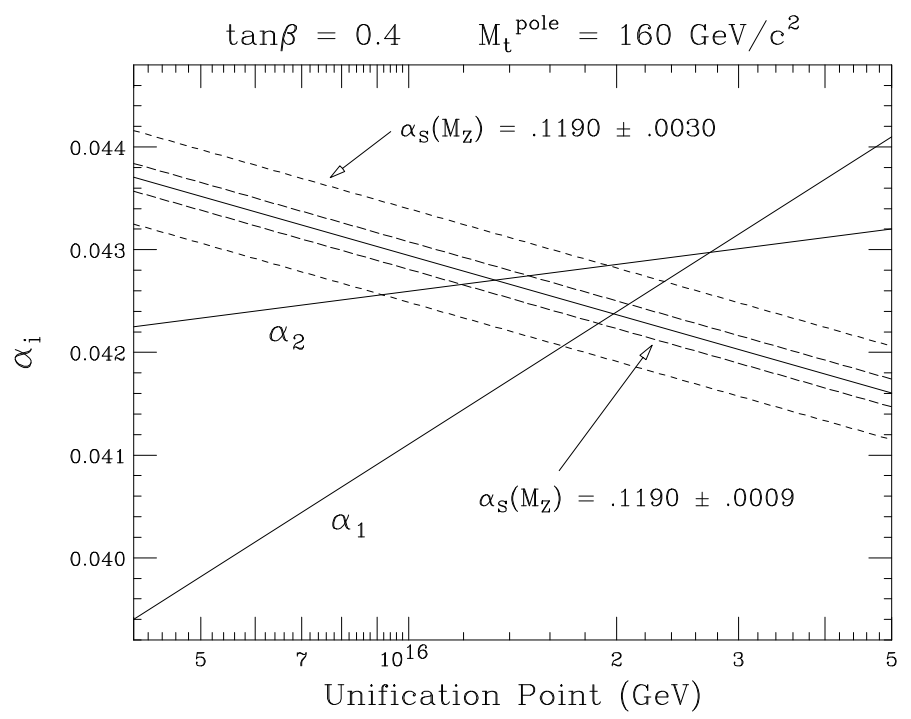

Figure 7.2: Improvement in the GUT scale constraint, assuming a $\pm 1 \%$ measurement of $\alpha_{s}\left(m_{Z}^{2}\right)$ at the linear collider. Renormalization group trajectories assume the MSSM with $\tan \beta=0.4$ and $m_{t}^{\text {pole }}=160 \mathrm{GeV}[12]$.

\subsection{Top quark strong moments}

The very large mass of the recently discovered top quark suggests the possibility that top plays a central role in physics beyond the Standard Model. If this is the case, it is likely that this new physics will manifest itself via anomalous top-quark moments, which represent the low-energy manifestation of effective higher-dimensional couplings. The measurement of the electroweak anomalous moments of the top quark is discussed in Chapter 6, Section 3.3.

In the case of the strong interactions of top, the lowest-dimensional gauge-invariant and CP-conserving extension to SM top quark couplings is the anomalous chromomagnetic moment, which we can parameterize via a dimensionless quantity $\kappa$. The corresponding chromoelectric moment, parameterized by $\tilde{\kappa}$, violates $\mathrm{CP}$ and arises from an operator of the same dimension. The resulting generalized three-point $t \bar{t} g$ vertex takes the form

$$
L=g_{s} \bar{t} T_{a}\left(\gamma_{\mu}+\frac{i}{2 m_{t}} \sigma_{\mu \nu}\left(\kappa-i \tilde{\kappa} \gamma_{5}\right) q^{\nu}\right) t G_{a}^{\mu},
$$


where $g_{s}$ is the $\mathrm{SU}(3)$ gauge coupling parameter, $m_{t}$ is the top quark mass, $T_{a}$ are the $\mathrm{SU}(3)$ color generators, $G_{a}^{\mu}$ are the vector gluon fields, and $q$ is the outgoing gluon four-momentum.

This interaction leads to a substantially different spectrum of gluon radiation for $e^{+} e^{-} \rightarrow t \bar{t}$ events above threshold than for the pure vector interaction case corresponding to $\kappa=\tilde{\kappa}=0$. Fits to this spectrum thus provide limits on the values of $\kappa$ and $\tilde{\kappa}$. Figure 7.3 , from Ref. [13], shows the limits in the $\kappa-\tilde{\kappa}$ plane that can be achieved with an integrated luminosity of 100 and $200 \mathrm{fb}^{-1}$ at $\sqrt{s}=1 \mathrm{TeV}$. Similar studies for the Tevatron and LHC [14] indicate that the corresponding sensitivities at hadron colliders will be substantially weaker, in particular for the case of $\kappa$, for which sensitivities of $|\kappa|<0.1$ will be difficult to achieve. In [15], the authors offer a technicolor model for which the unique capability of the LC to measure strong moments of top precisely would be a critical asset.

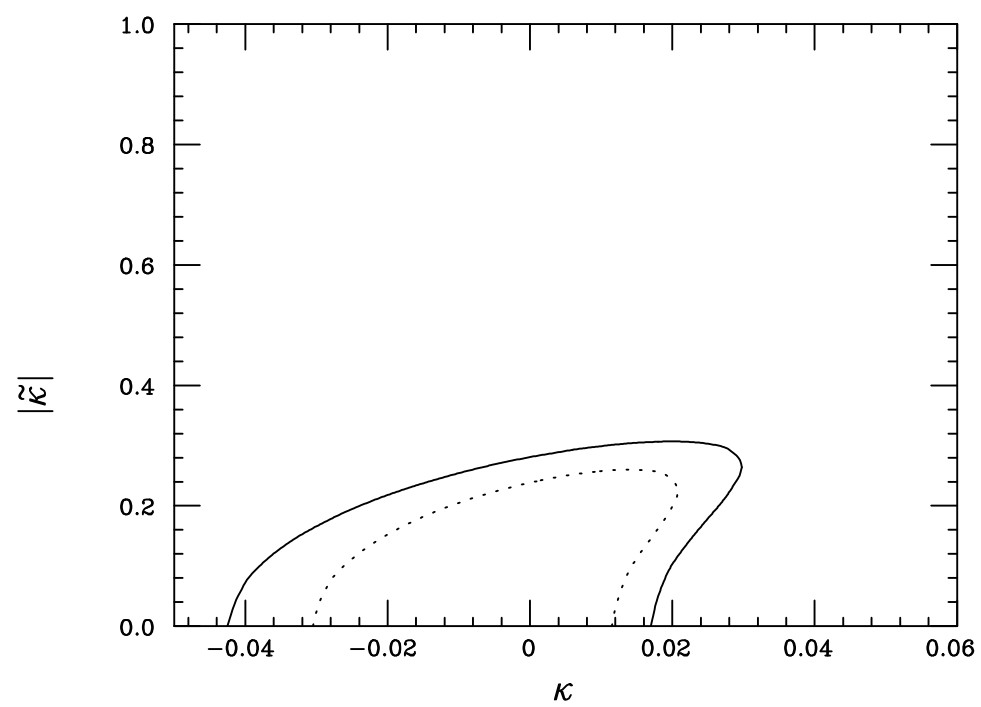

Figure 7.3: Constraints on anomalous strong moments of the top quark, derived from a LC sample of $100 \mathrm{fb}^{-1}$ (solid) and $200 \mathrm{fb}^{-1}$ (dotted) for $\sqrt{s}=1 \mathrm{TeV}$.

\section{Two-photon physics}

At a future $e^{+} e^{-}$linear collider, we will be able to study the two-photon processes $e^{+} e^{-} \rightarrow e^{+} e^{-}+\gamma^{(*)} \gamma^{(*)} \rightarrow e^{+} e^{-}+$hadrons for all combinations of real $(\gamma)$ and virtual $\left(\gamma^{*}\right)$ photons. Reactions of real photons can also be studied by using a dedicated backscattered-laser photon beam, as described in Chapter 13. These reactions test QCD in photon structure measurements and in the dynamics of parton distribution 
QCD and Two-Photon Physics

function evolution. Direct measurement of the photon structure function $F_{2}^{\gamma}\left(x, Q^{2}\right)$ in $\gamma \gamma^{*}$ collisions pushes into currently unattainable regimes of lower $x$ and higher $Q^{2}$, testing scaling behavior and $Q^{2}$ evolution. Extending the measurement of the total $\gamma \gamma$ cross section to higher $\sqrt{s}$ tests whether QCD-based models of parton emission describe photon interactions. By colliding two virtual photons, QCD dynamics can be studied in a relatively background-free environment. No other planned or anticipated future collider will be able to compete with an $e^{+} e^{-}$linear collider in these areas.

We now present a comprehensive plan for the study of photon structure through $e \gamma$ deep inelastic scattering (DIS) and $\gamma \gamma$ scattering, and through the study of QCD dynamics through $\gamma^{*} \gamma^{*}$ scattering. We discuss the relative merits of employing photons produced by bremsstrahlung and laser backscattering and the utility of having well-defined photon polarization.

\subsection{Experimental requirements}

Experimental issues related to two-photon physics are mainly concerned with instrumentation of the forward parts of the interaction region (IR), particularly inside the conical shielding masks. The cases in which the initial photons are produced by bremsstrahlung from $e^{+} e^{-}$and from laser backscattering have some differences, but also many similarities.

\subsection{Bremsstrahlung photon beam}

In an IR designed for $e^{+} e^{-}$collisions, the study of two-photon processes requires small-angle-tagging electromagnetic calorimeters in the forward regions. Some physics topics also require hadronic calorimetry from beampipe to beampipe.

Virtual photons are produced when, in the bremsstrahlung process, an $e^{+}$or $e^{-}$ transfers a significant amount of 4-momentum to the radiated photon. The virtuality,

$Q^{2}$, of the "tagged" photon is determined by measuring the energy and angle of the scattered lepton in an electromagnetic calorimeter via the relation

$$
Q^{2}=2 E_{e} E_{e}^{\prime}(1-\cos \theta),
$$

where $E_{e}$ is the incoming lepton beam energy, and $E_{e}^{\prime}$ and $\theta$ are the scattered lepton energy and angle, respectively. Since some physics analyses require that the measurement of $Q^{2}$ be as small as possible, the electromagnetic tagging calorimeters must be positioned as closely as possible to the outgoing beampipes on both sides of the interaction region and inside the shielding cone in order make the minimum measurable scattered lepton angle as small as possible, leading to the requirement of a compact design. Also, since $Q^{2} \simeq E_{e} E_{e}^{\prime} \theta^{2}$ at small angles, radial position resolution is an important consideration in $Q^{2}$ reconstruction, requiring fine-grained readout in the radial direction [16]. Fine-grained sampling calorimeters with these properties have been successfully used in photon-tagging experiments at LEP [17]. 
Almost-real photons $\left(Q^{2} \simeq 0\right)$ from the bremsstrahlung process are defined by anti-tags in the forward electromagnetic tagging calorimeters. For example, a single tag on one side of the IR, combined with an anti-tag on the other side with hadronic activity in the main detector, signals a $\gamma^{*} \gamma$ interaction ( $e \gamma$ DIS). Double anti-tags signal $\gamma \gamma$ interactions in which both interacting photons are almost real. It is important to note that the energy spectrum of bremsstrahlung-produced photons is dominated by low-energy photons. Furthermore, since the untagged photon energy is not known, it is important to have hadronic energy and angle measurement in the forward IR, to as small an angle as possible, in order to determine the kinematics of the interaction.

\subsubsection{Backscattered laser beam}

It would be desirable to create a beam of high-energy real photons by Compton backscattering of a high-power, high-repetition-rate laser from the electron beams. The technology for achieving this backscattered-laser photon beam is described in Chapter 13. To prepare the Compton-backscattered beam, $1 \mathrm{eV}$ laser photons backscatter from the incoming $250 \mathrm{GeV} e^{-}$beam, producing a beam of photons carrying about $75 \%$ of the electron beam energy with an energy spread of $5-10 \%$. Since the resulting photon beam energy spread is small, the kinematics of the high-energy photon interactions can be determined from the known photon energy. Also, since these are high-energy photons at nearly the incoming lepton beam energy, the mass of the twophoton system $W_{\gamma * \gamma}$ is much larger than that obtained from bremsstrahlung-produced photons, leading to the possibility of reaching very low $x$ in $e \gamma$ DIS.

In addition, the polarization state of the interacting photons and/or leptons can have a big effect on the physics impact of a measurement. For example, by combining the circular polarizations of the incoming leptons and the laser photons in an optimal way, the energy spread of the resulting backscattered photon beam can be reduced by almost a factor of 2 .

\subsection{Photon structure}

A real photon can interact both as a point-like particle, or as a collection of quarks and gluons, i.e., like a hadron. The structure of the photon is determined not by the traditional valence quark distributions as in a proton, but by fluctuations of the pointlike photon into a collection of partons. As such, the scaling behavior of the photon structure function, $d F_{2}^{\gamma} / d \ln Q^{2}$, is always positive. Single-tag and double-anti-tag events can be used to measure $F_{2}^{\gamma}$ directly and to constrain the relative quark/gluon fractions in the photon, testing predictions for this content and its behavior. 
QCD and Two-Photon Physics

\subsection{1 $\gamma^{*} \gamma$ scattering-e $\gamma$ DIS}

Direct measurement of the photon structure function $F_{2}^{\gamma}\left(x, Q^{2}\right)$ in $e \gamma$ DIS is accomplished by tagging a single virtual photon probe, anti-tagging an almost-real or real target photon, and requiring hadronic activity anywhere in the detector.

If the anti-tagged target photon is produced by bremsstrahlung from an incoming lepton, it has very small virtuality, $\left\langle Q^{2}\right\rangle \simeq 10^{-4} \mathrm{GeV}^{2}$, and low energy, neither of which is known. In order to determine the longitudinal momentum fraction, $x$, the mass $W_{\gamma^{*} \gamma}$ of the $\gamma^{*} \gamma$ system must be measured, which requires hadronic calorimetry to measure the energy and angle of all hadrons. The best measurements of $F_{2}^{\gamma}$ using bremsstrahlung photons as the target are done at relatively low $W_{\gamma^{*} \gamma}$ where it is well-measured away from the forward IR, which in kinematic space is at the high end of the $x, Q^{2}$ range. Physics topics that can best be addressed in this region are the scaling behavior of $F_{2}^{\gamma}$ as $x \rightarrow 1$ and its evolution with $Q^{2}$.

As $W_{\gamma^{*} \gamma}$ increases (towards low $x$ ), increasingly more of the hadronic mass escapes undetected in the beam direction and the mass of the observed hadrons, usually referred to as $W_{\text {vis }}$, begins to differ substantially from the true hadronic mass. Figure 7.4 illustrates this effect by comparing $W_{\text {vis }}$ with the true mass, $W_{\gamma^{*} \gamma}$.

Monte Carlo simulations of the fragmentation of the $\gamma^{*} \gamma$ system are used to correct $W_{\text {vis }}$ for this loss until the uncertainty in the correction begins to dominate the measurement. Eventually, this limits the low- $x$ range of the $F_{2}^{\gamma}$ measurement.

However, if the target photon is produced by laser backscattering, two advantages are realized: 1) the high $W_{\gamma^{*} \gamma}$ (low- $x$ ) region is enhanced since the real photon energy is high; and 2) the energy spread of the real photons is small enough that the error on $x$ caused by assuming a monochromatic photon does not dominate the systematics.

Figure 7.5 shows $F_{2}^{\gamma}$ versus $Q^{2}$ for various $x$ bins from possible measurements at a future $e^{+} e^{-}$linear collider [20]. The various points are differentiated according to the measurement method. The open squares represent the very low- $x$ region accessible only with photons produced by laser backscattering; open circles represent measurements with target photons from bremsstrahlung and with hadronic calorimetry built into a shielding mask down to $30 \mathrm{mrad}$; solid dots represent measurements with bremsstrahlung photons and with hadronic calorimetry only outside the mask. Note that there is enough overlap between the methods to provide cross-checks on the various measurements and experimental conditions.

With known polarization of both the target photon and the tagged virtual photon, polarized photon structure functions can be measured for the first time. The 'BFKL' terms involving $\ln (1 / x)$ in the unpolarized structure functions enter in polarized scattering as $\ln ^{2}(1 / x)$. These effects are then enhanced at low $x$ over the unpolarized case. Thus, in polarized $e \gamma$ DIS, forward particle and jet measurements, such as have been performed at HERA [21], can be done at a future $e^{+} e^{-}$linear collider with increased sensitivity to any BFKL effects. 


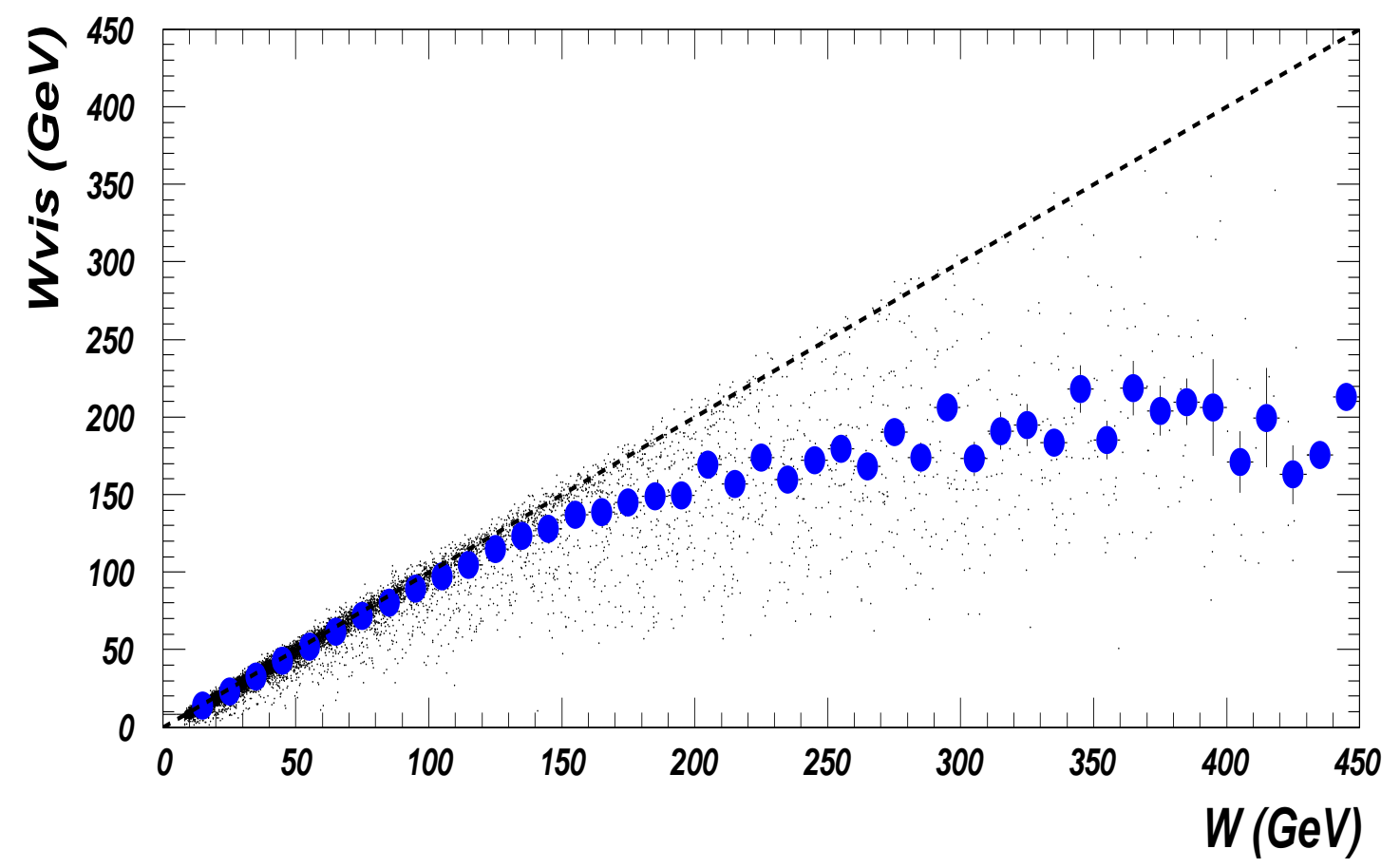

Figure 7.4: Comparison of $W_{\text {vis }}$ with $W_{\gamma^{*} \gamma}$ from PYTHIA [19] for a typical LC detector, including the average value (profile plot).

In addition to the $F_{2}^{\gamma}$ structure function, $e \gamma$ DIS can be used to test QCD in other ways. For example, dijet production in DIS can be used to extract the strong coupling parameter, $\alpha_{s}$, as is done at HERA [22]. At a future $e^{+} e^{-}$linear collider, $\alpha_{s}$ from $e^{+} e^{-}$event shapes and from dijets in DIS can be compared using the same detector.

\section{$3.4 \gamma \gamma$ scattering - total cross section}

Various models have been developed to describe the rise with energy of the total $\gamma \gamma$ cross section. These give either a fast rise driven by QCD effects such as minijets, or a slower rise based on reggeon exchange. To get to the highest $\sqrt{s}$ and $W_{\gamma \gamma}$, real photons from the laser backscattering process are required. Studies show that a precision of $\sim 20 \%$ on the total cross section will enable adequate discrimination of model types for energies up to $1 \mathrm{TeV}$ [23]. Figure 7.6 shows possible $\sigma_{\text {tot }}$ measurements at a $500 \mathrm{GeV}$ linear collider (large stars) compared to existing measurements at lower $\sqrt{s}$ and to various models. 
QCD and Two-Photon Physics

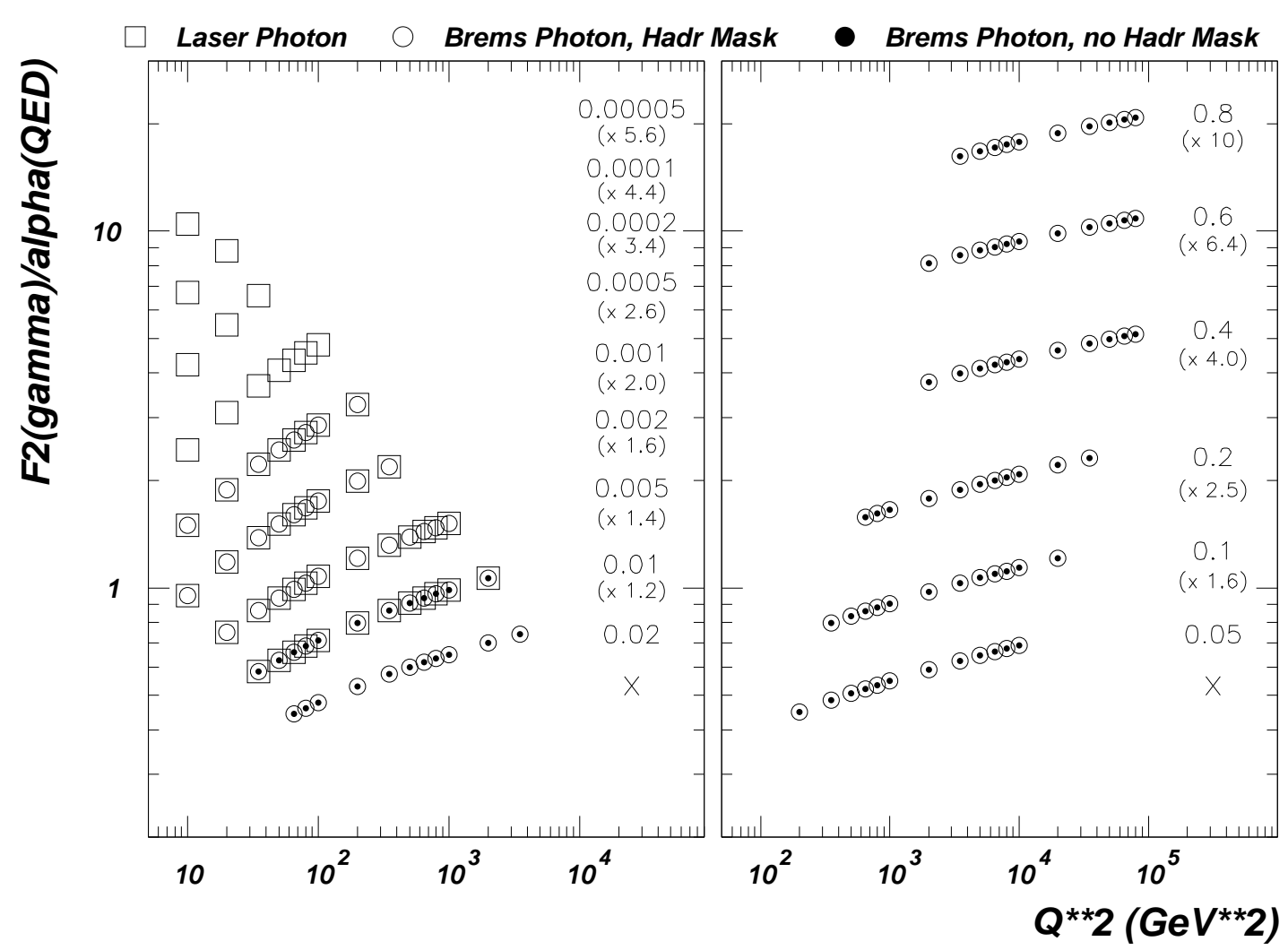

Figure 7.5: $F_{2}^{\gamma} / \alpha$ versus $Q^{2}$ in $x$ bins. Open squares: real photon target from laser backscattering; open circles: almost-real photon target from bremsstrahlung with smallangle hadronic calorimetry; solid dots: almost-real photon target from bremsstrahlung with hadronic calorimetry outside mask.

Using dijets from $\gamma \gamma$ scattering, the relative quark/gluon structure of the photon can be determined. Interactions between the almost-real photons produced by bremsstrahlung are determined primarily by interacting gluons in the ratio of approximately $70 \%$ gluons to $30 \%$ quarks. At higher $\sqrt{s}$, the gluon component should be more predominant. Thus, if real photons from laser backscattering are used, we expect to find an almost pure gluon-constituted photon $(90 \% \mathrm{~g} / 10 \% q)$ [24].

\section{5 $\gamma^{*} \gamma^{*}$ scattering - QCD dynamics}

Double-tagged virtual photon scattering completes the study of the photon at the linear collider by allowing the evolution of photon structure to be studied in an almost background-free environment. The $Q^{2}$ of each of the scattered leptons (denoted $Q_{1}^{2}$ and $Q_{2}^{2}$ ) is measured in the forward electromagnetic tagging calorimeters. By requir- 


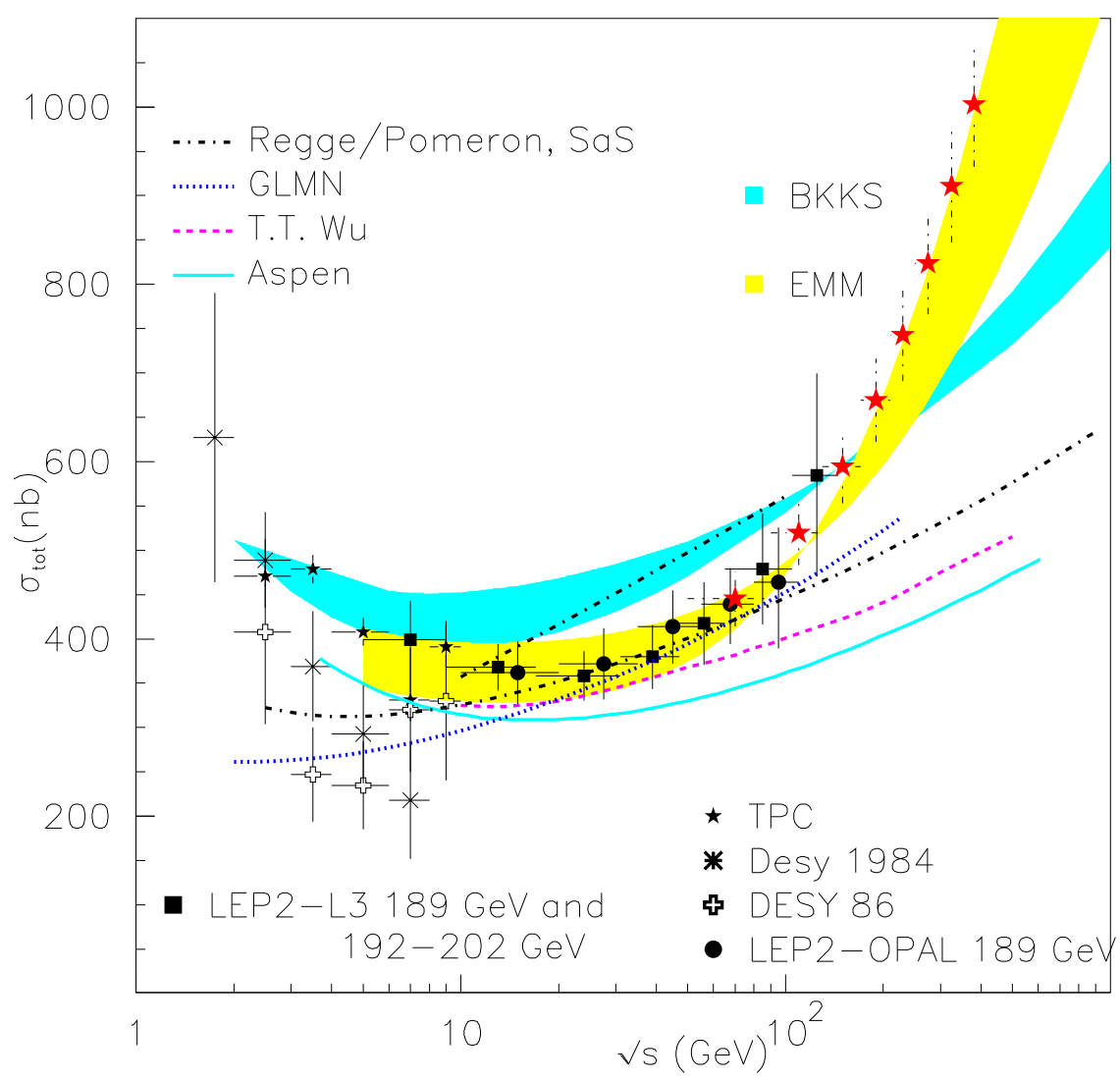

Figure 7.6: $\sigma_{\text {tot }}$ versus $\sqrt{s}$ at a LC (large stars) compared to existing data and various models.

ing the ratio $Q_{1}^{2} / Q_{2}^{2} \sim 1$, production of hadrons in the region between the two virtual photons through traditional DGLAP evolution is suppressed. This suppression grows stronger as the rapidity separation, $Y$, between the two virtual photons increases. At large values of $Y$, any signal above the small DGLAP background points to alternative forms of structure function evolution, e.g., to the $\ln (1 / x)$ evolution of BFKL [25]. Virtual photon scattering at a linear collider provides perhaps the cleanest environment in which to study BFKL physics $[26,27]$.

With total center-of-mass energy $\sqrt{s}$ and photon virtuality $Q^{2}$, BFKL effects are expected in the kinematic region where the square of the photon-photon invariant mass (or, equivalently, the hadronic final-state system) is large, and

$$
s \gg Q^{2} \gg \Lambda_{Q C D}^{2} .
$$

At fixed order in QCD, the dominant process is four-quark production with $t$-channel gluon exchange. Each photon couples to a quark box, and the quark boxes are 
QCD and Two-Photon Physics

connected via the gluon. The corresponding BFKL contribution arises from diagrams in which the $t$-channel gluon becomes a gluon ladder. At lepton-hadron or hadronhadron colliders, the presence of hadrons in the initial state can complicate or even mask BFKL effects.

The largest values of $Y$ are obtained at low $Q_{1,2}^{2}$, again emphasizing the need for the electromagnetic tagging calorimeters to be positioned as close to the beampipe as possible. Figure 7.7 shows the substantially greater reach in $Y$ available to the 500 $\mathrm{GeV}$ LC relative to that of LEP2 running at $189 \mathrm{GeV}$.

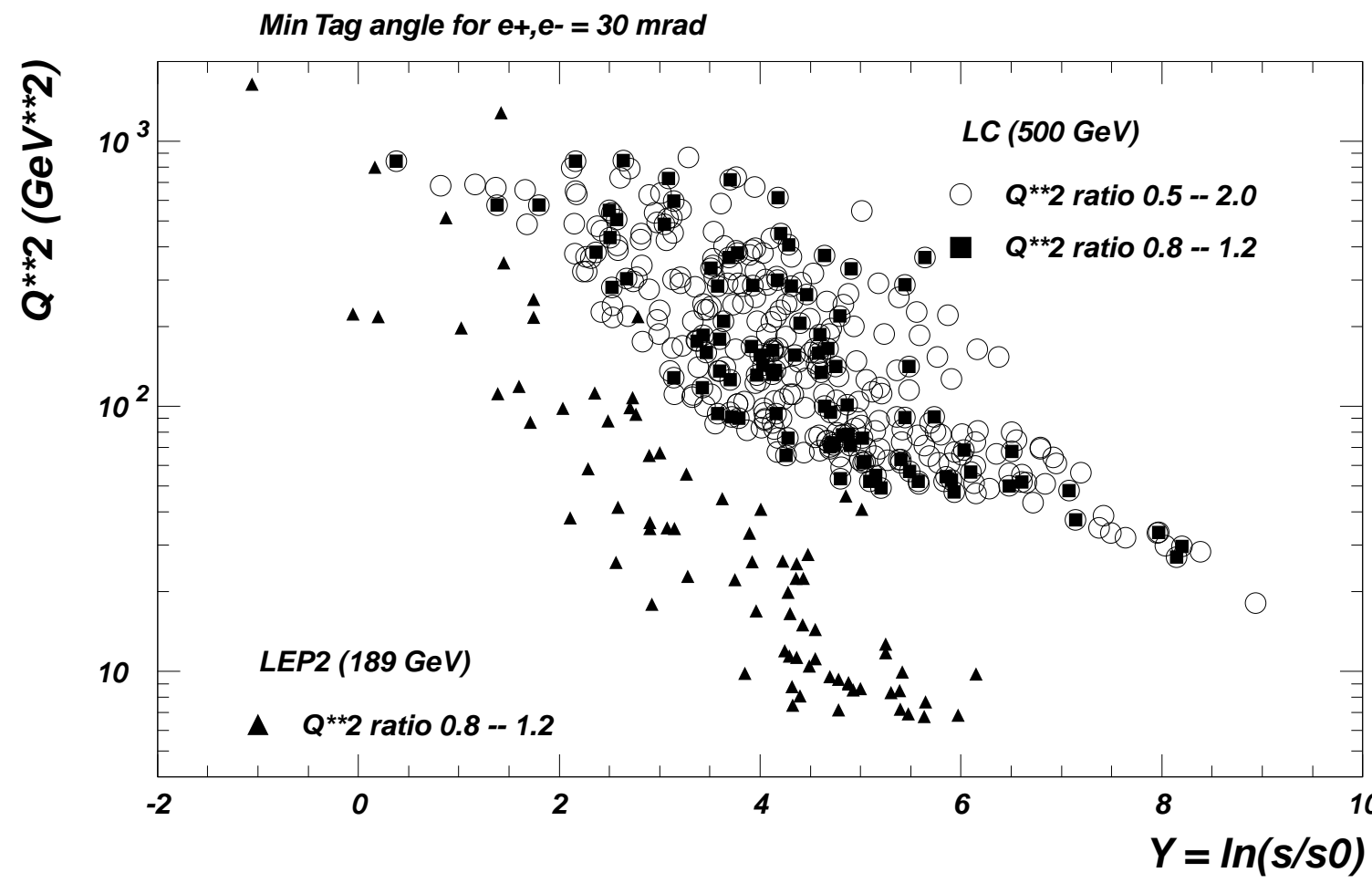

Figure 7.7: $Q^{2}$ versus $Y$ for a $500 \mathrm{GeV}$ LC compared to LEP2.

Experiments at LEP have looked for BFKL effects in virtual photon scattering [28]. The data tend to lie between the predictions of fixed-order QCD and analytic solutions to the BFKL equation (asymptotic full-order QCD). However, the data were compared to the asymptotic QCD prediction in a non-asymptotic regime [29], so the disagreement with QCD is not surprising. In contrast, a linear collider will be expected to reach closer to the asymptotic regime, providing a more definitive test of BFKL evolution. Improved predictions are also on the way with the development of BFKL Monte Carlo programs that incorporate kinematic constraints, such as [30]. On the more theoretical front, next-to-leading log corrections have been calculated 
and found to be large, but the source of the large corrections is understood and they are being brought under control; see [31] for a review and references.

\subsection{Summary of two-photon physics}

The study of two-photon physics from $e^{+} e^{-}$collisions has grown tremendously in the past several years of higher-energy LEP2 running and will continue to provide a wealth of precision measurements at a future $e^{+} e^{-}$linear collider. Using combinations of tagged and untagged bremsstrahlung photons, aspects of real and virtual photon structure will be addressed, especially $F_{2}^{\gamma}$ at high $Q^{2}$, the relative quark/gluon content of the photon from dijets, and possible BFKL effects in QCD evolution.

With laser-backscattered real photons, the highest energies available at the linear collider can be fully exploited. $F_{2}^{\gamma}$ can be measured at very low $x$, which in combination with high $Q^{2}$ measurements from bremsstrahlung photons, will map out a kinematic region in photon structure as extensive as that known for the proton. The total $\gamma \gamma$ cross section will also be measured at the highest $\sqrt{s}$ available at the linear collider, leading to understanding of the dominant mechanisms responsible for this interaction.

Finally, with combinations of lepton and photon polarization, BFKL effects can be enhanced and the first measurements of polarized structure functions of the photon can be made.

\section{Overall summary and conclusions}

The high-energy linear collider offers a unique program of QCD and related twophoton studies. The strong coupling constant $\alpha_{s}$ can be measured at high $Q^{2}$ to a precision approaching $\pm 1 \%$, free of the initial-state ambiguities that make the corresponding determination at a hadron collider substantially less precise, and allowing for substantial improvements in the determination of the running of the QCD coupling strength, as well as its extrapolation to the GUT scale. Constraints on the strong coupling properties of the top, providing sensitivity to a number of new physics scenarios inspired by the large mass of the top quark, can be made as much as an order of magnitude more stringent at an $e^{+} e^{-}$collider than at a proton collider of equivalent reach.

In two-photon reactions, the precisely defined state of the incoming electron and positron beams permits the kinematic properties of the interacting virtual and nearly on-shell photons to be inferred from the properties of the recoiling electrons. This in turn allows for a unique program of photon structure and strong-force dynamics which cannot be emulated by any other proposed facility. In addition, the possibility of precisely controlled real photon beams from the Compton backscattering of polarized laser light opens up further vistas in the exploration of photon structure, and may 
QCD and Two-Photon Physics

allow the resolution of long-standing questions regarding the energy evolution of the photon-photon total cross section. Again, these studies are only possible within the larger context of an $e^{+} e^{-}$linear collider program.

Together, these physics topics present a unique and compelling program of stronginteraction studies at a high-energy linear collider, one that adds substantial weight to the promise of the proposed linear collider physics program.

\section{References}

[1] See, e.g., S. Bethke, J. Phys. G26: R27 (2000).

[2] See, e.g., P. N. Burrows, in Proc. XXVIII International Conference on High Energy Physics, Warsaw, Poland, July 25-31 1996, Eds. Z. Adjuk, A. K. Wroblewski, World Scientific 1997, p. 797.

[3] S. Bethke, in Proc. Workshop on Physics and Experiments with Linear $\mathrm{e}^{+} \mathrm{e}^{-}$ Colliders, 26-30 April 1993, Waikoloa, Hawaii; World Scientific, Eds. F. A. Harris et al.

[4] B. Schumm, SCIPP-96-45, hep-ex/9612013 (1996).

[5] B. A. Schumm and A. S. Truitt, to appear in the proceedings of the 5th International Linear Collider Workshop (LCWS 2000), Fermilab, Batavia, Illinois, 24-28 Oct 2000; hep-ex/0102020.

[6] O. Biebel, PITHA 99/40, MPI-PhE/99-17, LC-PHSM-2000-003, hepex/9912051, Phys. Rept. 340, 165 (2001).

[7] See, e.g., OPAL Collaboration, OPAL Physics Note PN377.

[8] S. Catani et al., Nucl. Phys. B407, 3 (1993).

[9] Z. Bern, L. Dixon, and D.A. Kosower, JHEP 0001, 027 (2000).

[10] T. Gehrmann and E. Remiddi, Nucl. Phys. B601, 248, 287 (2001), hep$\mathrm{ph} / 0008287$, hep-ph/0101124.

[11] See, e.g., M. Neubert, Nucl. Phys. B463, 511 (1996); G. Altarelli, P. Nason, G. Ridolfi, Z. Phys. C68, 257 (1995).

[12] RG trajectories calculated in P. Langacker and N. Polonsky, Phys. Rev. D52, 3081 (1995).

[13] T. G. Rizzo, hep-ph/9605361.

[14] T. G. Rizzo, hep-ph/9506351.

[15] D. Atwood, A. Kagan, and T. G. Rizzo, Phys. Rev. D52, 6264 (1995).

[16] S. R. Magill, in Proc. Worldwide Study on Physics and Experiments with Future Linear $e^{+} e^{-}$Colliders, Sitges, Barcelona, Spain, April 28-May 5, 1066 (1999).

[17] R. Barate et al. (ALEPH), Phys. Lett. B458, 152 (1999); M. Acciarri et al. (L3), Phys. Lett. B436, 403 (1998); K. Ackerstaff et al. (OPAL), Phys. Lett. B412, 225 (1997); P. Abreu et al. (DELPHI), Zeit. Phys. C69, 223 (1996). 
[18] V. G. Serbo, Proc. International Workshop on High Energy Photon Colliders, DESY Hamburg, Germany, June 14-17, 2000, to be published in Nucl. Inst. Meth. A., and references therein.

[19] T. Sjöstrand, Comp. Phys. Comm. 82, 74 (1994).

[20] S. R. Magill, talk given at 2nd International Workshop on High Energy Photon Colliders, Fermilab, USA, March 14-17, 2001.

[21] J. Breitweg et al. (ZEUS), Eur. Phys. Jour. C6, 41 (1998); C. Adloff et al. (H1), Phys. Lett. B462, 440 (1999).

[22] J. Breitweg et al. (ZEUS), DESY 01-018 (February 2001), accepted by Phys. Lett. B; C. Adloff et al. (H1), DESY 00-181 (December 2000), submitted to Eur. Phys. J. C.

[23] R. M. Godbole and G. Pancheri, Proc. International Linear Collider Workshop (LCWS2000), Fermilab, USA, October 26-30, 2000.

[24] T. Wengler and A. De Roeck, Proc. International Workshop on High Energy Photon Colliders, DESY Hamburg, Germany, June 14-17, 2000, to be published in Nucl. Inst. Meth. A.

[25] L. N. Lipatov, Sov. J. Nucl. Phys. 23, 338 (1976); E. A. Kuraev, L. N. Lipatov and V. S. Fadin, Sov. Phys. JETP 45, 199 (1977); Ya. Balitsky and L. N. Lipatov, Sov. J. Nucl. Phys. 28, 822 (1978).

[26] S. J. Brodsky, F. Hautmann and D. E. Soper, Phys. Rev. D56, 6957 (1997).

[27] M. Boonekamp, A. De Roeck, C. Royon and S. Wallon, Nucl. Phys. B555, 540 (1999) [hep-ph/9812523].

[28] See, e.g., A. De Roeck, Nucl. Phys. Proc. Suppl. 99, 144 (2001) and references therein.

[29] L. H. Orr and W. J. Stirling, Proc. 30th International Conference on High-Energy Physics (ICHEP 2000), Osaka, Japan, 27 Jul - 2 Aug 2000, hep-ph/0012198.

[30] C. R. Schmidt, Phys. Rev. Lett. 784531 (1997); L. H. Orr and W. J. Stirling, Phys. Rev. D56 5875 (1997).

[31] G. P. Salam, Acta Phys. Polon. B30, 3679 (1999); Proc. 35th Rencontres de Moriond: QCD and High Energy Hadronic Interactions, Les Arcs, Savoie, France, 18-25 March 2000, hep-ph/0005304, and references therein. 


\section{Chapter 8 Precision Studies at the $Z$ and the $W W$ Threshold}

A high-precision program of electroweak and heavy-quark physics provides a natural complement to the direct searches for the Higgs boson and other new particles. The study of loop corrections to the electroweak parameters measured at the $Z$, in $p \bar{p}$ collisions and in neutrino experiments made impressive indirect predictions for the top quark mass, and constrains the mass range for a Standard Model Higgs. Limits on $\mathcal{B}\left(B \rightarrow X_{s} \gamma\right)$ provide the tightest mass limits on type II Higgs doublets. Because the new particles appear virtually in loops, the sensitivity extends over a much higher mass range than can be obtained in direct searches, though generally at the expense of some model-dependence.

While the physics program at $500 \mathrm{GeV}$ has the potential to be very rich, it is also possible that at this center-of-mass energy there is only one Higgs-like particle seen, or no such particle at all. Under either scenario, the constraints from the electroweak and heavy-quark studies can be powerful. In the case that we do see a plethora of new particles, the full spectrum of states predicted by any model must satisfy the rules dictated by the precision measurements. In the case that very little is seen directly, the precision low-energy measurements have a good chance of showing deviations from the Standard Model. These deviations will indicate the direction that future studies must take.

There remain open issues with respect to implementing a low-energy program at a linear collider. If only the basic electroweak program is undertaken, the goals may be met by devoting a modest amount of running time at low energy. A single facility for both the high-energy and the $Z$ running, however, requires incorporation of this capability into the design of the accelerator. For a broader program, including running at $W^{+} W^{-}$threshold and extended running at the $Z$ pole for heavy flavor physics, a low-energy facility that can operate in parallel with the high-energy may be required.

\section{Electroweak observables on the $Z$ resonance}

In principle, all measurements done at LEP and SLC can be repeated at the linear collider with much higher statistics. In about 100 days of running, it is possible to collect a sample of $10^{9} Z$ decays ('Giga-Z'), about 100 times the LEP or 1000 times the SLC statistics. A high degree of electron polarization seems certain and $\mathcal{P}_{\mathrm{e}^{-}}=80 \%$ will be assumed in the following. Positron polarization is desirable and the R\&D to 
achieve it is under way. Both options, with and without positron polarization, will be discussed. The issue of positron polarization is discussed further in Chapter 12 .

\subsection{Machine issues}

In the present designs, the linear collider can deliver a luminosity $\mathcal{L} \sim 5 \times$ $10^{33} \mathrm{~cm}^{-2} \mathrm{~s}^{-1}$ at the $Z$ resonance. The energy loss due to beamstrahlung for colliding particles is around $0.05 \%-0.1 \%$ and the depolarization in the interaction region is negligible. By sacrificing some luminosity, beamstrahlung can be reduced substantially, for example, by a factor three for a luminosity loss of a factor two [1].

Apart from the beamstrahlung there are several other effects that influence the precision of the measurements:

- The mean energies of the two beams have to be measured very precisely. A precision of $10^{-5}$ relative to the $Z$ mass might be needed to relate $A_{L R}$ to $\sin ^{2} \theta_{w}^{\text {eff }}$ with the desired precision.

- The beam energy spread of the machine plays a crucial role in the measurement of the total width of the $Z$. If the shape of the distribution is known, the width can be measured from the acolinearity of Bhabha events in the forward region as long as the energies of the two colliding particles are not strongly correlated.

- With the high luminosities planned, the $Z$ multiplicity in a train becomes high. This can influence $Z$ flavor tagging or even $Z$ counting.

- With positron polarization, the positron source must be able to switch polarizations on a time scale commensurate with the stability of the beam conditions.

The two main designs, $\mathrm{X}$-band and superconducting, differ in some aspects relevant for $Z$ running. For the X-band design a bunch train contains 190 bunches with $1.4 \mathrm{~ns}$ bunch spacing, for which over half of the $Z$ bosons are produced in the same train as at least one other $Z$. Typical event separation is about $150 \mathrm{~ns}$, but the experimental consequences merit some study. A TESLA bunch contains 2800 bunches with 280 ns bunch spacing. In this case bunch separation is not a problem, but data acquisition system requirements are higher. The smaller wakefields in the superconducting machine should reduce the beam energy spread. The larger bunch spacing may allow sufficient time for energy feedback, resulting in a smaller energy difference between the bunches in a train.

The LC design must accommodate the needs of the precision electroweak program in advance for the program to be viable. Suitable space in the beam delivery system for precise beam energy measurement and for polarimetry must be provided, or the beam energy measurement must be directly incorporated into the Final Focus magnet system. A measurement of these quantities behind the IP is also desirable, though it is difficult. A nonzero crossing angle might be needed. 
Precision Studies at the $Z$ and $W W$ Threshold

\begin{tabular}{ccc}
\hline \hline & LEP $/ \mathrm{SLC} / \mathrm{Tev}[2]$ & LC \\
\hline $\sin ^{2} \theta_{w}^{\mathrm{eff}}$ & $0.23146 \pm 0.00017$ & \pm 0.000013 \\
\hline lineshape observables: & \\
\hline$m_{Z}$ & $91.1875 \pm 0.0021 \mathrm{GeV}$ & $\pm 0.0021 \mathrm{GeV}$ \\
$\alpha_{s}\left(m_{Z}^{2}\right)$ & $0.1183 \pm 0.0027$ & \pm 0.0009 \\
$\Delta \rho_{\ell}$ & $(0.55 \pm 0.10) \times 10^{-2}$ & $\pm 0.05 \times 10^{-2}$ \\
$N_{\nu}$ & $2.984 \pm 0.008$ & \pm 0.004 \\
\hline heavy flavors: & \\
\hline $\mathcal{A}_{b}$ & $0.898 \pm 0.015$ & \pm 0.001 \\
$R_{b}^{0}$ & $0.21653 \pm 0.00069$ & \pm 0.00014 \\
\hline \hline
\end{tabular}

Table 8.1: Possible improvement in the electroweak physics quantities for $10^{9} Z$ 's collected at a linear collider. $N_{\nu}=3$ is assumed for $\alpha_{s}$ and $\Delta \rho_{\ell}$.

\section{$1.2 \quad$ Electroweak observables}

There are three classes of electroweak observables that can be measured during $Z$-running at a linear collider:

- observables related to the partial widths of the $Z$, measured in a $Z$ resonance scan;

- observables sensitive to the effective weak mixing angle;

- observables using quark flavor tagging.

Table 8.1 summarizes the present precision and the expectations for the linear collider for these quantities.

\subsubsection{Observables from the $Z$ resonance line scan}

From a scan of the $Z$ resonance curve the following quantities are measured:

- the mass of the $Z\left(m_{Z}\right)$;

- the total width of the $Z\left(\Gamma_{Z}\right)$;

- the hadronic pole cross section $\left(\sigma_{0}=\left(12 \pi / m_{Z}^{2}\right) \cdot\left(\Gamma_{e} \Gamma_{\text {had }} / \Gamma_{Z}^{2}\right)\right)$;

- the ratio of the hadronic to the leptonic width of the $Z\left(R_{\ell}=\frac{\Gamma_{\text {had }}}{\Gamma_{l}}\right)$.

From these parameters, two interesting physics quantities can be derived: the radiative correction parameter $\Delta \rho_{\ell}$ that normalizes the $Z$ leptonic width, and the strong coupling constant $\alpha_{s}$. 
The LEP measurements are already systematics-limited, so statistical improvement is not the issue. From LEP, $m_{Z}$ is known to $2 \times 10^{-5}$, and the other three parameters are all known to $10^{-3}$. To improve on $\alpha_{s}$ and especially on $\Delta \rho_{\ell}$, all three measured parameters must be improved. This requires one to understand the beam energy and the beam energy spread for $\Gamma_{Z}$, the hadronic and leptonic selection efficiencies for $R_{\ell}$, and the absolute luminosity for $\sigma_{0}$. With the better detectors and the higher statistics available for cross checks, the errors on the selection efficiency and on the luminosity might be improved by a factor of three relative to the best LEP experiment [3]. It is not clear whether the theory error on the luminosity can be improved beyond its present value of $0.05 \%$. These errors would improve the precision on $R_{\ell}$ by a factor of four and that on $\sigma_{0}$ by $30 \%$.

With a Møller spectrometer, one could possibly obtain a precision of $10^{-5}$ in the beam energy relative to $m_{Z}$. This would give a potential improvement of a factor of two in $\Gamma_{Z}$. However, because the second derivative of a Breit-Wigner curve at the maximum is rather large, $\Gamma_{Z}$ and $\sigma_{0}$ are significantly modified by beamstrahlung and beam energy spread. For illustration, the fitted $\Gamma_{Z}$ is increased by about $60 \mathrm{MeV}$ and $\sigma_{0}$ is decreased by $1.8 \%$ for the TESLA parameters. The energy spread dominates the effect, so this particularly needs to be understood to about $2 \%$ to avoid limiting the precision on $\Gamma_{Z}$ and $\Delta \rho_{\ell}$. There is a potential to achieve this precision with the acolinearity measurement of Bhabha events [4] or to extend the scan to five scan points and fit for the energy spread, but both options need further study.

\subsubsection{The effective weak mixing angle}

If polarized beams are available, the most sensitive quantity by far to the weak mixing angle is the left-right asymmetry:

$$
\begin{aligned}
A_{L R} & =\frac{1}{\mathcal{P}} \frac{\sigma_{L}-\sigma_{R}}{\sigma_{L}+\sigma_{R}} \\
& =\mathcal{A}_{e} \\
& =\frac{2 v_{e} a_{e}}{v_{e}^{2}+a_{e}^{2}} \\
v_{e} / a_{e} & =1-4 \sin ^{2} \theta_{w}^{\mathrm{eff}} .
\end{aligned}
$$

$A_{L R}$ is independent of the final state.

The $A_{L R}$ measurement has been analyzed for the linear collider environment in $[5,1]$. With $10^{9} Z$ 's, an electron polarization of $80 \%$ and no positron polarization, the statistical error is $\triangle A_{L R}=4 \times 10^{-5}$. The error from the polarization measurement is $\Delta A_{L R} / A_{L R}=\Delta \mathcal{P} / \mathcal{P}$. At SLC, $\Delta \mathcal{P} / \mathcal{P}=0.5 \%$ has been reached [6]. With some optimism a factor two improvement in $\Delta \mathcal{P} / \mathcal{P}$ is possible [1]. In combination with the improved statistics, this leads to $\Delta A_{L R}=3.8 \times 10^{-4}$. This precision is already more 
Precision Studies at the $Z$ and $W W$ Threshold
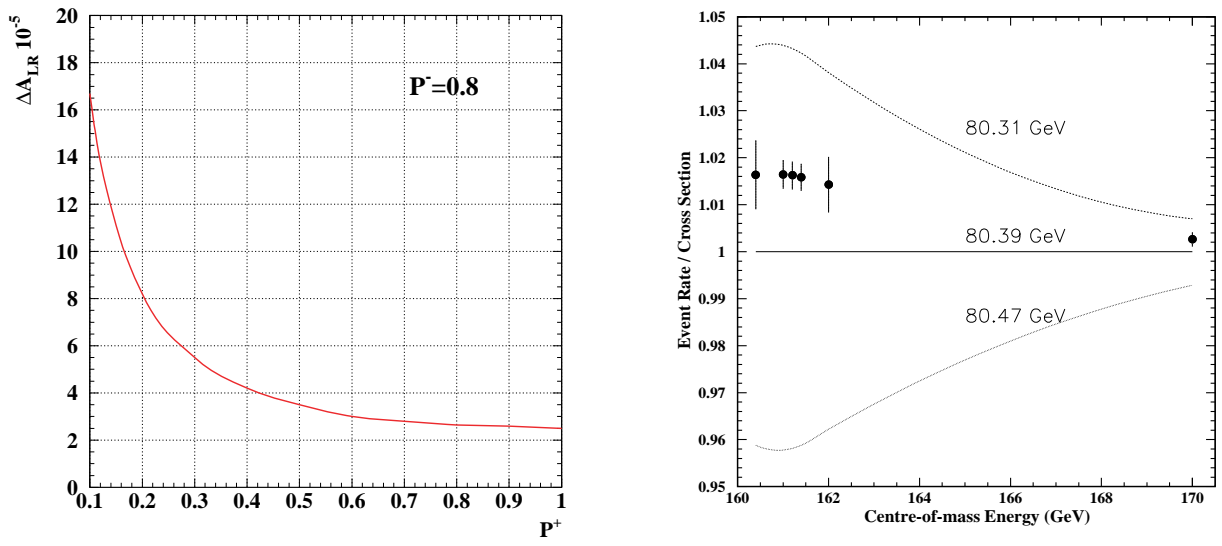

Figure 8.1: Left: Error of $A_{L R}$ as a function of the positron polarization for a luminosity corresponding to $10^{9}$ unpolarized $Z$ 's. The errors assume that switching of the positron polarization can be done on a time scale over which the beam conditions are suitably stable. Right: The ratio of the measured $W^{+} W^{-}$cross section to the predicted cross section for $M_{W}=80.39 \mathrm{GeV}$ (see Section 2). The data were generated using $M_{W}=80.36 \mathrm{GeV}$. The upper (lower) curves show the ratio of the predicted cross section for $M_{W}=80.31 \mathrm{GeV}$ $\left(M_{W}=80.47 \mathrm{GeV}\right)$ to that for $M_{W}=80.39 \mathrm{GeV}$.

than a factor of five improvement over the final SLD result for $\sin ^{2} \theta_{w}^{\text {eff }}$ and almost a factor of four over the combined LEP/SLD average.

If positron polarization is available, there is the potential to go much further using the 'Blondel scheme' [7]. This method of polarization measurement, and the associated techniques for obtaining polarized positrons, are described in more detail in Chapter 12. To summarize the results, the total cross section with both beams polarized is given as $\sigma=\sigma_{u}\left[1-\mathcal{P}_{\mathrm{e}^{+}} \mathcal{P}_{\mathrm{e}^{-}}+A_{L R}\left(\mathcal{P}_{\mathrm{e}^{+}}-\mathcal{P}_{\mathrm{e}^{-}}\right)\right]$, where $\sigma_{u}$ is the unpolarized cross section. If all four helicity combinations are measured, $A_{L R}$ can be determined without polarization measurement as

$$
A_{L R}=\sqrt{\frac{\left(\sigma_{++}+\sigma_{-+}-\sigma_{+-}-\sigma_{--}\right)\left(-\sigma_{++}+\sigma_{-+}-\sigma_{+-}+\sigma_{--}\right)}{\left(\sigma_{++}+\sigma_{-+}+\sigma_{+-}+\sigma_{--}\right)\left(-\sigma_{++}+\sigma_{-+}+\sigma_{+-}-\sigma_{--}\right)}} .
$$

Figure 8.1 shows the error on $A_{L R}$ as a function of the positron polarization. For $\mathcal{P}_{\mathrm{e}^{+}}>50 \%$ the dependence is relatively weak. For $10^{9} Z$ 's, the Blondel scheme with a positron polarization of $20 \%$ gives a better result than a polarization measurement of $0.1 \%$ and electron polarization only.

Polarimeters are still needed to resolve one remaining question. There could potentially be a difference between the absolute values of the polarization in the left- and right-handed states. If the two polarization values for electrons and positrons are written as $\mathcal{P}_{\mathrm{e}^{ \pm}}= \pm\left|\mathcal{P}_{\mathrm{e}^{ \pm}}\right|+\delta \mathcal{P}_{\mathrm{e}^{ \pm}}$, the dependence on this difference is $\mathrm{dA}_{\mathrm{LR}} / \mathrm{d} \delta \mathcal{P}_{\mathrm{e}^{ \pm}} \approx 0.5$. 
One therefore needs to understand $\delta \mathcal{P}_{\mathrm{e}^{ \pm}}$to $<10^{-4}$. If polarimeters with at least two channels are available, $\delta P$ can be measured together with other systematic effects intrinsic to the polarimeters in a way that does not increase the statistical error from the Blondel scheme.

Because of $\gamma-Z$ interference, the dependence of $A_{L R}$ on the beam energy is $\mathrm{dA}_{\mathrm{LR}} / \mathrm{d} \sqrt{\mathrm{s}}=2 \times 10^{-2} / \mathrm{GeV}$. The difference $\sqrt{s}-m_{Z}$ thus needs to be known to about $10 \mathrm{MeV}$ to match the measurement with electron polarization only, and to about $1 \mathrm{MeV}$ if polarized positrons are available. For the same reason beamstrahlung shifts $A_{L R}$. The shift is $9 \times 10^{-4}$ for TESLA and is larger for NLC/JLC [1]. The uncertainty can only be a few percent. If beamstrahlung in the $A_{L R}$ running is identical to that in the $Z$ scan used to calibrate the beam energy, the effect is absorbed into the mean energy measured in the calibration. In that case, practically no correction would be needed for $A_{L R}$. How well the beam parameters can be kept constant during the scan and how well the beamstrahlung can be measured still need further study. However, for $A_{L R}$, only the beamstrahlung and not the energy spread matters. If the beamstrahlung cannot be understood to the required level in the normal running mode one can still go to a mode with lower beamstrahlung at the expense of lower luminosity. The cost is an increase in the statistical error or the running time.

Finally, the rate at which the positron polarization must be switched, and the switching rates that are achievable are still unknown.

For the interpretation of the data it will be assumed that $\Delta A_{L R}=10^{-4}$ is possible. This leads to $\Delta \sin ^{2} \theta_{w}^{\text {eff }}=0.000013$. It must be kept in mind that this error will increase by a factor of four if no positron polarization is available.

\subsubsection{Observables with tagged quarks}

By the use of quark tagging in addition to the observables discussed above, the partial widths and forward-backward asymmetries for $b$ and $c$ quarks can be measured. These observables are sensitive to vertex corrections at the $Z q q$ vertex and to new Born-level effects that alter the SM relations between quarks and leptons. The $Z b b$ vertex is particularly interesting, since the $b$ is the partner of the heavy top quark, and since the vertex corrections are naturally enhanced with the quark mass.

To date, only the improvement to the $b$-quark observables has been estimated [5]. For the ratio $R_{b}$ of the $Z$ partial widths to $b$ quarks and to hadrons, an improvement of a factor five to the LEP/SLD average is possible. This improvement is due to the much better $b$ tagging than at LEP. The improved tagging results in a higher purity (over $99 \%$ for a 30\% efficiency) and a smaller energy dependence, which in turn reduces the hemisphere correlations.

The forward-backward asymmetry with unpolarized beams measures the product of the coupling parameters for the initial-state electrons and the final-state quarks: $A_{\mathrm{FB}}^{q}=\frac{3}{4} \mathcal{A}_{e} \mathcal{A}_{q}$, while the left-right forward-backward asymmetry with polarized 
beams measures the quark couplings directly: $A_{\mathrm{LR}, \mathrm{FB}}^{q}=\frac{3}{4} \mathcal{P} \mathcal{A}_{q}$. For this reason a factor 15 improvement on $\mathcal{A}_{b}$ relative to the LEP/SLC result is possible if polarized positrons are available, and if other systematic effects are relatively small. With polarized electrons only, the improvement is limited by the polarization error to a factor of six. For control of systematics, the improved $b$-tagging capabilities are essential here as well.

Though the SM predicts that $Z$ decays to quarks are flavor-diagonal to a very good approximation, loop effects of new physics can induce flavor-violating rare decays [8]. These could be searched for at a high-luminosity $Z$ factory. For $Z \rightarrow b \bar{s}$ decays, the SM predicts a branching ratio of $\mathcal{B}(Z \rightarrow b \bar{s}) \simeq 1.4 \cdot 10^{-8}$. To date, the direct experimental bound on this process is relatively weak, at the level of about $10^{-3}[9]$, though bounds from rare $b$ decays such as $b \rightarrow s \ell^{+} \ell^{-}$and $b \rightarrow s \nu \bar{\nu}$ lead to a bound $\mathcal{B}(Z \rightarrow b \bar{s}) \lesssim 5 \cdot 10^{-7}[8]$. Still, there is room for a new physics contribution that might be revealed in a large sample of $Z$ decays.

\section{$2 \quad m_{W}$ from $W W$ threshold running}

The mass $m_{W}$ of the $W$ boson plays a fundamental role in constraints on the Standard Model via comparison of direct measurement with the prediction based on other electroweak parameters. The electroweak measurements from LEP1 and Giga$\mathrm{Z}$ - combined with the Higgs boson and top quark mass measurements from the linear collider - allow $m_{W}$ to be predicted to about $3 \mathrm{MeV}$ within the SM. Measurements at the Tevatron and at LEP2 combine to give an $m_{W}$ precision of $34 \mathrm{MeV}$ [10]. The LEP2 experiments hope to reach a combined precision of $35 \mathrm{MeV}$. With Run II at the Tevatron, $30 \mathrm{MeV}$ per experiment appears feasible with $2 \mathrm{fb}^{-1}$, though systematics, correlated between experiments, will dominate [11]. The LHC experiments hope to reach an uncertainty of $20 \mathrm{MeV}$ each, for perhaps an overall uncertainty of 15 $\mathrm{MeV}$ [12]. Unfortunately, these uncertainties remain significantly larger than that expected for the indirect determination and would limit the power of the electroweak constraints.

A high-luminosity linear collider presents an opportunity to measure $m_{W}$ with a much higher precision. The two potential approaches [13] are a $W^{+} W^{-}$threshold scan and kinematic fitting of events with $W^{+} W^{-}$production. With expected linear collider luminosities, one could obtain $100 \mathrm{fb}^{-1}$ in one year $\left(10^{7} \mathrm{~s}\right)$ at $W^{+} W^{-}$threshold and about $1000 \mathrm{fb}^{-1}$ at $\sqrt{s}=500 \mathrm{GeV}$ in several years. The threshold scan requires precise determination of the absolute average beam energy and of the distortion of the luminosity spectrum by beamstrahlung. The kinematic fitting method also requires precise knowledge of the beam energy, since it relies on a beam energy constraint. The uncertainty from this parameter will grow with energy, since beam calibration will likely refer back to the $Z$ peak. Furthermore, the energy spread from beamstrahlung 
grows approximately as the square of the beam energy.

The four-quark $(4 q)$ channel ( $46 \%$ of the rate) cannot be used in the kinematic analysis because of theoretical uncertainties associated with final-state interactions between the decay products of the $W^{+}$and the $W^{-}$. This uncertainty contributes an error of $40-90 \mathrm{MeV}$ for the current LEP $4 q$ measurements [14-17]. Scaling of the LEP2 statistical precision for the remaining channels results in a $5 \mathrm{MeV} m_{W}$ precision at $500 \mathrm{GeV}$. However, significant reductions in systematics will be needed. In particular, the difficulties in beam energy calibration disfavor the direct reconstruction method.

\section{1 $m_{W}$ from a polarized threshold scan}

The extraction of $m_{W}$ from a threshold scan requires an accurate theoretical description of the cross-section dependence on $m_{W}$. The main corrections to the Born approximation near threshold come from QED. Fortunately, the dominant Coulomb correction (about 6\%) is already known to all orders [18]. To keep the theoretical uncertainty down to $2 \mathrm{MeV}$, however, the electroweak and QCD corrections to the cross section must be known to $0.12 \%$ (about the size of the second-order Coulomb contribution). While work is needed, this goal appears attainable.

Recent studies $[19,20]$ indicate that experimental systematics can be controlled to obtain a $5 \mathrm{MeV} m_{W}$ measurement with $100 \mathrm{fb}^{-1}$ of data if a polarization of $60 \%$ for the positron beam can be achieved. The strategy capitalizes on the domination of the $W^{+} W^{-}$cross section near threshold by the $t$-channel $\nu_{e}$ exchange process, which couples only to the $e_{R}^{+} e_{L}^{-}$helicity combination. The correct $e_{R}^{+} e_{L}^{-}$beam polarization enhances $W^{+} W^{-}$production relative to the background, while the $e_{L}^{+} e_{R}^{-}$polarization has almost negligible $W^{+} W^{-}$production and so can constrain the background levels.

A sample scan is illustrated in Fig. 8.1. This study assumes that the absolute luminosity and the reconstruction efficiencies can be determined with a relative (pointto-point) accuracy of $0.25 \%$. This is four times looser than that achieved for the LEP1 $Z$ line-shape scan. Beam polarizations are assumed known to $0.25 \%$, and are further constrained at each scan point by exploring various polarization combinations. About $90 \%$ of the luminosity is given to the main $e_{R}^{+} e_{L}^{-}$to $e_{R}^{+} e_{L}^{-}$configurations, in a 5:1 ratio, with the $10 \%$ devoted to the remaining configurations to determine the beam polarization. LEP signal efficiencies and background rates [21] are assumed; this should be conservative for a linear collider detector. The $W$ width $\Gamma_{W}$ is assumed to have the SM value. Under these assumptions, a precision on $m_{W}$ of $4.9 \mathrm{MeV}$ is predicted for $100 \mathrm{fb}^{-1}$ of data.

To reduce the dependence of the $m_{W}$ precision on the absolute beam polarization determination, 'radiative return' $\left(e^{+} e^{-} \rightarrow \gamma+Z\right)$ events can be incorporated into the analysis. They are sufficiently numerous $-10^{7}$ in $100 \mathrm{fb}^{-1}$ - that the Blondel scheme described in the previous section can be employed to measure the polarization. After fine tuning of the luminosity distribution among various helicity configurations, a scan can still determine $m_{W}$ to $5 \mathrm{MeV}$ without the $0.25 \%$ polarization calibration. 
Precision Studies at the $Z$ and $W W$ Threshold

The background from $e^{+} e^{-} \rightarrow q \bar{q}$ and its polarization asymmetry is neglected in this analysis. It is possible that the polarization asymmetry of the sample of background events that pass the $W W$ event selection cuts will be poorly known. In this case, the scan strategy above may not be optimal for control of the systematics. While further study is warranted, incorporation of a scan point below threshold should control the uncertainties without significantly degrading precision on $m_{W}$.

The beam-energy and beamstrahlung uncertainties of a $W^{+} W^{-}$threshold scan must be controlled to a few $\mathrm{MeV}$ to achieve the desired $m_{W}$ precision. One method [22] provides a direct measurement of the average $\sqrt{s}$ via reconstruction of $e^{+} e^{-} \rightarrow \gamma+Z$, $Z \rightarrow e^{+} e^{-} / \mu^{+} \mu^{-}$. This measurement includes the average beamstrahlung effect. A precision of $2.5 \mathrm{MeV}$ may be possible for $100 \mathrm{fb}^{-1}$. Absolute alignment of the detector polar angle to $10^{-5}$ and knowledge of the radiative corrections will be needed. One could also calibrate a precise beam spectrometer using the $Z$ line shape and extrapolate to the $W^{+} W^{-}$threshold. The uncertainty from the LEP1 $m_{Z}$ measurement will cancel in the $m_{W} / m_{Z}$ ratio. Beamstrahlung both reduces the effective $W^{+} W^{-}$ cross section at threshold and distorts the shape. To limit the effects to $2 \mathrm{MeV}$, the absolute induced distortion must be known to 0.1\%. Mapping of the distortion to this accuracy appears feasible by measurement of the distribution in the acolinearity angle in Bhabha scattering at forward angles [23]. All of these aspects of the precision energy determination will be challenging if one wishes to achieve a $2 \mathrm{MeV}$ error from this source.

\subsection{Conclusion}

The experimental systematics for an $m_{W}$ measurement near $W^{+} W^{-}$threshold appear to be under control at the few-MeV level. Issues related to beam energy and beamstrahlung deserve further attention, but cautious optimism is appropriate. Certainly the $m_{W}$ issues should be considered in the accelerator and interaction region design. Given the one year of running required to reach the order $5 \mathrm{MeV}$ accuracy in $m_{W}$, consideration of a dedicated low-energy facility seems appropriate. The feasibility of the measurement without positron polarization needs examination. A much longer running period would be necessary just to make up the loss in $W^{+} W^{-}$ production. The impact on control of the background level is currently unknown.

\section{Electroweak tests of the Standard Model}

The physics program outlined above opens new opportunities for high-precision physics in the electroweak sector. For reference, Table 8.2 [24] summarizes the present and anticipated precisions for the most relevant electroweak observables at the Tevatron-Run II $\left(2 \mathrm{fb}^{-1}\right)$ and TeV33 $\left(30 \mathrm{fb}^{-1}\right)$, the LHC, and a future linear collider without (LC) and with (Giga-Z) a low-energy program. 
Chapter 8

\begin{tabular}{cccrccc}
\hline \hline & now & Run II & \multicolumn{1}{c}{ TeV33 } & \multicolumn{1}{c}{ LHC } & \multicolumn{1}{c}{ LC } & Giga-Z \\
\hline$\delta \sin ^{2} \theta_{w}^{\text {eff }}\left(\times 10^{5}\right)$ & 17 & $50[28]$ & $13[28]$ & $21[28,30]$ & $(6)[28]$ & $1.3[5]$ \\
$\delta m_{W}[\mathrm{MeV}]$ & 37 & $30[11]$ & $15[12]$ & $15[12,30]$ & $15[32]$ & $6[34]$ \\
$\delta m_{t}[\mathrm{GeV}]$ & 5.1 & $4.0[28]$ & $2.0[28]$ & $2.0[28,31]$ & $0.2[33]$ & 0.2 \\
$\delta m_{h}[\mathrm{MeV}]$ & - & - & $2000[29]$ & $100[29]$ & $50[29]$ & $50[29]$ \\
\hline \hline
\end{tabular}

Table 8.2: The expected experimental precision from various collider programs for $\sin ^{2} \theta_{w}^{\text {eff }}$, $m_{W}, m_{t}$ and the Higgs boson mass, $m_{h}$, assuming $m_{h}=110 \mathrm{GeV}$. For the LC entry in parentheses, a fixed-target polarized Møller scattering experiment using the $e^{-}$beam has been assumed. The present uncertainty on $m_{W}$ will be improved with the final analysis of the LEP2 data.

The SM predictions for the electroweak precision observables are affected via loop corrections by contributions from the top quark mass, $m_{t}$, and the Higgs boson mass, $m_{h}$. The prediction for the $W$ boson mass is obtained from

$$
m_{W}=\frac{m_{Z}}{\sqrt{2}} \sqrt{1+\sqrt{\frac{4 \pi \alpha}{\sqrt{2} G_{F} m_{Z}^{2}}(1+\Delta r)}},
$$

where the loop corrections are contained in $\Delta r$ [25]. Beyond one-loop order, the QCD corrections are known at $\mathcal{O}\left(\alpha \alpha_{s}\right)$ [26] and $\mathcal{O}\left(\alpha \alpha_{s}^{2}\right)$ [27]. The electroweak two-loop corrections have recently been extended to include the complete fermionic contribution at $\mathcal{O}\left(\alpha^{2}\right)[35]$.

The effective leptonic weak mixing angle, $\sin ^{2} \theta_{w}^{\text {eff }}$, is defined through the effective couplings $g_{V}^{f}$ and $g_{A}^{f}$ of the $Z$ boson to fermions at the $Z$ resonance,

$$
\sin ^{2} \theta_{w}^{\mathrm{eff}}=\frac{1}{4 Q_{f}}\left(1-\frac{\operatorname{Re} g_{V}^{f}}{\operatorname{Re} g_{A}^{f}}\right)
$$

where the loop corrections enter through $g_{V, A}^{f}$. The radiative corrections entering the relations (8.2) and (8.3) depend quadratically on $m_{t}$, while the leading dependence on $m_{h}$ is only logarithmic.

The current theoretical uncertainties [36] are dominated by the uncertainties in the input parameters $m_{t}$ and $m_{h}$, and in the value of the running electromagnetic coupling constant evaluated at the scale $m_{Z}$. Let $\Delta \alpha=\alpha\left(m_{Z}\right)-\alpha(0)$. This difference results from electromagnetic vacuum polarization corrections due to the charged leptons and light quarks. The hadronic contributions to $\Delta \alpha$ currently give rise to an uncertainty $\delta \Delta \alpha \approx \pm 2 \times 10^{-4}$ [37]. If future low-energy $e^{+} e^{-}$experiments can measure the hadronic total cross section up to the $J / \psi$ to $1 \%$, it is possible to reduce this uncertainty to about $\delta \Delta \alpha= \pm 7 \times 10^{-5}$ [38]. As an estimate for the future theoretical uncertainties in the prediction of $m_{W}$ and $\sin ^{2} \theta_{w}^{\text {eff }}$ from unknown higher-order 
Precision Studies at the $Z$ and $W W$ Threshold

\begin{tabular}{lrrr}
\hline \hline & $m_{W}$ & $\sin ^{2} \theta_{w}^{\text {eff }}$ & all \\
\hline now & $200 \%$ & $62 \%$ & $60 \%$ \\
Run II & $77 \%$ & $46 \%$ & $41 \%$ \\
TeV33 & $39 \%$ & $28 \%$ & $26 \%$ \\
LHC & $28 \%$ & $24 \%$ & $21 \%$ \\
LC & $18 \%$ & $20 \%$ & $15 \%$ \\
Giga-Z & $12 \%$ & $7 \%$ & $7 \%$ \\
\hline \hline
\end{tabular}

Table 8.3: Cumulative expected precisions for the indirect determination of the Higgs boson mass, $\delta m_{h} / m_{h}$, taking into account the error projections in Table 8.2 and the theoretical uncertainties of $m_{W}$ and $\sin ^{2} \theta_{w}^{\text {eff }}$. The first two columns use $m_{W}$ and $\sin ^{2} \theta_{w}^{\text {eff }}$ constraints alone, while the last column uses the full set of precision observables.

corrections (including the uncertainties from $\delta \Delta \alpha$ ) we use

$$
\delta m_{W}(\text { theory })= \pm 3 \mathrm{MeV}, \quad \delta \sin ^{2} \theta_{w}^{\text {eff }}(\text { theory })= \pm 3 \times 10^{-5} \quad \text { (future) } .
$$

The experimental error on $m_{Z}\left(\delta m_{Z}= \pm 2.1 \mathrm{MeV}[10]\right)$ leads to an uncertainty in $\sin ^{2} \theta_{w}^{\text {eff }}$ of $\delta \sin ^{2} \theta_{w}^{\text {eff }}= \pm 1.4 \times 10^{-5}$. While this uncertainty can currently be neglected, it will have non-negligible impact given the precision obtainable at Giga-Z. The future experimental error in the top quark mass, $\delta m_{t}= \pm 130 \mathrm{MeV}$, induces further uncertainties $\delta m_{W}= \pm 0.8 \mathrm{MeV}$ and $\delta \sin ^{2} \theta_{w}^{\mathrm{eff}}= \pm 0.4 \times 10^{-5}$.

Comparison of an indirect determination of the SM Higgs boson mass, which would be significantly improved by Giga-Z $[39,24,40,5]$, with a future direct measurement will provide a sensitive test of the SM. Table 8.3 [24] summarizes both today's accuracy for the indirect prediction of $m_{h}$ and the accuracy available from the prospective improvements at forthcoming colliders listed in Table 8.2. The current accuracies assume $\delta \Delta \alpha= \pm 2 \times 10^{-4}$ [37], while the future cases assume $\delta \Delta \alpha= \pm 7 \times 10^{-5}$ [38] . The Giga-Z scenario allows an indirect determination of $m_{h}$ with an uncertainty of $\delta m_{h} / m_{h}= \pm 7 \%$ (about the level of the current indirect $m_{t}$ determination). This represents a factor of three improvement over the EW constraints that could be made using LHC measurements, while a linear collider running solely at high energy would provide only a modest gain.

Figure 8.2 compares the potential of Giga-Z for testing the electroweak theory with the present status from both theoretical and experimental standpoints. The SM prediction corresponds to an allowed $m_{h}$ interval of $113 \mathrm{GeV} \leq m_{h} \leq 400 \mathrm{GeV}$ and to an allowed $m_{t}$ interval within its measured uncertainty. The theoretical prediction assumes that the Higgs boson has been found, with masses of 120,150 and $180 \mathrm{GeV}$ considered. The uncertainty induced assuming $\delta m_{t}= \pm 200 \mathrm{MeV}$ and $\delta \Delta \alpha= \pm 7 \times$ $10^{-5}$ is indicated. The figure illustrates that the improved experimental accuracy at Giga-Z will allow tests of the internal consistency of the SM at an unprecedented 


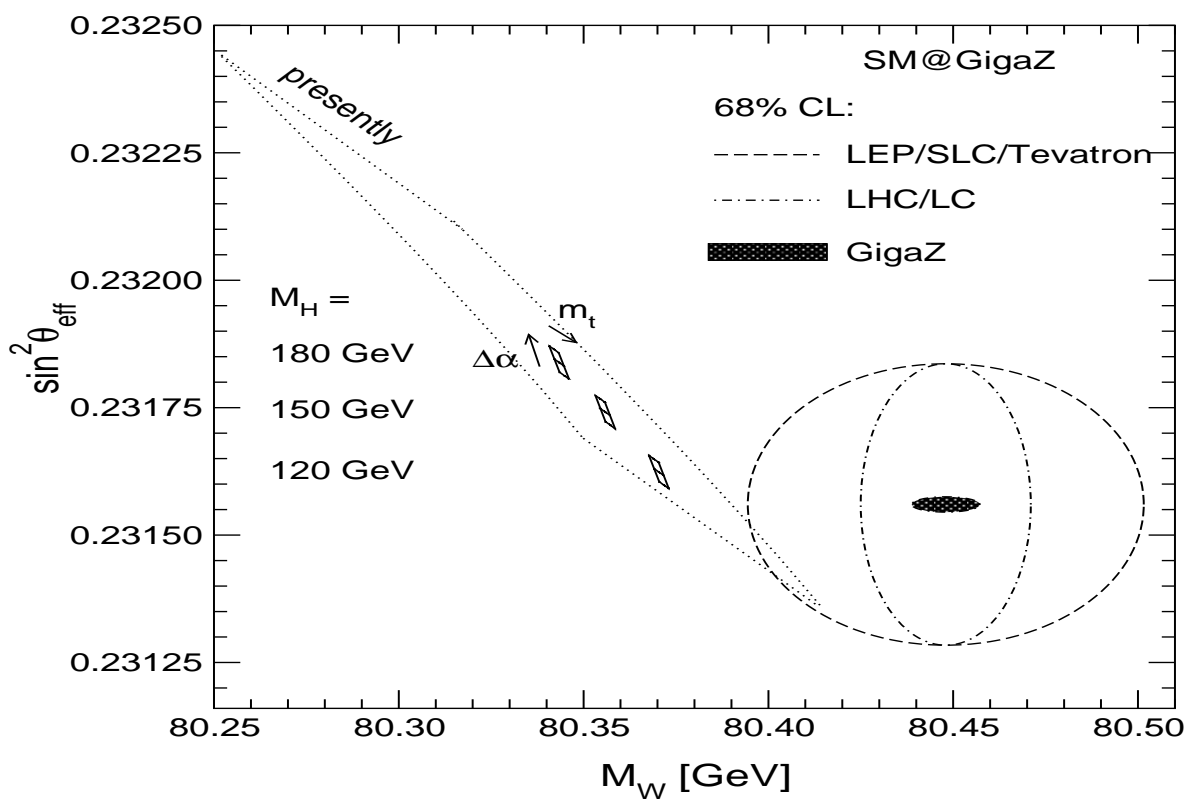

Figure 8.2: The present and prospective future theoretical predictions in the SM (for three $m_{h}$ values) are compared with the current experimental accuracies and those expected from LHC and Giga-Z (see Table 8.2). The future theoretical uncertainties arising from $\delta \Delta \alpha= \pm 7 \times 10^{-5}$ and $\delta m_{t}= \pm 200 \mathrm{MeV}$ are indicated.

level.

\subsection{Parameterizations of deviations from the Standard Model}

The precision achievable at Giga-Z allows for the exploration of possible effects of new physics with great sensitivity. This section is devoted to more general parameterizations of physics beyond the SM through the specific example of the $S, T$, $U$ parameters [41]. While these parameters are widely used, considerable confusion exists concerning their meaning and range of applicability. Because it is important to understand precisely how the effects of new physics can be probed in a sensible way given the potential Giga-Z accuracies, we briefly summarize the main points.

By definition, the $S, T, U$ parameters describe only the effects of new physics contributions that enter via vacuum-polarization effects (i.e., self-energy corrections) to the vector-boson propagators of the SM. That is, the new physics contributions are assumed to have negligible couplings to SM fermions. The parameters can be computed in new models as certain combinations of one-loop self-energies. Experimentally, their values are determined by comparing the measurements, $\mathcal{A}_{i}^{\exp }$, of a 
number of observables with their SM predictions, $\mathcal{A}_{i}^{\mathrm{SM}}$,

$$
\mathcal{A}_{i}^{\mathrm{exp}}=\mathcal{A}_{i}^{\mathrm{SM}}+f_{i}^{\mathrm{NP}}(S, T, U) .
$$

Here $\mathcal{A}_{i}^{\mathrm{SM}}$ contains all known radiative corrections in the SM evaluated at reference values of $m_{t}$ and $m_{h}$. The (linear) function $f_{i}^{\mathrm{NP}}(S, T, U)$ describes the contributions of new physics. For most precision observables, the corrections caused by a variation of $m_{t}$ and $m_{h}$ at one-loop order can also be absorbed into $S, T$, and $U$. A non-zero result for $S, T, U$ determined in this way indicates non-vanishing contributions of new physics (with respect to the SM reference value).

The $S, T, U$ parameters can only be applied for parameterizing effects of physics beyond the SM. To compute the SM predictions to which these parameters provide corrections, one must take into account the full contributions, which also contain vertex and box corrections, since these effects cannot be consistently absorbed into the $S, T, U$ parameters. For a more detailed discussion of this point, see [42]. Because the $S, T, U$ parameters are restricted to the leading-order contributions of new physics, they should only be applied for small deviations from the SM predictions. Their application to cases with large deviations from the SM, like extensions of the SM with a very heavy Higgs boson in the range of several $\mathrm{TeV}$, is questionable. The current experimental values [43] (assuming $m_{t}=173.4 \mathrm{GeV}$ and $m_{h}=100 \mathrm{GeV}$ ) are

$$
S=-0.07 \pm 0.11, \quad T=-0.10 \pm 0.14, \quad U=0.10 \pm 0.15 .
$$

Other parameterizations, defined via linear combinations of various observables without reference to the SM contribution, have been suggested (see, e.g., [44,45]). While any new physics model can be explored, it is not in all cases obvious that studying parameters is of advantage compared to studying the observables themselves. For this reason and for brevity, we restrict our discussion to the $S, T, U$ parameters.

Examples of new physics contributions that can be described in the framework of the $S, T, U$ parameters are contributions from a fourth generation of heavy fermions or effects from scalar quark loops (see Section 3.2). A counterexample going beyond the $S, T, U$ framework is given by corrections of the kind that could bring the prediction for the anomalous magnetic moment of the muon in agreement with the experimental value $[46,47]$.

While many SM extensions result in a vanishing or small contribution to the $U$ parameter (see Ref. [43] and references therein), sizable contributions to $S$ and $T$ can be expected from a number of models. For instance, the contribution of a heavy Higgs boson with $m_{h}=1 \mathrm{TeV}$ gives rise to a contribution in $S$ and $T$ of about $S \approx 0.1$, $T \approx-0.3$ [48] (see however the discussion above). In technicolor models one typically expects $S$ and $T$ to be positive and of order 1 [48]. Peskin and Wells [48] have also examined the 'topcolor seesaw' model of Dobrescu and Hill [49], which predicts little or no new physics observable at the LHC or LC. The Giga-Z scenario, however, would 
reveal a significant departure in the $(S, T)$ plane from the minimal SM with a light Higgs boson.

These additional contributions to the $S, T, U$ parameters have to be compared with the errors with which these parameters can be extracted at Giga-Z [24]:

$$
\Delta S= \pm 0.05, \quad \Delta T= \pm 0.06, \quad \Delta U= \pm 0.04
$$

These parameters are strongly correlated. Assuming $U=0$, as justified above, the anticipated errors in $S$ and $T$ would decrease to about

$$
\Delta S= \pm 0.02, \quad \Delta T= \pm 0.02 .
$$

The increased precision, compared to the present situation given in Eq. (8.6), will constrain or exclude of many possible extensions of the SM.

\subsection{Tests of supersymmetry}

We now explore the utility of the precision electroweak observables in a scenario with direct observation of new particles, by examining a specific example. Suppose that particles compatible with a MSSM Higgs boson and a light scalar top quark $\tilde{t}_{1}$ have been discovered at the Tevatron or the LHC, and further explored at an $e^{+} e^{-}$ linear collider. With the luminosity expected at a linear collider, the $\tilde{t}_{1}$ mass, $m_{\tilde{t}_{1}}$, and the mixing angle in the stop sector, $\cos \theta_{\tilde{t}}$, can be measured in the process $e^{+} e^{-} \rightarrow \tilde{t}_{1} \tilde{t}_{1}^{*}$ to a level below $1 \%[50,51]$.

The precision electroweak variables provide several constraints. First, the measurements and predictions for $m_{W}$ and $\sin ^{2} \theta_{w}^{\text {eff }}$ provide an indirect test of the MSSM, as they do for the SM. Comparison of the predicted to the measured value of the lightest CP-even MSSM Higgs boson mass, $m_{h}$, provides a further constraint. In the MSSM, $m_{h}$ is not a free parameter as in the SM; it is calculable from the other SUSY parameters. Furthermore, because $m_{W}, \sin ^{2} \theta_{w}^{\text {eff }}$ and $m_{h}$ are particularly sensitive to the SUSY parameters of the scalar top and bottom sector and of the Higgs sector, they provide an indirect probe of the masses of supersymmetric particles that might not be seen at the LHC or LC. In particular, the heavier scalar top quark, $\tilde{t}_{2}$, and the heavy Higgs bosons $A, H$ and $H^{ \pm}$could be outside the kinematic reach of the initial-stage LC, and background problems could preclude their observation at the LHC. Reference [24] explores this scenario and demonstrates that upper bounds on $M_{A}$ could be established through the SUSY contributions to $m_{W}$ and $\sin ^{2} \theta_{w}^{\text {eff }}$, just as the Higgs boson mass can be bounded in the SM.

Finally, we examine the indirect information on the mass of the heavier scalar top quark, $m_{\tilde{t}_{2}}$, that can be obtained by requiring consistency of the MSSM with measurements of $m_{W}, \sin ^{2} \theta_{w}^{\text {eff }}$, and $m_{h}$ in addition to those of $m_{\tilde{t}_{1}}$ and $\cos \theta_{\tilde{t}}$. The SUSY contributions to $m_{W}$ and $\sin ^{2} \theta_{w}^{\text {eff }}$ include the complete one-loop results in the 
MSSM [52] as well as the leading higher-order QCD corrections [53]. The prediction for $m_{h}$ is obtained with the program FeynHiggs [54], based on the Feynmandiagrammatic two-loop result of Ref. [55]. A future uncertainty in the theoretical prediction of $m_{h}$ of $\pm 0.5 \mathrm{GeV}$ is assumed.

We examine the scenarios for a $\mathrm{LC}$ with and without the Giga-Z option and for the LHC (see Table 8.2), taking $m_{\tilde{t}_{1}}=180 \pm 1.25 \mathrm{GeV}$ for LC/Giga-Z, and $180 \pm 18 \mathrm{GeV}$ for the LHC. The other parameters have been chosen according to the mSUGRA reference scenario 2 specified in Ref. [56], with the following accuracies: $M_{A}=257 \pm$ $10 \mathrm{GeV}, \mu=263 \pm 1 \mathrm{GeV}, M_{2}=150 \pm 1 \mathrm{GeV}, m_{\tilde{g}}=496 \pm 10 \mathrm{GeV}$. For $\tan \beta$ a lower bound of $\tan \beta>10$ has been taken. The central values for $m_{W}$ and $\sin ^{2} \theta_{w}^{\text {eff }}$ have been chosen in accordance with a non-zero contribution to the precision observables from SUSY loops.

As one can see in Fig. 8.3, the allowed parameter space in the $m_{\tilde{t}_{2}}-\left|\cos \theta_{\hat{t}}\right|$ plane is significantly reduced in the Giga- $Z$ scenario relative to the others. Using the direct information on $\left|\cos \theta_{\hat{t}}\right|$ from Ref. [51] allows an indirect determination of $m_{\tilde{t}_{2}}$ with a precision of better than $5 \%$ in the Giga-Z case. By comparing this indirect prediction for $m_{\tilde{t}_{2}}$ with direct experimental information on the mass of this particle, the MSSM could be tested at its quantum level in a sensitive and highly non-trivial way.

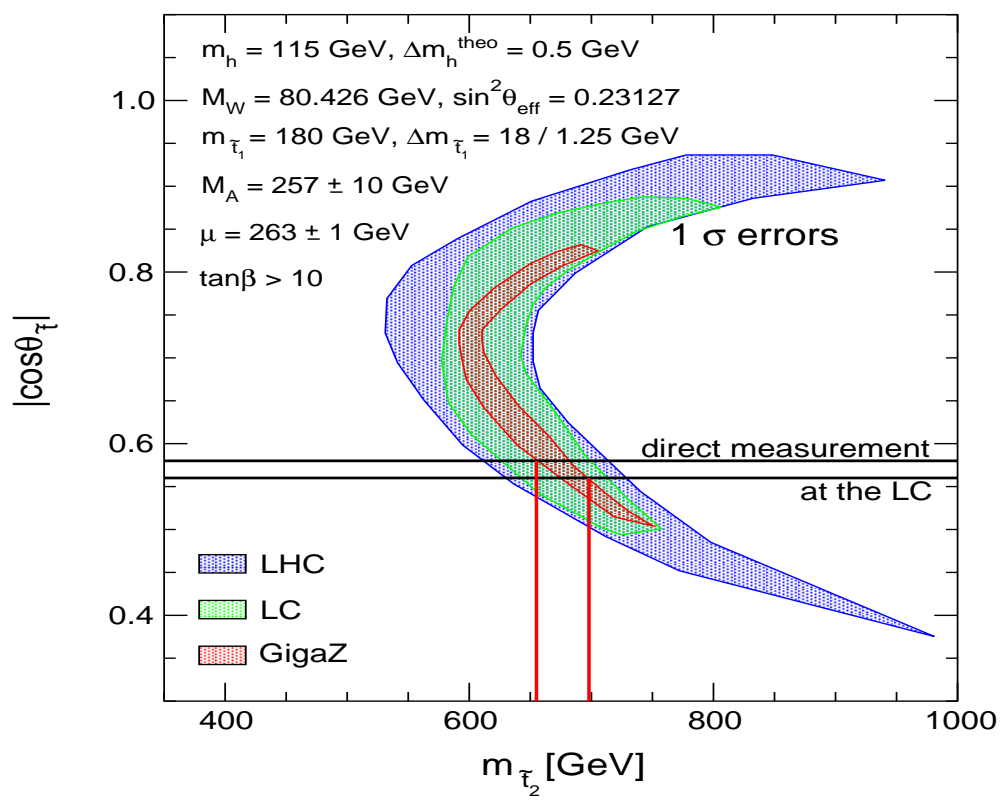

Figure 8.3: Indirect constraints on the MSSM parameter space in the $m_{\tilde{t}_{2}}-\left|\cos \theta_{\tilde{t}}\right|$ plane from measurements of $m_{h}, m_{W}, \sin ^{2} \theta_{w}^{\text {eff }}, m_{t}$ and $m_{\tilde{t}_{1}}$ at a LC with and without the Giga-Z option and at the LHC. The solid lines indicate the direct information on the mixing angle from a measurement at the LC and the corresponding indirect determination of $m_{\tilde{t}_{2}}$. 


\section{Heavy flavor physics}

The $Z$ pole has already been established as an excellent laboratory for the study of $b$ physics. The large boost and resulting detached vertices for the $b$ decays have amply compensated the relatively modest statistics of the LEP experiments, allowing them to make many competitive and important measurements. SLD, with much smaller statistics, has benefitted greatly from the SLC's beam polarization in the $b$ studies that require production tagging and has produced measurements competitive with LEP. The hadronic experiments, LHC-b and BTeV, will be faced with large backgrounds, with typical signal-to-noise ratios of $S / N \approx 5 \times 10^{-3}$ compared to $S / N \approx 0.21$ at the $Z$ (albeit with $10^{4}$ to $10^{5}$ more $b$ 's produced).

The $Z$-pole running will result in a very powerful $b$ experiment. With $80 \%$ and $60 \%$ polarizations for the electron and positron beams, respectively, production flavor tags that include the forward-backward production asymmetry should reach a signal $\times$ purity $\varepsilon D^{2}$ approaching 0.6 . (With $80 \%$ electron polarization and no positron polarization, one finds about half of this value.) For comparison, the $B$ factories have achieved $\varepsilon D^{2} \approx 0.25$ [57] while the hadronic facilities will have rather lower values. Coupled with the excellent resolution expected from the vertex detector for the linear collider, a reach in $\delta m_{s}$ of $40 \mathrm{ps}^{-1}$ is possible with $10^{9} Z$ 's, with a resolution limit of around $80 \mathrm{ps}^{-1}$.

The scenario in which $2 \times 10^{9} Z$ decays are produced, yielding about $6 \times 10^{8} \mathrm{~b}$ hadrons, has been investigated. This sample should be compared to the $\Upsilon(4 S)$ and hadronic $b$ samples that will be available in the same time period $[5,58]$. This section is largely based on a review of such work in [58]. With these statistics, $b$ studies at the $Z$ offer a number of measurements that are of fundamental importance for the comprehensive $b$-physics program that is being undertaken worldwide, but which cannot be addressed adequately at other existing or planned facilities. A longer running period at the $Z\left(10^{10} Z\right.$ 's) is necessary to improve upon the sensitivity for the 'canonical' measurements planned at other $b$ facilities, despite the combined advantages of tagging, boost and purity. Such a facility would be quite competitive. A precision on $\sin 2 \beta$ of about 0.01 would be obtainable, similar to that obtainable from LHC-b and $\mathrm{BTeV}$. If one translates the studies of $B \rightarrow \pi \pi$ to an effective value of $\sin 2 \alpha$, the uncertainty would be about 0.02, approaching that of $\mathrm{BTeV}$ and somewhat better than that expected from LHC-b.

The topics unique to a polarized $Z$ facility are the following:

1. The quark-level transition

$$
b \rightarrow q+\nu \bar{\nu}
$$

could well be affected significantly by new physics in ways quite different from $b \rightarrow q+l^{+} l^{-}$. Searching for $b \rightarrow q \nu \bar{\nu}$ in hadronic colliders appears hopeless. The searches also pose quite a challenge for an $\Upsilon(4 S)$ experiment because of 
Precision Studies at the $Z$ and $W W$ Threshold

the intermingling of the decay productions from the two $B$ decays [59].

2. The CKM elements $|V(c b)|$ and $|V(u b)|$, determined in semileptonic $B$ decay, suffer from a potentially considerable source of uncertainty due to limitations in the validity of quark-hadron duality, of which at present little is known for certain. Detailed comparisons of semileptonic $B_{s}$ and $B_{u, d}$ decays would be invaluable in this respect. The $\Upsilon(4 S)$ machines will not have $B_{s}$ samples, while the hadronic machines will have difficulty providing precise inclusive measurements.

3. The availability of polarized beams will allow production of a huge sample of highly polarized beauty baryons whose weak decays can be analyzed. In this way a determination of the handedness of a quark transition becomes feasible.

The canonical measurements for which $2 \times 10^{9} Z$ 's may be competitive include

1. The transition $b \rightarrow \tau \nu$ contains multiple neutrinos in the final state, with an experimental situation similar to that for $b \rightarrow q+\nu \bar{\nu}$. This measurement determines the product $F_{B}\left|V_{u b}\right|$, and would play a fundamental role in constraints of the CKM matrix. The reach at Giga-Z has not yet been studied.

2. The production flavor tagging from the $Z$ running might offer the most precise measurements of $\mathcal{B}\left(B^{0} \rightarrow \pi^{0} \pi^{0}\right)$ and $\mathcal{B}\left(\bar{B}^{0} \rightarrow \pi^{0} \pi^{0}\right)$, which are of great significance for extracting the angle $\phi_{2}$ or $\alpha$ from the measured CP asymmetry in $B^{0} \rightarrow \pi^{+} \pi^{-}$.

The following subsections elaborate on these points.

\subsection{Measurement prospects for $\mathcal{B}\left(B \rightarrow \pi^{0} \pi^{0}\right)$}

One of the promising strategies for measuring the CKM angle $\alpha$ is the study of the $\mathrm{CP}$ asymmetry in the decay $\mathrm{B}^{0} \rightarrow \pi^{+} \pi^{-}$. The presence of significant 'penguin' contributions to $\mathrm{B} \rightarrow \pi^{+} \pi^{-}$complicates the extraction of $\alpha$ from the measured timedependent $\mathrm{CP}$ asymmetry. The penguin and tree contributions can be separated by measuring the branching ratios $\mathcal{B}\left(\mathrm{B}^{0} \rightarrow \pi^{+} \pi^{-}\right), \mathcal{B}\left(\mathrm{B}^{+} \rightarrow \pi^{+} \pi^{0}\right)$ and $\mathcal{B}\left(\mathrm{B}^{0} \rightarrow \pi^{0} \pi^{0}\right)$ and the charge conjugate modes [60]. The first can be measured as a by-product of the CP-asymmetry analysis, but the other two are more difficult. The need to reconstruct $\pi^{0}$ s makes them extremely challenging for hadron machines. The expected branching ratios are also very small, of order $10^{-6}$, with experimental upper limits of $12.7 \times 10^{-6}$ $\left(\pi^{+} \pi^{0}\right)[61]$ and $9.3 \times 10^{-6}\left(\pi^{0} \pi^{0}\right)[62]$.

The feasibility of measuring these branching ratios at a linear collider was studied [5] using the fast Monte Carlo simulation SIMDET [63]. The reconstructed $B$ mass resolutions were found to be $150 \mathrm{MeV}\left(\pi^{0} \pi^{0}\right)$ and $120 \mathrm{MeV}\left(\pi^{+} \pi^{0}\right)$, dominated by the calorimeter angular resolution. Assuming signal branching ratios of a few 
$10^{-6}$ gives signal samples of about 200 events for $2 \times 10^{9} \mathrm{Z}^{0}$ decays, on top of several hundred events of combinatorial background. This would allow a flavor-independent measurement comparable to that of BABAR or BELLE with about $200 \mathrm{fb}^{-1}[5]$. For the separate $B$ versus the $\bar{B}$ branching fractions, which are needed for the $\alpha$ determination, the factor of two or more improvement in $\varepsilon D^{2}$ at the $Z$ relative to that for the $B$ factories makes these measurements with $10^{9} Z$ 's competitive with, if not better than, those obtainable at the $B$ factories. It should be emphasized that this study was performed with a very crude calorimeter simulation and further background rejection may certainly be possible after more detailed studies.

\section{$4.2 \quad B \rightarrow X_{q} \nu \bar{\nu}$}

The large backgrounds at hadronic machines make measurement of $B \rightarrow X_{q} \nu \bar{\nu}$ impossible there. In an $e^{+} e^{-}$threshold machine, such transitions could be found only at the cost of reconstructing one $B$ more or less fully. At Giga-Z, however, the relative cleanliness of the $Z$, the hemispheric separation of the $b$ quarks, and the well-defined initial state provide powerful tools for discovering and actually measuring properties of such transitions at the $Z$. This is illustrated by the fact that the current upper limit on this decay mode comes from LEP1:

$$
\mathrm{BR}\left(B \rightarrow X_{s} \nu \bar{\nu}\right) \leq 7.7 \times 10^{-4}(\mathrm{ALEPH}) .
$$

New physics can affect $b \rightarrow q l^{+} l^{-}$and $b \rightarrow q \nu \bar{\nu}$ in quite different way for various reasons [64]. For example, new contributions to an effective $b s Z$ vertex would enhance $b \rightarrow q \nu \bar{\nu}$ relative to $b \rightarrow q l^{+} l^{-}$by a large factor, and study of $b \rightarrow q \nu \bar{\nu}$ (with contributions from $b \rightarrow q \nu_{\tau} \bar{\nu}_{\tau}$ ) in addition to $b \rightarrow q e^{+} e^{-}$and $b \rightarrow q \mu^{+} \mu^{-}$can help disentangle new physics scenarios with generation-dependent couplings .

At the $Z$, the statistics will be high enough to make meaningful searches for $B \rightarrow X_{s} \nu \bar{\nu}$. With an inclusive branching fraction in the standard model of about $4 \times 10^{-5}$, and exclusive branching fractions to $K$ and $K^{*}$ of order $10^{-5}$ [64], one can expect a few times $10^{3}$ events in exclusive channels and about $10^{4}$ inclusively. The expected reach, including control of backgrounds such as $b \rightarrow \tau \nu$, is not known at this time, but warrants study.

\subsection{Semileptonic $B_{s}$ decays}

The CKM parameters $V_{c b}$ and $V_{u b}$ play a central role in the prediction of various $\mathrm{CP}$ asymmetries in $B$ and $K$ decays. With precision measurements, constraints on new physics scenarios would be obtained by comparison of the predictions with direct measurements. It is crucial for this program to have reliable determination of $V_{c b}$ and $V_{u b}$, obtained from semileptonic $B$ decays through observables in exclusive and inclusive modes. 
Precision Studies at the $Z$ and $W W$ Threshold

Inclusive measurements play an important role in these determinations. The known uncertainties are estimated at the $5 \%$ level for $V_{c b}$ and at the (10-15)\% level for $V_{u b}$. However, there may be an additional significant source of systematic uncertainty, the validity of quark-hadron duality, which underlies almost all applications of the $1 / m_{Q}$ expansions. A large body of folkloric or circumstantial evidence suggests that duality is a useful and meaningful concept. But for a full evaluation of the data from beauty physics it is essential to know with tested confidence whether the deviations from exact duality in semileptonic transitions arise at the $10 \%$, the $5 \%$, or the $1 \%$ level. It is quite unlikely that this question can be answered by theoretical means alone.

Experimentally, one can probe duality via an independent extraction of $\left|V_{c b}\right|$ in $B_{s}$ decays through measurement of $\Gamma_{S L}\left(B_{s}\right)$. One could also determine the rate for $B_{s} \rightarrow l \nu D_{s}^{*}$, extrapolate to zero recoil, and extract the product $\left|V(c b) F_{B_{s} \rightarrow D_{s}^{*}}(0)\right|$. The form factor can be obtained from the result of the Heavy Quark Expansion

$$
\left|F_{B_{s} \rightarrow D_{s}^{*}}(0)\right| \simeq\left|F_{B \rightarrow D^{*}}(0)\right|
$$

up to $S U(3)$ breaking corrections, which can be estimated.

The physical origin of duality violation would be the accidental presence of a nearby hadronic resonance with appropriate quantum numbers to affect the decay pattern for one of the $B$ mesons. On one hand, this resonance may affect $B_{d} \rightarrow l \nu X_{c}$ and $B_{u} \rightarrow l \nu X_{c}$, but not $B_{s} \rightarrow l \nu X_{c}$; conversely, it may affect $B_{s}$ transitions while having no impact on $B_{u, d}$ channels. If the same value emerged for $|V(c b)|$ in both cases, we would have verified the validity of duality in this case at least. If not, we would not know which, if any, of the values is the correct one, but we would be aware of a serious problem.

Duality violation could exhibit a different pattern in $B \rightarrow l \nu X_{u}$ channels. Here theory also calls for a detailed comparison of $B_{d}$ and $B_{u}$ modes, since one expects a difference in the endpoint region of $B_{d}$ and $B_{u}$ semileptonic decays [65]. Hadronic resonances could affect $B_{d} \rightarrow l \nu X_{u}$ and $B_{u} \rightarrow l \nu X_{u}$ quite differently. In addition, measurements of $B_{s} \rightarrow l \nu X_{u}$, both inclusive and exclusive, would provide crucial cross checks.

\subsection{Weak decays of polarized beauty baryons}

The large polarization asymmetry for $Z$ decay to $b$ quarks implies that beauty baryons produced in $Z$ decays are highly polarized. From $2 \times 10^{9} Z \mathrm{~s}$, one expects about $3 \times 10^{7}$ polarized $b$-flavored baryons. The study of the weak decays of these particles offers a whole new field of dynamical information. The existence of initialstate polarization in $\Lambda_{b}$ decays allows one to analyze the chirality of the quark coupling directly; it also leads to a new program of studying observables revealing direct $\mathrm{CP}$ violation. Charmed baryons also merit study. 
Chapter 8

\begin{tabular}{lcc}
\hline \hline Mode & Branching Ratio & Number of Events \\
\hline$\Lambda_{b} \rightarrow \Lambda_{c} \ell \bar{\nu}_{\ell}$ & $8 \times 10^{-2}$ & $5 \times 10^{6}$ \\
$\Lambda_{b} \rightarrow p \ell \bar{\nu}_{\ell}$ & $8 \times 10^{-4}$ & $5 \times 10^{4}$ \\
$\Lambda_{b} \rightarrow X_{s} \gamma$ & $3 \times 10^{-4}$ & 11000 \\
$\Lambda_{b} \rightarrow \Lambda \gamma$ & $5 \times 10^{-5}$ & 1400 \\
$\Lambda_{b} \rightarrow \Lambda \ell \ell$ & $1 \times 10^{-6}$ & 50 \\
\hline \hline
\end{tabular}

Table 8.4: Expected numbers of events for $\Lambda_{b}$ decays, based on the Standard Model estimates.

A generic analysis of $b \rightarrow s \gamma$ results in two transition operators, mediating the decays

$$
b_{R} \rightarrow s_{L} \gamma, b_{L} \rightarrow s_{R} \gamma
$$

While the second operator is highly suppressed in the SM, by a factor $m_{s} / m_{b}$, these operators could be of comparable size in new physics scenarios, for example, in LeftRight Symmetric models or the MSSM. While the decays of mesons realistically cannot distinguish between these two transitions, a study of the $\Lambda$ polarization in the decay $\Lambda_{b} \rightarrow \Lambda \gamma$ with polarized $\Lambda_{b}$ could probe the SM prediction that the ratio of left- to right-handed couplings is $r \lesssim 0.04$. One measures the asymmetry in the angular distribution defined between the $\Lambda_{b}$ spin and the photon in the parent baryon rest frame. Based on the statistics of Table 8.4, corresponding to roughly 750 fully reconstructed events, the measurement would be sensitive to values of $r$ between 0.5 and 1.9 at the $5 \sigma$ level. For comparison, the sensitivity extends from 0.2 and 4.1 with $10^{10} Z$ 's [66]. It should be noted that the angular asymmetry is a theoretically very clean observable and the extraction of $r$ is essentially limited only by statistics.

A significant non-vanishing contribution of $b_{L} \rightarrow s_{R} \gamma$ would signal the intervention of new physics. One can actually undertake an inclusive polarization study of $\Lambda_{b} \rightarrow \Lambda \gamma+X$ with large statistics; the clean environment of the $Z$ is crucial here. Corresponding studies can be performed with $\Lambda_{b} \rightarrow l^{+} l^{-} X$ with smaller statistics.

Although theoretically less clean, similar angular asymmetries in rare hadronic 2-body decays such as $\Lambda_{\mathrm{b}} \rightarrow \Lambda \phi$ offer a unique opportunity to probe for new physics contributions to four-quark penguin operators with chiralities opposite to those in the SM [66].

As an advantage over experiments with unpolarized $\Lambda_{b}$ baryons, spin correlations between the spin of the $\Lambda_{b}$ and the daughter baryon are fully accessible. It is possible, for example, to distinguish between pseudoscalar and vector transition form factors [67]. This allows for novel, powerful consistency checks of the Standard Model including its $\mathrm{CP}$ and chirality properties.

Semileptonic decays of polarized $\Lambda_{b}$ allow testing of the $V-A$ character of $b$ quarks with unprecedented accuracy and searches for $\mathrm{CP}$ asymmetries in the decay 
Precision Studies at the $Z$ and $W W$ Threshold

spectra. For example, comparison of

$$
\Lambda_{b} \rightarrow l^{-}(p+X)_{\text {no charm }} \quad \text { vs. } \quad \bar{\Lambda}_{b} \rightarrow l^{+}(\bar{p}+X)_{\text {no charm }},
$$

might reveal $\mathrm{CP}$ violation from new physics. In final states with at least three particles $\left(\Lambda_{b} \rightarrow A B C\right)$, one can also form $T$-odd correlations such as

$$
C_{T} \equiv\left\langle\vec{\sigma}_{\Lambda_{b}} \cdot\left(\vec{p}_{A} \times \vec{p}_{B}\right)\right\rangle
$$

with $\vec{p}_{A}, \vec{p}_{B}$ denoting the momenta of $A$ and $B$, respectively, and $\vec{\sigma}_{\Lambda_{b}}$ the $\Lambda_{b}$ polarization. A nonzero value of $C_{T}$ can be due either to $T$ violation or to final-state interactions. Measurement of $\bar{C}_{T}$ in the CP-conjugate process resolves the ambiguity. If $C_{T} \neq \bar{C}_{T}$, one has a signature of direct CP violation. Since these effects are typically quite suppressed in the Standard Model, such studies represent largely a search for new physics. They can be performed in nonleptonic modes

$$
\Lambda_{b}^{0} \rightarrow \Lambda_{c}^{+} \pi^{-} \pi^{0}, p \pi^{-} \pi^{0}, \Lambda K^{+} \pi^{-}
$$

as well as in semileptonic channels containing a $\tau$ lepton, since the effect is proportional to the lepton mass [68].

\section{Summary}

A sample of order $10^{9} Z$ 's will provide important and unique tools in the search for and constraint of physics beyond the Standard Model. The program available with polarized positron beams in particular provides dramatic improvement in the measurement precision of the electroweak observables at the $Z$. This improvement leads to markedly more powerful constraints on Standard Model and new physics scenarios. The polarized $b$-baryon program offers a unique window of exploration for new right-handed couplings. With the statistics and $b$-tagging capabilities available with two polarized beams, running for several years $\left(10^{10} Z\right.$ 's) could provide a $b$ physics program rivaling the proposed hadronic experiments in some fundamental CKM measurements.

Without positron polarization, significant gains can still be made. Much of the $b$ physics would suffer only from a decrease in statistics. Impact on the $\Lambda_{b}$ asymmetry measurements needs to be evaluated. The improvement in $\Delta A_{L R}$ is still significant and useful. The most damaging aspect could be the loss of the $m_{W}$ determination from threshold running, for which it is unclear that a $5-6 \mathrm{MeV}$ determination would be realistic without positron polarization. This impact still needs study. 


\section{References}

[1] P. C. Rowson and M. Woods, hep-ex/0012055.

[2] The LEP Collaborations, the LEP Electroweak Working Group, and the SLD Heavy Flavor and Electroweak Groups, A Combination of Preliminary Electroweak Measurements and Constraints on the Standard Model, CERN-EP/2001021.

[3] M. Winter, Determination of the strong coupling constant at Giga-Z, LC-PHSM2001-016.

[4] K. Mönig, Measurement of the Differential Luminosity using Bhabha events in the Forward-Tracking region at TESLA, LC-PHSM-2000-060.

[5] R. Hawkings and K. Monig, Eur. Phys. J. C8, 1 (1999) [hep-ex/9910022].

[6] K. Abe et al. [SLD Collaboration], Phys. Rev. Lett. 84, 5945 (2000) [hepex/0004026].

[7] A. Blondel, Phys. Lett. B202 (1988) 145.

[8] G. Buchalla, G. Hiller and G. Isidori, Phys. Rev. D63, 014015 (2001) [hep$\mathrm{ph} / 0006136]$.

[9] L3 note 2416, contributed paper to the Int. Europhysics Conference High Energy Physics 99, 15-21 July, 1999, Tampere, Finland.

[10] The LEP collaborations ALEPH, DELPHI, L3, OPAL, the LEP Electroweak Working Group and the SLD Flavor and Electroweak Group, CERN-EP/2000016.

[11] K. del Signore for the CDF and D $\varnothing$ Collaborations, The Future Collider Physics Program at Fermilab: Run II and TeV 33, preprint FERMILAB-Conf-98/221-E, published in the Proceedings of the 33rd Rencontres de Moriond: QCD and High Energy Hadronic Interactions, Les Arcs, France, 1998.

[12] ATLAS Collaboration, "ATLAS: Detector and physics performance technical design report. Volume 1,", CERN-LHCC-99-14; S. Keller and J. Womersley, hep-ph/9711304.

[13] Z. Kunszt et al., CERN 96-01, pp 141-206.

[14] R. Barate et al. [ALEPH Collaboration], Eur. Phys. J. C17, 241 (2000).

[15] P. Abreu et al. [DELPHI Collaboration], Phys. Lett. B462, 410 (1999).

[16] G. Abbiendi et al. [OPAL Collaboration], hep-ex/0009018.

[17] L3 Collaboration, L3 Note 2637, 2001.

[18] V. S. Fadin, V. A. Khoze, A. D. Martin and W. J. Stirling, Phys. Lett. B363, 112 (1995) [hep-ph/9507422].

[19] G. W. Wilson, Proceedings, Linear Collider Workshop, Sitges 1999, LC-PHSM2001-009. 
Precision Studies at the $Z$ and $W W$ Threshold

[20] G. W. Wilson, talk presented at "Linear Collider Workshop 2000", Fermi National Accelerator Laboratory, October, 2000.

[21] A. Ballestrero et al., J. Phys. G24, 365 (1998).

[22] G. W. Wilson, talk presented at ECFA/DESY workshop, Munich, 1996.

[23] R. Brinkmann et al., Nucl. Instrum. Meth. A406, 13 (1998) [hep-ex/9707017].

[24] J. Erler, S. Heinemeyer, W. Hollik, G. Weiglein, and P. M. Zerwas, Phys. Lett. B486, 125 (2000).

[25] A. Sirlin, Phys. Rev. D22, 971 (1980); W. Marciano and A. Sirlin, Phys. Rev. D22, 2695 (1980).

[26] A. Djouadi and C. Verzegnassi, Phys. Lett. B195, 265 (1987); A. Djouadi, Nuov. Cim. A100, 357 (1988); B. Kniehl, Nucl. Phys. B347, 89 (1990); F. Halzen and B. Kniehl, Nucl. Phys. B353, 567 (1991); B. Kniehl and A. Sirlin, Nucl. Phys. B371, 141 (1992); A. Djouadi and P. Gambino, Phys. Rev. D49, 3499 (1994).

[27] K. Chetyrkin, J. Kühn and M. Steinhauser, Phys. Lett. B351, 331 (1995); Phys. Rev. Lett. 75, 3394 (1995); L. Avdeev, J. Fleischer, S. Mikhailov and O. Tarasov, Phys. Lett. B336, 560 (1994); E: Phys. Lett. B349, 597 (1995).

[28] U. Baur and M. Demarteau, hep-ph/9611334, and references therein.

[29] J. F. Gunion et al., hep-ph/9703330.

[30] S. Haywood, P. R. Hobson, W. Hollik, Z. Kunzst et al., Electroweak Physics, hep-ph/0003275, in CERN-YR-2000/01, eds. G. Altarelli and M. L. Mangano and references therein.

[31] M. Beneke, I. Efthymiopoulos, M. L. Mangano, J. Womersley, Top Quark Physics, hep-ph/0003033, in CERN-YR-2000/01, eds. G. Altarelli and M. L. Mangano and references therein.

[32] H. Haber et al., hep-ph/9703391.

[33] R. Frey et al., hep-ph/9704243; P. Igo-Kemenes et al., Proceedings, LC Workshop 1991/93, DESY 92/93-123; A. Hoang et al., to appear in EPJdirect C, hepph/0001286.

[34] G. Wilson, Proceedings, Linear Collider Workshop, Sitges 1999, LC-PHSM-2001009.

[35] A. Freitas, W. Hollik, W. Walter and G. Weiglein, Phys. Lett. B495, 338 (2000); A. Freitas, S. Heinemeyer, W. Hollik, W. Walter and G. Weiglein, Nucl. Phys. Proc. Suppl. 89, 82 (2000); hep-ph/0101260.

[36] D. Bardin, M. Grünewald and G. Passarino, hep-ph/9802452.

[37] M. Davier and A. Höcker, Phys. Lett. B435, 427 (1998); J. Kühn and M. Steinhauser, Phys. Lett. B437, 425 (1998); J. Erler, Phys. Rev. D59, 054008 (1999); F. Jegerlehner, in Proceedings of the IVth International Symposium on Radiative Corrections, ed. J. Solà (World Scientific, Singapore, 1999), hep-ph/9901386; 
B. Pietrzyk, talk given at ICHEP 2000, Osaka, July 2000, to appear in the proceedings; A. D. Martin, J. Outhwaite and M.G. Ryskin, hep-ph/0012231.

[38] F. Jegerlehner, LC-TH-2001-035.

[39] S. Heinemeyer, T. Mannel and G. Weiglein, hep-ph/9909538.

[40] J. Erler and S. Heinemeyer, hep-ph/0102083.

[41] M. Peskin and T. Takeuchi, Phys. Rev. D46, 381 (1992).

[42] A. Denner, S. Dittmaier and G. Weiglein, in Proceedings of the Ringberg Workshop "Perspectives for electroweak interactions in $e^{+} e^{-}$collisions", ed. B. A. Kniehl (World Scientific, Singapore, 1995), p. 281, hep-ph/9505271.

[43] J. Erler and P. Langacker, Electroweak Model and Constraints on New Physics, in: Review of Particle Properties, Particle Data Group, Eur, Phys. J. 15 (2000).

[44] G. Altarelli and R. Barbieri, Phys. Lett. B253, 161 (1990); G. Altarelli, R. Barbieri and F. Caravaglios, Nucl. Phys. B405, 3 (1993).

[45] S. Dittmaier, K. Kolodziej, M. Kuroda and D. Schildknecht, Nucl. Phys. B426, 249E (1994): B446, 334 (1995); S. Dittmaier, M. Kuroda and D. Schildknecht, Nucl. Phys. B448, 3 (1995).

[46] H. N. Brown et al., Muon g-2 Collaboration, hep-ex/0102017.

[47] A. Czarnecki and W. J. Marciano, hep-ph/0102122.

[48] M. Peskin and J. Wells, hep-ph/0101342.

[49] B. A. Dobrescu and C. T. Hill, Phys. Rev. Lett. 81, 2634 (1998) [hepph/9712319]; R. S. Chivukula, B. A. Dobrescu, H. Georgi and C. T. Hill, Phys. Rev. D59, 075003 (1999) [hep-ph/9809470].

[50] G. Blair and U. Martyn, Proceedings, Linear Collider Workshop Sitges 1999, hep-ph/9910416.

[51] A. Bartl, H. Eberl, S. Kraml, W. Majerotto, W. Porod, and A. Sopczak, Z. Phys. C76, 549 (1997); A. Bartl, H. Eberl, S. Kraml, W. Majerotto and W. Porod, Proceedings, Linear Collider Workshop Sitges 1999, hep-ph/9909378; M. Berggren, R. Keränen, H. Nowak and A. Sopczak, Proceedings, Linear Collider Workshop Sitges 1999, hep-ph/9911345.

[52] P. Chankowski, A. Dabelstein, W. Hollik, W. Mösle, S. Pokorski and J. Rosiek, Nucl. Phys. B417, 101 (1994); D. Garcia and J. Solà, Mod. Phys. Lett. A9, 211 (1994); D. Garcia, R. Jiménez and J. Solà, Phys. Lett. B347, 309 (1995); A. Dabelstein, W. Hollik and W. Mösle, hep-ph/9506251; P. Chankowski and S. Pokorski, Nucl. Phys. B475, 3 (1996); W. de Boer, A. Dabelstein, W. Hollik, W. Mösle and U. Schwickerath, Z. Phys. C75, 625 (1997).

[53] A. Djouadi, P. Gambino, S. Heinemeyer, W. Hollik, C. Jünger and G. Weiglein, Phys. Rev. Lett. 78, 3626 (1997); Phys. Rev. D57, 4179 (1998).

[54] S. Heinemeyer, W. Hollik and G. Weiglein, Comp. Phys. Comm. 124, 76 (2000). 
[55] S. Heinemeyer, W. Hollik and G. Weiglein, Phys. Rev. D58, 091701 (1998); Phys. Lett. B440, 296 (1998); Eur. Phys. Jour. C9, 343 (1999).

[56] http://wwwhephy . oeaw.ac.at/susy/lcws.html

[57] B. Aubert et al. [BABAR Collaboration], hep-ex/0102030.

[58] A. Ali, D. Benson, I. Bigi, R. Hawking and T. Mannel, preprint hep-ph/0012218.

[59] J. Alexander, private communication.

[60] D. London, N. Sinha and R. Sinha, Phys. Rev. D63, 054015 (2001); M. Gronau and D. London, Phys. Rev. Lett. 65, 3381 (1990).

[61] CLEO collaboration, D. Cronin-Hennessy et al., Phys. Rev. Lett. 85, 515 (2000).

[62] CLEO collaboration, R. Godang et al., Phys. Rev. Lett. 80, 3456 (1998).

[63] SIMDET version 3, A Parametric Monte Carlo for a TESLA Detector, M. Pohl and H.J. Schreiber, DESY preprint 99-030.

[64] Y. Grossman, Z. Ligeti and E. Nardi, Nucl. Phys. B465, 369 (1996); A. Ali, C. Greub and T. Mannel, DESY-93-016, in Proc. of ECFA Workshop on the Physics of a B Meson Factory, eds. R. Aleksan, A. Ali, 1993.

[65] I. Bigi and N. Uraltsev, Nucl. Phys. B423, 33 (1994).

[66] G. Hiller and A. Kagan, SLAC-PUB-8752.

[67] S. Pakvasa, S.Tuan and S. Rosen, Phys. Rev. D42, 3746 (1990).

[68] D. Benson and I. Bigi, in preparation. 


\section{Pathways Beyond the Standard Model}




\section{Chapter 9 Pathways Beyond the Standard Model}

\section{Introduction}

Over the past 30 years or so, high energy physics experiments have systematically explored the behavior of the strong, electromagnetic and weak interactions. For the strong interactions, QCD is generally accepted as the correct description, and research on QCD has shifted to its application to special regimes such as diffractive and exclusive processes and the quark-gluon plasma. For the electromagnetic and weak interactions, the progress of the past decade on $W, Z$, top, and neutrino physics has demonstrated that their structure is understood with high precision.

Our current picture of the electroweak interactions requires spontaneous gauge symmetry breaking. As yet, there is no direct evidence on the means by which the gauge symmetry is broken. It is remarkable that all of the evidence accumulated to date is consistent with the Standard Model (SM) in which this symmetry breaking is due to a single elementary scalar field, the Higgs field, which generates the masses of the $W$ and $Z$ bosons and the quarks and leptons.

However, many features of this simple theory are inadequate. The Higgs field is an ad hoc addition to the SM. Its mass and symmetry-breaking expectation value are put in by hand. The quark and lepton masses are generated by arbitrary couplings to the Higgs field. The existence of three generations of quarks and leptons is not explained, nor is the dramatic lack of symmetry in the masses and mixings of these generations.

To explain these features, it is necessary to extend the SM. These extensions, in turn, predict new particles and phenomena. The compelling motivation for new experiments at the highest energies is to discover these phenomena and then to decipher them, so that we can learn the nature of the new laws of physics with which they are associated.

In this document, we are exploring the physics case for a next-generation $e^{+} e^{-}$ linear collider. To make this case, it is necessary to demonstrate that the linear collider can have an important impact on our understanding of these new phenomena. The argument should be made broadly for models of new physics covering the whole range of possibilities allowed from our current knowledge. It should take into account new information that we will learn from the Tevatron and LHC experiments which will be done before the linear collider is completed.

Our purpose in this chapter is to give an overview of possibilities for new physics beyond the SM. Our emphasis will be on general orientation to the pathways that one

might follow. We will then explain the relevance of the linear collider measurements 
to each possible scenario. We encourage the reader to consult the relevant chapter of the 'Sourcebook', Chapters 3-8, to see how each quantity we discuss is measured at a linear collider and why the experimental precision that we expect is justified.

The essay is organized as follows: In Section 2, we discuss the general principle that we use to organize models of new physics. In Sections 3 and 4, we discuss models of new physics in the typical dichotomy used since the 1980's: on the one hand, models with supersymmetry, on the other hand, models with new strong interactions at the $\mathrm{TeV}$ scale. In Section 5, we discuss a new class of models for which the key ingredient is the existence of extra spatial dimensions. It is now understood that these models stand on the same footing as the more traditional schemes and, in fact, address certain of their weaknesses. Section 6 gives some conclusions.

\section{Beyond the Standard Model}

We first discuss some general principles regarding physics beyond the Standard Model.

From an experimental point of view, it is necessary to study the interactions of the observed particles at higher energies and with higher accuracy. This may lead to the discovery of new particles, in which case we need to study their spectrum and determine their interactions. Alternatively, it may lead to the observation of anomalous properties of the observed particles, in which case we could infer the existence of new particles or phenomena responsible for these effects. After this information is obtained in experiments, we must attempt to reconstruct the structure of the underlying theory. The linear collider is a crucial complement to the LHC in ensuring that the experimental information is extensive and precise enough for this goal to be achieved.

From the theoretical point of view, different ideas lead to models that provide challenges to this experimental program. To discuss the range of possible models, an organizing principle is needed. We will organize our discussion around the major question that we believe most strongly motivates new physics at the $\mathrm{TeV}$ scale. This is the stability crisis in the SM explanation for electroweak symmetry breaking. In technical terms, this is the problem that the Higgs boson mass is extremely sensitive to physics at very high energy scales. In the SM, the effect of quantum fields at the energy scale $M$ is an additive contribution to the Higgs boson mass term of order $M^{2}$. More physically, this is the problem that not only the magnitude but even the sign of the Higgs boson mass term is not predicted in the SM, so that the SM cannot explain why the electroweak gauge symmetry is broken. From either perspective, this problem suggests that the SM is a dramatically incomplete picture of electroweak symmetry breaking. It is for this reason that we believe that new physics must appear at the $\mathrm{TeV}$ scale. We expect that the physics will be more exciting than simply the 
Pathways Beyond the Standard Model

production of some random new particles. The solution of the stability crisis will involve completely new principles of physics. These principles will be reflected in the spectrum and properties of the new particles, and in their interactions. Much as the discovery of the $J / \psi$ convincingly brought together many different elements of the SM in a coherent picture, so the discovery and study of these new states will spur us on to the construction of a new theory that will displace the SM.

We will use the idea of solving the stability crisis to guide our classification of the various models of new physics. The three approaches to this problem that have received the most study are supersymmetry, strongly coupled theories, and extra dimensions. The common theme in all three proposed solutions is that additional particle states and dynamics must be present near the electroweak scale. We briefly describe each approach, summarizing in each case the types of new interactions expected and the key experimental issues they raise.

Each possible model of new physics must be approached from the viewpoint expressed at the beginning of this section, that of dissecting experimentally the spectrum of new particles and their interactions. We take particular note of the important strengths that the linear collider brings to disentangling the physics of these models. We will see that, in most cases, the linear collider not only contributes but is essential to forming this experimental picture. Even if none of the specific models we discuss here is actually realized in Nature, this exercise illustrates the importance of the linear collider in unraveling the new world beyond the SM.

\section{Supersymmetry}

One attempt to cure the stability crisis of the Higgs field is to introduce a new symmetry - supersymmetry - which relates fermions and bosons. To realize this symmetry in Nature, there must exist supersymmetry partners for each of the known SM particles. Further, supersymmetry must be broken in the ground state so that these superpartners are more massive than ordinary particles. The Higgs mass terms are then not sensitive to mass scales above the superpartner masses. The Higgs field vacuum expectation value is naturally of order $100 \mathrm{GeV}$ if the superpartner masses are also near this energy scale.

The existence of superpartners implies a rich program for future accelerators. The phenomenology of supersymmetry has been studied in great detail in the literature. Dozens of papers have been written on the technical ability of linear collider experiments to discover and study supersymmetric theories of many different forms. This material is reviewed systematically in Chapter 4 of this book. Different patterns of supersymmetry breaking masses can yield substantially different phenomenology at a high-energy collider. Supersymmetry is not a dot on the theoretical landscape, but rather contains a tremendously varied range of possibilities to be searched for and 
studied at all available high-energy collider facilities.

In the remainder of this section, we summarize the most important issues for the study of supersymmetry and the relevant measurements that can be done at a linear collider. It is important to keep in mind that we are likely to be surprised with the spectrum that Nature ultimately gives us. The linear collider's ability to cleanly disentangle the superpartner mass spectrum and couplings would be extremely important when the surprises occur. Of course, this is relevant only if the linear collider has sufficiently high center-of-mass energy to produce the superpartners. Section 2 of Chapter 4 reviews the expectations for the masses of superpartners and gives estimates of what center-of-mass energies should be required.

Mass measurements of accessible sparticles. If supersymmetry is relevant for electroweak symmetry breaking, then some of the superpartners should be discovered at the LHC. Furthermore, the experiments at the LHC should be able to accurately measure some masses or mass differences of the SUSY spectrum. This issue is reviewed in Chapter 4, Section 7. However, the systematic measurement of the SUSY spectrum requires a linear collider.

Superpartner masses are measured at a linear collider in three main ways: from distributions of the products of an on-shell superpartner decay, from threshold scans, and from contributions of virtual superpartners to cross sections or decay amplitudes. When sleptons, charginos, and neutralinos are produced on-shell, their masses will typically be measured to within about $1 \%$. Even if the lightest neutralino LSP is not directly observed, its mass should be measurable to within $1 \%$ from these kinematic distributions. Threshold scans of sleptons in $e^{+} e^{-}$collisions and especially in $e^{-} e^{-}$ collisions may yield mass measurements to within one part in a thousand. Indirect off-shell mass measurements are more model-dependent but have power in specific applications. For example, the $t$-channel sneutrino contribution to chargino pair production may allow the presence of the sneutrino to be deduced when its mass is as high as twice the center-of-mass energy of the collider. These techniques are reviewed in more detail in Chapter 4, Section 3.

Slepton and squark quantum numbers and mixing angles. When sparticle mixing can be ignored, the cross sections for pair production of squarks and sleptons at a linear collider are precisely determined by the SM quantum numbers. This should allow unambiguous checks of the quantum numbers and spins for sparticles of the first two generations. In particular, it is straightforward to distinguish the superpartners of left- and right-handed species (e.g., $\tilde{e}_{L}$ from $\tilde{e}_{R}$ ) by cross section measurements with polarized beams. Third-generation sleptons and squarks are likely to be the most strongly mixed scalars of supersymmetry, forming mass eigenstates $\tilde{\tau}_{1,2}, \tilde{b}_{1,2}$, and $\tilde{t}_{1,2}$. Separation of these eigenstates and accurate measurement of their masses are difficult at the Tevatron and LHC but present no extraordinary problems to a linear collider. By combining direct mass measurements with polarization asymmetries for 
Pathways Beyond the Standard Model

the production of these sparticles, we can determine the mixing angle needed to form the observed mass eigenstates from the left- and right-handed weak-interaction eigenstates. The uncertainty in this determination depends on the parameters of the theory, but it has been demonstrated for some cases that the error is lower than $1 \%$.

Chargino/neutralino parameters. The neutralino and chargino states may be strongly mixed combinations of gauge boson and Higgs boson superpartners. The mass matrix is determined by four parameters of the underlying Lagrangian: $M_{1}$ (bino mass), $M_{2}$ (wino mass), $\mu$ (supersymmetric higgsino mass) and $\tan \beta$ (ratio of Higgs vacuum expectation values). Precision measurements of masses, mixing angles, and couplings associated with chargino and neutralino production can supply the information to determine these four important underlying parameters of supersymmetry. For example, measurements of chargino production alone can, in some cases, determine $\tan \beta$ to better than $10 \%$ with only $100 \mathrm{fb}^{-1}$ of data. The parameters $M_{1}$, $M_{2}$, and possibly $\mu$ can be determined at the percent level in large portions of the accessible supersymmetry parameter space.

Coupling relations. To establish supersymmetry as a principle of Nature, it is important to verify some of the symmetry relations that that principle predicts. An essential consequence of supersymmetry is that the couplings of sparticles to gauginos are equal to the corresponding couplings of particles to gauge bosons. It has been demonstrated that this equality can be tested at a linear collider to levels better than $1 \%$ for weakly interacting sparticles. The precision is sufficiently good that one can even contemplate measuring the tiny deviations from coupling equivalence that are caused by supersymmetry-breaking effects in loop corrections. This can give an estimate of the masses of unobserved sparticles with mass well above the collider energy, in the same way that the current precision measurements predict the mass of the Higgs. This issue is reviewed in Chapter 4, Section 4.

$C P$ violating phases. The SM apparently does not have enough $\mathrm{CP}$ violation to account for the baryon asymmetry in the universe. Supersymmetry has parameters that may introduce additional sources of $\mathrm{CP}$ violation into the theory. Testing for the existence of such phases would be an important part of a full supersymmetry program. It has been shown that the linear collider can determine evidence for additional non-zero CP-violating phases in supersymmetric theories if the phases are large enough $\left(\phi_{i} \sim 0.1\right)$, even accounting for the constraints from electric dipole moment measurements.

Lepton number violation. Recent data suggest that neutrinos have non-zero masses and mixings. This implies that non-zero lepton flavor angles should be present for leptons, in parallel with the CKM angles for the quarks. These rotation angles are difficult to measure using high-energy leptons because neutrinos are invisible and are summed over in most observables. However, these angles could be detected from 
superpartner decays, such as $\tilde{\mu}^{+} \tilde{\mu}^{-} \rightarrow e^{+} \mu^{-} \tilde{\chi}_{1}^{0} \tilde{\chi}_{1}^{0}$. A linear collider can use these measurements to probe the lepton flavor angles with greater sensitivity than any existing experiment in some parts of parameter space.

Complete spectrum. The LHC will be a wonderful machine for the discovery of many supersymmetric sparticles in large regions of parameter space. The linear collider can add to the superpartner discoveries at the LHC by detecting states that are not straightforward to observe in the $p p$ environment. The discovery abilities of the linear collider begin to be important at energies above LEPII and become increasingly important at energies of $500 \mathrm{GeV}$ and beyond. One example of this is slepton studies. Sleptons with masses above about $300 \mathrm{GeV}$ will be difficult to find at the LHC, especially if they are not produced copiously in the cascade decays of other strongly-interacting superpartners. Furthermore, if the left- and right-sleptons are close in mass to each other they will be difficult to resolve. The linear collider produces sleptons directly if the CM energy is sufficient. The two species of sleptons are readily distinguished using beam polarization and other observables. Another discovery issue arises in the case of a neutral wino or higgsino LSP, with a nearly degenerate charged $\widetilde{W}^{ \pm}$just above it in mass. The wino case occurs, for example, in anomaly-mediated and in $U(1)$-mediated supersymmetry breaking. In the limit in which all other superpartners are too massive to be produced at the LHC or LC, the linear collider with energy above $500 \mathrm{GeV}$ and $100 \mathrm{fb}^{-1}$ is expected to have a higher mass reach than the LHC for these states. There are other important cases, such as R-parity-violating supersymmetry, in which the linear collider is needed to discover or resolve states of the supersymmetry spectrum.

Supersymmetry and Higgs bosons. The minimal supersymmetric extension of the SM (MSSM) predicts that at least one scalar Higgs boson $\left(h^{0}\right)$ must have mass below about $135 \mathrm{GeV}$. The mass is controlled at tree-level by the $Z$-boson mass, and at one loop by the logarithm of superpartner masses. The prediction of a light Higgs boson has two virtues: it is a useful falsifiable test of the MSSM, and fits nicely within the upper bound from the current precision EW data. Over much of the parameter space, the light MSSM Higgs boson behaves very similarly to the SM Higgs boson.

The other physical scalar Higgs states of the MSSM are $H^{0}, A^{0}$, and $H^{ \pm}$. Unlike the $h^{0}$ state, these Higgs bosons receive tree-level masses directly from supersymmetry breaking parameters. Therefore, it is not possible to rigorously establish upper bounds to their masses. In large parts of parameter space, the masses of these particles are above $300 \mathrm{GeV}$, and the only important production processes in $e^{+} e^{-}$annihilation are the pair-production reactions $e^{+} e^{-} \rightarrow H^{+} H^{-}, H^{0} A^{0}$. Thus, these particles may not appear at the first-stage linear collider.

If the heavy Higgs boson are not seen directly, the effects of the more complicated Higgs sector of the MSSM can be observed by measuring slight deviations in the couplings of $h^{0}$ to fermions and gauge bosons from those predicted for a SM Higgs 
Pathways Beyond the Standard Model

boson. The more massive the heavy Higgs bosons are, the more $h^{0}$ behaves like the SM Higgs boson. Nevertheless, inconsistency with the SM can be discerned by precision measurements at the LC over much of the parameter space, even when $m_{A^{0}}$ is significantly higher than $\sqrt{s} / 2$ and out of reach of direct production. This issue is discussed in Chapter 3, Section 8. It demonstrates again the importance of precision Higgs boson measurements to pointing the way to new physics at higher mass scales.

Probing supersymmetry breaking. Finally, precision measurements of supersymmetry masses and mixing angles serve a purpose beyond simply determining what Lagrangian applies to the energy region around the weak interaction scale. Careful measurements can reveal a pattern characteristic of a more fundamental theory. For example, masses measured at the weak scale can be evolved using the renormalization group to a higher scale, where they might be seen to be unified or to fit another simple relation. A pattern that emerged from this study would point to a specific theory of supersymmetry breaking, indicating both the mechanism and scale at which it occurs. This study could also support or refute the hypothesis that our world is derived from a perturbative grand unified theory with an energy desert, a hypothesis that does seem to apply to the precisely known gauge couplings measured at $m_{Z}$. The ability of a linear collider to test these tantalizing ideas with precision measurements provides a route by which we can climb from the weak scale to a more profound theory operating at much higher energies.

\section{New strong interactions at the $\mathrm{TeV}$ scale}

A second way to cure the stability crisis of the Higgs field and to explain the origin of electroweak symmetry breaking is to introduce a new set of strong interactions that operate at the $\mathrm{TeV}$ scale of energies. In models of this type, symmetry breaking arises in the weak interactions in the same way that it arises in well-studied solidstate physics systems such as superconductors. Just as in those systems, the physics responsible for the symmetry breaking has many other consequences that lead to observable phenomena at the energy scale of the new interactions.

Two quite distinct implementations of this line of thought have been actively pursued. The first follows the possibility that the Higgs doublet (i.e., the four degrees of freedom which after electroweak symmetry breaking become the Higgs boson and the longitudinal components of the $W^{ \pm}$and $Z^{0}$ ) is a bound state that arises from a short-range strongly coupled force. Theories that have this behavior are generically called 'composite Higgs' models. These models are usually well approximated at low energies by the SM, and therefore are consistent with the electroweak data.

The second implementation follows the possibility that the new strong interactions do not generate a Higgs doublet, even as a bound state. This is possible if the 
electroweak symmetry is broken by the pair-condensation of some new strongly interacting particles. The prototype of such theories is 'technicolor', an asymptotically-free gauge interaction that becomes strong at the $\mathrm{TeV}$ scale. The behavior of technicolor theories below the $\mathrm{TeV}$ scale is typically very different from that of the SM. In most cases, there is no Higgs boson with an observable coupling to pairs of $Z$ bosons, and the new symmetry-breaking interactions generate substantial corrections to precision electroweak observables.

The linear collider experiments that directly test these two theoretical pictures are reviewed in detail in Chapter 5, Sections 3 and 4. In this section we briefly discuss the two ideas in general terms and discuss the relevance of the linear collider for uncovering and studying these new interactions.

\subsection{Composite Higgs models}

Several ways have been suggested in the literature to form a bound-state Higgs boson that mimics the properties of the Higgs particle of the SM. In the top-quark seesaw theory, the Higgs boson arises as a bound state of the left-handed top quark and the right-handed component of a new heavy vector-like quark. Although the composite Higgs boson mass is typically about $500 \mathrm{GeV}$, there is agreement with the precision electroweak data for a range of parameters in which new contributions from the additional heavy quark compensate the effects of a heavy Higgs boson. Depending on the binding interactions, an extended composite Higgs sector may form. In this case, mixing among the CP-even scalar bound states may bring the SM-like Higgs boson down to a mass below $200 \mathrm{GeV}$.

Another scenario that may lead to a composite Higgs boson is the SM in extra spatial dimensions, a case that we will discuss in more detail in the next section. Here the short-range strongly-coupled force is given by the Kaluza-Klein excited states of the $S U(3)_{C} \times S U(2)_{W} \times U(1)_{Y}$ gauge bosons. The Kaluza-Klein states of the top quark become the constituents of the Higgs boson. The Higgs boson in this scenario has a mass of order $200 \mathrm{GeV}$.

We now list a number of non-standard phenomena that are likely to appear in these theories at relatively low energies. Of course, these theories will ultimately be tested by going to the energy scale of the new interaction and determining its nature as a gauge theory or as a field theory of some other type.

Deviations in Higgs sector. In models in which the Higgs boson appears as a bound state, it is likely that additional composite scalar states will also be present at the $\mathrm{TeV}$ scale or below. If these states appear, their masses and couplings will provide important information on the nature of the constituents. Additional states with the quantum numbers of the Higgs boson can be produced at a linear collider in association with a $Z^{0}$ or singly in $\gamma \gamma$ collisions. Other states can be studied in pairproduction. In both cases, the precise measurement of their masses and branching 
Pathways Beyond the Standard Model

ratios will provide important information. In addition, it is possible at a linear collider to recognize even very small deviations of the properties of the Higgs boson from the predictions of the SM.

Extra fermions. The top-quark seesaw model implies the existence of an additional fermion whose left- and right-handed components have the same charges as the righthanded top quark, $t_{R}$. This quark could have a mass of many $\mathrm{TeV}$ with little loss in fine-tuning, making it hard to find directly at any of the next generation colliders, including the LHC. In this circumstance, however, the improved precision electroweak measurements described in Chapter 8 should show a clear deviation from the SM in the direction of positive $\rho$ parameter $(\Delta T>0)$. This would prove that the SM is incomplete and give a clue as to the nature of the new physics.

Heavy vector bosons. Both the top-quark seesaw theory and the extra-dimensional composite Higgs models imply the existence of heavy vector bosons. In the topcondensate scenario, the extra heavy vectors could arise from a topcolor gauge group. In addition, one often requires an additional gauge interaction that couples differently to $t_{R}$ and $b_{R}$ to explain why we see top quark but not bottom quark condensation. If a new vector boson couples with some strength to all three generations, it will appear as a resonance at the LHC, and its effects will be seen at the LC as a pattern of deviations in all of the polarized $e^{+} e^{-} \rightarrow f \bar{f}$ cross sections. In both cases, the experiments are sensitive to masses of $4 \mathrm{TeV}$ and above. This mass reach overlaps well with the expectation that the new physics should occur at a mass scale of several $\mathrm{TeV}$. The observation and characterization of new $Z$ bosons are described in Chapter 5 , Section 5.

\subsection{Technicolor theories}

Technicolor theories provide an alternative type of model with new strong interactions. These theories do not require a composite Higgs boson. Instead, they involve new chiral fermions and a confining gauge interaction that becomes strongly-coupled at an energy scale of order $1 \mathrm{TeV}$. The most robust prediction of these theories is that there is a vector resonance with mass below about $2 \mathrm{TeV}$ that couples with full strength to the $J=1 W^{+} W^{-}$scattering amplitude.

The general idea of technicolor is severely constrained by the precision electroweak measurements, which favor models with a light Higgs boson over models where this state is replaced by heavy resonances. In order to be viable, a technicolor model must provide some new contributions to the precision electroweak observables that compensate for the absence of the Higgs boson. This leads us away from models in which the new strong interactions mimic the behavior of QCD and toward models with a significantly different behavior. For such models, it is difficult to compute quantitatively and so we must look for qualitative predictions that can be tested at high-energy colliders. In this situation, the ability of the linear collider to discover new 
particles essentially independently of their decay schemes would play an important role.

We summarize some of the measurements that the linear collider can perform that are relevant to strongly-coupled theories of this type. Our approach is to identify qualitative features that are likely to result from technicolor dynamics. Because of the uncertainties in calculating the properties of such strongly-interacting theories, it is not possible to map out for what parameters a given model can be confirmed or ruled out. Nevertheless, the linear collider has the opportunity to identify key components of technicolor models.

Strong $W W$ scattering. As we have noted, the most robust qualitative prediction of technicolor theories is the presence of a resonance in $W W$ scattering in the vector $(J=1)$ channel. This particle is the analogue of the $\rho$ meson of QCD. For masses up to $2 \mathrm{TeV}$, the 'techni- $\rho$ ' should be seen as a mass peak in the $W^{+} W^{-}$invariant mass distribution observed at the LHC. In addition, the techni- $\rho$ will appear as a resonance in $e^{+} e^{-} \rightarrow W^{+} W^{-}$for longitudinal $W$ polarizations, for the same reason that in QCD the $\rho$ meson appears as a dramatic resonance in $e^{+} e^{-} \rightarrow \pi^{+} \pi^{-}$. The resonant effect is a very large enhancement of a well-understood SM process, so the effect should be unmistakable at the linear collider, even at $\sqrt{s}=500 \mathrm{GeV}$, well below the resonance. As with the case of a $Z^{\prime}$, the two different observations at the linear collider and the LHC can be put together to obtain a clear phenomenological picture of this new state. These issues are discussed further in Chapter 5, Section 3.

Anomalous gauge couplings. If there is no Higgs boson resonance below about $800 \mathrm{GeV}$, the unitarization of the $W W \rightarrow W W$ scattering cross-section by new strong interactions will lead to a large set of new effective interactions that alter the couplings of $W$ and $Z$. Some of these terms lead to anomalous contributions to the $W W \gamma$ and $W W Z$ vertices. Through the precision study of $e^{+} e^{-} \rightarrow W^{+} W^{-}$and related reactions, the $500 \mathrm{GeV}$ linear collider with $500 \mathrm{fb}^{-1}$ of integrated luminosity will detect these anomalous contributions or improve the limits by a factor of ten over those that will be set at the LHC. In the case that there are new strong interactions, the accuracy of the linear collider measurement is such as to make it possible to measure the coefficients of the effective Lagrangian that results from the new strong interactions. These measurements are discussed further in Chapter 5, Section 2. In addition, many technicolor models predict large anomalous contributions to the gauge interactions of the top quark particularly to the $t \bar{t} Z$ vertex function. The linear collider may provide the only way to measure this vertex precisely. The measurement is discussed in Chapter 6, Section 3.

Extra scalars. Just as, in QCD, where the strongly coupled quarks lead to octets of relatively light mesons, technicolor theories often imply the existence of a multiplet of pseudoscalar bosons that are relatively light compared to the $\mathrm{TeV}$ scale. These 
Pathways Beyond the Standard Model

bosons are composites of the underlying strongly coupled fermions. Since these particles have non-zero electroweak quantum numbers, they are pair-produced in $e^{+} e^{-}$ annihilation. The number of such bosons and their quantum numbers depend on the precise technicolor theory. Experimentally, these particles look like the particles of an extended Higgs sector, and their detection and study follow the methods discussed for that case in Chapter 2, Section 6. Particular models may include additional new particles. For example, in 'topcolor-assisted technicolor', there is a second doublet of Higgs bosons, with masses of $200-300 \mathrm{GeV}$, associated with top-quark mass generation.

\section{$5 \quad$ Extra spatial dimensions}

It is 'apparent' that the space we live in is three-dimensional, and in fact precise measurements are consistent with this even down to the small distances probed by LEP2 and the Tevatron. But one should not hastily conclude that the universe has no more than three dimensions, because two important loopholes remain. First, there could be extra spatial dimensions that are not accessible to SM particles such as the photon and the gluon. Second, there could be extra spatial dimensions that are compact, with a size smaller than $10^{-17} \mathrm{~cm}$. In both cases, it is possible to build models that are in agreement with all current data.

Besides being a logical possibility, the existence of extra spatial dimensions may explain key features of observed phenomena, ranging from the weakness of the gravitational interactions to the existence of three generations of quarks and leptons. Most importantly from the viewpoint of the stability problem of the Higgs field, the assumption that the universe contains more that three dimensions opens a number of new possibilities for models of electroweak symmetry breaking. In such models, the value of the weak-interaction scale results from the fact that some natural mass scale of gravity in higher dimensions, either the size of the new dimensions or the intrinsic mass scale of gravity, is of order $1 \mathrm{TeV}$. This, in turn, leads to new observable phenomena in high energy physics at energies near $1 \mathrm{TeV}$. These phenomena, and the possibility of their observation at a linear collider, are discussed in Chapter 5, Section 6 .

Once we have opened the possibility of new spatial dimensions, there are many ways to construct models. Most of the options can be classified by two criteria. First, we must specify which particles are allowed to propagate in the full space and which are restricted by some mechanism to live in a three-dimensional subspace. Second, we must specify whether the extra dimensions are flat, like the three dimensions we see, or highly curved. The latter case is referred to in the literature as a 'warped' geometry. Some ideas may require additional fields, beyond the SM fields, to solve certain problems (such as flavor violation or anomaly cancelation) that can arise from 
the hypothesized configuration of particles in the extra-dimensional space. We now give a brief overview of these possibilities and the role of the linear collider in each scenario.

\subsection{Flat extra dimensions, containing only gravity}

The first possibility is that all of the particles of the SM-quarks, leptons, and Higgs and gauge bosons - are localized on three-dimensional walls ('3-branes') in a higher-dimensional space. Gravity, however, necessarily propagates through all of space. Higher-dimensional gravity can be described in four-dimensional terms by using a momentum representation in the extra dimensions. If these extra dimensions are compact, the corresponding momenta are quantized. Each possible value of the extra-dimensional momentum gives a distinct particle in four dimensions. This particle has mass $m_{i}^{2}=\left(\vec{p}_{i}\right)^{2}$, where $\vec{p}_{i}$ is the quantized value of the extra-dimensional momenta. These four-dimensional particles arising from a higher-dimensional field are called Kaluza-Klein (KK) excitations. In the later examples, where we put SM fields also into the higher dimensions, these field will also acquire a KK spectrum.

If gravity propagates in the extra dimensions, the exchange of its KK excitations will increase the strength of the gravitational force at distances smaller than the size of the new dimensions. Then the fundamental mass scale $M_{*}$ at which gravity becomes a strong interaction is lower than the apparent Planck scale of $10^{19} \mathrm{GeV}$. It is possible that $M_{*}$ is as low as $1 \mathrm{TeV}$ if the volume of the extra dimensions is sufficiently large. In that case, there is no stability problem for the Higgs field. The Higgs expectation value is naturally of the order of $M_{*}$.

The KK gravitons can be produced in collider experiments. In $e^{+} e^{-}$collisions, one would look for $e^{+} e^{-}$annihilation into a photon plus missing energy. The cross section for this process has typical electroweak size as the CM energy approaches $M_{*}$ and the phase space for producing the KK gravitons opens up. The expected signals of extra dimensions are highly sensitive to the number of extra dimensions. Nevertheless, if the number of extra dimensions is less than or equal to six, the signal can be studied at a linear collider at CM energies that are a factor of 3-10 below $M_{*}$. The LHC can also study KK graviton production through processes such as $q \bar{q}$ annihilation to a jet plus missing energy. The sensitivity to $M_{*}$ is somewhat greater than that of a $1 \mathrm{TeV}$ linear collider, but it is not possible to measure the missing mass of the unobserved graviton.

The KK gravitons can also appear through their virtual exchange in processes such as $e^{+} e^{-} \rightarrow f \bar{f}, e^{+} e^{-} \rightarrow \gamma \gamma$, and $e^{+} e^{-} \rightarrow g g$. The graviton exchange leads to a spin-2 component that is distinct from the SM expectation. Although this indirect signal of KK gravitons is more model-dependent, it is expected that it can be seen even at $500 \mathrm{GeV}$ if $M_{*}$ is less than a few $\mathrm{TeV}$. 
Pathways Beyond the Standard Model

\subsection{Warped extra dimensions, containing only gravity}

If the extra dimensions are warped, the KK spectrum of gravitons has somewhat different properties. In the case of flat extra dimension, the KK particles are closely spaced in mass, but in the case of warped dimensions, the spacing is of order $1 \mathrm{TeV}$. In the simplest model, the KK gravitons have masses in a characteristic pattern given by the zeros of a Bessel function. The individual states appear as spin-2 resonances coupling with electroweak strength to $e^{+} e^{-}$and $q \bar{q}$. These resonances might be seen directly at the LHC or at a linear collider. If the resonances are very heavy, their effects can be seen from additional spin- 2 contact contributions to $e^{+} e^{-} \rightarrow f \bar{f}$, even for masses more than an order of magnitude above the collider CM energy.

\subsection{Flat extra dimensions, containing SM gauge fields}

It is often assumed that the quarks and leptons are localized on three-dimensional walls (3-branes) and therefore do not have KK modes, whereas the gauge bosons propagate in the extra-dimensional space. In this case, the KK modes of the electroweak gauge bosons contribute at tree level to the electroweak observables, so that a rather tight lower bound of about $4 \mathrm{TeV}$ can be imposed on the inverse size of the extra dimensions. The LHC should be able to see the first gauge boson KK resonance up to about $5 \mathrm{TeV}$, leaving a small window of available phase space for direct production of these states. On the other hand, precision measurements at a high-energy $e^{+} e^{-}$ linear collider can establish a pattern of deviations from the SM predictions for the reactions $e^{+} e^{-} \rightarrow f \bar{f}$ from KK resonances well beyond direct production sensitivities. The capability of an $e^{+} e^{-}$linear collider in identifying the rise in cross sections due to KK resonances improves when the center-of-mass energy is increased. High luminosity is also important. For example, with more than $100 \mathrm{fb}^{-1}$ of integrated luminosity at a $500 \mathrm{GeV}$, one could see the effects of resonance tails for $\mathrm{KK}$ masses above $10 \mathrm{TeV}$ in models with one extra dimension.

\subsection{Flat extra dimensions, containing all SM particles}

Finally, we consider the case of 'universal' extra dimensions, in which all SM particles are permitted to propagate. A distinctive feature of universal extra dimensions is that the quantized KK momentum is conserved at each vertex. Thus, the KK modes of electroweak gauge bosons do not contribute to the precision electroweak observables at the tree level. As a result, the current mass bound on the first KK states is as low as $300 \mathrm{GeV}$ for one universal extra dimension. If the $\mathrm{KK}$ states do indeed have a mass in the range 300-400 GeV, we would expect to observe the states at the Tevatron and the LHC. The linear collider, at a CM energy of $800 \mathrm{GeV}$, would become a KK factory that produces excited states of quarks, leptons, and gauge bosons. 


\section{Surprises}

Our brief discussion of pathways beyond the SM concentrated on three very different approaches that have been proposed to solve the conundrums of the SM. Although some of these ideas are more easily tested than others at the next-generation colliders, it is important to note that all three approaches have many new observable consequences. In all cases, we expect to see an explosion of new phenomena as we head to higher energies.

Though these three approaches are very different, we should not delude ourselves into thinking that they cover the full range of possibilities. Letting our imaginations run free, we could envision models in which quantum field theory itself breaks down at the weak interaction scale and an even more fundamental description takes over. Such a possibility would be viable only if it satisfies the constraint of giving back the predictions of the SM at energies below $100 \mathrm{GeV}$. String theory is an example of a framework that resembles the SM at low energies but, at the energies of the string scale, is dramatically different from a simple quantum field theory. Perhaps there are other alternatives to be found.

Exploring physics at shorter distances and with higher precision is an endeavor that implies the possibility of great surprises. Experiments at a linear collider will be a necessary and rewarding part of this program, and will constitute a major step in our quest to understand how Nature works. 


\section{Experimental Program Issues}




\section{Chapter 10 Scenarios for Linear Collider Running}

In the literature on physics studies at $e^{+} e^{-}$linear colliders, one typically finds each process analyzed in isolation with a specific choice of energy and polarization. This naturally raises the question of how the full program for the linear collider fits together and whether all of the important physics topics can actually be scheduled and investigated. In this chapter, we will examine this issue. We will suggest some simple run plans that accomplish the most important goals of the linear collider program under different physics scenarios.

Under almost any scenario, one would wish to run the linear collider at two or more different energies during the course of its program. Operation of the collider at energies lower than $500 \mathrm{GeV}$ typically yields lower luminosity, scaling roughly as $E_{\mathrm{CM}}$. In this chapter, we will craft scenarios using the following guidelines: We assume that the collider has a single interaction region that can run at any energy from $m_{Z}$ to $500 \mathrm{GeV}$, with instantaneous luminosity strictly proportional to the CM energy. We plan for a campaign equivalent to $1000 \mathrm{fb}^{-1}$ at $500 \mathrm{GeV}$, corresponding to 3-5 years at design luminosity. We then ask how the collider running should be allotted among the various possible conditions. These assumptions are rather simplistic, but they frame a problem whose solution is instructive. In Chapter 11, we describe in a more careful way how a collider with two interaction regions, sharing luminosity, would be configured for a flexible program covering a large dynamic range in CM energy.

\section{Preliminaries}

In designing a plan for linear collider running, we should consider the alternative strategies for energy and for polarization. In this paragraph, we consider these two topics in turn.

There are three different ways to choose the energy of an $e^{+} e^{-}$collider:

- Sit: Choose an energy that is optimal for a particular interesting process, and accumulate integrated luminosity at that point.

- Scan: Step through a threshold for pair-production of some particle, taking enough data to define the threshold behavior.

- Span: Go to the highest available energy, and take a large sample of data there.

In the application of $e^{+} e^{-}$colliders to the $J / \psi$ and $\Upsilon$ systems, and to the $Z^{0}$, the $e^{+} e^{-}$ annihilation cross section contained narrow structures that put great importance on 
the exact choice of the beam energy. For most of the important processes considered for study at the next-generation linear collider, the choice of energy should be less of an issue, since the Higgs boson, the top quark, supersymmetric particles, etc., will be studied mainly in continuum production of a pair of particles. These processes have cross sections that peak within $50-100 \mathrm{GeV}$ of the threshold and then fall as $E_{\mathrm{CM}}^{-2}$. This dependence is somewhat compensated by the higher collider luminosity at higher energy. Since the signatures of different particles seen in $e^{+} e^{-}$annihilation are distinctive, many different reactions can be studied at a single energy.

As an example, consider the measurement of Higgs boson branching ratios. For this study, the Higgs boson is produced in the reaction $e^{+} e^{-} \rightarrow Z^{0} h^{0}$. For a Higgs boson of mass $120 \mathrm{GeV}$, the peak of the cross section is at $250 \mathrm{GeV}$. However, taking into account the increase of luminosity with energy, the penalty in the total number of Higgs bosons in working at $500 \mathrm{GeV}$ instead of at the peak of the cross section is only a factor of 2. At higher energy, more reactions become accessible, and more effort must be made to isolate the Higgs sample. On the other hand, the Higgs production process has a distinctive signature, the monoenergetic $Z^{0}$. As the energy increases, the kinematics become more distinctive as the Higgs and the $Z^{0}$ are boosted into opposite hemispheres. We conclude that LC experimenters will continue to accumulate statistics for the Higgs branching ratio study as they move to higher energies. Thus, though concentration on this process would favor a sit at an energy below 300 $\mathrm{GeV}$, one could well adopt a span strategy if other physics required it. This example illustrates that it is important, in future studies of linear collider measurements, to evaluate explicitly how the quality of the measurement depends on CM energy.

Only a few reactions among those anticipated for the LC require a detailed scan of some energy region. These include the measurement of the top quark mass by a threshold scan, the precision measurement of supersymmetric particle masses (to the parts per mil rather than the percent level), and, in the precision electroweak program of Chapter 8, the measurement of the $W$ mass to $6 \mathrm{MeV}$. The top quark mass measurement actually becomes limited by theory errors after about $10 \mathrm{fb}^{-1}$ of data, though a longer run would be justified to obtain a precision measurement of the top quark width and the decay form factors. Other threshold scans require similarly small increments of luminosity, except for the cases of sleptons, where the threshold turns on very slowly, as $\beta^{3}$, and the $W$, where extreme precision is required.

As for the choice of beam polarization in LC running, it is important to understand how polarization will be implemented. The choice of a polarized or unpolarized electron source is not a limiting factor for the electron currents in the machine. So there is no penalty in choosing a polarization that is as large as possible $-80 \%$, with current technology. Polarized electrons are created by shining circularly polarized light on an appropriate cathode. In the SLD polarization program at the $Z^{0}$, the polarized light was created by passing a linearly polarized laser beam through a Pockels cell, a device that is effectively a quarter-wave plate whose sign is determined 
Scenarios for Linear Collider Running

by an applied voltage. The signal applied to the cell changed sign randomly at the 120 $\mathrm{Hz}$ repetition rate of the machine. This random sign was supplied to the experimenters and used to determine the initial-state polarization in detected events. We anticipate that the beam polarization will be created in a similar way at the LC. Thus, there will be no 'unpolarized' running. The normal running condition will be a half-andhalf mixture of left- and right-handed electron polarization, switching randomly at the repetition rate for bunch trains. In this arrangement, it is straightforward to measure polarization-averaged cross sections. The rapid switching allows polarization asymmetries to be measured with many systematic errors cancelling.

For certain processes, it is advantageous to take the bulk of the data in a single state of beam polarization. For example, the supersymmetric partners of the righthanded sleptons are most easily studied with a right-hand polarized electron beam, while $W W$ pair production and fusion processes such as $W^{+} W^{-} \rightarrow t \bar{t}$ receive most or all of their cross section from the left-handed electron beam. In contrast, $e^{+} e^{-} \rightarrow$ $Z^{0} h^{0}$ has only a weak polarization dependence. It is possible that our knowledge of physics at the time of the LC running will single out one such process as being of great importance and call for a run with an unequal (90\%/10\%) distribution of beam polarizations. As in the case of the energy choice, this is a shallow optimum, winning back, in the best case, less than a factor of 2 in luminosity.

\section{Illustrative scenarios}

With these considerations in mind, we now propose some sample run plans appropriate to different physics scenerios. For each plan, we quote the luminosity sample to be obtained at each energy and, in parentheses, the corresponding sample scaled to $500 \mathrm{GeV}$. These latter values are constrained to add up to $1000 \mathrm{fb}^{-1}$.

In most cases, the luminosity assigned below to $500 \mathrm{GeV}$ would be accumulated at the highest machine energy if higher energies were available. Many physics issues, including the measurement of the Higgs coupling to $t \bar{t}$ and the Higgs self-coupling in addition to studies of new heavy particles, benefit greatly from CM energies above $500 \mathrm{GeV}$. The integrated luminosities given are totals, which might be accumulated in any order. In the scenarios presented here, we omit, for simplicity, the possibility of positron polarization and $\gamma \gamma$ or $e^{-} e^{-}$running. These options are discussed in the later chapters of this section. In considering any of these options, it is important to keep in mind that these options entail trade-offs against $e^{+} e^{-}$integrated luminosity.

\subsection{A Higgs boson, but no other new physics, is seen at the LHC}

In this case, we would want to apply a substantial amount of luminosity to a precision study of the branching ratios of the known Higgs boson. It will also be important to search for Higgs bosons not seen at the LHC, to search for new particles 
with electroweak couplings that might have been missed at the LHC, and to measure the $W$ and top gauge couplings to look for the virtual influence of new particles. Thus:

- $300 \mathrm{GeV}: 250 \mathrm{fb}^{-1}\left(420 \mathrm{fb}^{-1}\right)$ sit

- $350 \mathrm{GeV}: 100 \mathrm{fb}^{-1}\left(140 \mathrm{fb}^{-1}\right)$ top threshold scan

- $500 \mathrm{GeV}: 440 \mathrm{fb}^{-1}\left(440 \mathrm{fb}^{-1}\right)$ span

This run plan gives a data sample for the Higgs boson branching ratio measurement equivalent to $600 \mathrm{fb}^{-1}$ at $350 \mathrm{GeV}$.

\subsection{No Higgs boson or other new particles are seen at the LHC}

In this case, we would want to apply the largest amount of luminosity to the highest available energy. The issues for this study would be the search for additional Higgs bosons not seen at the LHC and the search for new particles. The measurement of the $W$ and top gauge couplings would be of essential importance. Because the absence of a light Higgs conflicts with the precision electroweak fits within the SM, it will also be crucial in this case to include running at the $Z^{0}$ and the $W W$ threshold.

- $90 \mathrm{GeV}: 50 \mathrm{fb}^{-1}\left(280 \mathrm{fb}^{-1}\right)$ sit

- $160 \mathrm{GeV}: 70 \mathrm{fb}^{-1}\left(220 \mathrm{fb}^{-1}\right) W$ threshold scan

- $350 \mathrm{GeV}: 50 \mathrm{fb}^{-1}\left(70 \mathrm{fb}^{-1}\right)$ top threshold scan

- $500 \mathrm{GeV}: 430 \mathrm{fb}^{-1}\left(430 \mathrm{fb}^{-1}\right)$ span

\subsection{Light Higgs and superpartners are seen at the LHC}

In this case, it is necessary to compromise between the optimal energies to study each of the new states, the optimal energy for the Higgs study - since a light Higgs must also appear in supersymmetric models - and searches for new superparticles, such as the extended Higgs particles and the heavier charginos and neutralinos, that could have been missed at the LHC. The program will begin with extended running at $500 \mathrm{GeV}$, and perhaps also at a lower energy, to determine the superpartner masses to percent-level accuracy. This could be followed by detailed threshold scans.

Martyn and Blair [1] have studied a particular scenario in which the lightest neutralino has a mass of $70 \mathrm{GeV}$, the lighter charginos and sleptons lie at about 130 $\mathrm{GeV}$, and the heavier charginos and neutralinos are at about $350 \mathrm{GeV}$. Converting their suggested program to our rules, we have for this case: 
- $320 \mathrm{GeV}: 160 \mathrm{fb}^{-1}\left(250 \mathrm{fb}^{-1}\right)$ sit

- $500 \mathrm{GeV}: 245 \mathrm{fb}^{-1}\left(245 \mathrm{fb}^{-1}\right)$ span

- $255 \mathrm{GeV}: 20 \mathrm{fb}^{-1}\left(40 \mathrm{fb}^{-1}\right)$ chargino threshold scan

- $265 \mathrm{GeV}: 100 \mathrm{fb}^{-1}\left(190 \mathrm{fb}^{-1}\right)$ slepton $\left(\ell_{R}^{-} \ell_{R}^{+}\right)$threshold scan

- $310 \mathrm{GeV}: 20 \mathrm{fb}^{-1}\left(30 \mathrm{fb}^{-1}\right)$ slepton $\left(\ell_{L}^{-} \ell_{R}^{+}\right)$threshold scan

- $350 \mathrm{GeV}: 20 \mathrm{fb}^{-1}\left(30 \mathrm{fb}^{-1}\right)$ top threshold scan

- $450 \mathrm{GeV}$ : $100 \mathrm{fb}^{-1}\left(110 \mathrm{fb}^{-1}\right)$ neutralino $\left(\chi_{2}^{0} \chi_{3}^{0}\right)$ threshold scan

- $470 \mathrm{GeV}: 100 \mathrm{fb}^{-1}\left(105 \mathrm{fb}^{-1}\right)$ chargino $\left(\chi_{1}^{-} \chi_{2}^{+}\right)$threshold scan

The threshold scans would be done with the dominant beam polarization chosen, respectively, right, left, equal, left, left. The threshold with $\beta^{1}$ cross sections are given small amounts of running time; thresholds with $\beta^{3}$ cross sections or cross sections that are intrinsically small are given $100 \mathrm{fb}^{-1}$. The running time at the top threshold is more than sufficient to push the determination of $m_{t}$ to the systematics limit. While running at each threshold, pair production of all lighter species can also be studied. In particular, the total statistics for the Higgs branching ratio measurement is equivalent to about $700 \mathrm{fb}^{-1}$ at $350 \mathrm{GeV}$.

\section{References}

[1] H. Martyn and G. A. Blair, in Physics and Experiments at Future Linear $e^{+} e^{-}$ Colliders (LCWS99), E. Fernandez and A. Pacheco, eds. (Univ. Auton. de Barcelona, Bellaterra, 2000), hep-ph/9910416. 


\section{Chapter 11 Interaction Regions}

\section{Introduction}

The Standard Model has received considerable experimental attention in the past two decades, and much is known about its electroweak sector and about its flavor sector. Recent precision experiments have the sensitivity to look beyond the SM for new physics. However, the mechanism for symmetry breaking in the SM is still unknown, and many questions, such as the existence of SUSY, still are answered only by speculation. A future linear collider will provide the tools with which we may probe the mechanism of symmetry breaking and address the questions of new physics beyond the SM. We seek the best configuration of a linear collider facility that maximizes the potential for answering these questions.

The number of interaction regions is a very important issue, affecting the project cost, the physical footprint of the collider complex, the number of detectors that can be accommodated, the breadth of the physics program, and almost certainly the amount of enthusiasm and support the linear collider would receive in the world's high energy physics community. In this section we look at the nature and number of interaction regions to accompany the accelerator complex of a linear collider. The baseline configurations for TESLA and the NLC are briefly discussed here. This section gives only a brief overview of the technical designs. One must go to the relevant reports and documents to get more technical details.

Both the TESLA and the NLC designs for the IRs allow for two regions. The TESLA philosophy in its baseline design differs somewhat from that of the NLC. The baseline design for TESLA includes only one IR, with real estate available for a second IR and a second beam delivery system, if and when the funds become available. The NLC baseline design contains two IRs, as described below.

The arguments favoring the two-IR collider configuration come first from the physics program. The rich program of particle physics could best be investigated by two active IRs with two or more detectors. However, one must consider the tradeoff between the increased breadth of the physics program and the increased costs incurred. One of the "costs" encountered is the unavoidable sharing of the available luminosity between the two IRs. Strategies for simultaneous running in the NLC are briefly discussed.

However, it should be pointed out that the strongest motivation for two IRs may come from external factors. The future linear collider will surely be an international facility. In order for there to be international participation in the financing of the

collider, it would be wise to incorporate two IRs to facilitate broad participation 
in the detectors and the experimental program. This philosophy on international participation in the linear collider is surely part of the strategy for incorporating two IRs in the TESLA and NLC designs.

\section{The two interaction region design at TESLA}

TESLA has provision for two IRs, one which is in the baseline design, and a second which is not currently in the baseline, but may be added. The TESLA linear collider cannot serve two IRs with luminosity simultaneously. It is possible, however, to switch the beam between the two experimental stations. The primary IR will receive beams at a zero crossing angle, while the secondary IR will have a crossing angle of $34 \mathrm{mrad}$. If the secondary IR is run in the $e^{+} e^{-}$collider mode (with crab crossing), it is anticipated to have the same luminosity as the primary IR. The crossing angle also makes the secondary IR suitable for $\gamma \gamma$ and $e \gamma$ collider modes of operation using backscattered laser beams, as described in Chapter 13. Electron-electron collisions are possible at one or both IRs, by reversing magnet polarities and providing a second polarized electron source. This option is discussed in Chapter 14. The layout of the two IRs and their technical parameters can be found in the TESLA TDR [1].

\section{The dual-energy interaction region design at the NLC}

To allow for a collider design for the desired physics program that extends from the $Z$-pole to many TeV, the NLC group has introduced a dual-energy IR design [2]. The first IR is in a direct line with the main linacs that accelerate the beams. The second IR is reached by bending the beam away from this direct line. Both IRs have crossing angles, as described below. The IRs would be designed to operate in different energy ranges, the first from 250 to $1000 \mathrm{GeV}$, the second from 90 to $500 \mathrm{GeV}$.

There are two motivations for this choice. First, by having one of the two IRs in a direct line with the main linacs that accelerate the beams, this IR can operate at multi-TeV energies in subsequent machine energy upgrades. This layout eliminates the bending where incoherent synchrotron radiation would dilute the beam emittances. Second, Final Focus beamlines are naturally optimized to operate over roughly a factor of four to five in beam energy. At the high end of the range, the luminosity decays rapidly due to increasing synchrotron radiation. At lower energies, the luminosity scales proportionally to the collision energy until a limit is reached at roughly $25 \%$ of the maximum energy. Below this limit, the luminosity decays as the square of the collision energy due to increasing aberrations and limited vacuum and masking apertures. At either end, a smoother dependence of luminosity on energy can be retained by realigning the Final Focus components to change the total bending. 
Interaction Regions

The choices we have indicated, with two Final Focus systems of fixed configuration, give the NLC overlapping coverage of the energy region that is thought to be initially of interest.

Because the straight-ahead IR could support multi-TeV beam collisions, we refer to this as the 'high-energy' IR (HEIR). The bending required to reach the second IR limits the maximum energy attainable. Thus, we refer to this as the 'low-energy' IR (LEIR). Schematic plans of the NLC machine and the two-IR layout are shown in Figs. 11.1 and 11.2.

With this starting point, the collider layout is determined by the length of the beam delivery systems, the required transverse separation of the IRs, and the desired crossing angle in the interaction regions. Given the new Final Focus optics design which utilizes local chromatic correction, the Final Focus can be relatively short. The present NLC Final Focus design is 700 meters long. This length is sufficient up to $5 \mathrm{TeV}$ in the center of mass. In addition to the Final Focus optics itself, there are diagnostic regions and beam collimation regions upstream of the IP. Depending on the operating mode, these regions could likely be shared. In the present NLC design, these regions are roughly 1300 meters long for a total beam delivery system length of $2 \mathrm{~km}$ per side. This length could be reduced; however it is relatively inexpensive and provides a conservative solution to the beam optics and the beam collimation problems.

To attain reasonable transfer efficiency of the rf to the beam in a normal conducting linear collider, the bunches must be spaced together very closely. In this case, both IRs must have a non-zero crossing angle to prevent interactions between bunches at satellite crossings. Typical values for the crossing angle could range from $6 \mathrm{mrad}$ to $40 \mathrm{mrad}$. The larger angles result in easier beam extraction and IR integration but lead to more difficult tolerances. Simplifying the beam extraction is important if one believes that it is important to measure the beam energy spread and polarization after collision at the IP. The crossing angles allow for these measurements in the NLC but not at the primary IR at TESLA.

Without consideration of the extraction line, the minimum crossing angle is set by the 'multi-bunch kink' instability. At $\mathrm{CM}$ energies below $1.5 \mathrm{TeV}$, the minimum angle in a normal conducting design is roughly $2 \mathrm{mrad}$. However, studies of the CLIC $3 \mathrm{TeV}$ IR suggest that a minimum crossing angle of $15 \mathrm{mrad}$ is necessary at multi-TeV energies. For these reasons, a crossing angle of $20 \mathrm{mrad}$ at the HEIR and between $20-40 \mathrm{mrad}$ at the LEIR is suggested.

The IR halls have been sized assuming that one would house the NLC L or SD Detector and that one would house the P Detector. Table 11.1 gives a list of the hall parameters. The hall length (transverse to the beam) is large enough to allow assembly of the detector while a concrete wall shields the interaction point. The wall would also serve as radiation shielding if the detector is not deemed to be 'selfshielding'. If the detector were built in place on the beam line, and could be self- 


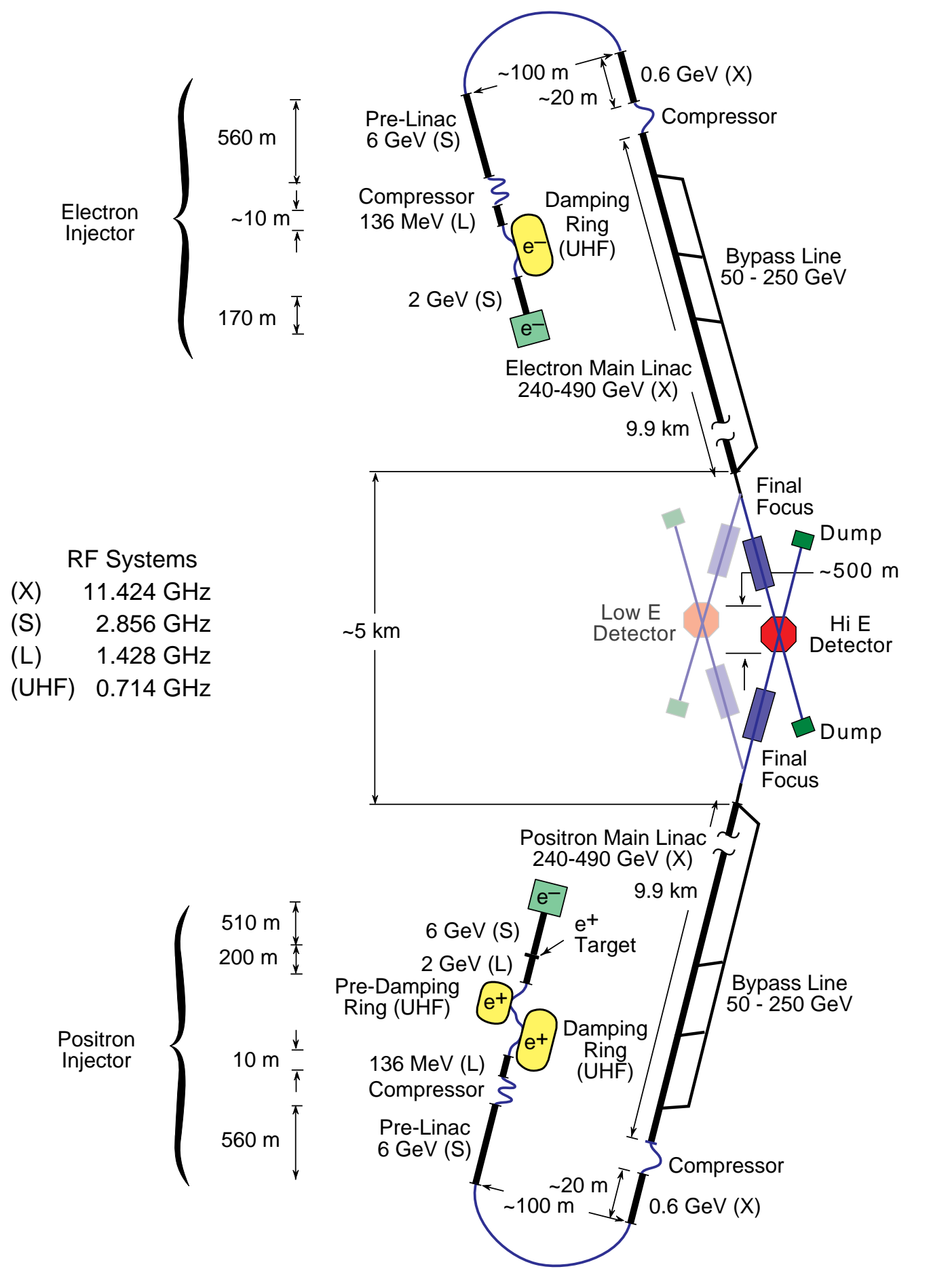

$10-2000$

8047A611

Figure 11.1: Schematic of the non-zero crossing angle of the two linacs and the Dual Energy IR layout. 


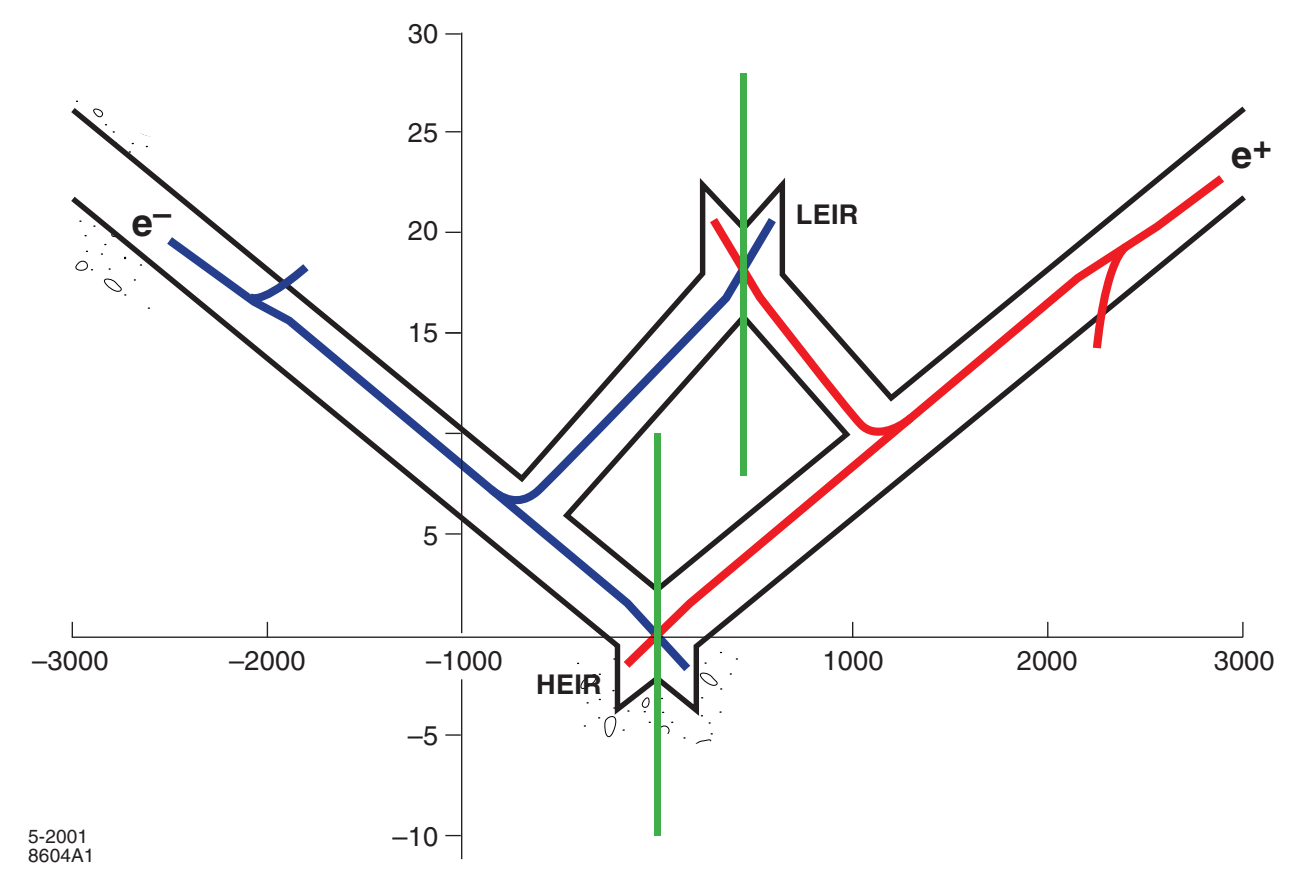

Figure 11.2: Schematic of the accelerator tunnels leading to the two interaction regions. The IRs are separated laterally by $25 \mathrm{~m}$ and longitudinally by $440 \mathrm{~m}$. The crossing angles at the HEIR and LEIR are $20 \mathrm{mrad}$ and $30 \mathrm{mrad}$, respectively. Note that the figure is extremely compressed in the horizontal direction; the detectors occupy the volume of the vertical rectangles that intersect the two beamlines at their crossing points.

shielding, the length could be reduced by roughly a factor of three. The hall width (parallel to the beamline) is set by the constraint that the doors open just enough to allow servicing of the inner detectors.

The baseline design assumes that the two IR halls are physically separated so that activities and mechanical equipment operating in one hall are seismically isolated from the other hall. For example, the LIGO facility has used $100 \mathrm{~m}$ as a minimum separation between rotating machinery and sensitive detectors. While the active detection and compensation of culturally induced ground vibration is a key element of the NLC R\&D program, passive compliance with vibration criteria is the ideal. In principle each of the IR halls could be designed to accommodate two detectors that share the beamline in a push-pull manner, thus increasing experimental opportunities, or the overall NLC layout could be changed to support only one push-pull IR at a considerable cost savings. In any push-pull scheme, major installation activities might need to be curtailed if they introduced uncompensated vibration of the final magnets producing data for the detector currently on the beam line.

All of these features are illustrated in the schematic designs shown in Figs. 11.1 and 


\begin{tabular}{|l|c|c|}
\hline \hline Parameter & Small Detector & Large Detector \\
\hline Detector footprint & $12 \times 11 \mathrm{~m}$ & $20 \times 20 \mathrm{~m}$ \\
\hline Pit length & $40 \mathrm{~m}$ & $62 \mathrm{~m}$ \\
\hline Pit width & $20 \mathrm{~m}$ & $30 \mathrm{~m}$ \\
\hline Pit depth below beamline & $5 \mathrm{~m}$ & $7 \mathrm{~m}$ \\
\hline Door height & $10 \mathrm{~m}$ & $13 \mathrm{~m}$ \\
\hline Door width & $10 \mathrm{~m}$ & $13 \mathrm{~m}$ \\
\hline Barrel weight & $2000 \mathrm{MT}$ & $7300 \mathrm{MT}$ \\
\hline Door weight & $500 \mathrm{MT}$ & $1900 \mathrm{MT}$ \\
\hline Total weight & $3100 \mathrm{MT}$ & $11100 \mathrm{MT}$ \\
\hline \hline
\end{tabular}

Table 11.1: The Baseline Interaction Region Parameters

11.2. The main linacs are aligned to provide the $20 \mathrm{mrad}$ crossing angle at the HEIR. The LEIR beamline is bent from the straight-ahead beams. The transverse separation between the two IR collision points is currently set at roughly 25 meters. However, roughly 440 meters longitudinal separation of the two IR halls has been provided for increased vibration isolation. In addition, bypass lines are installed along the side of the linac so that lower-energy beams can be transported to the Final Focus without passing through the downstream accelerator structures.

\subsection{The low-energy interaction region at the NLC}

The experimental program in the LEIR is determined by the range of accessible center-of-mass energies and the available luminosity. The amount of luminosity that should be dedicated to a particular $\sqrt{s}$ will depend on the physics that is revealed by the Tevatron and the LHC. This need for flexibility imposes the requirement that the LEIR have high performance at least over the range $m_{Z} \leq \sqrt{s} \leq 2 m_{t}$. Figure 11.3 shows the luminosity for the baseline design of the LEIR versus the center-of-mass energy. In the following, we outline the basic LEIR physics program as a function of increasing beam energy.

The lowest operating energy of the LEIR is determined by the requirement that high-statistics studies at the $Z$-pole be possible. The goal of a next-generation $Z$ pole experiment would be a significant reduction in the experimental errors in key electroweak parameters, as explained in Chapter 8. The success of this program relies on the availability of longitudinally polarized beams. Polarized electron beams will be available in the initial configuration. It would be desirable eventually to have positron polarization as well. Issues and technologies for positron polarization are discussed in Chapter 12. One feature pertaining to beam polarization in the LEIR is the need to account for the spin precession in the bends in the beam transport system. Another 
Interaction Regions

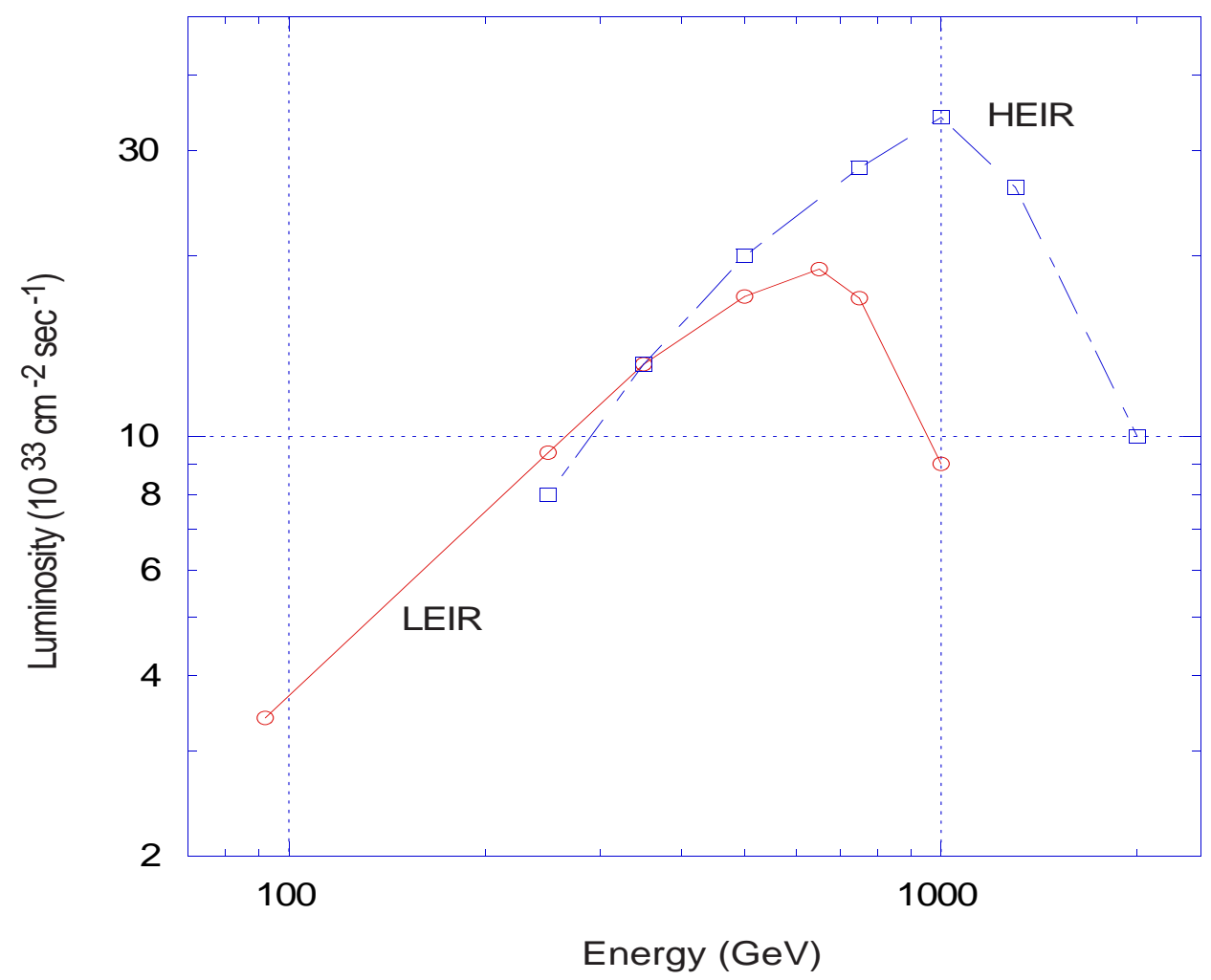

Figure 11.3: The baseline luminosity versus CM energy for the NLC LEIR and HEIR. The two IRs have been designed to have comparable performance in the region between $250 \mathrm{GeV}$ and $500 \mathrm{GeV}$, however, the NLC HEIR beam delivery system has been optimized for a maximum energy of $500 \mathrm{GeV}$, the HEIR for $1 \mathrm{TeV}$.

issue is the desire to account for the depolarization that arises during collision. For this reason, a crossing angle is desirable, since it eases the polarization measurement after the IP.

Precise determination of the electroweak parameters could be particularly valuable in understanding the SM and physics beyond, particularly at a time when the Higgs boson mass is experimentally determined. In the event that only a single Higgs boson is observed with no other direct evidence of new physics from the LHC programs, the precision electroweak measurements will be a crucial aspect of the NLC program. A benchmark for such a program would be to accumulate a sample of $10^{9} Z^{0}$ decays.

The $W$-pair threshold occurs near $\sqrt{s}=160 \mathrm{GeV}$ with the maximum production cross section at $\sqrt{s} \sim 200 \mathrm{GeV}$. In the event that a significantly improved measurement of the $W$ mass is required, it will be necessary to have dedicated running at the $W$-pair threshold. Studies have shown that an error on the $W$ mass of $6 \mathrm{MeV}$ would be obtainable with $100 \mathrm{fb}^{-1}$. Given the otherwise very limited physics program in 
this energy range, the need for high instantaneous luminosity is evident. ${ }^{1}$

Beyond the $W$-pair threshold, it is highly likely that next benchmark center-ofmass energy will be the production cross section peak for a light Higgs boson. Precise measurements of the Higgs mass, width, spin-parity, and branching fractions are essential to help understand the role this object would play in electroweak symmetry breaking. The associated production process $e^{+} e^{-} \rightarrow Z^{0} h^{0}$, with $Z^{0} \rightarrow \ell^{+} \ell^{-}$and $\ell$ an electron or muon, provides a model-independent tag of Higgs production. The Higgs signal is easily identifiable in the dilepton recoil mass distribution. The maximum cross section for associated production occurs at roughly $m_{Z}+\sqrt{2} m_{\mathrm{h}}$. In minimal SUSY, the mass of the lightest CP-even scalar is required to satisfy $m_{h} \lesssim 135 \mathrm{GeV}$. The precision electroweak fit to the SM calls for a Higgs boson with mass below $200 \mathrm{GeV}$. It is therefore essential that the LEIR design be capable of delivering high luminosity in the range $220 \lesssim \sqrt{s} \lesssim 340 \mathrm{GeV}$. The study of a light Higgs boson will also benefit from control of the beam polarization; for example, for the measurement of the $h W W$ coupling, one can exploit the large difference in the $\nu \bar{\nu} h^{0}$ production cross section for $e_{L}^{-}$and $e_{R}^{-}$beams. For some processes, positron polarization is also desirable. In many scenarios, the precision study of a light Higgs boson would be the principal focus of the LEIR program.

The $t \bar{t}$ threshold occurs near $350 \mathrm{GeV}$. The low-energy IR would be the natural facility to focus on this important topic. The threshold onset is a difficult process to study experimentally because of the resolution smearing caused by the natural energy spread from bremsstrahlung in the initial state, and from energy spread in the linear collider. The amount of dedicated running at the $t \bar{t}$ threshold will be dictated by the Higgs physics program. If a light Higgs is present, $m_{H} \lesssim 180 \mathrm{GeV}$, it may be desirable to run below the $t \bar{t}$ threshold to control physics backgrounds and to optimize the Higgs production rate. For the case where the physics of electroweak symmetry breaking has conspired to produce a heavy Higgs boson that somehow satisfies the precision constraints, the study of the top quark properties will assume a central importance. The integrated luminosity requirements for the LEIR at or above the $t \bar{t}$ threshold in such a scenario will be the order of $100 \mathrm{fb}^{-1}$ necessitating instantaneous luminosities of at least $5 \times 10^{33} \mathrm{~cm}^{-2} \mathrm{~s}^{-1}$.

Other physics options for the low-energy IR have been considered extensively. The region would serve well as the location for a 'second generation' detector for $\gamma \gamma$ collisions. Similarly, an $e^{-} e^{-}$program might be done in the LEIR, should the physics motivations lead in this direction.

In summary, a low-energy IR has many uses and advantages in an NLC program. It would provide considerable flexibility in the physics program, and would preserve many physics opportunities in scenarios in which the NLC is upgraded to multi-TeV

\footnotetext{
${ }^{1}$ Although investigation of $W$-boson properties will be an important goal of any NLC program, many of these studies, e.g., the determination of Triple Gauge Boson couplings, are best performed at the highest achievable center-of-mass energy. This issue is discussed in Chapter 5, Section 2.
} 
Interaction Regions

operations for high-energy studies in the other IR region.

\subsection{The high-energy interaction region at the NLC}

The design of the NLC allows for an IR region capable of upgrading to multi-TeV operations in an energy-upgraded NLC. To assure this possibility, the beam delivery systems are aligned in a straight-ahead configuration relative to their respective linacs, with very little bending of the incoming beams between the linear accelerator structure and the IR. To preserve the non-zero crossing angle required at the point of collisions, the two halves of the collider structure are not parallel but rather cross at an angle at the collision point. Figure 11.3 shows the luminosity versus CM energy for the baseline design of the HEIR.

The HEIR physics program is intimately related to the scenario that is realized in Nature for electroweak symmetry breaking. In the event that supersymmetry is discovered, the focus of the HEIR program will be the measurement of sparticle properties. It is unlikely that the full SUSY spectrum will be accessible at $\sqrt{s}=500$ $\mathrm{GeV}$; therefore, the energy reach of the HEIR should be upgradable to the multi-TeV region. Symmetry-breaking arising from some new strong dynamics would also be likely to put a premium on the energy reach. It is clear that in comparison to the LEIR, the physics requirements for the HEIR are, to first order, straightforward: the highest possible luminosity at the highest possible energy.

The energy span of the HEIR runs from $250 \mathrm{GeV}$ to $500 \mathrm{GeV}$ in the initial phase. Therefore the physics program can in principle include everything from $250 \mathrm{GeV}$ on up, a region which overlaps in energy with the LEIR. Studies of $W$-pairs, low-lying SUSY states, and the $t \bar{t}$ threshold could occur in the HEIR. Although, in the case of a light Higgs boson, much of the precision Higgs physics could be performed at the LEIR, there is Higgs physics unique to the HEIR. For a light Higgs boson consistent with the current theoretical and experimental constraints, the maximum cross section for the rare process $e^{+} e^{-} \rightarrow Z^{0} h^{0} h^{0}$ occurs at $\sqrt{s} \sim 500 \mathrm{GeV}$. This process is of great interest, since it enables measurement of the Higgs self-coupling which in turn can be related to the shape of the Higgs potential. The $W$-fusion process, $e^{+} e^{-} \rightarrow \nu \bar{\nu} h^{0}$, which is sensitive the $h W W$ vertex, has a cross section that increases with centerof-mass energy. The measurement of the Higgs self-coupling sets a benchmark for the accelerator performance. Depending on the exact mass, a measurement of this quantity requires integrated luminosities the order of $1000 \mathrm{fb}^{-1}$, which corresponds to 3-4 years at design luminosity.

Supersymmetry is a primary candidate for physics beyond the SM. Almost all versions of SUSY models result in low-lying states that would appear in $e^{+} e^{-}$annihilations below $500 \mathrm{GeV}$. Although the discovery phase for SUSY is likely to occur at the Tevatron or LHC, the NLC will play a key role in the detailed study of the sparticle spectrum and subsequent delineation of the soft SUSY-breaking Lagrangian. 
To exploit fully the physics potential of the NLC, a number of special operating conditions may be necessary for the HEIR. For example, in EWSB models with extended Higgs sectors, of which SUSY is the most widely studied, a $\gamma \gamma$ mode of operation for the HEIR may be crucial. For example, the $\gamma \gamma$ mode enables production of a single Higgs boson; for the case of a nominal $500 \mathrm{GeV}$ center-of-mass, this would effectively increase the mass reach from $250 \mathrm{GeV}$ to $400 \mathrm{GeV}$ for production of heavy neutral Higgs particles. Operation with transversely polarized photon beams allows separate production of the $\mathrm{CP}$-even and $\mathrm{CP}$-odd states. Control of the electron and positron beam polarization will also be extremely useful. For Higgs physics it can be used to increase the nominal production cross section for the self-coupling measurement. Beam polarization will also be useful in unraveling gaugino and slepton mixing. The need for an $e^{-} e^{-}$operating mode may be necessary to decipher selectron production.

It is likely and perhaps desirable that there be a staged evolution of the HEIR center-of-mass energy. Although the goal of the initial phase of the NLC is $500 \mathrm{GeV}$ for the HEIR, it may be possible to start physics earlier at a lower collision energy. An intermediate commissioning stage with $\sqrt{s} \sim 250 \mathrm{GeV}$ and modest luminosity could potentially be very relevant and exciting, especially if direct evidence from the LHC indicated the production of a light Higgs boson or a threshold for supersymmetric states. Another obvious commissioning stage could be the $t \bar{t}$ threshold at $350 \mathrm{GeV}$. Even at $10 \%$ of design luminosity, the physics program promises to be rich. For example, dedicating $10 \mathrm{fb}^{-1}$ to a scan of the $t \bar{t}$ threshold would already lead to a top quark mass measurement with a $200 \mathrm{MeV}$ error, as discussed in Chapter 6, Section 2 .

\subsection{Alternative interaction region scenarios}

The baseline scenario that we have assumed considers two interaction regions - a high-energy region limited only by the available accelerating structures and a second region that is limited in energy or by the support of $\gamma \gamma$ or other options. It is appropriate to discuss alternative scenarios and the interplay between the physics programs of the high- and low-energy interaction regions. The issue is complicated by the diversity of physics scenarios that may arise. An additional consideration is the possible staging of the maximum center-of-mass energy. The possibilities can be broadly classified into types:

a) Single interaction region with one detector;

b) Single interaction region with two detectors;

c) Two interaction regions, high-energy and low-energy;

d) Two high-energy interaction regions. 
Interaction Regions

For scenario (a), there is an obvious cost advantage; however, the NLC physics program could be unduly compromised. The physics program would be tightly coupled to the available center-of-mass energy. Depending on the details of the actual physics scenario, it may not be possible to simultaneously satisfy the various needs of a diverse user community. The resolution of mutually exclusive requirements for luminosity and choice of the center-of-mass energy may not be straightforward.

It is difficult to identify the merits of scenario (b), given the limitations of a single IR outlined above for scenario (a). Given that the total luminosity accumulated by both experiments will be comparable to that for a single experiment, this scenario would only be of interest if the two detectors were of sufficiently different capabilities or there were very strong sociological arguments for a second collaboration. One possible scenario where differences between detectors could arise is if there were a need to have a dedicated $\gamma \gamma$ collider program. In such a scenario, it would be more natural to consider a push-pull capability for one of the IRs in a two-IR facility. The two IR regions allow for a push-pull configuration in a least one of the two regions. The footprint of the push-pull IR hall must not infringe on the beamline of the adjacent region. In addition, access to the detector captured between the two beamlines must be possible, and adequate shielding must be provided to permit work in the IR hall when beams are alive in the machine. Scenarios for staging two detectors would have to be considered and understood. These are complicated issues that would involve assumptions that might not be appropriate at a future date. Nevertheless, provision for staging two detectors in a push-pull configuration would be a low-cost and effective means to keep open future possibilities for a unique and special-purpose detector.

The scenario that has been chosen as the baseline is (c); there are a number of considerations in its favor. It makes it possible to have parallel physics programs running simultaneously, a clearly desirable feature. The upgrade path for the HEIR is less complex. It provides for a lower-energy IR that can be dedicated to precision studies of the Higgs boson, $Z$-pole or $t \bar{t}$ system. Moreover, in this scenario both the HEIR and LEIR will cover the preferred energy range for the study of a light Higgs. The two-IR design adds a degree of flexibility that enables the NLC to address essentially any physics scenario that could arise.

The scenario (c) affords a natural context for energy staging. As mentioned in Section 2.2, staging the HEIR energy at the beginning of the NLC program would make it possible to perform an initial investigation of the region above $250 \mathrm{GeV}$. Commissioning of the LEIR program might follow the completion of the full complement of accelerating structures required to reach $500 \mathrm{GeV}$ though, with a bypass line, this might alternatively begin before the accelerator is complete. Many of the highluminosity measurements foreseen for the LEIR would benefit from longitudinally polarized positron beams, which are not likely to be available at the initial stages of running. 
Given the need to have minimal bending in the beam delivery system in order to preserve beam emittances, scenario (d), which has two high-energy IRs of similar performance, becomes technically challenging and more costly. Given the interest exhibited by many members of the physics community in the low-energy potential of the NLC, and the need to perform high-statistics studies of the $Z$-pole in a number of physics scenarios that could arise, it would seem prudent to have at least one IR capable of delivering that physics.

\subsection{Simultaneous operation}

The NLC design has in it the capability for simultaneous operations in the two IRs. In the baseline design, the accelerator delivers bunch trains at a rate of $120 \mathrm{~Hz}$. With pulsed magnets, the beams can be sent alternately to two IRs, resulting in an even split of $60-60 \mathrm{~Hz}$. Uneven splitting of the 120 pulses per second is technically more challenging, and is not envisioned as an option.

A higher pulse rate in the NLC is possible, but is not in the baseline design. It appears technically feasible, for example, to operate at $180 \mathrm{~Hz}$. This would require modifications to the damping rings and additional cooling for the klystrons and modulators in some regions of the accelerator. But these changes would allow operation, for example, with $60 \mathrm{~Hz}$ of low-energy beams in the LEIR and $120 \mathrm{~Hz}$ of beams in the HEIR. This mode of operation would clearly enhance the experimental program and augment the total luminosity delivered to the experimenters.

\section{References}

[1] TESLA Technical Design Report, http://tesla.desy.de/new_pages/TDR_CD/ start.html

[2] US NLC Collaboration, 2001 Report on the Next Linear Collider, FERMILABConf-01/075-E, LBNL-47935, SLAC-R-571. 


\section{Chapter 12 Positron Polarization}

\section{Introduction}

The baseline designs for NLC and TESLA include a polarized electron beam, but the positron beam is unpolarized. In this chapter, we investigate the physics merits of positron polarization and summarize the status of proposed polarized positron source designs. These questions have also been discussed in [1].

The importance of electron beam polarization has been demonstrated in $Z^{0}$ production at the Stanford Linear Collider (SLC), where $75 \%$ electron polarization was achieved. This level of electron polarization provided an effective luminosity increase of approximately a factor of 25 for many $Z$-pole asymmetry observables. In particular, it allowed the SLD experiment to make the world's best measurement of the weak mixing angle, which is a key ingredient for indirect predictions of the SM Higgs mass. The electron polarization at SLC also provided a powerful tool for bottom quark studies, providing a means for $b$ and $\bar{b}$ tagging from the large polarized forward-backward asymmetry, and for studies of parity violation in the $Z b \bar{b}$ vertex. At a $500 \mathrm{GeV}$ linear collider, electron polarization will increase sensitivity to form-factor studies of $W^{+} W^{-}$ and $t \bar{t}$ states, control the level of $W^{+} W^{-}$backgrounds in new physics searches, provide direct coupling to specific SUSY chiral states, and enhance sensitivity to new physics that would show up in the spin-zero channel.

But what will positron polarization add? First, the presence of appreciable positron polarization is equivalent to a boost in the effective electron polarization. Measured asymmetries that are proportional to the polarization will increase; fractional errors in these quantities will accordingly decrease. Second, cross sections for many processes will grow. Any process mediated by gauge bosons in the $s$-channel naturally wastes half the incident positrons. Left-handed electrons, for example, only annihilate on right-handed positrons. The same is true for $t$-channel exchanges with unique handedness in their couplings, such as neutrino exchange in $W$-pair production. By polarizing the positrons and coordinating their polarization with that of the electrons, the cross sections for these processes can double (in the limit of $100 \%$ polarization). Finally, polarimetry will benefit from positron polarization. As the effective polarization increases, its error decreases, allowing measurements with very small systematic errors. Such small errors are needed for high-precision work at the $Z$ pole and will benefit studies of production asymmetries for $W^{+} W^{-}$. And, by using measurements of rates with all four helicity states (RL,LR,RR,LL) the beam polarizations can be inferred directly without additional polarimetry.

What positron polarization can bring, poor yields of polarized positrons can take 
away, so the yield of any source of polarized positrons is very important. Several schemes have been advanced for polarizing positrons. All are ambitious, large systems which are mostly untested. R\&D is required before decisions are made about how and when to include positron polarization in linear collider design.

\section{The physics perspective}

\subsection{The structure of electroweak interactions at high energies}

The primary purpose of a linear collider will be to study the mechanism of electroweak symmetry breaking (EWSB). Beam polarization at a high-energy linear collider can play an important role in this endeavor because: (1) the electrons and positrons in the beams are essentially chirality eigenstates; (2) gauge boson interactions couple $e_{L}^{-} e_{R}^{+}$or $e_{R}^{-} e_{L}^{+}$but not $e_{L}^{-} e_{L}^{+}$or $e_{R}^{-} e_{R}^{+}$; and (3) the $S U(2)_{L}$ interaction involves only left-handed fermions in doublets, whereas right-handed fermions undergo only hypercharge $U(1)_{Y}$ interactions. At typical LC energies, where masses are small compared to $\sqrt{s}$, one can replace the exchange of $\gamma$ and $Z$ bosons with the $B$ and $W^{3}$ bosons associated with the unbroken $U(1)_{Y}$ and $S U(2)_{L}$.

As a concrete application of these points, consider $e^{+} e^{-} \rightarrow W^{+} W^{-}$production, which is a background to many new physics searches. There are three tree-level Feynman diagrams for this process, one involving the $t$-channel exchange of $\nu_{e}$ and the others involving the $s$-channel exchange of $\gamma$ and $Z$. The polarization choice $e^{+} e_{R}^{-}$will eliminate the first contribution, since $W$ bosons have only left-handed interactions. Decomposing the $s$-channel diagrams into a $W^{3}$ and a $B$ contribution, the $W^{3}$ diagram is also eliminated using $e_{R}^{-}$polarization for the same reason. The only remaining diagram now vanishes for symmetry reasons - the $B$ and $W$ bosons involve different interactions and do not couple to each other. In reality, there is a small but nonvanishing component to $W^{+} W^{-}$production, because of EWSB. The polarization choice $e_{R}^{+}$would eliminate this background at tree-level. Of course, it also important to consider the behavior of the signal process under the same choices of polarization and the fact that $100 \%$ beam polarization is difficult in practice.

In the example above, note how the polarization of only one beam had a dramatic effect. Once the electron polarization was chosen, only certain positron polarizations contributed. One can imagine also the case where the desired effect is to enhance the $W^{+} W^{-}$signal. Then, by judiciously choosing the polarization combination $e_{L}^{-} e_{R}^{+}$, the production rate is enhanced by a factor of four relative to the unpolarized case, and a factor of two beyond what is possible with only electron polarization. When either searching for rare processes or attempting precision measurements, such enhancements of signal and depletions of background can be quite important.

We use the convention that the sign of polarization is positive for right-handed polarization, both for electrons and for positrons. Then, for example, for the case of 
Positron Polarization

single gauge boson production, the production cross section is proportional to

$$
\left(1-\mathcal{P}_{-}\right)\left(1+\mathcal{P}_{+}\right) c_{L}^{2}+\left(1+\mathcal{P}_{-}\right)\left(1-\mathcal{P}_{+}\right) c_{R}^{2},
$$

where $c_{L}$ and $c_{R}$ are chiral couplings. Equation (12.1) is at the heart of the forwardbackward asymmetry that arises when $c_{L} \neq c_{R}$. If two measurements of the cross section are made with a different sign for the polarizations $\mathcal{P}_{-}$and $\mathcal{P}_{+}$, then the difference of the two measurements normalized to the sum is:

$$
\frac{N_{L}-N_{R}}{N_{L}+N_{R}}=\mathcal{P}_{\text {eff }} \frac{c_{L}^{2}-c_{R}^{2}}{c_{L}^{2}+c_{R}^{2}} \equiv \mathcal{P}_{\text {eff }} A_{L R},
$$

where

$$
\mathcal{P}_{\text {eff }}=\frac{\mathcal{P}_{-}-\mathcal{P}_{+}}{1-\mathcal{P}_{-} \mathcal{P}_{+}} .
$$

In $Z$ boson production, $A_{L R}$ depends on the difference between $1 / 4$ and $\sin ^{2} \theta_{W}$. Since the error in an asymmetry $A$ for a fixed number of events $N=N_{L}+N_{R}$ is given by $\delta A=\sqrt{\left(1-A^{2}\right) / N}$, increasing $\mathcal{P}_{\text {eff }}$ makes measurable asymmetries larger and reduces the error in the measured asymmetry significantly if $A^{2}$ is comparable to 1 . When only partial electron polarization is possible, a small positron polarization can substantially increase $\mathcal{P}_{\text {eff }}$, while also decreasing systematic errors. These asymmetry improvements utilizing polarized positrons are exploited in the Giga- $Z$ mode for a linear collider. With Giga-Z, polarized positrons are needed to take full advantage of the large statistics possible at a linear collider - 50 times more data than the integrated LEP-I data sample and 2000 times more data than SLD's sample. With a Giga-Z data sample, one expects to achieve a factor of 20 improvement over SLD's $A_{L R}$ and $A_{b}$ measurements. These improved measurements can be used to perform exquisite tests of the Standard Model. Together with a precise measurement of the top quark mass (to $100 \mathrm{MeV}$ from a threshold scan at a linear collider), the $A_{L R}$ measurement can be used to predict the Standard Model Higgs mass to 7\%. The Giga-Z program is discussed in more detail in Chapter 8.

Equation (12.1) is also applicable to other situations. In general, as long as a process has a helicity structure similar to that of $s$-channel gauge boson production, the rate is

$$
\left(1-\mathcal{P}_{-} \mathcal{P}_{+}\right) \sigma_{\text {unpol }}\left(1+\mathcal{P}_{\text {eff }} \frac{c_{L}^{2}-c_{R}^{2}}{c_{L}^{2}+c_{R}^{2}}\right)
$$

where $\sigma_{\text {unpol }}$ is the unpolarized cross section. Notice that polarization can increase the cross section by at most a factor of four, as can occur for $W^{+} W^{-}$production where $c_{R} \simeq 0$. 


\subsection{Standard Model-like Higgs boson}

One process of particular interest for a LC is Higgs boson production. The primary modes at a LC are associated production with a $Z$ boson $(Z h)$ and vector boson fusion $(\nu \bar{\nu} h)$. The $Z h$ process is particularly simple, since the direct coupling of the Higgs boson to electrons is negligible. Polarization effects appear only at the initial $e^{+} e^{-} Z$ vertex. The $Z$ process allows for the discovery and study of a Higgs boson with substantial couplings to the $Z$ boson independently of the Higgs boson decay mode, using the $Z$ recoil method. Therefore, the relative size of signal and background is of great interest.

\begin{tabular}{|c|c||c|c||c|c||c|c|}
\hline \hline \multicolumn{2}{|c||}{} & \multicolumn{3}{c||}{$\sigma(Z h)$} & \multicolumn{2}{c||}{$\sigma(Z Z)$} & \multicolumn{2}{c|}{$\sigma\left(W^{+} W^{-}\right)$} \\
\multicolumn{1}{|c|}{} & $c_{L}^{2}=.58$ & $c_{R}^{2}=.42$ & $c_{L}^{2}=.65$ & $c_{R}^{2}=.35$ & \multicolumn{2}{c|}{$c_{L}^{2} \simeq 1 \quad c_{R}^{2} \simeq 0$} \\
\cline { 2 - 8 } $\mathcal{P}_{-}$ & $\mathcal{P}_{+}$ & $P=1$ & $E=.8$ & $E=1$ & $E=.8$ & $E=1$ & $E=.8$ \\
\hline \hline 0 & 0 & 1 & 1 & 1 & 1 & 1 & 1 \\
\hline$+E$ & 0 & 0.84 & 0.87 & 0.69 & 0.75 & 0 & 0.2 \\
$-E$ & 0 & 1.16 & 1.13 & 1.31 & 1.25 & 2 & 1.8 \\
$+E$ & $-P$ & 1.68 & 1.26 & 1.37 & 1.05 & 0 & 0.08 \\
$-E$ & $+P$ & 2.32 & 1.70 & 2.62 & 1.91 & 4 & 2.88 \\
\hline \hline
\end{tabular}

Table 12.1: Behavior of various Standard Model cross sections relevant for Higgs boson studies as a function of polarization for full and partial electron and positron polarization. The numbers listed are normalized to the unpolarized cross section.

At tree-level, the $Z h$ cross section depends on polarization as indicated in Eq. (12.1) with the couplings $c_{L}=-\frac{1}{2}+\sin ^{2} \theta_{w}, c_{R}=\sin ^{2} \theta_{w}$. Numerically, the two squared coupling factors appear with the relative weights (normalized to unity) 0.58 to 0.42. Table 12.1 shows the relative behavior of the $Z h$ cross section for full $(100 \%)$ and partial electron (80\%) and positron (60\%) polarization. Even for partial polarization, a substantial increase to the production cross section occurs over the unpolarized case. Other Higgs boson production processes, such as $e^{+} e^{-} \rightarrow H A$ in the MSSM or $e^{+} e^{-} \rightarrow Z h h$ in the SM or MSSM (relevant for measuring the Higgs self-coupling), proceed through the $Z$ resonance and have the same chiral structure.

Significant backgrounds to the $Z h$ search can arise from $W^{+} W^{-}$and $Z Z$ production. The polarization dependence of these processes is also shown in Table 12.1. The physics of the $W^{+} W^{-}$background was discussed previously. It is relevant to note from Table 12.1 that without full polarization - which may be difficult to obtain in practice - the $W^{+} W^{-}$background cannot be fully eliminated. On the other hand, the partial polarization of both beams can approximately recover the benefits of full polarization, since the effective polarization $\mathcal{P}_{\text {eff }}$ is close to 1 . Another potential 
Positron Polarization

background, $Z Z$ production, has a similar behavior as the signal $Z h$, except that an additional $Z$ must be attached to the incoming $e^{+} e^{-}$. Therefore, the relative weight of the different polarization pieces goes as the square of those for $Z h$ production. For the case of partial polarization of both beams and $\left(\mathcal{P}_{-}=+80 \%, \mathcal{P}_{+}=-60 \%\right)$, where the $W^{+} W^{-}$background is substantially decreased, there is a small increase in $\sigma(Z h) / \sigma(Z Z)$. The efficacy of polarization will depend on the most significant background. Note that for a Higgs boson mass that is significantly different from $m_{Z}$, propagator effects and non-resonant diagrams need to be included, but the results should not be significantly different from those shown here.

The other Higgs production process of interest is $W W$ fusion, which has a similar behavior to the $W W$ background. When operating at energies where $Z h$ and $W W$ fusion are comparable, polarization can be used to dial off the fusion contribution. This may be important for the study of inclusive Higgs production using the recoil technique.

\subsection{Supersymmetric particle production}

The production and study of new particles with electroweak quantum numbers should be the forte of a linear collider, where the major backgrounds are also electroweak in strength. Supersymmetry is a concrete example of physics beyond the SM that predicts a spectrum of new electroweak states related to the SM ones by a spin transformation. We now discuss some aspects of supersymmetry measurements affected by beam polarization. For further discussion of supersymmetry mass and coupling measurements, see Chapter 4.

\subsubsection{Slepton and squark production}

One of the simplest sparticle production processes to consider is $\widetilde{\mu}$ pair production, where the interaction eigenstates $\widetilde{\mu}_{R}$ and $\widetilde{\mu}_{L}$ are expected to be nearly mass eigenstates. Gauge bosons couple to the combinations $\widetilde{\mu}_{R} \widetilde{\mu}_{R}^{*}$ and $\widetilde{\mu}_{L} \widetilde{\mu}_{L}^{*}$. $\widetilde{\mu}_{R}$ has only couplings to the hypercharge boson $B$. The initial $e^{+} e^{-}$state has different hypercharge depending on the electron polarization: $e_{L}^{-}$has $Y=-1 / 2$, whereas $e_{R}^{-}$has $Y=-1$. The production cross section depends on $Y^{2}$ and thus is four times larger for $e_{R}^{-}$than for $e_{L}^{-}$. Furthermore, the choice $e_{R}^{-}$significantly reduces the background from $W^{+} W^{-}$ production, which comes both from decays to $\mu^{+} \nu_{\mu} \mu^{-} \bar{\nu}_{\mu}$ and from feed-down from decays to $\tau$. Since $e_{R}^{-} e_{R}^{+}$components do not contribute to the signal, left-polarizing the positron beam doubles the signal rate. $\widetilde{\mu}_{L}$ pair production depends on both $B$ and $W^{3}(\gamma$ and $Z)$ components. Switching the electron polarization will emphasize different combinations. In all, a judicious choice of the positron polarization will make more efficient use of the beam, increase the cross section, and suppress the backgrounds. 
For third-generation sparticles such as $\widetilde{\tau}$ and $\widetilde{t}$, there may be significant mixing between the mass and interaction eigenstates, leading to new observables. As for the $\widetilde{\mu}$ case, the production cross section itself is sensitive to the electron polarization. However, increased sensitivity to the mixing may be obtained from a measurement of the left-right asymmetry. For $\tilde{t}$ production, the addition of $60 \%$ polarization in the positron beam increases the accuracy of the mixing angle measurement by $25 \%$, while decreasing systematic errors [2]. Of course, the former effect can be achieved with only $e^{-}$polarization by increasing the integrated luminosity.

Selectron production may benefit more from positron polarization because of the $e^{+} e^{-}$initial state at a LC. The exchange of neutralinos $\widetilde{\chi}^{0}$ in the $t$-channel introduces more structure beyond the $s$-channel exchange of $\gamma$ and $Z$. The processes $e_{L}^{-} e_{L}^{+} \rightarrow \widetilde{e}_{L} \widetilde{e}_{R}^{*}$ and $e_{R}^{-} e_{R}^{+} \rightarrow \widetilde{e}_{R} \widetilde{e}_{L}^{*}$ proceed through $\tilde{\chi}^{0}$ exchange only. Considering the case that $\widetilde{e}_{L}$ and $\widetilde{e}_{R}$ are close in mass, the polarization of both beams can play an essential role in disentangling the different interaction states. For example, $e_{L}^{-} e_{L}^{+}$ polarization will only produce the negatively-charged $\widetilde{e}_{L}$ and the positively-charged $\widetilde{e}_{R}^{*}$. Switching the polarization of both beams will produce only negatively-charged $\widetilde{e}_{R}$ and positively-charged $\widetilde{e}_{L}^{*}$. Since the endpoints of the lepton spectrum can be used to reconstruct the selectron and neutralino masses, the electrons and positrons yield separate information about $\widetilde{e}_{L}$ and $\widetilde{e}_{R}$. Without the positron polarization, one would always have contamination from $\widetilde{e}_{L} \widetilde{e}_{L}^{*}$ and $\widetilde{e}_{R} \widetilde{e}_{R}^{*}$ production. Conversely, the observation of the switch from one species to another with the change in positron polarization would give more weight to the SUSY interpretation of the events. The study of $t$-channel exchange in selectron production is an important method for studying neutralino mixing, since the components of the neutralinos that are Higgsino-like do not contribute. Therefore, it is valuable to be able to isolate the $t$-channel exchanges experimentally by using polarization.

\subsubsection{Chargino and neutralino production}

The study of chargino pair production $e^{+} e^{-} \rightarrow \tilde{\chi}^{-} \tilde{\chi}^{+}$gives access to the parameters $M_{2}, \mu, \tan \beta, m_{\widetilde{\nu}_{e}}$. It is conservative to assume that only the lightest chargino is kinematically accessible. In this case, studies have considered the case of extracting the SUSY parameters from the measurement of cross sections for full $e_{L}^{-} e_{R}^{+}\left(\sigma_{L}\right)$, $e_{R}^{-} e_{L}^{+}\left(\sigma_{R}\right)$ and transverse $\left(\sigma_{T}\right)$ polarizations [3]. By analyzing $\sigma_{R}$ and $\sigma_{L}$, the two mixing parameters of the chargino sector can be determined up to at most a four-fold ambiguity, provided that the electron sneutrino mass is known and one assumes the supersymmetric relation between couplings in the interaction Lagrangian. The addition of transverse polarization allows the ambiguity to be resolved and gives a handle on the sneutrino mass. The role of transverse polarization is to allow interference between two different helicity states so that a product of two mixing factors appears in a physical observable instead of sums of squares of individual mixing factors, resolving 
Positron Polarization

the sign ambiguity. Given the measurement of the chargino mass and the mixing parameters, the Lagrangian parameters $M_{2}, \mu, \tan \beta$ can be determined up to two-fold ambiguity in modulus and a $2 \pi$ ambiguity in the phase combination $\arg \left(m_{2}\right)+\arg (\mu)$. Such studies need to be redone with more detail, considering partial beam polarization, backgrounds, cuts, and the likely absence of transverse polarization, but there is promise that SUSY parameters can be extracted from real data.

Other investigations have considered the consequences of partial longitudinal polarization at a purely theoretical level, focusing on the case $\left|\mathcal{P}_{-}\right|=.85,\left|\mathcal{P}_{+}\right|=.60$, and studying production cross sections near threshold [4]. Comparing a gaugino-like and Higgsino-like chargino, the total cross sections including the decay $\widetilde{\chi}^{-} \rightarrow e^{-} \bar{\nu} \widetilde{\chi}_{1}^{0}$ are calculated as a function of electron and positron polarization. For an unpolarized positron beam, the cross sections from $e_{L}^{-}$are larger than those from $e_{R}^{-}$for both the gaugino and Higgsino cases. However, the addition of positron polarization gives access to more detailed information. For example, one has the relation that $\sigma\left(e_{R}^{-} e_{L}^{+}\right)$ is less than the unpolarized cross section for gaugino-like charginos, and greater for Higgsino-like charginos. The sensitivity of the forward-backward asymmetry $A_{F B}$ to polarization, and how this effect can be used to bound the sneutrino mass, has also been discussed [5]. Similar considerations can be applied to the case of $\widetilde{\chi}^{0} \widetilde{\chi}^{0}$ production. These analyses would benefit from more detailed studies, including backgrounds and addressing the issue of measuring branching ratios.

\subsection{Some other new physics}

Contact interactions can arise from many sources of new physics, such as compositeness, a heavy $Z^{\prime}$, leptoquarks, KK excitations, etc. The low-energy effect of such physics can be parameterized in an effective Lagrangian as

$$
\mathcal{L}_{\text {eff }}=\frac{\tilde{g}^{2}}{\Lambda_{\alpha \beta}} \eta_{\alpha \beta}\left(\bar{e}_{\alpha} \gamma_{\mu} e_{\alpha}\right)\left(\bar{f}_{\beta} \gamma_{\mu} f_{\beta}\right), f \neq e, t .
$$

The chiral components are extracted by varying $P_{\text {eff }}= \pm P$ (this is just $A_{L R}$ ). Positron polarization increases the reach on $\Lambda_{\alpha \beta}$ by $20-40 \%$ depending on the nature of the couplings [6].

Low-energy signatures of string theory may include spin-zero resonances with non-negligible couplings to the electron and sizable amplitudes [7], i.e., $\mathcal{A}\left(e_{R}^{-} e_{R}^{+} \rightarrow\right.$

$\left.\gamma_{03}^{*}\right)=\sqrt{2} e M_{S}$ and $\mathcal{A}\left(e_{L}^{-} e_{L}^{+} \rightarrow \gamma_{04}^{*}\right)=\sqrt{2} e M_{S}$. With positron polarization, the SM backgrounds to these processes should be negligible.

\subsection{Transverse polarization}

Finally, we should comment on transverse polarization, which has been considered in some chargino studies. Transversely polarized beams are linear combinations of different helicities with equal weight. Transverse polarization can introduce an azimuthal 
dependence into production cross sections, proportional to the degree of polarization. However, all such effects in the SM are negligible upon azimuthal averaging for an $e^{-} e^{+}$collider, because of the small electron mass and Yukawa coupling [8]. Thus, transverse polarization can be used as a probe of physics beyond the SM, when small amplitudes from new physics interfere with larger SM ones. Without the positron polarization, however, there is no visible effect.

\section{$3 \quad$ Experimental issues}

\subsection{Polarimetry}

The baseline NLC design includes a laser-backscattering Compton polarimeter to measure the electron beam polarization with an expected accuracy of $1 \%$ or better $[9,10]$. For the Giga-Z physics program, an accuracy of $0.25 \%$ should be achievable in an optimized setup, which is a factor two improvement over SLD's Compton polarimeter. Above the $W$-pair threshold, the SM asymmetry in forward $W$ pairs can also be used [9]. Sub-1\% polarimetry using this technique will require reduction of the background to the $W$-pair sample below $1 \%$.

If the positron beam can also be polarized, significant improvements in polarimetry are possible. At Giga-Z, the polarimetry error can be improved to $0.1 \%$ using the 'Blondel scheme'. In this method, one measures the three independent asymmetries $[11,12]$ :

$$
\begin{aligned}
A_{1} & =\frac{N_{L L}-N_{R R}}{N_{L L}+N_{R R}} \\
A_{2} & =\frac{N_{R R}-N_{L R}}{N_{R R}+N_{L R}} \\
A_{3} & =\frac{N_{L R}-N_{R L}}{N_{L R}+N_{R L}}=\mathcal{P}_{\text {eff }} A_{L R},
\end{aligned}
$$

where $\mathcal{P}_{\text {eff }}$ is given by Eq. (12.3). From these three measurements, one can determine $A_{L R}$ (and hence the weak mixing angle) along with $\mathcal{P}_{-}$and $\mathcal{P}_{+}$. It should be noted that $\mathcal{P}_{\text {eff }}$ is typically substantially higher than either $\mathcal{P}_{-}$or $\mathcal{P}_{+}$and has a smaller uncertainty. For example, if $\mathcal{P}_{-}=80 \%$ and $\mathcal{P}_{+}=-60 \%$, then $\mathcal{P}_{\text {eff }}=94.6 \%$, and the error on $\mathcal{P}_{\text {eff }}$ is proportional to the difference from $100 \%$. With a Giga-Z sample using these polarization values, $A_{L R}$ can be determined to an accuracy of $10^{-4}$ and the beam polarizations to an accuracy of $10^{-3}$. These estimates are derived in Chapter 8 , Section 1. An advantage of the Blondel scheme for polarimetry is that the luminosityweighted polarization, $P_{e}^{\text {lum }}$, is directly measured. A Compton polarimeter measures the average beam polarization and small corrections may be needed to extract $P_{e}^{\text {lum }}$.

It should be noted that a Compton polarimeter is still needed to measure the difference 
Positron Polarization

between the right-handed and left-handed beam polarizations. One also needs to understand the relative luminosities for the four beam polarization states (at the level $10^{-4}$ for Giga-Z).

Away from the $Z$-pole, the Blondel scheme with polarized positrons can also be applied to $W$-pair events. Using $W$ pairs when both beams are polarized, an error on the beam polarizations of $0.1 \%$ should be achievable. The large $W$-pair physics asymmetry can be fit together with the beam polarizations, without sensitivity to backgrounds or assumptions about the polarization asymmetry in $W$ interactions.

\subsection{Frequency of spin flips}

Depending on the method for producing polarized positrons, it may be difficult to achieve fast reversals of the positron helicity. For the polarized electron source, helicity reversals are easily done at the train frequency $(120 \mathrm{~Hz}$ for NLC or $5 \mathrm{~Hz}$ for TESLA) using an electro-optic Pockels cell in the polarized source laser system. At SLC, the $120 \mathrm{~Hz}$ random helicity was very useful in controlling possible small left-right asymmetries in luminosity. Helicity reversals that are fast compared to any time constants for machine feedbacks are desirable. If fast helicity reversals are not possible, then relative integrated luminosities for the different polarization states need to be measured to better than $10^{-4}$ for Giga-Z. This should be achievable using forward detectors for Bhabha and radiative Bhabha events.

\subsection{Run time strategy for LL, LR, RL, RR}

One of the advantages of polarizing the positron beam is the increase in event rate by running in the (higher cross section) LR or RL polarization states. However, to take advantage of the Blondel technique for polarimetry and $A_{L R}$ measurements, it is necessary also to accumulate data in the LL and RR states. However, it has been shown that only $10 \%$ of the running time has to be spent in the lower-event rate LL and RR states to achieve adequate statistics for the asymmetry measurements [13]. One anticipates equal run times for the LR and RL configurations, even though some physics analyses may benefit most from selecting one of these configurations for enhancing or suppressing $W$ pairs or to enhance a cross section for a new process. Of course, some new physics searches will benefit from choosing those configurations that are suppressed in the SM.

\section{Sources of polarized positrons}

Several techniques have been suggested for producing polarized positrons for a linear collider. Present designs are largely conceptual, and much work remains before they can be realized. 
In 1979, Mikhailichenko and Balakin [14] proposed generating circularly polarized photons by running a high-energy electron beam through a helical undulator. These photons are directed onto a thin target, where they produce $e^{+} e^{-}$pairs. Selecting positrons near the high-energy end of the spectrum gives a sample with appreciable polarization. Okugi et al. [15] have proposed generating polarized photons by colliding intense circularly polarized laser pulses with few-GeV electron beams. Variations on this theme have been proposed in an attempt to mitigate the rather extreme requirements on laser power by using an optical cavity to concentrate and store multiple laser pulses [16,17]. Finally, Potylitsin [18] has proposed directing a $50 \mathrm{MeV}$ beam of polarized electrons onto a thin target.

\subsection{Helical undulator}

In the baseline TESLA design, unpolarized positrons are generated by photons produced when the full-energy electron beam is passed through a $100 \mathrm{~m}$ long wiggler prior to collision. The photon beam is directed to a thin, rotating target where $e^{+} e^{-}$ pairs are produced, and the positrons are subsequently captured, accelerated, and damped. This novel approach reduces the power dissipated in the positron target to manageable levels and significantly reduces radiation in the target area.

Replacing the wiggler with a helical undulator would in principle allow polarized positrons to be produced. The magnetic field created by a helical undulator has two transverse components that vary sinusoidally down the length of the device, the vertical component shifted in phase by $90^{\circ}$ from the horizontal. Such a field is created by two interleaved helical coils of the same handedness, driven by equal and opposite currents. Typical fields are of order $1 \mathrm{~T}$; the period of the sinusoidal field variation is about $1 \mathrm{~cm}$. The resulting electron trajectory for a $150 \mathrm{GeV}$ beam is a helix whose axis coincides with that of the undulator; the radius of curvature is measured in nanometers! The undulator coils must be quite compact, with an internal radius of several millimeters and an outer radius of about 1 centimeter [19].

Efficient positron production requires photon energies of about $20 \mathrm{MeV}$, which in turn necessitates electron beam energies of approximately $150-200 \mathrm{GeV}$. The photons produced within $\theta \approx 1 / \gamma$ have high average polarization. Collimators which are arranged to absorb the radiation at larger angles remove about $80 \%$ of the flux. To compensate this loss, the undulator length must be about 200 meters, somewhat longer than that of the wigglers used in the TESLA positron source. The undulator requires a very low-emittance electron beam, which probably prevents reuse of the electron beam after it has been used for high-energy collisions. It is possible that one could direct the primary high-energy electron beam through the undulator prior to collision. A drift space of about 200 meters between the undulator and the target is required to achieve the required photon beam size.

The highly polarized photons produced in the undulator are directed against a 0.4 $X_{0}$ target, where pair production can occur. Positrons produced with energies above 
Positron Polarization

$15 \mathrm{MeV}$ are highly polarized. With this energy cut, roughly $0.025 e^{+} /$incident photon is collected and $60 \%$ polarization is obtained [19]. Collection of the positrons requires solenoidal magnets, rf acceleration, and a predamping ring to handle the enlarged phase space. On paper, the scheme can generate the needed positron bunch currents.

The undulator scheme makes excellent use of the high-energy electron beam as the source of polarized photons. The low emittance requirements probably preclude the use of the post-collision beam. Whether the primary, pre-collision beam should be run through the undulator, or a dedicated beam should be generated for the sole purpose of positron production is a choice still being debated. A helical undulator generates positrons of a single helicity, so other means must be developed to flip the spin, and preferably to do so rapidly. Many of the photons could be absorbed in the undulator coil, so a workable design must accommodate many kilowatts of power dissipation.

\subsection{Backscattered laser}

A second method for producing highly polarized photons with enough energy to produce electron-positron pairs on a thin target involves backscattering an intense circularly polarized laser beam on a high-energy electron beam. The highest energy photons are strongly polarized and have helicity opposite to that of the incident laser light. As above, positrons are produced when these photons intercept a thin target. The highest-energy positrons are strongly polarized.

Omori and his collaborators have made a conceptual design of a laser-backscattering polarized positron source suitable for NLC/JLC [20]. They arrange for multiple collisions between polarized laser pulses from $50 \mathrm{CO}_{2}$ lasers and a high-current $5.8 \mathrm{GeV}$ electron beam. The laser system must provide $250 \mathrm{~kW}$ of average optical power, which is regarded as extremely ambitious. Positron production is accomplished just as in the helical undulator scheme above. Simulations indicate that $9.4 \%$ of the incident photons produce a positron above $20 \mathrm{MeV}, 26 \%$ of which are accepted into the pre-damping ring, with an average polarization of $60 \%$ [20].

This scheme makes production of polarized positrons independent of the highenergy electron beam, hence independent of its energy, but does so at the very considerable expense of a dedicated high-current linac and a very complex laser system. The estimated power required by those systems is roughly $10 \%$ of that required for the whole collider facility.

\section{Conclusions}

A polarized positron beam at a $\mathrm{LC}$ would be a powerful tool for enhancing signalto-background, increasing the effective luminosity, improving asymmetry measurements with increased statistical precision and reduced systematic errors, and improv- 
ing sensitivity to non-standard couplings. Suppression of $W$-pair backgrounds can be improved by a factor 3 with $60 \%$ positron polarization. By limiting the running time allotted for LL and RR modes to $10 \%$, the effective luminosity for annihilation processes can be enhanced by $50 \%$. For asymmetry measurements, the effective polarization is substantially increased (e.g., from $80 \%$ to $95 \%$ ) and the systematic precision is improved by a factor 3 . With these features, a polarized positron beam may provide critical information for clarifying the interpretation of new physics signals. Polarized positrons are needed to realize the full potential for precision measurements, especially those anticipated for Giga-Z $Z$ running at the $Z$-pole.

Designs of polarized positron sources have not reached maturity. Several approaches have been proposed, the most promising of which uses a helical undulator, but to date no real engineering designs, cost estimates, or experimental proofs of principle are available. Since much of the benefit of a polarized positron source would be negated if luminosity were compromised, it is very important that eventual designs have some margin on projected yields. Also, the source needs to be available for all collision energies. The helicity of a polarized positron source may be difficult to switch quickly and provision needs to be made to allow this, with a strong motivation to have helicity-switching capability at the train frequency. Present designs must be further developed and additional $R \& D$ is needed to pursue new schemes, some of which have been mentioned here.

Though a polarized positron source is not yet advanced enough to be included as part of the baseline linear collider design, it is an attractive feature that should be pursued as an upgrade. Site layout and engineering for a linear collider baseline design should accommodate such an upgrade at a later date. This has been done for the TESLA design and needs to be done for the NLC design as well.

\section{References}

[1] G. Moortgat-Pick and H. Steiner, EPJdirect C6, 1 (2001), DESY-00-178.

[2] A. Bartl, H. Eberl, H. Fraas, S. Kraml, W. Majerotto, G. Moortgat-Pick and W. Porod, hep-ph/0010018.

[3] S. Y. Choi, M. Guchait, J. Kalinowski and P. M. Zerwas, Phys. Lett. B479, 235 (2000) [hep-ph/0001175].

[4] G. Moortgat-Pick, A. Bartl, H. Fraas and W. Majerotto, Eur. Phys. J. C18, 379 (2000) [hep-ph/0007222].

[5] G. Moortgat-Pick and H. Fraas, Acta Phys. Polon. B30, 1999 (1999) [hep$\mathrm{ph} / 9904209]$.

[6] A. A. Babich, P. Osland, A. A. Pankov and N. Paver, Phys. Lett. B481, 263 (2000) [hep-ph/0003253]. 
[7] S. Cullen, M. Perelstein and M. E. Peskin, Phys. Rev. D62, 055012 (2000) [hep$\mathrm{ph} / 0001166]$.

[8] K. Hikasa, Phys. Rev. D33, 3203 (1986).

[9] M. Woods, Int. J. Mod. Phys. A15, 2529 (2000).

[10] P. C. Rowson and M. Woods, SLAC-PUB-8745 (2000).

[11] A. Blondel, Phys. Lett. B202, 145 (1988).

[12] F. Cuypers and P. Gambino, Phys. Lett. B388, 211 (1996); F. Cuypers and P. Gambino, PSI-PR-96-27 (1996).

[13] R. Hawkings and K. Monig, DESY 99-157 (1999).

[14] V. E. Balakin and A. A. Mikhailichenko, The Conversion System for Obtaining High Polarized Electrons and Positrons, INP 79-85 (1979).

[15] T. Okugi et al., Jpn. J. Appl. Phys. 35, (1996).

[16] J. Frisch, in Proceedings of the Workshop on New Kinds of Positron Sources for Linear Colliders, SLAC Report 502, 125 (1997).

[17] A. Potylitsin, Single Pass Laser Polarizatrion of Ultrarelativistic Positrons, arXiv:physics/0001004 (2000).

[18] A. P. Potylitsin, Nucl. Inst. and Meth. A398, 395 (1997).

[19] K. Flottmann, S. G. Wipf, Field Enhancement of a Superconducting Helical Undulator with Iron, TESLA 96-05. K. Flottmann, Investigations toward the Development of Polarized and Unpolarized High Intensity Positron Sources for Linear Colliders, DESY 93-161a (1993).

[20] T. Omori, A Concept of a Polarized Positron Source for a Linear Collider, KEK 99-188 (2000). 


\section{Chapter 13 Photon Collider}

\section{Introduction}

The concept of producing $\gamma \gamma$ collisions through Compton backscattering of laser photons in a linear collider [1,2] was proposed in 1981. The available laser technology was barely adequate for the accelerators operating at that time. The linear colliders proposed since then are orders of magnitude more ambitious and require equivalent improvements in laser technology to produce a $\gamma \gamma$ collider. Fortunately, breakthroughs in laser technology have made feasible lasers capable of delivering the $10 \mathrm{~kW}$ of average power in short pulses of $1 \mathrm{TW}$ peak power that are required for the NLC. The problem of obtaining such high peak power was resolved in 1985 with the invention of Chirped-Pulse Amplification (CPA). The high average power requirement could not be met without a long technology campaign that involved the development of diode-pumped lasers, adaptive optics and high-power multilayer optics, plus all of the associated engineering for thermal management. Nevertheless, today the laser and optics technology is finally in hand to proceed with an engineering design of a photon collider.

In the past few years there has been a crescendo of interest and theoretical activity in $\gamma \gamma$. This work has focused particularly on the precision measurement of the radiative width of the Higgs, the study of heavy neutral Higgs bosons, and on detailed studies of supersymmetric particles and the top quark. The $\gamma \gamma$ channel is also highly sensitive to new physics such as large extra dimensions and the appearance of strong gravity at the $10 \mathrm{TeV}$ scale.

With the publication of the TESLA Technical Design Report (TDR) and the development of the NLC/JLC toward full conceptual design, it was appropriate therefore to bring the photon collider from its highly schematic state into parity with the mature design of the rest of the accelerator. A year ago, a team of scientists and engineers from LLNL, SLAC, and UC Davis along with a FNAL-Northwestern theory consortium began to develop a complete design that would be required for full incorporation in the future NLC Conceptual Design Report. This effort involved a tightly integrated effort of particle theory and modeling, accelerator physics, optics, laser technology and engineering. The guiding principle was to develop a design that was robust, relied on existing technology, involved a minimum of R\&D, and posed the least risk. Considerations of elegance, power efficiency and cost, while not unimportant, were relegated to second place. A satisfactory design was also required to stay well away from compounding detector backgrounds, and to involve minimal modification to the existing Final Focus and detector geometries. While this is still 
a work-in-progress, the conclusion of the study so far is that a photon collider can be built with confidence on existing technology, satisfying these guidelines and criteria. This chapter describes the principal physics drivers for the $\gamma \gamma$ IR, and the basic design and technologies to implement it.

\section{Physics Studies at a $\gamma \gamma$ Collider}

\subsection{Production of Higgs bosons}

Perhaps the most important physics that can be done at a $\gamma \gamma$ collider is in probing the properties of the Higgs boson(s). At such colliders the Higgs bosons of the SM and the MSSM can be singly produced as $s$-channel resonances through one-loop triangle diagrams. They will be observed in their subsequent decay to $b \bar{b}, \tau^{+} \tau^{-}, W W^{*}, Z Z$, etc. Contributions to this type of loop graph arise from all charged particles that receive mass from the produced Higgs. In the SM, the loop contributions are dominated by the $W$ and top. SUSY contributions may be as large as $10 \%$ of the SM amplitude. In addition, other currently unknown particles may also contribute to the loop and their existence may be probed indirectly by observing a deviation from the SM value. (Since other particles, such as gravitons, can also appear in the $s$-channel, it will also be necessary to determine the spin of any resonances that are produced.) By combining measurements at both $e^{+} e^{-}$and $\gamma \gamma$ colliders it will be possible to determine both the quantity $\Gamma_{\gamma \gamma}$ and the Higgs total width [3,4].

A light Higgs $\left(m_{H} \leq 135 \mathrm{GeV}\right)$ can be detected in the $b \bar{b}$ mode, with the main background due to the conventional QED $\gamma \gamma \rightarrow b \bar{b}, c \bar{c}$ continuum [3,4]. Because of the relatively large $c \bar{c}$ cross section, excellent $b$ tagging is necessary. The two initial photon polarizations can be chosen to produce spin-zero resonant states and to simultaneously reduce the cross section for the background which, at tree level, is suppressed by $m_{q}^{2} / m_{h}^{2}$. Unfortunately, both QCD and QED radiative corrections remove this strong helicity suppression and must be well accounted for in both the Higgs and QED channels when comparing anticipated signals and backgrounds. Several detailed Monte Carlo simulations have been performed for this channel, with some typical results shown in Fig. 13.1 [5]; these have demonstrated that the quantity $\Gamma_{\gamma \gamma} B(h \rightarrow b \bar{b})$ can be determined with a relative error of $2 \%$. Assuming that the $b \bar{b}$ branching fraction can be measured to the level of $1 \%$ by combining $e^{+} e^{-}$and $\gamma \gamma$ data, $\Gamma_{\gamma \gamma}$ will be determined at the level of $2 \%$. This level of accuracy is sufficient to distinguish the SM and MSSM Higgs and to see contributions of additional heavy states to the triangle loop graph. If $e^{+} e^{-}$colliders can also provide the branching fraction for $h \rightarrow \gamma \gamma$ at the $\sim 10 \%$ level, the total Higgs width can be determined with a comparable level of uncertainty. A similar analysis can be performed using either the $W W^{*}$ or $Z Z$ final state for Higgs masses up to $350-400 \mathrm{GeV}$, with comparable results [4]. 
Photon Collider
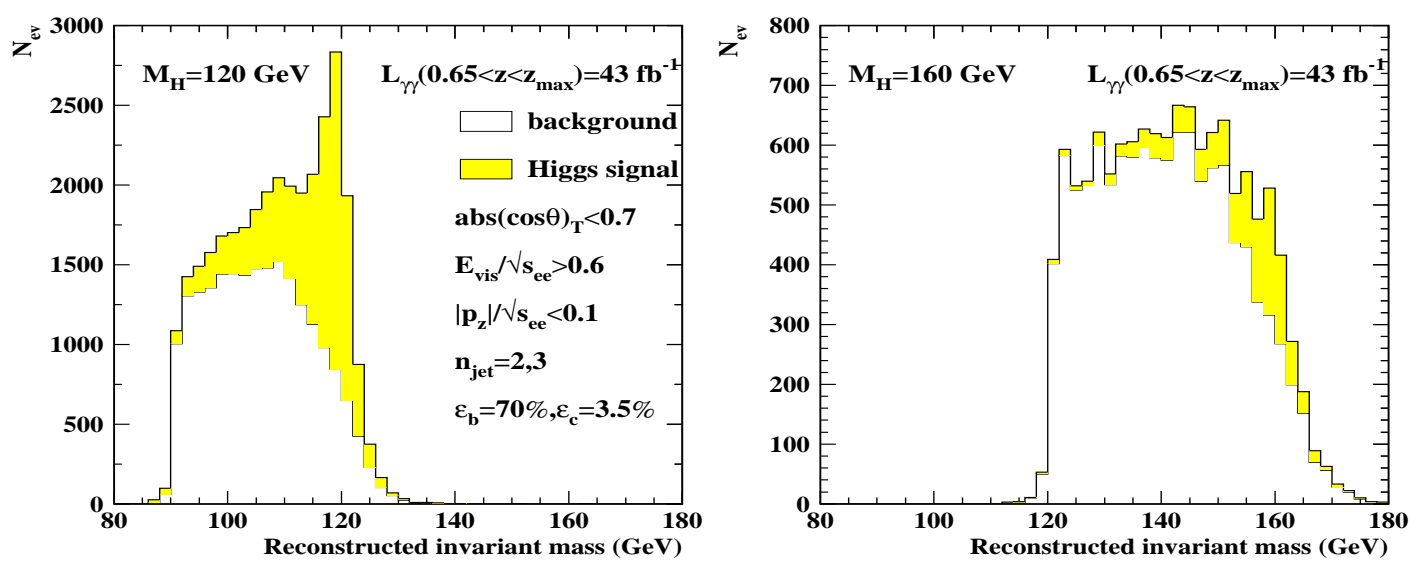

Figure 13.1: Mass distributions for the Higgs signal and heavy quark background for a Higgs mass of 120(left) and 160(right) GeV from Söldner-Rembold and Jikia [5]. The reduced signal-to-background at $160 \mathrm{GeV}$ reflects the diminished branching ratio to $b \bar{b}$ near the $W W$ threshold.

Very heavy Higgs bosons, such as those present in the MSSM, can also be produced as $s$-channel resonances in $\gamma \gamma$ collisions. In the MSSM, these heavy states have suppressed couplings to gauge bosons and may be most easily observed in $b \bar{b}$ or $t \bar{t}$ final states. These states may escape discovery at the LHC for intermediate values of $\tan \beta$. At $e^{+} e^{-}$colliders they can only be produced via associated production, $e^{+} e^{-} \rightarrow H A$, and thus lie outside the kinematic reach of the machine if their mass exceeds $240 \mathrm{GeV}$. The single production mode of the $\gamma \gamma$ collider allows the discovery reach to be extended to over $400 \mathrm{GeV}$. The $\gamma \gamma$ collider also allows one to separate degenerate $H$ and $A$ states and to study possible CP-violating mixing between $H$ and $A$ using linear polarization.

\subsection{Supersymmetric particle production}

For production significantly above threshold, sfermion and charged Higgs boson pairs have production cross sections in $\gamma \gamma$ collisions that are larger than those in $e^{+} e^{-}$ annihilation. Thus, $\gamma \gamma$ collisions can provide an excellent laboratory for their detailed study. In addition, $\gamma \gamma$ production isolates the electromagnetic couplings of these particles, whereas in $e^{+} e^{-}$the $Z$ and possible $t$-channel exchanges are also present. Thus complementary information can be obtained by combining data extracted from the two production processes. It should be noted that the search reach for new particles is typically somewhat greater in $e^{+} e^{-}$because of the kinematic cut-off of the photon spectra. However, the SUSY process $\gamma e \rightarrow \tilde{e}_{L, R} \chi_{1}^{0}$ shows that there are exceptions to this rule; the threshold for this process can be significantly below that for $\tilde{e}$ pair production in $e^{+} e^{-}$collisions when the $\chi_{1}^{0}$ is light. In the study of this reaction, both the $\tilde{e}$ and $\chi_{1}^{0}$ masses can be determined. 


\section{$2.3 \quad \gamma \gamma \rightarrow W^{+} W^{-}$and $\gamma e \rightarrow W \nu$}

New physics beyond the SM can affect the expected values of the trilinear and quartic couplings of gauge bosons. These couplings can be studied in the reactions $\gamma e \rightarrow W \nu$ and $\gamma \gamma \rightarrow W W$, as well as in $e^{+} e^{-} \rightarrow W W$ [6]. It is noteworthy that the photon collider reactions isolate the anomalous photon couplings to the $W$, while $e^{+} e^{-} \rightarrow W W$ also involves anomalous $Z$ couplings. In addition, the process $\gamma \gamma \rightarrow$ $W^{+} W^{-}$allows access to the quartic $\gamma \gamma W^{+} W^{-}$coupling. The complementarity of the three reactions in determining the anomalous couplings is illustrated in Fig. 13.2, taken from [6]. Since the time of this study, it has been understood how to achieve bounds on the anomalous couplings from $e^{+} e^{-} \rightarrow W W$ that are a factor of 30 better than those shown in the figure, by taking advantage of more systematic event analysis and higher luminosities. Methods for that analysis are described in Chapter 5, Section 2. A similar improvement should be possible for the constraints from $\gamma e \rightarrow W \nu$ and $\gamma \gamma \rightarrow W W$, though the detailed study remains to be done.

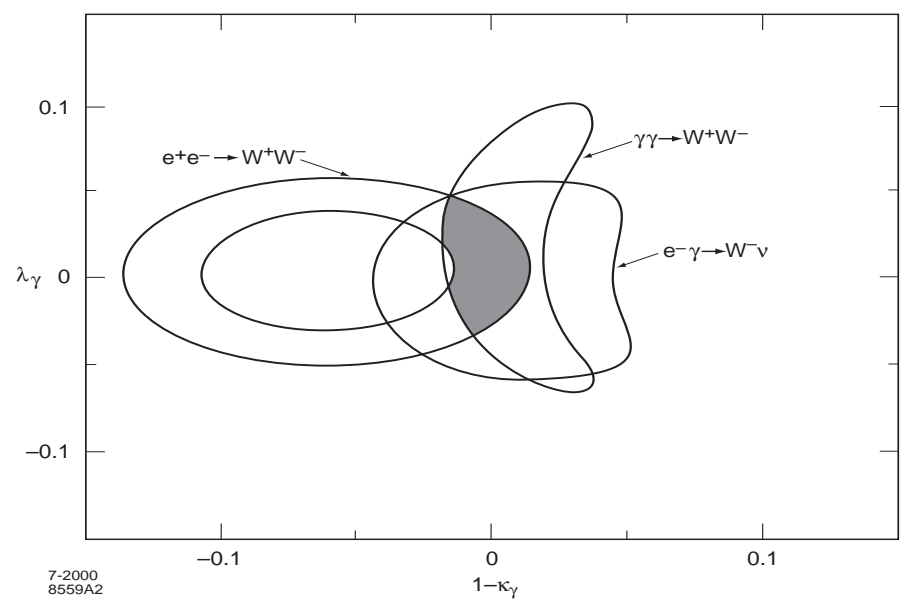

Figure 13.2: Allowed overlapping regions in the $\Delta \kappa_{\gamma}-\lambda_{\gamma}$ anomalous coupling plane, from the analysis of Choi and Schrempp [6].

The reaction $\gamma \gamma \rightarrow W^{+} W^{-}$is also highly sensitive to other forms of new physics such as the exchange of virtual towers of gravitons that occurs in models of millimeterscale extra dimensions $[7,8]$. It has been shown that this is the most sensitive process to graviton exchange of all those so far examined. Such exchanges can lead to substantial alterations in cross sections, angular distributions, asymmetries and $W$ polarizations. These effects make it possible to probe the associated gravitational mass scale, $M_{s}$, to values as high as $13 \sqrt{s}$ for the correctly chosen set of initial laser and electron polarizations. (For comparison, the reach in $e^{+} e^{-}$is about $7 \sqrt{s}$.) The search reach as a function of the $\gamma \gamma$ luminosity is shown in Fig. 13.3 for the various 
polarization choices. This same process can be used to search for graviton resonances such as those predicted in the Randall-Sundrum model [9].

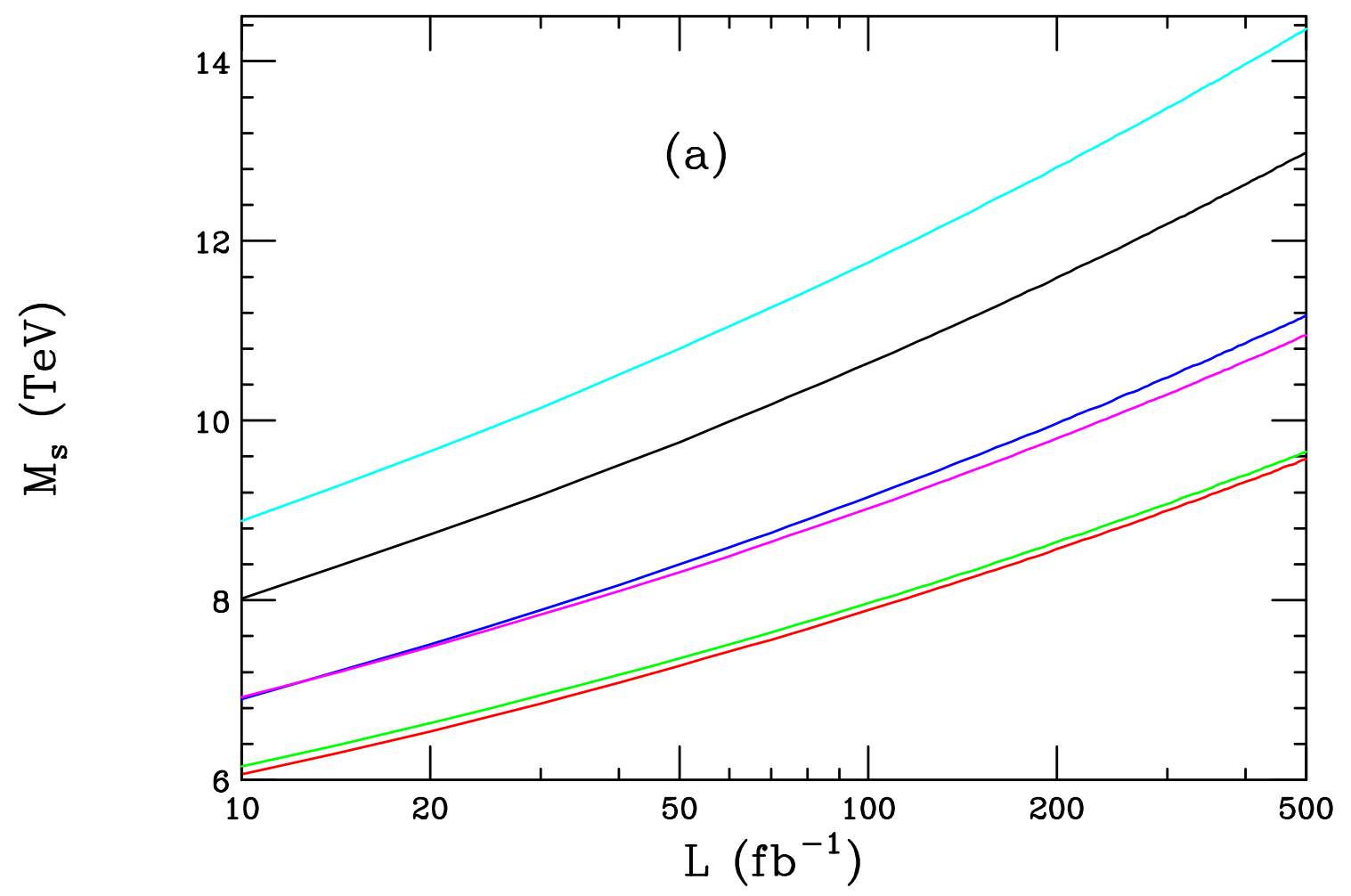

Figure 13.3: $M_{s}$ reach for the process $\gamma \gamma \rightarrow W^{+} W^{-}$at a $1 \mathrm{TeV} e^{+} e^{-}$collider as a function of the integrated luminosity for the different initial and final state polarizations. From top to bottom on the right hand side of the figure the polarizations are $(-++-),(+---)$, $(++--),(+-+-),(+---)$, and $(++++)$.

\section{$2.4 \gamma \gamma \rightarrow t \bar{t}$}

Since the top quark is the heaviest SM fermion, with a Yukawa coupling that is quite close to unity, one might expect that its properties may be the most sensitive to new physics beyond the SM. For example, the top may have anomalous couplings to the SM gauge bosons, including the photon. The cross section for top pairs in $\gamma \gamma$ collisions is somewhat larger than in $e^{+} e^{-}$, thus this process may provide the best laboratory to probe new physics couplings to the top. In addition, while both $e^{+} e^{-}$ and $\gamma \gamma$ colliders can probe the anomalous $\gamma t \bar{t}$ couplings, these are more easily isolated in $\gamma \gamma$ collisions. As shown in [10], there are 4 form factors that describe this vertex, one of which is CP-violating and corresponds to the top quark electric dipole moment. By measurements of the $t \bar{t}$ angular distribution significant constraints on these form 
factors are possible with sensitivities to both electric and magnetic dipole moment couplings that are about an order of magnitude better in $\gamma \gamma$ colliders than in $e^{+} e^{-}$ machines. In addition, CP-violating couplings can be directly probed through the use of polarization asymmetries and limits superior to those obtainable from $e^{+} e^{-}$ colliders are possible.

\subsection{Other processes}

There are many other interesting processes that one can study in $\gamma \gamma$ collisions. As far as new physics is concerned, the $Z \gamma$ and $Z Z$ final states can be used to probe anomalous $Z Z \gamma$ and $Z \gamma \gamma$ couplings [11] while the $\gamma \gamma$ final state can be used to search for non-commutativity and violations of Lorentz invariance in QED [12]. The couplings of leptoquarks discovered in $e^{+} e^{-}$collisions can be more easily disentangled by using data from both $\gamma \gamma$ and $\gamma e$ collisions [13]. It may also be possible to form resonances of stoponium, the supersymmetric version of toponium, with production rates that are significantly higher than in $e^{+} e^{-}[14]$.

Within the SM there are a number of interesting QCD processes that can also be examined to obtain information on topics such as the gluon and quark content of the photon, the spin-dependent part of the photon structure function, and the QCD pomeron. These topics are reviewed in Chapter 7, Section 3.

\section{Compton Backscattering for $\gamma \gamma$ Collisions}

\subsection{Introduction}

High-energy photons can be produced through two-body scattering of laser photons from a high-energy electron beam. For example, the scattering of $1 \mathrm{eV}$ laser photons from an electron beam of $250 \mathrm{GeV}$ can produce gammas of up to $200 \mathrm{GeV}$. An electron linear collider can be converted to a $\gamma \gamma$ collider if a high-power laser pulse intersects the electron beam just before the interaction point (IP). The point where the laser beam intersects the electron beam - the conversion point $(\mathrm{CP})$ - can be within $1 \mathrm{~cm}$ of the IP. A high $\gamma \gamma$ luminosity comparable to that of $e^{+} e^{-}$can be achieved, since the photons will focus to about the same spot size as the electron beam. The principles are reviewed in detail elsewhere [15].

\subsection{Photon spectra}

For the case mentioned above- $1 \mathrm{eV}$ laser photons and $250 \mathrm{GeV}$ electrons - the energy spectrum of the backscattered photons ranges from 0 up to 0.8 of the incoming beam energy. Two-body kinematics creates a correlation between the photon energy and the angle between the outgoing photon and the incoming electron. The maxi- 

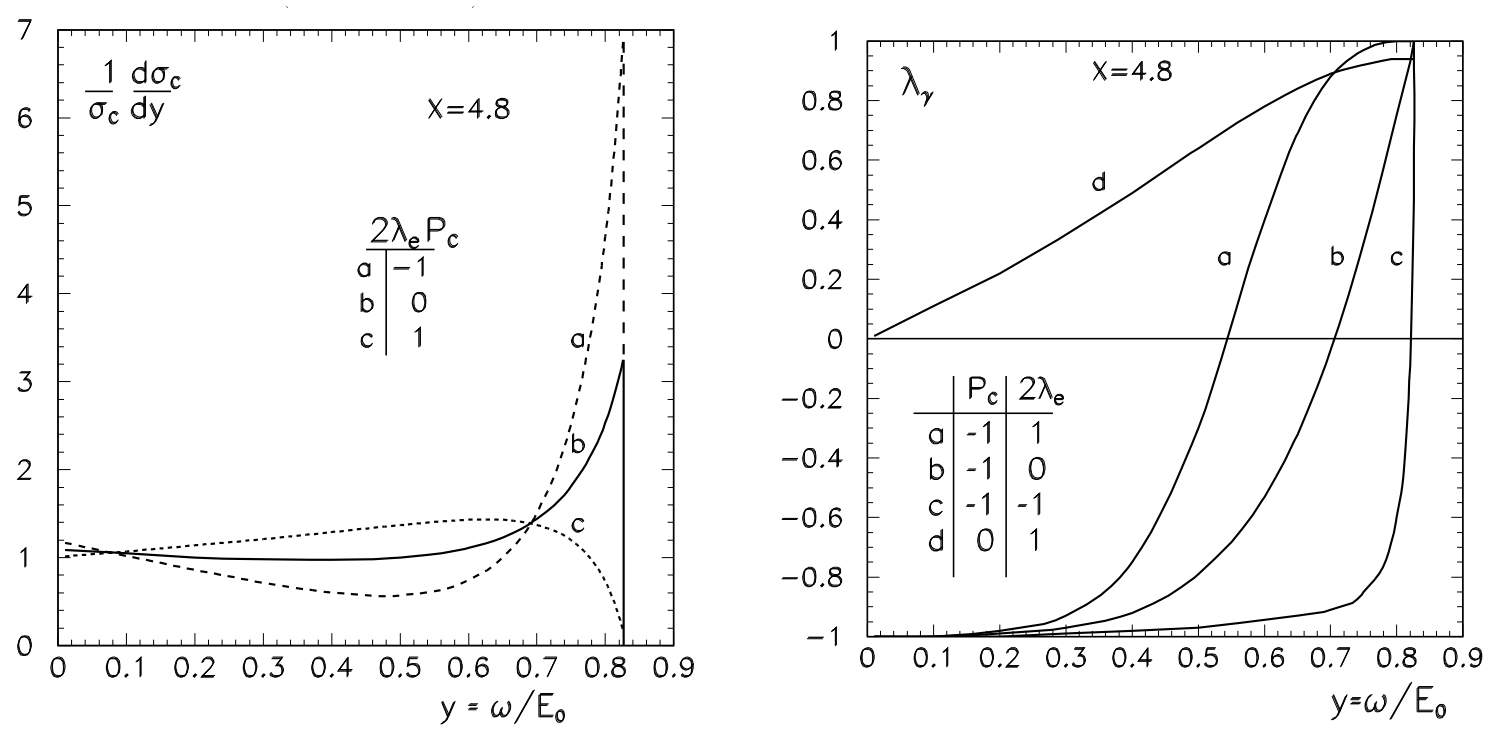

Figure 13.4: The energy spectrum and helicity spectrum of the Compton-backscattered photons for various helicities of the incoming electron beam with circularly polarized incoming photons [15]. The variable $y$ is the photon energy as a fraction of the electron beam energy. The laser photon and electron helicities are designated by $P_{c}$ and $\lambda_{e}$. The parameter $x=4 E_{e} \omega_{0} / m^{2} c^{4}$.

mum photon energy occurs when the produced photon is collinear with the incoming electron.

The exact energy spectrum is a function of the polarization of the incoming electron and laser beams. Figure 13.4 shows the energy spectrum of the backscattered photons for circularly polarized laser photons. The population of the high-energy peak is maximized when the electron beam is fully polarized and of opposite helicity to the laser beam. For that situation, the high-energy photons are also fully circularly polarized. While the lasers naturally produce linearly polarized photons, any combination of circular and linear polarization can be produced through the use of quarter-wave plates.

From Fig. 13.4 it can be seen that the ability to polarize the incoming electron beam is crucial for producing high-energy $\gamma \gamma$ collisions with polarized gammas. Currently it is foreseen that the electron beams will achieve $80 \%$ polarization while positrons will be unpolarized. This makes it attractive to run in an $e^{-} e^{-}$mode rather than $e^{+} e^{-}$. Many Standard Model backgrounds are also suppressed by choosing $e^{-} e^{-}$ running.

Calculating the $\gamma \gamma$ luminosity spectrum at the IP is not as simple as convoluting the single-scattering energy spectrum with itself. There are additional sources of $\gamma^{\prime}$ s that must be included. An electron can Compton backscatter multiple times as it 
passes through the laser beam. This leads to a tail of low-energy photons, as can be seen in Fig. 13.5. Also, the leftover electron beam arrives at the IP coincident with the photons. When the two electron beams interact they produce a large number of beamstrahlung photons. All of these contribute to the $\gamma \gamma$ luminosity.
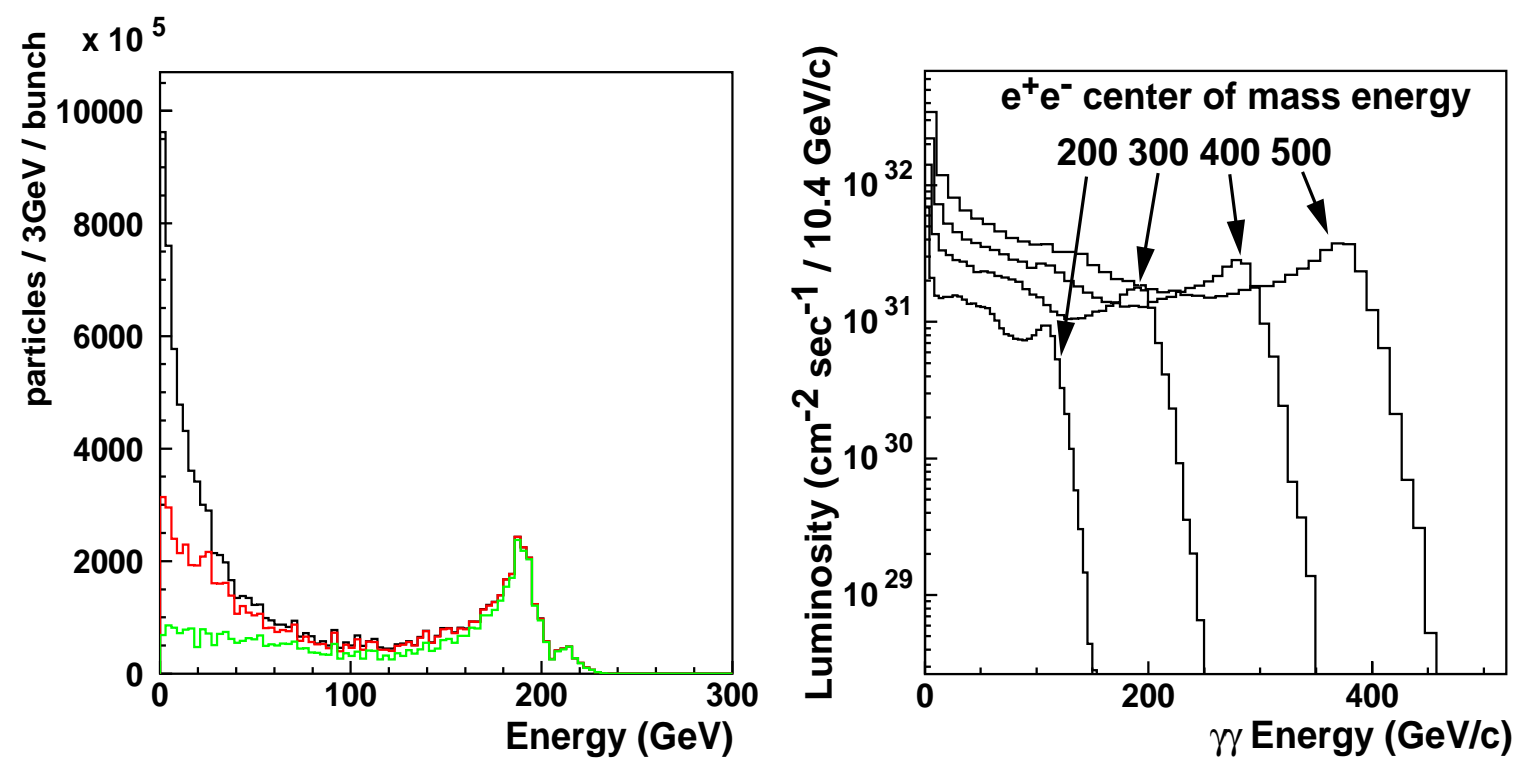

Figure 13.5: The first plot shows the energy spectrum from Compton backscattering when (respectively, from the bottom curve to the top) primary, secondary, and all higher scatters are taken into account. The second plot shows the $\gamma \gamma$ luminosity for $e^{-} e^{-}$center-of-mass energies of 500, 400, 300, and $200 \mathrm{GeV}$ for the NLC-B machine parameters.

As a result of the energy-angle correlation, the spot size for collision of soft photons will be larger than that for the collision of harder photons. Thus the luminosity spectrum may be hardened by increasing the distance between the CP and IP. In the following, we chose the $\mathrm{CP}$ to be $5 \mathrm{~mm}$ from the IP.

To compute the $\gamma \gamma$, e $\gamma$ and $e e$ luminosities, we use the program CAIN [16], which models all of the processes just described. Results for various incident electron beam energies are shown in Fig. 13.5. The luminosity spectrum peaks at $\gamma \gamma \mathrm{CM}$ energies close to 0.8 times of the $e^{-} e^{-}$CM energy. The decrease of luminosity with decreasing CM energy, apparent from the plot, is primarily caused by the increased spot size of the electron beams and, secondarily, by a softer Compton-backscattering spectrum. For a $120 \mathrm{GeV}$ Higgs this leads to a situation where higher luminosities can be achieved by running at $500 \mathrm{GeV} e^{-} e^{-} \mathrm{CM}$ energy at the cost of having unpolarized photons. For measurements requiring definite states of $\gamma \gamma$ polarization, on-peak running with $150 \mathrm{GeV} e^{-} e^{-} \mathrm{CM}$ energy is required. 
Photon Collider

\subsubsection{Accelerator modifications}

While no changes to the accelerator are required to produce $\gamma \gamma$ collisions, some changes can optimize performance. Beam-beam interactions are a major concern for $e^{+} e^{-}$but are not present in $\gamma \gamma$ collisions. Therefore the $\beta$ functions of the Final Focus should be as small as possible to achieve a minimum spot size and maximum luminosity. The luminosity improvements from small $\beta$ functions are limited by chromatic aberrations in the Final Focus and the hourglass effect, in which the $\beta$ function becomes comparable to the longitudinal spot size. In addition, a small transverse spot size tends to select unboosted events because of the correlation between the energy and production angle of the high-energy $\gamma$ 's. A Final Focus design with rounder beams simplifies the final doublet stabilization and has been shown to recover nearly a factor of two in luminosity by increasing the contribution of boosted events. However, these boosted events suffer from reduced reconstruction efficiency and we have not yet optimized the design for this effect.

Achieving rounder beams requires only a change in the strength of the Final Focus magnets. It is useful also to cut the number of bunches in half and double the bunch charge, to better match the laser technology. This nominally increases the luminosity by a factor of two, although this is not fully achieved due to the increased emittance growth and the increased longitudinal spot size. The parameters we use are shown in Table 13.1. These have been reviewed and approved by the NLC machine group. When we reduce the $e^{-} e^{-} \mathrm{CM}$ energy such that the $\gamma \gamma$ peak is at $120 \mathrm{GeV}$ for Higgs running, the $\gamma \gamma$ luminosity becomes $2.9 \times 10^{31} \mathrm{~cm}^{2} / \mathrm{s} / \mathrm{GeV}$ at $\sqrt{s_{\gamma \gamma}}=120 \mathrm{GeV}$, with $80 \%$ of events being spin 0 .

\begin{tabular}{|l|c|}
\hline \hline$e^{-} e^{-}$CM Energy $(\mathrm{GeV})$ & 490 \\
Luminosity & $1.23 \times 10^{33} @>65 \% e^{-} e^{-}$energy \\
Bunch Charge & $1.5 \times 10^{10}$ \\
Bunches / pulse & 95 \\
Bunch separation & $2.8 \mathrm{~ns}$ \\
$\gamma \epsilon_{x}$ at IP & $360 \times 10^{-8} \mathrm{~m}-\mathrm{rad}$ \\
$\gamma \epsilon_{y}$ at IP & $7.1 \times 10^{-8} \mathrm{~m}-\mathrm{rad}$ \\
$\beta_{x} / \beta_{y}$ at IP & $0.76 / 1.81 \mathrm{~mm}$ \\
$\sigma_{x} / \sigma_{y}$ at IP & $76 / 16 \mathrm{~nm}$ \\
$\sigma_{Z}$ at IP & $0.150 \mathrm{~mm}$ \\
\hline \hline
\end{tabular}

Table 13.1: NLC-G parameter set. Unless otherwise noted parameters are identical to NLC-H. 


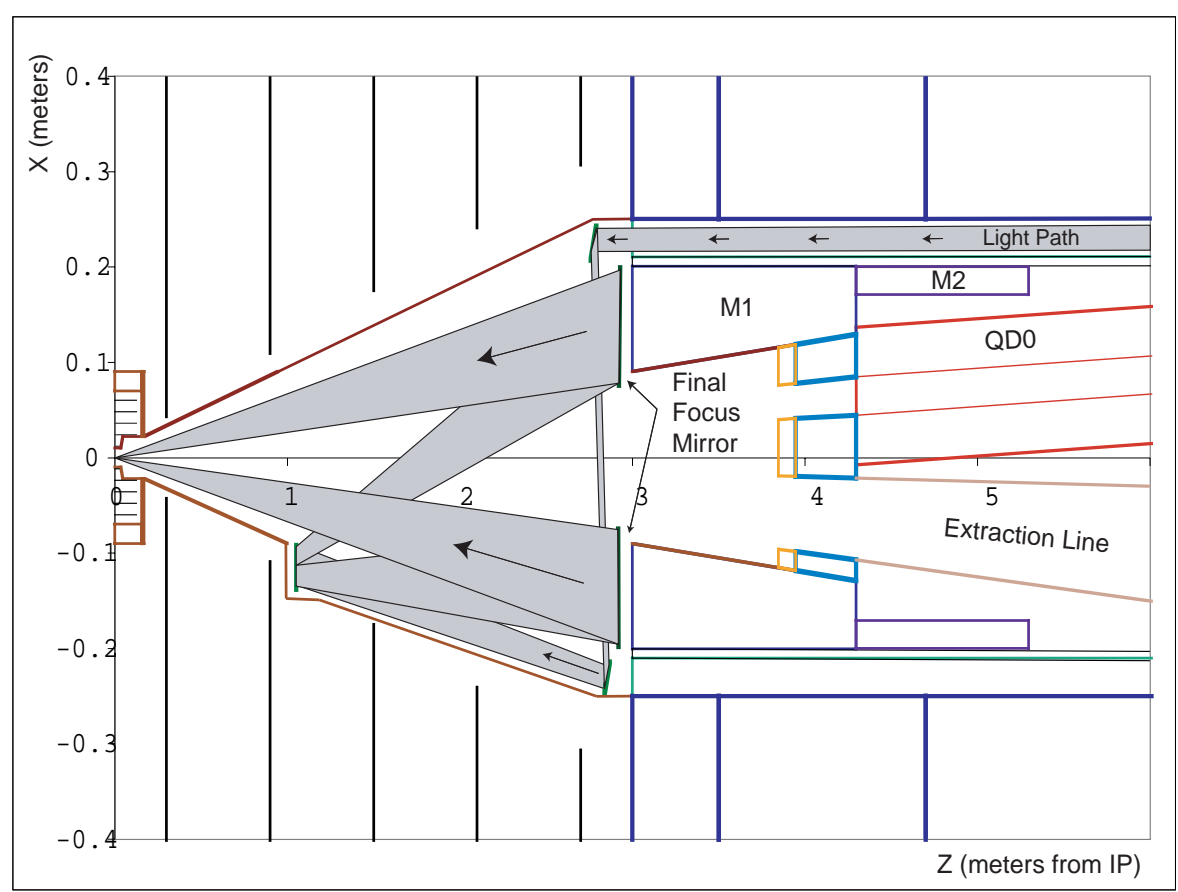

Figure 13.6: Optical configuration to inject the laser light into the Interaction Region. The high subpulse intensity requires all these optics to be reflective and mounted inside the vacuum enclosure.
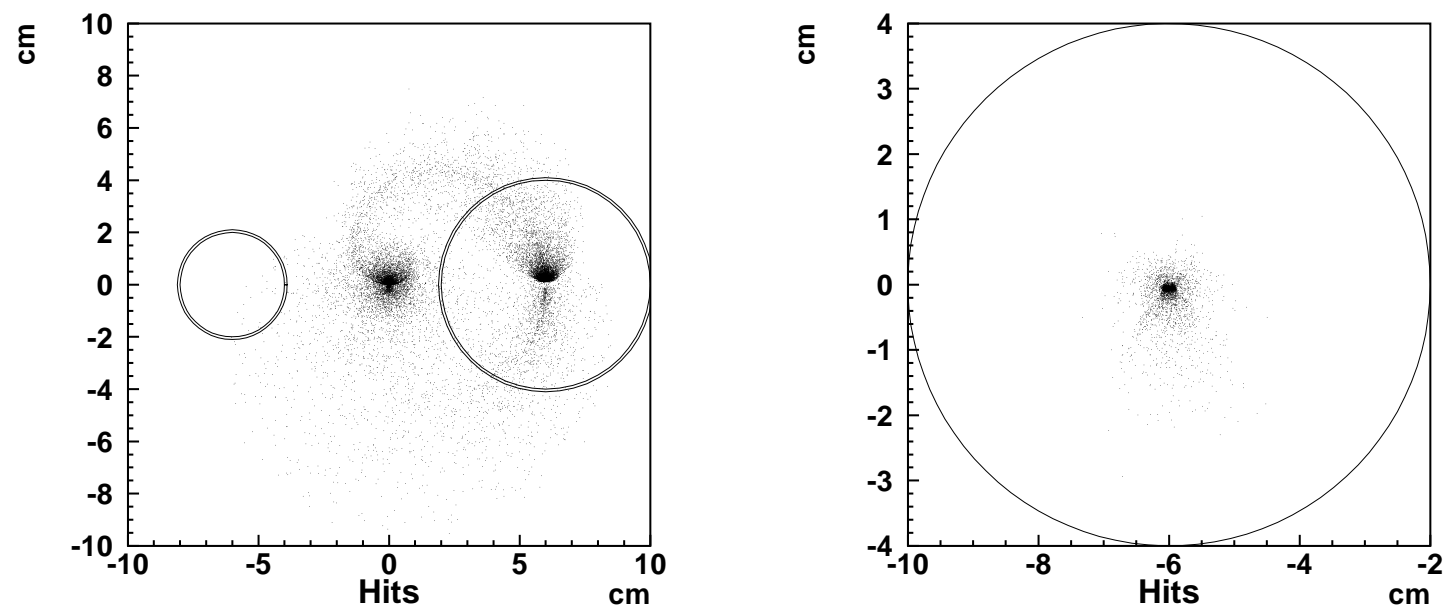

Figure 13.7: The front face of the magnet at $z=4$ meters. The first plot shows the impact point of the pair background. High-energy particles travel out the extraction line. Lowenergy particles spiral in the solenoidal magnetic field of the detector. The second plot is an expanded view of the extraction line aperture showing the location of the outgoing beam. 
Photon Collider

\subsection{Interaction region design and backgrounds}

Figure 13.6 shows the interaction region for a $\gamma \gamma$ collider. The design begins from the IR for $e^{+} e^{-}$collisions, but many modifications are needed to accommodate the laser beams. The first of these is the system of mirrors required to bring laser light into the IR, described in detail in Section 4.1. The mirrors have been carefully placed to be outside the path of the beams and the pair background. The pair background consists of low-energy electrons and positrons which spiral in the magnetic field of the detector. Their transverse location at $z=4$ meters can be seen in Fig. 13.7. No additional backgrounds are generated by the presence of the mirrors.

The extraction lines for the spent beams must be modified for the $\gamma \gamma$ interaction region. The energy spectrum of electrons leaving the conversion point is composed of unscattered electrons at the beam energy and scattered electrons which peak around $1 / 5$ the beam energy. The low-energy electrons receive a larger angular deflection from the beam-beam interaction at the IP, necessitating an increased aperture for the extraction line. Additionally, just as in the case of the pair background, the low-energy electrons spiral in the magnetic field. Figure 13.7 shows the position of the outgoing electrons at the entrance to the extraction line. An aperture of \pm 10 milliradians accommodates these particles. In order to prevent mechanical interference between the extraction line and the last focusing quadrupole, the crossing angle has been increased from 20 to 30 milliradians.

Increasing the extraction line aperture has a detrimental effect on the neutron background levels at the IP. The Silicon Vertex Detector, $1.2 \mathrm{~cm}$ away from the IP, now has a direct line of sight back to the beam dump 150 meters away. It experiences a fluence of $10^{11}$ neutrons $/ \mathrm{cm}^{2} /$ year. The standard CCD technology chosen for the $e^{+} e^{-}$IR cannot withstand it. The $\gamma \gamma$ IR would need a rad-hard CCD or pixel design.

We foresee no impact to the detector aside from the need for a rad-hard vertex detector. The machine backgrounds in the $\gamma \gamma$ IR are comparable to the $e^{+} e^{-}$IR. Still to be evaluated is the effect of resolved photon events from the higher $\gamma \gamma$ luminosity.

\section{IR optical system}

\subsection{Optics design}

The function of the optical system is to bring the laser beam to the IR while also minimizing the required laser pulse energy. The requirement for efficient conversion of the electrons sets the laser photon density required at the interaction point. The optical system will focus the laser beam at a point near the interaction point to maximize the conversion probability. The size of the laser focal spot will be much larger than the electron beam; therefore the size of the focal point should be minimized in order to minimize the required laser pulse energy. 
When bringing a beam to a focus, the size of the focal point is determined by the f-number of the focusing optic, defined as the ratio of the focal length to the optic diameter. The size of the useful laser spot is approximately the wavelength times the f-number. There is a limit, however, to how small one can usefully make the f-number. The focal spot has a limited depth of focus. When the electron-photon interaction region becomes longer than the depth of focus, the required laser energy becomes independent of the f-number. Lowering the f-number beyond this point results in no decrease in the required laser pulse energy. Optimally, the laser pulse length should be the same as the electron bunch length to minimize the required pulse energy. However, at such high intensities, non-linear effects degrade the purity of the photon polarization. We choose a pulse length of 2 ps FWHM, which is well matched to the available laser technology. For such a 2 ps laser pulse, decreasing the f-number below 7 gains little further energy reduction. For the reference design the f-number is 8 .

Figure 13.6 shows the optical design near the interaction region. The final focusing optic is located at the $3 \mathrm{~m}$ station and is mounted adjacent to (or on) the $40 \mathrm{~cm}$ diameter tungsten plug (M1). The optic has a $300 \mathrm{~cm}$ focal length and a $38 \mathrm{~cm}$ diameter, giving it an f-number of 8 . The central $15 \mathrm{~cm}$ hole provides a space for the electron beams and high-energy scattered electrons to pass through the Final Focus optic. The secondary optic is mounted off-axis to minimize the obscuration of the laser beam. Additional turning optics provide centering and pointing capabilities as well as beam injection to the secondary optic. The high subpulse intensity requires all these optics to be reflective and mounted inside the vacuum chamber.

The laser beam enters the IR from one side. A symmetric set of optics (not shown in Fig. 13.6) takes the beam to a mirror that sends the beam back to a focus intersecting the second electron beam. The difference in the image plane of the focal spots as well as the difference in arrival times can then be used to separate the incoming and exiting laser beams in the beam transport system.

\subsection{Beam pipe modifications}

The short pulse format of the laser results in beam intensities that cannot be propagated through air or transmissive optics. The pulse compression, beam transport and IR injection optics will all be reflective optics inside vacuum enclosures. The small vacuum pipe that transports the electron beam must be expanded in the IR to contain the laser injection optics (as shown in Fig. 13.6). The level of vacuum required will be determined by the electron beam since it will be higher than needed by the laser. It should be noted that the vacuum requirement of the electron beam may place restrictions on the materials that can be used in the optics mounts and controls.

The laser beam transport pipe will contain isolation gate valves that will be open when the laser is operating. These valves can be closed during maintenance and 
Photon Collider

other operations when the laser in not operating. They can also be used to prevent contamination or accidental pressurization of the linac and IR during shutdowns.

The optics and vacuum enclosures will be mounted on the same structures as the electron beam transport system. The electron beam transport system in the IR region has not been designed in sufficient detail to begin the design of the laser system interfaces. The seismic requirements for the laser optics are not as stringent as for the Final Focus magnets. If both systems use the same supports, it will be important that the laser system does not feed excessive acoustic energy into the final quadrupole support structure.

\section{$5 \quad$ Laser system}

\subsection{Requirements and overview}

The laser system must match the pulse format of the electron beam and supply an adequate photon density at the IR to backscatter the laser photons efficiently to gamma rays. For efficient conversion of $250 \mathrm{GeV}$ electrons, the optimal laser wavelength is one micron. The laser requirements for the NLC are summarized in Table 13.2 .

A picosecond-duration laser pulse cannot be amplified to the joule level directly. The combination in the laser subpulse of a high pulse energy $(1 \mathrm{~J})$ and a short pulse duration (2 ps) generates field intensities that will damage laser materials. This problem is solved by first stretching a very low-energy laser subpulse to 3 ns and then amplifying this long pulse. The pulse is then compressed back to 2 ps for use in the IR. The procedure for stretching and compressing the laser pulse with diffraction gratings, known as Chirped-Pulse-Amplification (CPA) [17], is discussed below. The procedure requires the laser medium to have significant gain bandwidth.

Efficiently energizing a laser with the very low required duty factor (300 ns/8 ms) requires the use of a 'storage laser' material. Generally storage lasers are solid-state and, when used in a high-pulse-rate application, they are strongly limited by heatremoval capabilities. LLNL has been developing a solid-state Yb:S-FAP laser with diode pump lasers and rapid helium gas cooling to address this issue as part of its Inertial Fusion Energy program. The Mercury Laser Project is currently assembling a prototype. The default Mercury laser pulse format differs from that required for $\gamma \gamma$ operation. The necessary modifications of the laser are described below.

\subsection{Laser system front end}

The laser system front end must generate a low-power laser signal with a temporal format matched to that of the electron linac. This signal will then be delivered to the Mercury amplifiers to generate the high pulse energies needed to interact with the electron pulses. 
A laser oscillator will be required with an approximately $350 \mathrm{MHz}$ pulse rate and 2 ps pulse duration. With pulse energies of $1.0 \mathrm{~nJ}$, the average power will only be only $0.35 \mathrm{~W}$. The laser must be tuned to the 1.047 micron wavelength which overlaps the gain bandwidth of the Yb:S-FAP laser amplifiers. Commercial Ti-sapphire lasers will be appropriate for this task. The laser oscillator must have high frequency stability and must be locked to the master clock of the linac so that the laser pulse timing matches that of the electron pulses.

The beam from the oscillator will pass through a Pockels cell slicer that will cut out $300 \mathrm{~ns}$ pulse trains at $120 \mathrm{~Hz}$. These batches will match the electron bunch trains, which contain approximately 100 subpulses. The pulse trains will then be passed through an electro-optic modulator that will impose a moderately increasing amplitude ramp on the macro-pulse. This amplitude ramp is designed to offset the decreasing gain ramp that will be experienced in the amplifier as the stored energy is extracted during the laser macro-pulse. The low power (about $1 \mu \mathrm{W}$ ) is easily handled by current EO modulators.

The gain in the amplifier will have frequency variations as well as amplitude diminution during the macro-pulse. To avoid strong amplitude variations at different frequencies in the amplified laser signal, the amplitude of the input laser beam will be sculpted in frequency space [18] to offset the effects of the gain variation. The short pulse length of the subpulses gives them a frequency bandwidth such that a diffraction grating will spread the beam over a range of angles. The different frequencies are then passed through a programmable liquid crystal display that provides different attenuation for different positions (frequencies) in the beam.

The laser beam is next passed through a diffraction grating pulse stretcher, described in a later section, that stretches the 2 ps subpulses to 3 ns. The spectral sculpting and pulse stretching might be combined into a single device if appropriate.

The stretched laser pulses can now be passed through a high-gain, low-power preamplifier. A laser optical parametric amplifier (OPA) will provide the high bandwidth needed to preserve the frequency profile of the laser pulse. A high-pulse energy green laser will pump a BBO crystal to provide the gain needed. The laser beam will be amplified to $500 \mu \mathrm{J} /$ subpulse. The beam will be split into twelve $10 \mathrm{~Hz}$ beams and then injected into the Mercury amplifiers.

\begin{tabular}{|l|c||l|c|}
\hline \hline Wavelength & $1 \mu$ & Format & $\sim 100$ subpulses/macro-pulse \\
Subpulse energy & $1 \mathrm{~J}$ & Repetition rate & $120 \mathrm{~Hz}$ \\
Subpulse separation & $2.8 \mathrm{~ns}$ & Gain bandwidth & $10 \mathrm{~nm}$ \\
Subpulse duration & $2 \mathrm{ps}$ & Beam quality & $<1.5$ diffraction limit \\
\hline \hline
\end{tabular}

Table 13.2: $\gamma \gamma$ collider laser requirements. 


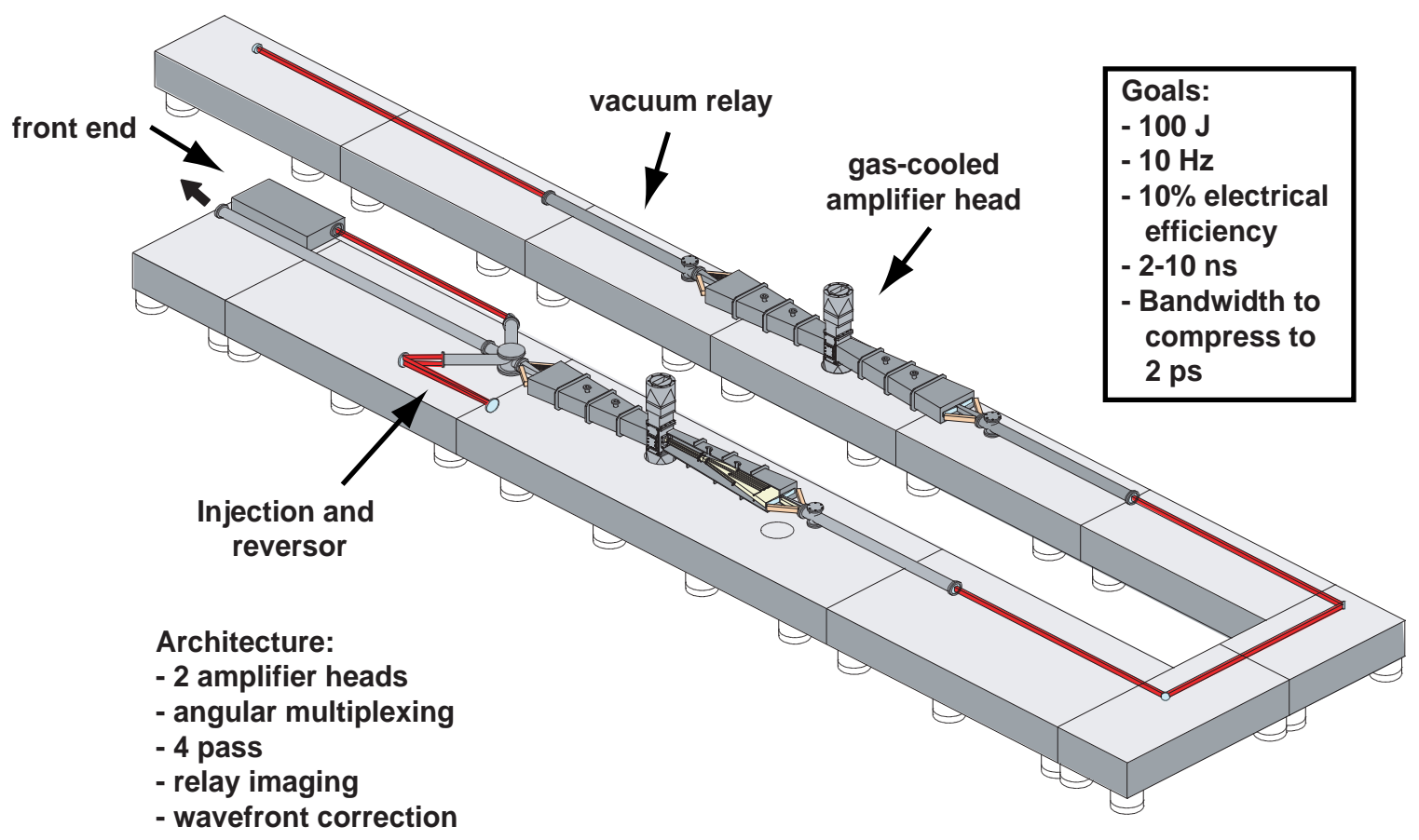

Figure 13.8: The diode-pumped solid state Mercury laser is a high-pulse rate, nextgeneration laser fusion driver.

\subsection{Mercury amplifier}

The Mercury laser (Fig. 13.8) will operate at $10 \mathrm{~Hz}$ with $100 \mathrm{~J}$ pulses. Twelve such lasers would have to be time-multiplexed to achieve the $\gamma \gamma$ laser requirements. The major challenge will be the modification of the Mercury laser pulse format, which is currently a single several-nanosecond-long pulse. Achieving the desired diffractionlimited beam quality will also be an important challenge.

The Mercury laser utilizes three primary innovations to achieve the goal of a highefficiency, high-repetition-rate laser driver for laser fusion experiments. The first is that the removal of heat from the laser media is accomplished by flowing helium at high speed over the surface of thin laser slabs. The thermal gradients in the laser media are oriented both in the short dimension, for effective conductive cooling, and in the direction of the laser propagation, to minimize the optical distortion. The low index of refraction of helium minimizes the helium thermal-optical distortions that must later be removed with adaptive optics. Figure 13.9 shows the arrangement of thin laser slabs embedded in flow vanes within the helium flow duct. Full-scale demonstrations have validated the flow and thermal models have confirmed that the design meets the optical system requirements.

The second innovation is the use of diode lasers rather than flash lamps to energize 

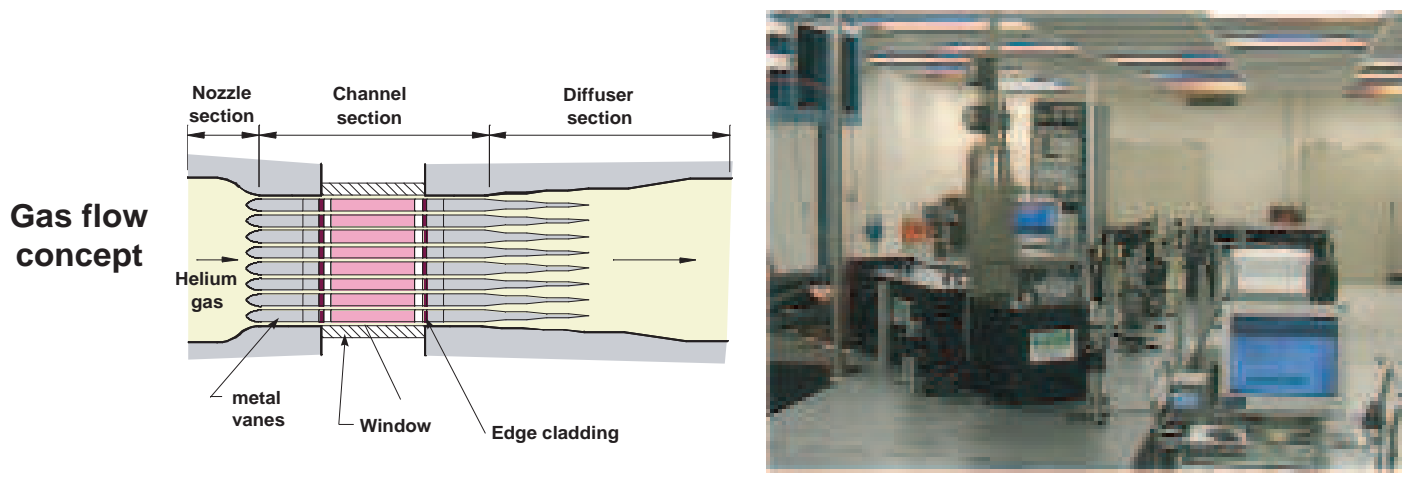

Mercury Lab

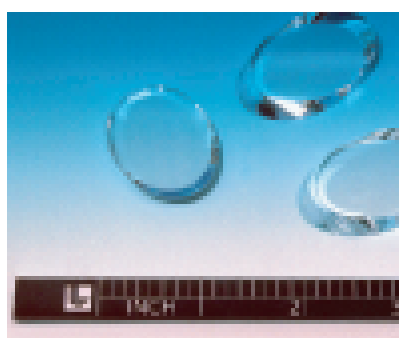

Yb:S-FAP crystals

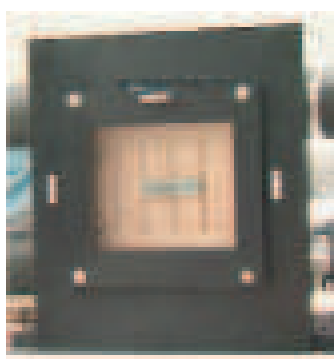

Diode array capable of $160 \mathrm{~kW}$

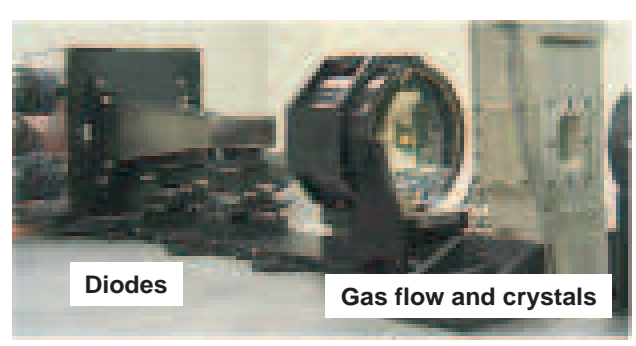

Figure 13.9: The Mercury laser will utilize three key technologies: gas cooling, diodes, and Yb:S-FAP crystals to deliver $100 \mathrm{~J}$ at $10 \mathrm{~Hz}$ with $10 \%$ efficiency.

the laser media. The narrow frequency output of the diode laser is matched to the absorption band of the laser media. The efficient coupling and the efficiency of diode lasers result in significantly higher pumping efficiency of the laser media and also significantly lower waste heat that must be removed by the helium cooling system. The primary challenge for the diode laser design is minimizing the high capital cost of the diode laser and its packaging design. LLNL has developed a low-cost packaging design that also efficiently couples the diode light into the laser slabs. This design has been produced under commercial contract and will be tested this year in the Mercury laser laboratory.

The third innovation is the use of Yb:S-FAP as the laser media instead of the usual Nd-glass. This crystalline media has better thermal conductivity for cooling, longer storage lifetime for efficient pumping, and a high quantum efficiency to minimize waste heat. The growth of these new crystals (Fig. 13.9) with adequate size and optical quality has been the primary technical challenge in the Mercury project. Crystals 
Photon Collider

grown recently may satisfy these requirements, but some testing remains to be done.

The Mercury laser has two amplifier heads and a four-pass optical system. This year one amplifier head and the full optical configuration will be tested in the Mercury laboratory. A second amplifier head must be constructed before full-power extraction can be demonstrated.

\subsection{Multiplexer and beam transport}

The beams from twelve Mercury lasers, each operating at $10 \mathrm{~Hz}$, must be combined into a single co-aligned beam to produce the required $120 \mathrm{~Hz}$ beam. The beam combination should occur before the pulse compressor to minimize the stress on the combiner optics. At these low pulse rates the simplest beam combination scheme is a simple rotating faceted optic.

The beam combination optic is a $4 \mathrm{~cm}$-diameter optic with twelve flat facets each covering a thirty degree sector. Each facet is ground at a slightly different angle. The optic is rotated on its axis at $10 \mathrm{~Hz}(600 \mathrm{rpm})$. The twelve incoming laser beams arrive at slightly different angle, such that they are all aligned after reflection off the optic. The angle differences are sufficiently large to allow the incoming laser beams to be projected from spatially separated optics. The incident laser beam diameter of $0.5 \mathrm{~cm}$ will give a power density of $5 \mathrm{~kW} / \mathrm{cm}^{2}$ on the optic. This will be below the damage threshold of $10 \mathrm{~kW} / \mathrm{cm}^{2}$. The optic can be made larger if a larger damage margin is desired.

The combined beam is then transported to the pulse compressor. The pulse compressor can be located in the laser facility or close to the detector, just prior to the final transport optics into the IR. For the reference design it is assumed that the compressor is located in the laser facility and that the laser facility is located a nominal 100 meters from the detector hall. The transport of the laser beams will be in vacuum pipes from the exit of the Mercury laser modules. To minimize the evolution of amplitude variations due to diffraction or phase aberrations, the laser beam will be expanded to a nominal $10 \mathrm{~cm}$ and image-relayed. The vacuum tubes should be $15 \mathrm{~cm}$ to allow for errors in initial alignment procedures.

\subsection{Compressor / stretcher}

The basic concept of compressing long pulses into short pulses after amplification is well known and widely used $[19,20]$. The challenge is in designing and fabricating high-efficiency gratings that can handle high-power laser beams. The specifications for the stretcher and compressor systems are given in Table 13.3.

The subpulses from the oscillator are 2 ps and $1.0 \mathrm{~nJ}$. Their transform-limited full-width-half-maximum is $0.9 \mathrm{~nm}$. The gratings in the stretcher give the beam an angular spread. Light of different wavelengths within the bandwidth of the laser follows optical paths of different length, thus introducing a frequency-time correlation 


\begin{tabular}{|l|l|l|}
\hline \hline & Stretcher & Compressor \\
\hline Substrate material & silica & silica \\
Coating material & gold & Multi-layer \\
First grating size $(\mathrm{cm})$ & $4 \times 15$ & $30 \times 84$ \\
Second grating size $(\mathrm{cm})$ & $4 \times 15$ & $30 \times 84$ \\
Roof mirror size $(\mathrm{cm})$ & $4 \times 8$ (flat) & $30 \times 40$ \\
Grating separation $(\mathrm{m})$ & 5 & 15 \\
Lines per mm & 1740 & 1740 \\
Laser beam diameter $(\mathrm{cm})$ & 1 & 10 \\
Cut bandwidth $(\mathrm{nm})$ & 2.0 & 2.0 \\
Exit subpulse duration $(\mathrm{ps})$ & 3000 & 2.2 \\
Efficiency-single bounce $(\%)$ & 90. & 96.0 \\
System efficiency $(\%)$ & 60 & 80 \\
Laser macro-pulse fluence $\left(\mathrm{J} / \mathrm{cm}^{2}\right)$ & $10^{-7}$ & 1.3 \\
Damage fluence $\left(\mathrm{J} / \mathrm{cm}^{2}\right)$ & 0.4 & 2.0 \\
\hline \hline
\end{tabular}

Table 13.3: Specifications for stretcher and compressor optical systems.

to the subpulse ("chirping"). The laser subpulse has a 3 ns halfwidth duration upon exiting. The finite size of the grating results in the truncation of some frequencies and gives the exiting pulse a truncated spectral distribution and a temporal pulse with side lobes. The 100 subpulses that are separated by 2.8 ns will overlap to form a 300 ns macro-pulse that has some $(\sim 10 \%)$ time/amplitude modulation. Since the beam in the stretcher is of such low power, there are no technical issues with this system. The system efficiency will be limited by the reflectivity of the gratings in the first order and the frequency clipping due to finite grating size.

The compressor gratings must be designed to handle the full $100 \mathrm{~J}$ macro-pulses without damage. The $100 \mathrm{~Hz}$ pulse rate will also generate an average-power thermal concern. The large gold coatings used in laser fusion experiments (Fig. 13.10) have too large an absorption and would have thermal distortion problems. LLNL has also developed multi-layer dielectric diffraction gratings with high efficiency [21]. Their low absorptivity removes the thermal concerns while also increasing the system efficiency. Figure 13.10 shows the design of these gratings. Alternating layers of hafnia and silica are placed on the substrate to give a high-reflectivity, high-damage fluence coating. The grating is etched in the silica overcoating.

\subsection{Laser facility, systems design and risk reduction}

The general layout of the laser facility is shown in Fig. 13.11. The facility is dominated by the operating bays for the laser amplifiers and their utilities. The operating strategy will be to do no laser repairs in these operation bays. The laser 

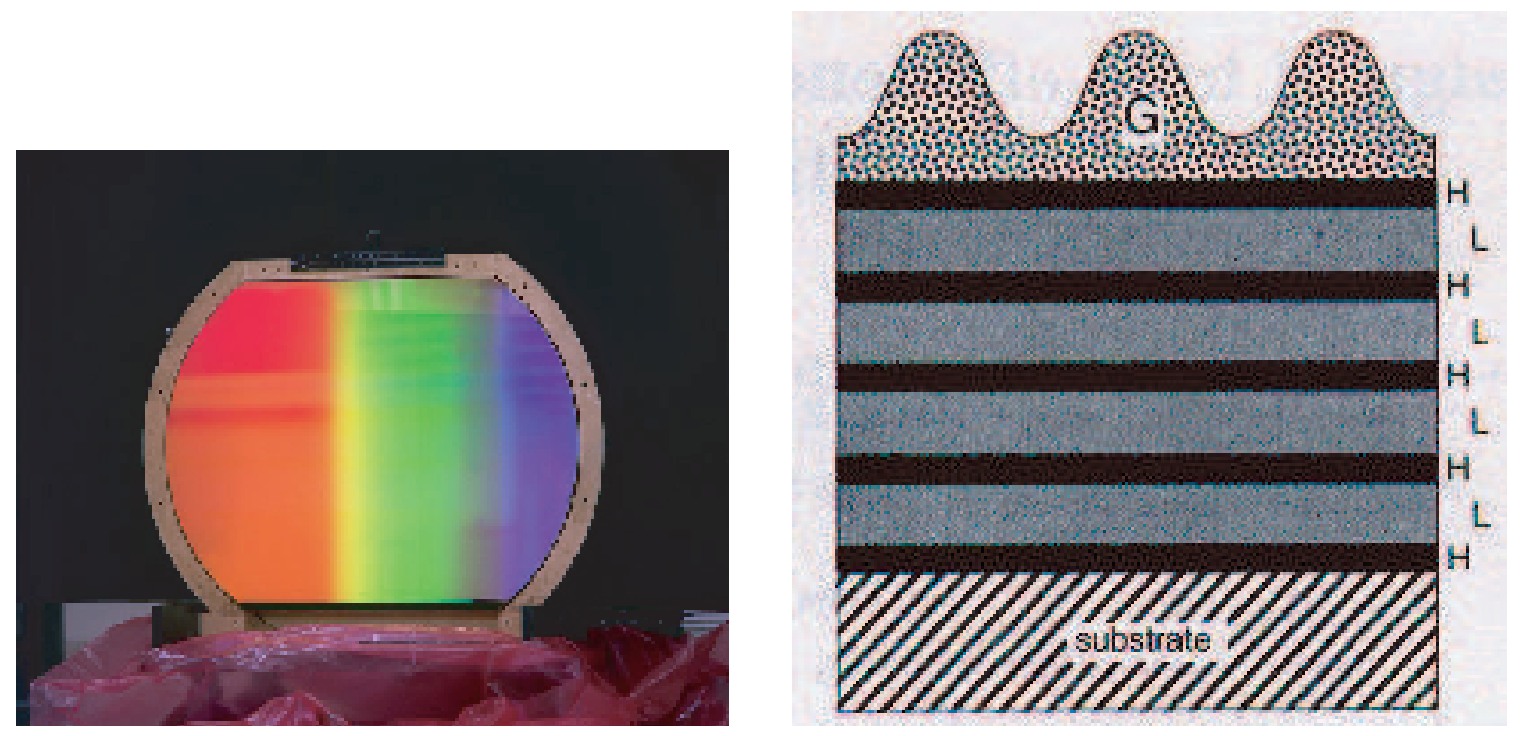

Figure 13.10: The $94 \mathrm{~cm}$ aperture gold-coated diffraction grating used for pulse compression on the Petawatt laser is shown on the left. A multilayer dielectric grating design of highindex $(\mathrm{H})$ and low-index $(\mathrm{L})$ layers and groove corrugations $(\mathrm{G})$ is shown on the right. Layers form a high-reflectivity stack under the corrugations.

systems will be designed with quickly removable Line Replaceable Units (LRUs) for all the major subsystems, as in the NIF project. The equipment will be monitored by computer during operation. When a system needs special or preventive maintenance, the LRU is quickly removed and moved to a separate repair facility. A new LRU is inserted, and the laser is immediately returned to service. This repair strategy allows for high system availability without requiring excessive component lifetimes or redundancy. Some long-lifetime components such as the optics vacuum chamber may have to be occasionally repaired in place.

A systematic cost estimate has not yet been done. The expected capital cost is of order of $\$ 200 \mathrm{M}$, and the operating budget of approximately $\$ 20 \mathrm{M} / \mathrm{yr}$. The largest uncertainties in the capital costs are the diode costs and the laser size needed to meet the performance requirements. The operating cost uncertainties are dominated by diode laser lifetime and cost uncertainties.

The cost risk reduction strategy is to identify the main cost drivers. Since diode lifetime is expected to be the primary cost risk driver, efforts will be made to acquire diode lifetime data.

The technical risks are dominated by the laser beam quality uncertainties and the lack of prototype demonstrations of some of the subsystems. The Mercury laser being built for the fusion program will serve as the main laser amplifier prototype. Other critical systems such as the laser system front end will be prototyped as part of a risk 


\section{$\gamma \gamma$ Laser Facility}

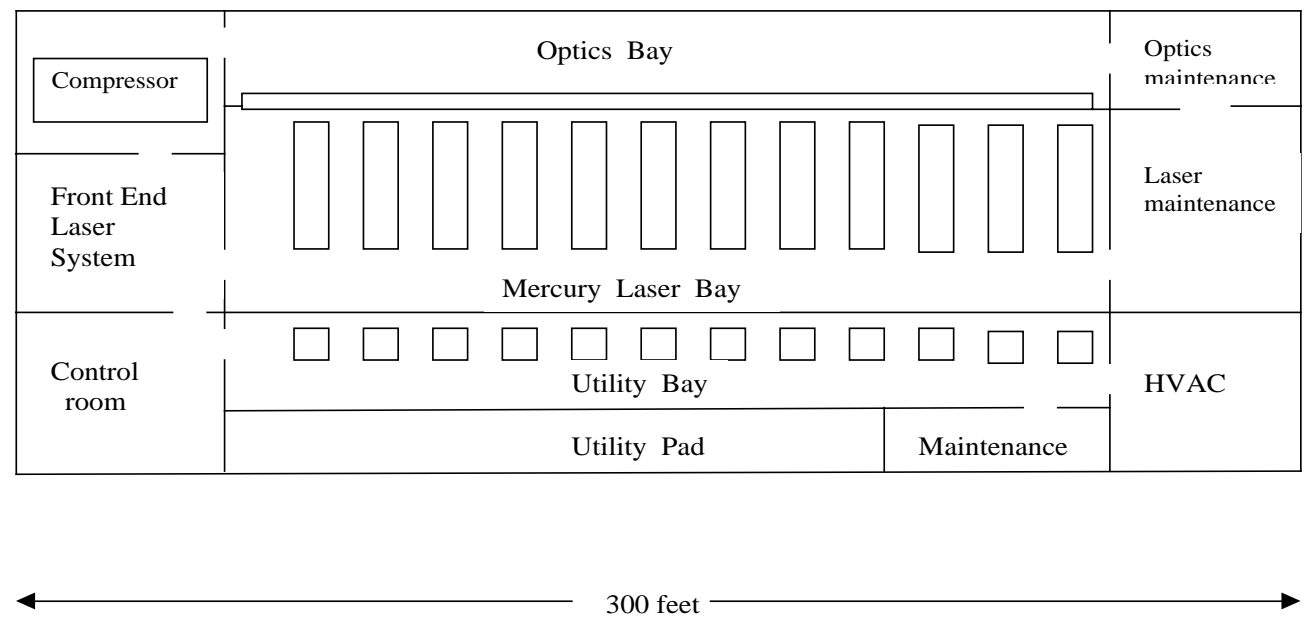

Figure 13.11: Floor plan of the laser physical plant.

reduction program.

\section{References}

[1] I. F. Ginzburg, G. L. Kotkin, V. G. Serbo and V. I. Telnov, Pizma ZhETF 34, 514 (1981); JETP Lett. 34, 491 (1982) (Preprint INF 81-50, Novosibirsk (1981) in English).

[2] I. F. Ginzburg, G. L. Kotkin, V. G. Serbo and V. I. Telnov, Nucl. Instrum. Meth. 205, 47 (1983).

[3] D. L. Bordon, D. A. Bauer, and D. O. Caldwell, Phys. Rev. D48, 4018 (1993); T. Ohgaki, T. Takahashi and I. Wantanabe, Phys. Rev. D56, 1723 (1997); T. Ohgaki, T. Takahashi, I. Wantanabe and T. Tauchi, Int. J. Mod. Phys. A13, 2411 (1998); I. Wantanabe et al., KEK Report 97-17 (1998).

[4] J. F. Gunion et al., hep-ph/9703330; H. E. Haber, hep-ph/9505240; A. Djouadi, V. Driesen, W. Hollik and J. I. Illana, E. Phys. J. C1, 149 (1998); B. Grzadkowski and J. F. Gunion, Phys. Lett. B291, 361 (1992); J. F. Gunion and J. G. Kelly, Phys. Lett. B333, 110 (1994).

[5] See, for example, S. Söldner-Rembold and G. Jikia, in Proc. Internat. Workshop on High-Energy Photon Colliders, Hamburg, Germany, 14-17 June 2000; G. Jikia 
Photon Collider

and A. Tkabladze, Phys. Rev. D54, 2030 (1996); M. Melles, W. J. Stirling and V. A. Khoze, Phys. Rev. D61, 054015 (2000) and M. Melles, hep-ph/0008125; M. Battaglia, hep-ph/9910271.

[6] E. Yehudai, Phys. Rev. D41, 33 (1990) and D44, 3434 (1991); S. Y. Choi and F. Schrempp, Phys. Lett. B272, 149 (1991); S. J. Brodsky, T. G. Rizzo and I. Schmidt, Phys. Rev. D52, 4929 (1995); T. Takahashi, in Physics and Experiments with Linear Colliders, ed. A. Miyamoto et al. (World Scientific, Singapore, 1996); M. Baillargeon, G. Belanger and F. Boudjema, Nucl. Phys. B500, 224 (1997). For a review, see H. Aihara et al. in Electroweak Symmetry Breaking and Beyond the Standard Model, ed. T. Barklow et al., (World Scientific, Singapore, 1996).

[7] N. Arkani-Hamed, S. Dimopoulos and G. Dvali, Phys. Lett. B429, 263 (1998) and Phys. Rev. D59, 086004 (1999); I. Antoniadis, N. Arkani-Hamed, S. Dimopoulos and G. Dvali, Phys. Lett. B436, 257 (1998); G. F. Giudice, R. Rattazzi and J. D. Wells, Nucl. Phys. B544, 3 (1999); T. Han, J. D. Lykken and R. Zhang, Phys. Rev. D59, 105006 (1999), E. A. Mirabelli, M. Perelstein and M. E. Peskin, Phys. Rev. Lett. 82, 2236 (1999); J. L. Hewett, Phys. Rev. Lett. 82, 4765 (1999); T. G. Rizzo, Phys. Rev. D59, 115010 (1999).

[8] T. G. Rizzo, Phys. Rev. D60, 115010 (1999).

[9] L. Randall and R. Sundrum, Phys. Rev. Lett. 83, 3370 (1999). H. Davoudiasl, J. L. Hewett and T. G. Rizzo, Phys. Lett. B473, 43 (2000), hep-ph/0006041 and Phys. Rev. Lett. 84, 2080 (2000).

[10] A. Djouadi, Proc. of the Workshop on $e^{+} e^{-}$collisions at $500 \mathrm{GeV}$ : the Physics Potential, edited by P. Zerwas, DESY Report 92-123B; P. Poulose and S. D. Rindani, Phys. Lett. B452, 347 (1999); S. Y. Choi and Hagiwara, Phys. Lett. B359, 369 (1995); M. S. Baek, S. Y. Choi and C. S. Kim, Phys. Rev. D56, 6835 (1997).

[11] For a recent review and original references, see G. J. Gounaris, P. I. Porfyiadis and F.M. Renard, hep-ph/0010006.

[12] J. L. Hewett, F. J. Petriello and T. G. Rizzo, hep-ph/0010354.

[13] For a review, see A. Djoudai, J. Ng and T. G. Rizzo, in Electroweak Symmetry Breaking and Beyond the Standard Model, ed. T. Barklow et al., (World Scientific, Singapore, 1996); W. Buchmüller, R. Rückl and D. Wyler, Phys. Lett. B191, 442 (1987); J. L. Hewett and T. G. Rizzo, Phys. Rev. 183, 193 (1989) and Phys. Rev. D56, 5709 (1997); J. Blümlein and R. Rückl, Phys. Lett. B304, 337 (1993); S. Davidson, D. Bailey, and B. A. Campbell, Z. Phys. C61, 613 (1994); M. Leurer, Phys. Rev. D50, 536 (1994), and D49, 333 (1994).

[14] For a recent summary, see D. S. Gorbunov, V. A. Ilyin and V. I. Telnov, hep$\mathrm{ph} / 0012175$.

[15] V. Telnov, Nucl. Instr. and Meth. A 355, 3 (1995). 
[16] K. Yokoya, "A Computer Simulation Code for the Beam-Beam Interaction in Linear Colliders", KEK report 85-9, Oct. 1985.

[17] D. Strickland and G. Mourou, Opt. Commun. 56, 219 (1985).

[18] M. D. Perry, F. G. Patterson, and J. Weston, Opt. Lett. 15, 381 (1990)

[19] E. B. Treacy, IEEE J.Quantum Electron. QE-5, 454 (1969).

[20] M. D. Perry and G. Mourou, Science 264, 917 (1994).

[21] B. W. Shore, M. D. Perry, J. A. Britten, R. D. Boyd, M. D. Feit, H. T. Nguyen, R. Chow, G. E. Loomis and Lifeng Li, J. Opt. Soc. Am., 14, No.5, 1124 (May 1997). 


\section{Chapter $14 e^{-} e^{-}$Collisions}

\section{General characteristics of $e^{-} e^{-}$collisions}

The primary goal of the linear collider program will be to elucidate new physics at the weak scale. The $e^{-} e^{-}$collider brings a number of strengths to this program. Electron-electron collisions are characterized by several unique features:

- Exactly Specified Initial States and Flexibility. For precision measurements, complete knowledge of the initial state is a great virtue. This information is provided optimally in $e^{-} e^{-}$collisions. The initial state energy is well-known for both $e^{+}$and $e^{-}$ beams, despite small radiative tails due to initial state radiation and beamstrahlung. For $e^{-}$beams, however, $85 \%$ polarization is routinely obtainable now, and $90 \%$ appears to be within reach for linear colliders. The three possible polarization combinations allow one to completely specify the spin $S_{z}$, weak isospin $I_{w}^{3}$, and hypercharge $Y$ of the initial state. One may also switch between these combinations with ease and incomparable flexibility.

- Extreme Cleanliness. Backgrounds are typically highly suppressed in $e^{-} e^{-}$collisions. The typical annihilation processes of $e^{+} e^{-}$collisions are absent. In addition, processes involving $W$ bosons, often an important background in $e^{+} e^{-}$collisions, may be greatly suppressed by right-polarizing both beams.

- Dictatorship of Leptons. In $e^{+} e^{-}$collisions, particles are produced 'democratically'. In contrast, the initial state of $e^{-} e^{-}$collisions has lepton number $L=2$, electron number $L_{e}=2$, and electric charge $Q=-2$.

With respect to the first two properties, the $e^{-} e^{-}$collider takes the linear collider concept to its logical end. The third property precludes many processes available in $e^{+} e^{-}$interactions, but also provides unique opportunities for the study of certain types of new physics, such as supersymmetry. The physics motivations for the $e^{-} e^{-}$ collider have been elaborated in a series of workshops over the past six years [1-3]. In the following, we briefly describe a number of possibilities for new physics in which $e^{-} e^{-}$collisions provide information beyond what is possible in other experimental settings. We then review the accelerator and experimental issues relevant for $e^{-} e^{-}$ collisions. 


\section{Physics at $e^{-} e^{-}$colliders}

\subsection{Møller scattering}

The process $e^{-} e^{-} \rightarrow e^{-} e^{-}$is, of course, present in the standard model. At $e^{-} e^{-}$ colliders, the ability to polarize both beams makes it possible to exploit this process fully.

One may, for example, define two left-right asymmetries

$$
\begin{aligned}
A_{L R}^{(1)} & \equiv \frac{d \sigma_{L L}+d \sigma_{L R}-d \sigma_{R L}-d \sigma_{R R}}{d \sigma_{L L}+d \sigma_{L R}+d \sigma_{R L}+d \sigma_{R R}} \\
A_{L R}^{(2)} & \equiv \frac{d \sigma_{L L}-d \sigma_{R R}}{d \sigma_{L L}+d \sigma_{R R}},
\end{aligned}
$$

where $d \sigma_{i j}$ is the differential cross section for $e_{i}^{-} e_{j}^{-} \rightarrow e^{-} e^{-}$scattering. There are four possible beam polarization configurations. The number of events in each of the four configurations, $N_{i j}$, depends on the two beam polarizations $P_{1}$ and $P_{2}$. Given the standard model value for $A_{L R}^{(1)}$, the values of $N_{i j}$ allow one to simultaneously determine $P_{1}, P_{2}$, and $A_{L R}^{(2)}$. For polarizations $P_{1} \simeq P_{2} \simeq 90 \%$, integrated luminosity $10 \mathrm{fb}^{-1}$, and $\sqrt{s}=500 \mathrm{GeV}$, the beam polarizations may be determined to $\Delta P / P \approx 1 \%[4,5]$. Such a measurement is comparable to precisions achieved with Compton polarimetry, and has the advantage that it is a direct measurement of beam polarization at the interaction point.

This analysis also yields a determination of $A_{L R}^{(2)}$, as noted above. Any inconsistency with the standard model prediction is then a signal of new physics. For example, one might consider the possibility of electron compositeness, parameterized by the dimension-six operator $\mathcal{L}_{\text {eff }}=\frac{2 \pi}{\Lambda^{2}} \bar{e}_{L} \gamma^{\mu} e_{L} \bar{e}_{L} \gamma_{\mu} e_{L}$. With $\sqrt{s}=1 \mathrm{TeV}$ and an $82 \mathrm{fb}^{-1}$ event sample, an $e^{-} e^{-}$collider is sensitive to scales as high as $\Lambda=150 \mathrm{TeV}$ [6]. The analogous reach for Bhabha scattering at $e^{+} e^{-}$colliders with equivalent luminosity is roughly $\Lambda=100 \mathrm{TeV}$.

\subsection{Higgs bosons}

The Higgs boson production mechanism $e^{+} e^{-} \rightarrow Z h$ in the $e^{+} e^{-}$mode is complemented by production through $W W$ and $Z Z$ fusion in both $e^{+} e^{-}$and $e^{-} e^{-}$colliders. The study of $e^{-} e^{-} \rightarrow e^{-} e^{-} h^{0}$ through $Z Z$ fusion has a number of advantages [7,8]. The cross section is large at high energy, since it does not fall off as $1 / s$. The usual backgrounds from $e^{+} e^{-}$annihilation are absent. The final electrons typically have transverse momenta of order $m_{Z}$. Thus, one can reconstruct the recoil mass and observe the Higgs boson in this distribution, as shown in Fig. 14.1. Invisible decays of the Higgs boson, and branching ratios more generally, can be studied by this technique. 


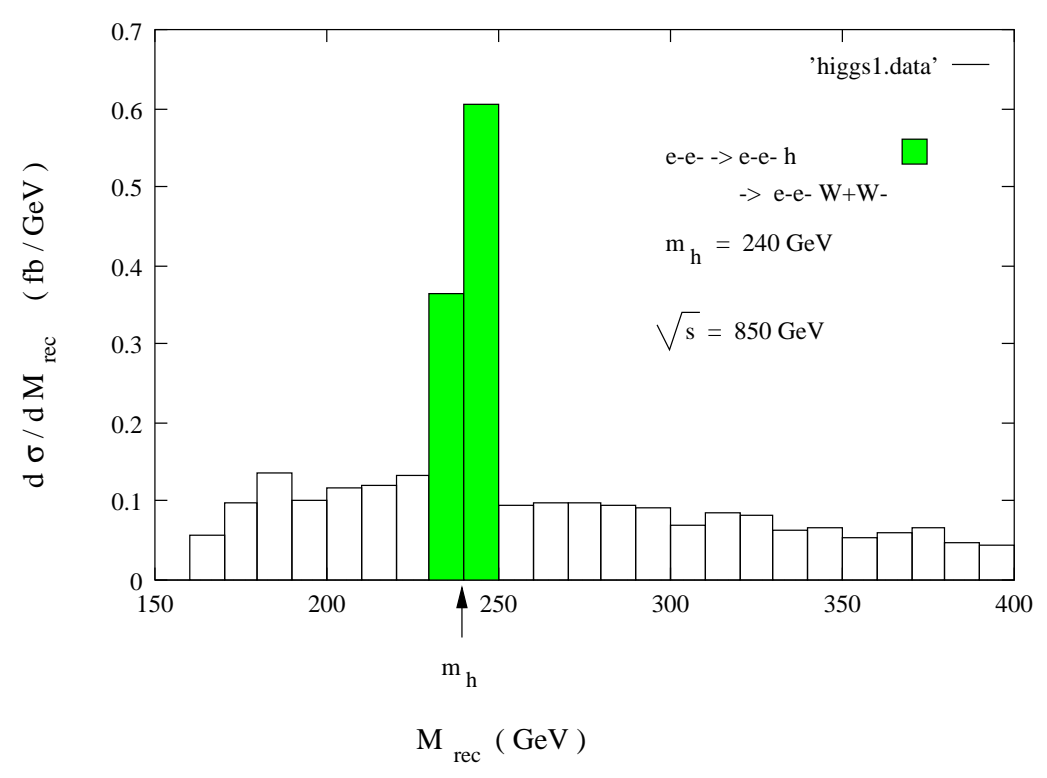

Figure 14.1: Differential cross sections as functions of recoil mass for $e^{-} e^{-} \rightarrow e^{-} e^{-} h$ and its principal standard model background $e^{-} e^{-} \rightarrow e^{-} e^{-} W^{+} W^{-}$. The Higgs boson mass is $m_{h}=240 \mathrm{GeV}, \sqrt{s}=850 \mathrm{GeV}$, and each electron satisfies an angular cut $\theta_{e^{-}}>5^{\circ}$. From [7].

\subsection{Supersymmetry}

The $e^{-} e^{-}$mode is an ideal setting for studies of sleptons. All supersymmetric models contain Majorana fermions that couple to electrons - the electroweak gauginos $\widetilde{B}$ and $\widetilde{W}$. Slepton pair production is therefore always possible [9], while all potential backgrounds are absent or highly suppressed. Precision measurements of slepton masses, slepton flavor mixings, and slepton couplings in the $e^{-} e^{-}$mode are typically far superior to those possible in the $e^{+} e^{-}$mode. Studies of all of these possibilities are reviewed in Chapter 4, Section 6.1.

The $e^{-} e^{-}$collider may also be used to determine the properties of other superpartners. For example, the production of right-handed selectron pairs is highly sensitive to the Majorana Bino mass $M_{1}$ that enters in the $t$-channel (see Fig. 14.2). As a consequence, extremely high Bino masses $M_{1}$ may be measured through the cross section of $\tilde{e}_{R}^{-}$pair production [10]. This region of parameter space is difficult to access in other ways.

\subsection{Bileptons}

The peculiar initial state quantum numbers of $e^{-} e^{-}$colliders make them uniquely suited for the exploration of a variety of exotic phenomena. Among these are bilep- 


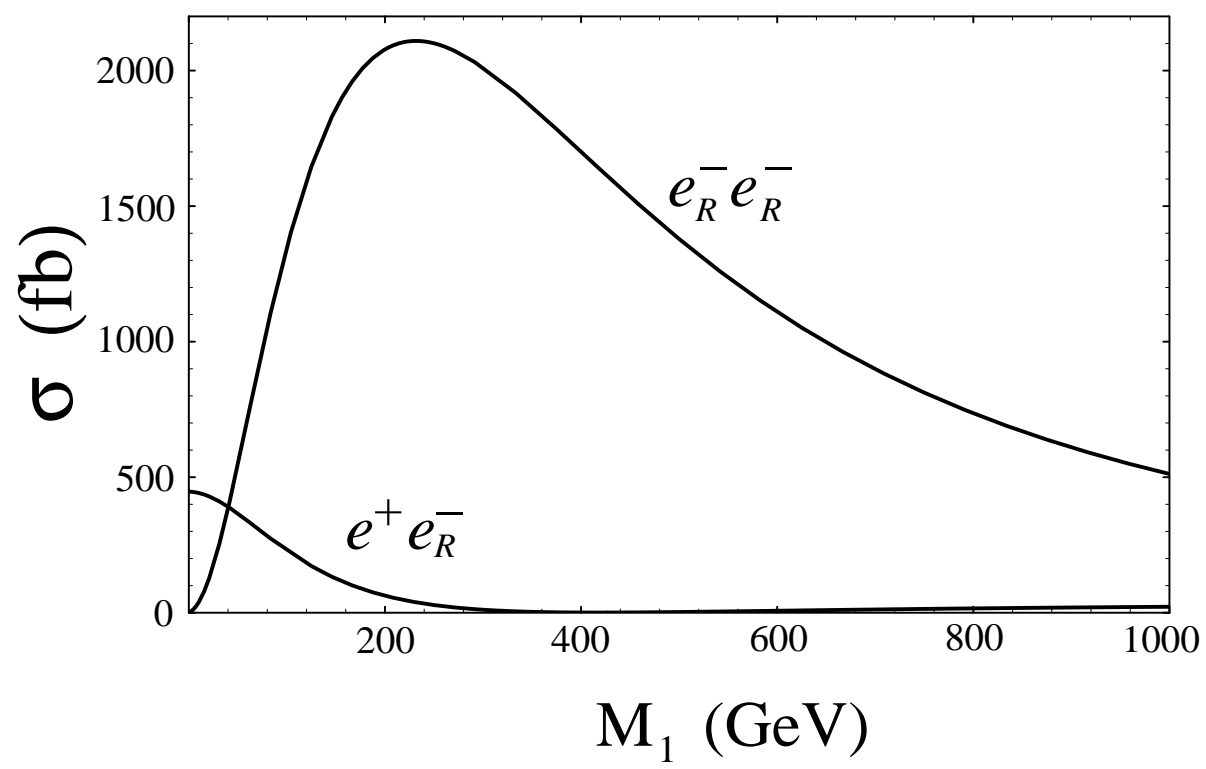

Figure 14.2: The total selectron pair production cross sections for the $e_{R}^{-} e_{R}^{-}$and $e^{+} e_{R}^{-}$modes with $m_{\tilde{e}_{R}}=150 \mathrm{GeV}$ and $\sqrt{s}=500 \mathrm{GeV}$, as functions of the Bino mass $M_{1}$. From [10].

tons, particles with lepton number $L= \pm 2$. Such particles appear, for example, in models where the $\mathrm{SU}(2)_{L}$ gauge group is extended to $\mathrm{SU}(3)$ [11], and the Lagrangian contains the terms

$$
\mathcal{L} \supset\left(\begin{array}{lll}
\ell^{-} & \nu & \ell^{+}
\end{array}\right)_{L}^{*}\left(\begin{array}{ccc} 
& & Y^{--} \\
& & Y^{-} \\
Y^{++} & Y^{+} &
\end{array}\right)\left(\begin{array}{c}
\ell^{-} \\
\nu \\
\ell^{+}
\end{array}\right)_{L},
$$

where $Y$ are new gauge bosons. $Y^{--}$may then be produced as an $s$-channel resonance at $e^{-} e^{-}$colliders, mediating background-free events like $e^{-} e^{-} \rightarrow Y^{--} \rightarrow \mu^{-} \mu^{-}$. Clearly the $e^{-} e^{-}$collider is ideal for such studies.

Bileptons may also appear in models with extended Higgs sectors that contain doubly charged Higgs bosons $H^{--}$. In these models, both types of particles are produced as resonances in $e^{-} e^{-}$scattering. However, the types of states are clearly distinguished by initial state polarization: bileptons are produced from initial polarization states with $\left|J_{z}\right|=1$, while doubly charged Higgs particles are produced in channels with $J_{z}=0$. The potential of $e^{-} e^{-}$colliders to probe the full spectrum of these models is reviewed in [12].

\subsection{Other physics}

In addition to these topics, the potential of $e^{-} e^{-}$colliders has also been studied as a probe of strong $W^{-} W^{-}$scattering, anomalous trilinear and quartic gauge boson 
$e^{-} e^{-}$Collider

couplings, heavy Majorana neutrinos, leptoquarks, heavy $Z^{\prime}$ bosons, TeV-scale gravity and Kaluza-Klein states, and non-commuting spacetime observables. These topics and other possibilities are discussed in [1-3].

\section{Accelerator and experimental issues}

\subsection{Machine design}

There are at present two well-developed approaches to linear collider architecture in the 0.35 to $1 \mathrm{TeV}$ energy range: the NLC/JLC and TESLA designs. Both approaches are easily adaptable to make both $e^{+} e^{-}$and $e^{-} e^{-}$collisions available with relatively little overhead.

The general layout of the NLC design is given in Fig. 14.3. The careful inclusion of the $e^{-} e^{-}$design is described in [13]. The installation of a second polarized electron source presents no difficulty, but magnet polarity reversals and potential spin rotators need to be carefully optimized.

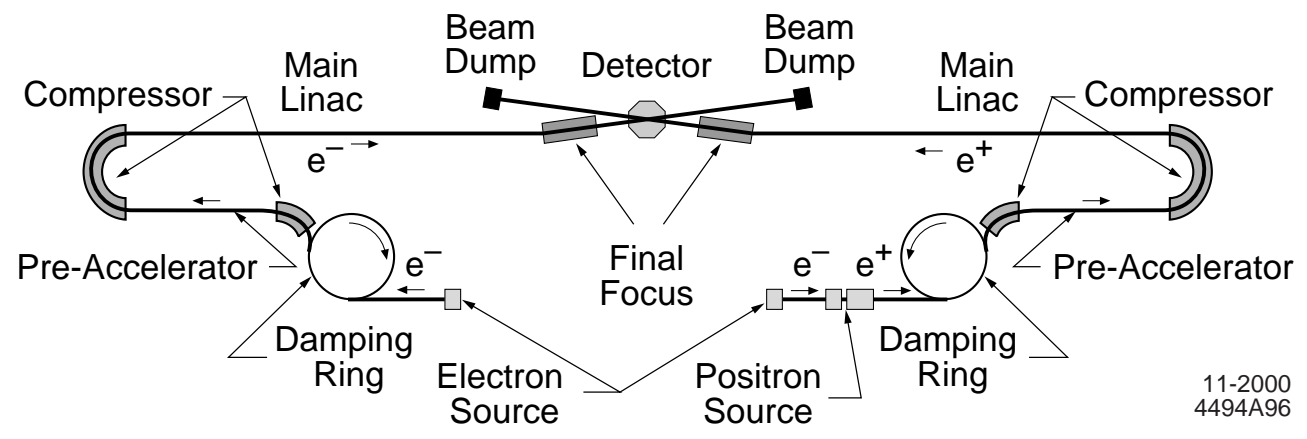

Figure 14.3: Schematic of the NLC. From [13].

Three different modifications for the injection area on the "positron" side have been investigated [14]. We show one of these in Fig. 14.4. In this scheme, the damping ring and bunch compressor for the $e^{+}$beam are used for an $e^{-}$beam which circulates in the opposite direction. A new electron gun and some additional components for injection and extraction are needed, but the cost of these is modest, and the switchover from $e^{+}$to $e^{-}$operation can be accomplished without significant manual intervention.

For the TESLA project, it is even simpler to introduce polarized $e^{-}$through the $e^{+}$injection system. A new polarized electron source is needed, and new components are needed for injection and extraction from the existing positron 'dogbone' damping ring [15]. The positions of these new devices mirror the positions of the electron injection and extraction points on the other side of the machine. 


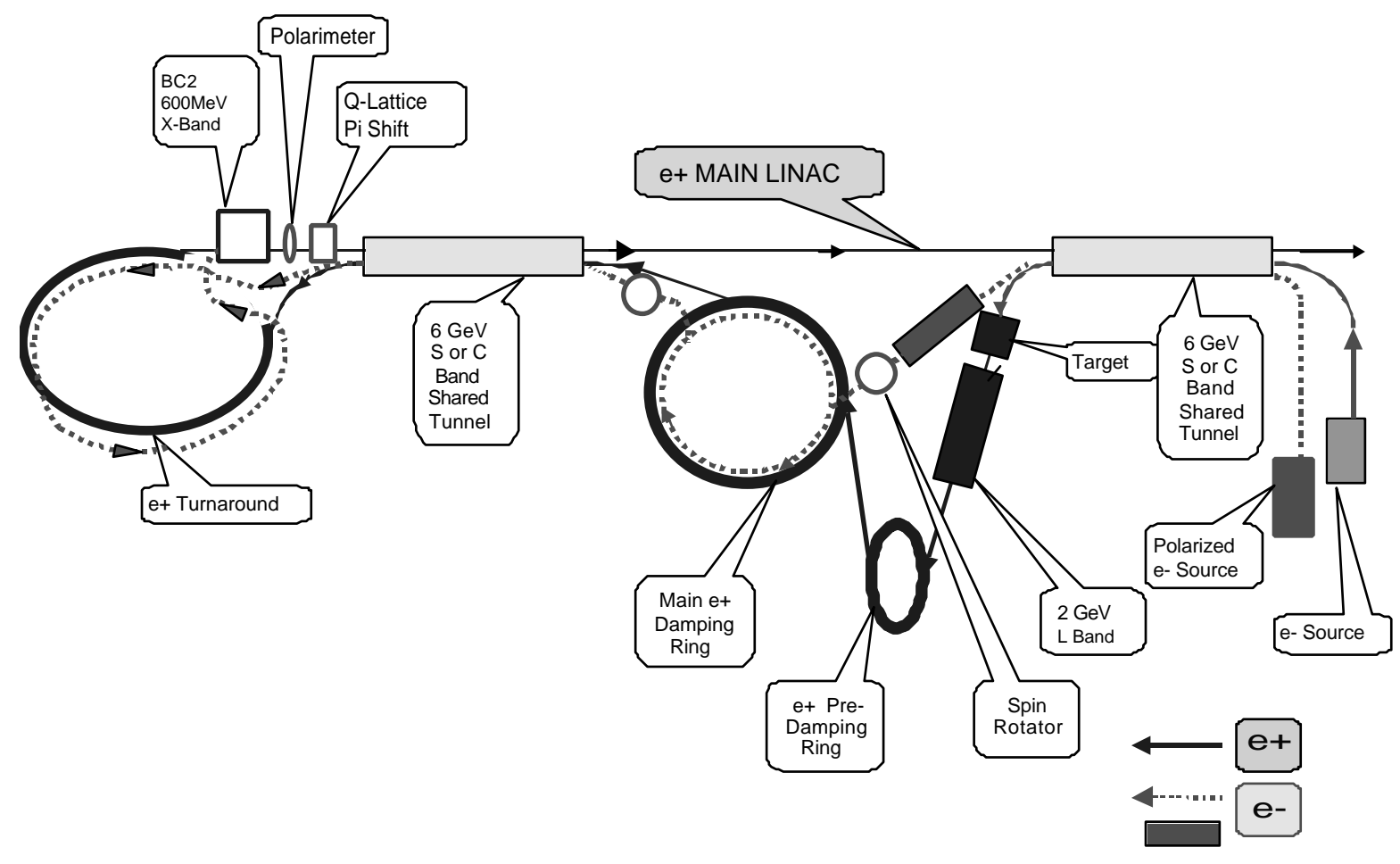

Figure 14.4: The direction reversal model. From [14].

Similar considerations apply to the higher-energy CLIC proposal [16]. As with NLC/JLC and TESLA, the main difficulties involve the injection scheme; once appropriate components are provided, the acceleration of $e^{-}$beams and the switchover from $e^{+} e^{-}$to $e^{-} e^{-}$should be straightforward.

\subsection{Interaction region}

Although $e^{-} e^{-}$operation is straightforwardly incorporated in linear collider designs, experimentation at $e^{-} e^{-}$colliders is not entirely equivalent to that at $e^{+} e^{-}$ colliders. This is because the luminosity of the collider is decreased significantly by beam disruption due to the electromagnetic repulsion of the two $e^{-}$beams.

Clever manipulation of the beam parameters can minimize the relative luminosity loss; see, for example, [17]. The resulting parameters give about a factor 3 loss for NLC/JLC and a factor 5 loss for TESLA, and do not much reduce the merits of the proposed $e^{-} e^{-}$studies. A plasma lens $[18,19]$ has been proposed to reduce the disruption effects, but this would introduce a serious level of beam-gas backgrounds.

The beamstrahlung effect in $e^{-} e^{-}$is somewhat larger than that in $e^{+} e^{-}$due to the larger disruption, leading to a stronger effective field from the opposite beam. The effect is still modest in size for $500 \mathrm{GeV}$ CM energy. Figure 14.5 shows a comparison of the $e^{-} e^{-}$and $e^{+} e^{-}$cases for the TESLA machine design [20]. 
$e^{-} e^{-}$Collider

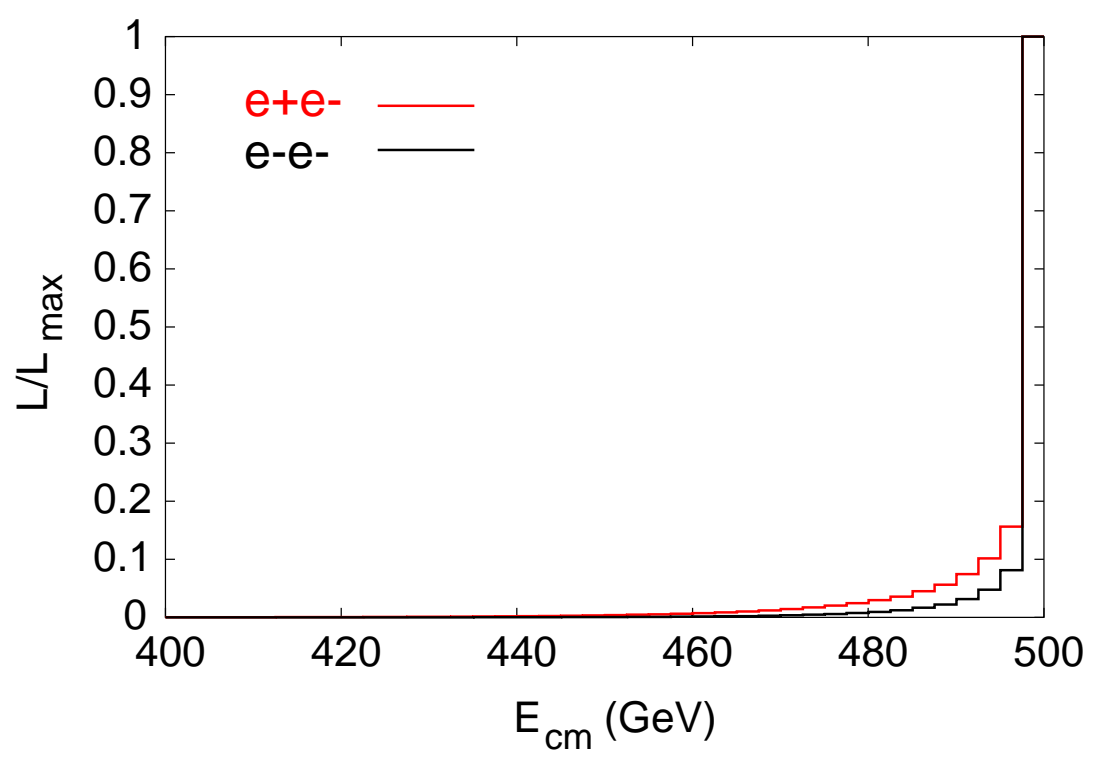

Figure 14.5: Normalized luminosity spectrum for $e^{-} e^{-}$collisions compared to $e^{+} e^{-}$. From [20].

\subsection{Detectors}

It is important to realize that the detector configuration is easily shared for $e^{+} e^{-}$ and $e^{-} e^{-}$experimentation. A caveat exists for beam disposal downstream of the interaction point: if there is any bend upstream of this point, like-sign incoming beams will not follow the incoming trajectories of the opposite side, and special beam dumps may have to be configured.

If the linear collider program plans to incorporate $e \gamma$ and $\gamma \gamma$ collisions, with backscattered photon beams, the photon beams must be created from $e^{-}$rather than $e^{+}$beams, so that the electron beam polarization can be used to optimize the energy spectrum and polarization of the photon beams. Photon colliders of course have their own, very different, requirements for interaction regions and detectors. These are described in Chapter 13, Section 3.

\section{Conclusions}

For a number of interesting physics scenarios, the unique properties of $e^{-} e^{-}$colliders will provide additional information through new channels and observables. While the specific scenario realized in nature is yet to be determined, these additional tools may prove extremely valuable in elucidating the physics of the weak scale and beyond. Given the similarities of the $e^{+} e^{-}$and $e^{-} e^{-}$colliders, it should be possible with some 
thought in advance to guarantee the compatibility of these two modes of operation and the ease of switching between them. For many possibilities for new physics in the energy region of the linear collider, the small effort to ensure the availability of $e^{-} e^{-}$collisions should reap great benefits.

\section{References}

[1] Proceedings of the 1st International Workshop on Electron-Electron Interactions at TeV Energies ( $e^{-} e^{-}$95), Santa Cruz, California, 5-6 September 1995, ed. C. A. Heusch, Int. J. Mod. Phys. A11, 1523-1697 (1996).

[2] Proceedings of the 2nd International Workshop on Electron-Electron Interactions at TeV Energies ( $e^{-} e^{-97}$ ), Santa Cruz, California, 22-24 September 1997, ed. C. A. Heusch, Int. J. Mod. Phys. A13, 2217-2549 (1998).

[3] Proceedings of the 3nd International Workshop on Electron-Electron Interactions at TeV Energies ( $e^{-} e^{-99}$ ), Santa Cruz, California, 10-12 December 1999, ed. C. A. Heusch, Int. J. Mod. Phys. A15, 2347-2628 (2000).

[4] F. Cuypers and P. Gambino, Phys. Lett. B388, 211 (1996) [hep-ph/9606391].

[5] A. Czarnecki and W. J. Marciano, Int. J. Mod. Phys. A13, 2235 (1998) [hep$\mathrm{ph} / 9801394]$.

[6] T. L. Barklow, Int. J. Mod. Phys. A11, 1579 (1996).

[7] P. Minkowski, Int. J. Mod. Phys. 13, 2255 (1998).

[8] J. F. Gunion, T. Han and R. Sobey, Phys. Lett. B429, 79 (1998) [hep$\mathrm{ph} / 9801317]$.

[9] W. Y. Keung and L. Littenberg, Phys. Rev. D28, 1067 (1983).

[10] J. L. Feng, Int. J. Mod. Phys. A13, 2319 (1998) [hep-ph/9803319].

[11] P. H. Frampton, Int. J. Mod. Phys. A13, 2345 (1998) [hep-ph/9711281].

[12] J. F. Gunion, Int. J. Mod. Phys. A13, 2277 (1998) [hep-ph/9803222].

[13] P. Tenenbaum, Int. J. Mod. Phys. A15, 2461 (2000).

[14] R. S. Larsen, Int. J. Mod. Phys. A15, 2477 (2000).

[15] J. Andruszkow, et al., "TESLA Technical Design Report" (DESY 2001011/ECFA 2001-209) Vol. II, Chapter 5.

[16] J. P. Delahaye et al., CERN/PS 98-009 (1998).

[17] K. A. Thompson, Int. J. Mod. Phys. A15, 2485 (2000).

[18] P. Chen, A. Spitkovsky and A. W. Weidemann, Int. J. Mod. Phys. A11, 1687 (1996).

[19] J. S. Ng et al., SLAC-PUB-8565, Invited talk presented at the 9th Workshop on Advanced Accelerator Concepts, Santa Fe, New Mexico, 11-16 June 2000.

[20] I. Reyzl and S. Schreiber, Int. J. Mod. Phys. A15, 2495 (2000). 
Detectors for the Linear Collider 


\section{Chapter 15 Detectors for the Linear Collider}

\section{Introduction}

The linear collider detector must be optimized for physics performance, taking consideration of its special environment. To plan for this detector, we consider the physics requirements of the linear collider and build on the experience of operating SLD at the SLC.

The detector must be hermetic, with good charged-track momentum and impact parameter resolution. The calorimeter must provide good resolution, with good granularity, particularly in the electromagnetic section. Electron and muon identification must be done efficiently.

The beamline conditions of the linear collider motivate a strong solenoidal magnetic field to contain the vast number of low-energy electron-positron pairs. There must be provision for an accurate measurement of the differential luminosity, and for timing information that will be useful to separate interactions from separate bunches within a bunch train.

This chapter begins with a discussion of the major issues for the linear collider detector, starting from the beamline conditions and working through the subsys-

tems. Following this discussion, three potential detectors developed for the NLC are described, two designed for the higher-energy IR, and the third for the second IR, where the lower-energy operation is foreseen. Other detectors have been considered in Europe [1] and Asia [2].

These detector studies have been undertaken to understand how well the diverse physics measurements at a linear collider can be accomplished, to provide preliminary guidance on costs, and to highlight areas where R\&D is needed. The specific choices of technology and full detector optimization will await the formation of LC experimental collaborations.

\section{Interaction region issues for the detector}

\subsection{Time structure.}

The NLC is expected to operate with trains of 190 bunches with 1.4 ns bunch spacing. This time structure requires that the beams cross at an angle. It also affects the number of bunches seen within the integration time of any detector subcomponent and has a strong influence on the types of feedback schemes that can be used to keep the beams in collision. 
Crossing angle and parasitic collisions. In order to avoid parasitic collisions, a crossing angle between the colliding beams is required. The minimum angle acceptable for this beam-beam limit is approximately $4 \mathrm{mrad}$ for the NLC parameters. A larger angle is desirable because it permits a more straightforward extraction of the spent beams (see Fig. 15.1), but an excessively large crossing angle will result in a luminosity loss. The angle between the beams chosen in the NLC design is 20 mrad.

The bunches must interact head-to-head or there will be a substantial loss of luminosity. RF cavities that rotate each bunch transversely will be located 10-20 m on either side of the IP. At 20 mrad crossing angle, the relative phasing of the two $\mathrm{RF}$ pulses must be accurate to within $10 \mu \mathrm{m}$ to limit the luminosity loss to less than $2 \%$. This corresponds to 0.04 degrees of phase at S-band $(2.8 \mathrm{GHz})$. The achievable resolution is about 0.02 degrees, which sets an upper limit on the crossing angle of 40 mrad.

Solenoid field effects. The crossing angle in the $x-z$ plane causes the beam to see a transverse component of the detector's solenoid field. If uncorrected, this field will deflect the beams so they do not collide. Likewise, the deflection would cause dispersion that would blow up the beam spot size. Both of these effects can be cancelled by judiciously offsetting the position of the last quadrupole, QD0, and steering the beam appropriately. Synchrotron radiation emission in the transverse field leads to an irreducible increase in spot size. This effect is proportional to $\left(L^{*} B_{S} \theta_{C}\right)^{5 / 2}$, where $L^{*}$ is the distance between the IP face of the last magnet and the interaction point. While it is small at the values of $L^{*}, B_{S}$, and $\theta_{C}$ considered to date, this effect might someday limit the design of the detector and IR.

After the beams collide at the IP, they are further bent by the solenoid field. Since compensating for this energy-dependent position and angle change with independent dipoles is difficult, the extraction line must be adjusted appropriately for the chosen beam energy. Realignment will be required if the extraction line does not have adequate dynamic aperture to accommodate the full range of beam energies used in experiments.

Finally, if the permeability of QD0 is not exactly unity, the field gradient of the solenoid in the detector endcap region will result in forces on QD0 that will need to be compensated. This may influence the schemes considered to compensate for nanometer-level vibration compensation of the magnet.

\subsection{IR layout}

Magnet technology. The NLC/JLC and TESLA designs have chosen to use different technologies for the final quadrupole doublet. The choices are dictated by the choice of crossing angle, and by the scheme to extract the spent beam after the collision. The NLC approach is to extract outside the outer radius of a compact Rare Earth Cobalt (REC) magnet into an extraction line that begins $6 \mathrm{~m}$ from the IP. The $\mathrm{REC}$ of choice is $\mathrm{Sm}_{2} \mathrm{Co}_{17}$, because of its radiation-resistant properties. Since the 


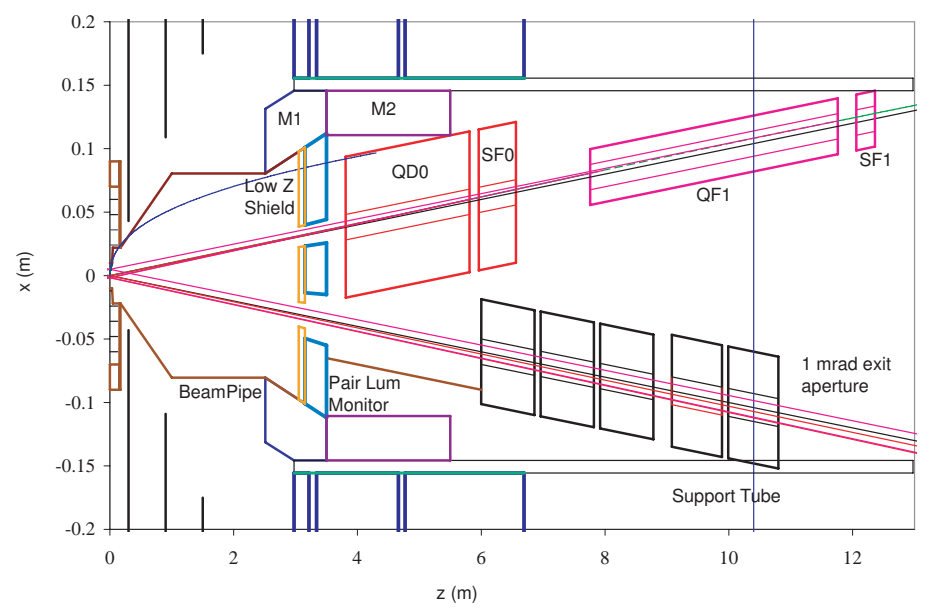

Figure 15.1: IR Layout for the NLC Large Detector.

final quadrupoles can be made light and stiff and have no external power connections, they are well suited for vibration stabilization. The downside to this choice is a lack of flexibility. Other issues that need to be explored further are the compatibility of the REC material with the solenoid field of the detector and the variation of the magnetic field with temperature.

In the current design $L^{*}=3.8 \mathrm{~m}$. An additional $30 \mathrm{~cm}$ of free space has been left in front of the pair luminosity monitor to allow for different magnet configurations as the beam energy is increased. Increasing $L^{*}$ provides more transverse space for the final quads and moves their mounting points further outside the detector, where they can presumably be better stabilized. By keeping $L^{*}$ larger than the minimum $z$ of the endcap calorimeter, the heavy W/Si-instrumented mask described below can be better incorporated into the detector's acceptance and mechanical structure. By increasing the distance between the IP and the first piece of high- $Z$ material seen by the beam, one can minimize the effect of backscattered debris from the interaction of off-energy $e^{+} e^{-}$pairs created when the beams interact.

On the other hand, increasing $L^{*}$ tightens the tolerances of the Final Focus optics and reduces its bandwidth. Synchrotron radiation produced by beam halo particles in the final lenses determines the minimum radius of the beam pipe inside the vertex detector. The larger $L^{*}$ is, the larger will be the fan of photons shining on the vertex detector.

Masks. The electrons and positrons produced in pairs in the beam-beam interaction have a mean energy of about $13 \mathrm{GeV}$ at $\sqrt{s}=1 \mathrm{TeV}$. These off-energy particles spiral in the detector's solenoid field and strike the pair-luminosity monitor or the inner bore of the extraction line magnet. The main purpose of the masking is to 
shield the detector from the secondary particle debris produced when an $e^{ \pm}$interacts. There are three masks foreseen. M1 begins at the back of the pair luminosity monitor and extends $0.64 \mathrm{~m}$ in $z$ beyond its front face; its inner angle is set by the requirement that it stay just outside the so-called "dead cone" through which the pairs coming from the IP travel. With the mask tip at $2.5 \mathrm{~m}$, a $3 \mathrm{~T}$ field requires an inner angle of $32 \mathrm{mrad}$. This mask would ideally be made of $\mathrm{W} / \mathrm{Si}$ and be fully integrated with the detector's calorimetry. M2 is a simple tungsten cylinder. The last mask near the IP is a $10-50 \mathrm{~cm}$ layer of low- $Z$ material (e.g., Be or C) that absorbs low energy charged particles and neutrons produced when the pairs hit the front face of the W/Si pair luminosity monitor. The very low-energy charged secondaries would otherwise flow back along the solenoid's field lines toward the vertex detector (VXD) and produce unacceptable backgrounds.

\subsection{Small spot size issues}

The beams must be held stable with respect to one another in the vertical plane at the level of one nanometer. Measurements in existing detectors imply that the mounting of the final quadrupoles may have to correct as much as $50 \mathrm{~nm}$ of vibration, caused mostly by local vibration sources and to a much lesser extent by naturally occurring seismic ground motion. Concerns about vibrations caused by moving fluids lead to the choice of permanent magnets for QD0 and QF1. These magnets will be mounted in cam-driven mover assemblies and the beam-beam interaction used to control their position to compensate for disturbances at frequencies below about $f / 20$, where $f$ is the beam repetition rate of $120 \mathrm{~Hz}$.

For frequencies above 5-6 Hz, the NLC strategy for stabilizing luminosity relies on a combination of passive compliance (minimizing and passively suppressing vibration sources while engineering to avoid resonant behavior) and active suppression techniques. Quad motion will be measured either optically relative to the surrounding bedrock or inertially, and a correction will be applied to either the final doublet position (via an independent set of magnet movers) or its field center (via a corrector coil). Finally, there will be feedback based on the measured beam-beam deflection. Such a system can respond sufficiently rapidly (within $15 \mathrm{ns)}$ ) to correct the trailing bunches in a train, once the first few are used to measure any collision offset.

\subsection{The beam-beam interaction}

The two main experimental consequences of the beam-beam interaction are a broadening of the energy distribution, due to the emission of photons by one beam in the field of the oncoming beam, and the subsequent background generated by interactions of those photons. The beamstrahlung contribution to the energy spread must be considered together with the intrinsic energy spread of the accelerator and the effect of initial state radiation. These effects have been taken into account in the 
Detectors for the Linear Collider

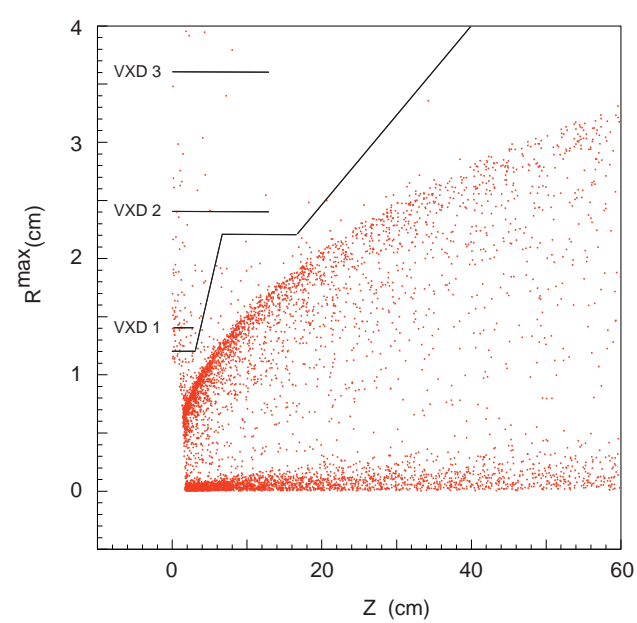

Figure 15.2: $R_{\max }$ vs. $z$ distribution of pairs in a 3 Tesla solenoid field. $R_{\max }$ is the maximum radius the particle travels from the IP, plotted at the $z$ corresponding to the first apex of its helical trajectory.

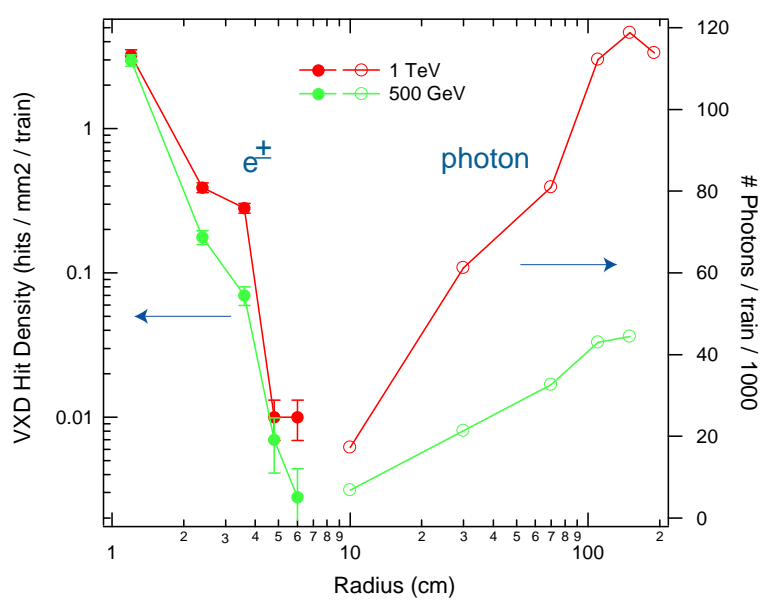

Figure 15.3: Charged particle hit density per train in the VXD, and the absolute number of photons per train entering the TPC within $|\cos \theta|<0.92$, as function of radius.

discussion of the various physics process. Below we discuss the beam-beam interaction as a potential source of backgrounds.

$e^{+} e^{-}$pairs and the minimum solenoid field. The incoherent production of $e^{+} e^{-}$ pairs arising from Bethe-Heitler $\left(e^{ \pm} \gamma \rightarrow e^{ \pm} e^{+} e^{-}\right)$, Breit-Wheeler $\left(\gamma \gamma \rightarrow e^{+} e^{-}\right)$, and Landau-Lifshitz $\left(e^{+} e^{-} \rightarrow e^{+} e^{-} e^{+} e^{-}\right)$processes is the main source of background at the present generation of planned linear colliders. At $\mathrm{CM}$ energies of $1 \mathrm{TeV}$, roughly $10^{5}$ particles are produced each bunch crossing, with a mean energy of $13 \mathrm{GeV}$. Very few particles are produced at a large angle and the dominant deflection is due to the collective field of the oncoming beam. The so-called 'dead cone' that is filled by these particles is clear in the $R_{\max }$ vs. $z$ plot in Fig. 15.2. The beam pipe inside the VXD innermost layer must be large enough and short enough that it does not intersect this region.

Secondary particles and their sources. Secondary particle backgrounds - from neutrons, photons, and charged particles - can be a problem for the detector whenever primary particles or particles from the collision are lost close to the IP. The main purpose of the masking described earlier is to limit the backgrounds these secondaries produce. Figure 15.3 shows the charged particle hit density per train in the VXD as a function of radius, and the absolute number of photons per train entering the TPC within $|\cos \theta|<0.92$. The most important sources of secondary particles are as follows:

- $e^{+} e^{-}$pairs striking the pair luminosity monitor are the most important source 
of secondaries as the pairs are well off the nominal beam energy, spiral in the detector's field and strike high-Z materials close to the IP. Backgrounds from this process are controlled by the masks described above.

- Radiative Bhabhas are a source of off-energy particles that are outside the energy acceptance of the extraction line. However, they are sufficiently few in number and leave the beam line sufficiently far from the IP that they are not an important background for the main detector elements.

- The low-energy tail of the disrupted beam cannot be transported all the way to the dump. The current design of the extraction line includes a chicane to move the charged beam transversely relative to the neutral beam of beamstrahlung photons. The bends at the beginning and the end of the chicane are the primary locations where particles are lost. The number of particles lost, $\sim 0.25 \%$ of the beam, and the separation of the loss point from the IP makes this an unimportant background source for the main detector, but calls into question the viability of sophisticated instrumentation, such as a polarimeter and an energy spectrometer, in the extraction line.

- Neutrons shining back on the detector from the dump are controlled by shielding immediately surrounding the dump, placing concrete plugs at the tunnel mouths, maximizing the distance from the dump to the IP, and minimizing window penetrations in the concrete. The detector of most concern is the VXD, which can look into the dump with an aperture equal to that provided to accommodate the outgoing beamstrahlung photons and synchrotron radiation.

Beamstrahlung photons. At $500 \mathrm{GeV}, 5 \%$ of the beam power is transformed into beamstrahlung photons; this rises to $10 \%$ at $1 \mathrm{TeV}$. The IR is designed so that these photons pass unimpeded to a dump. This consideration, along with the angular spread of the synchrotron radiation (SR) photons, determines the exit aperture of the extraction line, currently set at $1 \mathrm{mrad}$. The maximum transverse size of the dump window that can be engineered and the beamstrahlung angular spread set the maximum distance the dump can be located from the IP. That distance and the size of the aperture in the concrete blockhouse surrounding the dump determine the level of neutron backshine at the detector.

Hadrons from $\gamma \gamma$ interactions. Beamstrahlung photon interactions will also produce hadrons. For the TESLA $500 \mathrm{GeV}$ IP parameters it is estimated that there is a $2 \%$ probability per bunch crossing of producing a hadronic event with $p_{T_{\min }}>$ $2.2 \mathrm{GeV}$ [3]. The average number of charged tracks is 17 per hadronic $\gamma \gamma$ event, with $100 \mathrm{GeV}$ deposited in the calorimeter. This study needs to be repeated for the NLC IP parameters and detector acceptance. Nonetheless, we can estimate the severity of this background by scaling the rate from the TESLA study by the square of $n_{\gamma}$, the 
Detectors for the Linear Collider

average number of photons produced by beamstrahlung, giving a factor $\left((1.2 / 1.6)^{2}\right)$, and also taking the bunch structure (190/1) into account. This leads to an event probability of 2.2 events/train with $220 \mathrm{GeV}$ in the calorimeter at $\sqrt{s}=500 \mathrm{GeV}$. It would clearly be advantageous to be able to time-stamp the hit calorimeter cells and tracks with the bunch number that produced them and thereby limit the background affecting a physics event of interest.

Muons and synchrotron radiation. SR photons arise from the beam halo in the final doublet, as shown in Fig. 15.4. The limiting apertures of the IR layout determine the maximum angular divergence of the charged particles that can be tolerated. Particles above the maximum divergence must be removed by the accelerator's collimation system. If the VXD radius is too small, the apertures in the collimation system required to remove the beam halo will be unreasonably small and will produce wakefields that will lead to beam spot size increases and a loss of luminosity. As particles are scraped off by the collimation system, muons are produced. Depending on the level of the halo and the robustness of the detector against background muons, a magnetic muon spoiler system may be required.
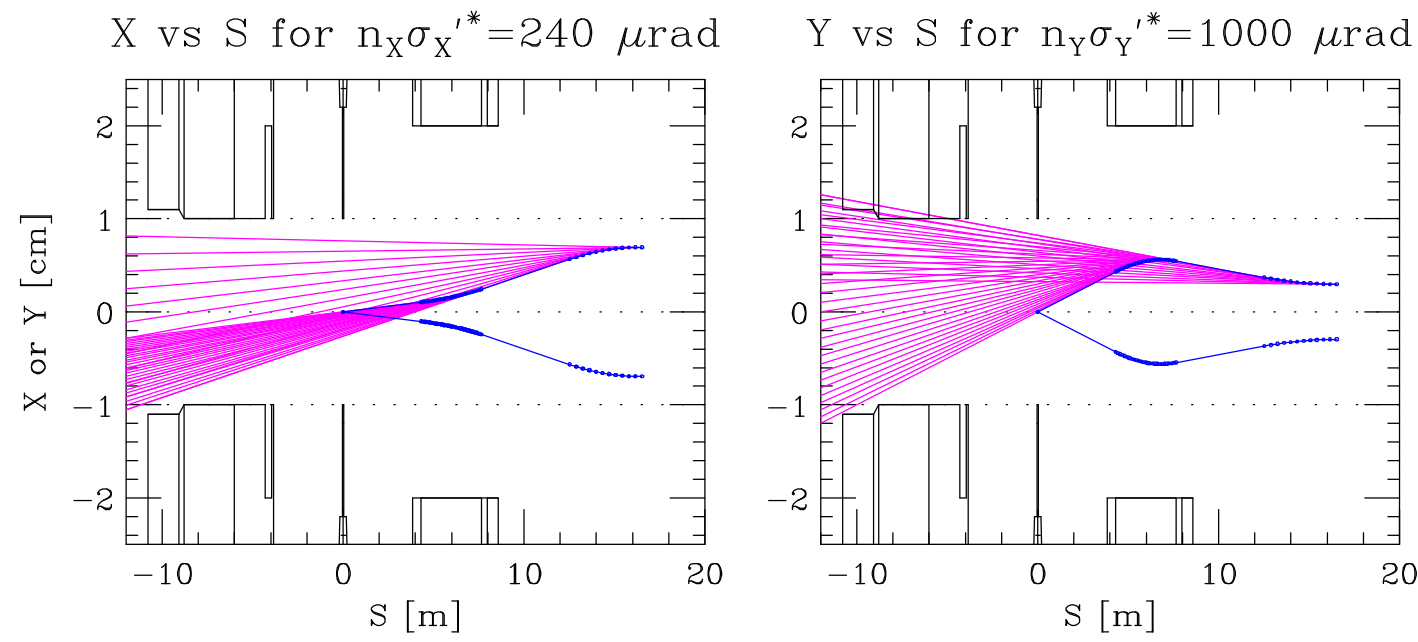

Figure 15.4: Synchrotron radiation fans from beam halo particles .

\section{Subsystem considerations}

\subsection{Vertex detector}

Recent experiments have benefited enormously from investments in excellent vertex detectors. An important lesson has been the immense value of a pixelated detector. This technology enabled SLD to match many of the physics measurements 
at LEP with a much smaller data sample. The physics goals of the linear collider will also demand optimal vertex detection. The physics signals are rich in secondary vertices, and event rates are limited, demanding highly pure and efficient tagging.

Physics processes requiring vertex detection include the Higgs branching ratios, SUSY Higgs searches such as $\mathrm{A} \rightarrow \tau^{+} \tau^{-}$, searches for staus, top studies, improved measurement of $W$ pairs, $Z^{\prime}$ studies such as $\tau$ polarization, and $Z$ pole physics. Some processes will involve several heavy quark decays, complicating the reconstruction, and increasing the demand for pixelated detectors. The physics will require highly efficient and pure $b$ and $c$ tagging, including tertiary vertex reconstruction, and charge tagging (as needed for $b / \bar{b}$ discrimination, for example). Optimal performance calls for point resolutions better than $4 \mu \mathrm{m}$, ladder thickness under $0.2 \% X_{0}$, inner layers within 2 or $3 \mathrm{~cm}$ of the interaction point, coverage at least over $|\cos \theta|<0.9$, and good central tracking linked to the vertex detector. The accelerator time structure and radiation environment will constrain the design, and must be carefully considered.

A pixel CCD vertex detector was developed at the SLC. The SLD vertex detector, VXD3 [4], comprised 307 million pixels on 96 detectors, and achieved $3.8 \mu \mathrm{m}$ point resolution throughout this large system. With such exceptional precision, extremely pure and efficient flavor tagging at the $Z$-pole was possible: $60 \% b$ tagging efficiency with $>98 \%$ purity, and better than $20 \% c$ tagging efficiency with $60 \%$ purity. SLD also achieved exceptional charge separation between $b$ and $\bar{b}$. The value of the pixel detector has been clearly established, even in the relatively clean environment of the SLC, where the hit occupancy in VXD3 was about $10^{-4}$. These successes motivate the choice of CCDs for the next-generation linear collider, where even better performance is foreseen.

The main weaknesses of the CCD approach to vertex detection are the slow readout speed and the radiation sensitivity. The speed issue can be managed at the linear collider, as SLD demonstrated. The hit density is maximal at the inner radius, where one expects about 3 per $\mathrm{mm}^{2}$ per bunch train at $1.2 \mathrm{~cm}$. This rate of $\sim 10^{-3}$ per pixel is challenging, but manageable, especially when the inner layer hits are matched to tracks reconstructed outside this layer.

With regard to the radiation background, the neutrons create the major challenge. Fluences greater than $10^{9} / \mathrm{cm}^{2} /$ year are expected. CCDs are expected to withstand this level of radiation. However, since the neutron backgrounds could be larger, CCDs with engineered rad-hard enhancements are being studied [5].

Despite the established performance of the CCD vertex detector, active pixels do provide interesting alternatives. They can be inherently less sensitive to radiation damage (hence the interest in using them at the LHC), but generally have been less precise, and they contain more material leading to multiple scattering. Efforts are underway to close the gap between the demonstrated CCD performance and the state of the art in active pixels. These efforts will be followed closely.

Central tracking is vital to the performance of the vertex detector. With severely 
Detectors for the Linear Collider

limited momentum resolution of its own, the vertex detector relies on the momentum measurement of the tracker for inward projection of tracks.

\section{$3.2 \quad$ Tracking}

Tracking of high-energy isolated charged particles will be important at a linear $e^{+} e^{-}$collider. Isolated leptons are prevalent in many new physical processes, including production of sleptons, heavy leptons, and leptoquarks, and in many interesting Standard Model processes, notably in associated $h Z$ production where the $Z$ decays into charged leptons. While the calorimeter may provide a good measure of electron energy (but not electric charge), excellent tracking will be needed to measure high muon energies and the charged decay products of $\tau$ 's.

Reconstruction of hadron jets will also be important, both in searching for new physical processes and in understanding Standard Model channels. Compared to the high-energy leptons discussed above, charged hadrons in jets have much lower average energies, relaxing the asymptotic $\sigma\left(1 / p_{t}\right)$ requirements. But tracking these hadrons well requires good two-track separation in both azimuth $(\phi)$ and polar angle $(\theta)$. Aggressive jet energy flow measurement also requires unambiguous extrapolation of tracks into the electromagnetic calorimeter, again demanding good two-track separation and also good absolute precision.

Forward-angle tracking is expected to be more important at a linear collider than has been traditionally the case for $e^{+} e^{-}$detectors. Some supersymmetry processes have strongly forward-peaked cross sections. Furthermore, in order to monitor beamstrahlung adequately, it is likely that precise differential luminosity measurement will be necessary, including accurate $(0.1 \mathrm{mrad})$ polar angle determination of low-angle scattered electrons and positrons [6].

The central tracker cannot be considered in isolation. Its outer radius drives the overall detector size and cost. Given a desired momentum resolution the tracker's spatial resolution and sampling drive the required magnetic field. This affects the solenoid design, including the flux return volume.

For a detector with a compact silicon vertex detector and a large gas chamber for central tracking, an intermediate tracking layer can improve momentum resolution, provide timing information for bunch tagging, and serve as a trigger device for a linear collider with a long spill time.

The most important technical issue for the tracking system is designing to meet a desired resolution in $1 / p_{t}$ of order $10^{-5} \mathrm{GeV}^{-1}$. This goal is driven by mass resolution on dileptons in Higgsstrahlung events and by end-point resolution in leptonic supersymmetry decays. There are tradeoffs among intrinsic spatial resolution, the number of sampling layers, the tracking volume size, and the magnetic field. The choices affect many other issues. For example, pattern recognition is more prone to ambiguities for a small number of sampling layers, with in-flight decays a particular problem. Matching to the vertex detector and achieving good two-track separation is more 
difficult for large intrinsic spatial resolution. A large magnetic field distorts electron drift trajectories for several tracking technologies. High accelerator backgrounds may lead to space charge buildup in a time projection chamber (TPC), degrading field uniformity and hence resolution. More generally, though, high backgrounds tend to favor choosing a TPC or another device which makes 3-dimensional space point measurements (such as a silicon drift detector) over a device with 2-dimensional projective measurements (such as an axial drift chamber or silicon microstrips). On the other hand, a pixel-based vertex detector may provide adequate 'seeds' for tracks, even in the presence of large backgrounds.

Material in the tracker degrades momentum resolution for soft tracks and increases tracker occupancy from accelerator backgrounds due to Compton scattering and conversions. Because front-end electronics can be a significant source of material, readout configuration can be quite important, affecting detector segmentation and stereo-angle options. Achieving polar angle resolution comparable to the azimuthal angle resolution may be expensive and technically difficult.

As mentioned above, accelerator backgrounds can degrade track reconstruction via excessive channel occupancy. One possible way to ameliorate the effects of this background is via bunch tagging (or bunch-group tagging) of individual tracking hits, but such tagging may place strong demands on the tracker readout technology.

\subsection{Calorimetry}

\subsubsection{Energy flow}

The first question for calorimetry at the linear collider is one that not only influences the overall philosophy of this system but also has ramifications for other detector subsystems and for the overall cost: Should the calorimeter be optimized to use the 'energy flow' technique for jet reconstruction? The promise of substantial improvement in resolution using this technique is appealing. However, quantitative measures of this improvement are still being developed, and it is likely that an energy flow calorimeter will be relatively complicated and expensive because of the fine segmentation and high channel count.

Clearly, multi-jet final states will be important for LC physics. Examples from the physics program include separation of $W W, Z Z$, and $Z h$ in hadronic final states, identification of $Z h h$, and $t \bar{t} h$ in hadronic decays, and full reconstruction of $t \bar{t}$ and $W W$ events in studies of anomalous couplings and strongly-coupled EWSB. A further example comes at high energy from the processes $e^{+} e^{-} \rightarrow \nu \bar{\nu} W W$ and $e^{+} e^{-} \rightarrow \nu \bar{\nu} t \bar{t}$, where because of low statistics and backgrounds, one would need good jet-jet mass resolution without the benefit of a beam energy constraint. Indeed, one of the oftenstated advantages of the $e^{+} e^{-}$environment is the possibility to reconstruct many types of final states accurately. In some instances, this is the key to the physics performance. 
Detectors for the Linear Collider

The energy flow (EF) technique makes use of the fact that the modest momenta of charged hadrons within jets are more precisely determined in the tracking detectors, than with a calorimeter. On the other hand, good energy resolution for photons (from $\pi^{0}$ decay) is achieved using any standard technique for electromagnetic calorimetry. Long-lived neutral hadrons (mostly $K_{L}^{0}$ ) are problematic using any technique, but they cannot be ignored. Therefore, a calorimeter designed to take advantage of EF must efficiently separate neutral from charged particle energy depositions. Such designs are characterized by a large tracking detector (radius $R$ ), a large central magnetic field $(B)$, and an electromagnetic calorimeter highly segmented in 3-D. A figure of merit describing the ability to separate charged hadrons from photons within a jet is $B R^{2} / R_{m}$, where $R_{m}$ is the Moliere radius of the electromagnetic calorimeter (EMCal). The EMCal's transverse segmentation should then be less than $R_{m}$ in order to localize the photon showers accurately and distinguish them from charged particles. Similarly, the separation of the long-lived neutral hadrons from charged hadrons improves with $B R^{2}$ and a finely segmented hadron calorimeter (HCal). The reconstruction process involves pattern recognition to perform the neutral-charged separation in the calorimeter, followed by a substitution of the charged energy with the corresponding measurement from the tracker.

The advantage of $\mathrm{EF}$ is clear in principle. Whether the advantage is borne out with realistic simulation is not yet resolved, as the tools required to do justice to the technique are still under development. With their silicon/tungsten EMCal, the TESLA group currently finds [7] $40 \% / \sqrt{E}$ for jet energy resolution (where $E$ is the jet energy in $\mathrm{GeV}$ ). They expect this to improve to $30 \% / \sqrt{E}$ with progress in pattern recognition. Assuming that such good performance is indeed achievable with EF, it is useful to identify how this would improve the physics outlook, and at what cost.

\subsubsection{Resolution, segmentation, and other requirements}

There is no compelling argument from LC physics that demands outstanding photon energy resolution, resulting for example from an EMCal using high- $Z$ crystals. Furthermore, such an optimization would not be consistent with the high degree of segmentation required for excellent jet reconstruction. Instead, the requirements for calorimetry from LC physics are jet energy and spatial resolution, and multi-jet invariant mass resolution. The required jet energy resolution depends, of course, on specific physics goals. A recent study [8] indicates that a resolution of $40 \% / \sqrt{E}$ is necessary to measure the Higgs self-coupling using $Z h h$ final states. One benchmark for jet-jet mass resolution is the separation of $W$ and $Z$ hadronic decays in $W W$, $Z Z$, and $Z h$ events. Both of these requirements may be achievable using energy flow reconstruction.

Segmentation is a critical parameter, since an EF design requires efficient separation of charged hadrons and their showers from energy depositions due to neutrals. 
The typical charged-neutral separation, $\Delta x$, is dervied from the particle density in jets after they pass through the tracking detectors. This depends upon the physics process and $\sqrt{s}$, as well as the tracker radius and the detector magnetic field. Studies show that the minimum $\Delta x$ is typically $1-4 \mathrm{~cm}$ in the EMCal and about $5-10 \mathrm{~cm}$ in the HCal. The EMCal should be very dense, with Moliere radius of a few $\mathrm{cm}$ or less, and should have transverse segmentation that is smaller still, in order to localize the photon showers accurately. Fine longitudinal segmentation, with each layer read out, is also essential in order to track the charged particles through the EMCal and to allow charged-neutral separation in 3-D. This will also benefit the energy resolution for photons and electrons. There is no reason to organize the layers in towers, and, in fact, this probably should be avoided. The fine transverse segmentation provides excellent electron identification and photon direction reconstruction. The latter is also useful for measuring photons which result from a secondary vertex. This is relevant, for example, in gauge-mediated SUSY, which can lead to secondary vertices with a photon as the only visible decay particle.

For EF in the HCal, it is desirable to track MIPs throughout. One would need to identify shower positions with a resolution of a few $\mathrm{cm}$. Because of the relatively diffuse distribution of deposited energy for hadron-initiated showers, the solution for charged/neutral identification is not as obvious as for the EMCal case, and different ideas are under consideration. In any scheme, one requires a high degree of segmentation. This might be implemented, for example, using scintillator tiles roughly $5-10$ $\mathrm{cm}$ on a side. Another idea is to push to finer segmentation, using, for example resistive plate chambers (RPCs), but without providing pulse height in the readout. Such a 'digital' hadron calorimeter is one of the options being considered for TESLA. This provides increased resolution for pattern recognition, but perhaps with poorer neutral hadron energy resolution.

As with this segmentation issue, many of the other properties of the HCal in an EF calorimeter remain uncertain. One example is the necessary total calorimeter depth in interaction lengths. Another is the placement of the solenoid coil. Since the fields are typically large, and the coils are at large radius, their thickness is not negligible. Qualitatively, for good performance one would prefer to have the coil outside the HCal. But the tradeoffs are not yet well understood quantitatively.

The EF jet resolution is dominated by the tracker momentum resolution, the calorimeter pattern recognition efficiency, and by the purity of charged/neutral identification. Hence, single-particle resolutions are less important. However, the current EF designs yield energy resolution $A / \sqrt{E}$ in the range $A=12-20 \%$ for photons, and in the range $A=40-50 \%$ for single hadrons.

For a detector not designed to use energy flow, there are, of course, many traditional choices available. Assuming that jets are to be reconstructed using the calorimeter only, one might choose a compensating, sampling calorimeter with a tower geometry. One or more layers of detector with finer segmentation may be required 
Detectors for the Linear Collider

at the front of the EMCal, or at shower maximum, to aid with electron and photon identification. Such a calorimeter would certainly be cheaper than an EF device at a similar radius. At low $\sqrt{s}$, especially at the $Z$, this may suffice.

One also needs to consider Bhabha scattering in the calorimeter design. First, the final state $e^{ \pm}$at $\sqrt{s} / 2$ determines the upper end of the dynamic range of the EMCal readout. For example, for a dense EMCal, the ratio of deposited energy for Bhabha electrons to MIPs can be $10^{3}$ to $10^{4}$, depending on segmentation. Secondly, the Bhabhas are used for luminosity measurements of two types. First, the Bhabha rate can be used to measure the absolute luminosity. Since this rate at intermediate to large angles (endcap and barrel) will be large compared to (known) physics processes, it would not be necessary to rely on a small-angle luminosity monitor (LUM), although a LUM would still be useful for crosschecks and operations. Running at the $Z$ is an exceptional case where a precise LUM would be required. The Bhabhas also provide probably the best measurement of the luminosity spectrum, $d \mathcal{L} / d E$, because the Bhabha acolinearity is closely related to the beam energy loss. This is ideally measured at intermediate angles, and the EMCal endcap will need to be able to aid the tracker with this measurement.

In addition to Bhabha scattering, two other types of measurement have been discussed for the small-angle region. One is a measurement of the flux of pairs produced in the collision beam-beam interactions. This would provide immediate feedback to operators of a quantity closely related to the instantaneous luminosity. The other is small-angle tagging of the forward-scattered electron or positron resulting from a two-photon interaction. This is useful both in the study of the two-photon process itself and in reducing background in the study of processes such as slepton pair production which resemble two-photon reactions. Such a device would need to tag a single high-energy electron within the angular region flooded by low-energy pairs from the beam-beam interaction.

Finally, the small-angle elements of any calorimeter design must reflect the requirement to limit the detector contribution to the missing transverse momentum resolution. This contribution is roughly $E_{b} \theta_{\min }$, where $E_{b}$ is the beam energy. Given the limited angular coverage of the central tracking systems, one should consider carefully what type of calorimetry should be used near $\theta_{\min }$.

\subsubsection{Technology options}

For the dense, finely segmented electromagnetic calorimeter required for energy flow, layers composed of a tungsten radiator with silicon detectors $(\mathrm{Si} / \mathrm{W})$ are a natural choice. The Moliere radius of tungsten is small $(9 \mathrm{~mm})$, and the silicon is thin and easily segmented transversely. Si/W EMCal's are currently incorporated in two LC detector designs, the TESLA detector and the NLC SD detector described in Section 4.2. This option has one outstanding drawback, the cost of the silicon detectors. 
Both TESLA and SD assume that a cost of roughly $\$ 3 / \mathrm{cm}^{2}$ can be achieved in the future with a very large order. This is about a factor two cheaper than current costs. There are a number of cost and performance optimization possibilities. For example, one would probably not need to sample the EMCal uniformly in depth, reducing the sampling frequency after about $12 X_{0}$. One could also improve the photon energy resolution by sampling with thicker silicon, at some small loss of Moliere radius.

Perhaps it is possible to design a competitive energy flow electromagnetic calorimeter at lower cost using an alternative to silicon, for example, scintillator tiles. The transverse segmentation is limited using present techniques by the inability to couple sufficient light to a readout fiber. Perhaps this can be improved. However, given the larger cells, sufficiently large $B$ and $R$ may compensate for the segmentation disadvantage. This is the rationale for the NLC L design described in Section 4.1. Another alternative being considered for TESLA is a Shashlik EMCal. Beam test results [9], using fibers of two lifetimes in order to achieve some longitudinal segmentation, have been impressive, but it is unclear whether the segmentation is sufficient for EF.

The hadron calorimeter for an EF detector is not as highly constrained as the EMCal. Here, scintillator tiles can be of size similar to present applications, say 8-10 $\mathrm{cm}$ on a side, with coupling to an optical fiber. Such a scheme is under consideration for the TESLA and NLC L and P designs. (The last of these is described in Section 4.3.) Other possibilities include the 'digital' option mentioned above, which might use, for example, double-gap RPC readout layers or extruded scintillator. The spatial resolution per layer might be about $1 \mathrm{~cm}$.

If it were possible to relax the need for precise jet reconstruction, then one might forego EF, and save some money with a more traditional calorimeter. For example, the NLC P design uses modestly segmented towers built up from $\mathrm{Pb} /$ scintillator layers. This might also be implemented using liquid argon.

\subsection{Muon detection}

The main purpose of the LC muon system is to identify muons and provide a software muon trigger. A secondary purpose is to use the muon detector as backup calorimetry for those particles that penetrate beyond the normal hadron calorimeter. The signature for muons is their penetration through the calorimetry and the instrumented iron flux-return for the solenoid field.

The momentum of muons is determined from the central and forward tracking systems. This requires the association of tracks found in the instrumented flux-return with hits/tracks in the central and forward tracking detectors. Two conditions permit this: a reasonable density of hits in the inner layers of the tracking detectors and limited confusion from multiple scattering due to the electromagnetic and hadronic calorimeters between the inner tracking detectors and the front face of the muon detectors. These conditions are satisfied, since the maximum density of tracks, at a radius of $3 \mathrm{~m}$, is about $1 / \mathrm{cm}^{2}$ [10] and the $r-\phi$ rms multiple scattering of a $10 \mathrm{GeV} / \mathrm{c}$ 
muon is approximately $2 \mathrm{~cm}$. The number of radiation lengths $X_{0}$ of material in front of the muon system for the three candidate detectors L, SD and P are 200, 88 and 125 , respectively.

Muons are identified by their ionization in tracking chamber panels [10] or scintillator strips [11] in $2 \mathrm{~cm}$ gaps between 5 or $10 \mathrm{~cm}$ thick Fe plates that make up the barrel and end sections of the Fe return yoke for the central solenoidal magnetic field. RPCs are taken as the example technology. These planar devices can be built with appropriate perimeter shapes, and they do not contain wires that could break. Tracking hits from the avalanche produced in the RPC gaps are read out with strip electrodes that run in the $\phi$ and $z$ directions. The spatial resolution of these strips is $1 \mathrm{~cm}$ per detector plane.

For the case of the L detector, it can be seen in Fig. 15.5 that the number of hits as a function of momentum for $W$ pair production, plateaus at about $5 \mathrm{GeV}$ with 25 instrumented gaps. The plot shows that in the $3 \mathrm{~T}$ field there will be very good efficiency if 15 or more hits are required in the muon tracking algorithm.

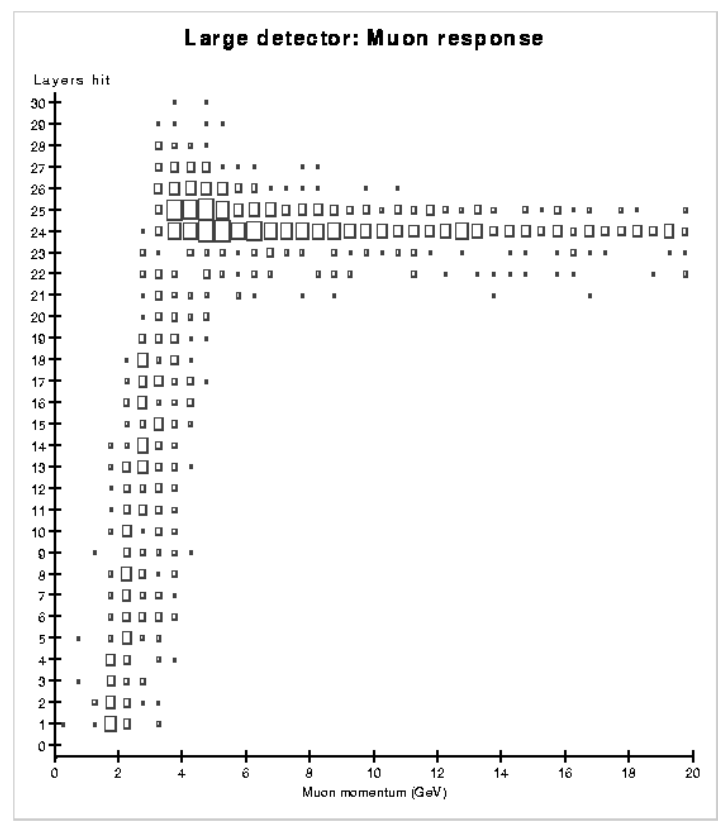

Figure 15.5: Hits in the muon system as a function of momentum for the $\mathrm{L}$ detector. The pllot shows $10000 e^{+} e^{-} \rightarrow W^{+} W^{-}$events in which one $W$ decays to a muon.

The Fe plate and strip readout for the muon system can be used as additional 
coarse hadron calorimetry, since the number of interaction lengths $\lambda$ for the L, SD and $\mathrm{P}$ options are, respectively, 6.6, 6.1 and 3.9. The muon Fe adds 7, 6, and $6 \lambda$ that can be used in the determination of residual hadronic energy with a resolution that will be about $1 / \sqrt{E}$.

\subsection{Solenoid}

The detector is assumed to be a classical solenoidal design. The field in the tracking region ranges from 3 to $5 \mathrm{~T}$ for the various designs. The solenoid is assumed to be of the CMS type, based on a relatively thick, multi-layer superconducting coil. The radial thickness of the complete assembly is about $85 \mathrm{~cm}$. The CMS vacuum shell has a total thickness of $12 \mathrm{~cm}$, and a cold mass thickness of $31 \mathrm{~cm}$ (aluminum). It is likely that the cold mass thickness will scale roughly as $B^{2} R$. Then, the coil of the SD detector would be about $35 \%$ thicker.

The iron serves as the flux return, the absorber for the muon tracker, and the support structure for the detector. The (perhaps debatable) requirement of returning most of the flux drives the scale of the detector. At this stage of preliminary design, it is assumed that the steel is in laminations of $5 \mathrm{~cm}$ with $1.5 \mathrm{~cm}$ gaps.

The door structure very likely runs along the beamline past $\mathrm{L}^{*}$, the position of the downstream face of the last machine quadrupole. Thus it is essentially certain that the Final Doublet (FD) is inside the detector, and quite possibly within the Hadronic Calorimeter. For this reason, the FD cannot be mounted on a massive column going directly to bedrock.

\subsection{Particle ID}

The physics topics of the linear collider do not demand hadron ID in a direct way, though the information may prove valuable for some analyses. Pions, kaons and protons are produced in the ratio of about 8:1:0.6 in high-energy $e^{+} e^{-}$colliders. The momentum spectrum of kaons in $q \bar{q}$ events at $\sqrt{s}=500 \mathrm{GeV}$ extends up to 150-200 $\mathrm{GeV} / \mathrm{c}$, posing a possibly unsurmountable ID measurement challenge. However, the average kaon momentum is only $10-17 \mathrm{GeV} / \mathrm{c}$, and more than half of all kaons have momenta below $7 \mathrm{GeV} / \mathrm{c}$. In $t$-quark and multi- $b$ jet Higgs events, the multiplicity is higher, and so kaons have a slightly lower mean momentum.

The measurement of particle species distributions provides information on QCD processes and permits model tests, but the most important use of hadron ID may be to assist the application of other techniques, such as $B$ tagging. As an example, two studies $[12,13]$ have discussed the use of net kaon charge to tag the flavor of neutral $B$ mesons produced in $q \bar{q}$ events. They find that with perfect knowledge of decay product identities in vertex-tagged neutral $B$ mesons, roughly a quarter are correctly tagged by the net charge of kaons. The efficiency is much lower if all undiscriminated hadrons are used. It is a detailed, and so far unanswered, question whether the use of 
Detectors for the Linear Collider

hadron ID with realistic detector efficiencies can be an important tool to unscramble complex events that contain multiple $b$ - or $c$-quark jets.

The geometric and, ultimately, the cost constraints limit the choice of technology for a hadron ID system of a linear collider detector. Ideally, it should take up no space and introduce no additional mass in front of the calorimeter. Traditional ionization measurement $(d E / d x)$ in gas-based tracking chambers comes close to meeting these criteria.

The Time Projection Chamber (TPC) technology that appears in the TESLA and L tracker designs may be an optimal choice for combined tracking and ionization measurements for particle ID. The energy resolution that has been achieved with existing non-pressurized TPCs (e.g., ALEPH at LEP) is $4.5 \%$, which would yield $\pi / \mathrm{K}$ separation of better than $2 \sigma$ for $p<0.8 \mathrm{GeV} / \mathrm{c}$ and $2-3 \sigma$ for $1.7<p<65$ $\mathrm{GeV} / \mathrm{c}$. One can improve the capability of a TPC by using pressurized gas to achieve $2.5 \%$ resolution, as demonstrated by the TPC at PEP. According to a recent model [14], this could provide $4 \sigma \pi / K$ separation in the range $1.75<p<30 \mathrm{GeV} / \mathrm{c}$.

In practice, experiments that desire a high degree of species separation have supplemented ionization measurements with specialized devices such as time-of-flight, threshold Cerenkov or ring-imaging Cerenkov devices. The major drawback of a specialized hadron ID subsystem is its collateral impact on the tracking and calorimetry. All supplementary techniques take up radial space between the tracker and calorimeter, which means either shorter tracking volume or increased calorimeter radius with consequent cost and performance implications. Without a clearly defined need for the capability, it is difficult to justify a significant impact on the rest of the detector. For example, in the $B^{0}$ tagging study, even though the best performance was provided by an SLD-style CRID or a high-pressure TPC, relatively inexpensive improvements to an ALEPH-type TPC could achieve a sensitivity within a factor of two of these more complicated options but with little impact on the calorimetry.

In summary, at this stage there is no compelling argument to include a specialized hadron ID system in the high energy detector design, though in the process of optimizing the design this assumption may be reexamined.

\subsection{Electronics and data acquisition}

The NLC beam consists of 190 bunches spaced 1.4 ns apart, in trains that repeat at $120 \mathrm{~Hz}$. There are variations with a doubled bunch spacing and an increased train frequency of $180 \mathrm{~Hz}$, but these variations do not affect the basic theme. For most of the detector subsystems it will neither be possible, nor particularly desirable, to resolve bunches in a train. The train repetition rate of $120 \mathrm{~Hz}$ is a low frequency compared with Level 1 or Level 2 trigger rates at many other machines. There is no need for a hardware trigger, and (zero-suppressed, calibration-corrected) data can flow from the detector at this rate. A traditional Level 3 Trigger (software on a small set of processors) can select events for storage. 
The time horizon for a detector is roughly 8 to 10 years away, which is at least 5 Moore's Law generations. To be sure, Moore's Law refers to computing power per dollar, but there are clearly related trends in most areas of silicon technology. At this time it seems most appropriate to sketch plausible architectures to help generate cost estimates, and to avoid detailed designs.

Perhaps the clearest distinction that should be made is the role of interconnections that are not on silicon. Rather inexpensive systems have been developed for large CCD detectors. The costs strongly reflect the number of output nodes that must be serviced, and correspond only weakly to the number of pixels being transmitted through that node. In addition, because of the train spacing, there is no penalty to serial multiplexing of the data from very large numbers of pixels. This is in contrast to the LHC, where there are many interactions associated with each beam crossing, which occurs every 25 ns. This is not to say there are no limits to the serial multiplexing. The readout of the SLD Vertex Detector crossed about 8 beam crossings at SLC, and it would be desirable to avoid this at the next-generation linear collider.

Consequently, we have developed the concept of clusters rather than channels. A cluster is a set of detector elements that can conveniently be processed and serialized into a single data stream, presumably an optical fiber. In the CCD example, each node might correspond to a cluster, although it might even be possible to handle multiple nodes in a single cluster. For the CCD case, we think of an ASIC located millimeters from the CCD and bonded to the CCD. This ASIC might handle the clock generation and the gate drives as well as the amplification and digitization of the CCD data. For silicon strip detectors, we foresee a single chip servicing a cluster of strips, presumably a complete detector a few $\mathrm{cm}$ wide. For a calorimeter utilizing scintillator and Hybrid Photo Diodes or Multi-Anode Phototubes, a cluster would correspond to all the outputs from each such device. In all cases, we avoid, as much as possible, all low-level cables and interconnects. The cluster reflects the mechanical nature of the detector. Some cases are less obvious. For a tungsten-silicon calorimeter, a cluster might correspond to a large area board carrying many close packed wafers of silicon diodes. It may cover perhaps a square meter or so. Variations on this concept would cover readout sectors of the TPC and the muon tracking detectors.

Thus the detector proper carries all the front end processing, and a relatively modest set of fibers carries data off the detector. We envision the fibers delivering the data to processors, perhaps based on VME, although there are hints that crate systems based on optical serial backplanes may arrive in time. These processor arrays would complete the signal processing, build the events, and pass those events to the system responsible for the Level 3 decision. 
Detectors for the Linear Collider

\section{Detectors}

Three detector models are now being studied as potential detectors for the NLC. These include two options for the high-energy IR, called L and SD, and one for the lower-energy, second IR, called P. Here we describe each of these detectors, and present some of their performance curves.

\subsection{L detector for the high-energy IR}

The $\mathrm{L}$ detector design is driven by the desire to provide a large tracking volume, to optimize tracking precision. This leads to a large-radius calorimeter and limits the magnetic field strength to about 3 Tesla.

The $\mathrm{L}$ detector is illustrated in Fig. 15.6. Table 15.1 presents the dimensions of the $\mathrm{L}$ detector, along with those for the SD and $\mathrm{P}$ detectors, described below.

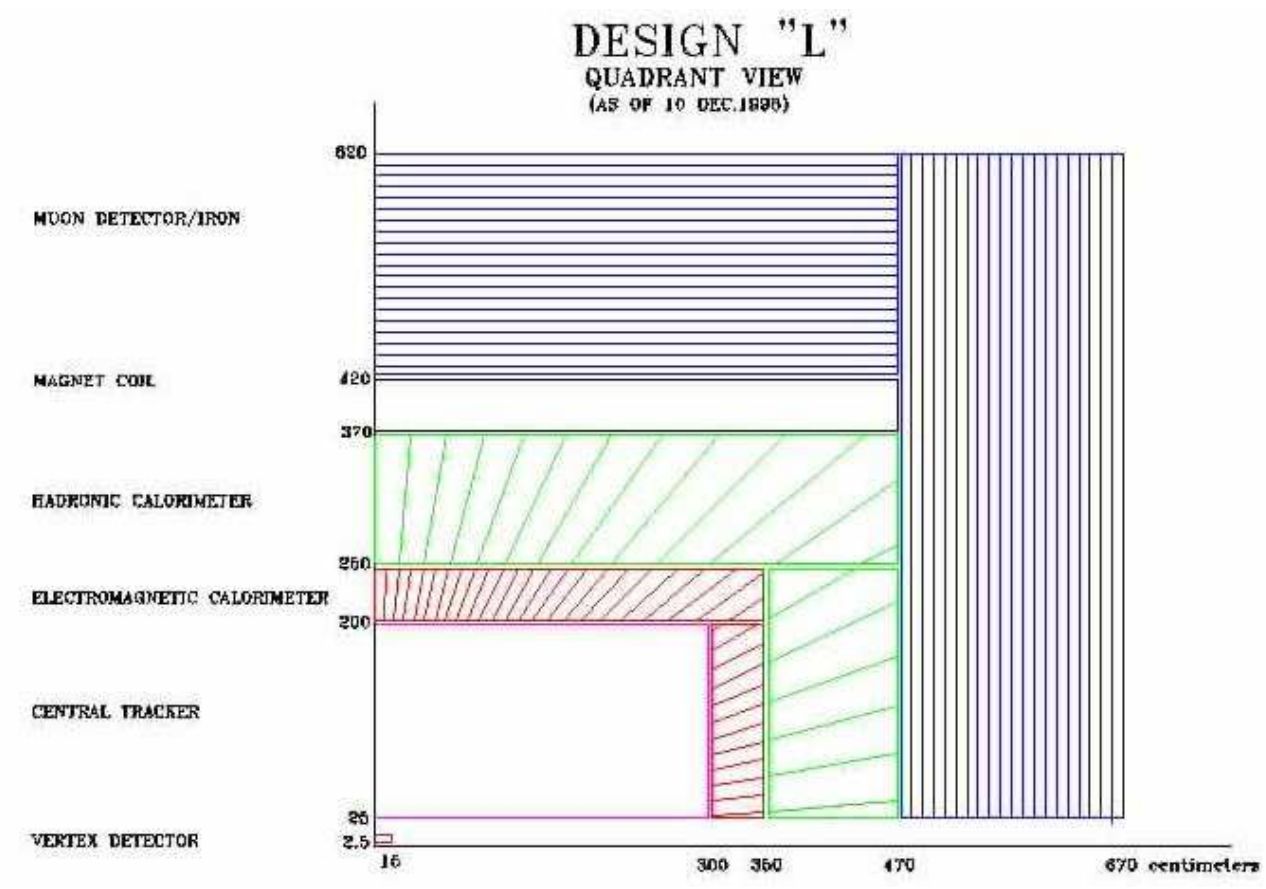

Figure 15.6: Quadrant view of the L detector.

The vertex detector is a five-barrel CCD vertex detector, based on the technology developed for SLD. The beam pipe radius of $1 \mathrm{~cm}$ allows the inner barrel of the detector to reside $1.2 \mathrm{~cm}$ from the IP. The inner barrel extends over $5 \mathrm{~cm}$ longitudinally. The other barrels have radii of $2.4 \mathrm{~cm}, 3.6 \mathrm{~cm}, 4.8 \mathrm{~cm}$, and $6.0 \mathrm{~cm}$, and they each extend $25 \mathrm{~cm}$ longitudinally. The barrel thicknesses are $0.12 \% X_{0}$ and the precision 
Chapter 15

\begin{tabular}{|l|cc|cc||cc|cc||cc|cc|}
\hline \hline \multirow{2}{*}{ Component } & \multicolumn{4}{|c||}{ L Detector } & \multicolumn{4}{c||}{ SD Detector } & \multicolumn{4}{c|}{ P Detector } \\
& $\mathrm{R}(\mathrm{cm})$ & \multicolumn{2}{c|}{$\mathrm{Z}(\mathrm{cm})$} & \multicolumn{2}{c|}{$\mathrm{R}(\mathrm{cm})$} & \multicolumn{2}{c|}{$\mathrm{Z}(\mathrm{cm})$} & \multicolumn{2}{c|}{$\mathrm{R}(\mathrm{cm})$} & \multicolumn{2}{c|}{$\mathrm{Z}(\mathrm{cm})$} \\
& Min & Max & Min & Max & Min & Max & Min & Max & Min & Max & Min & Max \\
\hline Vertex Det. & 1.0 & 10 & 0 & 15 & 1.0 & 10 & 0 & 15 & 1.0 & 10 & 0 & 15 \\
C.Track. & 25 & 200 & 0 & 300 & 20 & 125 & 0 & 125 & 25 & 150 & 0 & 200 \\
ECal & & & & & & & & & & & & \\
$\quad$ Barrel & 200 & 250 & 0 & 350 & 127 & 142 & 0 & 187 & 150 & 185 & 0 & 235 \\
$\quad$ EndCap & 25 & 200 & 300 & 350 & 20 & 125 & 172 & 187 & 25 & 150 & 205 & 240 \\
HCal & & & & & & & & & & & & \\
Barrel & 250 & 370 & 0 & 470 & 143 & 245 & 0 & 289 & 215 & 295 & 0 & 320 \\
$\quad$ EndCap & 25 & 250 & 350 & 470 & 20 & 125 & 172 & 187 & 25 & 175 & 240 & 320 \\
Magnet & 370 & 420 & 0 & 470 & 248 & 308 & 0 & 289 & 185 & 215 & 0 & 235 \\
Iron/Muon & & & & & & & & & & & & \\
$\quad$ Barrel & 420 & 620 & 0 & 470 & 311 & 604 & 0 & 290 & 295 & 425 & 0 & 320 \\
$\quad$ EndCap & 25 & 620 & 470 & 670 & 20 & 604 & 290 & 583 & 25 & 425 & 320 & 450 \\
\hline \hline
\end{tabular}

Table 15.1: Dimensions of the L, SD, and P Linear Collider Detectors.

is assumed to be $5 \mu \mathrm{m}$. (This is taken as a conservative assumption, since SLD has achieved $3.8 \mu \mathrm{m}$.) The entire system comprises $670,000,000$ pixels of $20 \times 20 \times 20 \mu \mathrm{m}^{3}$.

Figure 15.7 illustrates this system. The detector operates in an ambient temperature of $190^{\circ} \mathrm{K}$, created by boil-off nitrogen. It is enclosed within a low mass foam cryostat. The same five-barrel CCD design has been assumed for the SD and P detectors below.

The performance of the vertex detector is illustrated in Figs. 15.8 and 15.9, where it is also compared to the SD and P Detector performance. The impact parameter resolution shown in Fig. 15.8 is shown to surpass the performance of SLD's VXD3. The bottom and charm tagging performance, shown in Fig. 15.9, is also seen to be exceptional.

The L detector central and forward trackers consist of a large-volume TPC, an intermediate silicon tracking layer (silicon drift detector or double-sided silicon microstrips), and five layers of double-sided, silicon microstrip disks in the forward regions. An additional scintillating-fiber intermediate tracker option has also been proposed to provide precise bunch timing. Figure 15.10 shows a sketch of the L detector tracking system.

A large-volume TPC with three-dimensional space point measurements along charged particle trajectories provides excellent pattern recognition (including recognition of long-lived particles that decay in the tracking volume) and good particle identification via $d E / d x$ measurements. The baseline L detector TPC [15] has 144 tracking layers enclosed in a cylindrical volume of inner and outer radii $=50$ and 200 $\mathrm{cm}$, respectively, and of half-length $290 \mathrm{~cm}$. The assumed resolutions on each hit are $150 \mu \mathrm{m}$ in $r-\phi$ and $0.5 \mathrm{~mm}$ in $r-z$. A GEM-based readout has the potential to reduce 


\section{Suggested layout of Vertex Detector for future}

$\mathbf{e}^{+} \mathbf{e}^{-}$Linear Collider (Updated November 1998)

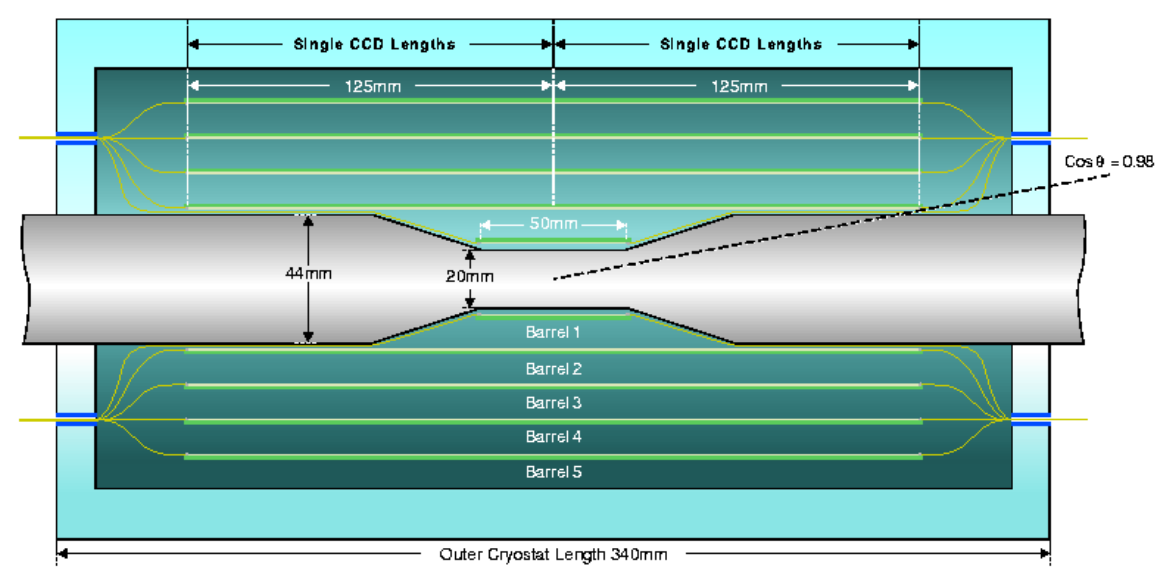

Figure 15.7: The five-barrel CCD vertex detector proposed for the linear collider.

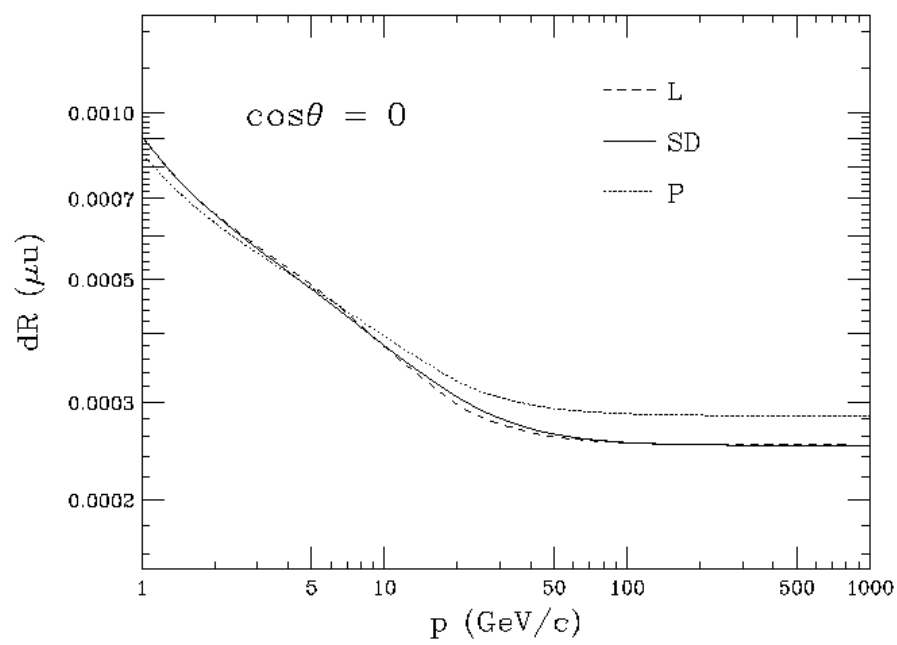

Figure 15.8: Impact parameter resolution versus momentum for the vertex detector shown in Fig. 15.7. 

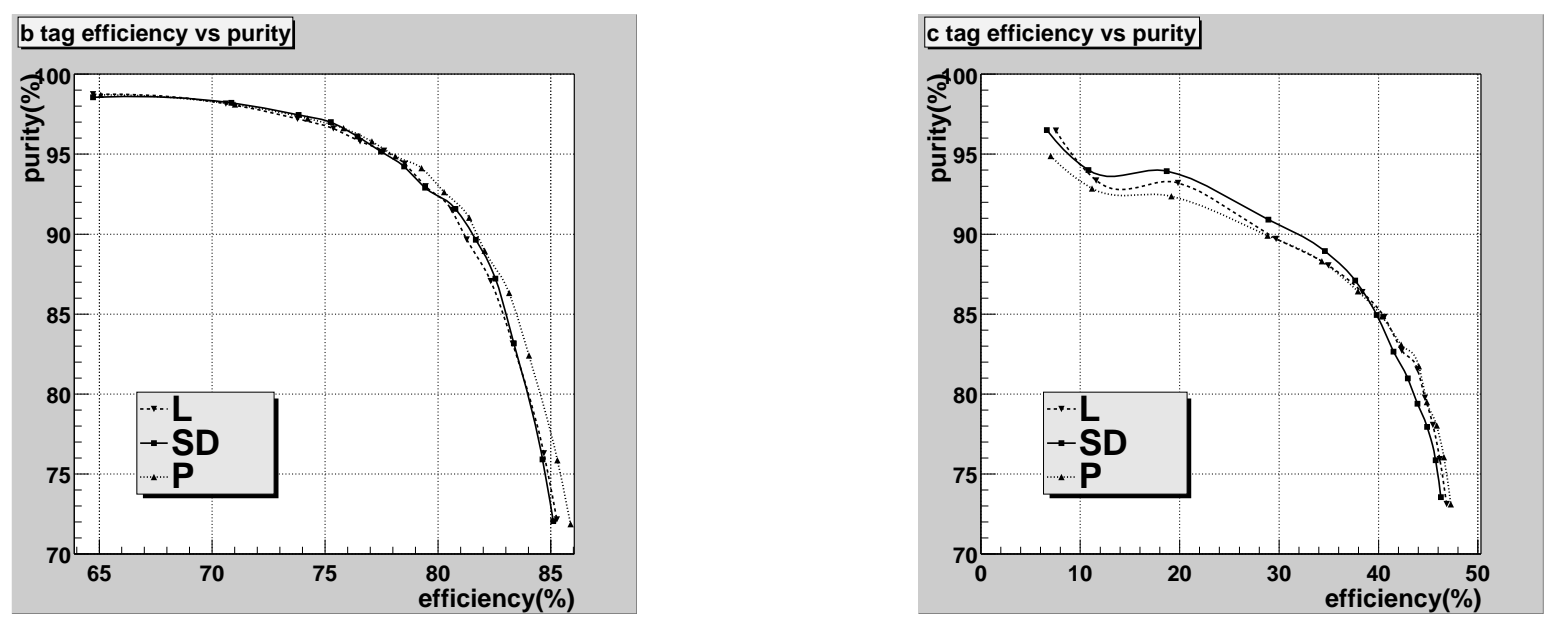

Figure 15.9: Vertex-tagging purity versus efficiency for $b$ (left) and $c$ (right), evaluated for decays of the $Z^{0}$ at $E_{\mathrm{CM}}=91.26 \mathrm{GeV}$.

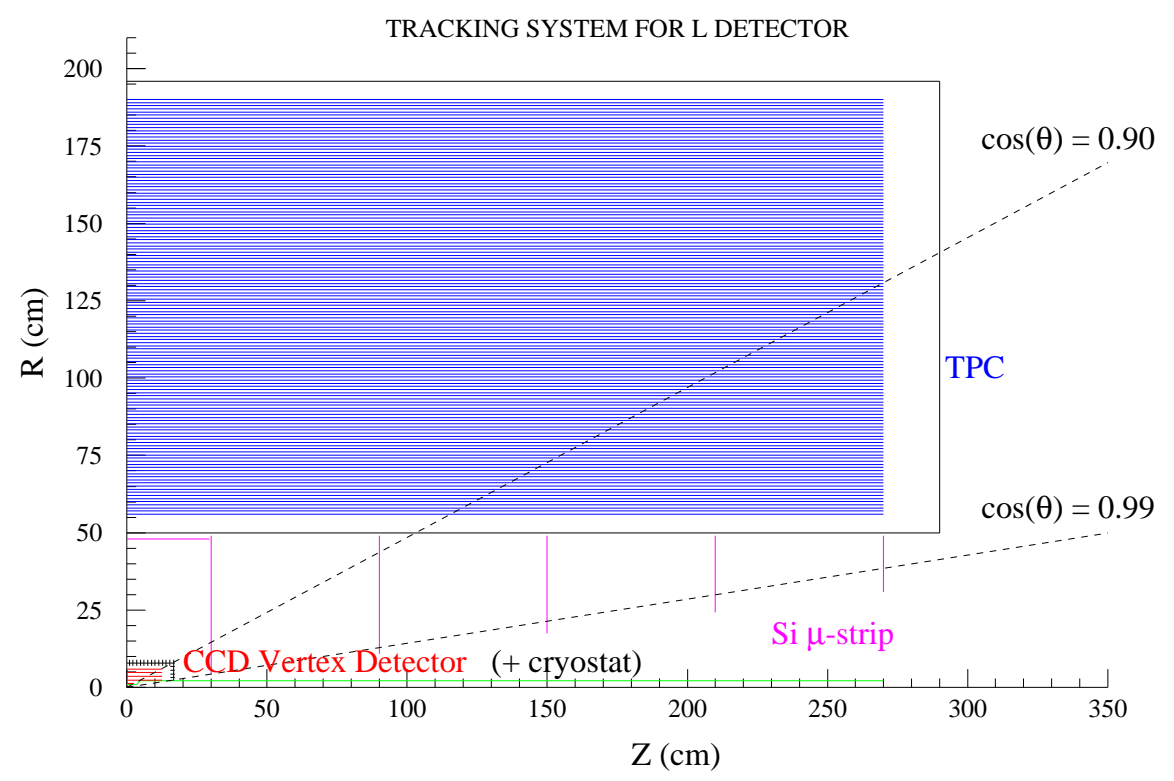

Figure 15.10: Sketch of L detector tracking system.

the $r-\phi$ resolution to $100 \mu \mathrm{m}$. The small transverse diffusion for TPC operation in the $3 \mathrm{~T}$ magnetic field requires very narrow cathode pads and large total channel counts. Longer pads or the use of induced signal on adjacent pads may be considered to reduce the channel count. Good track timing resolution is obtained by requiring individual charged tracks to point back to a reconstructed vertex in the $r-z$ plane. This timing resolution helps in reducing accelerator backgrounds. 
The TPC in the STAR detector at RHIC has over 138,000 electronics channels and includes several design innovations. To reduce the required cable plant, low-noise lowpower front end electronics are mounted on the TPC end planes. The analog signals are amplified, sampled and digitized before being sent to the DAQ system over fiber optics. A similar scheme is assumed here, with $20-\mathrm{MHz}$ sampling, a 200-ns peaking time and 9-bit digitization.

The TPC analog front end electronics would consist of a high-bandwidth preamplifier and shaper amplifier (8-16 channels/ASIC chip), providing a 200 nsec peaking time pulse to the analog sampling and digitization section. The analog signals from the preamplifier and shaper amplifier would be sampled and stored with a highfrequency $20-\mathrm{MHz}$ clock as they come in, and then digitized on a longer $(10 \mu \mathrm{sec})$ time scale as new samples are being taken. The recognition of charge cluster signals on a central cathode-pad channel triggers a switched capacitor array (SCA) to sample the channel and its nearest neighbors.

Gas mixtures of argon with methane and carbon dioxide are being considered, with $\operatorname{Ar}(90 \%): \mathrm{CH}_{4}(5 \%): \mathrm{CO}_{2}(5 \%)$ being quite attractive in balancing safety concerns, neutron-background quenching, and drift velocity. Positive ions feeding back from endplane gas amplification can be mitigated by the installation of a gating grid.

A silicon intermediate tracking detector just inside the TPC inner radius provides nearly a factor of two improvement in momentum resolution for high- $p_{t}$ tracks and offers a pattern recognition bridge between the TPC and the vertex detector. Two silicon options are under consideration: a silicon drift detector and a double-sided silicon microstrip layer. In each case the layer would have a half-length of $29.5 \mathrm{~cm}$ and an average radius of $48 \mathrm{~cm}$. The estimated space-point resolutions in $r-\phi$ and $r-z$ are $7 \mu \mathrm{m}$ and $10 \mu \mathrm{m}$, respectively, for the silicon drift detector option, with both at $7 \mu \mathrm{m}$ for the double-sided microstrip option.

An additional or alternative intermediate tracker constructed from scintillating fibers offers high-precision timing to allow the matching of tracks to individual beam bunches. The current NLC accelerator design provides beams composed of trains of bunches with bunch spacings of 1.4 ns. Large rates of two-photon interactions are expected both from interactions of virtual photons and from real photons created by beamstrahlung. The overlap of the two-photon events with $e^{+} e^{-}$annihilation events results in additional 'mini-jets', which can be a problem if tracks created in different bunch crossings are not separated. A scintillating-fiber intermediate tracker, coupled by clear fiber to visible light photon counters and read out by the SVXIIe chip [16] can achieve time resolutions on the order of $1 \mathrm{~ns}$ to associate tracks with individual bunches, as well as to complement time measurements in the TPC. Appropriate Si:As devices manufactured by Boeing [17] have a fast response time of less than 100 ps. One possible system consists of two axial layers and two $3^{\circ}$-stereo layers with a halflength of $29.5 \mathrm{~cm}$ at an average radius of $48 \mathrm{~cm}$, supported by a carbon fiber cylinder. Scintillating fibers of diameter $800 \mu \mathrm{m}$ would provide individual measurements to 


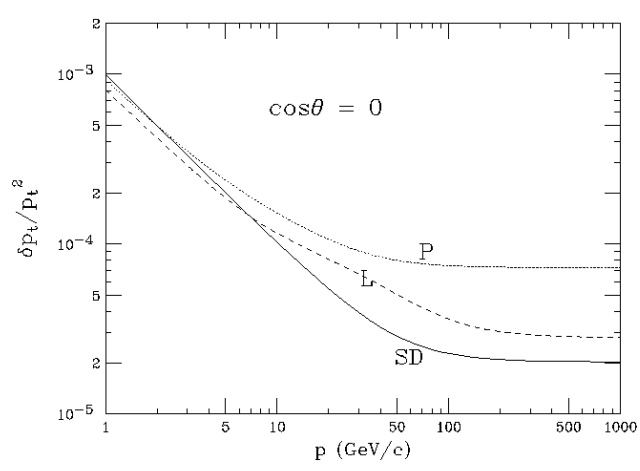

Figure 15.11: Expected fractional momentum resolution vs. momentum for the $\mathrm{L}$, $\mathrm{SD}$, and $\mathrm{P}$ central trackers for tracks transverse to the beam direction.

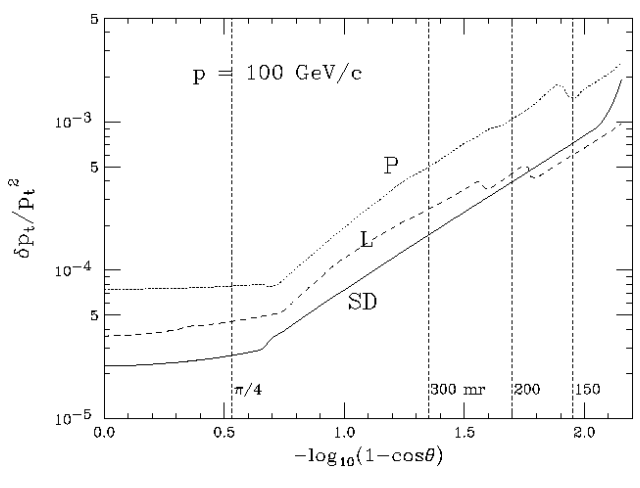

Figure 15.12: Expected fractional momentum resolution vs. $\cos \theta$ for the $\mathrm{L}, \mathrm{SD}$, and $\mathrm{P}$ central trackers for $100 \mathrm{GeV}$ tracks.

$230 \mu \mathrm{m}$ and a combined point measurement with a precision of $\sim 100 \mu \mathrm{m}$, resulting in a system with 15,000 channels.

As currently envisioned, the five layers of the L detector forward disk system are double-sided silicon microstrips, at distances of $30 \mathrm{~cm}$ to $270 \mathrm{~cm}$ from the interaction point, with fixed outer radii at $48 \mathrm{~cm}$. Each side provides counterposing $\pm 20 \mathrm{mrad} r-$ $\phi$ stereo information, with a point resolution of $7 \mu \mathrm{m}$. For high-momentum tracks at $\theta=300 \mathrm{mrad}(|\cos \theta|=0.955)$, this small-angle stereo geometry provides a resolution in $\theta$ of about $\pm 300 \mu \mathrm{rad}$. If large-angle $\left(90^{\circ}\right)$ stereo were used instead, the $\theta$ resolution would improve to about $\pm 100 \mu \mathrm{rad}$. Although the layout of silicon strip detectors is more naturally suited to small-angle stereo, the demands placed on the $\theta$ resolution by the determination of the differential luminosity spectrum may force the consideration of large-angle stereo.

The performance of the $\mathrm{L}$ detector tracking system, including the CCD vertex detector, is summarized in Fig. 15.11, which shows fractional momentum resolution vs. momentum for tracks transverse to the beam direction $(\cos \theta=0)$. Figure 15.12 shows the fractional momentum resolution vs. $\cos \theta$ for tracks of momentum $100 \mathrm{GeV}$. In the limit of high-momentum tracks, the $\mathrm{L}$ tracking resolution in $1 / p_{t}$ is $3 \times 10^{-5}$ $\mathrm{GeV}^{-1}$. Figure 15.13 shows the expected distribution in recoil mass from dimuons in the Higgsstrahlung process $e^{+} e^{-} \rightarrow h Z \rightarrow X \mu^{+} \mu^{-}$at $\sqrt{s}=350 \mathrm{GeV}$ for the nominal $\mathrm{L}$ detector baseline and for several globally rescaled resolutions in $1 / p_{t}$.

The electromagnetic calorimeter of the L Detector is a lead-scintillator laminate with $4 \mathrm{~mm}$ lead followed by $1 \mathrm{~mm}$ scintillator for 40 layers. This results in 28.6 radiation lengths with a $2.1 \mathrm{~cm}$ Moliere radius. One layer of $1 \mathrm{~cm}^{2}$ silicon pads is foreseen near shower maximum. The transverse segmentation of the scintillator is $5.2 \mathrm{~cm} \times 5.2 \mathrm{~cm}$. The barrel of the electromagnetic calorimeter has an inner radius 
Detectors for the Linear Collider

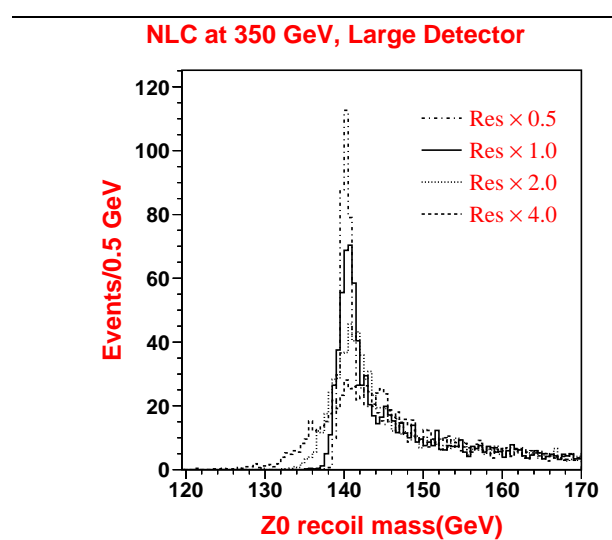

Figure 15.13: Expected recoil mass distribution in recoil mass from dimuons in the Higgsstrahlung process $e^{+} e^{-} \rightarrow h Z \rightarrow$ $X \mu^{+} \mu^{-}$at $E_{c m}=350 \mathrm{GeV}$ for the nominal $\mathrm{L}$ detector baseline and for several globally rescaled resolutions in $1 / p_{t}$.

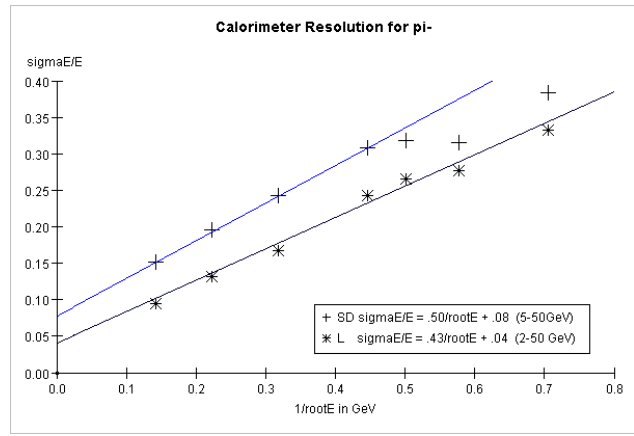

Figure 15.14: Expected $\pi^{-}$energy resolution in the $\mathrm{L}\left(\sigma_{E} / E=0.43 / \sqrt{E}+0.04\right)$ and $\operatorname{SD}\left(\sigma_{E} / E=0.50 / \sqrt{E}+0.08\right)$ Detectors.

of $200 \mathrm{~cm}$. The electromagnetic energy resolution is expected to be $17 \% / \sqrt{E}$.

The hadronic calorimeter is 120 layers of $8 \mathrm{~mm}$ lead layers with $2 \mathrm{~mm}$ scintillator sampling. The entire calorimeter comprises 6.6 interaction lengths. The transverse segmentation of the scintillator in the hadronic calorimeter is $19 \mathrm{~cm} \times 19 \mathrm{~cm}$. Figure 15.14 presents the expected $\pi^{-}$energy resolution.

The hope is that the large $B R^{2}$ of the $\mathrm{L}$ design will allow jet reconstruction using energy flow at a more modest cost than $\mathrm{Si} / \mathrm{W}$, overcoming the limited transverse segmentation possible with scintillator and the larger Moliere radius of lead. But, since the transverse segmentation of the EMCal is much larger than the Moliere radius, it is not clear whether energy flow can be effectively carried out for L. This is in contrast to the SD case, where the fine segmentation allows one to have some confidence that an efficient EF reconstruction can be carried out. This is clearly an area where additional work with full shower simulations is required.

Since shower reconstruction for an EF algorithm for the American detectors is still in its infancy, one can in the meantime use parameterizations of calorimeter performance using a fast simulation. One would expect that the performance from full reconstructions will eventually approach that of the fast simulation. Therefore, for the following performance plots we apply the energy flow technique, but assume a perfect charged-neutral separation in the calorimeters. The appropriate charged track resolutions and EMCal resolutions are then applied. This assumption is not unreasonable for $\mathrm{SD}$, but for $\mathrm{L}$ it is probably too idealized. In any case, our method should indicate the asymptotic limit of performance. 
To examine jet energy resolution, we used $e^{+} e^{-} \rightarrow q \bar{q}$ events without ISR or beamstrahlung, and demanded that exactly two jets be reconstructed. Hence, $E_{\text {jet }}=$ $\sqrt{s} / 2=E_{\text {beam }}$. An example distribution of the reconstructed jet energy, for $\sqrt{s}=100$ $\mathrm{GeV}$ is given in Fig. 15.15. Only events with $\left|\cos \theta_{\text {thrust }}\right|<0.8$ are included. The tail of the distribution is due to QCD and jet-finding effects, whereas the resolution we are interested in here is given by the Gaussian distribution near $E_{\text {beam }}$, and we take the $\sigma$ of this fit as the resolution. Figure 15.16 gives the resolution (the asymptotic limit of performance without accounting for non-Gaussian tails, as described above) as a function of $E_{\text {jet }}$. A fit to these data gives

$$
\frac{\sigma_{E_{\mathrm{jet}}}}{E_{\mathrm{jet}}}=\frac{0.18}{\sqrt{E_{\mathrm{jet}}}} .
$$

One should not expect to actually achieve this idealized resolution with the L calorimeter.

Another important and general measurement of performance is the jet-jet mass resolution. To examine this, we examine the process $e^{+} e^{-} \rightarrow Z Z \rightarrow$ hadrons. Exactly four final-state jets were required. To get a distribution with little background, we require that one 2-jet combination have mass near $M_{Z}$, then plot the mass of the other jet pair, $M_{j j}$. An example $M_{j j}$ distribution is given in Fig. 15.17 for $\sqrt{s}=350 \mathrm{GeV}$. Again, we fit a Gaussian to the distribution near $E_{Z}=E_{\text {beam }}$ to extract a measure of the $M_{j j}$ resolution. This resolution, $\sigma_{M j j} / M_{j j}$, is plotted vs. $E_{Z}=\sqrt{s} / 2 \approx E_{j j}$ in Fig. 15.18. A fit to the data of the form $\left(A / \sqrt{E_{Z}}\right) \oplus B$ gives

$$
\frac{\sigma_{M_{j j}}}{M_{j j}}=\frac{0.64}{\sqrt{E_{Z}}},
$$

with negligible constant term. To the extent that the dijets from a $Z$ are perfectly identified and that no color connection or jet merging effects occur, the sampling term constant here should approach that for the single jet energy resolution given in (15.1). The degradation of dijet mass resolution from this ideal limit requires more study.

The $3 \mathrm{~T}$ solenoidal coil is located outside the hadronic calorimeter to optimize calorimeter performance. The inner radius of the solenoidal coil is $370 \mathrm{~cm}$.

The muon system consists of 24 layers of $5 \mathrm{~cm}$ iron plates, with $3 \mathrm{~cm}$ gaps for $\mathrm{RPC}$ detectors. Axial strips of $3 \mathrm{~cm}$ pitch measure the $\phi$ coordinate to $1 \mathrm{~cm}$ precision in all 24 gaps, and every sixth gap provides azimuthal strips for a measurement of the $z$ coordinate to $1 \mathrm{~cm}$ precision. The barrel muon system begins at a radius of 420 $\mathrm{cm}$. Figure 15.5 illustrates the expected performance for the L detector.

\subsection{SD detector for the high energy IR}

The strategy of the 'Silicon Detector' (SD) is based on the assumption that energy flow calorimetry will be important. While this has not yet been demonstrated in 
Detectors for the Linear Collider

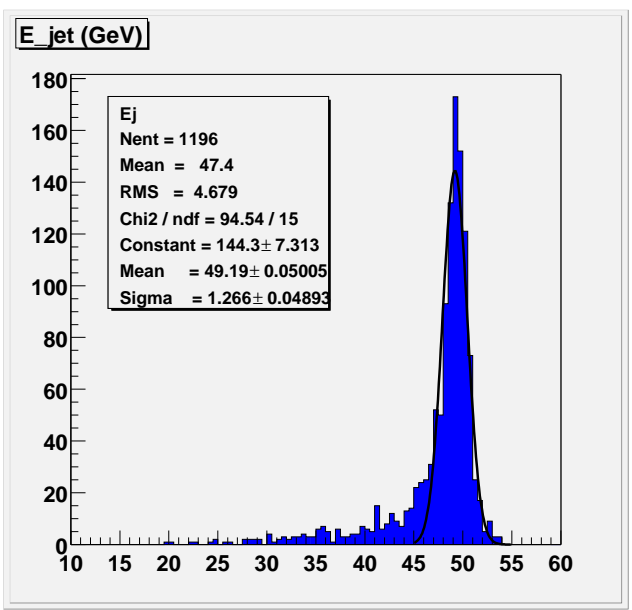

Figure 15.15: Reconstructed jet energy with the $\mathrm{L}$ detector for $50 \mathrm{GeV}$ beam energy.

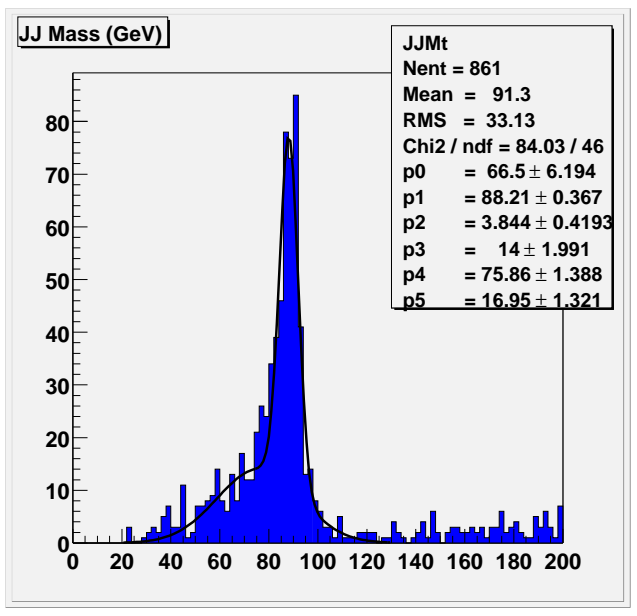

Figure 15.17: Reconstructed jet-jet mass for $Z$ candidates in $e^{+} e^{-} \rightarrow Z Z \rightarrow$ hadrons at $350 \mathrm{GeV}$ for the $\mathrm{L}$ detector.

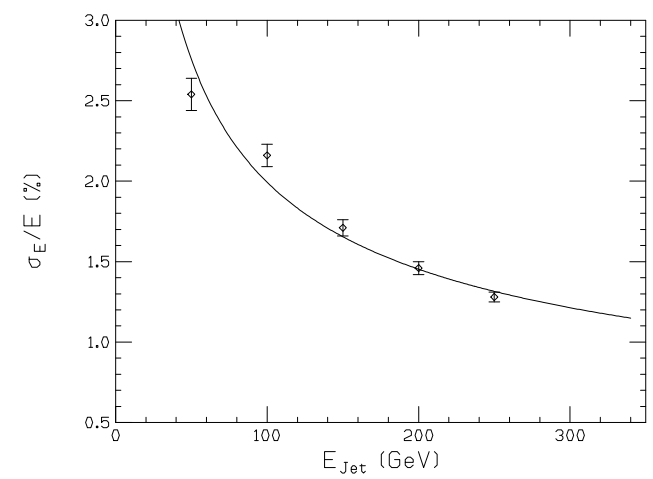

Figure 15.16: Jet energy resolution (in \%) vs. jet energy for the $\mathrm{L}$ detector. The curve is the fit described in the text.

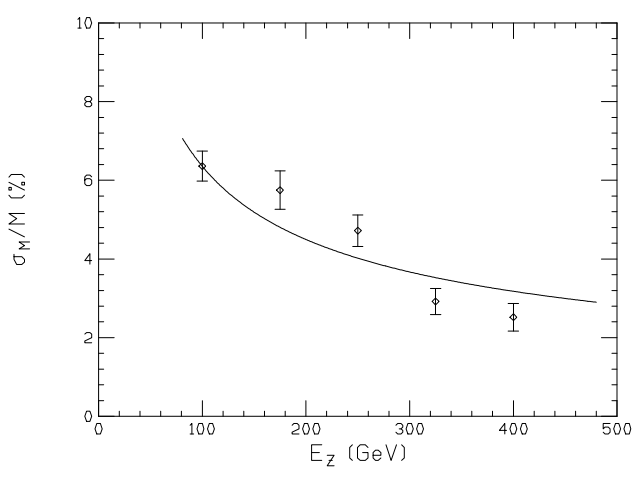

Figure 15.18: Jet-jet mass resolution (in $\%$ ) for $Z \rightarrow 2$ jets vs. $Z$ energy for the $\mathrm{L}$ detector in $e^{+} e^{-} \rightarrow Z Z \rightarrow$ hadrons events. The curve is the fit described in the text.

simulation by the American groups, the TESLA Collaboration has accepted it. This assumption then leads directly to a reasonably large value of $B R^{2}$ to provide chargedneutral separation in a jet, and to an electromagnetic calorimeter (EMCal) design with a small Moliere radius and small pixel size. Additionally, it is desirable to read out each layer of the EMCal to provide maximal information on shower development. This leads to the same nominal solution as TESLA: a series of layers of about 0.5 


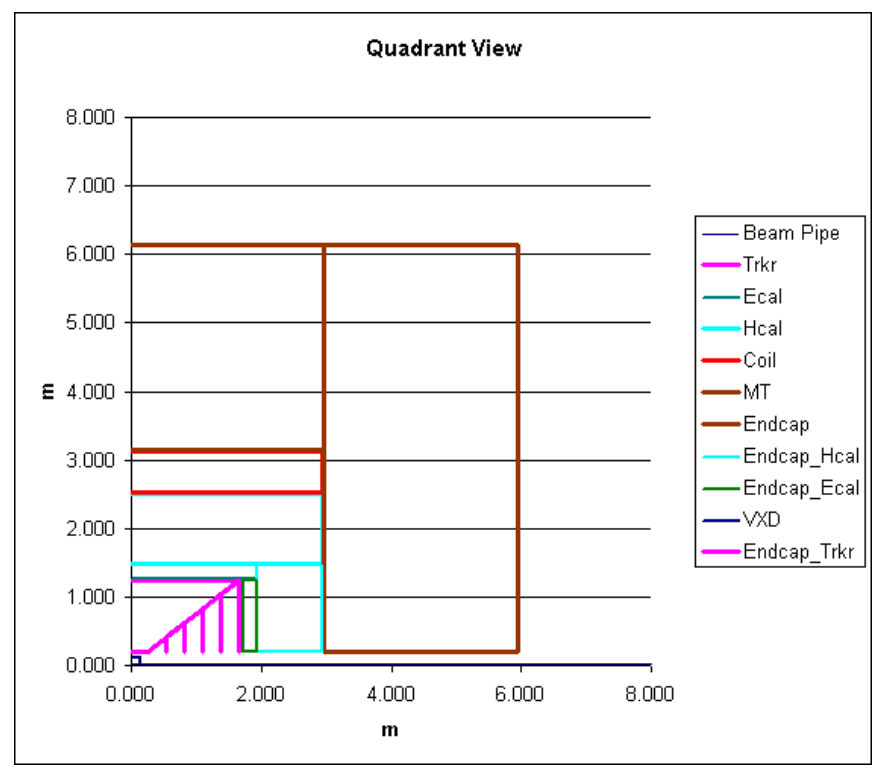

Figure 15.19: Quadrant view of the SD detector.

$X_{0}$ tungsten sheets alternating with silicon diodes. Such a calorimeter is expensive; its cost is moderated by keeping the scale of the inner detectors down. This has two implications: the space point resolution of the tracker should be excellent to meet momentum resolution requirements in a detector of modest radius, and the design should admit high-performance endcaps so that the barrel length (or $\cos \theta_{\text {Barrel }}$ ) will be small. Obviously it is desirable to minimize multiple scattering in the tracker, but compromises will be needed and must be tested with detailed simulation. The last real strategic question is whether the Hadronic Calorimeter ( $\mathrm{HCal}$ ) will be inside or outside the coil. Locating the $\mathrm{HCal}$ inside the coil permits reasonably hermetic calorimetry, but requires a larger, more expensive coil and more iron to return the flux. It is assumed that the detector will have an ultra-high-performance vertex detector based on CCD's or an equivalent thin, small pixel technology, as we have discussed for the L detector. A muon tracker will be interleaved in the iron flux return utilizing reliable RPC's or equivalent.

These considerations lead to a trial design with a tracking radius of $1.25 \mathrm{~m}$ and a field of $5 \mathrm{~T}$. This is a $B R^{2}$ of 8 , compared to 10 for TESLA and 12 for the L detector. The tracker is 5 layers of silicon strips with a $\cos \theta_{\text {Barrel }}$ of 0.8 . Sets of five disks with silicon strips are arranged as endcaps to complete the acceptance. The HCal is inside the coil. The quadrant view is shown in Fig. 15.19, and the major dimensions are tabulated in Table 15.1.

The SD detector relies entirely upon silicon tracking in a $5 \mathrm{~T}$ solenoidal field in the central and forward regions. Its central and forward trackers consist of a 5-layer 
silicon barrel - a silicon drift detector (SDD) or microstrips - and five layers of doublesided silicon microstrip forward disks. Figure 15.20 shows a sketch of the SD detector tracking system. The inner/outer radii of the barrel layers are $20 / 125 \mathrm{~cm}$. The inner and outer disks are at $40 \mathrm{~cm}$ and $167 \mathrm{~cm}$ from the interaction point. The boundary between the barrel and disk system lies at $|\cos \theta|=0.8$.

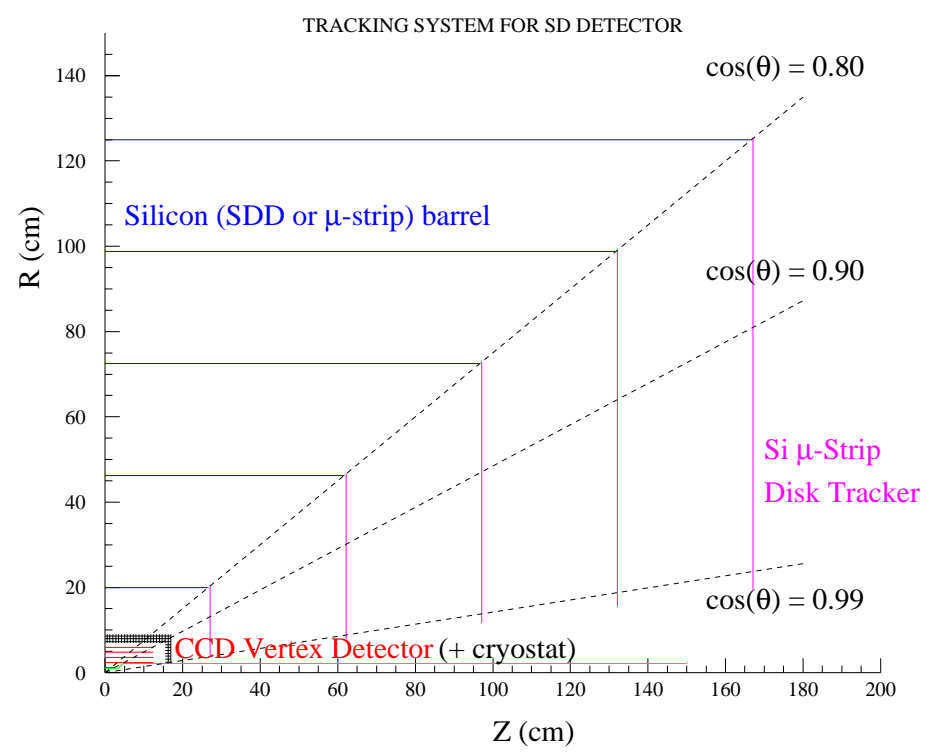

Figure 15.20: Sketch of SD detector tracking system.

The SDD option provides a solid-state analog to a time projection chamber. A potential gradient is applied via implanted cathodes in the silicon in order to force the generated electron cloud to drift through the bulk of the silicon to a collection anode. The highest voltage supplied to a single cathode can be up to $2500 \mathrm{~V}$. By measuring the cloud distribution across the collection anodes and the drift time to the anodes in parallel, one records three-dimensional position information with a one-dimensional electronics readout. Three-dimensional position resolutions below $10 \mu \mathrm{m}$ in each dimension can be achieved with an anode spacing between 200 and $300 \mu \mathrm{m}$. Thus, the electronics cost is considerably reduced compared to other semiconductor detector options. Recently, a three-barrel SDD Tracker, using 216 largearea Silicon Drift wafers, was successfully completed and has been installed in the STAR experiment at RHIC.

Compared to the STAR detector the following modifications would be made to build a linear collider tracker: 1) increase the wafer size to $10 \mathrm{~cm} \times 10 \mathrm{~cm}$; 2) reduce the wafer thickness from 300 to $150 \mu \mathrm{m}$; and 3) redesign the front-end electronics for lower power to eliminate water cooling. The detector contains $56 \mathrm{~m}^{2}$ of active silicon, 
requiring about 6000 wafers and 4.4 million channels distributed over 229 ladders constructed from carbon-fiber material.

The silicon strip detector (SSD) option makes use of what is at this time a very mature tracking technology. Nevertheless, several avenues for further R\&D are discussed below. It should be possible to exploit the small (order $10^{-5}$ ) duty cycle of the linear collider to reduce the power dissipated by the readout electronics by switching to a quiescent state in between trains. This would substantially reduce the heat load, leading to a great reduction in the complexity and material burden of the mechanical structure.

In order to improve the robustness of the detector against linear collider backgrounds, it should be possible to develop a microstrip readout with a short shaping time, with timing resolution of order 5-10 nsec. This would allow out-of-time background hits to be eliminated from the bunch train with a rejection factor of better than 10:1.

On the other hand, the high granularity of microstrip detectors would make an SSD central tracker fairly robust against backgrounds even in the absence of intratrain timing. If instead it is felt that low- and intermediate-momentum track parameter resolution is more important than timing resolution, the use of a readout with a very long shaping-time should make it possible to implement detector ladders of substantially greater length than that of the 10-20 cm ladders of conventional strip detector systems. The AMS collaboration has developed a slow readout [19] with 6 electrons equivalent noise per $\mathrm{cm}$ of detector length. This may allow single ladders to stretch the entire half-length of the outermost silicon layer, and for the inner layers to be thinned. This, combined with a space frame that derives much of its support from the ladders themselves, would lead to a substantial reduction in the material burden, and give an overall low-momentum track parameter resolution on par with that of the $\mathrm{L}$ detector.

The forward disks for the SD tracker would have the same intrinsic performance as those described above for the L detector.

The performance of the SD detector tracking system, including the CCD vertex detector, is summarized in Fig. 15.11 and Fig. 15.12. In the limit of high-momentum tracks, the SD tracking resolution in $1 / p_{t}$ is $2 \times 10^{-5} \mathrm{GeV}^{-1}$.

The EMCal consists of layers of tungsten with gaps sufficient for arrays of silicon diode detectors mounted on G10 mother boards. The thickness of these gaps is a major issue, in that it drives the Moliere radius of the calorimeter. A thickness of $4 \mathrm{~mm}$ seems quite comfortable, accommodating a $0.3-0.5 \mathrm{~mm}$ silicon wafer, a $2 \mathrm{~mm}$ G10 carrier, and $1.5 \mathrm{~mm}$ of clearance. Conversely, $1.5 \mathrm{~mm}$ seems barely plausible, and probably implies a stacked assembly rather than insertion into a slot. For now, we assume a $2.5 \mathrm{~mm}$ gap.

It is expected that the readout electronics from preamplification through digitization and zero suppression can be integrated into the same wafer as the detectors. A 
Detectors for the Linear Collider

fallback would be to bump- or diffusion-bond a separate chip to the wafer. Thus it is expected that the pixel size on the wafer will not affect the cost directly. A pixel size between 5 and $10 \mathrm{~mm}$ on a side is expected. Shaping times would be optimized for the (small) capacitance of the depleted diode, but will probably be too long to provide any significant bunch localization within the train.

The HCal is chosen to lie inside the coil. This choice permits much better hermeticity for the HCal, and extends the solenoid to the endcap flux return. This makes a more uniform field for the track finding, and simplifies the coil design. The $\mathrm{HCal}$ absorber is a non-magnetic metal, probably copper or stainless steel. Lead is possible, but is mechanically more difficult. The detectors could be 'digital', with high-reliability RPC's assumed. The HCal is assumed to be $4 \lambda$ thick, with 34 layers of radiator $2 \mathrm{~cm}$ thick alternating with $1 \mathrm{~cm}$ gaps.

We have examined performance for the SD detector model in the same way as the $\mathrm{L}$ detector, calculating the asymptotic limit of performance. (See the corresponding discussion in Section 4.1 for the limitations of this analysis.) The electromagnetic energy resolution is expected to be $18 \% / \sqrt{E}$. Figure 15.14 presents the expected $\pi^{-}$ energy resolution. The resolution for jet energy reconstruction is given in Fig. 15.21. A fit to these data gives for the asymptotic limit

$$
\frac{\sigma_{E_{\mathrm{jet}}}}{E_{\mathrm{jet}}}=\frac{0.15}{\sqrt{E_{\mathrm{jet}}}} .
$$

As previously, we fit a Gaussian to the distribution near $E_{Z}=E_{\text {beam }}$ to extract a measure of the $M_{j j}$ resolution. This resolution, $\sigma_{M j j} / M_{j j}$, is plotted vs. $E_{Z}$ in Fig. 15.22. A fit to the data of the form $\left(A / \sqrt{E_{Z}}\right) \oplus B$ gives

$$
\frac{\sigma_{M j j}}{M_{j j}}=\frac{0.72}{\sqrt{E_{Z}}},
$$

with negligible constant term. These idealized studies are not yet precise enough to conclude that this is significantly worse than the L Detector performance.

The coil concept is based on the CMS design, with two layers of superconductor and stabilizer. The stored energy is $1.4 \mathrm{GJ}$, compared to about 2.4 GJ for the TESLA detector and 1.7 GJ for the $\mathrm{L}$ detector. The coil thickness is $60 \mathrm{~cm}$, which is probably conservative.

The flux return and muon tracker is designed to return the flux from the solenoid, although the saturation field for the iron is assumed to be $1.8 \mathrm{~T}$, which may be optimistic. The iron is laminated in $5 \mathrm{~cm}$ slabs with $1.5 \mathrm{~cm}$ gaps for detectors.

\subsection{P detector for the lower-energy IR}

The P Detector is proposed as a lower-cost detector for the second IR, capable of the performance required for lower-energy operation, including the $Z$-pole physics. 


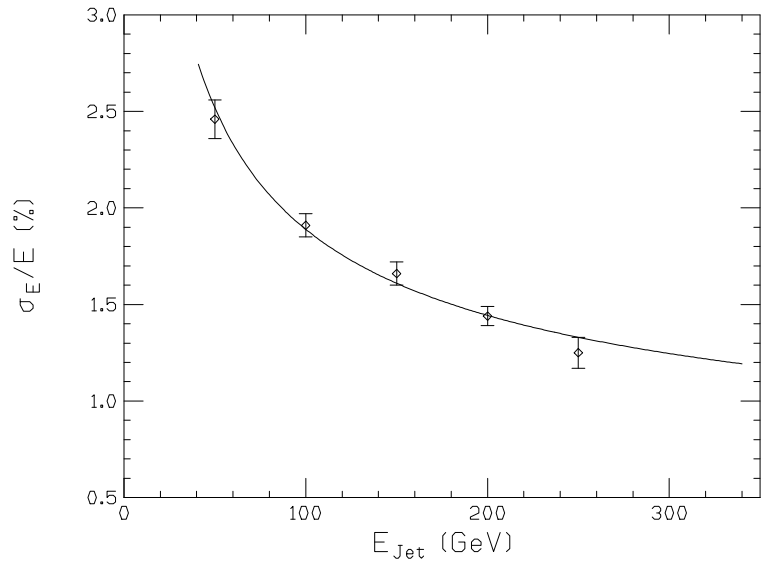

Figure 15.21: Jet energy resolution (in \%) vs. jet energy for the SD detector. The curve is the fit described in the text.

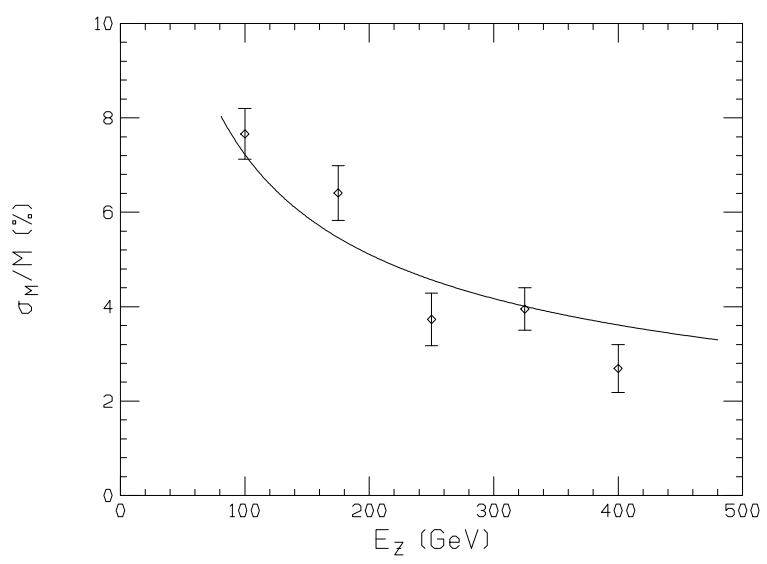

Figure 15.22: Jet-jet mass resolution (in \%) for $Z \rightarrow 2$ jets vs. $Z$ energy for the SD detector in $e^{+} e^{-} \rightarrow Z Z \rightarrow$ hadrons events. The curve is the fit described in the text.

The $\mathrm{P}$ detector is illustrated in Fig. 15.23. The dimensions of the $\mathrm{P}$ Detector are presented in Table 15.1.

The $\mathrm{P}$ detector employs the same CCD vertex detector design described for the L detector above, illustrated in Fig. 15.7.

The $\mathrm{P}$ detector's tracker design is modelled very closely upon that of the $\mathrm{L}$ detector. Since it is meant to operate at lower center-of-mass energies, its required resolution in $1 / p_{t}$ is correspondingly less severe, allowing for a smaller tracking system and therefore a smaller, cheaper overall detector design. Figure 15.24 shows a sketch of the $\mathrm{P}$ detector tracking system.

Briefly, the $\mathrm{P}$ central tracker consists of a 120-layer TPC, of inner/outer radii $=$ $25 / 150 \mathrm{~cm}$ and half-length $200 \mathrm{~cm}$. Again, one or more intermediate tracking layers of silicon or scintillating fiber just inside the inner TPC radius may be desirable. The forward tracker consists of five silicon microstrip disks similar to those in the L and $\mathrm{SD}$ detectors. The performance of the $\mathrm{P}$ detector tracking system in a $3 \mathrm{~T}$ solenoidal field, including the CCD vertex detector, is summarized in Fig. 15.11 and Fig. 15.12. In the limit of high-momentum tracks, the $\mathrm{P}$ tracking resolution in $1 / p_{t}$ is $6 \times 10^{-5}$ $\mathrm{GeV}^{-1}$.

The 3 Telsa solenoidal coil is located outside the electromagnetic calorimeter and inside the hadronic calorimeter. This compromise (over the desire to move the coil outside the hadronic calorimeter) contains the cost of the $\mathrm{P}$ detector. The inner radius of the solenoid is $185 \mathrm{~cm}$.

The electromagnetic calorimeter of the P Detector consists of 32 layers of leadscintillator laminate, with $4 \mathrm{~mm}$ lead layers followed by $3 \mathrm{~mm}$ scintillator, for 22.8 


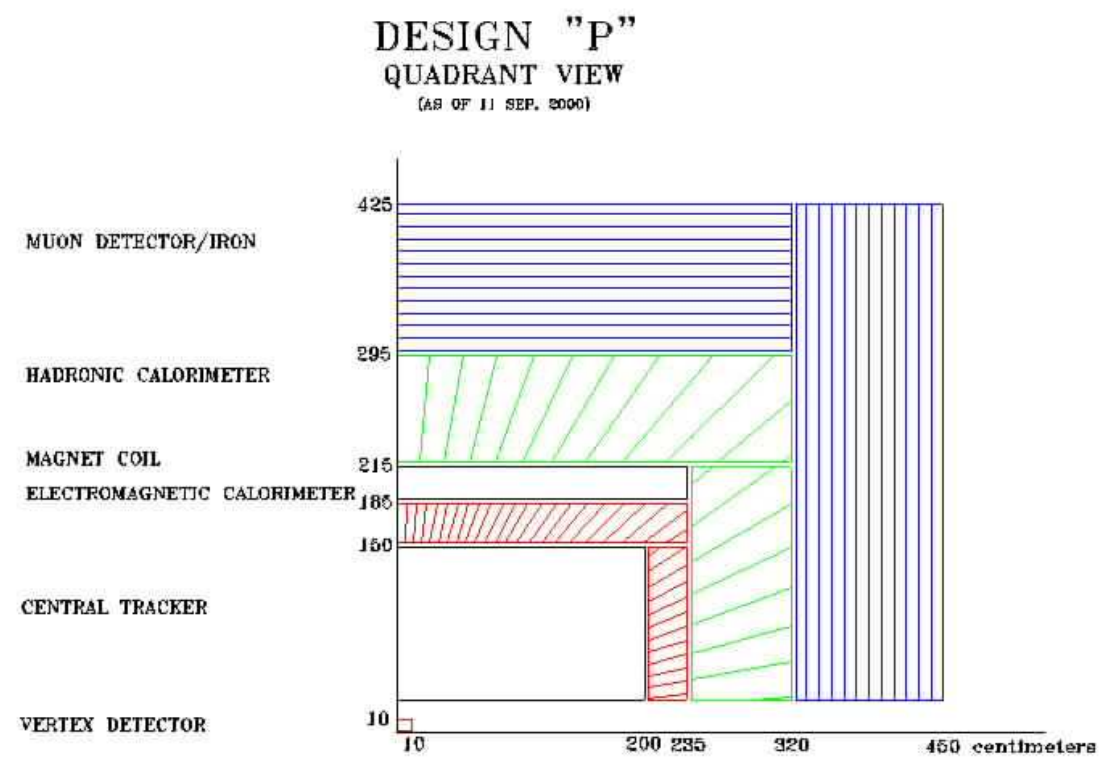

Figure 15.23: Quadrant view of the P detector.

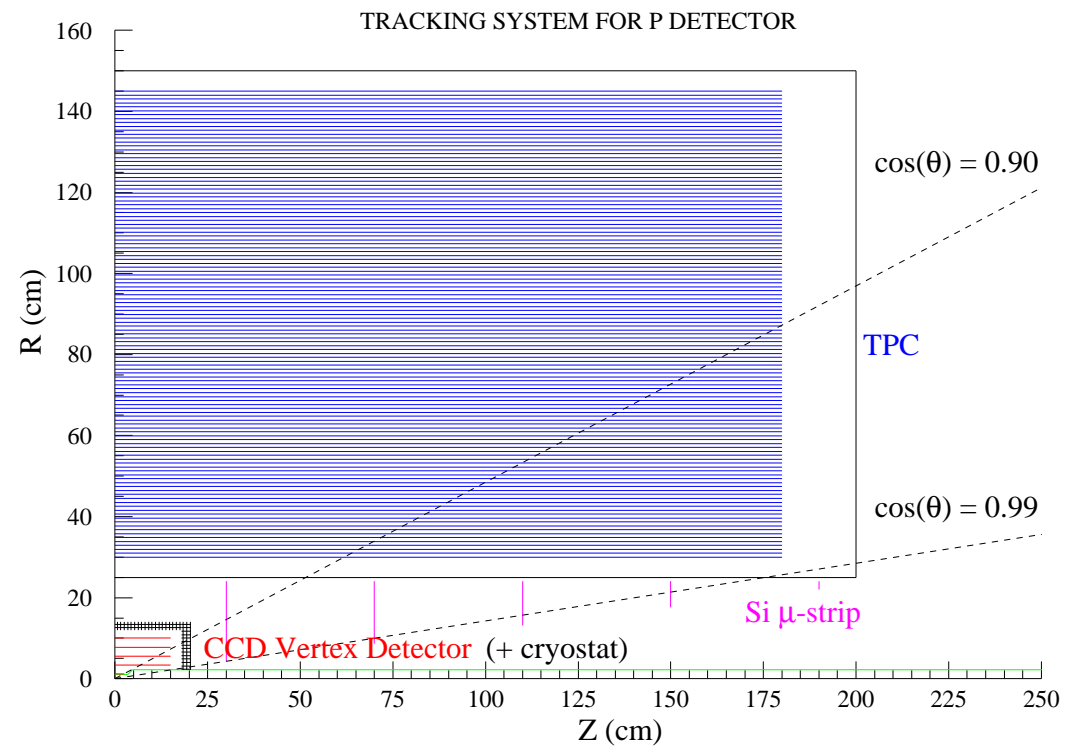

Figure 15.24: Sketch of P detector tracking system. 
radiation lengths. These layers are ganged in pairs, giving 16 readout layers. One layer of $1 \mathrm{~cm}^{2}$ silicon pads is forseen near the EMCal shower maximum. The transverse segmentation of the scintillator is 2 degrees $\times 2$ degrees. It has an inner radius of 150 $\mathrm{cm}$.

The hadronic calorimeter is 65 layers of $8 \mathrm{~mm}$ lead layers with $3 \mathrm{~mm}$ scintillator sampling. These layers are ganged to produce 8 independent samples. The inner radius of the hadronic calorimeter barrel is $215 \mathrm{~cm}$. The entire calorimeter (electromagnetic and hadronic) comprises 3.9 interaction lengths. The transverse segmentation of the scintillator in the hadronic calorimeter is 4 degrees $\times 4$ degrees.

Given its segmentation, the P detector would not be well-suited for using energy flow in jet reconstruction. Unlike $\mathrm{L}$ and $\mathrm{SD}$, the segmentation is organized as towers of constant $\theta$ and $\phi$. For running at the $Z$, excellent jet reconstruction is probably not an important issue. However, at higher energy, for light Higgs or $W$-pair physics, for example, this conclusion is less clear. Jet reconstruction for $\mathrm{P}$ would most likely be carried out using the calorimeter alone (or the tracker alone). Note, however, that the Pb-scintillator ratio, as currently proposed, would not be expected to give good compensation of electromagnetic and hadronic energy depositions. Performance results for jet reconstruction, similar to those given for $\mathrm{L}$ and $\mathrm{SD}$, have not yet been carried out. The results would provide an interesting point of comparison to the energy flow performance of SD.

The muon system consists of 10 layers of $10 \mathrm{~cm}$ iron plates, with $3 \mathrm{~cm}$ gaps for $\mathrm{RPC}$ detectors. Axial strips of $3 \mathrm{~cm}$ pitch measure the $\phi$ coordinate to $1 \mathrm{~cm}$ precision in all 10 gaps, and two gaps (5 and 10) provide azimuthal strips for a measurement of the $z$ coordinate to $1 \mathrm{~cm}$ precision.

\subsection{Cost estimates}

The costs of the subsystems of each of the three detectors have been estimated based on past experience and escalation to FY01. The three cost estimates are shown in Table 15.2. Approximately 40\% contingency is assumed for each of the detectors, resulting in a total cost estimate of $\$ 359$ million for the $\mathrm{L}$ detector, $\$ 326$ million for the SD detector, and $\$ 210$ million for the $\mathrm{P}$ detector.

\section{References}

[1] TESLA Technical Design Report, http://tesla.desy.de/new_pages/TDR_CD/ start.html

[2] T. Matsui, in Physics and Experiments with Future Linear Colliders (LCWS99), ed. E. Fernandez and A. Pacheco. (Univ. Auton. de Barcelona, Bellaterra, 2000).

[3] C. Hensel, LC-DET-2000-001, Table 7 (2000). 
Detectors for the Linear Collider

\begin{tabular}{l|ccc}
\hline \hline Detector & $\mathrm{L}$ & $\mathrm{SD}$ & $\mathrm{P}$ \\
\hline 1.1 Vertex & 4.0 & 4.0 & 4.0 \\
1.2 Tracking & 34.6 & 19.7 & 23.4 \\
1.3 Calorimeter & 48.9 & 60.2 & 40.7 \\
1.3.1 EM & $(28.9)$ & $(50.9)$ & $(23.8)$ \\
1.3.2 Had & $(19.6)$ & $(8.9)$ & $(16.5)$ \\
1.3.3 Lum & $(0.4)$ & $(0.4)$ & $(0.4)$ \\
1.4 Muon & 16.0 & 16.0 & 8.8 \\
1.5 DAQ & 27.4 & 52.2 & 28.4 \\
1.6 Magnet \& support & 110.8 & 75.6 & 30.5 \\
1.7 Installation & 7.3 & 7.4 & 6.8 \\
1.8 Management & 7.4 & 7.7 & 7.4 \\
\hline SUBTOTAL & 256.4 & 242.8 & 150.0 \\
1.9 Contingency & 102.6 & 83.4 & 60.0 \\
\hline Total & 359.0 & 326.2 & 210.0 \\
\hline \hline
\end{tabular}

Table 15.2: $e^{+} e^{-}$linear collider detector budgets (WBS to subsystem level) in M\$ FY01.

[4] The SLD Collaboration, Nucl. Inst. and Meth. A400, 287 (1997).

[5] J. E. Brau and N. Sinev, IEEE Trans. Nucl. Sci. 47, 1898 (2000).

[6] M. N. Frary and D. J. Miller, DESY 92-123A, Vol I, 1992, p. 379; D. Cinabro, in Physics and Experiments with Future Linear Colliders, ed. E. Fernandez and A. Pacheco. (Univ. Auton. de Barcelona, Bellaterra, 2000).

[7] J.-C. Brient, in Physics and Experiments with Future Linear $e^{+} e^{-}$Colliders (LCWS 2000), ed. A. Para. (AIP Conference Proceedings, 2001).

[8] P. Gay, in Physics and Experiments with Future Linear $e^{+} e^{-}$Colliders (LCWS 2000), ed. A. Para. (AIP Conference Proceedings, 2001).

[9] P. Checchia, in Physics and Experiments with Future Linear $e^{+} e^{-}$Colliders (LCWS 2000), ed. A. Para. (AIP Conference Proceedings, 2001).

[10] T. Behnke, S. Bertolucci, R. Heuer, R. Settles, eds. DESY Pub: 2001-011 March 2001 Part III, A Detector for TESLA, p. IV-107.

[11] A. Para, in Physics and Experiments with Future Linear $e^{+} e^{-}$Colliders (LCWS 2000), ed. A. Para. (AIP Conference Proceedings, 2001).

[12] R. J. Wilson, in Physics and Experiments with Future Linear Colliders (LCWS99), ed. E. Fernandez and A. Pacheco. (Univ. Auton. de Barcelona, Bellaterra, 2000).

[13] R. J. Wilson, in Physics and Experiments with Future Linear $e^{+} e^{-}$Colliders (LCWS 2000), ed. A. Para. (AIP Conference Proceedings, 2001). 
[14] H. Yamamoto, in Physics and Experiments with Future Linear Colliders (LCWS99), ed. E. Fernandez and A. Pacheco. (Univ. Auton. de Barcelona, Bellaterra, 2000).

[15] M. Ronan and R. Settles, to appear in Proceedings of the Vienna Instrumentation Conference, Vienna, Austria, Feb 19-23, 2001.

[16] R. Yarema et al., Fermilab-TM-1892, June 1994 (revised Oct. 1996).

[17] Boeing Electronic Systems, 3370 Miraloma Ave, Anaheim, CA 92803; M. D. Petroff et al., Appl. Phys. Lett. 51, 406 (1987).

[18] For a cost breakdown, see http://www-mhp. physics.lsa.umich.edu/ keithr/LC/trackercosts.html.

[19] M. Fauluzzi, Nucl. Inst. and Meth. 383, 35 (1996). 
Questions for Further Study 


\section{Chapter 16 Suggested Study Questions on LC Physics and Experimentation}

\section{Physics issues}

\subsection{Higgs physics}

For further information on this section, consult with: Jack Gunion, Howard Haber, Andreas Kronfeld, Rick van Kooten.

1. Perform a fully simulated study of the precision to which Higgs branching ratios can be determined for $m_{h}=115 \mathrm{GeV}$; for $m_{h}=140 \mathrm{GeV}$; for $m_{h}=200 \mathrm{GeV}$. How do these precisions depend on $\mathrm{CM}$ energy?

2. Is $\gamma \gamma$ needed to measure the total Higgs width, for low mass Higgs?

3. Outline the necessary experimental program to determine the spin/parity of a putative Higgs state.

4. Optimize a program for determination of the Higgs self-couplings. What requirements does this study impose on the dijet invariant mass resolution?

5. What is the utility of positron polarization for Higgs measurements?

6. From knowledge of measured Higgs branching ratios (fermion pairs, $Z Z, W W$, $g g, \gamma \gamma)$, the total width, and the couplings $g_{Z Z h}, g_{W W h}$, what reach is available to detect the presence of the SUSY states $H, A$ ? What is the relative importance of errors in each measurement?

7. To what extent can one measure $\tan \beta$ for the SUSY Higgs from Higgs sector measurements alone? Is it possible to do so in a truly model-independent way for the most general sets of MSSM parameters?

8. How will one disentangle $H^{0}$ and $A^{0}$ in the decoupling limit where the masses are nearly degenerate?

9. Contrast the use of $e^{+} e^{-}$and $e^{-} e^{-}$beams for the $\gamma \gamma \rightarrow h$ measurement. The use of $e^{+} e^{-}$admits numerous physics backgrounds that are absent for $e^{-} e^{-}$. Is it critical to avoid these backgrounds? Can the advantage of $e^{-} e^{-}$over $e^{+} e^{-}$be compensated by higher integrated luminosity? 
10. The dominant backgrounds to $\gamma \gamma \rightarrow h \rightarrow b \bar{b}$ are $\gamma \gamma \rightarrow b \bar{b}(g)$ and $\gamma \gamma \rightarrow c \bar{c}(g)$. The production cross section for $c \bar{c}(g)$ is about 25 times larger than for $b \bar{b}(g)$. The background can be suppressed, first, by improved $b$ tagging, and second, by improved Higgs (two-jet) mass resolution. With this in mind, what is the optimal strategy for isolating the Higgs peak from the background?

11. Contrast the use of $e^{+} e^{-}$and $e^{-} e^{-}$beams, in the same way, for a broadband search for a heavy Higgs $s$-channel resonance in $\gamma \gamma$.

\section{$1.2 \quad$ Supersymmetry}

For further information on this section, consult with: Jonathan Feng, Uriel Nauenberg, Frank Paige, James Wells.

1. Develop a plan for measuring the chargino mass matrix, including mixing, for the most general sets of MSSM parameters.

2. Do the same for the neutralino, stau and stop mixing matrices.

3. Is there a program by which one could, at least in principle, measure all 105 independent MSSM parameters?

4. What can LC measurements tell us, and with what precision, about the nature of the SUSY model and the SUSY breaking mechanism and scale? What can be learned about the scale and physics of grand unification?

5. Evaluate the benefit of positron polarization for SUSY measurements.

6. For what questions of SUSY spectroscopy are $\gamma \gamma, e \gamma$, and $e^{-} e^{-}$beams of special importance?

7. How well can CP-violating effects be studied in supersymmetry? How do these compare and connect to those made in the $B$ factories or $K$ decays?

8. What limits can be set on lepton flavor violation in slepton reactions? Is it possible to measure quark flavor violation effects that are associated with SUSY parameters and independent of CKM mixing?

9. What measurements from the LC would be required to verify the neutralino origin of cosmological dark matter?

10. What information encoded in the SUSY parameters can provide information about the nature of string/M theory? 
Suggested Study Questions on LC Physics and Experimentation

\subsection{New physics at the TeV scale}

For further information on this section, consult with: Tim Barklow, Bogdan Dobrescu, JoAnne Hewett, Slawek Tkaczyk.

1. What precision can eventually be reached on anomalous $W W V, Z Z V$ and $t \bar{t} V$ couplings? What machine parameters are needed?

2. For the broad range of strong coupling models that obey existing precision EW constraints, what are the observable consequences at a $500 \mathrm{GeV}$ LC? At 1000 GeV? At $1500 \mathrm{GeV}$ ? Are there models of strong coupling for which there are no observable consequences at $500 \mathrm{GeV}$ ?

3. Is it possible for models of a strong-coupling Higgs sector to mimic predictions of supersymmetry or extended Higgs models in a way that these models cannot be distinguished at the LHC? What $e^{+} e^{-}$measurements would be most important in these cases?

4. What is the utility of $\gamma \gamma$ or $e^{-} e^{-}$operation for probing the strong coupling models?

5. Develop general classification of models with large extra dimensions.

6. How can measurements at the TeV scale constrain string/M-theory models with string or quantum gravity scales much less than $10^{19} \mathrm{GeV}$ ?

7. Describe the reach of a LC for seeing large extra dimensions as a function of energy and luminosity in various scenarios. To what extent does the higher precision of a $500 \mathrm{GeV}$ LC complement the higher energy reach of the LHC?

8. What is the role of $\gamma \gamma, e \gamma$, and $e^{-} e^{-}$experiments in probing models with extra dimensions?

9. What would be the role of the LC in understanding the nature of cosmological dark matter in models not related to supersymmetry?

10. In what way can LC measurements constrain gravitational effects such as Hawking black hole radiation?

\subsection{Top quark physics}

For further information on this section, consult with: Ulrich Baur, David Gerdes.

1. How well can the top quark width be determined from threshold measurements? A full analysis should include the threshold shape, the top quark momentum distribution, and the forward-backward asymmetry from S-P mixing. Are there additional effects that can contribute to this determination? 
2. Can one determine the top quark Yukawa coupling at the $t \bar{t}$ threshold? With what precision?

3. Can CP violation associated with the top quark be probed at the $t \bar{t}$ threshold?

4. Can a high-precision top quark mass be obtained from continuum $t \bar{t}$ production? Is there an infrared-safe definition of $m_{t}$ that can be applied to this analysis?

5. How well can the top quark Yukawa coupling be determined in $e^{+} e^{-} \rightarrow t \bar{t} h$ ? What backgrounds arise from other top quark production processes $\left(e . g ., e^{+} e^{-} \rightarrow\right.$ $t \bar{t} g)$ ? Are spin correlations derived from kinematic fitting useful in this analysis?

6. How well can one measure the vector and axial $t \bar{t} Z$ couplings?

7. How well can one measure the $t \bar{t} \gamma$ form factors and the top anomalous magnetic moment?

8. How well can one measure the $(V+A)$ decay of the top quark?

9. What ambiguities arise when one fits for more than one anomalous coupling at a time? Can polarization or spin correlation measurements resolve these ambiguities?

\subsection{QCD and two-photon physics}

For further information on this section, consult with: Bruce Schumm, Lynne Orr.

1. What is the precision that can be obtained for $\alpha_{s}$ from $e^{+} e^{-}$annihilation? In particular, can it be definitively demonstrated that detector systematics are less than $\pm 1 \%$ ?

2. What is the precision that can be obtained for $\alpha_{s}$ from measurements on the top quark?

3. Outline the program for obtaining the photon structure functions. What energies of operation are desired, and are special beam conditions required?

4. How can the LC make definitive studies of all-orders BFKL resummation?

\subsection{Precision electroweak measurements}

For further information on this section, consult with: Lawrence Gibbons, Bill Marciano.

1. Evaluate the need for Giga-Z in various scenarios in which there do or do not exist light Higgs particles. 
Suggested Study Questions on LC Physics and Experimentation

2. Evaluate the need for Giga-Z in scenarios in which new light particles from supersymmetry or other new physics are discovered.

3. Are there strategies for further improving the precision for measuring $\sin ^{2} \theta_{w}$ using $Z$-pole observables? How can the various systematics limits described in the text be avoided?

4. Evaluate the precision of $W$ and top quark mass measurements. What special measurements of the accelerator parameters will be needed to achieve this precision?

5. What are the systematic limits on $B$ physics measurements, including CKM parameters and rare $B$ decay rates, at a polarized $Z$ factory?

\section{Accelerator issues}

\section{$2.1 \quad$ Running scenarios}

For further information on this section, consult with: Joel Butler, Paul Grannis, Michael Peskin.

1. What elements should be present in a charge to a future international technical panel established to compare linear collider technical proposals? What emphasis should be given to risk analysis, needed $\mathrm{R} \& \mathrm{D}$, upgradability in energy or luminosity, cost comparison?

2. For a physics-rich scenario (e.g., low mass Higgs and SUSY with observable $\left.\widetilde{\chi}_{1}^{0}, \widetilde{\chi}_{2}^{0}, \widetilde{\chi}_{1}^{+}, \widetilde{t}, \widetilde{\tau}\right)$ outline the desired run plan, giving the required integrated luminosity for all necessary beam energies, beam polarizations, beam particles. What compromises can be envisioned to limit the number of distinct machine parameters without undue effect on the physics results?

3. Do the same for a thinner physics scenario (e.g., with Higgs mass of $180 \mathrm{GeV}$ and no supersymmetry or other new particle observation).

\subsection{Machine configuration}

For further information on this section, consult with: Charles Prescott, Tor Raubenheimer, Andre Turcot.

1. Evaluate an IR scheme with IR1 capable of operation at $E_{C M} \leq 250 \mathrm{GeV}$ and IR2 capable of operation at $E_{C M}<500(1000) \mathrm{GeV}$. Contrast this configuration with one in which two detectors share an IR in push-pull mode. 
2. How important is it that the LEIR be able to operate at energies of $500 \mathrm{GeV}$ or higher?

3. Evaluate the benefits from simultaneous operations at two IRs (with interleaved pulse trains). What are the constraints on the collider design?

4. What are the requirements imposed on the first-phase accelerator design to permit upgrade to multi-TeV energies?

5. What constraints and opportunities are brought by including a free electron laser facility with the NLC? Are there other non-HEP uses of the linear accelerator that could be contemplated?

\subsection{Positron polarization}

For further information on this section, consult with: John Jaros, Steve Mrenna, Mike Woods.

1. Evaluate the need for positron polarization in accomplishing the physics program. What polarization (and error), energy (and error), luminosity are required for the relevant physics topics?

\subsection{Photon collider}

For further information on this section, consult with: Jeff Gronberg, Adam Para, Tom Rizzo, Karl van Bibber.

1. Compile the list of physics topics for which $\gamma \gamma$ operation is essential or desirable.

2. Typically $\gamma \gamma$ luminosity and $e \gamma$ luminosity are comparable at a $\gamma \gamma$ collider. Identify $e \gamma$ processes that might be problematic backgrounds for $\gamma \gamma$ physics analyses.

3. How can a detector be made compatible with both $\gamma \gamma$ and $e^{+} e^{-}$operation?

4. Is it sufficient to provide $\gamma \gamma$ collisions only for $E_{C M}(\gamma \gamma)<400 \mathrm{GeV}$ (i.e., at the low energy IR)?

5. Evaluate the prospects for high-power lasers and the configuration of the $\gamma \gamma$ IR. Is R\&D needed on the most important IR components (e.g., mirrors, masking, beam stability)? 
Suggested Study Questions on LC Physics and Experimentation

\section{$2.5 e^{-} e^{-}$}

For further information on this section, consult with: Jonathan Feng, Clem Heusch.

1. Compile the list of physics topics for which $e^{-} e^{-}$operation is essential or desirable.

\subsection{Fixed Target}

For further information on this section, consult with: Mike Woods.

1. What experiments could be done using the $e^{-}$or $e^{+}$beam of a linear collider for fixed target experiments? For example, can Møller scattering of a fixed target beam be used to obtain $\sin ^{2} \theta_{w}$ with very high precision? Can the spent beams that have passed through the interaction region be used in these experiments?

2. What are the relative advantages of $e^{-} v s . e^{+}$beams?

3. What experiments could be done using the polarized $\gamma$ beams from laser backscattering for fixed target experiments? Can fixed target experiments be done with the spent beams while the collider is operating in $\gamma \gamma$ mode?

\section{Detector issues}

\subsection{Detectors}

For further information on this section, consult with: Jim Brau, Marty Breidenbach, Gene Fisk, Ray Frey, Tom Markiewicz, Keith Riles.

1. What are the physics reasons for wanting exceptional jet energy (mass) resolution? How do signal/backgrounds and sensitivities vary as a function of resolution? Is mass discrimination of $W$ and $Z$ in the dijet decay mode feasible, and necessary?

2. How does energy flow calorimetry resolution depend on such variables as Moliere radius, $\Delta \theta / \Delta \phi$ segmentation, depth segmentation, inner radius, $B$ field, number of radiation lengths in tracker, etc.?

3. What benefits arise from very high-precision tracking (e.g., silicon strip tracker)? What are the limitations imposed by having relatively few samples, and by the associated radiation budget? What minimum radius tracker would be feasible?

4. Evaluate the dependence of physics performance on solenoidal field strength and radius. 
